\title{
Long-Time Asymptotics of Solutions to the Cauchy Problem for the Defocusing Non-Linear Schrödinger Equation with Finite-Density Initial Data. I. Solitonless Sector
}

\author{
A. H. Vartanian* \\ Department of Mathematics \\ Winthrop University \\ Rock Hill, South Carolina 29733 \\ U. S. A.
}

3 December 2001

\begin{abstract}
The methodology of the Riemann-Hilbert (RH) factorisation approach for Lax-pair isospectral deformations is used to derive, in the solitonless sector, the leading-order asymptotics as $t \rightarrow \pm \infty(x / t \sim \mathcal{O}(1))$ of solutions to the Cauchy problem for the defocusing non-linear Schrödinger equation ( $\left.\mathrm{D}_{f} \mathrm{NLSE}\right), \mathrm{i} \partial_{t} u+\partial_{x}^{2} u-2\left(|u|^{2}-1\right) u=0$, with (finite-density) initial data $u(x, 0)={ }_{x \rightarrow \pm \infty} \exp \left(\frac{\mathrm{i}(1 \mp 1) \theta}{2}\right)(1+o(1)), \theta \in[0,2 \pi)$. A limiting case of these asymptotics related to the $\mathrm{RH}$ problem for the Painlevé II equation, or one of its special reductions, is also identified.
\end{abstract}

2000 Mathematics Subject Classification. (Primary) 35Q15, 37K40, 35Q55, 37K15: (Secondary) 30E20, 30E25, 81U40

Abbreviated Title. Asymptotics of the Defocusing NLSE

Key Words. Asymptotics, direct and inverse scattering, reflection coefficient, Riemann-Hilbert problems, singular integral equations

${ }^{*}$ E-mail: vartaniana@arthur.winthrop.edu. Current address: Department of Mathematics, Duke University, Durham, North Carolina 27708, U. S. A., e-mail: arthur@math.duke.edu 


\section{Introduction}

In the optical fibre literature, the mathematical model, in normalised and dimensionless form, describing dark soliton pulse propagation (which consists of a rapid dip in the intensity of a broad pulse of a continuous wave background) in polarisation preserving, single-mode optical fibres in the picosecond time scale is the following non-linear Schrödinger equation (NLSE) 11, 2, 3], i $\partial_{z} q+\partial_{\tau}^{2} q-2 q|q|^{2}=0$, where $q=q(\tau, z)$ is the slowly varying amplitude of the complex field envelope, $z$ is the distance along the fibre length, and $\tau$ is the retarded time measured in a reference frame moving along the fibre at the group velocity, with non-vanishing boundary conditions $q(\tau, z)={ }_{\tau \rightarrow \pm \infty} \varrho \mathrm{e}^{\mathrm{i}\left(\varphi_{\infty}^{ \pm}-2 \varrho^{2} z\right)}$, where $\varrho(>0)$ is the so-called density, and $\varphi_{\infty}^{ \pm}(\in[0,2 \pi))$ are the asymptotic phases. Mapping, isomorphically, the physical variables onto the mathematical variables, $(\tau, z) \mapsto(\widetilde{x}, \widetilde{t})$, setting $q(\widetilde{x}, \widetilde{t}):=\widehat{q}(\widetilde{x}, \widetilde{t}) \mathrm{e}^{-2 \mathrm{i} \varrho^{2} \widetilde{t}}$, scaling according to the rule $\widetilde{t} \rightarrow \varrho^{-2} t, \widetilde{x} \rightarrow \varrho^{-1} x$, and $\widehat{q} \rightarrow \varrho u(x, t) \mathrm{e}^{\mathrm{i} \varphi_{\infty}^{+}}$, and defining $\theta:=\varphi_{\infty}^{-}-\varphi_{\infty}^{+}$, one arrives at considering solutions of the following non-linear evolution equation (NLEE), hereafter referred to as the defocusing non-linear Schrödinger equation $\left(\mathrm{D}_{f} \mathrm{NLSE}\right)$, with finite-density initial data,

$$
\begin{array}{r}
\mathrm{i} \partial_{t} u+\partial_{x}^{2} u-2\left(|u|^{2}-1\right) u=0, \quad(x, t) \in \mathbb{R} \times \mathbb{R}, \\
u(x, 0):=u_{o}(x) \underset{x \rightarrow \pm \infty}{=} \exp \left(\frac{\mathrm{i}(1 \mp 1) \theta}{2}\right)(1+o(1)),
\end{array}
$$

where $u_{o}(x) \in \mathbf{C}^{\infty}(\mathbb{R}), \theta \in[0,2 \pi)$ (see Lemma 2.2), and the $o(1)$ term is to be understood in the sense that, $\forall(k, l) \in \mathbb{Z}_{\geqslant 0} \times \mathbb{Z}_{\geqslant 0},|x|^{k}\left(\frac{\mathrm{d}}{\mathrm{d} x}\right)^{l}\left(u_{o}(x)-\exp \left(\frac{\mathrm{i}(1 \mp 1) \theta}{2}\right)\right)={ }_{x \rightarrow \pm \infty} 0$. Only for initial data satisfying $|x|^{k}\left(\frac{\mathrm{d}}{\mathrm{d} x}\right)^{l}\left(u_{o}(x)-\exp \left(\frac{\mathrm{i}(1 \mp 1) \theta}{2}\right)\right)={ }_{x \rightarrow \pm \infty} 0,(k, l) \in \mathbb{Z}_{\geqslant 0} \times \mathbb{Z}_{\geqslant 0}$, is it true that the closure of the set of reflectionless (soliton) potentials of the $\mathrm{D}_{f} \mathrm{NLSE}$ in the topology of uniform convergence of functions on compact sets of $\mathbb{R}$ (denoted by $\mathcal{B}$ ) remains an invariant set of this model for $t \neq 0$ (a solution of the $\mathrm{D}_{f}$ NLSE with finite density initial data, in the above-defined sense, remains in $\mathcal{B} \forall t \in \mathbb{R}$, and not just for $t=0$ ) 四.

It is instructive to study the asymptotics of solutions to the Cauchy problem for the $\mathrm{D}_{f} \mathrm{NLSE}$ for finite-density initial data having a decomposition of the form $u_{o}(x):=u_{\mathrm{sol}}(x)+$ $u_{\text {rad }}(x), x \in \mathbb{R}$, where $u_{o}(x)$ satisfies the conditions stated heretofore, $u_{\text {sol }}(x)$ is responsible for "generating" the multi- or $N$-dark soliton solution, and $u_{\text {rad }}(x)$ is the "small" non-darksoliton component manifesting as the asymptotically decaying dispersive component of the solution. In fact, this is the principal objective of the present series of works devoted to the asymptotic analysis of solutions to the $\mathrm{D}_{f}$ NLSE for finite-density initial data; in particular, in this work, the case $u_{\text {sol }}(x) \equiv 0$ and $u_{\text {rad }}(x) \not \equiv 0$ is treated, and the case $\left(u_{\text {sol }}(x), u_{\text {rad }}(x)\right) \not \equiv(0,0)$ is presently under study. Another objective of this series of works, which will be pursued elsewhere, is to use the results obtained herein to derive an explicit asymptotic expression for the transfer matrix for an $N$-dark soliton $\mathrm{X}$ junction [5].

It is well-known that, within the framework of the inverse scattering method (ISM) 6, 7, 81, the $\mathrm{D}_{f}$ NLSE is a completely integrable NLEE with an explicit representation as an infinite-dimensional Hamiltonian system [9]. Even though the analysis of NLS-like NLEEs with rapidly decaying, e.g., Schwartz class, initial data on $\mathbb{R}$ has received the vast majority of the attention in the context of direct and inverse spectral treatments, there have been a handful of, in some cases seminal, works devoted exclusively to the direct and inverse scattering analysis of completely integrable NLEEs belonging to the ZS-AKNS class with non-vanishing values of the initial data [10, 11, 12]. As shown in Part 1 of [9], a two-sheeted Riemann surface plays a central role in the direct/inverse spectral formulation associated with the $\mathrm{D}_{f}$ NLSE for finite-density initial data. Other interesting classes of finite-density (or non-vanishing)-type initial data for completely integrable NLEEs, e.g., NLS, derivative and modified NLS, KdV, and sinh/e-Gordon, have also been considered [13, 14, 15, 16, 17, 18]. To the best of the author's knowledge as at the time of the presents, the first to consider the asymptotics of solutions to the $\mathrm{D}_{f}$ NLSE for finite-density initial data were Its et al. [19, 20].

In the framework of the ISM, the asymptotic analysis of solutions to the Cauchy problem for the $\mathrm{D}_{f}$ NLSE with finite-density initial data is divided into two steps: (1) the analysis of the solitonless (continuum) component of the solution; and (2) the inclusion of the 
$N$-dark soliton component via the application of a "dressing" procedure to the solitonless background/component [21, 22]. In this work, stage (1) of the above-mentioned two-step asymptotic paradigm, which is the more technical of the two, is carried out systematically using the methodology of the Riemann-Hilbert (RH) factorisation [23] approach to the ISM [6, 8, 24, 25, 26].

This paper is organized as follows. In Section 2, starting from the Lax-pair isospectral deformation condition associated with the $\mathrm{D}_{f} \mathrm{NLSE}$, all necessary formulae from the direct and inverse scattering analyses associated with the solution of the Cauchy problem for the $\mathrm{D}_{f}$ NLSE with finite-density initial data are derived, the corresponding (matrix) RiemannHilbert problem (RHP) is formulated, and the particular case of this RHP studied asymptotically in this work is stated. In Section 3, a self-contained synopsis of the Beals-Coifman [24 construction for the solution of a matrix RHP on an oriented contour is given, a detailed account of the Deift-Zhou 27] non-linear steepest descent method for the asymptotic analysis of the RHP stated in Section 2 is presented, and the results of this paper are summarised in Theorems 3.1-3.3. In Section 4, as $t \rightarrow+\infty(x / t \sim \mathcal{O}(1))$, the RHP is reformulated as an auxiliary RHP on an augmented contour which is then dissected to produce an equivalent RHP on a truncated contour. In Section 5, it is shown that, to leading order as $t \rightarrow+\infty(x / t \sim \mathcal{O}(1))$, modulo terms that are $\mathcal{O}\left(t^{-1 / 2} \ln t\right)$, the solution of the equivalent RHP on the truncated contour "tends to" the solution of an explicitly solvable model RHP on a contour which consists of the disjoint union of two rotated crosses. In Section 6 , as $t \rightarrow+\infty(x / t \sim \mathcal{O}(1))$, the model $\mathrm{RHP}$ on the disjoint union of the two rotated crosses is reformulated as an asymptotic system of linear singular integral equations which are then solved explicitly to yield the asymptotics of solutions (and related integrals of solutions) to the Cauchy problem for the $\mathrm{D}_{f}$ NLSE. In Section 7 , the above asymptotic paradigm is succinctly reworked for the case when $t \rightarrow-\infty$ $(x / t \sim \mathcal{O}(1))$. The paper concludes with an Appendix.

\section{The Direct/Inverse Scattering Analysis and the Riemann- Hilbert Problem}

The necessary facts from the direct/inverse scattering analysis of the Lax pair (see Proposition 2.1) associated with the $\mathrm{D}_{f}$ NLSE for finite-density initial data are derived, the corresponding RHP is formulated, and the particular case of this RHP which is analysed asymptotically as $t \rightarrow \pm \infty(x / t \sim \mathcal{O}(1))$ in this work is stated. Before proceeding, however, the notation/nomenclature used throughout this work is summarised.

\section{$\underline{\text { Notational Conventions }}$}

(1) $\mathrm{I}=\left(\begin{array}{ll}1 & 0 \\ 0 & 1\end{array}\right)$ is the $2 \times 2$ identity matrix, $\sigma_{1}=\left(\begin{array}{ll}0 & 1 \\ 1 & 0\end{array}\right), \sigma_{2}=\left(\begin{array}{cc}0 & -\mathrm{i} \\ \mathrm{i} & 0\end{array}\right)$, and $\sigma_{3}=\left(\begin{array}{cc}1 & 0 \\ 0 & -1\end{array}\right)$ are the Pauli matrices, $\sigma_{+}=\left(\begin{array}{ll}0 & 1 \\ 0 & 0\end{array}\right)$ and $\sigma_{-}=\left(\begin{array}{ll}0 & 0 \\ 1 & 0\end{array}\right)$ are, respectively, the raising and lowering matrices, $\operatorname{sgn}(z):=+1$ if $z>0,0$ if $z=0$, and -1 if $z<0, \mathbb{R}_{ \pm}:=\{x ; \pm x>0\}$, and $\pm \mathrm{i}:=\exp ( \pm \mathrm{i} \pi / 2)$

(2) for a scalar $\varpi$ and a $2 \times 2$ matrix $\Upsilon, \varpi^{\operatorname{ad}\left(\sigma_{3}\right)} \Upsilon:=\varpi^{\sigma_{3}} \Upsilon \varpi^{-\sigma_{3}}$;

(3) for each segment of an oriented contour $\mathcal{D}$, according to the given orientation, the "+" side is to the left and the "-" side is to the right as one traverses the contour in the direction of orientation, i.e., for a matrix $\mathcal{A}_{i j}(\cdot), i, j \in\{1,2\},\left(\mathcal{A}_{i j}(\cdot)\right)_{ \pm}$denote the non-tangential limits $\left(\mathcal{A}_{i j}(z)\right)_{ \pm}:=\lim _{\substack{z^{\prime} \rightarrow z \\ z^{\prime} \in \pm \text { side of } \mathcal{D}}} \mathcal{A}_{i j}\left(z^{\prime}\right)$

(4) for a matrix $\mathcal{A}_{i j}(\cdot), i, j \in\{1,2\}$, to have boundary values in the $\mathcal{L}^{2}$ sense on an oriented contour $\mathcal{D}$, it is meant that $\lim _{\substack{z^{\prime} \in \pm \text { side of } \mathcal{D} \\ z^{\prime} \rightarrow z}} \int_{\mathcal{D}}\left|\mathcal{A}\left(z^{\prime}\right)-(\mathcal{A}(z))_{ \pm}\right|^{2}|\mathrm{~d} z|=0$, where $|\mathcal{A}(\cdot)|$ denotes the Hilbert-Schmidt norm, $|\mathcal{A}(\cdot)|:=\left(\sum_{i, j=1}^{2} \overline{\mathcal{A}_{i j}(\cdot)} \mathcal{A}_{i j}(\cdot)\right)^{1 / 2}$, with $\overline{(\bullet)}$ denoting complex conjugation of $(\bullet)$, i.e., if, say, $\mathcal{D}=\mathbb{R}$ oriented from $+\infty$ to $-\infty$, then $\mathcal{A}(\cdot)$ has $\mathcal{L}^{2}$ boundary values on $\mathcal{D}$ means that $\lim _{\varepsilon \downarrow 0} \int_{\mathbb{R}}\left|\mathcal{A}(x \mp \mathrm{i} \varepsilon)-(\mathcal{A}(x))_{ \pm}\right|^{2} \mathrm{~d} x=0$; 
(5) for $1 \leqslant p<\infty$ and $\mathcal{D}$ some point set,

$$
\mathcal{L}_{\mathrm{M}_{2}(\mathbb{C})}^{p}(\mathcal{D}):=\left\{f: \mathcal{D} \rightarrow \mathrm{M}(2, \mathbb{C}) ;\|f(\cdot)\|_{\mathcal{L}_{\mathrm{M}_{2}(\mathbb{C})}^{p}(\mathcal{D})}:=\left(\int_{\mathcal{D}}|f(z)|^{p}|\mathrm{~d} z|\right)^{1 / p}<\infty\right\},
$$

and, for $p=\infty$,

$$
\mathcal{L}_{\mathrm{M}_{2}(\mathbb{C})}^{\infty}(\mathcal{D}):=\left\{g: \mathcal{D} \rightarrow \mathrm{M}(2, \mathbb{C}) ;\|g(\cdot)\|_{\mathcal{L}_{\mathrm{M}_{2}(\mathbb{C})}^{\infty}(\mathcal{D})}:=\max _{i, j \in\{1,2\}} \sup _{z \in \mathcal{D}}\left|g_{i j}(z)\right|<\infty\right\}
$$

(6) for $D$ an unbounded domain of $\mathbb{R}, \mathcal{S}_{\mathbb{C}}(D)$ (respectively $\mathcal{S}_{\mathrm{M}_{2}(\mathbb{C})}(D)$ ) denotes the Schwartz space on $D$, namely, the space of all infinitely continuously differentiable (smooth) $\mathbb{C}$ valued (respectively $\mathrm{M}(2, \mathbb{C})$-valued) functions which together with all their derivatives tend to zero faster than any positive power of $|\bullet|^{-1}$ as $|\bullet| \rightarrow \infty$, that is, $\mathcal{S}_{\mathbb{C}}(D):=\mathbf{C}^{\infty}(D) \cap\left\{f: D \rightarrow \mathbb{C} ;\|f(\cdot)\|_{k, l}:=\sup _{x \in \mathbb{R}}\left|x^{k}\left(\frac{\mathrm{d}}{\mathrm{d} x}\right)^{l} f(x)\right|<\infty \forall(k, l) \in \mathbb{Z}_{\geqslant 0} \times\right.$ $\left.\mathbb{Z}_{\geqslant 0}\right\}$ and $\mathcal{S}_{\mathrm{M}_{2}(\mathbb{C})}(D):=\left\{F: D \rightarrow \mathrm{M}(2, \mathbb{C}) ; F_{i j}(\cdot) \in \mathbf{C}^{\infty}(D), i, j \in\{1,2\}\right\} \cap\{G: D \rightarrow$ $\left.\mathrm{M}(2, \mathbb{C}) ;|| G_{i j}(\cdot) \|_{k, l}:=\sup _{x \in \mathbb{R}}\left|x^{k}\left(\frac{\mathrm{d}}{\mathrm{d} x}\right)^{l} G_{i j}(x)\right|<\infty \forall(k, l) \in \mathbb{Z}_{\geqslant 0} \times \mathbb{Z}_{\geqslant 0}, i, j \in\{1,2\}\right\}$, and $\mathbf{C}_{0}^{\infty}(*):=\cap_{k=0}^{\infty} \mathbf{C}_{0}^{k}(*)$;

(7) for $D$ an unbounded domain of $\mathbb{R}, \mathcal{S}_{\mathbb{C}}^{1}(D):=\mathcal{S}_{\mathbb{C}}(D) \cap\left\{h(z) ;\left\|h(\cdot)\left|\|_{\mathcal{L}^{\infty}(D)}:=\sup _{z \in D}\right| h(z) \mid\right.\right.$ $<1\}$;

(8) $\left\|\left.\mathcal{F}(\cdot)\right|_{\cap_{p \in \mathfrak{J}} \mathcal{L}_{\mathrm{M}_{2}(\mathbb{C})}^{p}(*)}:=\sum_{p \in \mathfrak{J}}\right\| \mathcal{F}(\cdot) \|_{\mathcal{L}_{\mathrm{M}_{2}(\mathbb{C})}^{p}(*)}$, where $\mathfrak{J}$ is a finite index set;

(9) for $(\mu, \widetilde{\nu}) \in \mathbb{R} \times \mathbb{R}$, the function $(\bullet-\mu)^{\mathrm{i} \widetilde{\nu}}: \mathbb{C} \backslash(-\infty, \mu) \rightarrow \mathbb{C}: \bullet \mapsto \mathrm{e}^{\mathrm{i} \widetilde{\nu} \ln (\bullet-\mu)}$, with the branch cut taken along $(-\infty, \mu)$ and the principal branch of the logarithm chosen, $\ln (\bullet-\mu):=\ln |\bullet-\mu|+\mathrm{i} \arg (\bullet-\mu), \arg (\bullet-\mu) \in(-\pi, \pi)$;

(10) a contour, $\mathcal{D}$, say, which is the finite union of piecewise smooth simple closed curves, is said to be orientable if its complement, $\mathbb{C} \backslash \mathcal{D}$, can always be divided into two, possibly disconnected, disjoint open sets $\mho^{+}$and $\mho^{-}$, either of which has finitely many components, such that $\mathcal{D}$ admits an orientation so that it can either be viewed as a positively oriented boundary $\mathcal{D}^{+}$for $\mho^{+}$or as a negatively oriented boundary $\mathcal{D}^{-}$for $\mho^{-}[28]$, i.e., the (possibly disconnected ) components of $\mathbb{C} \backslash \mathcal{D}$ can be coloured by + or - in such a way that the + regions do not share boundary with the - regions, except, possibly, at finitely many points [29].

Proposition 2.1 ( of the following linear system (Lax-pair), for arbitrary $\zeta \in \mathbb{C}$,

$$
\partial_{x} \Psi(x, t ; \zeta)=\mathcal{U}(x, t ; \zeta) \Psi(x, t ; \zeta), \quad \partial_{t} \Psi(x, t ; \zeta)=\mathcal{V}(x, t ; \zeta) \Psi(x, t ; \zeta)
$$

where

$$
\begin{aligned}
& \mathcal{U}(x, t ; \zeta)=-\mathrm{i} \lambda(\zeta) \sigma_{3}+\left(\begin{array}{cc}
0 & u \\
\bar{u} & 0
\end{array}\right), \\
& \mathcal{V}(x, t ; \zeta)=-2 \mathrm{i}(\lambda(\zeta))^{2} \sigma_{3}+2 \lambda(\zeta)\left(\begin{array}{cc}
0 & u \\
\bar{u} & 0
\end{array}\right)-\mathrm{i}\left(\begin{array}{cc}
u \bar{u}-1 & \partial_{x} u \\
\partial_{x} \bar{u} & u \bar{u}-1
\end{array}\right) \sigma_{3},
\end{aligned}
$$

and $\lambda(\zeta):=\frac{1}{2}\left(\zeta+\frac{1}{\zeta}\right)$, with $\operatorname{tr}(\mathcal{U}(x, t ; \zeta))=\operatorname{tr}(\mathcal{V}(x, t ; \zeta))=0$, is that $u=u(x, t)$ satisfies the $\mathrm{D}_{f} \mathrm{NLSE}$.

Proof. Invoking the isospectral deformation condition, $\partial_{*} \zeta=0, * \in\{x, t\}$, one shows that the $\mathrm{D}_{f} \mathrm{NLSE}$ is the Frobenius compatibility, or zero-curvature, condition for system (2), $\partial_{t} \mathcal{U}(x, t ; \zeta)-\partial_{x} \mathcal{V}(x, t ; \zeta)+[\mathcal{U}(x, t ; \zeta), \mathcal{V}(x, t ; \zeta)]=\left(\begin{array}{cc}0 & 0 \\ 0 & 0\end{array}\right), \zeta \in \mathbb{C}$, where $[\mathfrak{A}, \mathfrak{B}]:=\mathfrak{A} \mathfrak{B}-\mathfrak{B A}$ is the matrix commutator. 
Proposition 2.2. Let $u(x, t)$ be a solution of the $\mathrm{D}_{f} \mathrm{NLSE}$ and $\Psi(x, t ; \zeta)$ the corresponding solution of system $(2)$. Then $\Psi(x, t ; \zeta):=\Psi(x, t ; \zeta) \mathcal{Q}(\zeta)$, with $\mathcal{Q}(\zeta) \in \mathrm{M}(2, \mathbb{C})$, is also a solution of system (2).

Proof. Let $u(x, t)$ be a solution of the $\mathrm{D}_{f}$ NLSE and $\Psi(x, t ; \zeta)$ the corresponding solution of system (2). Multiply system (2) on the right by $\mathcal{Q} \in \mathrm{M}(2, \mathbb{C})$ and define $\Psi(x, t ; \zeta)$ as in the Proposition.

As a consequence of Proposition $2.2, \Psi(x, t ; \zeta)$ becomes the principal object of study. The ISM analysis for the $\mathrm{D}_{f} \mathrm{NLSE}$ is based on the direct scattering problem for the (self-adjoint) operator (cf. Proposition 2.1) $\mathcal{O}^{\mathcal{D}}:=\mathrm{i} \sigma_{3} \partial_{x}-\left(\begin{array}{cc}0 & \mathrm{i} u_{o}(x) \\ \mathrm{i} u_{o}(x) & 0\end{array}\right)-\operatorname{diag}\left(\frac{1}{2}\left(\zeta+\frac{1}{\zeta}\right)\right)$, where $u(x, 0):=$ $u_{o}(x)$ satisfies $u_{o}(x)={ }_{x \rightarrow \pm \infty} u_{o}( \pm \infty)(1+o(1))$, with $u_{o}( \pm \infty):=\exp \left(\frac{\mathrm{i}(1 \mp 1) \theta}{2}\right), \theta \in[0,2 \pi)$ (see Lemma 2.2), $u_{o}(x) \in \mathbf{C}^{\infty}(\mathbb{R})$, and $u_{o}(x)-u_{o}( \pm \infty) \in \mathcal{S}_{\mathbb{C}}\left(\mathbb{R}_{ \pm}\right)$.

Proposition 2.3. Let $u(x, t)$ be a solution of the $\mathrm{D}_{f} \operatorname{NLSE}$ and $\Psi(x, t ; \zeta)$ the corresponding solution of system (2) defined in Proposition 2.2. Then $\Psi(x, t ; \zeta)$ satisfies the symmetry reductions $\sigma_{1} \overline{\Psi(x, t ; \bar{\zeta})} \sigma_{1}=\Psi(x, t ; \zeta) \mathrm{M}_{1}(\zeta)$ and $\Psi\left(x, t ; \frac{1}{\zeta}\right)=\Psi(x, t ; \zeta) \mathrm{M}_{2}(\zeta)$, where $\mathrm{M}_{i}(\zeta) \in$ $\mathrm{GL}(2, \mathbb{C}), i \in\{1,2\}$.

Proof. For the $\zeta \rightarrow \bar{\zeta}$ (respectively $\zeta \rightarrow \frac{1}{\zeta}$ ) involution, one shows that $\partial_{x}\left(\sigma_{1} \overline{\Psi(x, t ; \bar{\zeta})} \sigma_{1}\right)=$ $\mathcal{U}(x, t ; \zeta) \sigma_{1} \overline{\Psi(x, t ; \bar{\zeta})} \sigma_{1}$ and $\partial_{t}\left(\sigma_{1} \overline{\Psi(x, t ; \bar{\zeta})} \sigma_{1}\right)=\mathcal{V}(x, t ; \zeta) \sigma_{1} \overline{\Psi(x, t ; \bar{\zeta})} \sigma_{1}$ (respectively $\partial_{x} \Psi(x$, $\left.t ; \frac{1}{\zeta}\right)=\mathcal{U}(x, t ; \zeta) \Psi\left(x, t ; \frac{1}{\zeta}\right)$ and $\left.\partial_{t} \Psi\left(x, t ; \frac{1}{\zeta}\right)=\mathcal{V}(x, t ; \zeta) \Psi\left(x, t ; \frac{1}{\zeta}\right)\right)$; hence, $\exists \mathrm{M}_{1}(\zeta) \in \mathrm{GL}(2, \mathbb{C})$ (respectively $\left.\mathrm{M}_{2}(\zeta) \in \mathrm{GL}(2, \mathbb{C})\right)$ such that $\sigma_{1} \overline{\Psi(x, t ; \bar{\zeta})} \sigma_{1}=\Psi(x, t ; \zeta) \mathrm{M}_{1}(\zeta)$ (respectively $\Psi\left(x, t ; \frac{1}{\zeta}\right)$ $\left.=\Psi(x, t ; \zeta) \mathrm{M}_{2}(\zeta)\right)$ solves system $(2)$.

Definition 2.1. Let $u(x, t)$ be a solution of the $\mathrm{D}_{f} \mathrm{NLSE}$ with $u(x, 0):=u_{o}(x)={ }_{x \rightarrow \pm \infty}$ $u_{o}( \pm \infty)(1+o(1))$, where $u_{o}( \pm \infty):=\exp \left(\frac{\mathrm{i}(1 \mp 1) \theta}{2}\right), \theta \in[0,2 \pi)$ (see Lemma 2.2$), u_{o}(x) \in \mathbf{C}^{\infty}(\mathbb{R})$, and $u_{o}(x)-u_{o}( \pm \infty) \in \mathcal{S}_{\mathbb{C}}\left(\mathbb{R}_{ \pm}\right)$. Define the $\mathrm{M}(2, \mathbb{C})$-valued functions $\Psi^{ \pm}(x, 0 ; \zeta)$ as the (Jost) solutions of the first equation of system $(2), \mathcal{O}^{\mathcal{D}} \Psi^{ \pm}(x, 0 ; \zeta)=\left(\begin{array}{cc}0 & 0 \\ 0 & 0\end{array}\right)$, with the following asymptotics,

$$
\Psi^{ \pm}(x, 0 ; \zeta) \underset{x \rightarrow \pm \infty}{=}\left(\mathrm{e}^{\frac{\mathrm{i}(1 \mp 1) \theta}{4} \sigma_{3}}\left(\begin{array}{cc}
1 & -\mathrm{i} \zeta^{-1} \\
\mathrm{i} \zeta^{-1} & 1
\end{array}\right)+o(1)\right) \mathrm{e}^{-\mathrm{i} k(\zeta) x \sigma_{3}}
$$

where $k(\zeta):=\frac{1}{2}\left(\zeta-\frac{1}{\zeta}\right)$

Corollary 2.1. $\sigma_{1} \overline{\Psi(x, t ; \bar{\zeta})} \sigma_{1}=\Psi(x, t ; \zeta)$ and $\Psi\left(x, t ; \frac{1}{\zeta}\right)=\zeta \Psi(x, t ; \zeta) \sigma_{2}$.

Proof. Since, from Definition $2.1, \Psi^{ \pm}(x, 0 ; \zeta)$ satisfy $\mathcal{O}^{\mathcal{D}} \Psi^{ \pm}(x, 0 ; \zeta)=\left(\begin{array}{cc}0 & 0 \\ 0 & 0\end{array}\right)$, and, as a consequence of Proposition 2.3, $\sigma_{1} \overline{\Psi^{ \pm}(x, 0 ; \bar{\zeta})} \sigma_{1}=\Psi^{ \pm}(x, 0 ; \zeta) \mathrm{M}_{1}(\zeta)$ (respectively $\Psi^{ \pm}\left(x, 0 ; \frac{1}{\zeta}\right)=$ $\left.\Psi^{ \pm}(x, 0 ; \zeta) \mathrm{M}_{2}(\zeta)\right)$, one uses the asymptotics for $\Psi^{ \pm}(x, 0 ; \zeta)$ given in Definition 2.1 and the fact that $\overline{k(\bar{\zeta})}=k(\zeta)$ (respectively $k\left(\frac{1}{\zeta}\right)=-k(\zeta)$ ) to deduce that $\mathrm{M}_{1}(\zeta)=\mathrm{I}$ (respectively $\mathrm{M}_{2}(\zeta)=$ $\left.\zeta \sigma_{2}\right)$.

Proposition 2.4. Set $\Psi^{ \pm}(x, 0 ; \zeta):=\left(\begin{array}{l}\Psi_{11}^{ \pm}(\zeta) \Psi_{12}^{ \pm}(\zeta) \\ \Psi_{21}^{ \pm}(\zeta) \Psi_{22}^{ \pm}(\zeta)\end{array}\right)$. Then $\left(\begin{array}{l}\Psi_{12}^{+}(\zeta) \\ \Psi_{22}^{+}(\zeta)\end{array}\right)$ and $\left(\begin{array}{l}\Psi_{11}^{-}(\zeta) \\ \Psi_{21}^{-}(\zeta)\end{array}\right)$ have analytic continuation to $\mathbb{C}_{+}$(respectively $\left(\begin{array}{l}\Psi_{11}^{+}(\zeta) \\ \Psi_{21}^{+}(\zeta)\end{array}\right)$ and $\left(\begin{array}{l}\Psi_{12}^{-}(\zeta) \\ \Psi_{22}^{-}(\zeta)\end{array}\right)$ have analytic continuation to $\left.\mathbb{C}_{-}\right)$, the monodromy (scattering) matrix, $\mathrm{T}(\zeta)$, is defined by $\Psi^{-}(x, 0 ; \zeta):=\Psi^{+}(x, 0 ; \zeta) \mathrm{T}(\zeta)$,

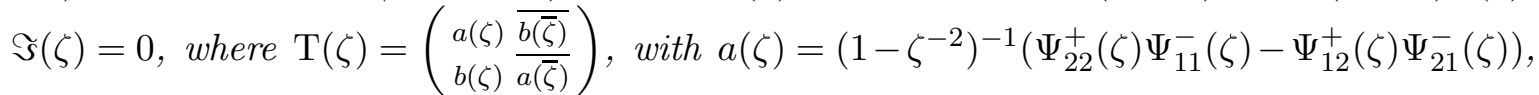
$b(\zeta)=\left(1-\zeta^{-2}\right)^{-1}\left(\overline{\Psi_{22}^{+}(\bar{\zeta})} \Psi_{21}^{-}(\zeta)-\overline{\Psi_{12}^{+}(\bar{\zeta})} \Psi_{11}^{-}(\zeta)\right),|a(\zeta)|^{2}-|b(\zeta)|^{2}=1, a\left(\frac{1}{\zeta}\right)=\overline{a(\bar{\zeta})}, b\left(\frac{1}{\zeta}\right)=-\overline{b(\bar{\zeta})}$, and $\left.\operatorname{det}\left(\Psi^{ \pm}(x, 0 ; \zeta)\right)\right|_{\zeta= \pm 1}=0$. 
Proof. The analytic continuation of the respective columns of $\Psi^{ \pm}(x, 0 ; \zeta)$ to $\mathbb{C}_{ \pm}$follows from Definition 2.1. Introduce the monodromy matrix according to $\Psi^{+}(x, 0 ; \zeta) \mathrm{T}(\zeta)=$ $\Psi^{-}(x, 0 ; \zeta), \Im(\zeta)=0$, where $\mathrm{T}(\zeta)=\left(\begin{array}{c}a(\zeta) \widetilde{b}(\zeta) \\ b(\zeta)\end{array}\right)$. From the $\sigma_{1}$ symmetry reduction $\sigma_{1} \overline{\Psi^{ \pm}(x, 0 ; \bar{\zeta})}$ - $\sigma_{1}=\Psi^{ \pm}(x, 0 ; \zeta)$, it follows that $\widetilde{a}(\zeta)=\overline{a(\bar{\zeta})}$ and $\widetilde{b}(\zeta)=\overline{b(\bar{\zeta})}$; hence, the expression for $\mathrm{T}(\zeta)$ given in the Proposition. Since $\left(\Psi^{+}(x, 0 ; \zeta)\right)^{-1} \Psi^{-}(x, 0 ; \zeta)=\mathrm{T}(\zeta)=\left(\begin{array}{l}a(\zeta) \\ b(\zeta) \frac{\overline{b(\bar{\zeta})}}{a(\bar{\zeta})}\end{array}\right)$ and $\operatorname{det}\left(\Psi^{+}(x, 0 ; \zeta)\right)=\operatorname{det}\left(\Psi^{-}(x, 0 ; \zeta)\right)=1-\zeta^{-2}$, namely, $\operatorname{det}(\mathrm{T}(\zeta))=1$, one deduces the expressions for $a(\zeta)$ and $b(\zeta)$ given in the Proposition, and, using the unimodularity of $\mathrm{T}(\zeta)$, one deduces that $a(\zeta) \overline{a(\bar{\zeta})}-b(\zeta) \overline{b(\bar{\zeta})}=1, \Im(\zeta)=0$. Using the $\sigma_{2}$ symmetry reduction, $\Psi^{ \pm}\left(x, 0 ; \frac{1}{\zeta}\right)=\zeta \Psi^{ \pm}(x, 0 ; \zeta) \sigma_{2}$, and the expression for $\mathrm{T}(\zeta)$ given in the Proposition, one shows that $a\left(\frac{1}{\zeta}\right)=\overline{a(\bar{\zeta})}$ and $b\left(\frac{1}{\zeta}\right)=-\overline{b(\bar{\zeta})}$ : finally, since $\operatorname{det}\left(\Psi^{ \pm}(x, 0 ; \zeta)\right)=1-\zeta^{-2}$, setting $\zeta= \pm 1$, the degeneracy of $\Psi^{ \pm}(x, 0 ; \zeta)$ at $\zeta= \pm 1$ follows.

Corollary 2.2. Let the reflection coefficient associated with the direct scattering problem for the operator $\mathcal{O}^{\mathcal{D}}$ be defined by $r(\zeta):=\frac{b(\zeta)}{a(\zeta)}$. Then $r\left(\frac{1}{\zeta}\right)=-\overline{r(\bar{\zeta})}$.

Proof. The relation $r\left(\frac{1}{\zeta}\right)=-\overline{r(\bar{\zeta})}$ is an immediate consequence of the definition of $r(\zeta)$ given in the Corollary and the properties of $a(\zeta)$ and $b(\zeta)$ given in Proposition 2.4.

Remark 2.1. Note that, from Proposition 2.4, even though $a(\zeta)$ (respectively $a^{*}(\zeta):=\overline{a(\bar{\zeta})}$ ) has an analytic continuation off $\Im(\zeta)=0$ to $\mathbb{C}_{+}$(respectively $\mathbb{C}_{-}$) and is continuous on $\overline{\mathbb{C}}_{+}$ (respectively $\overline{\mathbb{C}}_{-}$), in general, $b(\zeta)$ does not have an analytic continuation off $\Im(\zeta)=0$ : in this work, $b(\zeta)$ has an analytic continuation to (compact subsets of) $\{\zeta ;|\zeta| \leqslant 1\}$; in particular, to rays of the form $r_{n} \mathrm{e}^{ \pm \mathrm{i} \phi_{n}}, n \in\{1,2, \ldots, N\}$, where $\left(r_{n}, \phi_{n}\right) \in[0,1] \times(0, \pi)$.

Lemma 2.1. Let $u(x, t)$ be the solution of the Cauchy problem for the $\mathrm{D}_{f} \mathrm{NLSE}$ with finitedensity initial data and $\Psi^{ \pm}(x, 0 ; \zeta)$ the corresponding (Jost) solutions of $\mathcal{O}^{\mathcal{D}} \Psi^{ \pm}(x, 0 ; \zeta)=\left(\begin{array}{cc}0 & 0 \\ 0 & 0\end{array}\right)$ given in Definition 2.1. Then $\Psi^{ \pm}(x, 0 ; \zeta)$ have the following asymptotics:

$$
\begin{aligned}
& \Psi^{-}(x, 0 ; \zeta) \underset{\zeta \rightarrow \infty}{=} \mathrm{e}^{\frac{\mathrm{i} \theta}{2} \sigma_{3}}\left(\mathrm{I}+\frac{1}{\zeta}\left(\begin{array}{cc}
\mathrm{i} \int_{-\infty}^{x} \frac{\left(\left|u_{o}(\xi)\right|^{2}-1\right) \mathrm{d} \xi}{\mathrm{i} \overline{u_{o}(x)}} \mathrm{e}^{\mathrm{i} \theta} & -\mathrm{i} \int_{o}(x) \mathrm{e}^{-\mathrm{i} \theta} \\
\Psi^{+}\left(\left|u_{o}(\xi)\right|^{2}-1\right) \mathrm{d} \xi
\end{array}\right)+\mathcal{O}\left(\zeta^{-2}\right)\right) \mathrm{e}^{-\mathrm{i} k(\zeta) x \sigma_{3}}, \\
& \Psi^{-}(x, 0 ; \zeta) \underset{\zeta \rightarrow \infty}{=}\left(\mathrm{I}+\frac{1}{\zeta}\left(\begin{array}{cc}
\mathrm{i} \int_{+\infty}^{x}\left(\left|u_{o}(\xi)\right|^{2}-1\right) \mathrm{d} \xi & -\mathrm{i} u_{o}(x) \\
\overline{\mathrm{i} u_{o}(x)} & -\mathrm{i} \int_{+\infty}^{x}\left(\left|u_{o}(\xi)\right|^{2}-1\right) \mathrm{d} \xi
\end{array}\right)+\mathcal{O}\left(\zeta^{-2}\right)\right) \mathrm{e}^{-\mathrm{i} k(\zeta) x \sigma_{3}}, \\
& \left.\Psi_{\zeta} \sigma_{2} \mathrm{e}^{-\frac{\mathrm{i} \theta}{2} \sigma_{3}}+\mathcal{O}(1)\right) \mathrm{e}^{-\mathrm{i} k(\zeta) x \sigma_{3}}, \quad \Psi^{+}(x, 0 ; \zeta) \underset{\zeta \rightarrow 0}{=}\left(\frac{1}{\zeta} \sigma_{2}+\mathcal{O}(1)\right) \mathrm{e}^{-\mathrm{i} k(\zeta) x \sigma_{3}}
\end{aligned}
$$

Proof. From the asymptotics of the (Jost) solutions of $\mathcal{O}^{\mathcal{D}} \Psi^{ \pm}(x, 0 ; \zeta)=\left(\begin{array}{ll}0 & 0 \\ 0 & 0\end{array}\right)$ given in Definition 2.1, the $\sigma_{1}$ symmetry reduction (Corollary 2.1), and a Volterra-type integral rep-

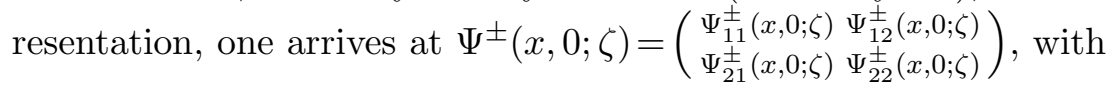

$$
\begin{aligned}
& \Psi_{11}^{-}(x, 0 ; \zeta)=\overline{\Psi_{22}^{-}(x, 0 ; \bar{\zeta})}=\exp \left(\frac{\mathrm{i} \theta}{2}\right) \exp \left(\mathrm{i}\left(\int_{-\infty}^{x} \mathcal{S}(\xi ; \zeta) \mathrm{d} \xi-k(\zeta) x\right)\right) \\
& \Psi_{21}^{-}(x, 0 ; \zeta)=\overline{\Psi_{12}^{-}(x, 0 ; \bar{\zeta})}=\exp \left(-\frac{\mathrm{i} \theta}{2}\right) \mathcal{A}(x ; \zeta) \exp \left(\mathrm{i}\left(\int_{-\infty}^{x} \mathcal{S}(\xi ; \zeta) \mathrm{d} \xi-k(\zeta) x\right)\right) \\
& \Psi_{12}^{+}(x, 0 ; \zeta)=\overline{\Psi_{21}^{+}(x, 0 ; \bar{\zeta})}=\mathcal{B}(x ; \zeta) \exp \left(\mathrm{i}\left(\int_{+\infty}^{x} \mathcal{T}(\xi ; \zeta) \mathrm{d} \xi+k(\zeta) x\right)\right) \\
& \Psi_{22}^{+}(x, 0 ; \zeta)=\overline{\Psi_{11}^{+}(x, 0 ; \bar{\zeta})}=\exp \left(\mathrm{i}\left(\int_{+\infty}^{x} \mathcal{T}(\xi ; \zeta) \mathrm{d} \xi+k(\zeta) x\right)\right)
\end{aligned}
$$

where, in the neighbourhood of the singular points, namely, the origin $(\zeta=0)$ and the point at infinity $(\zeta=\infty), \mathcal{A}(x ; \zeta), \mathcal{B}(x ; \zeta), \mathcal{S}(x ; \zeta)$ and $\mathcal{T}(x ; \zeta)$ have the asymptotic expansions given 
below. Since $\Psi^{ \pm}(x, 0 ; \zeta)$ are the solutions of $\mathcal{O}^{\mathcal{D}} \Psi^{ \pm}(x, 0 ; \zeta)=\left(\begin{array}{ll}0 & 0 \\ 0 & 0\end{array}\right)$ with the asymptotics given in Definition 2.1, using the matrix representations for $\Psi^{ \pm}(x, 0 ; \zeta)$ given above, one arrives at the following system of equations (as well as their complex conjugates):

$$
\begin{gathered}
\mathcal{A}(x ; \zeta)\left(-\frac{\mathrm{i}}{2}\left(\zeta+\frac{1}{\zeta}\right)+u_{o}(x) \mathrm{e}^{-\mathrm{i} \theta} \mathcal{A}(x ; \zeta)\right)+\partial_{x} \mathcal{A}(x ; \zeta)=\frac{\mathrm{i}}{2}\left(\zeta+\frac{1}{\zeta}\right) \mathcal{A}(x ; \zeta)+\overline{u_{o}(x)} \mathrm{e}^{\mathrm{i} \theta}, \\
\mathcal{B}(x ; \zeta)\left(\frac{\mathrm{i}}{2}\left(\zeta+\frac{1}{\zeta}\right)+\overline{u_{o}(x)} \mathcal{B}(x ; \zeta)\right)+\partial_{x} \mathcal{B}(x ; \zeta)=-\frac{\mathrm{i}}{2}\left(\zeta+\frac{1}{\zeta}\right) \mathcal{B}(x ; \zeta)+u_{o}(x), \\
\mathrm{i} \mathcal{S}(x ; \zeta)=-\frac{\mathrm{i}}{\zeta}+u_{o}(x) \mathrm{e}^{-\mathrm{i} \theta} \mathcal{A}(x ; \zeta), \quad \mathrm{i} \mathcal{T}(x ; \zeta)=\frac{\mathrm{i}}{\zeta}+\overline{u_{o}(x)} \mathcal{B}(x ; \zeta) .
\end{gathered}
$$

In the neighbourhood of $\zeta=\infty$, one has the following (formal) asymptotic expansions (see Theorem 2.9 in [29]: Note, $v(x)$ in Eq. (2.10) of [29] should be changed to $v(z)$ ), $\mathcal{A}(x ; \zeta)=\sum_{n=1}^{\infty} \widetilde{I}_{n}^{a}[u, \bar{u}] \zeta^{-n}+\mathcal{O}\left(|\zeta|^{-\infty}\right), \mathcal{B}(x ; \zeta)=\sum_{n=1}^{\infty} \widetilde{I}_{n}^{b}[u, \bar{u}] \zeta^{-n}+\mathcal{O}\left(|\zeta|^{-\infty}\right), \mathcal{S}(x ; \zeta)=$ $\sum_{n=1}^{\infty} \widetilde{I}_{n}^{s}[u, \bar{u}] \zeta^{-n}+\mathcal{O}\left(|\zeta|^{-\infty}\right)$, and $\mathcal{T}(x ; \zeta)=\sum_{n=1}^{\infty} \widetilde{I}_{n}^{t}[u, \bar{u}] \zeta^{-n}+\mathcal{O}\left(|\zeta|^{-\infty}\right)$, where $\widetilde{I}_{n}^{\star}[u, \bar{u}]:=$ $\star^{\infty}(x), \star \in\{a, b, s, t\}$, are functionals of $u_{o}(x)$ and $\overline{u_{o}(x)}$, and, in the neighbourhood of $\zeta=0$, one has the following (formal) asymptotic expansions, $\mathcal{A}(x ; \zeta)=\sum_{n=-1}^{\infty} \widehat{I}_{n}^{a}[u, \bar{u}] \zeta^{n}+\mathcal{O}\left(|\zeta|^{+\infty}\right)$, $\widehat{I}_{-1}^{a}[u, \bar{u}] \not \equiv 0, \mathcal{B}(x ; \zeta)=\sum_{n=-1}^{\infty} \widehat{I}_{n}^{b}[u, \bar{u}] \zeta^{n}+\mathcal{O}\left(|\zeta|^{+\infty}\right), \widehat{I}_{-1}^{b}[u, \bar{u}] \not \equiv 0, \mathcal{S}(x ; \zeta)=\sum_{n=-1}^{\infty} \widehat{I}_{n}^{s}[u, \bar{u}] \zeta^{n}+$ $\mathcal{O}\left(|\zeta|^{+\infty}\right)$, and $\mathcal{T}(x ; \zeta)=\sum_{n=-1}^{\infty} \widehat{I}_{n}^{t}[u, \bar{u}] \zeta^{n}+\mathcal{O}\left(|\zeta|^{+\infty}\right)$, where $\widehat{I}_{n}^{\star}[u, \bar{u}]:=\star^{0}(x)$ are functionals of $u_{o}(x)$ and $\overline{u_{o}(x)}$. Substituting the above asymptotic expansions as $\zeta \rightarrow \infty$ into system (L2.1a), one arrives at (for the first few-leading-terms), with $d_{x}^{n}:=\left(\frac{\mathrm{d}}{\mathrm{d} x}\right)^{n}$,

$$
\begin{aligned}
& \mathcal{O}(1):-\mathrm{i} a_{1}^{\infty}(x)=\overline{u_{o}(x)} \mathrm{e}^{\mathrm{i} \theta} \Rightarrow a_{1}^{\infty}(x)=\overline{\mathrm{i} u u_{o}(x)} \mathrm{e}^{\mathrm{i} \theta}, \quad \mathrm{i} b_{1}^{\infty}(x)=u_{o}(x) \Rightarrow b_{1}^{\infty}(x)=-\mathrm{i} u_{o}(x), \\
& \mathcal{O}\left(\frac{1}{\zeta}\right):-\mathrm{i} a_{2}^{\infty}(x)+d_{x} a_{1}^{\infty}(x)=0 \Rightarrow a_{2}^{\infty}(x)=\mathrm{e}^{\mathrm{i} \theta} d_{x} \overline{u_{o}(x)}, \\
& \mathrm{i} b_{2}^{\infty}(x)+d_{x} b_{1}^{\infty}(x)=0 \Rightarrow b_{2}^{\infty}(x)=d_{x} u_{o}(x) \text {, } \\
& \mathrm{i} s_{1}^{\infty}(x)=-\mathrm{i}+u_{o}(x) \mathrm{e}^{-\mathrm{i} \theta} a_{1}^{\infty}(x) \Rightarrow s_{1}^{\infty}(x)=-1+\left|u_{o}(x)\right|^{2}, \\
& \mathrm{i} t_{1}^{\infty}(x)=\mathrm{i}+u_{o}(x) b_{1}^{\infty}(x) \Rightarrow t_{1}^{\infty}(x)=1-\left|u_{o}(x)\right|^{2}, \\
& \mathcal{O}\left(\frac{1}{\zeta^{2}}\right):-\mathrm{i} a_{3}^{\infty}(x)-\mathrm{i} a_{1}^{\infty}(x)+u_{o}(x) \mathrm{e}^{-\mathrm{i} \theta}\left(a_{1}^{\infty}(x)\right)^{2}+d_{x} a_{2}^{\infty}(x)=0 \Rightarrow \\
& a_{3}^{\infty}(x)=-\mathrm{ie}^{\mathrm{i} \theta}\left(\overline{u_{o}(x)}-\left|u_{o}(x)\right|^{2} \overline{u_{o}(x)}+d_{x}^{2} u_{o}(x)\right), \\
& \mathrm{i} b_{3}^{\infty}(x)+\mathrm{i} b_{1}^{\infty}(x)+\overline{u_{o}(x)}\left(b_{1}^{\infty}(x)\right)^{2}+d_{x} b_{2}^{\infty}(x)=0 \Rightarrow \\
& b_{3}^{\infty}(x)=\mathrm{i}\left(u_{o}(x)-\left|u_{o}(x)\right|^{2} u_{o}(x)+d_{x}^{2} u_{o}(x)\right), \\
& \mathrm{i} s_{2}^{\infty}(x)=u_{o}(x) \mathrm{e}^{-\mathrm{i} \theta} a_{2}^{\infty}(x) \Rightarrow s_{2}^{\infty}(x)=-\mathrm{i} u_{o}(x) d_{x} \overline{u_{o}(x)} \text {, } \\
& \mathrm{i} t_{2}^{\infty}(x)=\overline{u_{o}(x)} b_{2}^{\infty} \Rightarrow t_{2}^{\infty}(x)=-\mathrm{i} \overline{u_{o}(x)} d_{x} u_{o}(x) \text {; }
\end{aligned}
$$

hence, from these $\zeta \rightarrow \infty$ results and the Volterra-type integral representation for $\Psi^{ \pm}(x, 0 ; \zeta)$ given at the beginning of the Lemma, one shows that

$$
\begin{aligned}
& \Psi^{-}(x, 0 ; \zeta) \underset{\zeta \rightarrow \infty}{=} \mathrm{e}^{\frac{\mathrm{i} \theta}{2} \sigma_{3}}\left(\left(\begin{array}{cc}
\mathrm{e}^{\frac{\mathrm{i}}{\zeta} \int_{-\infty}^{x}\left(\left|u_{o}(\xi)\right|^{2}-1\right) \mathrm{d} \xi} & \frac{1}{\zeta}\left(-\mathrm{i} u_{o}(x)\right) \mathrm{e}^{-\mathrm{i}\left(\theta+\frac{1}{\zeta} \int_{-\infty}^{x}\left(\left|u_{o}(\xi)\right|^{2}-1\right) \mathrm{d} \xi\right)} \\
\frac{1}{\zeta}(\overline{\mathrm{i} u o(x)}) \mathrm{e}^{\mathrm{i}\left(\theta+\frac{1}{\zeta} \int_{-\infty}^{x}\left(\left|u_{o}(\xi)\right|^{2}-1\right) \mathrm{d} \xi\right)} & \mathrm{e}^{-\frac{\mathrm{i}}{\zeta} \int_{-\infty}^{x}\left(\left|u_{o}(\xi)\right|^{2}-1\right) \mathrm{d} \xi}
\end{array}\right)\right. \\
& \left.+\mathcal{O}\left(\zeta^{-2}\right)\right) \mathrm{e}^{-\mathrm{i} k(\zeta) x \sigma_{3}}, \\
& \Psi^{+}(x, 0 ; \zeta) \underset{\zeta \rightarrow \infty}{=}\left(\left(\begin{array}{cc}
\mathrm{e}^{\frac{\mathrm{i}}{\zeta} \int_{+\infty}^{x}\left(\left|u_{o}(\xi)\right|^{2}-1\right) \mathrm{d} \xi} & \frac{1}{\zeta}\left(-\mathrm{i} u_{o}(x)\right) \mathrm{e}^{-\frac{\mathrm{i}}{\zeta} \int_{+\infty}^{x}\left(\left|u_{o}(\xi)\right|^{2}-1\right) \mathrm{d} \xi} \\
\frac{1}{\zeta}\left(\mathrm{i} u_{o}(x)\right) \mathrm{e}^{\frac{\mathrm{i}}{\zeta} \int_{+\infty}^{x}\left(\left|u_{o}(\xi)\right|^{2}-1\right) \mathrm{d} \xi} & \mathrm{e}^{-\frac{\mathrm{i}}{\zeta} \int_{+\infty}^{x}\left(\left|u_{o}(\xi)\right|^{2}-1\right) \mathrm{d} \xi}
\end{array}\right)\right. \\
& \left.+\mathcal{O}\left(\zeta^{-2}\right)\right) \mathrm{e}^{-\mathrm{i} k(\zeta) x \sigma_{3}}:
\end{aligned}
$$

finally, expanding the exponentials in power series in $\zeta^{-1}$, one obtains the $\zeta \rightarrow \infty$ asymptotics stated in the Lemma. Similarly, substituting the asymptotic expansions as $\zeta \rightarrow 0$ into 
system (L2.1a), one arrives at (for the first few-leading — terms),

$$
\begin{aligned}
& \mathcal{O}\left(\frac{1}{\zeta^{2}}\right):-\mathrm{i} a_{-1}^{0}(x)+\left(a_{-1}^{0}(x)\right)^{2} u_{o}(x) \mathrm{e}^{-\mathrm{i} \theta}=0 \Rightarrow a_{-1}^{0}(x)=\mathrm{i} \mathrm{e}^{\mathrm{i} \theta}\left(u_{o}(x)\right)^{-1}, \\
& \mathrm{i} b_{-1}^{0}(x)+\left(b_{-1}^{0}(x)\right)^{2} \overline{u_{o}(x)}=0 \Rightarrow b_{-1}^{0}(x)=-\mathrm{i}\left(\overline{u_{o}(x)}\right)^{-1}, \\
& \mathcal{O}\left(\frac{1}{\zeta}\right):-\mathrm{i} a_{0}^{0}(x)+2 u_{o}(x) \mathrm{e}^{-\mathrm{i} \theta} a_{-1}^{0}(x) a_{0}^{0}(x)+d_{x} a_{-1}^{0}(x)=0 \Rightarrow a_{0}^{0}(x)=-\mathrm{e}^{\mathrm{i} \theta} d_{x}\left(\left(u_{o}(x)\right)^{-1}\right) \text {, } \\
& \mathrm{i} b_{0}^{0}(x)+2 \overline{u_{o}(x)} b_{-1}^{0}(x) b_{0}^{0}(x)+d_{x} b_{-1}^{0}(x)=0 \Rightarrow b_{0}^{0}(x)=-d_{x}\left(\left(\overline{u_{o}(x)}\right)^{-1}\right), \\
& \mathrm{i} s_{-1}^{0}(x)=-\mathrm{i}+u_{o}(x) \mathrm{e}^{-\mathrm{i} \theta} a_{-1}^{0}(x) \Rightarrow s_{-1}^{0}(x)=0, \\
& \mathrm{i} t_{-1}^{0}(x)=\mathrm{i}+\overline{u_{o}(x)} b_{-1}^{0}(x) \Rightarrow t_{-1}^{0}(x)=0 \text {, } \\
& \mathcal{O}(1): \quad \mathrm{i} s_{0}^{0}(x)=u_{o}(x) \mathrm{e}^{-\mathrm{i} \theta} a_{0}^{0}(x) \Rightarrow s_{0}^{0}(x)=-\mathrm{i} d_{x} \ln \left(u_{o}(x)\right), \\
& \mathrm{i} t_{0}^{0}(x)=\overline{u_{o}(x)} b_{0}^{0}(x) \Rightarrow t_{0}^{0}(x)=-\mathrm{i} d_{x} \ln \left(\overline{u_{o}(x)}\right) \text {, }
\end{aligned}
$$

and the expressions for $a_{1}^{0}(x)$ and $b_{1}^{0}(x)$, resulting from the $\mathcal{O}(1)$ terms, have not been written as they will not actually be used; hence, from these $\zeta \rightarrow 0$ results, and the Volterra-type integral representation for $\Psi^{ \pm}(x, 0 ; \zeta)$ given at the beginning of the Lemma, one shows that

$$
\begin{aligned}
& \Psi^{-}(x, 0 ; \zeta) \underset{\zeta \rightarrow 0}{=} \mathrm{e}^{\frac{\mathrm{i} \theta}{2} \sigma_{3}}\left(\begin{array}{cc}
\mathcal{O}(1) & -\frac{1}{\zeta} \overline{\frac{\mathrm{ie}^{-\mathrm{i} \theta}}{u u_{o}(x)}} \mathrm{e}^{\left.\int_{-\infty}^{x} d_{\xi} \ln \overline{\left(u_{o}(\xi)\right.}\right) \mathrm{d} \xi}+\mathcal{O}(1) \\
\frac{1}{\zeta} \frac{\mathrm{i}^{\mathrm{i}^{\mathrm{i} \theta}}}{u_{o}(x)} \mathrm{e}^{\int_{-\infty}^{x} d_{\xi} \ln \left(u_{o}(\xi) \mathrm{d} \xi\right.}+\mathcal{O}(1) & \mathcal{O}(1)
\end{array}\right) \mathrm{e}^{-\mathrm{i} k(\zeta) x \sigma_{3}}
\end{aligned}
$$

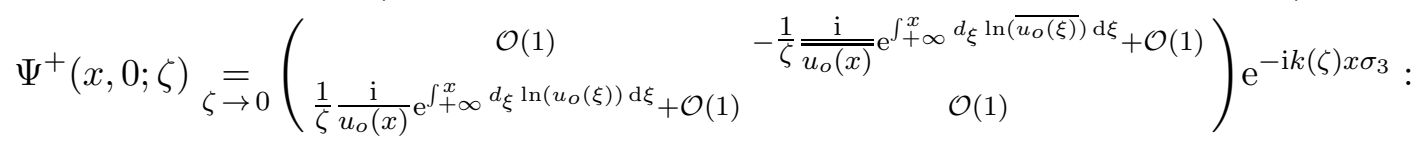

finally, using the fact that $\int_{ \pm \infty}^{x} d_{\xi} \ln \left(u_{o}(\xi)\right) \mathrm{d} \xi=\ln \left(u_{o}(x)\right)-\ln \left(u_{o}( \pm \infty)\right)=\ln \left(u_{o}(x)\right)-\frac{\mathrm{i}(1 \mp 1) \theta}{2}$ (having taken the principal branch for $\ln (\cdot)$ ), one obtains the $\zeta \rightarrow 0$ asymptotics stated in the Lemma.

Corollary 2.3. The following asymptotics are valid:

$$
\begin{gathered}
a(\zeta) \underset{\zeta \rightarrow \infty}{=} \mathrm{e}^{\mathrm{i} \theta}\left(1+\left(\mathrm{i} \int_{-\infty}^{+\infty}\left(\left|u_{o}(\xi)\right|^{2}-1\right) \mathrm{d} \xi\right) \zeta^{-1}+\mathcal{O}\left(\zeta^{-2}\right)\right), \quad a(\zeta) \underset{\zeta \rightarrow 0}{=} \mathrm{e}^{-\frac{\mathrm{i} \theta}{2}}(1+\mathcal{O}(\zeta)), \\
r(\zeta) \underset{\zeta \rightarrow \infty}{=} \mathcal{O}\left(\zeta^{-1}\right), \quad r(\zeta) \underset{\zeta \rightarrow 0}{=} \mathcal{O}(\zeta) .
\end{gathered}
$$

Proof. From Proposition 2.4,

$$
\begin{aligned}
& a(\zeta)=\frac{\zeta^{2}}{\zeta^{2}-1}\left(\Psi_{22}^{+}(x, 0 ; \zeta) \Psi_{11}^{-}(x, 0 ; \zeta)-\Psi_{12}^{+}(x, 0 ; \zeta) \Psi_{21}^{-}(x, 0 ; \zeta)\right), \\
& b(\zeta)=\frac{\zeta^{2}}{\zeta^{2}-1}\left(\overline{\Psi_{22}^{+}(x, 0 ; \bar{\zeta})} \Psi_{21}^{-}(x, 0 ; \zeta)-\overline{\Psi_{12}^{+}(x, 0 ; \bar{\zeta})} \Psi_{11}^{-}(x, 0 ; \zeta)\right) .
\end{aligned}
$$

Using the $\zeta \rightarrow \infty$ and $\zeta \rightarrow 0$ asymptotics for $\Psi_{i j}^{ \pm}(x, 0 ; \zeta), i, j \in\{1,2\}$, given in Lemma 2.1, one obtains the asymptotics for $a(\zeta)$ stated in the Corollary, and also $b(\zeta)={ }_{\zeta \rightarrow \infty} \mathcal{O}\left(\zeta^{-1}\right)$ and $b(\zeta)={ }_{\zeta \rightarrow 0} \mathcal{O}(\zeta)$; hence, since $r(\zeta):=\frac{b(\zeta)}{a(\zeta)}$, one arrives at the asymptotics for $r(\zeta)$ stated in the Corollary (in particular, $r(0)=0$ ).

Remark 2.2. Although the technical details of this argument are not presented here, using the fact that $u_{o}(x) \in \mathbf{C}^{\infty}(\mathbb{R})$ and $u_{o}(x)-u_{o}( \pm \infty) \in \mathcal{S}_{\mathbb{C}}\left(\mathbb{R}_{ \pm}\right)$, one shows that, using the Volterratype integral representation for the elements of $\Psi^{ \pm}(x, 0 ; \zeta)$ given in Lemma 2.1, based on a successive approximations (Neumann series-type) argument, $r(\zeta) \in \mathcal{S}_{\mathbb{C}}(\mathbb{R})$ (see the second article in [12] for complete details; see, also, Part 1 of [9]). 
Lemma 2.2. Let $u(x, t)$ be the solution of the Cauchy problem for the $\mathrm{D}_{f} \mathrm{NLSE}$ with finitedensity initial data, and $\Psi^{ \pm}(x, 0 ; \zeta)$ the (Jost) solutions of $\mathcal{O}^{\mathcal{D}} \Psi^{ \pm}(x, 0 ; \zeta)=\left(\begin{array}{ll}0 & 0 \\ 0 & 0\end{array}\right)$ with the asymptotics stated in Definition 2.1 and satisfying the symmetry reductions of Corollary 2.1. In the absence of spectral singularities, denote the discrete and continuous spectra of the direct scattering problem for the operator $\mathcal{O}^{\mathcal{D}}$ by $\sigma_{d}$ and $\sigma_{c}$, respectively, with $\sigma_{\mathcal{O}^{\mathcal{D}}}:=\operatorname{spec}\left(\mathcal{O}^{\mathcal{D}}\right)$ partitioned such that $\sigma_{\mathcal{O}^{\mathcal{D}}}=\sigma_{d} \cup \sigma_{c}$, and $\sigma_{d} \cap \sigma_{c}=\emptyset$. Then, for $r(\zeta) \in \mathcal{S}_{\mathbb{C}}(\mathbb{R}), \sigma_{d}=\Delta_{a} \cup \overline{\Delta_{a}}$, with $\Delta_{a}:=\left\{\varsigma_{n} ;\left.a(\zeta)\right|_{\zeta=\varsigma_{n}}=0, \varsigma_{n}=\mathrm{e}^{\mathrm{i} \phi_{n}}, \phi_{n} \in(0, \pi), n \in\{1,2, \ldots, N\}\right\}, \Delta_{a} \cap \overline{\Delta_{a}}=\emptyset$, and $\operatorname{card}\left(\sigma_{d}\right)=2 N<\infty$, and $\sigma_{c}=\{\zeta ; \Im(\zeta)=0\}$, with $\operatorname{card}\left(\sigma_{c}\right)=\infty$. Furthermore,

$$
a(\zeta)=\mathrm{e}^{\mathrm{i} \theta} \frac{\left(\zeta-\varsigma_{n}\right)}{2} \prod_{n=1}^{N} \frac{\left(\zeta-\overline{\varsigma_{n}}\right)}{(\zeta)}\left(-\int_{-\infty}^{+\infty} \frac{\ln \left(1-|r(\mu)|^{2}\right)}{(\mu-\zeta)} \frac{\mathrm{d} \mu}{2 \pi \mathrm{i}}\right), \quad \zeta \in \mathbb{C}_{+},
$$

and

$$
0 \leqslant \theta=-2 \sum_{n=1}^{N} \phi_{n}-\int_{-\infty}^{+\infty} \frac{\ln \left(1-|r(\mu)|^{2}\right)}{\mu} \frac{\mathrm{d} \mu}{2 \pi}<2 \pi .
$$

Proof. From classical complex analysis, a function $f(z)$ analytic and of finite order $\eta$ for $z \in \mathbb{C}_{+}$admits the inner-outer factorisation

$$
\begin{aligned}
f(z) & =\exp \left(\mathrm{i}\left(\sum_{k=0}^{q} b_{k} z^{k}\right)+\int_{-1}^{1} \frac{\ln (|f(\mu)|)}{(\mu-z)} \frac{\mathrm{d} \mu}{\pi \mathrm{i}}+\int_{-1}^{1} \frac{\mathrm{d} \tau(\mu)}{(\mu-z)} \frac{1}{\pi \mathrm{i}}\right) \prod_{\left|z_{n}\right|<1} \frac{\left(z-z_{n}\right)}{\left(z-\overline{z_{n}}\right)} \\
& \times \exp \left(z^{q+1}\left(\int_{|\mu| \geqslant 1} \frac{\ln (|f(\mu)|)}{\mu^{q+1}(\mu-z)} \frac{\mathrm{d} \mu}{\pi \mathrm{i}}+\int_{|\mu| \geqslant 1} \frac{\mathrm{d} \tau(\mu)}{\mu^{q+1}(\mu-z)} \frac{1}{\pi \mathrm{i}}\right)\right) \prod_{\left|z_{n}\right| \geqslant 1} D_{q}\left(z, z_{n}\right),
\end{aligned}
$$

where $q=[\eta]$, with $[\bullet]$ denoting the greatest integer less than or equal to $\bullet, b_{k} \in \mathbb{R}_{\geqslant 0}, \tau(\cdot)$ is a singular boundary function, $z_{n}:=r_{n} \mathrm{e}^{\mathrm{i} \theta_{z}^{n}}$, with $\left(r_{n}, \theta_{z}^{n}\right) \in \mathbb{R}_{+} \times(0, \pi)$, are the zeros of $f(z)$, $D_{q}(u, v):=\frac{E(u / v, q)}{E(u / \bar{v}, q)}$ is the canonical Nevanlinna factor, with

$$
E(w, q)= \begin{cases}1-w, & q=0 \\ (1-w) \exp \left(w+\frac{w^{2}}{2}+\cdots+\frac{w^{q}}{q}\right), & q \in \mathbb{N}\end{cases}
$$

the canonical Weierstrass factor, and all integrals and finite products converge absolutely (hence converge), $\sum_{r_{n} \leqslant 1} r_{n} \sin \theta_{z}^{n}<\infty, \sum_{r_{n}>1} r_{n}^{-\mu-\epsilon} \sin \theta_{z}^{n}<\infty, \int_{-\infty}^{+\infty} \frac{|\ln | f(\xi)||}{1+|\xi|^{1+\mu+\epsilon}} \mathrm{d} \xi<\infty$, and $\int_{-\infty}^{+\infty} \frac{|\mathrm{d} \tau(\xi)|}{1+|\xi|^{1+\mu+\epsilon}}<\infty$, where $\mu:=\max (\eta, 1)$, and $\epsilon$ is an arbitrary positive (real) number. Specialise the above to $a(\zeta)$. Let $\varsigma_{n}$ be the (necessarily simple: see below) zeros of $a(\zeta)$ : since $u_{o}(x) \in \mathbf{C}^{\infty}(\mathbb{R}), u_{o}(x)-u_{o}( \pm \infty) \in \mathcal{S}_{\mathbb{C}}\left(\mathbb{R}_{ \pm}\right)$, and there are no spectral singularities, thus only a finite number of simple poles (see the paragraph preceding Section 3 in [28]), it follows from an argument in 24] that $\operatorname{card}\left\{\varsigma_{n} ;\left.a(\zeta)\right|_{\zeta=\varsigma_{n}}=0\right\}:=N<\infty$. Since $a(\zeta)$ has an analytic continuation to $\mathbb{C}_{+}$, and, from Proposition 2.4, $a\left(\frac{1}{\zeta}\right)=\overline{a(\bar{\zeta})}$, it follows that $\left\{\varsigma_{n}\right\}_{n=1}^{N}$ are distributed along $\mathbb{C}_{+} \cap(\{z ;|z|=1\} \backslash\{ \pm 1\})$; hence, $\sigma_{d}$, the discrete spectrum of $\mathcal{O}^{\mathcal{D}}$, is given by $\sigma_{d}=\Delta_{a} \cup \overline{\Delta_{a}}$, where $\Delta_{a}$ is defined in the Lemma. Since $\Delta_{a} \cap \overline{\Delta_{a}}=\emptyset$, it follows (from the Inclusion-Exclusion Principle) that $\operatorname{card}\left(\Delta_{a} \cup \overline{\Delta_{a}}\right)=2 N$. From 24], the continuous spectrum of $\mathcal{O}^{\mathcal{D}}$ is given by $\sigma_{c}=\{\zeta ; \Re(-\mathrm{i} \lambda(\zeta))=\Re(\mathrm{i} \lambda(\zeta))\}=\{\zeta ; \Im(\zeta)=0\}$ (oriented from $-\infty$ to $+\infty)$. As there are no spectral singularities [28], $\tau(\cdot) \equiv 0$; hence, from the above argument, the $\zeta \rightarrow \infty$ asymptotics for $a(\zeta)$ given in Corollary 2.3 (in which case $\eta=0$, that is, $q=[0]=0)$, the unimodularity relation $|a(\zeta)|^{2}-|b(\zeta)|^{2}=0, \Im(\zeta)=0$, given in Proposition 2.4, and the definition of the reflection coefficient, $r(\zeta)$, given in Corollary 2.2, it follows that, for $b_{0}:=\left(\frac{1}{2} \theta+2 \sum_{n=1}^{N} \phi_{n}+\int_{|\mu| \geqslant 1} \frac{\ln \left(1-|r(\mu)|^{2}\right)}{\mu} \frac{\mathrm{d} \mu}{2 \pi}\right) \bmod (2 \pi), a(\zeta)$ has the simplified representation $a(\zeta)=\mathrm{e}^{\frac{\mathrm{i} \theta}{2}} \prod_{\varsigma_{n} \in \Delta_{a}} \frac{\left(\zeta-\varsigma_{n}\right)}{\left(\zeta-\overline{\zeta_{n}}\right)} \exp \left(-\int_{\sigma_{c}} \frac{\ln \left(1-|r(\mu)|^{2}\right)}{(\mu-\zeta)} \frac{\mathrm{d} \mu}{2 \pi \mathrm{i}}\right), \zeta \in \mathbb{C}_{+}$. Writing $a(\zeta)=$ 
$\mathrm{e}^{\frac{\mathrm{i} \theta}{2}} \prod_{n=1}^{N} \frac{\left(\zeta-\varsigma_{n}\right)}{\left(\zeta-\overline{\zeta_{n}}\right)} \exp \left(-\int_{-\infty}^{+\infty} \frac{\ln \left(1-|r(\mu)|^{2}\right)}{(\mu-\zeta)} \frac{\mathrm{d} \mu}{2 \pi \mathrm{i}}\right), \zeta \in \mathbb{C}_{+}$, and using the $\zeta \rightarrow 0$ asymptotics for $a(\zeta)$ given in Corollary 2.3, one obtains the expression for $\theta$ given in the Lemma; moreover, with this $\theta$, one also shows that $a(\zeta)$ satisfies the involution $a\left(\frac{1}{\zeta}\right)=\overline{a(\bar{\zeta})}$, and, since $r(\zeta) \in \mathcal{S}_{\mathbb{C}}(\mathbb{R})$, $\int_{-\infty}^{+\infty} \frac{\ln \left(1-|r(\xi)|^{2}\right)}{1+|\xi|^{2+\epsilon}} \mathrm{d} \xi<\infty$.

Lemma 2.3. For $r(\zeta) \in \mathcal{S}_{\mathbb{C}}^{1}(\mathbb{R})$,

$$
a(s+\mathrm{i} \varepsilon) \underset{\varepsilon \downarrow 0}{=} \frac{(-s)^{N} \mathrm{e}^{\mathrm{i}\left(\frac{\theta}{2}+\sum_{n=1}^{N} \phi_{n}\right)} \exp \left(-\mathrm{P} . \mathrm{V} \cdot \int_{\mathbb{R} \backslash\{s\}} \frac{\ln \left(1-|r(\mu)|^{2}\right)}{(\mu-s)} \frac{\mathrm{d} \mu}{2 \pi \mathrm{i}}\right)}{\left(1-|r(s)|^{2}\right)^{\varkappa_{\operatorname{sgn}}(s)}}(1+o(1)), \quad s \in\{ \pm 1\},
$$

where P.V. $\int$ denotes the principal value integral, and $\varkappa_{ \pm}$are real, possibly zero, constants.

Proof. This result will be proved via two independent approaches: (1) using the representation for $a(\zeta)$ given in Lemma 2.2 (METHOD (i)); and (2) analysing the expression for $a(\zeta)$ given in Proposition 2.4 (Method (ii)).

Method (i). One starts with the representation for $a(\zeta)$ given in Lemma 2.2, namely, $a(\zeta)=\mathrm{e}^{\mathrm{i} \theta} \frac{{ }^{2}}{2} \prod_{n=1}^{N} \frac{\left(\zeta-\varsigma_{n}\right)}{\left(\zeta-\overline{\varsigma_{n}}\right)} \exp \left(-\int_{-\infty}^{+\infty} \frac{\ln \left(1-|r(\mu)|^{2}\right)}{(\mu-\zeta)} \frac{\mathrm{d} \mu}{2 \pi \mathrm{i}}\right), \zeta \in \mathbb{C}_{+}$, where $\theta \in[0,2 \pi)$ is given in Lemma 2.2, $\varsigma_{n}=\mathrm{e}^{\mathrm{i} \phi_{n}}, \phi_{n} \in(0, \pi), n \in\{1,2, \ldots, N\}$, and $r(\zeta) \in \mathcal{S}_{\mathbb{C}}(\mathbb{R})$. The fact that ||$r(\cdot)||_{\mathcal{L}^{\infty}(\mathbb{R})}:=\sup _{z \in \mathbb{R}}|r(z)|<1$ is essential for the proof: this will be proved in Lemma 2.4 below. To study the behaviour of $a(\zeta)$ as $\zeta \rightarrow \pm 1$ from above $\left(\mathbb{C}_{+}\right)$, set $\zeta= \pm 1+\epsilon \mathrm{e}^{\mathrm{i} \beta}$, $\beta \in(0, \pi)$, and consider the limit as $\epsilon \downarrow 0$. Set $\mathbb{U}_{\varepsilon}( \pm 1):=\{z ;|z \mp 1|<\varepsilon\}$, where $\varepsilon$ is an arbitrarily fixed, sufficiently small positive real number. Write $I=\int_{-\infty}^{+\infty} \frac{\ln \left(1-|r(\mu)|^{2}\right)}{(\mu-\zeta)} \frac{\mathrm{d} \mu}{2 \pi \mathrm{i}}=$ $I_{o}^{r}+\left(\int_{\mathbb{U}_{\varepsilon}(-1)}+\int_{\mathbb{U}_{\varepsilon}(+1)}\right) \frac{\ln \left(1-|r(\mu)|^{2}\right)}{(\mu-\zeta)} \frac{\mathrm{d} \mu}{2 \pi \mathrm{i}}$, where $I_{o}^{r}:=\int_{\mathbb{R} \backslash \mathbb{U}_{\varepsilon}( \pm 1)} \frac{\ln \left(1-|r(\mu)|^{2}\right)}{(\mu-\zeta)} \frac{\mathrm{d} \mu}{2 \pi \mathrm{i}}$. Since $r(\zeta) \in \mathcal{S}_{\mathbb{C}}(\mathbb{R})$, one Taylor expands $\left(1-|r(\zeta)|^{2}\right)$ to show that, for $\zeta \in \mathbb{U}_{\varepsilon}( \pm 1),\left(1-|r(\zeta)|^{2}\right)=1-r_{o}( \pm 1)+\widehat{r}_{1}( \pm 1)(\zeta \mp$ $1)+\frac{\widehat{r}_{2}( \pm 1)}{2 !}(\zeta \mp 1)^{2}+\mathcal{O}\left((\zeta \mp 1)^{3}\right)$, where $r_{o}( \pm 1):=|r( \pm 1)|^{2}(\neq 1)$, and $\widehat{r}_{j}( \pm 1), j \in\{1,2\}$, are some $\mathbb{C}$-valued constants: now, using the fact that $\|r(\cdot)\|_{\mathcal{L}^{\infty}(\mathbb{R})}<1$, and the expansion $\ln (1+x)=|x|<1$ $x+\mathcal{O}\left(x^{2}\right)$, one shows that $I=I_{o}^{r}+\sum_{l \in\{ \pm 1\}}\left(\int_{\mathbb{U}_{\varepsilon}(l)} \frac{\ln \left(1-r_{o}(l)\right)}{(\mu-\zeta)} \frac{\mathrm{d} \mu}{2 \pi \mathrm{i}}+\int_{\mathbb{U}_{\varepsilon}(l)} \frac{\left(\widetilde{r}_{1}(l)(\mu-l)+\mathcal{O}\left((\mu-l)^{2}\right)\right)}{(\mu-\zeta)} \frac{\mathrm{d} \mu}{2 \pi \mathrm{i}}\right)$, where $\widetilde{r}_{j}(l), j \in\{1,2\}, l \in\{ \pm 1\}$, are some $\mathbb{C}$-valued constants. Using the distributional identities $\left(x-\left(x_{o} \pm \mathrm{i} 0\right)\right)^{-1}=$ P.V. $\left(x-x_{o}\right)^{-1} \pm \mathrm{i} \pi \delta\left(x-x_{o}\right)$, where P.V. denotes the principal value integral and $\delta\left(x-x_{o}\right)$ is the Dirac delta function, and $\int_{x_{1}}^{x_{2}} f(x) \delta\left(x-x_{o}\right) \mathrm{d} x= \begin{cases}f\left(x_{o}\right), & x_{o} \in\left(x_{1}, x_{2}\right), \\ 0, & x_{o} \in \mathbb{R} \backslash\left(x_{1}, x_{2}\right),\end{cases}$ one shows that, with the choice $|\epsilon \cos \beta|<\varepsilon(\forall \beta \in(0, \pi))$, and taking the principal branch, for $|r( \pm 1)| \neq 1, I={ }_{\varepsilon \downarrow 0} \mathrm{P} . \mathrm{V} . \int_{\mathbb{R} \backslash\{ \pm 1\}} \frac{\ln \left(1-|r(\mu)|^{2}\right)}{(\mu \mp 1)} \frac{\mathrm{d} \mu}{2 \pi \mathrm{i}}+\sum_{l \in\{ \pm 1\}} \varkappa_{\operatorname{sgn}(l)} \ln \left(1-|r(l)|^{2}\right)+o(1)$, with $\varkappa_{\operatorname{sgn}(l)}$, $l \in\{ \pm 1\}$, some $\mathbb{R}$-valued, possibly zero, constants. One also shows that $\prod_{n=1}^{N} \frac{\left( \pm 1+\mathrm{i} \varepsilon-\zeta_{n}\right)}{\left( \pm 1+\mathrm{i} \varepsilon-\overline{\zeta_{n}}\right)}={ }_{\varepsilon \downarrow 0}$ $(\mp 1)^{N} \exp \left(\mathrm{i} \sum_{n=1}^{N} \phi_{n}\right)(1+o(1))$; hence, from the above estimates, it follows that $a( \pm 1+\mathrm{i} \varepsilon)={ }_{\varepsilon \downarrow 0}$ $\frac{\exp \left(\frac{\mathrm{i} \theta}{2}\right) \exp \left(-\mathrm{P} . \mathrm{V} \cdot \int_{\mathbb{R} \backslash\{ \pm 1\}} \frac{\ln \left(1-|r(\mu)|^{2}\right)}{(\mu \mp 1)} \frac{\mathrm{d} \mu}{2 \pi \mathrm{i}}\right)(\mp 1)^{N} \exp \left(\mathrm{i} \sum_{n=1}^{N} \phi_{n}\right)}{\left(1-|r( \pm 1)|^{2}\right)^{\varkappa} \pm}(1+o(1))$.

Method (ii). From Proposition 2.4, $a(\zeta)=\frac{\zeta^{2}}{\zeta^{2}-1}\left(\Psi_{22}^{+}(\zeta) \Psi_{11}^{-}(\zeta)-\Psi_{12}^{+}(\zeta) \Psi_{21}^{-}(\zeta)\right), \zeta \in \mathbb{C}_{+}$, where, from Lemma 2.1,

$$
\begin{aligned}
& \Psi^{-}(\zeta):=\Psi^{-}(x, 0 ; \zeta)=\left(\begin{array}{cc}
\mathrm{e}^{\mathrm{i} \frac{\mathrm{i} \theta}{2}} \mathrm{e}^{\mathrm{i}\left(\int_{-\infty}^{x} \delta(\xi ; \zeta) \mathrm{d} \xi-k(\zeta) x\right)} & \mathrm{e}^{\mathrm{i} \theta} \overline{\mathcal{A}(x ; \bar{\zeta})} \mathrm{e}^{-\mathrm{i}\left(\int_{-\infty}^{x} \overline{\delta(\xi ; \bar{\zeta})} \mathrm{d} \xi-k(\zeta) x\right)} \\
\mathrm{e}^{\mathrm{i} \frac{\mathrm{i} \theta}{2}} \mathcal{A}(x ; \zeta) \mathrm{e}^{\mathrm{i}\left(\int_{-\infty}^{x} \delta(\xi ; \zeta) \mathrm{d} \xi-k(\zeta) x\right)} & \mathrm{e}^{-\frac{\mathrm{i} \theta}{2}} \mathrm{e}^{-\mathrm{i}\left(\int_{-\infty}^{x} \overline{\delta(\xi ; \bar{\zeta})} \mathrm{d} \xi-k(\zeta) x\right)}
\end{array}\right), \\
& \Psi^{+}(\zeta):=\Psi^{+}(x, 0 ; \zeta)=\left(\begin{array}{cc}
\mathrm{e}^{-\mathrm{i}\left(\int_{+\infty}^{x} \overline{\mathcal{T}(\xi ; \bar{\zeta})} \mathrm{d} \xi+k(\zeta) x\right)} & \mathcal{B}(x ; \zeta) \mathrm{e}^{\mathrm{i}\left(\int_{+\infty}^{x} \mathcal{T}(\xi ; \zeta) \mathrm{d} \xi+k(\zeta) x\right)} \\
\overline{\mathcal{B}(x ; \bar{\zeta})} \mathrm{e}^{-\mathrm{i}\left(\int_{+\infty}^{x} \overline{\mathcal{T}(\xi ; \bar{\zeta})} \mathrm{d} \xi+k(\zeta) x\right)} & \mathrm{e}^{\mathrm{i}\left(\int_{+\infty}^{x} \mathcal{T}(\xi ; \zeta) \mathrm{d} \xi+k(\zeta) x\right)}
\end{array}\right),
\end{aligned}
$$

with $\mathcal{A}(x ; \zeta), \mathcal{B}(x ; \zeta), \mathcal{S}(x ; \zeta)$, and $\mathcal{T}(x ; \zeta)$ satisfying system (L2.1a), and $\theta$ given in Lemma 2.2 . Near \pm 1 , let the small parameters be defined by $\mu_{ \pm}:=\zeta \mp 1$ : since $r(\zeta) \in \mathcal{S}_{\mathbb{C}}(\mathbb{R})$, it follows from a result of Zhou [29] that $\mathcal{A}\left(x ; \mu_{ \pm}\right)=\sum_{n=0}^{\infty} a_{n}^{ \pm}[u, \bar{u}] \mu_{ \pm}^{n}+\mathcal{O}\left(\left|\mu_{ \pm}\right|^{+\infty}\right)$ and $\mathcal{B}\left(x ; \mu_{ \pm}\right)=$ 
$\sum_{n=0}^{\infty} b_{n}^{ \pm}[u, \bar{u}] \mu_{ \pm}^{n}+\mathcal{O}\left(\left|\mu_{ \pm}\right|^{+\infty}\right)$, where $\star_{n}^{ \pm}[u, \bar{u}]:=\star_{n}^{ \pm}$, with $\star \in\{a, b\}$, are functionals of $u_{o}(x)$ and $\overline{u_{o}(x)}$. Substituting these asymptotic expansions into the first two equations of system (L2.1a) and using the geometric progression $(1 \pm z)^{-1}=\sum_{n=0}^{\infty}(\mp 1)^{n} z^{n},|z|<1$, one shows that, for $\zeta \approx \pm 1$

$$
\begin{aligned}
& \mp \mathrm{i}\left(2+\mu_{ \pm}^{2} \mp \mu_{ \pm}^{3}+\cdots\right)\left(a_{0}^{ \pm}+a_{1}^{ \pm} \mu_{ \pm}+a_{2}^{ \pm} \mu_{ \pm}^{2}+a_{3}^{ \pm} \mu_{ \pm}^{3}+\cdots\right)+d_{x}\left(a_{0}^{ \pm}+a_{1}^{ \pm} \mu_{ \pm}+a_{2}^{ \pm} \mu_{ \pm}^{2}+a_{3}^{ \pm} \mu_{ \pm}^{3}+\cdots\right) \\
& +u_{o}(x) \mathrm{e}^{-\mathrm{i} \theta}\left(a_{0}^{ \pm}+a_{1}^{ \pm} \mu_{ \pm}+a_{2}^{ \pm} \mu_{ \pm}^{2}+a_{3}^{ \pm} \mu_{ \pm}^{3}+\cdots\right)\left(a_{0}^{ \pm}+a_{1}^{ \pm} \mu_{ \pm}+a_{2}^{ \pm} \mu_{ \pm}^{2}+a_{3}^{ \pm} \mu_{ \pm}^{3}+\cdots\right)=\overline{u_{o}(x)} \mathrm{e}^{\mathrm{i} \theta} \\
& \quad \pm \mathrm{i}\left(2+\mu_{ \pm}^{2} \mp \mu_{ \pm}^{3}+\cdots\right)\left(b_{0}^{ \pm}+b_{1}^{ \pm} \mu_{ \pm}+b_{2}^{ \pm} \mu_{ \pm}^{2}+b_{3}^{ \pm} \mu_{ \pm}^{3}+\cdots\right)+d_{x}\left(b_{0}^{ \pm}+b_{1}^{ \pm} \mu_{ \pm}+b_{2}^{ \pm} \mu_{ \pm}^{2}+b_{3}^{ \pm} \mu_{ \pm}^{3}+\cdots\right) \\
& \quad \overline{u_{o}(x)}\left(b_{0}^{ \pm}+b_{1}^{ \pm} \mu_{ \pm}+b_{2}^{ \pm} \mu_{ \pm}^{2}+b_{3}^{ \pm} \mu_{ \pm}^{3}+\cdots\right)\left(b_{0}^{ \pm}+b_{1}^{ \pm} \mu_{ \pm}+b_{2}^{ \pm} \mu_{ \pm}^{2}+b_{3}^{ \pm} \mu_{ \pm}^{3}+\cdots\right)=u_{o}(x) .
\end{aligned}
$$

From Eqs. (L2.2a) and (L2.2b), one has, from the $\mathcal{O}(1)$ terms,

$$
\begin{aligned}
& -2 \mathrm{i} a_{0}^{+}+d_{x} a_{0}^{+}+u_{o}(x) \mathrm{e}^{-\mathrm{i} \theta}\left(a_{0}^{+}\right)^{2}=\overline{u_{o}(x)} \mathrm{e}^{\mathrm{i} \theta}, \quad 2 \mathrm{i} b_{0}^{+}+d_{x} b_{0}^{+}+\overline{u_{o}(x)}\left(b_{0}^{+}\right)^{2}=u_{o}(x), \\
& 2 \mathrm{i} a_{0}^{-}+d_{x} a_{0}^{-}+u_{o}(x) \mathrm{e}^{-\mathrm{i} \theta}\left(a_{0}^{-}\right)^{2}=\overline{u_{o}(x)} \mathrm{e}^{\mathrm{i} \theta}, \quad-2 \mathrm{i} b_{0}^{-}+d_{x} b_{0}^{-}+\overline{u_{o}(x)}\left(b_{0}^{-}\right)^{2}=u_{o}(x) .
\end{aligned}
$$

Eqs. (L2.2c) and (L2.2d) are non-linear ordinary differential equations (ODEs) of the Riccati type for $a_{0}^{ \pm}$and $b_{0}^{ \pm}$: for the purposes of this proof, their explicit solutions are not necessary (they can also be transformed into linear 2nd-order non-constant coefficient ODEs). From Eqs. (L2.2c) and (L2.2d), one shows that $a_{0}^{ \pm} b_{0}^{ \pm}=\mathrm{e}^{\mathrm{i} \theta}$; hence, with the representations $a_{0}^{ \pm}:=\left|a_{0}^{ \pm}(x)\right| \exp \left(\mathrm{i} \phi_{ \pm}^{a}(x)\right)$ and $b_{0}^{ \pm}:=\left|b_{0}^{ \pm}(x)\right| \exp \left(\mathrm{i} \phi_{ \pm}^{b}(x)\right.$ ), with $\left|a_{0}^{ \pm}(x)\right|: \mathbb{R} \rightarrow \mathbb{R}_{+}$(respectively $\left|b_{0}^{ \pm}(x)\right|: \mathbb{R} \rightarrow \mathbb{R}_{+}$) and $\phi_{ \pm}^{a}(x): \mathbb{R} \rightarrow \mathbb{R} \backslash\{0\}$ (respectively $\phi_{ \pm}^{b}(x): \mathbb{R} \rightarrow \mathbb{R} \backslash\{0\}$ ), it follows that $\left|a_{0}^{ \pm}(x)\right|\left|b_{0}^{ \pm}(x)\right|=1$ and $\phi_{ \pm}^{a}(x)+\phi_{ \pm}^{b}(x)=\theta \bmod (2 \pi)$ : one also requires that (zero-integral conditions) $\int_{\mathbb{R}}\left|u_{o}(\xi)\right|\left(\left|a_{0}^{ \pm}(\xi)\right|+\left|a_{0}^{ \pm}(\xi)\right|^{-1}\right) \cos \left(\arg \left(u_{o}(\xi)\right)-\theta+\phi_{ \pm}^{a}(\xi)\right) \mathrm{d} \xi=0$ and $\int_{\mathbb{R}}\left|u_{o}(\xi)\right|\left(\left|a_{0}^{ \pm}(\xi)\right|-\left|a_{0}^{ \pm}(\xi)\right|^{-1}\right) \sin \left(\arg \left(u_{o}(\xi)\right)-\theta+\phi_{ \pm}^{a}(\xi)\right) \mathrm{d} \xi=0$. From Eqs. (L2.2a) and (L2.2b), one has, from the $\mathcal{O}\left(\mu_{ \pm}\right)$terms,

$$
\begin{gathered}
d_{x} a_{1}^{+}+a_{1}^{+}\left(2 u_{o}(x) \mathrm{e}^{-\mathrm{i} \theta} a_{0}^{+}-2 \mathrm{i}\right)=0, \quad d_{x} b_{1}^{+}+b_{1}^{+}\left(2 \overline{u_{o}(x)} b_{0}^{+}+2 \mathrm{i}\right)=0, \\
d_{x} a_{1}^{-}+a_{1}^{-}\left(2 u_{o}(x) \mathrm{e}^{-\mathrm{i} \theta} a_{0}^{-}+2 \mathrm{i}\right)=0, \quad d_{x} b_{1}^{-}+b_{1}^{-}\left(2 \overline{u_{o}(x)} b_{0}^{-}-2 \mathrm{i}\right)=0 .
\end{gathered}
$$

Eqs. (L2.2e) and (L2.2f) are linear 1st-order ODEs for $a_{1}^{ \pm}$and $b_{1}^{ \pm}$which can be solved explicitly to yield $a_{1}^{ \pm}=c_{ \pm} \exp \left(-\int_{-\infty}^{x}\left(2 u_{o}(\xi) \mathrm{e}^{-\mathrm{i} \theta} a_{0}^{ \pm} \mp 2 \mathrm{i}\right) \mathrm{d} \xi\right)$ and $b_{1}^{ \pm}=d_{ \pm} \exp \left(-\int_{+\infty}^{x}\left(2 \overline{u_{o}(\xi)} b_{0}^{ \pm} \pm\right.\right.$ $2 \mathrm{i}) \mathrm{d} \xi)$, with $\left(c_{ \pm}, d_{ \pm}\right) \in \mathbb{C} \times \mathbb{C}$. Once again, since $r(\zeta) \in \mathcal{S}_{\mathbb{C}}(\mathbb{R})$, it follows from a result of Zhou [29] that, near \pm 1 , with $\mu_{ \pm}:=\zeta \mp 1, \mathcal{S}\left(x ; \mu_{ \pm}\right)=\sum_{n=0}^{\infty} s_{n}^{ \pm}[u, \bar{u}] \mu_{ \pm}^{n}+\mathcal{O}\left(\left|\mu_{ \pm}\right|^{+\infty}\right)$ and $\mathcal{T}\left(x ; \mu_{ \pm}\right)=\sum_{n=0}^{\infty} t_{n}^{ \pm}[u, \bar{u}] \mu_{ \pm}^{n}+\mathcal{O}\left(\left|\mu_{ \pm}\right|^{+\infty}\right)$, where $\star_{n}^{ \pm}[u, \bar{u}]:=\star_{n}^{ \pm}$, with $\star \in\{s, t\}$, are functionals of $u_{o}(x)$ and $\overline{u_{o}(x)}$. Substituting these asymptotic expansions into the third equation of system (L2.1a) and using the geometric progression $(1 \pm z)^{-1}=\sum_{n=0}^{\infty}(\mp 1)^{n} z^{n},|z|<1$, one shows that, for $\zeta \approx \pm 1$,

$$
\begin{gathered}
\mathrm{i}\left(s_{0}^{ \pm}+s_{1}^{ \pm} \mu_{ \pm}+s_{2}^{ \pm} \mu_{ \pm}^{2}+\cdots\right)=\mp \mathrm{i}\left(1 \mp \mu_{ \pm}+\mu_{ \pm}^{2}+\cdots\right)+u_{o}(x) \mathrm{e}^{-\mathrm{i} \theta}\left(a_{0}^{ \pm}+a_{1}^{ \pm} \mu_{ \pm}+\cdots\right), \\
\mathrm{i}\left(t_{0}^{ \pm}+t_{1}^{ \pm} \mu_{ \pm}+t_{2}^{ \pm} \mu_{ \pm}^{2}+\cdots\right)= \pm \mathrm{i}\left(1 \mp \mu_{ \pm}+\mu_{ \pm}^{2}+\cdots\right)+\overline{u_{o}(x)}\left(b_{0}^{ \pm}+b_{1}^{ \pm} \mu_{ \pm}+\cdots\right) .
\end{gathered}
$$

Solving, algebraically, Eqs. (L2.2g) and (L2.2h) for the first two non-zero terms, one arrives at $s_{0}^{ \pm}=\mp 1-\mathrm{i} u_{o}(x) \mathrm{e}^{-\mathrm{i} \theta} a_{0}^{ \pm}, t_{0}^{ \pm}= \pm 1-\mathrm{i} \overline{u_{o}(x)} b_{0}^{ \pm}, s_{1}^{ \pm}=1-\mathrm{i} u_{o}(x) \mathrm{e}^{-\mathrm{i} \theta} a_{1}^{ \pm}$, and $t_{1}^{ \pm}=-1-\overline{\mathrm{i} u_{o}(x)} b_{1}^{ \pm}$, with $a_{0}^{ \pm}$and $b_{0}^{ \pm}$(respectively $a_{1}^{ \pm}$and $b_{1}^{ \pm}$) as stated (respectively given) above. Substituting the above asymptotic expansions into the matrix representations for $\Psi^{ \pm}(x, 0 ; \zeta)$ given at the beginning of METHOD (ii), isolating the elements $\Psi_{22}^{+}(\zeta), \Psi_{11}^{-}(\zeta), \Psi_{12}^{+}(\zeta)$, and $\Psi_{21}^{-}(\zeta)$, recalling that $a_{0}^{ \pm} b_{0}^{ \pm}=\mathrm{e}^{\mathrm{i} \theta}$, choosing $c_{ \pm}$and $d_{ \pm}$judiciously, along with the convergence conditions $\int_{-\infty}^{+\infty}\left|u_{o}(\xi)\right|\left|a_{0}^{ \pm}(\xi)\right| \cos \left(\arg \left(u_{o}(\xi)\right)-\theta+\phi_{ \pm}^{a}(\xi)\right) \mathrm{d} \xi<\infty$ and $\int_{-\infty}^{+\infty}\left(\left|u_{o}(\xi)\right|\left|a_{0}^{ \pm}(\xi)\right| \sin \left(\arg \left(u_{o}(\xi)\right)-\right.\right.$ $\left.\left.\theta+\phi_{ \pm}^{a}(\xi)\right) \mp 1\right) \mathrm{d} \xi<\infty$ (so as to remove secular terms), invoking the zero-integral conditions, and substituting, finally, into the expression for $a(\zeta)$ given at the beginning of METHOD (ii), 
one shows that, for $\zeta \approx \pm 1, \pm 2(\zeta \mp 1) a(\zeta)=\exp \left(\mathcal{K}^{ \pm}+\mathcal{O}(\zeta \mp 1)\right)(\underbrace{\mathrm{e}^{\mathrm{i} \theta}-\mathrm{e}^{\frac{\mathrm{i} \theta}{2}}}_{0}+\mathcal{O}(\zeta \mp 1))$, with $\mathcal{K}^{ \pm} \in \mathbb{C} \backslash\{0\}$ and $\mathcal{O}(1)$; hence, $a(\zeta)={ }_{\zeta \rightarrow \pm 1} \widehat{a}_{o}^{ \pm}+\mathcal{O}(\zeta \mp 1)$, where $\widehat{a}_{o}^{ \pm}$are some $\mathbb{C}$-valued $\mathcal{O}(1)$ constants (whose explicit expressions are not given here).

Remark 2.3. The analysis for the singular limit $|r( \pm 1)|=1$ is not addressed in this work: it is assumed throughout that $|r( \pm 1)| \neq 1$.

The $t$-dependence is re-introduced into the analysis by studying the $\partial_{t} \Psi(x, t ; \zeta)=\mathcal{V}(x, t ; \zeta)$ - $\Psi(x, t ; \zeta)$ component of system $(2)$. It is shown in [9] that the scattering map $(S) u_{o}(\cdot) \mapsto$ $r(\zeta)=\mathcal{R}\left(u_{o}(\cdot)\right)$, which is a bijection for $u_{o}(x)$ and $r(\zeta)$ belonging to the spaces defined heretofore, linearises the $\mathrm{D}_{f} \mathrm{NLSE}$ flow. This leads to the following

Proposition $2.5(\sqrt[9]{\mathbf{9}}, \mathbf{3 0})$. Let $u(x, t)$ be the solution of the Cauchy problem for the $\mathrm{D}_{f} \mathrm{~N}$ LSE with finite-density initial data and $\Psi(x, t ; \zeta)$ the corresponding solution of system (2) defined in Proposition 2.2. Then $a(\zeta, t)=a(\zeta)$ and $b(\zeta, t)=b(\zeta) \exp (4 \mathrm{i} k(\zeta) \lambda(\zeta) t)$.

From Proposition 2.5, it follows that, since $a(\zeta, t)$ is independent of $t, a(\zeta)$ is the "generator" of the integrals of motion of the model, and, from the definition $r(\zeta, t):=\frac{b(\zeta, t)}{a(\zeta, t)}$, it follows that $r(\zeta, t)$ evolves in the scattering data phase space according to the rule $r(\zeta, t)=$ $r(\zeta) \exp (4 \mathrm{i} k(\zeta) \lambda(\zeta) t)$, with $r(\zeta) \in \mathcal{S}_{\mathbb{C}}^{1}(\mathbb{R})$. Assembling the above, one arrives at the following (normalised at $\infty)$ RHP for the $\mathrm{M}(2, \mathbb{C})$-valued function $m(x, t ; \zeta)$.

Lemma 2.4. Let $u(x, t)$ be the solution of the Cauchy problem for the $\mathrm{D}_{f} \mathrm{NLSE}$ with finitedensity initial data $u(x, 0):=u_{o}(x)={ }_{x \rightarrow \pm \infty} u_{o}( \pm \infty)(1+o(1))$, where $u_{o}( \pm \infty):=\exp \left(\frac{\mathrm{i}(1 \mp 1) \theta}{\mathbb{C}^{2}}\right)$, $\theta$ is given in Lemma $2.2, u_{o}(x) \in \mathbf{C}^{\infty}(\mathbb{R})$, and $u_{o}(x)-u_{o}( \pm \infty) \in \mathcal{S}_{\mathbb{C}}\left(\mathbb{R}_{ \pm}\right)$. For $\zeta \in \mathbb{C}_{+}$, set $\Phi(x, t ; \zeta):=\left(\begin{array}{cc}\frac{\Psi_{11}^{-}(x, t ; \zeta)}{a(\zeta)} & \Psi_{12}^{+}(x, t ; \zeta) \\ \frac{\Psi_{21}^{-}(x, t ; \zeta)}{a(\zeta)} & \Psi_{22}^{+}(x, t ; \zeta)\end{array}\right)$, and, for $\zeta \in \mathbb{C}_{-}$, set $\Phi(x, t ; \zeta):=\left(\begin{array}{c}\Psi_{11}^{+}(x, t ; \zeta) \frac{\Psi_{12}^{-}(x, t ; \zeta)}{\overline{a(\bar{\zeta})}} \\ \Psi_{21}^{+}(x, t ; \zeta) \frac{\Psi_{22}^{-}(x, t ; \zeta)}{\overline{a(\bar{\zeta})}}\end{array}\right)$, where $a(\zeta)$ is given in Lemma $2.2, \Psi^{ \pm}(x, t ; \zeta)$ are the solutions of system (2) defined in Proposition 2.2 , and $\Psi^{ \pm}(x, 0 ; \zeta)$ solve $\mathcal{O}^{\mathcal{D}} \Psi^{ \pm}(x, 0 ; \zeta)=\left(\begin{array}{cc}0 & 0 \\ 0 & 0\end{array}\right)$ with the asymptotics stated in Definition 2.1. Set $m(x, t ; \zeta):=\Phi(x, t ; \zeta) \exp \left(\mathrm{i} k(\zeta)(x+2 \lambda(\zeta) t) \sigma_{3}\right)$. Then: (1) the bounded discrete set $\sigma_{d}$ is finite; (2) the poles of $m(x, t ; \zeta)$ are simple; (3) the first (respectively second) column of $m(x, t ; \zeta)$ has poles in $\mathbb{C}_{+}$(respectively $\left.\mathbb{C}_{-}\right)$at $\left\{\varsigma_{n}\right\}_{n=1}^{N}$ (respectively $\left\{\overline{\varsigma_{n}}\right\}_{n=1}^{N}$ ), $\varsigma_{n}:=\mathrm{e}^{\mathrm{i} \phi_{n}}, \phi_{n} \in(0, \pi)$; and (4) $m(x, t ; \zeta): \mathbb{C} \backslash\left(\sigma_{d} \cup \sigma_{c}\right) \rightarrow \mathrm{M}(2, \mathbb{C})$ solves the following RHP:

(i) $m(x, t ; \zeta)$ is piecewise meromorphic $\forall \zeta \in \mathbb{C} \backslash \sigma_{c}$;

(ii) $m_{ \pm}(x, t ; \zeta):=\lim _{\varepsilon \downarrow 0} m(x, t ; \zeta \pm \mathrm{i} \varepsilon)$ satisfy the jump condition

$$
m_{+}(x, t ; \zeta)=m_{-}(x, t ; \zeta) \mathcal{G}(x, t ; \zeta), \quad \zeta \in \mathbb{R}
$$

where $\mathcal{G}(x, t ; \zeta):=\exp \left(-\mathrm{i} k(\zeta)(x+2 \lambda(\zeta) t) \operatorname{ad}\left(\sigma_{3}\right)\right)\left(\begin{array}{cc}1+r(\zeta) r\left(\frac{1}{\zeta}\right) & r\left(\frac{1}{\zeta}\right) \\ r(\zeta) & 1\end{array}\right)$, and $r(\zeta)$, the reflection coefficient of the direct scattering problem for $\mathcal{O}^{\mathcal{D}}$, satisfies $r(\zeta)=\zeta_{\zeta \rightarrow 0} \mathcal{O}(\zeta)$, $r(\zeta)={ }_{\zeta \rightarrow \infty} \mathcal{O}\left(\zeta^{-1}\right), r\left(\frac{1}{\zeta}\right)=-\overline{r(\bar{\zeta})}$, and $r(\zeta) \in \mathcal{S}_{\mathbb{C}}^{1}(\mathbb{R}) ;$

(iii) for the simple poles of $m(x, t ; \zeta)$ at $\left\{\varsigma_{n}\right\}_{n=1}^{N}$ and $\left\{\overline{\varsigma_{n}}\right\}_{n=1}^{N}$, there exist nilpotent matrices, with degree of nilpotency 2 , such that the residues of $m(x, t ; \zeta)$ satisfy the polar conditions

$$
\begin{aligned}
& \operatorname{res}\left(m(x, t ; \zeta) ; \varsigma_{n}\right)=\lim _{\zeta \rightarrow \varsigma_{n}} m(x, t ; \zeta) g_{n}(x, t) \sigma_{-}, \quad n \in\{1,2, \ldots, N\}, \\
& \operatorname{res}\left(m(x, t ; \zeta) ; \overline{\varsigma_{n}}\right)=\sigma_{1} \overline{\operatorname{res}\left(m(x, t ; \zeta) ; \varsigma_{n}\right)} \sigma_{1}, \quad n \in\{1,2, \ldots, N\},
\end{aligned}
$$

where $g_{n}(x, t):=g_{n} \mathrm{e}^{2 \mathrm{i} k\left(\varsigma_{n}\right)\left(x+2 \lambda\left(\varsigma_{n}\right) t\right)}$, with $g_{n}=b_{n}\left(\varsigma_{n}-\overline{\varsigma_{n}}\right) \mathrm{e}^{-\mathrm{i} \widehat{\theta}\left(\varsigma_{n}\right)} \prod_{\substack{k=1 \\ k \neq n}}^{N}\left(\frac{\varsigma_{n}-\overline{\varsigma_{k}}}{\varsigma_{n}-\varsigma_{k}}\right)$ and $\widehat{\theta}(z):=\frac{\theta}{2}+\int_{-\infty}^{+\infty} \frac{\ln \left(1-|r(\mu)|^{2}\right)}{(\mu-z)} \frac{\mathrm{d} \mu}{2 \pi}$, and $b_{n}:=\left|b_{n}\right| \mathrm{e}^{\mathrm{i} \theta_{b_{n}}}, \theta_{b_{n}} \in\left\{ \pm \frac{\pi}{2}\right\}$, are (pure imaginary) constants associated with the direct scattering problem for the operator $\mathcal{O}^{\mathcal{D}}$; 
(iv) $\left.\operatorname{det}(m(x, t ; \zeta))\right|_{\zeta= \pm 1}=0$

(v) $m(x, t ; \zeta) \underset{\zeta \rightarrow 0}{=} \frac{1}{\zeta} \sigma_{2}+\mathcal{O}(1)$

(vi) as $\zeta \rightarrow \infty, \zeta \in \mathbb{C} \backslash\left(\sigma_{d} \cup \sigma_{c}\right), m(x, t ; \zeta)=\mathrm{I}+\mathcal{O}\left(\zeta^{-1}\right)$;

(vii) $m(x, t ; \zeta)$ possesses the following symmetry reductions, $m(x, t ; \zeta)=\sigma_{1} \overline{m(x, t ; \bar{\zeta})} \sigma_{1}$ and $m\left(x, t ; \frac{1}{\zeta}\right)=\zeta m(x, t ; \zeta) \sigma_{2}$.

For $r(\zeta) \in \mathcal{S}_{\mathbb{C}}^{1}(\mathbb{R}):(I)$ the RHP for $m(x, t ; \zeta)$ formulated above is uniquely (asymptotically) solvable; and $(I I) \Phi(x, t ; \zeta):=m(x, t ; \zeta) \exp \left(-\mathrm{i} k(\zeta)(x+2 \lambda(\zeta) t) \sigma_{3}\right)$ solves system (2), with

$$
u(x, t):=\mathrm{i} \lim _{\substack{\zeta \rightarrow \infty \\ \zeta \in \mathbb{C} \backslash\left(\sigma_{d} \cup \sigma_{c}\right)}}(\zeta(m(x, t ; \zeta)-\mathrm{I}))_{12}
$$

the solution of the Cauchy problem for the $\mathrm{D}_{f} \mathrm{NLSE}$, and

$$
\int_{+\infty}^{x}\left(|u(\xi, t)|^{2}-1\right) \mathrm{d} \xi:=-\mathrm{i} \lim _{\substack{\zeta \rightarrow \infty \\ \zeta \in \mathbb{C} \backslash\left(\sigma_{d} \cup \sigma_{c}\right)}}(\zeta(m(x, t ; \zeta)-\mathrm{I}))_{11}
$$

Sketch of Proof. From the definition of $\Phi(x, t ; \zeta), \zeta \in \mathbb{C} \backslash \mathbb{R}$, given in the Lemma, the $\zeta \rightarrow \infty$ and $\zeta \rightarrow 0$ asymptotics for $\Psi^{ \pm}(x, 0 ; \zeta)$ (respectively $a(\zeta)$ ) given in Lemma 2.1 (respectively Corollary 2.3), the (evolution) rule $r(\zeta) \rightarrow r(\zeta) \exp (4 \mathrm{i} k(\zeta) \lambda(\zeta) t)$, and the fact that $a(\zeta)$ is independent of $t$, one shows that

$$
\begin{aligned}
& \Phi(x, t ; \zeta) \underset{\zeta \rightarrow \infty}{=}\left(\mathrm{I}+\frac{1}{\zeta}\left(\begin{array}{cc}
\mathrm{i} \int_{+\infty}^{x}\left(|u(\xi, t)|^{2}-1\right) \mathrm{d} \xi & -\mathrm{i} u(x, t) \\
\mathrm{i} \overline{u(x, t)} & -\mathrm{i} \int_{+\infty}^{x}\left(|u(\xi, t)|^{2}-1\right) \mathrm{d} \xi
\end{array}\right)+\mathcal{O}\left(\frac{1}{\zeta^{2}}\right)\right) \mathrm{e}^{-\mathrm{i} k(\zeta)(x+2 \lambda(\zeta) t) \sigma_{3}}, \\
& \Phi(x, t ; \zeta) \underset{\zeta \rightarrow 0}{=}\left(\frac{1}{\zeta} \sigma_{2}+\mathcal{O}(1)\right) \mathrm{e}^{-\mathrm{i} k(\zeta)(x+2 \lambda(\zeta) t) \sigma_{3}}:
\end{aligned}
$$

this can also be obtained by mimicking calculations similar to those given in the proof of Lemma 2.1. From the monodromy and unimodularity relations given in Proposition 2.4, one shows that $\Phi(x, t ; \zeta)$, with $\Phi_{ \pm}(x, t ; \zeta):=\lim _{\varepsilon \downarrow 0} \Phi(x, t ; \zeta \pm \mathrm{i} \varepsilon)$, satisfies the "jump" relation

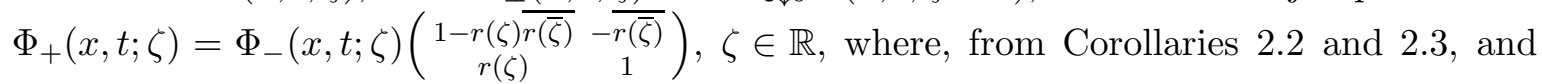
Remark 2.2, $r\left(\frac{1}{\zeta}\right)=-\overline{r(\bar{\zeta})}, r(\zeta)={ }_{\zeta \rightarrow \infty} \mathcal{O}\left(\zeta^{-1}\right), r(\zeta)={ }_{\zeta \rightarrow 0} \mathcal{O}(\zeta)$, and $r(\zeta) \in \mathcal{S}_{\mathbb{C}}(\mathbb{R})$. From the definition of $\Phi(x, t ; \zeta), \zeta \in \mathbb{C} \backslash \mathbb{R}$, and the representation for $a(\zeta)$ given in Lemma 2.2, one shows that, for $\zeta \in \mathbb{C}_{+}, \Phi(x, t ; \zeta)=\left(\begin{array}{cc}\mathrm{e}^{-\mathrm{i} \hat{\theta}(\zeta)} \prod_{n=1}^{N} \frac{\left(\zeta-\overline{\zeta_{n}}\right)}{\left(\zeta-\varsigma_{n}\right)} \Psi_{11}^{-}(x, t ; \zeta) & \Psi_{12}^{+}(x, t ; \zeta) \\ \mathrm{e}^{-\mathrm{i} \hat{\theta}(\zeta)} \prod_{n=1}^{N} \frac{\left(\zeta-\overline{\zeta_{n}}\right)}{\left(\zeta-\varsigma_{n}\right)} \Psi_{21}^{-}(x, t ; \zeta) & \Psi_{22}^{+}(x, t ; \zeta)\end{array}\right)$, and, for $\zeta \in \mathbb{C}_{-}, \Phi(x, t ; \zeta)=\left(\begin{array}{ll}\Psi_{11}^{+}(x, t ; \zeta) & \mathrm{e}^{\mathrm{i} \hat{\theta}(\zeta)} \prod_{n=1}^{N} \frac{\left(\zeta-\varsigma_{n}\right)}{\left(\zeta-\overline{\zeta_{n}}\right)} \Psi_{12}^{-}(x, t ; \zeta) \\ \Psi_{21}^{+}(x, t ; \zeta) & \mathrm{e}^{\mathrm{i} \hat{\theta}(\zeta)} \prod_{n=1}^{N} \frac{\left(\zeta-\varsigma_{n}\right)}{\left(\zeta-\overline{\zeta_{n}}\right)} \Psi_{22}^{-}(x, t ; \zeta)\end{array}\right)$, with $\widehat{\theta}(z)$ as defined in the Lemma; hence, it follows that the polar structure of $\Phi(x, t ; \zeta)$ is such that, for $\zeta \in \mathbb{C}_{+}$(respectively $\zeta \in \mathbb{C}_{-}$), the first (respectively second) column of $\Phi(x, t ; \zeta)$ has (a finitely denumerable number of) simple poles at $\left\{\varsigma_{n}\right\}_{n=1}^{N}$ (respectively $\left.\left\{\bar{\varsigma}_{n}\right\}_{n=1}^{N}\right)$, where $\varsigma_{n}:=\mathrm{e}^{\mathrm{i} \phi_{n}}, \phi_{n} \in(0, \pi)$. Since $\left.a(\zeta)\right|_{\zeta=\varsigma_{n}}=0, n \in\{1,2, \ldots, N\}$, it follows from the monodromy relation given in Proposition 2.4 that $\Psi^{-}\left(x, t ; \varsigma_{n}\right)=\Psi^{+}\left(x, t ; \varsigma_{n}\right)\left(\begin{array}{cc}0 & \overline{b\left(\overline{\varsigma_{n}}\right)} \\ b\left(\varsigma_{n}\right) & 0\end{array}\right)$; hence, from this relation and the definition of $\Phi(x, t ; \zeta)$ for $\zeta \in \mathbb{C}_{+}$, it follows that, with the choice $g_{n}=b_{n}\left(\varsigma_{n}-\overline{\varsigma_{n}}\right) \mathrm{e}^{-\mathrm{i} \widehat{\theta}\left(\varsigma_{n}\right)} \prod_{\substack{k=1 \\ k \neq n}}^{N} \frac{\left(\varsigma_{n}-\overline{\varsigma_{k}}\right)}{\left(\varsigma_{n}-\varsigma_{k}\right)}$, $\operatorname{res}\left(\Phi(x, t ; \zeta) ; \varsigma_{n}\right)=\lim _{\zeta \rightarrow \varsigma_{n}} \Phi(x, t ; \zeta) g_{n} \sigma_{-}, n \in\{1,2, \ldots, N\}$ : using the fact that $\widehat{\theta}\left(\overline{\varsigma_{n}}\right)=\overline{\widehat{\theta}\left(\varsigma_{n}\right)}$, a similar argument shows that $\operatorname{res}\left(\Phi(x, t ; \zeta) ; \overline{\varsigma_{n}}\right)=\sigma_{1} \overline{\operatorname{res}\left(\Phi(x, t ; \zeta) ; \varsigma_{n}\right)} \sigma_{1}, n \in\{1,2, \ldots, N\}$. From the definition of $\Phi(x, t ; \zeta), \zeta \in \mathbb{C} \backslash \mathbb{R}$, the jump condition for $\Phi(x, t ; \zeta)$, and the 
fact that $\Psi(x, t ; \zeta)$ satisfies the $\sigma_{1}$ symmetry reduction $\Psi(x, t ; \zeta)=\sigma_{1} \overline{\Psi(x, t ; \bar{\zeta})} \sigma_{1}$, it follows that $\sigma_{1} \overline{\Phi_{ \pm}(x, t ; \bar{\zeta})} \sigma_{1}=\Phi_{\mp}(x, t ; \zeta)$, namely, $\Phi(x, t ; \zeta)=\sigma_{1} \overline{\Phi(x, t ; \bar{\zeta})} \sigma_{1}$ : a similar argument, along with the fact that $r\left(\frac{1}{\zeta}\right)=-\overline{r(\bar{\zeta})}$, shows that $\Phi\left(x, t ; \frac{1}{\zeta}\right)=\zeta \Phi(x, t ; \zeta) \sigma_{2}$. The fact that $\left.\operatorname{det}(\Phi(x, t ; \zeta))\right|_{\zeta= \pm 1}=0$ is a consequence of the definition of $\Phi(x, t ; \zeta)$, the asymptotics (proof of Lemma 2.3) $a(\zeta)={ }_{\zeta \rightarrow \pm 1} \widehat{a}_{o}^{ \pm}+\mathcal{O}(\zeta \mp 1)$, with $\widehat{a}_{o}^{ \pm} \neq 0$, and the degeneracy condition $\left.\operatorname{det}(\Psi(x, t ; \zeta))\right|_{\zeta= \pm 1}=0$ (Proposition 2.4). Now, setting $m(x, t ; \zeta):=\Phi(x, t ; \zeta) \exp (\mathrm{i} k(\zeta)(x+$ $2 \lambda(\zeta) t) \sigma_{3}$ ), one arrives at items (ii)-(vii) of the Lemma: item (i) is an immediate consequence of the polar structure of $m(x, t ; \zeta)$ and the fact that $m(x, t ; \zeta)$ has a jump discontinuity across $\mathbb{R}$ (oriented from $-\infty$ to $+\infty$ ). Since the solution of the RHP for $m(x, t ; \zeta)$ can be written as the ordered factorisation $m(x, t ; \zeta)=\left(\mathrm{I}+\Delta_{o}(x, t) \zeta^{-1}\right) \mathcal{P}(x, t ; \zeta) m_{d}(x, t ; \zeta) m^{c}(x, t ; \zeta)$, where $m^{c}(x, t ; \zeta)\left(=\sigma_{1} \overline{m^{c}(x, t ; \bar{\zeta})} \sigma_{1}\right) \in \mathrm{SL}(2, \mathbb{C}), m_{d}(x, t ; \zeta)\left(=\sigma_{1} \overline{m_{d}(x, t ; \bar{\zeta})} \sigma_{1}\right) \in \mathrm{SL}(2, \mathbb{C})$ has

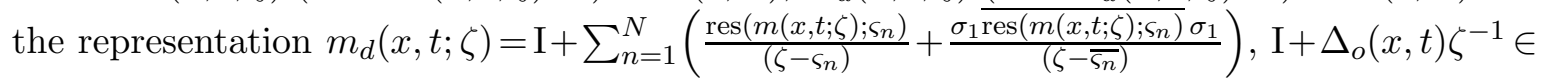
$\mathrm{M}(2, \mathbb{C})$, with $\Delta_{o}(x, t)\left(=\sigma_{1} \overline{\Delta_{o}(x, t)} \sigma_{1}\right) \in \mathrm{GL}(2, \mathbb{C})$, is analytic in a punctured neighbourhood of the origin, and $\mathcal{P}(x, t ; \zeta)\left(=\sigma_{1} \overline{\mathcal{P}(x, t ; \bar{\zeta})} \sigma_{1}\right) \in \mathrm{GL}(2, \mathbb{C})$ is chosen so that $\Delta_{o}(x, t)$ $\left(=\mathcal{P}(x, t ; 0) m_{d}(x, t ; 0) m^{c}(x, t ; 0) \sigma_{2}\right)$ is idempotent, and, for ||$r(\cdot) \|_{\mathcal{L}^{\infty}(\mathbb{R})}:=\sup _{\zeta \in \mathbb{R}}|r(\zeta)|<1$, $\frac{1}{2}\left(\mathcal{G}^{\dagger}(x, t ; \bar{\zeta})+\mathcal{G}(x, t ; \zeta)\right)$, with ${ }^{\dagger}$ denoting Hermitian conjugation, is positive definite, it follows that, either due to a classical result of Gohberg and Krein (see [23] for details) or Zhou's skew Schwarz reflection invariant symmetry principle (see Theorem 9.3 in [31] and Theorem 2.2.10 in [28]: see, also, [32]), and the fact that $\sum_{j=1}^{2} \mathrm{k}_{j}=\frac{1}{2 \pi} \int_{\mathbb{R}} \mathrm{d}(\arg (\operatorname{det}(\mathcal{G}(x, t ; \xi))))=0$, where $\mathrm{k}_{j}$, $j \in\{1,2\}$, denote the partial indices of the RHP factorisation for $m(x, t ; \zeta)$, and the winding number of $(1-r(\zeta) \overline{r(\bar{\zeta})})$ vanishes (see Proposition 1.38 in [33]), that is, $\mathrm{W}_{\mathbb{R}}(1-r(\zeta) \overline{r(\bar{\zeta})})=$ $\sum_{l \in\{ \pm\}} \mathrm{s}(l) \mathrm{n}(l)=0$, where $\mathrm{s}(+)=-\mathrm{s}(-)=1$ and $\mathrm{n}( \pm):=$ card $\left\{\varsigma_{n} ; \mp \Im\left(\varsigma_{n}\right)>0\right\}$, the RHP for $m(x, t ; \zeta)$ is asymptotically solvable: uniqueness follows from a standard argument (see, for example, Chapter 7 of [25]). The fact that $u(x, t)$, defined by Eq. (3), satisfies the $\mathrm{D}_{f} \mathrm{NLSE}^{-}$ follows from the $\zeta \rightarrow \infty$ asymptotics of $m(x, t ; \zeta)$ (see the $\zeta \rightarrow \infty$ asymptotics for $\Phi(x, t ; \zeta)$ given at the beginning of the proof and recall the definition of $m(x, t ; \zeta)$ in terms of $\Phi(x, t ; \zeta))$. The fact that $\Phi(x, t ; \zeta)=m(x, t ; \zeta) \exp \left(-\mathrm{i} k(\zeta)(x+2 \lambda(\zeta) t) \sigma_{3}\right)$ satisfies system $(2)$ is a straightforward calculation.

Remark 2.4. The precise sense in which the limits for $m(x, t ; \zeta)$ are taken is explained in detail in Chapter 7 of [25]. It is convenient to define the formal (space of) scattering data as $\mathcal{S D}:=\left\{\left(\begin{array}{c}1-r(\zeta) \overline{r(\bar{\zeta})}-\overline{r(\bar{\zeta})} \\ r(\zeta)\end{array}\right), \zeta \in \mathbb{R}\right\} \cup\left(\cup_{n=1}^{N}\left\{\phi_{n}, g_{n}:=\left|g_{n}\right| \mathrm{e}^{\mathrm{i} \phi_{g_{n}}}\right\}\right)\left(\subset \mathcal{M}_{\infty} \times \mathbb{R}^{3 n}\right.$, where $\mathcal{M}_{\infty}$ is some, unspecified for the time being, infinite-dimensional space): setting $\mathcal{S} \mathcal{D}_{o}:=$ $\left\{\left(\begin{array}{c}1-r(\zeta) \overline{r(\bar{\zeta})}-\overline{r(\bar{\zeta})} \\ r(\zeta)\end{array}\right), \zeta \in \mathbb{R}\right\} \cup\left(\cup_{n=1}^{N}\left\{\phi_{n}\right\}\right)(\subset \mathcal{S D})$, one notes from the expression for $\theta$ given in Lemma 2.2 that $\theta: \mathcal{S} \mathcal{D}_{o} \rightarrow[0,2 \pi)$, namely, $\{r(\zeta), \zeta \in \mathbb{R}\} \cup\left(\cup_{n=1}^{N}\left\{\phi_{n}\right\}\right) \mapsto-2 \sum_{n=1}^{N} \phi_{n}-$ $\int_{-\infty}^{+\infty} \frac{\ln \left(1-|r(\mu)|^{2}\right)}{\mu} \frac{\mathrm{d} \mu}{2 \pi}$.

Since $a(\zeta)$ is independent of $t$, it follows from a well-known result [9] that $\ln (a(\zeta))$ is the generating function for the integrals of motion, namely, $\ln (a(\zeta))=\underset{\zeta \in \mathbb{C}_{+}}{\substack{\operatorname{m} \\ \sum_{n=0}^{\infty}}} \mathrm{I}_{n}[u, \bar{u}] \zeta^{-n}+$ $\mathcal{O}\left(|\zeta|^{-\infty}\right)$, where $\mathrm{I}_{n}[u, \bar{u}]$ are local functionals of $u(x, t)$ and $\overline{u(x, t)}$. This leads to the following (trace identity)

Proposition 2.6.

$$
\int_{-\infty}^{+\infty}\left(|u(\xi, t)|^{2}-1\right) \mathrm{d} \xi=-2 \sum_{n=1}^{N} \sin \left(\phi_{n}\right)-\int_{-\infty}^{+\infty} \ln \left(1-|r(\mu)|^{2}\right) \frac{\mathrm{d} \mu}{2 \pi} .
$$

Proof. From Corollary 2.3, it follows that, for $\zeta \in \mathbb{C}_{+}, \ln (a(\zeta))={ }_{\zeta \rightarrow \infty} \frac{\mathrm{i} \theta}{2}+\left(\mathrm{i} \int_{-\infty}^{+\infty}\left(|u(\xi, t)|^{2}-\right.\right.$ 1) $\mathrm{d} \xi) \zeta^{-1}+\mathcal{O}\left(\zeta^{-2}\right)$. Using the representation for $a(\zeta)$ given in Lemma 2.2 and the fact that $r(\zeta) \in \mathcal{S}_{\mathbb{C}}(\mathbb{R})$, one deduces that, for $\zeta \in \mathbb{C}_{+}, \ln (a(\zeta))={ }_{\zeta \rightarrow \infty} \frac{\mathrm{i} \theta}{2}+\left(\sum_{n=1}^{N}\left(\overline{\varsigma_{n}}-\varsigma_{n}\right)+\int_{-\infty}^{+\infty} \ln (1-\right.$ 
$\left.\left.|r(\mu)|^{2}\right) \frac{\mathrm{d} \mu}{2 \pi \mathrm{i}}\right) \zeta^{-1}+\mathcal{O}\left(\zeta^{-2}\right)$ : noting that $\sum_{n=1}^{N}\left(\overline{\varsigma_{n}}-\varsigma_{n}\right)=-2 \mathrm{i} \sum_{n=1}^{N} \sin \left(\phi_{n}\right)$, with $\left|\sum_{n=1}^{N}\left(\overline{\varsigma_{n}}-\varsigma_{n}\right)\right| \leqslant$ $2 N$, equating the above (two) $\zeta \rightarrow \infty$ asymptotics for $\ln (a(\zeta)$ ), one obtains the result stated in the Proposition.

Heretofore, the discussion has focused on the general case when $\sigma_{\mathcal{O}^{\mathcal{D}}}:=\operatorname{spec}\left(\mathcal{O}^{\mathcal{D}}\right)=\sigma_{d} \cup \sigma_{c}$, with $\sigma_{d} \cap \sigma_{c}=\emptyset$, where $\sigma_{d}$ and $\sigma_{c}$ subsume, respectively, the " $N$-dark soliton" (discrete spectrum) and "continuum" (continuous spectrum) contributions to the solution of the Cauchy problem for the $\mathrm{D}_{f} \mathrm{NLSE}$ : in this work, only the asymptotics of solutions to the Cauchy problem for the $\mathrm{D}_{f}$ NLSE in the so-called solitonless sector, that is, $\sigma_{d} \equiv \emptyset$ and $\sigma_{c} \not \equiv \emptyset$, are studied (the asymptotic analysis for the general case $\left\{\sigma_{d}, \sigma_{c}\right\} \not \equiv\{\emptyset, \emptyset\}$ is in progress). Essentially, in the construction of the asymptotic solution of the RHP for the $\mathrm{M}(2, \mathbb{C})$-valued function $m(x, t ; \zeta)$ stated in Lemma 2.4, $\sigma_{d} \equiv \emptyset$ means dropping all reference to the polar structure of $m(x, t ; \zeta)$ at $\left\{\varsigma_{n}, \overline{\varsigma_{n}}\right\}_{n=1}^{N}$ : all terms depending, in any way, on $\left\{\varsigma_{n}, \overline{\varsigma_{n}}\right\}_{n=1}^{N}$ are absent from the specification of the RHP for $m(x, t ; \zeta)$; in particular, $a(\zeta)=\mathrm{e}^{\mathrm{i} \frac{\theta}{2}} \exp \left(-\int_{-\infty}^{+\infty} \frac{\ln \left(1-|r(\mu)|^{2}\right)}{(\mu-\zeta)} \frac{\mathrm{d} \mu}{2 \pi \mathrm{i}}\right)$, $\zeta \in \mathbb{C}_{+}$, with $0 \leqslant \theta=-\int_{-\infty}^{+\infty} \frac{\ln \left(1-|r(\mu)|^{2}\right)}{\mu} \frac{\mathrm{d} \mu}{2 \pi}<2 \pi, m_{d}(x, t ; \zeta) \equiv \mathrm{I}$, and it is sufficient to take $\mathcal{P}(x, t ; \zeta) \equiv \mathrm{I}$. One is lead to the following (normalised at $\infty$ ) RHP for $m(x, t ; \zeta)$ :

Lemma 2.5. Let $u(x, t)$ be the solution of the Cauchy problem for the $\mathrm{D}_{f} \mathrm{NLSE}$ with finitedensity initial data $u(x, 0):=u_{o}(x)={ }_{x \rightarrow \pm \infty} u_{o}( \pm \infty)(1+o(1))$, where $u_{o}( \pm \infty):=\exp \left(\frac{\mathrm{i}(1 \mp 1) \theta}{2}\right)$, $0 \leqslant \theta=-\int_{-\infty}^{+\infty} \frac{\ln \left(1-|r(\mu)|^{2}\right)}{\mu} \frac{\mathrm{d} \mu}{2 \pi}<2 \pi, u_{o}(x) \in \mathbf{C}^{\infty}(\mathbb{R})$, and $u_{o}(x)-u_{o}( \pm \infty) \in \mathcal{S}_{\mathbb{C}}\left(\mathbb{R}_{ \pm}\right)$. Then $m(x, t ; \zeta): \mathbb{C} \backslash \sigma_{c} \rightarrow \mathrm{M}(2, \mathbb{C})$ solves the following $\mathrm{RHP}$ :

(i) $m(x, t ; \zeta)$ is piecewise holomorphic $\forall \zeta \in \mathbb{C} \backslash \sigma_{c}$;

(ii) $m_{ \pm}(x, t ; \zeta):=\lim _{\varepsilon \downarrow 0} m(x, t ; \zeta \pm \mathrm{i} \varepsilon)$ satisfy the jump condition

$$
m_{+}(x, t ; \zeta)=m_{-}(x, t ; \zeta) \mathcal{G}(x, t ; \zeta), \quad \zeta \in \mathbb{R}
$$

where $\mathcal{G}(x, t ; \zeta):=\exp \left(-\mathrm{i} k(\zeta)(x+2 \lambda(\zeta) t) \operatorname{ad}\left(\sigma_{3}\right)\right)\left(\begin{array}{cc}1-r(\zeta) \overline{r(\bar{\zeta})} & -\overline{r(\bar{\zeta})} \\ r(\zeta) & 1\end{array}\right)$, and $r(\zeta)$, the reflection coefficient of the direct scattering problem for $\mathcal{O}^{\mathcal{D}}$, satisfies $r(\zeta)={ }_{\zeta \rightarrow 0} \mathcal{O}(\zeta)$, $r(\zeta)={ }_{\zeta \rightarrow \infty} \mathcal{O}\left(\zeta^{-1}\right), r\left(\frac{1}{\zeta}\right)=-\overline{r(\bar{\zeta})}$, and $r(\zeta) \in \mathcal{S}_{\mathbb{C}}^{1}(\mathbb{R}) ;$

(iii) $\left.\operatorname{det}(m(x, t ; \zeta))\right|_{\zeta= \pm 1}=0$;

(iv) $m(x, t ; \zeta) \underset{\zeta \rightarrow 0}{=} \frac{1}{\zeta} \sigma_{2}+\mathcal{O}(1)$

(v) as $\zeta \rightarrow \infty, \zeta \in \mathbb{C} \backslash \sigma_{c}, m(x, t ; \zeta)=\mathrm{I}+\mathcal{O}\left(\zeta^{-1}\right)$;

(vi) $m(x, t ; \zeta)$ possesses the following symmetry reductions, $m(x, t ; \zeta)=\sigma_{1} \overline{m(x, t ; \bar{\zeta})} \sigma_{1}$ and $m\left(x, t ; \frac{1}{\zeta}\right)=\zeta m(x, t ; \zeta) \sigma_{2}$.

For $r(\zeta) \in \mathcal{S}_{\mathbb{C}}^{1}(\mathbb{R}):(I)$ the RHP for $m(x, t ; \zeta)$ formulated above is uniquely (asymptotically) solvable; and $(I I) \Phi(x, t ; \zeta):=m(x, t ; \zeta) \exp \left(-\mathrm{i} k(\zeta)(x+2 \lambda(\zeta) t) \sigma_{3}\right)$, with $\Phi(x, t ; \zeta)$ in terms of $\Psi^{ \pm}(x, t ; \zeta)$ as defined in Lemma 2.4 for $a(\zeta)=\exp \left(\frac{\mathrm{i} \theta}{2}\right) \exp \left(-\int_{-\infty}^{+\infty} \frac{\ln \left(1-|r(\mu)|^{2}\right)}{(\mu-\zeta)} \frac{\mathrm{d} \mu}{2 \pi \mathrm{i}}\right), \zeta \in \mathbb{C}_{+}$, solves system (2),

$$
u(x, t):=\mathrm{i} \lim _{\substack{\zeta \rightarrow \infty \\ \zeta \in \mathbb{C} \backslash \sigma_{c}}}(\zeta(m(x, t ; \zeta)-\mathrm{I}))_{12}
$$

is the solution of the Cauchy problem for the $\mathrm{D}_{f} \mathrm{NLSE}$, and

$$
\int_{+\infty}^{x}\left(|u(\xi, t)|^{2}-1\right) \mathrm{d} \xi:=-\mathrm{i} \lim _{\substack{\zeta \rightarrow \infty \\ \zeta \in \mathbb{C} \backslash \sigma_{c}}}(\zeta(m(x, t ; \zeta)-\mathrm{I}))_{11} .
$$


Corollary 2.4. In the solitonless sector $\left(\sigma_{d} \equiv \emptyset\right)$,

$$
\int_{-\infty}^{+\infty}\left(|u(\xi, t)|^{2}-1\right) \mathrm{d} \xi=-\int_{-\infty}^{+\infty} \ln \left(1-|r(\mu)|^{2}\right) \frac{\mathrm{d} \mu}{2 \pi} .
$$

Proof. Since, in the solitonless sector, $\Delta_{a} \equiv \emptyset$ (Lemma 2.2), it follows that $\sum_{n=1}^{N} \sin \left(\phi_{n}\right)=$ 0 : the result stated in the Corollary is now a consequence of Proposition 2.6.

Lemma 2.6. The solution of the RHP for $m(x, t ; \zeta): \mathbb{C} \backslash \sigma_{c} \rightarrow \mathrm{M}(2, \mathbb{C})$ formulated in Lemma 2.5 can be written as the following ordered product,

$$
m(x, t ; \zeta)=\left(\mathrm{I}+\Delta_{o}(x, t) \zeta^{-1}\right) m^{c}(x, t ; \zeta),
$$

where $\Delta_{o}(x, t) \in \mathrm{GL}(2, \mathbb{C})$, $\left.\operatorname{det}\left(\mathrm{I}+\Delta_{o}(x, t) \zeta^{-1}\right)\right|_{\zeta= \pm 1}=0, \sigma_{1} \overline{\Delta_{o}(x, t)} \sigma_{1}=\Delta_{o}(x, t)$, with finite, order 2 , matrix involutive structure,

$$
\Delta_{o}(x, t)=\left(\begin{array}{cc}
\Delta_{o}^{11}(x, t) \mathrm{e}^{\mathrm{i}(k+1 / 2) \pi} & \sqrt{1+\left(\Delta_{o}^{11}(x, t)\right)^{2}} \mathrm{e}^{-\mathrm{i} \vartheta(x, t)} \\
\sqrt{1+\left(\Delta_{o}^{11}(x, t)\right)^{2}} \mathrm{e}^{\mathrm{i} \vartheta(x, t)} & \Delta_{o}^{11}(x, t) \mathrm{e}^{-\mathrm{i}(k+1 / 2) \pi}
\end{array}\right), \quad k \in \mathbb{Z},
$$

and $\Delta_{o}^{11}(x, t): \mathbb{R} \times \mathbb{R} \rightarrow \mathbb{R}$ and $\vartheta(x, t): \mathbb{R} \times \mathbb{R} \rightarrow \mathbb{R} \backslash\{0\}$ obtained from the determining relation $\Delta_{o}(x, t) m^{c}(x, t ; 0)=\sigma_{2}\left(\operatorname{det}\left(\Delta_{o}(x, t)\right)=\operatorname{det}\left(\sigma_{2}\right)=-1\right)$, and $m^{c}(x, t ; \zeta): \mathbb{C} \backslash \sigma_{c} \rightarrow$ $\mathrm{SL}(2, \mathbb{C})$ solves the following RHP: (1) $\mathrm{m}^{c}(x, t ; \zeta)$ is piecewise holomorphic $\forall \zeta \in \mathbb{C} \backslash \sigma_{c}$; (2) $m_{+}^{c}(x, t ; \zeta)=m_{-}^{c}(x, t ; \zeta) \mathcal{G}(x, t ; \zeta), \zeta \in \mathbb{R}$, where $\mathcal{G}(x, t ; \zeta)$ is defined in Lemma 2.5; (3) as $\zeta \rightarrow \infty, \zeta \in \mathbb{C} \backslash \sigma_{c}, m^{c}(x, t ; \zeta)=\mathrm{I}+\mathcal{O}\left(\zeta^{-1}\right)$; and (4) $m^{c}(x, t ; \zeta)$ satisfies the symmetry reduction $m^{c}(x, t ; \zeta)=\sigma_{1} \overline{m^{c}(x, t ; \bar{\zeta})} \sigma_{1}$ and the condition $\left(m^{c}(x, t ; 0) \sigma_{2}\right)^{2}=\mathrm{I}$.

Proof. Follows from the ordered (matrix) product given in Eq. (7) and Lemma 2.5.

Remark 2.5. The $\mathrm{M}(2, \mathbb{C})$-valued function $\mathrm{I}+\Delta_{o}(x, t) \zeta^{-1}$, with $\Delta_{o}(x, t) \in \mathrm{GL}(2, \mathbb{C})$, which is analytic in a punctured neighbourhood of the origin, plays a role somewhat analogous to the (analytic) matrix-valued function $E(z)$ in [34. One can take a Laurent expansion of the form I $+\sum_{j \in \mathbb{Z}} \widetilde{\Delta}_{j}(x, t) \zeta^{-j}$, with $\sigma_{1}{\widetilde{\widetilde{\Delta}_{j}}(x, t)}_{\sigma_{1}}=\widetilde{\Delta}_{j}(x, t)$ and $\widetilde{\Delta}_{j}(x, t) \in \operatorname{GL}(2, \mathbb{C})$, for the first factor on the right-hand side of the ordered product in Eq. (7); however, in order to satisfy the conditions imposed by Lemma 2.5 on the solution of the RHP for $m(x, t ; \zeta)$, one shows that $\widetilde{\Delta}_{j}(x, t)=\left(\begin{array}{ll}0 & 0 \\ 0 & 0\end{array}\right), j \in \mathbb{Z} \backslash\{1\}$, and only the $\widetilde{\Delta}_{1}(x, t):=\Delta_{o}(x, t)$ term is non-zero.

Hence, the main technical thrust revolves around the explicit (asymptotic) construction of $m^{c}(x, t ; \zeta)$, and the determination of $\Delta_{o}(x, t)$ is relegated to a purely algebraic computation, namely, $\Delta_{o}(x, t)=\sigma_{2}\left(m^{c}(x, t ; 0)\right)^{-1}$.

\section{The Beals-Coifman Construction, the Deift-Zhou Non-Lin- ear Steepest-Descent Method, and Summary of Results}

In this section, the Beals-Coifman (BC) construction [24] for the representation of the solution of the (matrix) RHP for $m^{c}(x, t ; \zeta)$ on $\mathbb{R}$ stated in Lemma 2.6 is succinctly recapitulated, the Deift-Zhou (DZ) non-linear steepest-descent method [27] for the asymptotic analysis of the RHP for $m^{c}(x, t ; \zeta)$ is discussed in detail, and the results of this work are summarised in Theorems 3.1-3.3.

The solution framework for matrix RHPs of the type stated in Section 2 (for $m^{c}(x, t ; \zeta)$ ) is constructed according to the BC formulation [24], a self-contained synopsis of which, with some requisite preamble, follows (explicit $x, t$ dependences are temporarily suppressed). One agrees to call a contour $\Gamma^{\sharp}$ oriented if: (1) $\mathbb{C} \backslash \Gamma^{\sharp}$ has finitely many open connected components; (2) $\mathbb{C} \backslash \Gamma^{\sharp}$ is the disjoint union of two, possibly disconnected, open regions, denoted by $\mho^{+}$ and $\mho^{-}$; and (3) $\Gamma^{\sharp}$ may be viewed as either the positively oriented boundary for $\mho^{+}$or the negatively oriented boundary for $\mho^{-}\left(\mathbb{C} \backslash \Gamma^{\sharp}\right.$ is coloured by two colours, namely, \pm ). Let $\Gamma^{\sharp}$, 
as a closed set, be the union of finitely many oriented simple piecewise-smooth arcs. Denote the set of all self-intersections of $\Gamma^{\sharp}$ by $\widehat{\Gamma}^{\sharp}$ (with $\operatorname{card}\left(\widehat{\Gamma}^{\sharp}\right)<\infty$ assumed throughout). Set $\widetilde{\Gamma}^{\sharp}:=\Gamma^{\sharp} \backslash \widehat{\Gamma}^{\sharp}$. The BC construction for the solution of a (matrix) RHP, in the absence of a discrete spectrum and spectral singularities [28], on an oriented contour $\Gamma^{\sharp}$ consists of finding an $\mathrm{M}(2, \mathbb{C})$-valued function $\mathcal{X}(\lambda)$ such that: $(1) \mathcal{X}(\lambda)$ is piecewise holomorphic $\forall \lambda \in \mathbb{C} \backslash \Gamma^{\sharp}$, $\left.\mathcal{X}(\lambda)\right|_{\mathbb{C} \backslash \Gamma^{\sharp}}$ has continuous extension to $\widetilde{\Gamma}^{\sharp}$, and $\lim _{\substack{\lambda^{\prime} \in \pm \text { side of } \tilde{\Gamma}^{\sharp} \\ \lambda^{\prime} \rightarrow \lambda}} \int_{\widetilde{\Gamma}^{\sharp}}\left|\mathcal{X}\left(\lambda^{\prime}\right)-\mathcal{X}_{ \pm}(\lambda)\right|^{2}|\mathrm{~d} \lambda|=0 ;(2)$ $\mathcal{X}_{+}(\lambda)=\mathcal{X}_{-}(\lambda) v(\lambda), \lambda \in \widetilde{\Gamma}^{\sharp}$, for some "jump" matrix $v(\lambda): \widetilde{\Gamma}^{\sharp} \rightarrow \mathrm{GL}(2, \mathbb{C})$; and (3) uniformly as $\lambda \rightarrow \infty, \lambda \in \mathbb{C} \backslash \Gamma^{\sharp}, \mathcal{X}(\lambda)=\mathrm{I}+\mathcal{O}\left(\lambda^{-1}\right)$. Let $v(\lambda):=\left(\mathrm{I}-w_{-}(\lambda)\right)^{-1}\left(\mathrm{I}+w_{+}(\lambda)\right), \lambda \in \widetilde{\Gamma}^{\sharp}$, be a factorisation for $v(\lambda)$, where $w_{ \pm}(\lambda)$ are some upper/lower or lower/upper triangular (depending on the orientation of $\Gamma^{\sharp}$ ) nilpotent matrices, with degree of nilpotency 2, and $w_{ \pm}(\lambda) \in \cap_{p \in\{2, \infty\}} \mathcal{L}_{\mathrm{M}_{2}(\mathbb{C})}^{p}\left(\widetilde{\Gamma}^{\sharp}\right)$ (if $\widetilde{\Gamma}^{\sharp}$ is unbounded, one requires that $w_{ \pm}(\lambda)=\underset{\substack{\lambda \rightarrow \infty \\ \lambda \in \Gamma^{\sharp}}}{\left(\begin{array}{ll}0 & 0 \\ 0 & 0\end{array}\right) \text { ). Define }}$ $w(\lambda):=w_{+}(\lambda)+w_{-}(\lambda)$, and introduce the Cauchy operators on $\mathcal{L}_{\mathrm{M}_{2}(\mathbb{C})}^{2}\left(\Gamma^{\sharp}\right),\left(C_{ \pm} f\right)(\lambda):=$ $\lim \underset{\lambda^{\prime} \in \pm \text { side of } \Gamma^{\sharp}}{\lambda^{\prime} \rightarrow \lambda} \int_{\Gamma^{\sharp}} \frac{f(z)}{\left(z-\lambda^{\prime}\right)} \frac{\mathrm{d} z}{2 \pi \mathrm{i}}$, where $\lambda^{\prime} \rightarrow \lambda\left(\in \Gamma^{\sharp}\right), \lambda^{\prime} \in \pm$ side of $\Gamma^{\sharp}$, denotes the non-tangential limits from the \pm -sides of $\Gamma^{\sharp}$ at $\lambda \in \Gamma^{\sharp}, f(\cdot) \in \mathcal{L}_{\mathrm{M}_{2}(\mathbb{C})}^{2}\left(\Gamma^{\sharp}\right)$, with $C_{ \pm}: \mathcal{L}_{\mathrm{M}_{2}(\mathbb{C})}^{2}\left(\Gamma^{\sharp}\right) \rightarrow \mathcal{L}_{\mathrm{M}_{2}(\mathbb{C})}^{2}\left(\Gamma^{\sharp}\right)$ bounded in operator norm, namely, $\left\|C_{ \pm}\right\|_{\mathcal{N}\left(\Gamma^{\sharp}\right)}<\infty$, where $\mathcal{N}(*)$ denotes the space of all bounded linear operators acting from $\mathcal{L}_{\mathrm{M}_{2}(\mathbb{C})}^{2}(*)$ into $\mathcal{L}_{\mathrm{M}_{2}(\mathbb{C})}^{2}(*)$, and $\left\|\left(C_{ \pm} f\right)(\cdot)\right\|_{\mathcal{L}_{\mathrm{M}_{2}(\mathbb{C})}^{2}(*)} \leqslant$ const. $\|f(\cdot)\|_{\mathcal{L}_{\mathrm{M}_{2}(\mathbb{C})}^{2}(*)}$. Introduce the BC operator on $\mathcal{L}_{\mathrm{M}_{2}(\mathbb{C})}^{2}(*), C_{w} f:=C_{+}\left(f w_{-}\right)+C_{-}\left(f w_{+}\right)$; moreover, since $\mathbb{C} \backslash \Gamma^{\sharp}$ can be coloured by two colours, $\pm C_{ \pm}$are complementary projections [28], $C_{+}^{2}=C_{+}, C_{-}^{2}=-C_{-}, C_{+} C_{-}=C_{-} C_{+}=\mathbf{0}$, and $C_{+}-C_{-}=\mathbf{1}$ : in the case that $C_{+}$and $-C_{-}$ are complementary, the contour $\Gamma^{\sharp}$ can always be oriented in such a way that the \pm regions lie on the \pm sides of the contour, respectively. Re-introducing $x, t$ dependences, specialising the $\mathrm{BC}$ formulation to the solution of the RHP for $m^{c}(x, t ; \zeta)$ on $\sigma_{c}=\mathbb{R}$ (oriented from $-\infty$ to $+\infty)$, and defining $\mathcal{G}(x, t ; \zeta):=\left(\mathrm{I}-w_{-}^{\mathcal{G}}(x, t ; \zeta)\right)^{-1}\left(\mathrm{I}+w_{+}^{\mathcal{G}}(x, t ; \zeta)\right)$, the integral representation for $m^{c}(x, t ; \zeta)$ is given by the following

\section{Lemma 3.1 (Beals and Coifman [24]). Let}

$$
\mu^{\mathcal{G}}(x, t ; \zeta)=m_{+}^{c}(x, t ; \zeta)\left(\mathrm{I}+w_{+}^{\mathcal{G}}(x, t ; \zeta)\right)^{-1}=m_{-}^{c}(x, t ; \zeta)\left(\mathrm{I}-w_{-}^{\mathcal{G}}(x, t ; \zeta)\right)^{-1} .
$$

If $\mu^{\mathcal{G}}(x, t ; \zeta) \in \mathrm{I}+\mathcal{L}_{\mathrm{M}_{2}(\mathbb{C})}^{2}\left(\sigma_{c}\right)$ 凹: $=\left\{\mathrm{I}+h(\cdot) ; h(\cdot) \in \mathcal{L}_{\mathrm{M}_{2}(\mathbb{C})}^{2}\left(\sigma_{c}\right)\right\}$ solves the linear singular integral equation

$$
\left(\mathbf{1}-C_{w^{\mathcal{G}}}\right)\left(\mu^{\mathcal{G}}(x, t ; \zeta)-\mathrm{I}\right)=C_{w^{\mathcal{G}}} \mathrm{I}=C_{+}\left(w_{-}^{\mathcal{G}}(x, t ; \zeta)\right)+C_{-}\left(w_{+}^{\mathcal{G}}(x, t ; \zeta)\right), \quad \zeta \in \sigma_{c},
$$

where 1 is the identity operator on $\mathrm{I}+\mathcal{L}_{\mathrm{M}_{2}(\mathbb{C})}^{2}\left(\sigma_{c}\right)$, then the solution of the RHP for $m^{c}(x, t ; \zeta)$ is

$$
m^{c}(x, t ; \zeta)=\mathrm{I}+\int_{\sigma_{c}} \frac{\mu^{\mathcal{G}}(x, t ; z) w^{\mathcal{G}}(x, t ; z)}{(z-\zeta)} \frac{\mathrm{d} z}{2 \pi \mathrm{i}}, \quad \zeta \in \mathbb{C} \backslash \sigma_{c},
$$

where $\mu^{\mathcal{G}}(x, t ; z):=\left(\left(\mathbf{1}-C_{w^{\mathcal{G}}}\right)^{-1} \mathrm{I}\right)(x, t ; z)$, and $w^{\mathcal{G}}(x, t ; z):=\sum_{l \in\{ \pm\}} w_{l}^{\mathcal{G}}(x, t ; z)$.

Remark 3.1. The central difficulty subsumed in the analysis is concerned with the existence and invertibility of the operator $\mathbf{1}-C_{w^{\mathcal{G}}}$, in particular $\left(\mathbf{1}-C_{w^{\mathcal{G}}}\right)^{-1}$, as an operator from $\mathcal{L}_{\mathrm{M}_{2}(\mathbb{C})}^{2}\left(\sigma_{c}\right) \rightarrow \mathcal{L}_{\mathrm{M}_{2}(\mathbb{C})}^{2}\left(\sigma_{c}\right)$. In Sections 4 and 5 , it is shown by explicit construction that, asymptotically, $\left.\operatorname{ker}\left(\mathbf{1}-C_{w^{\mathcal{G}}}\right)\right|_{\mathcal{L}_{\mathrm{M}_{2}(\mathbb{C})}^{2}\left(\sigma_{c}\right)}=\emptyset$, or, alternatively, the Fredholm index of $\mathbf{1}-C_{w^{\mathcal{G}}}$ on $\mathcal{L}_{\mathrm{M}_{2}(\mathbb{C})}^{2}\left(\sigma_{c}\right)$ is zero, that is,

$$
\left.i\left(\mathbf{1}-C_{w^{\mathcal{G}}}\right)\right|_{\mathcal{L}_{\mathrm{M}_{2}(\mathbb{C})}^{2}\left(\sigma_{c}\right)}:=\left.\left(\operatorname{dim} \operatorname{ker}\left(\mathbf{1}-C_{w^{\mathcal{G}}}\right)-\operatorname{dim} \operatorname{coker}\left(\mathbf{1}-C_{w^{\mathcal{G}}}\right)\right)\right|_{\mathcal{L}_{\mathrm{M}_{2}(\mathbb{C})}^{2}}\left(\sigma_{c}\right)=0 .
$$

\footnotetext{
${ }^{1}$ For $f(\zeta) \in \mathrm{I}+\mathcal{L}_{\mathrm{M}_{2}(\mathbb{C})}^{2}(*),\|f(\cdot)\|_{\mathrm{I}+\mathcal{L}_{\mathrm{M}_{2}(\mathbb{C})}^{2}(*)}:=\left(|f(\infty)|^{2}+\|f(\cdot)-f(\infty)\|_{\mathcal{L}_{\mathrm{M}_{2}(\mathbb{C})}^{2}(*)}^{2}\right)^{1 / 2}[31]$.
} 
From Lemma 2.6, the ordered factorisation of Eq. (7), and Eq. (5), one shows that

$$
\begin{gathered}
u(x, t)=\mathrm{i}\left(\Delta_{o}(x, t)\right)_{12}+\int_{\sigma_{c}}\left(\mu^{\mathcal{G}}(x, t ; z)\right)_{11} \overline{r(\bar{z})} \exp \left(-2 \mathrm{i} t \theta^{u}(z)\right) \frac{\mathrm{d} z}{2 \pi} \\
\theta^{u}(\zeta):=\frac{1}{2}\left(\zeta-\frac{1}{\zeta}\right)\left(z_{o}+\zeta+\frac{1}{\zeta}\right), \quad z_{o}:=\frac{x}{t}
\end{gathered}
$$

(with an analogous expression for $\int_{+\infty}^{x}\left(|u(\xi, t)|^{2}-1\right) \mathrm{d} \xi$ ), where $(\star)_{i j}$ denotes the $(i j)$-element of $\star$. It is clear from Eq. (8) that the determination of $u(x, t)$ comes down to an asymptotic analysis of the resolvent kernel, $\mu^{\mathcal{G}}(x, t ; z)$, for which no a priori explicit information regarding its analytical properties is available: the remedy to this is achieved via the application of the DZ (non-linear steepest-descent) method [27], complemented by the explicit asymptotic solution of an auxiliary system of linear singular integral equations. In analogy with the method of steepest descents, the DZ method begins by examining the saddle point(s) of the phase function, $\theta^{u}(\zeta)$. The following cases evince themselves: (i) $t \rightarrow \pm \infty$ and $x \rightarrow \mp \infty$ such that $z_{o}<-2, \partial_{\zeta} \theta^{u}(\zeta)=\zeta^{-3}\left(\zeta-\zeta_{1}\right)\left(\zeta-\zeta_{2}\right)\left(\zeta-\zeta_{3}\right)\left(\zeta-\zeta_{4}\right)$, where $\left\{\zeta_{i}\right\}_{i=1}^{4}$ are defined in Theorem 3.1, Eqs. (16) and (17); (ii) $t \rightarrow \pm \infty$ and $x \rightarrow \pm \infty$ such that $z_{o}>2, \partial_{\zeta} \theta^{u}(\zeta)=$ $\zeta^{-3}\left(\zeta-\aleph_{1}\right)\left(\zeta-\aleph_{2}\right)\left(\zeta-\aleph_{3}\right)\left(\zeta-\aleph_{4}\right)$, where $\left\{\aleph_{i}\right\}_{i=1}^{4}$ are defined in Theorem 3.1, Eq. (24); (iii) $t \rightarrow \pm \infty$ and $x \rightarrow \mp \infty$ (respectively $x \rightarrow \pm \infty$ ) such that $z_{o} \in(-2,0)$ (respectively $z_{o} \in(0,2)$ ), $\partial_{\zeta} \theta^{u}(\zeta)=\zeta^{-3}\left(\zeta-\zeta_{1}^{\sharp}\right)\left(\zeta-\overline{\zeta_{1}^{\sharp}}\right)\left(\zeta-\zeta_{3}^{\sharp}\right)\left(\zeta-\overline{\zeta_{3}^{\sharp}}\right)$, where $\zeta_{n}^{\sharp}, n \in\{1,3\}$, are defined in Theorem 3.2, Eqs. (36) and (37); (iv) $t \rightarrow \pm \infty$ and $x \rightarrow \mp \infty$ (respectively $x \rightarrow \pm \infty$ ) such that $z_{0} \rightarrow 0^{-}$ (respectively $\left.z_{o} \rightarrow 0^{+}\right), \partial_{\zeta} \theta^{u}(\zeta)=\zeta^{-3}\left(\zeta-\mathrm{e}^{\mathrm{i} \pi} \frac{1}{4}\right)\left(\zeta-\mathrm{e}^{-\frac{\mathrm{i} \pi}{4}}\right)\left(\zeta-\mathrm{e}^{\frac{3 \pi \mathrm{i}}{4}}\right)\left(\zeta-\mathrm{e}^{-\frac{3 \pi \mathrm{i}}{4}}\right) ;(\mathrm{v}) t \rightarrow \pm \infty$ and $x \rightarrow \mp \infty$ such that $z_{o}=-2, \partial_{\zeta} \theta^{u}(\zeta)=\zeta^{-3}(\zeta-1)^{2}\left(\zeta-\mathrm{e}^{\frac{2 \pi \mathrm{i}}{3}}\right)\left(\zeta-\mathrm{e}^{-\frac{2 \pi \mathrm{i}}{3}}\right)$; and (vi) $t \rightarrow \pm \infty$ and $x \rightarrow \pm \infty$ such that $z_{o}=2, \partial_{\zeta} \theta^{u}(\zeta)=\zeta^{-3}(\zeta+1)^{2}\left(\zeta-\mathrm{e}^{\mathrm{i} \pi} \frac{1}{3}\right)\left(\zeta-\mathrm{e}^{-\frac{\mathrm{i} \pi}{3}}\right)$. Cases (i) and (ii) correspond to oscillatory asymptotics, cases (iii) and (iv) give rise to exponentially decaying asymptotics, and cases (v) and (vi) give rise to asymptotics which are related to those of the transcendent of the Painlevé II equation (PII) [35, 36, 37, 38, 39]. In this work, only cases (i)-(iv) are considered, and cases (v) and (vi) will be studied elsewhere. Hereafter, the discussion will focus exclusively on how the DZ method is applied to the subcase $t \rightarrow+\infty$ and $x \rightarrow-\infty$ such that $z_{0}<-2$ of case (i): analogous statements/arguments, with corresponding modifications, apply to cases (ii)-(vi). Succinctly, the DZ method for (oscillatory) RHPs is based on a succession of transformations (involving judicious factorisations of the jump matrix), and deformations of $\sigma_{c}$ (orientations, too), which, as $t \rightarrow+\infty$, convert the original RHP into an equivalent RHP (in the sense that a solution of the equivalent RHP gives a solution of the original RHP and vice versa $\left.\left.\right|^{2}\right)$ with jump matrix $\mathcal{G}_{\text {equiv }}(x, t ; \zeta)$ of the form $\mathcal{G}_{\text {equiv }}(x, t ; \zeta)=\mathcal{G}_{\text {model }}(x, t ; \zeta)+\mathcal{G}_{\text {error }}(x, t ; \zeta)$, where $\mathcal{G}_{\text {model }}(x, t ; \zeta)$ is the jump matrix for an explicitly solvable model RHP, and $\mathcal{G}_{\text {error }}(x, t ; \zeta)={ }_{t \rightarrow+\infty} \mathrm{I}+o(1)$. Modulo error estimates, the solution of the original RHP "tends to" the solution of the model RHP. The delineation of the DZ method is as follows:

(i) decompose the complex plane of the spectral parameter $\zeta$ according to the signature of $\Re\left(\mathrm{i} t \theta^{u}(\zeta)\right)$ (see Section 4 , Figure 1 ), where $\pm \leftrightarrow \Re\left(\mathrm{i} t \theta^{u}(\zeta)\right) \gtrless 0$, and $\left\{\zeta_{2}, \zeta_{1}\right\}$ and $\left\{\zeta_{3}, \overline{\zeta_{3}}\right\}$ are, respectively, the real and complex first-order saddle points (note that $\mathbb{C} \backslash \sigma_{c}$ is partitioned into the disjoint union of two disconnected domains coloured, respectively, by \pm$)$;

(ii) reorient $\sigma_{c}=\mathbb{R}$ (oriented from $-\infty$ to $+\infty$ ) according to, and consistent with, the signature of $\Re\left(\mathrm{i} t \theta^{u}(\zeta)\right)$, denoted by $\sigma_{c}^{\prime}$ (see Section 4, Figure 2), "conjugate" the RHP for $m^{c}(x, t ; \zeta)$ according to $\widehat{m}^{c}(x, t ; \zeta):=m^{c}(x, t ; \zeta)(\delta(\zeta))^{-\sigma_{3}}$, where $\delta(\zeta)$ solves the scalar

\footnotetext{
${ }^{2}$ In particular, if there are two RHPs, $\left(\mathcal{X}_{1}(\lambda), v_{1}(\lambda), \Gamma_{1}\right)$ and $\left(\mathcal{X}_{2}(\lambda), v_{2}(\lambda), \Gamma_{2}\right)$, say, with $\Gamma_{2} \subset \Gamma_{1}$ and $v_{1}(\lambda) \Gamma_{\Gamma_{1} \backslash \Gamma_{2}}={ }_{t \rightarrow+\infty} \mathrm{I}+o(1)$, then, within the BC framework (Lemma 3.1), and modulo $o(1)$ estimates, their solutions, $\mathcal{X}_{1}(\lambda)$ and $\mathcal{X}_{2}(\lambda)$, respectively, are (asymptotically) equal.
} 
discontinuous RHP

$$
\begin{aligned}
\delta_{+}(\zeta) & = \begin{cases}\delta_{-}(\zeta)(1-r(\zeta) \overline{r(\bar{\zeta})}), & \Re(\zeta) \in(-\infty, 0) \cup\left(\zeta_{2}, \zeta_{1}\right), \\
\delta_{-}(\zeta)=\delta(\zeta), & \Re(\zeta) \in\left(0, \zeta_{2}\right) \cup\left(\zeta_{1},+\infty\right),\end{cases} \\
\delta(\zeta) & =1+\mathcal{O}\left(\zeta^{-1}\right),
\end{aligned}
$$

with solution $\delta(\zeta)=\left(\frac{\zeta-\zeta_{1}}{\zeta-\zeta_{2}}\right)^{\mathrm{i} \nu} \exp \left(\int_{-\infty}^{0} \frac{\ln \left(1-|r(\mu)|^{2}\right)}{(\mu-\zeta)} \frac{\mathrm{d} \mu}{2 \pi \mathrm{i}}+\int_{\zeta_{2}}^{\zeta_{1}} \ln \left(\frac{1-|r(\mu)|^{2}}{1-\left|r\left(\zeta_{1}\right)\right|^{2}}\right) \frac{1}{(\mu-\zeta)} \frac{\mathrm{d} \mu}{2 \pi \mathrm{i}}\right)$, where $\nu:=-\frac{1}{2 \pi} \ln \left(1-\left|r\left(\zeta_{1}\right)\right|^{2}\right), \delta(\zeta) \overline{\delta(\bar{\zeta})}=1$, and $\delta(\zeta) \delta\left(\frac{1}{\zeta}\right)=\delta(0)$, and derive the following RHP for $\widehat{m}^{c}(x, t ; \zeta): \mathbb{C} \backslash \sigma_{c}^{\prime} \rightarrow \mathrm{SL}(2, \mathbb{C})$,

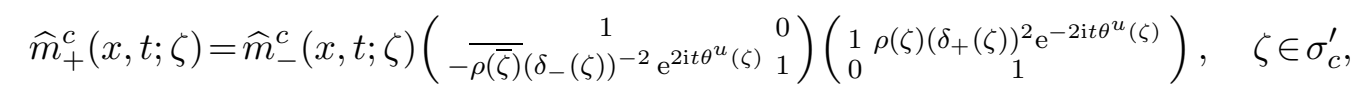

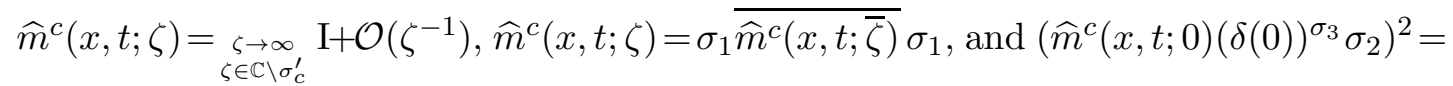
I, where $\rho(\zeta):= \begin{cases}\overline{r(\bar{\zeta})}, & \Re(\zeta) \in\left(0, \zeta_{2}\right) \cup\left(\zeta_{1},+\infty\right), \\ -\overline{r(\bar{\zeta})}(1-r(\zeta) \overline{r(\bar{\zeta})})^{-1}, & \Re(\zeta) \in(-\infty, 0) \cup\left(\zeta_{2}, \zeta_{1}\right) ;\end{cases}$

(iii) on each interval $(-\infty, 0),\left(0, \zeta_{2}\right),\left(\zeta_{2}, \zeta_{1}\right)$, and $\left(\zeta_{1},+\infty\right)$, replace $r(\zeta)$ by rational functions, and deform and augment $\sigma_{c}^{\prime}$ to the (oriented) contour $\Sigma^{\prime}$ (see Section 4, Figure 3 ) such that the respective jump matrices on $\sigma_{c}^{\prime} \subset \Sigma^{\prime}$ and the finite triangular humps (respectively linear segments) of $\Sigma^{\prime} \backslash \sigma_{c}^{\prime}$, away from the union of the neighbourhoods of the real first-order saddle points $\zeta_{2}$ and $\zeta_{1}$ (respectively complex first-order saddle points $\zeta_{3}$ and $\left.\overline{\zeta_{3}}\right)$, tend to I as $t \rightarrow+\infty$, and rewrite the RHP for $\widehat{m}^{c}(x, t ; \zeta)$ on $\sigma_{c}^{\prime}$ as an equivalent RHP for an $\mathrm{SL}(2, \mathbb{C})$-valued function $m^{\sharp}(x, t ; \zeta)$ (see Section 4 , Lemma 4.3 for the explicit transformation from $\widehat{m}^{c}(x, t ; \zeta)$ to $\left.m^{\sharp}(x, t ; \zeta)\right)$ on $\Sigma^{\prime}$ (this is possible due to the fact that the index of the RHP is a topological invariant [23]);

(iv) showing that the contribution of $\zeta_{3}$ (respectively $\overline{\zeta_{3}}$ ) to the leading-order asymptotics of $m^{\sharp}(x, t ; \zeta)$ is $\mathcal{O}\left(\exp \left(-\widehat{g}\left(z_{o}\right) t\right)\right)$, where $\widehat{g}\left(z_{o}\right)>0$, truncate $\Sigma^{\prime}$ to the (oriented) contour $\Sigma^{\sharp}$ (see Section 4, Figure 4) partitioned such that $\Sigma^{\sharp}=\Sigma_{A^{\prime}} \cup \Sigma_{B^{\prime}}$ and $\Sigma_{A^{\prime}} \cap \Sigma_{B^{\prime}}=\emptyset$, with $\operatorname{dist}\left(\Sigma_{A^{\prime}}, \Sigma_{B^{\prime}}\right)={ }_{t \rightarrow+\infty} \mathcal{O}(1)$;

(v) localising the jump matrices of the most rapidly descented RHPs on the union of the truncated disjoint crosses to the open neighbourhoods of $\zeta_{1}$ and $\zeta_{2}$, introducing the scaling-shifting operators (see Section 5, Eqs. (105) and (106)) $\mathcal{N}_{A}, f(\zeta) \mapsto\left(\mathcal{N}_{A} f\right)(\widetilde{w})=$ $f\left(\zeta_{2}+\widetilde{w} / \frac{\left|\zeta_{2}-\zeta_{3}\right|}{\zeta_{2}} \sqrt{\frac{2 t\left(\zeta_{1}-\zeta_{2}\right)}{\zeta_{2}}}\right)$, and $\mathcal{N}_{B}, g(\zeta) \mapsto\left(\mathcal{N}_{B} g\right)(\widetilde{w})=g\left(\zeta_{1}+\widetilde{w} / \frac{\left|\zeta_{1}-\zeta_{3}\right|}{\zeta_{1}} \sqrt{\frac{2 t\left(\zeta_{1}-\zeta_{2}\right)}{\zeta_{1}}}\right)$, which scale and shift (centre) $\Sigma_{A^{\prime}}$ and $\Sigma_{B^{\prime}}$, respectively, to $\widetilde{w}=0$, deducing that the leading-order asymptotics are $\mathcal{O}\left(t^{-1 / 2}\right)$, and showing that the higher order interaction between the crosses is $\mathcal{O}\left(t^{-1 / 2} \ln t\right)$, one separates out the contributions of the crosses and shows that $u(x, t)$ can be written as the linear superposition of the contributions of the various (disjoint) crosses, and, with additional transformations and scalings, reduce the RHPs on the crosses to model RHPs (on $\mathbb{R}$ ) which can be solved in closed form in terms of parabolic cylinder functions.

Remark 3.2. Throughout this work, $M \in \mathbb{R}_{>1}$ denotes a fixed, bounded constant, and the "symbols" $c^{\mathcal{S}}(\diamond), \underline{c}(b, \underline{\natural}, \sharp), \underline{c}\left(z_{1}, z_{2}, z_{3}, z_{4}\right), \underline{c}(\bullet)$, and $\underline{c}$ appearing in the various error estimates are to be understood as follows: (1) for $\pm \diamond>0, c^{\overline{\mathcal{S}}}(\diamond) \in \mathcal{S}_{\mathbb{C}}\left(\mathbb{R}_{ \pm}\right) ;(2)$ for $\pm b>0, \underline{c}(b, \underline{\natural}, \sharp) \in$ $\mathcal{L}_{\mathbb{C}}^{\infty}\left(\mathbb{R}_{ \pm} \times \mathbb{C}^{*} \times \overline{\mathbb{C}^{*}}\right)$, where $\mathbb{C}^{*}:=\mathbb{C} \backslash\{0\}$ (and bounded); (3) for $\left(z_{1}, z_{2}\right) \in \mathbb{R}_{ \pm} \times \mathbb{R}_{ \pm}, \underline{c}\left(z_{1}, z_{2}, z_{3}, z_{4}\right) \in$ $\mathcal{L}_{\mathbb{C}}^{\infty}\left(\mathbb{R}_{ \pm}^{2} \times \mathbb{C}^{*} \times \overline{\mathbb{C}^{*}}\right) ;(4)$ for $\pm \bullet>0, \underline{c}(\bullet) \in \mathcal{L}_{\mathbb{C}}^{\infty}\left(\mathrm{D}_{ \pm}\right)$, where $\mathrm{D}_{+}:=(0,2)$ and $\mathrm{D}_{-}:=(-2,0)$; and (5) $\underline{c} \in \mathbb{C}^{*}$. Even though the symbols $c^{\mathcal{S}}(\diamond), \underline{c}(b, \underline{\natural}, \sharp), \underline{c}\left(z_{1}, z_{2}, z_{3}, z_{4}\right), \underline{c}(\bullet)$, and $\underline{c}$ appearing in the error estimations are not, in general, equal, and should properly be denoted as $c_{1}(\cdot), c_{2}(\cdot)$, etc., 
the simplified notations $c^{\mathcal{S}}(\diamond), \underline{c}(b, \underline{\natural}, \sharp), \underline{c}\left(z_{1}, z_{2}, z_{3}, z_{4}\right), \underline{c}(\bullet)$, and $\underline{c}$ are retained throughout in order to eschew a flood of superfluous notation, as well as to maintain consistency with the main theme of this work, namely, to derive explicitly the leading order asymptotics and the classes to which the errors belong without regard to their precise $z_{o}$-dependence.

Remark 3.3. In Eqs. (9) and (18) below, one should keep, everywhere, the upper (respectively lower) signs as $t \rightarrow+\infty$ (respectively $t \rightarrow-\infty$ ).

Theorem 3.1. For $r(\zeta) \in \mathcal{S}_{\mathbb{C}}(\mathbb{R}) \cap\left\{h(z) ; \|\left. h(\cdot)\right|_{\mathcal{L}^{\infty}(\mathbb{R})}:=\sup _{z \in \mathbb{R}}|h(z)|<1\right\}$, let $m(x, t ; \zeta)$ be the solution of the Riemann-Hilbert problem formulated in Lemma 2.5. Let $u(x, t)$, the solution of the Cauchy problem for the $\mathrm{D}_{f} \mathrm{NLSE}$ with finite-density initial data $u(x, 0):=$ $u_{o}(x)={ }_{x \rightarrow \pm \infty} u_{o}( \pm \infty)(1+o(1))$, where $u_{o}( \pm \infty):=\exp \left(\frac{\mathrm{i}(1 \mp 1) \theta}{2}\right), 0 \leqslant \theta=-\int_{-\infty}^{+\infty} \frac{\ln \left(1-|r(\mu)|^{2}\right)}{\mu} \frac{\mathrm{d} \mu}{2 \pi}<$ $2 \pi, u_{o}(x) \in \mathbf{C}^{\infty}(\mathbb{R})$, and $u_{o}(x)-u_{o}( \pm \infty) \in \mathcal{S}_{\mathbb{C}}\left(\mathbb{R}_{ \pm}\right)$, be defined by Eq. (5). Then as $t \rightarrow \pm \infty$ and $x \rightarrow \mp \infty$ such that $z_{o}:=x / t<-2$,

$$
\begin{aligned}
u(x, t) & =\mathrm{e}^{-\mathrm{i} \theta^{ \pm}(1)}\left(1+\frac{\mathrm{i} \sqrt{\nu\left(\zeta_{1}\right)}}{\sqrt{|t|\left(\zeta_{1}-\zeta_{2}\right)}\left(z_{o}^{2}+32\right)^{1 / 4}}\left(\zeta_{1} \mathrm{e}^{\mp \mathrm{i}\left(\Theta^{ \pm}\left(z_{o}, t\right) \pm(2 \mp 1) \frac{\pi}{4}\right)}+\zeta_{2} \mathrm{e}^{ \pm \mathrm{i}\left(\Theta^{ \pm}\left(z_{o}, t\right) \pm(2 \mp 1) \frac{\pi}{4}\right)}\right)\right. \\
& \left.+\mathcal{O}\left(\left(\frac{c^{\mathcal{S}}\left(\zeta_{1}\right) \underline{c}\left(\zeta_{2}, \zeta_{3}, \zeta_{4}\right)}{\sqrt{\zeta_{1}\left(z_{o}^{2}+32\right)}}+\frac{c^{\mathcal{S}}\left(\zeta_{2}\right) \underline{c}\left(\zeta_{1}, \zeta_{3}, \zeta_{4}\right)}{\sqrt{\zeta_{2}\left(z_{o}^{2}+32\right)}}\right) \frac{\ln |t|}{\left(\zeta_{1}-\zeta_{2}\right) t}\right)\right)
\end{aligned}
$$

where

$$
\begin{gathered}
\theta^{+}(j):=\int_{-\infty}^{0} \frac{\ln \left(1-|r(\mu)|^{2}\right)}{\mu^{j}} \frac{\mathrm{d} \mu}{2 \pi}+\int_{\zeta_{2}}^{\zeta_{1}} \frac{\ln \left(1-|r(\mu)|^{2}\right)}{\mu^{j}} \frac{\mathrm{d} \mu}{2 \pi}, \quad j \in\{0,1\}, \\
\theta^{-}(l):=\int_{0}^{\zeta_{2}} \frac{\ln \left(1-|r(\mu)|^{2}\right)}{\mu^{l}} \frac{\mathrm{d} \mu}{2 \pi}+\int_{\zeta_{1}}^{+\infty} \frac{\ln \left(1-|r(\mu)|^{2}\right)}{\mu^{l}} \frac{\mathrm{d} \mu}{2 \pi}, \quad l \in\{0,1\}, \\
\nu(z):=-\frac{1}{2 \pi} \ln \left(1-|r(z)|^{2}\right), \\
\Theta^{ \pm}\left(z_{o}, t\right):= \pm \arg r\left(\zeta_{1}\right)-\arg \Gamma\left(\mathrm{i} \nu\left(\zeta_{1}\right)\right) \pm t\left(\zeta_{1}-\zeta_{2}\right)\left(z_{o}+\zeta_{1}+\zeta_{2}\right)+\nu\left(\zeta_{1}\right) \ln |t| \\
+3 \nu\left(\zeta_{1}\right) \ln \left(\zeta_{1}-\zeta_{2}\right)+\frac{1}{2} \nu\left(\zeta_{1}\right) \ln \left(z_{o}^{2}+32\right) \mp \Omega^{ \pm}\left(\zeta_{1}\right) \pm \frac{1}{2} \Omega^{ \pm}(0), \\
\Omega^{+}(z)=\frac{1}{\pi} \int_{-\infty}^{0} \ln |\mu-z| \mathrm{d} \ln \left(1-|r(\mu)|^{2}\right)+\frac{1}{\pi} \int_{\zeta_{2}}^{\zeta_{1}} \ln |\mu-z| \mathrm{d} \ln \left(1-|r(\mu)|^{2}\right), \\
\Omega^{-}(z)=\frac{1}{\pi} \int_{0}^{\zeta_{2}} \ln |\mu-z| \mathrm{d} \ln \left(1-|r(\mu)|^{2}\right)+\frac{1}{\pi} \int_{\zeta_{1}}^{+\infty} \ln |\mu-z| \mathrm{d} \ln \left(1-|r(\mu)|^{2}\right), \\
\zeta_{1}:=\frac{1}{2}\left(-a_{1}+\sqrt{a_{1}^{2}-4}\right), \quad \zeta_{2}=\frac{1}{\zeta_{1}}, \quad \zeta_{3}:=\frac{1}{2}\left(-a_{2}+\mathrm{i} \sqrt{4-a_{2}^{2}}\right), \quad \zeta_{4}=\overline{\zeta_{3}}, \\
a_{1}=\frac{1}{4}\left(z_{o}-\sqrt{z_{o}^{2}+32}\right), \quad a_{2}=\frac{1}{4}\left(z_{o}+\sqrt{z_{o}^{2}+32}\right),
\end{gathered}
$$

$0<\zeta_{2}<\zeta_{1},\left|\zeta_{3}\right|^{2}=1, a_{1} a_{2}=-2$, and $\Gamma(\cdot)$ is the gamma function [40], and as $t \rightarrow \pm \infty$ and $x \rightarrow \pm \infty$ such that $z_{o}>2$,

$$
\begin{aligned}
u(x, t) & =-\mathrm{e}^{-\mathrm{i} \phi^{ \pm}(1)}\left(1+\frac{\mathrm{i} \sqrt{\nu\left(\aleph_{4}\right)}}{\sqrt{|t|\left(\aleph_{3}-\aleph_{4}\right)}\left(z_{o}^{2}+32\right)^{1 / 4}}\left(\aleph_{4} \mathrm{e}^{\mp \mathrm{i}\left(\Phi^{ \pm}\left(z_{o}, t\right) \pm(2 \mp 1) \frac{\pi}{4}\right)}+\aleph_{3} \mathrm{e}^{ \pm \mathrm{i}\left(\Phi^{ \pm}\left(z_{o}, t\right) \pm(2 \mp 1) \frac{\pi}{4}\right)}\right)\right. \\
& \left.+\mathcal{O}\left(\left(\frac{c^{\mathcal{S}}\left(\aleph_{3}\right) \underline{c}\left(\aleph_{4}, \aleph_{1}, \aleph_{2}\right)}{\sqrt{\left|\aleph_{3}\right|\left(z_{o}^{2}+32\right)}}+\frac{c^{\mathcal{S}}\left(\aleph_{4}\right) \underline{c}\left(\aleph_{3}, \aleph_{1}, \aleph_{2}\right)}{\sqrt{\left|\aleph_{4}\right|\left(z_{o}^{2}+32\right)}}\right) \frac{\ln |t|}{\left(\aleph_{3}-\aleph_{4}\right) t}\right)\right)
\end{aligned}
$$


where

$$
\begin{gathered}
\phi^{+}(j):=\int_{-\infty}^{\aleph_{4}} \frac{\ln \left(1-|r(\mu)|^{2}\right)}{\mu^{j}} \frac{\mathrm{d} \mu}{2 \pi}+\int_{\aleph_{3}}^{0} \frac{\ln \left(1-|r(\mu)|^{2}\right)}{\mu^{j}} \frac{\mathrm{d} \mu}{2 \pi}, \quad j \in\{0,1\}, \\
\phi^{-}(l):=\int_{\aleph_{4}}^{\aleph_{3} \ln \left(1-|r(\mu)|^{2}\right)} \frac{\mathrm{d} \mu}{2 \pi}+\int_{0}^{+\infty} \frac{\ln \left(1-|r(\mu)|^{2}\right)}{\mu^{l}} \frac{\mathrm{d} \mu}{2 \pi}, \quad l \in\{0,1\}, \\
\Phi^{ \pm}\left(z_{o}, t\right):= \pm \arg r\left(\aleph_{4}\right)-\arg \Gamma\left(\mathrm{i} \nu\left(\aleph_{4}\right)\right) \pm t\left(\aleph_{4}-\aleph_{3}\right)\left(z_{o}+\aleph_{3}+\aleph_{4}\right)+\nu\left(\aleph_{4}\right) \ln |t| \\
+3 \nu\left(\aleph_{4}\right) \ln \left(\aleph_{3}-\aleph_{4}\right)+\frac{1}{2} \nu\left(\aleph_{4}\right) \ln \left(z_{o}^{2}+32\right) \mp \Lambda^{ \pm}\left(\aleph_{4}\right) \pm \frac{1}{2} \Lambda^{ \pm}(0), \\
\Lambda^{+}(z)=\frac{1}{\pi} \int_{-\infty}^{\aleph_{4}} \ln |\mu-z| \mathrm{d} \ln \left(1-|r(\mu)|^{2}\right)+\frac{1}{\pi} \int_{\aleph_{3}}^{0} \ln |\mu-z| \mathrm{d} \ln \left(1-|r(\mu)|^{2}\right), \\
\Lambda^{-}(z)=\frac{1}{\pi} \int_{\aleph_{4}}^{\aleph_{3}} \ln |\mu-z| \mathrm{d} \ln \left(1-|r(\mu)|^{2}\right)+\frac{1}{\pi} \int_{0}^{+\infty} \ln |\mu-z| \mathrm{d} \ln \left(1-|r(\mu)|^{2}\right), \\
\aleph_{1}:=\frac{1}{2}\left(-a_{1}+\mathrm{i} \sqrt{4-a_{1}^{2}}\right), \quad \aleph_{2}=\bar{\aleph}_{1}, \quad \aleph_{3}:=\frac{1}{2}\left(-a_{2}+\sqrt{a_{2}^{2}-4}\right), \quad \aleph_{4}=\frac{1}{\aleph_{3}},
\end{gathered}
$$

$\aleph_{4}<\aleph_{3}<0$, and $\left|\aleph_{1}\right|^{2}=1$. For $u(x, t)$ as defined and given above, let $\int_{+\infty}^{x}\left(|u(\xi, t)|^{2}-1\right) \mathrm{d} \xi$ be defined by Eq. (6). Then: (i) as $t \rightarrow+\infty$ and $x \rightarrow-\infty$ such that $z_{o}<-2$,

$$
\begin{aligned}
\int_{+\infty}^{x}\left(|u(\xi, t)|^{2}-1\right) \mathrm{d} \xi & =\theta^{+}(0)-\frac{2 \sqrt{\nu\left(\zeta_{1}\right)} \cos \left(\Theta^{+}\left(z_{o}, t\right)+\frac{\pi}{4}\right)}{\sqrt{t\left(\zeta_{1}-\zeta_{2}\right)}\left(z_{o}^{2}+32\right)^{1 / 4}} \\
& +\mathcal{O}\left(\left(\frac{c^{\mathcal{S}}\left(\zeta_{1}\right) \underline{c}\left(\zeta_{2}, \zeta_{3}, \zeta_{4}\right)}{\sqrt{\zeta_{1}\left(z_{o}^{2}+32\right)}}+\frac{c^{\mathcal{S}}\left(\zeta_{2}\right) \underline{c}\left(\zeta_{1}, \zeta_{3}, \zeta_{4}\right)}{\sqrt{\zeta_{2}\left(z_{o}^{2}+32\right)}}\right) \frac{\ln t}{\left(\zeta_{1}-\zeta_{2}\right) t}\right) \\
\int_{-\infty}^{x}\left(|u(\xi, t)|^{2}-1\right) \mathrm{d} \xi & =-\theta^{-}(0)-\frac{2 \sqrt{\nu\left(\zeta_{1}\right)} \cos \left(\Theta^{+}\left(z_{o}, t\right)+\frac{\pi}{4}\right)}{\sqrt{t\left(\zeta_{1}-\zeta_{2}\right)}\left(z_{o}^{2}+32\right)^{1 / 4}} \\
& +\mathcal{O}\left(\left(\frac{c^{\mathcal{S}}\left(\zeta_{1}\right) \underline{c}\left(\zeta_{2}, \zeta_{3}, \zeta_{4}\right)}{\sqrt{\zeta_{1}\left(z_{o}^{2}+32\right)}}+\frac{c^{\mathcal{S}}\left(\zeta_{2}\right) \underline{c}\left(\zeta_{1}, \zeta_{3}, \zeta_{4}\right)}{\sqrt{\zeta_{2}\left(z_{o}^{2}+32\right)}}\right) \frac{\ln t}{\left(\zeta_{1}-\zeta_{2}\right) t}\right)
\end{aligned}
$$

(ii) as $t \rightarrow-\infty$ and $x \rightarrow+\infty$ such that $z_{o}<-2$,

$$
\begin{aligned}
\int_{+\infty}^{x}\left(|u(\xi, t)|^{2}-1\right) \mathrm{d} \xi & =\theta^{-}(0)-\frac{2 \sqrt{\nu\left(\zeta_{1}\right)} \cos \left(\Theta^{-}\left(z_{o}, t\right)-\frac{3 \pi}{4}\right)}{\sqrt{|t|\left(\zeta_{1}-\zeta_{2}\right)}\left(z_{o}^{2}+32\right)^{1 / 4}} \\
& +\mathcal{O}\left(\left(\frac{c^{\mathcal{S}}\left(\zeta_{1}\right) \underline{c}\left(\zeta_{2}, \zeta_{3}, \zeta_{4}\right)}{\sqrt{\zeta_{1}\left(z_{o}^{2}+32\right)}}+\frac{c^{\mathcal{S}}\left(\zeta_{2}\right) \underline{c}\left(\zeta_{1}, \zeta_{3}, \zeta_{4}\right)}{\sqrt{\zeta_{2}\left(z_{o}^{2}+32\right)}}\right) \frac{\ln |t|}{\left(\zeta_{1}-\zeta_{2}\right) t}\right) \\
\int_{-\infty}^{x}\left(|u(\xi, t)|^{2}-1\right) \mathrm{d} \xi & =-\theta^{+}(0)-\frac{2 \sqrt{\nu\left(\zeta_{1}\right)} \cos \left(\Theta^{-}\left(z_{o}, t\right)-\frac{3 \pi}{4}\right)}{\sqrt{|t|\left(\zeta_{1}-\zeta_{2}\right)}\left(z_{o}^{2}+32\right)^{1 / 4}} \\
& +\mathcal{O}\left(\left(\frac{c^{\mathcal{S}}\left(\zeta_{1}\right) \underline{c}\left(\zeta_{2}, \zeta_{3}, \zeta_{4}\right)}{\sqrt{\zeta_{1}\left(z_{o}^{2}+32\right)}}+\frac{c^{\mathcal{S}}\left(\zeta_{2}\right) \underline{c}\left(\zeta_{1}, \zeta_{3}, \zeta_{4}\right)}{\sqrt{\zeta_{2}\left(z_{o}^{2}+32\right)}}\right) \frac{\ln |t|}{\left(\zeta_{1}-\zeta_{2}\right) t}\right)
\end{aligned}
$$


(iii) as $t \rightarrow+\infty$ and $x \rightarrow+\infty$ such that $z_{o}>2$,

$$
\begin{aligned}
\int_{+\infty}^{x}\left(|u(\xi, t)|^{2}-1\right) \mathrm{d} \xi & =\phi^{-}(0)+\frac{2 \sqrt{\nu\left(\aleph_{4}\right)} \cos \left(\Phi^{+}\left(z_{o}, t\right)+\frac{\pi}{4}\right)}{\sqrt{t\left(\aleph_{3}-\aleph_{4}\right)}\left(z_{o}^{2}+32\right)^{1 / 4}} \\
& +\mathcal{O}\left(\left(\frac{c^{\mathcal{S}}\left(\aleph_{3}\right) \underline{c}\left(\aleph_{4}, \aleph_{1}, \aleph_{2}\right)}{\sqrt{\left|\aleph_{3}\right|\left(z_{o}^{2}+32\right)}}+\frac{c^{\mathcal{S}}\left(\aleph_{4}\right) \underline{c}\left(\aleph_{3}, \aleph_{1}, \aleph_{2}\right)}{\sqrt{\left|\aleph_{4}\right|\left(z_{o}^{2}+32\right)}}\right) \frac{\ln t}{\left(\aleph_{3}-\aleph_{4}\right) t}\right), \\
\int_{-\infty}^{x}\left(|u(\xi, t)|^{2}-1\right) \mathrm{d} \xi & =-\phi^{+}(0)+\frac{2 \sqrt{\nu\left(\aleph_{4}\right)} \cos \left(\Phi^{+}\left(z_{o}, t\right)+\frac{\pi}{4}\right)}{\sqrt{t\left(\aleph_{3}-\aleph_{4}\right)}\left(z_{o}^{2}+32\right)^{1 / 4}} \\
& +\mathcal{O}\left(\left(\frac{c^{\mathcal{S}}\left(\aleph_{3}\right) \underline{c}\left(\aleph_{4}, \aleph_{1}, \aleph_{2}\right)}{\sqrt{\left|\aleph_{3}\right|\left(z_{o}^{2}+32\right)}}+\frac{c^{\mathcal{S}}\left(\aleph_{4}\right) \underline{c}\left(\aleph_{3}, \aleph_{1}, \aleph_{2}\right)}{\sqrt{\left|\aleph_{4}\right|\left(z_{o}^{2}+32\right)}}\right) \frac{\ln t}{\left(\aleph_{3}-\aleph_{4}\right) t}\right) ;
\end{aligned}
$$

and (iv) as $t \rightarrow-\infty$ and $x \rightarrow-\infty$ such that $z_{0}>2$,

$$
\begin{aligned}
& \int_{+\infty}^{x}\left(|u(\xi, t)|^{2}-1\right) \mathrm{d} \xi=\phi^{+}(0)+\frac{2 \sqrt{\nu\left(\aleph_{4}\right)} \cos \left(\Phi^{-}\left(z_{o}, t\right)-\frac{3 \pi}{4}\right)}{\sqrt{|t|\left(\aleph_{3}-\aleph_{4}\right)}\left(z_{o}^{2}+32\right)^{1 / 4}} \\
&+\mathcal{O}\left(\left(\frac{c^{\mathcal{S}}\left(\aleph_{3}\right) \underline{c}\left(\aleph_{4}, \aleph_{1}, \aleph_{2}\right)}{\sqrt{\left|\aleph_{3}\right|\left(z_{o}^{2}+32\right)}}+\frac{c^{\mathcal{S}}\left(\aleph_{4}\right) \underline{c}\left(\aleph_{3}, \aleph_{1}, \aleph_{2}\right)}{\sqrt{\left|\aleph_{4}\right|\left(z_{o}^{2}+32\right)}}\right) \frac{\ln |t|}{\left(\aleph_{3}-\aleph_{4}\right) t}\right), \\
& \int_{-\infty}^{x}\left(|u(\xi, t)|^{2}-1\right) \mathrm{d} \xi=-\phi^{-}(0)+\frac{2 \sqrt{\nu\left(\aleph_{4}\right)} \cos \left(\Phi^{-}\left(z_{o}, t\right)-\frac{3 \pi}{4}\right)}{\sqrt{|t|\left(\aleph_{3}-\aleph_{4}\right)}\left(z_{o}^{2}+32\right)^{1 / 4}} \\
&+\mathcal{O}\left(\left(\frac{c^{\mathcal{S}}\left(\aleph_{3}\right) \underline{c}\left(\aleph_{4}, \aleph_{1}, \aleph_{2}\right)}{\sqrt{\left|\aleph_{3}\right|\left(z_{o}^{2}+32\right)}}+\frac{c^{\mathcal{S}}\left(\aleph_{4}\right) \underline{c}\left(\aleph_{3}, \aleph_{1}, \aleph_{2}\right)}{\sqrt{\left|\aleph_{4}\right|\left(z_{o}^{2}+32\right)}}\right) \frac{\ln |t|}{\left(\aleph_{3}-\aleph_{4}\right) t}\right) .
\end{aligned}
$$

Theorem 3.2. For $r(\zeta) \in \mathcal{S}_{\mathbb{C}}(\mathbb{R}) \cap\left\{h(z) ;\|h(\cdot)\|_{\mathcal{L}^{\infty}(\mathbb{R})}:=\sup _{z \in \mathbb{R}}|h(z)|<1\right\}$, let $m(x, t ; \zeta)$ be the solution of the Riemann-Hilbert problem formulated in Lemma 2.5. Let $u(x, t)$, the solution of the Cauchy problem for the $\mathrm{D}_{f} \mathrm{NLSE}$ with finite-density initial data $u(x, 0):=u_{o}(x)={ }_{x \rightarrow \pm \infty}$ $u_{o}( \pm \infty)(1+o(1))$, where $u_{o}( \pm \infty):=\exp \left(\frac{\mathrm{i}(1 \mp 1) \theta}{2}\right), 0 \leqslant \theta=-\int_{-\infty}^{+\infty} \frac{\ln \left(1-|r(\mu)|^{2}\right)}{\mu} \frac{\mathrm{d} \mu}{2 \pi}<2 \pi, u_{o}(x) \in$ $\mathbf{C}^{\infty}(\mathbb{R})$, and $u_{o}(x)-u_{o}( \pm \infty) \in \mathcal{S}_{\mathbb{C}}\left(\mathbb{R}_{ \pm}\right)$, be defined by Eq. (5). Set $s_{1}:=\zeta_{1}^{\sharp}=\exp \left(\mathrm{i} \widehat{\varphi}_{1}\right)$ and $s_{2}:=\zeta_{3}^{\sharp}=\exp \left(\mathrm{i} \widehat{\varphi}_{3}\right)$, where $\zeta_{n}^{\sharp}$ and $\widehat{\varphi}_{n}, n \in\{1,3\}$, are defined in Eqs. (36) and (37). Then: (i) for $r\left(s_{1}\right)=\exp \left(-\frac{\mathrm{i} \varepsilon_{1} \pi}{2}\right)\left|r\left(s_{1}\right)\right|, \varepsilon_{1} \in\{ \pm 1\}, r\left(\overline{s_{2}}\right)=\exp \left(\frac{\mathrm{i} \varepsilon_{2} \pi}{2}\right)\left|r\left(\overline{s_{2}}\right)\right|, \varepsilon_{2} \in\{ \pm 1\}, 0<r\left(s_{2}\right) \overline{r\left(\overline{s_{2}}\right)}<1$, and $\varepsilon_{1}=\varepsilon_{2}$, as $t \rightarrow+\infty$ and $x \rightarrow-\infty$ such that $z_{0}:=x / t \in(-2,0)$,

$$
\begin{aligned}
& u(x, t)=\mathrm{e}^{-\mathrm{i} \psi^{+}(1)}\left(1+\frac{\mathrm{e}^{\frac{\mathrm{i}}{2}\left(\varepsilon_{1} \pi-\left(\widehat{\varphi}_{1}-\widehat{\varphi}_{3}\right)\right)} \mathrm{e}^{-\left(\widetilde{a}_{+} t+\widetilde{c}_{+}\right)} \beth_{+}}{\widetilde{b} \sqrt{t}}\left(\mathrm { i } \operatorname { s i n } ( \frac { 1 } { 2 } ( \widehat { \varphi } _ { 1 } + \widehat { \varphi } _ { 3 } ) ) \operatorname { s i n h } \left(\widetilde{a}_{-} t+\widetilde{c}_{-}\right.\right.\right. \\
& \left.\left.+\frac{1}{8} \ln \left(\frac{4-a_{1}^{2}}{4-a_{2}^{2}}\right)-\ln \beth_{-}\right)+\cos \left(\frac{1}{2}\left(\widehat{\varphi}_{1}+\widehat{\varphi}_{3}\right)\right) \cosh \left(\widetilde{a}_{-} t+\widetilde{c}_{-}+\frac{1}{8} \ln \left(\frac{4-a_{1}^{2}}{4-a_{2}^{2}}\right)-\ln \beth_{-}\right)\right) \\
& \left.+\mathcal{O}\left(\frac{\underline{c}\left(z_{o}\right) \mathrm{e}^{-4 \alpha t}}{\beta t}\right)\right) \text {, }
\end{aligned}
$$

and, for $\varepsilon_{1}=-\varepsilon_{2}$,

$$
\begin{aligned}
& u(x, t)=\mathrm{e}^{-\mathrm{i} \psi^{+}(1)}\left(1-\frac{\mathrm{e}^{\frac{\mathrm{i}}{2}\left(\varepsilon_{1} \pi-\left(\widehat{\varphi}_{1}-\widehat{\varphi}_{3}\right)\right)} \mathrm{e}^{-\left(\widetilde{a}_{+} t+\widetilde{c}_{+}\right)} \beth_{+}}{\widetilde{b} \sqrt{t}}\left(\mathrm { i } \operatorname { s i n } ( \frac { 1 } { 2 } ( \widehat { \varphi } _ { 1 } + \widehat { \varphi } _ { 3 } ) ) \operatorname { c o s h } \left(\widetilde{a}_{-} t+\widetilde{c}_{-}\right.\right.\right. \\
& \left.\left.+\frac{1}{8} \ln \left(\frac{4-a_{1}^{2}}{4-a_{2}^{2}}\right)-\ln \beth_{-}\right)+\cos \left(\frac{1}{2}\left(\widehat{\varphi}_{1}+\widehat{\varphi}_{3}\right)\right) \sinh \left(\widetilde{a}_{-} t+\widetilde{c}_{-}+\frac{1}{8} \ln \left(\frac{4-a_{1}^{2}}{4-a_{2}^{2}}\right)-\ln \beth_{-}\right)\right) \\
& \left.+\mathcal{O}\left(\frac{\underline{c}\left(z_{o}\right) \mathrm{e}^{-4 \alpha t}}{\beta t}\right)\right) \text {, }
\end{aligned}
$$


where

$$
\begin{gathered}
\psi^{+}(l):=\int_{-\infty}^{0} \frac{\ln \left(1-|r(\mu)|^{2}\right)}{\mu^{l}} \frac{\mathrm{d} \mu}{2 \pi}, \quad l \in\{0,1\}, \\
\zeta_{1}^{\sharp}:=-\frac{1}{2}\left(a_{1}-\mathrm{i}\left(4-a_{1}^{2}\right)^{1 / 2}\right)=\mathrm{e}^{\mathrm{i} \widehat{\varphi}_{1}}, \quad \widehat{\varphi}_{1}=\arctan \left(\frac{\left(4-a_{1}^{2}\right)^{1 / 2}}{\left|a_{1}\right|}\right) \in\left(0, \frac{\pi}{2}\right), \quad a_{1}<0, \quad\left|a_{1}\right|<2, \\
\zeta_{3}^{\sharp}:=-\frac{1}{2}\left(a_{2}-\mathrm{i}\left(4-a_{2}^{2}\right)^{1 / 2}\right)=\mathrm{e}^{\mathrm{i} \widehat{\varphi}_{3}}, \quad \widehat{\varphi}_{3}=-\arctan \left(\frac{\left(4-a_{2}^{2}\right)^{1 / 2}}{\left|a_{2}\right|}\right) \in\left(\frac{\pi}{2}, \pi\right), \quad a_{2}>0, \quad\left|a_{2}\right|<2, \\
\widetilde{a}_{ \pm}:=\frac{1}{2} z_{o}\left(\left(4-a_{1}^{2}\right)^{1 / 2} \mp\left(4-a_{2}^{2}\right)^{1 / 2}\right)-\frac{1}{2}\left(a_{1}\left(4-a_{1}^{2}\right)^{1 / 2} \mp a_{2}\left(4-a_{2}^{2}\right)^{1 / 2}\right), \quad \widetilde{a}_{+}>0, \\
\widetilde{c}_{ \pm}:=\sin \left(\widehat{\varphi}_{1}\right) \int_{-\infty}^{0} \frac{\ln \left(1-|r(\mu)|^{2}\right)}{\left(\mu-\cos \widehat{\varphi}_{1}\right)^{2}+\sin ^{2} \widehat{\varphi}_{1}} \frac{\mathrm{d} \mu}{2 \pi} \mp \sin \left(\widehat{\varphi}_{3}\right) \int_{-\infty}^{0} \frac{\ln \left(1-|r(\mu)|^{2}\right)}{\left(\mu-\cos \widehat{\varphi}_{3}\right)^{2}+\sin ^{2} \widehat{\varphi}_{3}} \frac{\mathrm{d} \mu}{2 \pi}, \\
\beth_{+}:=2^{-1 / 2}\left(z_{o}^{2}+32\right)^{1 / 4}\left(4-a_{1}^{2}\right)^{1 / 8}\left(4-a_{2}^{2}\right)^{1 / 8}, \\
\left(\frac{\left|r\left(s_{1}\right)\right|\left|r\left(\overline{s_{2}}\right)\right|}{\left(1-r\left(s_{2}\right) \overline{r\left(\overline{s_{2}}\right)}\right)}\right)^{1 / 2}, \quad \beth_{-}:=\left(\frac{\left|r\left(s_{1}\right)\right|\left(1-r\left(s_{2}\right) \overline{r\left(\overline{s_{2}}\right)}\right)}{\left|r\left(\overline{s_{2}}\right)\right|}\right)^{1 / 2}, \\
\alpha:=\min \left\{\frac{1}{2}\left(z_{o}-a_{1}\right)\left(4-a_{1}^{2}\right)^{1 / 2},-\frac{1}{2}\left(z_{o}-a_{2}\right)\left(4-a_{2}^{2}\right)^{1 / 2}\right\} \quad(>0), \\
\beta:=\min \left\{\frac{1}{2}\left(z_{o}^{2}+32\right)^{1 / 2}\left(4-a_{1}^{2}\right)^{1 / 2}, \frac{1}{2}\left(z_{o}^{2}+32\right)^{1 / 2}\left(4-a_{2}^{2}\right)^{1 / 2}\right\} \quad(>0),
\end{gathered}
$$

and $a_{1}$ and $a_{2}$ are given in Theorem 3.1, Eq. (17); (ii) for $r\left(\overline{s_{1}}\right)=\exp \left(\frac{\mathrm{i} \varepsilon_{1} \pi}{2}\right)\left|r\left(\overline{s_{1}}\right)\right|, \varepsilon_{1} \in\{ \pm 1\}$, $r\left(s_{2}\right)=\exp \left(-\frac{\mathrm{i} \varepsilon_{2} \pi}{2}\right)\left|r\left(s_{2}\right)\right|, \varepsilon_{2} \in\{ \pm 1\}, 0<r\left(s_{1}\right) \overline{r\left(\overline{s_{1}}\right)}<1$, and $\varepsilon_{1}=\varepsilon_{2}$, as $t \rightarrow-\infty$ and $x \rightarrow+\infty$ such that $z_{o} \in(-2,0)$,

$$
\begin{aligned}
& u(x, t)=\mathrm{e}^{-\mathrm{i} \psi^{-}(1)}\left(1+\frac{\left.\mathrm{e}^{\frac{\mathrm{i}}{2}\left(\varepsilon_{1} \pi+\left(\widehat{\varphi}_{1}-\widehat{\varphi}_{3}\right)\right)} \mathrm{e}^{-\left(\widetilde{a}_{+}|t|-\widehat{c}_{+}\right)}\right\rceil_{+}}{\widetilde{b} \sqrt{|t|}}\left(\mathrm { i } \operatorname { s i n } ( \frac { 1 } { 2 } ( \widehat { \varphi } _ { 1 } + \widehat { \varphi } _ { 3 } ) ) \operatorname { s i n h } \left(\widetilde{a}_{-}|t|-\widehat{c}_{-}\right.\right.\right. \\
& \left.\left.\left.\left.\quad+\frac{1}{8} \ln \left(\frac{4-a_{1}^{2}}{4-a_{2}^{2}}\right)+\ln \right\urcorner_{-}\right)-\cos \left(\frac{1}{2}\left(\widehat{\varphi}_{1}+\widehat{\varphi}_{3}\right)\right) \cosh \left(\widetilde{a}_{-}|t|-\widehat{c}_{-}+\frac{1}{8} \ln \left(\frac{4-a_{1}^{2}}{4-a_{2}^{2}}\right)+\ln \right\urcorner_{-}\right)\right) \\
& \left.\quad+\mathcal{O}\left(\frac{\underline{c}\left(z_{o}\right) \mathrm{e}^{-4 \alpha|t|}}{\beta t}\right)\right)
\end{aligned}
$$

and, for $\varepsilon_{1}=-\varepsilon_{2}$,

$$
\begin{aligned}
u(x, t) & =\mathrm{e}^{-\mathrm{i} \psi^{-}(1)}\left(1-\frac{\left.\mathrm{e}^{\frac{\mathrm{i}}{2}\left(\varepsilon_{1} \pi+\left(\widehat{\varphi}_{1}-\widehat{\varphi}_{3}\right)\right)} \mathrm{e}^{-\left(\widetilde{a}_{+}|t|-\widehat{c}_{+}\right)}\right\rceil_{+}}{\widetilde{b} \sqrt{|t|}}\left(\mathrm { i } \operatorname { s i n } ( \frac { 1 } { 2 } ( \widehat { \varphi } _ { 1 } + \widehat { \varphi } _ { 3 } ) ) \operatorname { c o s h } \left(\widetilde{a}_{-}|t|-\widehat{c}_{-}\right.\right.\right. \\
& \left.\left.\left.\left.+\frac{1}{8} \ln \left(\frac{4-a_{1}^{2}}{4-a_{2}^{2}}\right)+\ln \right\rceil_{-}\right)-\cos \left(\frac{1}{2}\left(\widehat{\varphi}_{1}+\widehat{\varphi}_{3}\right)\right) \sinh \left(\widetilde{a}_{-}|t|-\widehat{c}_{-}+\frac{1}{8} \ln \left(\frac{4-a_{1}^{2}}{4-a_{2}^{2}}\right)+\ln \right\rceil_{-}\right)\right) \\
& \left.+\mathcal{O}\left(\frac{\underline{c}\left(z_{o}\right) \mathrm{e}^{-4 \alpha|t|}}{\beta t}\right)\right),
\end{aligned}
$$

where

$$
\begin{gathered}
\psi^{-}(l):=\int_{0}^{+\infty} \frac{\ln \left(1-|r(\mu)|^{2}\right)}{\mu^{l}} \frac{\mathrm{d} \mu}{2 \pi}, \quad l \in\{0,1\}, \\
\widehat{c}_{ \pm}:=\sin \left(\widehat{\varphi}_{1}\right) \int_{0}^{+\infty} \frac{\ln \left(1-|r(\mu)|^{2}\right)}{\left(\mu-\cos \widehat{\varphi}_{1}\right)^{2}+\sin ^{2} \widehat{\varphi}_{1}} \frac{\mathrm{d} \mu}{2 \pi} \mp \sin \left(\widehat{\varphi}_{3}\right) \int_{0}^{+\infty} \frac{\ln \left(1-|r(\mu)|^{2}\right)}{\left(\mu-\cos \widehat{\varphi}_{3}\right)^{2}+\sin ^{2} \widehat{\varphi}_{3}} \frac{\mathrm{d} \mu}{2 \pi} \\
\left.\neg_{+}:=\left(\frac{\left|r\left(\overline{s_{1}}\right)\right|\left|r\left(s_{2}\right)\right|}{\left(1-r\left(s_{1}\right) \overline{r\left(\overline{s_{1}}\right)}\right)}\right)^{1 / 2}, \quad\right\urcorner_{-}:=\left(\frac{\left|r\left(s_{2}\right)\right|\left(1-r\left(s_{1}\right) \overline{r\left(\overline{s_{1}}\right)}\right)}{\left|r\left(\overline{s_{1}}\right)\right|}\right)^{1 / 2} ;
\end{gathered}
$$


(iii) for $r\left(\overline{s_{2}}\right)=\exp \left(-\frac{\mathrm{i} \varepsilon_{1} \pi}{2}\right)\left|r\left(\overline{s_{2}}\right)\right|, \varepsilon_{1} \in\{ \pm 1\}, r\left(s_{1}\right)=\exp \left(\frac{\mathrm{i} \varepsilon_{2} \pi}{2}\right)\left|r\left(s_{1}\right)\right|, \varepsilon_{2} \in\{ \pm 1\}, 0<$ $r\left(s_{1}\right) \overline{r\left(\overline{s_{1}}\right)}<1$, and $\varepsilon_{1}=\varepsilon_{2}$, as $t \rightarrow+\infty$ and $x \rightarrow+\infty$ such that $z_{o} \in(0,2)$,

$$
\begin{aligned}
u(x, t) & =-\mathrm{e}^{-\mathrm{i} \psi^{+}(1)}\left(1+\frac{\mathrm{e}^{\frac{\mathrm{i}}{2}\left(\varepsilon_{1} \pi-\left(\widehat{\varphi}_{1}-\widehat{\varphi}_{3}\right)\right)} \mathrm{e}^{-\left(\widetilde{a}_{+} t+\widetilde{c}_{+}\right)} \widetilde{\beth}_{+}}{\widetilde{b} \sqrt{t}}\left(\mathrm { i } \operatorname { s i n } ( \frac { 1 } { 2 } ( \widehat { \varphi } _ { 1 } + \widehat { \varphi } _ { 3 } ) ) \operatorname { s i n h } \left(\widetilde{a}_{-} t+\widetilde{c}_{-}\right.\right.\right. \\
& \left.\left.+\frac{1}{8} \ln \left(\frac{4-a_{1}^{2}}{4-a_{2}^{2}}\right)+\ln \widetilde{\beth}_{-}\right)+\cos \left(\frac{1}{2}\left(\widehat{\varphi}_{1}+\widehat{\varphi}_{3}\right)\right) \cosh \left(\widetilde{a}_{-} t+\widetilde{c}_{-}+\frac{1}{8} \ln \left(\frac{4-a_{1}^{2}}{4-a_{2}^{2}}\right)+\ln \widetilde{\beth}_{-}\right)\right) \\
& \left.+\mathcal{O}\left(\frac{\underline{c}\left(z_{o}\right) \mathrm{e}^{-4 \alpha t}}{\beta t}\right)\right),
\end{aligned}
$$

and, for $\varepsilon_{1}=-\varepsilon_{2}$,

$$
\begin{aligned}
u(x, t) & =-\mathrm{e}^{-\mathrm{i} \psi^{+}(1)}\left(1+\frac{\mathrm{e}^{\frac{\mathrm{i}}{2}\left(\varepsilon_{1} \pi-\left(\widehat{\varphi}_{1}-\widehat{\varphi}_{3}\right)\right)} \mathrm{e}^{-\left(\widetilde{a}_{+} t+\widetilde{c}_{+}\right)}}{\widetilde{b} \sqrt{t}}\left(\mathrm { i } \operatorname { s i n } ( \frac { 1 } { 2 } ( \widehat { \varphi } _ { 1 } + \widehat { \varphi } _ { 3 } ) ) \operatorname { c o s h } \left(\widetilde{a}_{-} t+\widetilde{c}_{-}\right.\right.\right. \\
& \left.\left.+\frac{1}{8} \ln \left(\frac{4-a_{1}^{2}}{4-a_{2}^{2}}\right)+\ln \widetilde{\beth}_{-}\right)+\cos \left(\frac{1}{2}\left(\widehat{\varphi}_{1}+\widehat{\varphi}_{3}\right)\right) \sinh \left(\widetilde{a}_{-} t+\widetilde{c}_{-}+\frac{1}{8} \ln \left(\frac{4-a_{1}^{2}}{4-a_{2}^{2}}\right)+\ln \widetilde{\beth}_{-}\right)\right) \\
& \left.+\mathcal{O}\left(\frac{c}{\left(z_{o}\right) \mathrm{e}^{-4 \alpha t}}\right)\right),
\end{aligned}
$$

where

$$
\widetilde{\mathfrak{I}}_{+}:=\left(\frac{\left|r\left(\overline{s_{2}}\right)\right|\left|r\left(s_{1}\right)\right|}{\left(1-r\left(s_{1}\right) \overline{r\left(\overline{s_{1}}\right)}\right)}\right)^{1 / 2}, \quad \widetilde{\mathfrak{I}}_{-}:=\left(\frac{\left|r\left(\overline{s_{2}}\right)\right|\left(1-r\left(s_{1}\right) \overline{r\left(\overline{s_{1}}\right)}\right)}{\left|r\left(s_{1}\right)\right|}\right)^{1 / 2} ;
$$

and (iv) for $r\left(s_{2}\right)=\exp \left(\frac{\mathrm{i} \varepsilon_{1} \pi}{2}\right)\left|r\left(s_{2}\right)\right|, \varepsilon_{1} \in\{ \pm 1\}, r\left(\overline{s_{1}}\right)=\exp \left(-\frac{\mathrm{i} \varepsilon_{2} \pi}{2}\right)\left|r\left(\overline{s_{1}}\right)\right|, \varepsilon_{2} \in\{ \pm 1\}, 0<$ $r\left(s_{2}\right) \overline{r\left(\overline{s_{2}}\right)}<1$, and $\varepsilon_{1}=\varepsilon_{2}$, as $t \rightarrow-\infty$ and $x \rightarrow-\infty$ such that $z_{o} \in(0,2)$,

$$
\begin{aligned}
u(x, t) & =-\mathrm{e}^{-\mathrm{i} \psi^{-}(1)}\left(1+\frac{\mathrm{e}^{\frac{\mathrm{i}}{2}\left(\varepsilon_{1} \pi+\left(\widehat{\varphi}_{1}-\widehat{\varphi}_{3}\right)\right)} \mathrm{e}^{-\left(\widetilde{a}_{+}|t|-\widehat{c}_{+}\right) \widehat{\beth}_{+}}}{\widetilde{b} \sqrt{|t|}}\left(\mathrm { i } \operatorname { s i n } ( \frac { 1 } { 2 } ( \widehat { \varphi } _ { 1 } + \widehat { \varphi } _ { 3 } ) ) \operatorname { s i n h } \left(\widetilde{a}_{-}|t|-\widehat{c}_{-}\right.\right.\right. \\
& \left.\left.+\frac{1}{8} \ln \left(\frac{4-a_{1}^{2}}{4-a_{2}^{2}}\right)-\ln \widehat{\Xi}_{-}\right)-\cos \left(\frac{1}{2}\left(\widehat{\varphi}_{1}+\widehat{\varphi}_{3}\right)\right) \cosh \left(\widetilde{a}_{-}|t|-\widehat{c}_{-}+\frac{1}{8} \ln \left(\frac{4-a_{1}^{2}}{4-a_{2}^{2}}\right)-\ln \widehat{\beth}_{-}\right)\right) \\
& \left.+\mathcal{O}\left(\frac{c\left(z_{o}\right) \mathrm{e}^{-4 \alpha|t|}}{\beta t}\right)\right),
\end{aligned}
$$

and, for $\varepsilon_{1}=-\varepsilon_{2}$,

$$
\begin{aligned}
u(x, t) & =-\mathrm{e}^{-\mathrm{i} \psi^{-}(1)}\left(1+\frac{\mathrm{e}^{\frac{\mathrm{i}}{2}\left(\varepsilon_{1} \pi+\left(\widehat{\varphi}_{1}-\widehat{\varphi}_{3}\right)\right)} \mathrm{e}^{-\left(\widetilde{a}_{+}|t|-\widehat{c}_{+}\right) \widehat{\beth}_{+}}}{\widetilde{b} \sqrt{|t|}}\left(\mathrm { i } \operatorname { s i n } ( \frac { 1 } { 2 } ( \widehat { \varphi } _ { 1 } + \widehat { \varphi } _ { 3 } ) ) \operatorname { c o s h } \left(\widetilde{a}_{-}|t|-\widehat{c}_{-}\right.\right.\right. \\
& \left.\left.+\frac{1}{8} \ln \left(\frac{4-a_{1}^{2}}{4-a_{2}^{2}}\right)-\ln \widehat{\beth}_{-}\right)-\cos \left(\frac{1}{2}\left(\widehat{\varphi}_{1}+\widehat{\varphi}_{3}\right)\right) \sinh \left(\widetilde{a}_{-}|t|-\widehat{c}_{-}+\frac{1}{8} \ln \left(\frac{4-a_{1}^{2}}{4-a_{2}^{2}}\right)-\ln \widehat{\beth}_{-}\right)\right) \\
& \left.+\mathcal{O}\left(\frac{\frac{c}{\left(z_{o}\right) \mathrm{e}^{-4 \alpha|t|}}}{\beta t}\right)\right),
\end{aligned}
$$

where

$$
\widehat{\mathrm{I}}_{+}:=\left(\frac{\left|r\left(s_{2}\right)\right|\left|r\left(\overline{s_{1}}\right)\right|}{\left(1-r\left(s_{2}\right) \overline{r\left(\overline{s_{2}}\right)}\right)}\right)^{1 / 2}, \quad \widehat{\mathrm{I}}_{-}:=\left(\frac{\left|r\left(\overline{s_{1}}\right)\right|\left(1-r\left(s_{2}\right) \overline{r\left(\overline{s_{2}}\right)}\right)}{\left|r\left(s_{2}\right)\right|}\right)^{1 / 2} .
$$

For $u(x, t)$ as defined and given above, let $\int_{+\infty}^{x}\left(|u(\xi, t)|^{2}-1\right) \mathrm{d} \xi$ be defined by Eq. (6). Then: (i) for $r\left(s_{1}\right)=\exp \left(-\frac{\mathrm{i} \varepsilon_{1} \pi}{2}\right)\left|r\left(s_{1}\right)\right|, \varepsilon_{1} \in\{ \pm 1\}, r\left(\overline{s_{2}}\right)=\exp \left(\frac{\mathrm{i} \varepsilon_{2} \pi}{2}\right)\left|r\left(\overline{s_{2}}\right)\right|, \varepsilon_{2} \in\{ \pm 1\}, 0<r\left(s_{2}\right) \overline{r\left(\overline{s_{2}}\right)}<1$, 
and $\varepsilon_{1}=\varepsilon_{2}$, as $t \rightarrow+\infty$ and $x \rightarrow-\infty$ such that $z_{o} \in(-2,0)$,

$$
\begin{aligned}
\int_{+\infty}^{x}\left(|u(\xi, t)|^{2}-1\right) \mathrm{d} \xi & =\psi^{+}(0)-\frac{\operatorname{sgn}\left(\varepsilon_{1}\right) \mathrm{e}^{-\left(\widetilde{a}_{+} t+\widetilde{c}_{+}\right)} \beth_{+}}{\widetilde{b} \sqrt{t}} \cosh \left(\widetilde{a}_{-} t+\widetilde{c}_{-}+\frac{1}{8} \ln \left(\frac{4-a_{1}^{2}}{4-a_{2}^{2}}\right)-\ln \beth_{-}\right) \\
& +\mathcal{O}\left(\frac{\underline{c}\left(z_{o}\right) \mathrm{e}^{-4 \alpha t}}{\beta t}\right) \\
\int_{-\infty}^{x}\left(|u(\xi, t)|^{2}-1\right) \mathrm{d} \xi & =-\psi^{-}(0)-\frac{\operatorname{sgn}\left(\varepsilon_{1}\right) \mathrm{e}^{-\left(\widetilde{a}_{+} t+\widetilde{c}_{+}\right)} \beth_{+}}{\widetilde{b} \sqrt{t}} \cosh \left(\widetilde{a}_{-} t+\widetilde{c}_{-}+\frac{1}{8} \ln \left(\frac{4-a_{1}^{2}}{4-a_{2}^{2}}\right)-\ln \beth_{-}\right) \\
& +\mathcal{O}\left(\frac{c}{\beta t}\right)
\end{aligned}
$$

and, for $\varepsilon_{1}=-\varepsilon_{2}$,

$$
\begin{aligned}
\int_{+\infty}^{x}\left(|u(\xi, t)|^{2}-1\right) \mathrm{d} \xi & =\psi^{+}(0)+\frac{\operatorname{sgn}\left(\varepsilon_{1}\right) \mathrm{e}^{-\left(\widetilde{a}_{+} t+\widetilde{c}_{+}\right)} \beth_{+}}{\widetilde{b} \sqrt{t}} \sinh \left(\widetilde{a}_{-} t+\widetilde{c}_{-}+\frac{1}{8} \ln \left(\frac{4-a_{1}^{2}}{4-a_{2}^{2}}\right)-\ln \beth_{-}\right) \\
& +\mathcal{O}\left(\frac{\underline{c}\left(z_{o}\right) \mathrm{e}^{-4 \alpha t}}{\beta t}\right) \\
\int_{-\infty}^{x}\left(|u(\xi, t)|^{2}-1\right) \mathrm{d} \xi & =-\psi^{-}(0)+\frac{\operatorname{sgn}\left(\varepsilon_{1}\right) \mathrm{e}^{-\left(\widetilde{a}_{+} t+\widetilde{c}_{+}\right)} \beth_{+}}{\widetilde{b} \sqrt{t}} \sinh \left(\widetilde{a}_{-} t+\widetilde{c}_{-}+\frac{1}{8} \ln \left(\frac{4-a_{1}^{2}}{4-a_{2}^{2}}\right)-\ln \beth_{-}\right) \\
& +\mathcal{O}\left(\frac{\underline{c}\left(z_{o}\right) \mathrm{e}^{-4 \alpha t}}{\beta t}\right) ;
\end{aligned}
$$

(ii) for $r\left(\overline{s_{1}}\right)=\exp \left(\frac{\mathrm{i} \varepsilon_{1} \pi}{2}\right)\left|r\left(\overline{s_{1}}\right)\right|, \varepsilon_{1} \in\{ \pm 1\}, r\left(s_{2}\right)=\exp \left(-\frac{\mathrm{i} \varepsilon_{2} \pi}{2}\right)\left|r\left(s_{2}\right)\right|, \varepsilon_{2} \in\{ \pm 1\}, 0<$ $r\left(s_{1}\right) \overline{r\left(\overline{s_{1}}\right)}<1$, and $\varepsilon_{1}=\varepsilon_{2}$, as $t \rightarrow-\infty$ and $x \rightarrow+\infty$ such that $z_{o} \in(-2,0)$,

$$
\begin{aligned}
\int_{+\infty}^{x}\left(|u(\xi, t)|^{2}-1\right) \mathrm{d} \xi & \left.=\psi^{-}(0)+\frac{\left.\operatorname{sgn}\left(\varepsilon_{1}\right) \mathrm{e}^{-\left(\widetilde{a}_{+}|t|-\widehat{c}_{+}\right)}\right\rceil_{+}}{\widetilde{b} \sqrt{|t|}} \cosh \left(\widetilde{a}_{-}|t|-\widehat{c}_{-}+\frac{1}{8} \ln \left(\frac{4-a_{1}^{2}}{4-a_{2}^{2}}\right)+\ln \right\rceil_{-}\right) \\
& +\mathcal{O}\left(\frac{\underline{c}\left(z_{0}\right) \mathrm{e}^{-4 \alpha|t|}}{\beta t}\right), \\
\int_{-\infty}^{x}\left(|u(\xi, t)|^{2}-1\right) \mathrm{d} \xi & \left.=-\psi^{+}(0)+\frac{\left.\operatorname{sgn}\left(\varepsilon_{1}\right) \mathrm{e}^{-\left(\widetilde{a}_{+}|t|-\widehat{c}_{+}\right)}\right\rceil_{+}}{\widetilde{b} \sqrt{|t|}} \cosh \left(\widetilde{a}_{-}|t|-\widehat{c}_{-}+\frac{1}{8} \ln \left(\frac{4-a_{1}^{2}}{4-a_{2}^{2}}\right)+\ln \right\urcorner_{-}\right) \\
& +\mathcal{O}\left(\frac{c\left(z_{o}\right) \mathrm{e}^{-4 \alpha|t|}}{\beta t}\right)
\end{aligned}
$$

and, for $\varepsilon_{1}=-\varepsilon_{2}$,

$$
\begin{aligned}
\int_{+\infty}^{x}\left(|u(\xi, t)|^{2}-1\right) \mathrm{d} \xi & \left.=\psi^{-}(0)-\frac{\left.\operatorname{sgn}\left(\varepsilon_{1}\right) \mathrm{e}^{-\left(\widetilde{a}_{+}|t|-\widehat{c}_{+}\right)}\right\urcorner_{+}}{\widetilde{b} \sqrt{|t|}} \sinh \left(\widetilde{a}_{-}|t|-\widehat{c}_{-}+\frac{1}{8} \ln \left(\frac{4-a_{1}^{2}}{4-a_{2}^{2}}\right)+\ln \right\urcorner_{-}\right) \\
& +\mathcal{O}\left(\frac{\underline{c}\left(z_{o}\right) \mathrm{e}^{-4 \alpha|t|}}{\beta t}\right), \\
\int_{-\infty}^{x}\left(|u(\xi, t)|^{2}-1\right) \mathrm{d} \xi & \left.=-\psi^{+}(0)-\frac{\left.\operatorname{sgn}\left(\varepsilon_{1}\right) \mathrm{e}^{-\left(\widetilde{a}_{+}|t|-\widehat{c}_{+}\right)}\right\rceil_{+}}{\widetilde{b} \sqrt{|t|}} \sinh \left(\widetilde{a}_{-}|t|-\widehat{c}_{-}+\frac{1}{8} \ln \left(\frac{4-a_{1}^{2}}{4-a_{2}^{2}}\right)+\ln \right\urcorner_{-}\right) \\
& +\mathcal{O}\left(\frac{c}{\beta t}\right) ;
\end{aligned}
$$


(iii) for $r\left(\overline{s_{2}}\right)=\exp \left(-\frac{\mathrm{i} \varepsilon_{1} \pi}{2}\right)\left|r\left(\overline{s_{2}}\right)\right|, \varepsilon_{1} \in\{ \pm 1\}, r\left(s_{1}\right)=\exp \left(\frac{\mathrm{i} \varepsilon_{2} \pi}{2}\right)\left|r\left(s_{1}\right)\right|, \varepsilon_{2} \in\{ \pm 1\}, 0<$ $r\left(s_{1}\right) \overline{r\left(\overline{s_{1}}\right)}<1$, and $\varepsilon_{1}=\varepsilon_{2}$, as $t \rightarrow+\infty$ and $x \rightarrow+\infty$ such that $z_{o} \in(0,2)$,

$$
\begin{aligned}
\int_{+\infty}^{x}\left(|u(\xi, t)|^{2}-1\right) \mathrm{d} \xi & =\psi^{-}(0)+\frac{\operatorname{sgn}\left(\varepsilon_{1}\right) \mathrm{e}^{-\left(\widetilde{a}_{+} t+\widetilde{c}_{+}\right)} \widetilde{\Xi}_{+}}{\widetilde{b} \sqrt{t}} \cosh \left(\widetilde{a}_{-} t+\widetilde{c}_{-}+\frac{1}{8} \ln \left(\frac{4-a_{1}^{2}}{4-a_{2}^{2}}\right)+\ln \widetilde{\Xi}_{-}\right) \\
& +\mathcal{O}\left(\frac{\underline{c}\left(z_{o}\right) \mathrm{e}^{-4 \alpha t}}{\beta t}\right) \\
\int_{-\infty}^{x}\left(|u(\xi, t)|^{2}-1\right) \mathrm{d} \xi & =-\psi^{+}(0)+\frac{\operatorname{sgn}\left(\varepsilon_{1}\right) \mathrm{e}^{-\left(\widetilde{a}_{+} t+\widetilde{c}_{+}\right)}}{\widetilde{\boldsymbol{\Xi}} \sqrt{t}} \cosh \left(\widetilde{a}_{-} t+\widetilde{c}_{-}+\frac{1}{8} \ln \left(\frac{4-a_{1}^{2}}{4-a_{2}^{2}}\right)+\ln \widetilde{\Xi}_{-}\right) \\
& +\mathcal{O}\left(\frac{\underline{c}\left(z_{o}\right) \mathrm{e}^{-4 \alpha t}}{\beta t}\right),
\end{aligned}
$$

and, for $\varepsilon_{1}=-\varepsilon_{2}$,

$$
\begin{aligned}
\int_{+\infty}^{x}\left(|u(\xi, t)|^{2}-1\right) \mathrm{d} \xi & =\psi^{-}(0)+\frac{\operatorname{sgn}\left(\varepsilon_{1}\right) \mathrm{e}^{-\left(\widetilde{a}_{+} t+\widetilde{c}_{+}\right)}}{\widetilde{\boldsymbol{I}} \sqrt{t}} \sinh \left(\widetilde{a}_{-} t+\widetilde{c}_{-}+\frac{1}{8} \ln \left(\frac{4-a_{1}^{2}}{4-a_{2}^{2}}\right)+\ln \widetilde{\beth}_{-}\right) \\
& +\mathcal{O}\left(\frac{\underline{c}\left(z_{0}\right) \mathrm{e}^{-4 \alpha t}}{\beta t}\right) \\
\int_{-\infty}^{x}\left(|u(\xi, t)|^{2}-1\right) \mathrm{d} \xi & =-\psi^{+}(0)+\frac{\operatorname{sgn}\left(\varepsilon_{1}\right) \mathrm{e}^{-\left(\widetilde{a}_{+} t+\widetilde{c}_{+}\right)}}{\widetilde{\beth} \sqrt{t}} \sinh \left(\widetilde{a}_{-} t+\widetilde{c}_{-}+\frac{1}{8} \ln \left(\frac{4-a_{1}^{2}}{4-a_{2}^{2}}\right)+\ln \widetilde{\Xi}_{-}\right) \\
& +\mathcal{O}\left(\frac{c}{\beta t}\right)
\end{aligned}
$$

and (iv) for $r\left(s_{2}\right)=\exp \left(\frac{\mathrm{i} \varepsilon_{1} \pi}{2}\right)\left|r\left(s_{2}\right)\right|, \varepsilon_{1} \in\{ \pm 1\}, r\left(\overline{s_{1}}\right)=\exp \left(-\frac{\mathrm{i} \varepsilon_{2} \pi}{2}\right)\left|r\left(\overline{s_{1}}\right)\right|, \varepsilon_{2} \in\{ \pm 1\}, 0<$ $r\left(s_{2}\right) \overline{r\left(\overline{s_{2}}\right)}<1$, and $\varepsilon_{1}=\varepsilon_{2}$, as $t \rightarrow-\infty$ and $x \rightarrow-\infty$ such that $z_{o} \in(0,2)$,

$$
\begin{aligned}
\int_{+\infty}^{x}\left(|u(\xi, t)|^{2}-1\right) \mathrm{d} \xi & =\psi^{+}(0)-\frac{\operatorname{sgn}\left(\varepsilon_{1}\right) \mathrm{e}^{-\left(\widetilde{a}_{+}|t|-\widehat{c}_{+}\right) \widehat{\beth}_{+}}}{\widetilde{b} \sqrt{|t|}} \cosh \left(\widetilde{a}_{-}|t|-\widehat{c}_{-}+\frac{1}{8} \ln \left(\frac{4-a_{1}^{2}}{4-a_{2}^{2}}\right)-\ln \widehat{\Xi}_{-}\right) \\
& +\mathcal{O}\left(\frac{\underline{c}\left(z_{o}\right) \mathrm{e}^{-4 \alpha|t|}}{\beta t}\right), \\
\int_{-\infty}^{x}\left(|u(\xi, t)|^{2}-1\right) \mathrm{d} \xi & =-\psi^{-}(0)-\frac{\operatorname{sgn}\left(\varepsilon_{1}\right) \mathrm{e}^{-\left(\widetilde{a}_{+}|t|-\widehat{c}_{+}\right) \widehat{\beth}_{+}}}{\widetilde{b} \sqrt{|t|}} \cosh \left(\widetilde{a}_{-}|t|-\widehat{c}_{-}+\frac{1}{8} \ln \left(\frac{4-a_{1}^{2}}{4-a_{2}^{2}}\right)-\ln \widehat{\Xi}_{-}\right) \\
& +\mathcal{O}\left(\frac{c\left(z_{o}\right) \mathrm{e}^{-4 \alpha|t|}}{\beta t}\right),
\end{aligned}
$$

and, for $\varepsilon_{1}=-\varepsilon_{2}$,

$$
\begin{aligned}
\int_{+\infty}^{x}\left(|u(\xi, t)|^{2}-1\right) \mathrm{d} \xi & =\psi^{+}(0)-\frac{\operatorname{sgn}\left(\varepsilon_{1}\right) \mathrm{e}^{-\left(\widetilde{a}_{+}|t|-\widehat{c}_{+}\right) \widehat{\beth}_{+}}}{\widetilde{b} \sqrt{|t|}} \sinh \left(\widetilde{a}_{-}|t|-\widehat{c}_{-}+\frac{1}{8} \ln \left(\frac{4-a_{1}^{2}}{4-a_{2}^{2}}\right)-\ln \widehat{\beth}_{-}\right) \\
& +\mathcal{O}\left(\frac{c}{\beta t}\right), \\
\int_{-\infty}^{x}\left(|u(\xi, t)|^{2}-1\right) \mathrm{d} \xi & =-\psi^{-4 \alpha|t|}(0)-\frac{\operatorname{sgn}\left(\varepsilon_{1}\right) \mathrm{e}^{-\left(\widetilde{a}_{+}|t|-\widehat{c}_{+}\right) \widehat{\beth}_{+}}}{\widetilde{b} \sqrt{|t|}} \sinh \left(\widetilde{a}_{-}|t|-\widehat{c}_{-}+\frac{1}{8} \ln \left(\frac{4-a_{1}^{2}}{4-a_{2}^{2}}\right)-\ln \widehat{\Xi}_{-}\right) \\
& +\mathcal{O}\left(\frac{c\left(z_{o}\right) \mathrm{e}^{-4 \alpha|t|}}{\beta t}\right) .
\end{aligned}
$$


Theorem 3.3. For $r(\zeta) \in \mathcal{S}_{\mathbb{C}}(\mathbb{R}) \cap\left\{h(z) ; \|\left. h(\cdot)\right|_{\mathcal{L}^{\infty}(\mathbb{R})}:=\sup _{z \in \mathbb{R}}|h(z)|<1\right\}$, let $m(x, t ; \zeta)$ be the solution of the Riemann-Hilbert problem formulated in Lemma 2.5. Let $u(x, t)$, the solution of the Cauchy problem for the $\mathrm{D}_{f} \mathrm{NLSE}$ with finite-density initial data $u(x, 0):=$ $u_{o}(x)={ }_{x \rightarrow \pm \infty} u_{o}( \pm \infty)(1+o(1))$, where $u_{o}( \pm \infty):=\exp \left(\frac{\mathrm{i}(1 \mp 1) \theta}{2}\right), 0 \leqslant \theta=-\int_{-\infty}^{+\infty} \frac{\ln \left(1-|r(\mu)|^{2}\right)}{\mu} \frac{\mathrm{d} \mu}{2 \pi}<$ $2 \pi, u_{o}(x) \in \mathbf{C}^{\infty}(\mathbb{R})$, and $u_{o}(x)-u_{o}( \pm \infty) \in \mathcal{S}_{\mathbb{C}}\left(\mathbb{R}_{ \pm}\right)$, be defined by Eq. (5). Set $s_{1}:=\exp \left(\frac{\mathrm{i} \pi}{4}\right)$ and $s_{2}:=\exp \left(\frac{3 \pi \mathrm{i}}{4}\right)$. Then: (i) for $r\left(s_{1}\right)=\exp \left(-\frac{\mathrm{i} \varepsilon_{1} \pi}{2}\right)\left|r\left(s_{1}\right)\right|, \varepsilon_{1} \in\{ \pm 1\}, r\left(\overline{s_{2}}\right)=\exp \left(\frac{\mathrm{i} \varepsilon_{2} \pi}{2}\right)\left|r\left(\overline{s_{2}}\right)\right|$, $\varepsilon_{2} \in\{ \pm 1\}, 0<r\left(s_{2}\right) \overline{r\left(\overline{s_{2}}\right)}<1$, and $\varepsilon_{1}=\varepsilon_{2}$, as $t \rightarrow+\infty$ and $x \rightarrow-\infty$ such that $z_{o}:=x / t \rightarrow 0^{-}$,

$$
u(x, t)=\mathrm{e}^{-\mathrm{i} \psi^{+}(1)}\left(1+\frac{\mathrm{e}^{\mathrm{i} \pi} \frac{\left(\varepsilon_{1}+\frac{3}{2}\right)}{\mathrm{e}^{-\left(2 t+\widetilde{\mathfrak{c}}_{+}\right)} \mathfrak{b}_{+}}}{2 \sqrt{t}} \sinh \left(\widetilde{\mathfrak{c}}_{-}-\ln \mathfrak{b}_{-}\right)+\mathcal{O}\left(\frac{\underline{c} \mathrm{e}^{-4 t}}{t}\right)\right),
$$

and, for $\varepsilon_{1}=-\varepsilon_{2}$,

$$
u(x, t)=\mathrm{e}^{-\mathrm{i} \psi^{+}(1)}\left(1-\frac{\mathrm{e}^{\frac{\mathrm{i} \pi}{2}\left(\varepsilon_{1}+\frac{3}{2}\right)} \mathrm{e}^{-\left(2 t+\tilde{\mathfrak{c}}_{+}\right)} \mathfrak{b}_{+}}{2 \sqrt{t}} \cosh \left(\widetilde{\mathfrak{c}}_{-}-\ln \mathfrak{b}_{-}\right)+\mathcal{O}\left(\frac{\underline{c} \mathrm{e}^{-4 t}}{t}\right)\right),
$$

where

$$
\begin{gathered}
\widetilde{\mathfrak{c}}_{ \pm}:=\frac{1}{\sqrt{2}} \int_{-\infty}^{0} \frac{\ln \left(1-|r(\mu)|^{2}\right)}{(\sqrt{2} \mu-1)^{2}+1} \frac{\mathrm{d} \mu}{\pi} \mp \frac{1}{\sqrt{2}} \int_{-\infty}^{0} \frac{\ln \left(1-|r(\mu)|^{2}\right)}{(\sqrt{2} \mu+1)^{2}+1} \frac{\mathrm{d} \mu}{\pi}, \\
\mathfrak{b}_{+}:=\left(\frac{\left|r\left(s_{1}\right)\right|\left|r\left(\overline{s_{2}}\right)\right|}{\left(1-r\left(s_{2}\right) \overline{r\left(\overline{s_{2}}\right)}\right)}\right)^{1 / 2}, \quad \mathfrak{b}_{-}:=\left(\frac{\left|r\left(s_{1}\right)\right|\left(1-r\left(s_{2}\right) \overline{r\left(\overline{s_{2}}\right)}\right)}{\left|r\left(\overline{s_{2}}\right)\right|}\right)^{1 / 2},
\end{gathered}
$$

and $\psi^{+}(\cdot)$ is defined in Theorem 3.2, Eq. (35); (ii) for $r\left(\overline{s_{1}}\right)=\exp \left(\frac{\mathrm{i} \varepsilon_{1} \pi}{2}\right)\left|r\left(\overline{s_{1}}\right)\right|, \varepsilon_{1} \in\{ \pm 1\}$, $r\left(s_{2}\right)=\exp \left(-\frac{\mathrm{i} \varepsilon_{2} \pi}{2}\right)\left|r\left(s_{2}\right)\right|, \varepsilon_{2} \in\{ \pm 1\}, 0<r\left(s_{1}\right) \overline{r\left(\overline{s_{1}}\right)}<1$, and $\varepsilon_{1}=\varepsilon_{2}$, as $t \rightarrow-\infty$ and $x \rightarrow+\infty$ such that $z_{0} \rightarrow 0^{-}$,

$$
u(x, t)=\mathrm{e}^{-\mathrm{i} \psi^{-}(1)}\left(1-\frac{\mathrm{e}^{\frac{\mathrm{i} \pi}{2}\left(\varepsilon_{1}+\frac{1}{2}\right)} \mathrm{e}^{-\left(2|t|-\widehat{\mathfrak{c}}_{+}\right)} \mathfrak{d}_{+}}{2 \sqrt{|t|}} \sinh \left(\widehat{\mathfrak{c}}_{-}-\ln \mathfrak{d}_{-}\right)+\mathcal{O}\left(\frac{\underline{c} \mathrm{e}^{-4|t|}}{t}\right)\right),
$$

and, for $\varepsilon_{1}=-\varepsilon_{2}$,

$$
u(x, t)=\mathrm{e}^{-\mathrm{i} \psi^{-}(1)}\left(1+\frac{\mathrm{e}^{\frac{\mathbf{i} \pi}{2}\left(\varepsilon_{1}-\frac{3}{2}\right)} \mathrm{e}^{-\left(2|t|-\widehat{\mathfrak{c}}_{+}\right)} \mathfrak{d}_{+}}{2 \sqrt{|t|}} \cosh \left(\widehat{\mathfrak{c}}_{-}-\ln \mathfrak{d}_{-}\right)+\mathcal{O}\left(\frac{\underline{c} \mathrm{e}^{-4|t|}}{t}\right)\right),
$$

where

$$
\begin{gathered}
\widehat{\mathfrak{c}}_{ \pm}:=\frac{1}{\sqrt{2}} \int_{0}^{+\infty} \frac{\ln \left(1-|r(\mu)|^{2}\right)}{(\sqrt{2} \mu-1)^{2}+1} \frac{\mathrm{d} \mu}{\pi} \mp \frac{1}{\sqrt{2}} \int_{0}^{+\infty} \frac{\ln \left(1-|r(\mu)|^{2}\right)}{(\sqrt{2} \mu+1)^{2}+1} \frac{\mathrm{d} \mu}{\pi} \\
\mathfrak{d}_{+}:=\left(\frac{\left|r\left(\overline{s_{1}}\right)\right|\left|r\left(s_{2}\right)\right|}{\left(1-r\left(s_{1}\right) \overline{r\left(\overline{s_{1}}\right)}\right)}\right)^{1 / 2}, \quad \mathfrak{d}_{-}:=\left(\frac{\left|r\left(s_{2}\right)\right|\left(1-r\left(s_{1}\right) \overline{r\left(\overline{s_{1}}\right)}\right)}{\left|r\left(\overline{s_{1}}\right)\right|}\right)^{1 / 2},
\end{gathered}
$$

and $\psi^{-}(\cdot)$ is defined in Theorem 3.2, Eq. (46); (iii) for $r\left(\overline{s_{2}}\right)=\exp \left(-\frac{\mathrm{i} \varepsilon_{1} \pi}{2}\right)\left|r\left(\overline{s_{2}}\right)\right|, \varepsilon_{1} \in\{ \pm 1\}$, $r\left(s_{1}\right)=\exp \left(\frac{\mathrm{i} \varepsilon_{2} \pi}{2}\right)\left|r\left(s_{1}\right)\right|, \varepsilon_{2} \in\{ \pm 1\}, 0<r\left(s_{1}\right) \overline{r\left(\overline{s_{1}}\right)}<1$, and $\varepsilon_{1}=\varepsilon_{2}$, as $t \rightarrow+\infty$ and $x \rightarrow+\infty$ such that $z_{o} \rightarrow 0^{+}$,

$$
u(x, t)=-\mathrm{e}^{-\mathrm{i} \psi^{+}(1)}\left(1+\frac{\mathrm{e}^{\frac{\mathrm{i} \pi}{2}\left(\varepsilon_{1}+\frac{3}{2}\right)} \mathrm{e}^{-\left(2 t+\widetilde{\mathfrak{c}}_{+}\right)} \widetilde{\mathfrak{g}}_{+}}{2 \sqrt{t}} \sinh \left(\widetilde{\mathfrak{c}}_{-}+\ln \widetilde{\mathfrak{g}}_{-}\right)+\mathcal{O}\left(\frac{\underline{c} \mathrm{e}^{-4 t}}{t}\right)\right)
$$


and, for $\varepsilon_{1}=-\varepsilon_{2}$,

$$
u(x, t)=-\mathrm{e}^{-\mathrm{i} \psi^{+}(1)}\left(1-\frac{\mathrm{e}^{\frac{\mathrm{i} \pi}{2}\left(\varepsilon_{1}-\frac{1}{2}\right)} \mathrm{e}^{-\left(2 t+\widetilde{\mathfrak{c}}_{+}\right)} \widetilde{\mathfrak{g}}_{+}}{2 \sqrt{t}} \cosh \left(\widetilde{\mathfrak{c}}_{-}+\ln \widetilde{\mathfrak{g}}_{-}\right)+\mathcal{O}\left(\frac{\underline{c} \mathrm{e}^{-4 t}}{t}\right)\right),
$$

where

$$
\widetilde{\mathfrak{g}}_{+}:=\left(\frac{\left|r\left(\overline{s_{2}}\right)\right|\left|r\left(s_{1}\right)\right|}{\left(1-r\left(s_{1}\right) \overline{r\left(\overline{s_{1}}\right)}\right)}\right)^{1 / 2}, \quad \widetilde{\mathfrak{g}}_{-}:=\left(\frac{\left|r\left(\overline{s_{2}}\right)\right|\left(1-r\left(s_{1}\right) \overline{r\left(\overline{s_{1}}\right)}\right)}{\left|r\left(s_{1}\right)\right|}\right)^{1 / 2}
$$

and (iv) for $r\left(s_{2}\right)=\exp \left(\frac{\mathrm{i} \varepsilon_{1} \pi}{2}\right)\left|r\left(s_{2}\right)\right|, \varepsilon_{1} \in\{ \pm 1\}, r\left(\overline{s_{1}}\right)=\exp \left(-\frac{\mathrm{i} \varepsilon_{2} \pi}{2}\right)\left|r\left(\overline{s_{1}}\right)\right|, \varepsilon_{2} \in\{ \pm 1\}, 0<$ $r\left(s_{2}\right) \overline{r\left(\overline{s_{2}}\right)}<1$, and $\varepsilon_{1}=\varepsilon_{2}$, as $t \rightarrow-\infty$ and $x \rightarrow-\infty$ such that $z_{o} \rightarrow 0^{+}$,

$$
u(x, t)=-\mathrm{e}^{-\mathrm{i} \psi^{-}(1)}\left(1-\frac{\mathrm{e}^{\frac{\mathrm{i} \pi}{2}\left(\varepsilon_{1}+\frac{1}{2}\right)} \mathrm{e}^{-\left(2|t|-\widehat{\mathfrak{c}}_{+}\right)} \widehat{\mathfrak{g}}_{+}}{2 \sqrt{|t|}} \sinh \left(\widehat{\mathfrak{c}}_{-}+\ln \widehat{\mathfrak{g}}_{-}\right)+\mathcal{O}\left(\frac{\underline{c} \mathrm{e}^{-4|t|}}{t}\right)\right),
$$

and, for $\varepsilon_{1}=-\varepsilon_{2}$,

$$
u(x, t)=-\mathrm{e}^{-\mathrm{i} \psi^{-}(1)}\left(1+\frac{\mathrm{e}^{\frac{\mathrm{i} \pi}{2}\left(\varepsilon_{1}+\frac{1}{2}\right)} \mathrm{e}^{-\left(2|t|-\widehat{\mathfrak{c}}_{+}\right)} \widehat{\mathfrak{g}}_{+}}{2 \sqrt{|t|}} \cosh \left(\widehat{\mathfrak{c}}_{-}+\ln \widehat{\mathfrak{g}}_{-}\right)+\mathcal{O}\left(\frac{\underline{c} \mathrm{e}^{-4|t|}}{t}\right)\right),
$$

where

$$
\widehat{\mathfrak{g}}_{+}:=\left(\frac{\left|r\left(s_{2}\right)\right|\left|r\left(\overline{s_{1}}\right)\right|}{\left(1-r\left(s_{2}\right) \overline{r\left(\overline{s_{2}}\right)}\right)}\right)^{1 / 2}, \quad \widehat{\mathfrak{g}}_{-}:=\left(\frac{\left|r\left(\overline{s_{1}}\right)\right|\left(1-r\left(s_{2}\right) \overline{r\left(\overline{s_{2}}\right)}\right)}{\left|r\left(s_{2}\right)\right|}\right)^{1 / 2} .
$$

For $u(x, t)$ as defined and given above, let $\int_{+\infty}^{x}\left(|u(\xi, t)|^{2}-1\right) \mathrm{d} \xi$ be defined by Eq. (6). Then: (i) for $r\left(s_{1}\right)=\exp \left(-\frac{\mathrm{i} \varepsilon_{1} \pi}{2}\right)\left|r\left(s_{1}\right)\right|, \varepsilon_{1} \in\{ \pm 1\}, r\left(\overline{s_{2}}\right)=\exp \left(\frac{\mathrm{i} \varepsilon_{2} \pi}{2}\right)\left|r\left(\overline{s_{2}}\right)\right|, \varepsilon_{2} \in\{ \pm 1\}, 0<r\left(s_{2}\right) \overline{r\left(\overline{s_{2}}\right)}<1$, and $\varepsilon_{1}=\varepsilon_{2}$, as $t \rightarrow+\infty$ and $x \rightarrow-\infty$ such that $z_{o} \rightarrow 0^{-}$,

$$
\begin{aligned}
& \int_{+\infty}^{x}\left(|u(\xi, t)|^{2}-1\right) \mathrm{d} \xi=\psi^{+}(0)-\frac{\operatorname{sgn}\left(\varepsilon_{1}\right) \mathrm{e}^{-\left(2 t+\widetilde{\mathfrak{c}}_{+}\right)} \mathfrak{b}_{+}}{2 \sqrt{t}} \cosh \left(\widetilde{\mathfrak{c}}_{-}-\ln \mathfrak{b}_{-}\right)+\mathcal{O}\left(\frac{\underline{c} \mathrm{e}^{-4 t}}{t}\right) \\
& \int_{-\infty}^{x}\left(|u(\xi, t)|^{2}-1\right) \mathrm{d} \xi=-\psi^{-}(0)-\frac{\operatorname{sgn}\left(\varepsilon_{1}\right) \mathrm{e}^{-\left(2 t+\widetilde{\mathfrak{c}}_{+}\right)} \mathfrak{b}_{+}}{2 \sqrt{t}} \cosh \left(\widetilde{\mathfrak{c}}_{-}-\ln \mathfrak{b}_{-}\right)+\mathcal{O}\left(\frac{\underline{c} \mathrm{e}^{-4 t}}{t}\right)
\end{aligned}
$$

and, for $\varepsilon_{1}=-\varepsilon_{2}$,

$$
\begin{aligned}
& \int_{+\infty}^{x}\left(|u(\xi, t)|^{2}-1\right) \mathrm{d} \xi=\psi^{+}(0)+\frac{\operatorname{sgn}\left(\varepsilon_{1}\right) \mathrm{e}^{-\left(2 t+\widetilde{\mathfrak{c}}_{+}\right)} \mathfrak{b}_{+}}{2 \sqrt{t}} \sinh \left(\widetilde{\mathfrak{c}}_{-}-\ln \mathfrak{b}_{-}\right)+\mathcal{O}\left(\frac{\underline{c} \mathrm{e}^{-4 t}}{t}\right) \\
& \int_{-\infty}^{x}\left(|u(\xi, t)|^{2}-1\right) \mathrm{d} \xi=-\psi^{-}(0)+\frac{\operatorname{sgn}\left(\varepsilon_{1}\right) \mathrm{e}^{-\left(2 t+\widetilde{\mathfrak{c}}_{+}\right)} \mathfrak{b}_{+}}{2 \sqrt{t}} \sinh \left(\widetilde{\mathfrak{c}}_{-}-\ln \mathfrak{b}_{-}\right)+\mathcal{O}\left(\frac{\underline{c} \mathrm{e}^{-4 t}}{t}\right)
\end{aligned}
$$

(ii) for $r\left(\overline{s_{1}}\right)=\exp \left(\frac{\mathrm{i} \varepsilon_{1} \pi}{2}\right)\left|r\left(\overline{s_{1}}\right)\right|, \varepsilon_{1} \in\{ \pm 1\}, r\left(s_{2}\right)=\exp \left(-\frac{\mathrm{i} \varepsilon_{2} \pi}{2}\right)\left|r\left(s_{2}\right)\right|, \varepsilon_{2} \in\{ \pm 1\}, 0<$ $r\left(s_{1}\right) \overline{r\left(\overline{s_{1}}\right)}<1$, and $\varepsilon_{1}=\varepsilon_{2}$, as $t \rightarrow-\infty$ and $x \rightarrow+\infty$ such that $z_{o} \rightarrow 0^{-}$,

$$
\begin{aligned}
& \int_{+\infty}^{x}\left(|u(\xi, t)|^{2}-1\right) \mathrm{d} \xi=\psi^{-}(0)+\frac{\operatorname{sgn}\left(\varepsilon_{1}\right) \mathrm{e}^{-\left(2|t|-\widehat{\mathfrak{c}}_{+}\right)} \mathfrak{d}_{+}}{2 \sqrt{|t|}} \cosh \left(\widehat{\mathfrak{c}}_{-}-\ln \mathfrak{d}_{-}\right)+\mathcal{O}\left(\frac{\underline{c} \mathrm{e}^{-4|t|}}{t}\right), \\
& \int_{-\infty}^{x}\left(|u(\xi, t)|^{2}-1\right) \mathrm{d} \xi=-\psi^{+}(0)+\frac{\operatorname{sgn}\left(\varepsilon_{1}\right) \mathrm{e}^{-\left(2|t|-\widehat{\mathfrak{c}}_{+}\right)} \mathfrak{d}_{+}}{2 \sqrt{|t|}} \cosh \left(\widehat{\mathfrak{c}}_{-}-\ln \mathfrak{d}_{-}\right)+\mathcal{O}\left(\frac{\underline{c} \mathrm{e}^{-4|t|}}{t}\right),
\end{aligned}
$$


and, for $\varepsilon_{1}=-\varepsilon_{2}$,

$$
\begin{aligned}
& \int_{+\infty}^{x}\left(|u(\xi, t)|^{2}-1\right) \mathrm{d} \xi=\psi^{-}(0)+\frac{\operatorname{sgn}\left(\varepsilon_{1}\right) \mathrm{e}^{-\left(2|t|-\widehat{\mathfrak{c}}_{+}\right)} \mathfrak{d}_{+}}{2 \sqrt{|t|}} \sinh \left(\widehat{\mathfrak{c}}_{-}-\ln \mathfrak{d}_{-}\right)+\mathcal{O}\left(\frac{\underline{c} \mathrm{e}^{-4|t|}}{t}\right), \\
& \int_{-\infty}^{x}\left(|u(\xi, t)|^{2}-1\right) \mathrm{d} \xi=-\psi^{+}(0)+\frac{\operatorname{sgn}\left(\varepsilon_{1}\right) \mathrm{e}^{-\left(2|t|-\widehat{\mathfrak{c}}_{+}\right)} \mathfrak{d}_{+}}{2 \sqrt{|t|}} \sinh \left(\widehat{\mathfrak{c}}_{-}-\ln \mathfrak{d}_{-}\right)+\mathcal{O}\left(\frac{\underline{c} \mathrm{e}^{-4|t|}}{t}\right) ;
\end{aligned}
$$

(iii) for $r\left(\overline{s_{2}}\right)=\exp \left(-\frac{\mathrm{i} \varepsilon_{1} \pi}{2}\right)\left|r\left(\overline{s_{2}}\right)\right|, \varepsilon_{1} \in\{ \pm 1\}, r\left(s_{1}\right)=\exp \left(\frac{\mathrm{i} \varepsilon_{2} \pi}{2}\right)\left|r\left(s_{1}\right)\right|, \varepsilon_{2} \in\{ \pm 1\}, 0<$ $r\left(s_{1}\right) \overline{r\left(\overline{s_{1}}\right)}<1$, and $\varepsilon_{1}=\varepsilon_{2}$, as $t \rightarrow+\infty$ and $x \rightarrow+\infty$ such that $z_{o} \rightarrow 0^{+}$,

$$
\begin{aligned}
& \int_{+\infty}^{x}\left(|u(\xi, t)|^{2}-1\right) \mathrm{d} \xi=\psi^{-}(0)+\frac{\operatorname{sgn}\left(\varepsilon_{1}\right) \mathrm{e}^{-\left(2 t+\widetilde{\mathfrak{c}}_{+}\right)} \widetilde{\mathfrak{g}}_{+}}{2 \sqrt{t}} \cosh \left(\widetilde{\mathfrak{c}}_{-}+\ln \widetilde{\mathfrak{g}}_{-}\right)+\mathcal{O}\left(\frac{\underline{c} \mathrm{e}^{-4 t}}{t}\right) \\
& \int_{-\infty}^{x}\left(|u(\xi, t)|^{2}-1\right) \mathrm{d} \xi=-\psi^{+}(0)+\frac{\operatorname{sgn}\left(\varepsilon_{1}\right) \mathrm{e}^{-\left(2 t+\widetilde{\mathfrak{c}}_{+}\right)} \widetilde{\mathfrak{g}}_{+}}{2 \sqrt{t}} \cosh \left(\widetilde{\mathfrak{c}}_{-}+\ln \widetilde{\mathfrak{g}}_{-}\right)+\mathcal{O}\left(\frac{\underline{c} \mathrm{e}^{-4 t}}{t}\right)
\end{aligned}
$$

and, for $\varepsilon_{1}=-\varepsilon_{2}$,

$$
\begin{aligned}
& \int_{+\infty}^{x}\left(|u(\xi, t)|^{2}-1\right) \mathrm{d} \xi=\psi^{-}(0)+\frac{\operatorname{sgn}\left(\varepsilon_{1}\right) \mathrm{e}^{-\left(2 t+\widetilde{\mathfrak{c}}_{+}\right)} \widetilde{\mathfrak{g}}_{+}}{2 \sqrt{t}} \sinh \left(\widetilde{\mathfrak{c}}_{-}+\ln \widetilde{\mathfrak{g}}_{-}\right)+\mathcal{O}\left(\frac{\underline{c} \mathrm{e}^{-4 t}}{t}\right) \\
& \int_{-\infty}^{x}\left(|u(\xi, t)|^{2}-1\right) \mathrm{d} \xi=-\psi^{+}(0)+\frac{\operatorname{sgn}\left(\varepsilon_{1}\right) \mathrm{e}^{-\left(2 t+\widetilde{\mathfrak{c}}_{+}\right)} \widetilde{\mathfrak{g}}_{+}}{2 \sqrt{t}} \sinh \left(\widetilde{\mathfrak{c}}_{-}+\ln \widetilde{\mathfrak{g}}_{-}\right)+\mathcal{O}\left(\frac{\underline{c} \mathrm{e}^{-4 t}}{t}\right)
\end{aligned}
$$

and (iv) for $r\left(s_{2}\right)=\exp \left(\frac{\mathrm{i} \varepsilon_{1} \pi}{2}\right)\left|r\left(s_{2}\right)\right|, \varepsilon_{1} \in\{ \pm 1\}, r\left(\overline{s_{1}}\right)=\exp \left(-\frac{\mathrm{i} \varepsilon_{2} \pi}{2}\right)\left|r\left(\overline{s_{1}}\right)\right|, \varepsilon_{2} \in\{ \pm 1\}, 0<$ $r\left(s_{2}\right) \overline{r\left(\overline{s_{2}}\right)}<1$, and $\varepsilon_{1}=\varepsilon_{2}$, as $t \rightarrow-\infty$ and $x \rightarrow-\infty$ such that $z_{o} \rightarrow 0^{+}$,

$$
\begin{aligned}
& \int_{+\infty}^{x}\left(|u(\xi, t)|^{2}-1\right) \mathrm{d} \xi=\psi^{+}(0)-\frac{\operatorname{sgn}\left(\varepsilon_{1}\right) \mathrm{e}^{-\left(2|t|-\widehat{\mathfrak{c}}_{+}\right)}}{2 \sqrt{|t|}} \cosh \left(\widehat{\mathfrak{c}}_{-}+\ln \widehat{\mathfrak{g}}_{-}\right)+\mathcal{O}\left(\frac{\underline{c} \mathrm{e}^{-4|t|}}{t}\right), \\
& \int_{-\infty}^{x}\left(|u(\xi, t)|^{2}-1\right) \mathrm{d} \xi=-\psi^{-}(0)-\frac{\operatorname{sgn}\left(\varepsilon_{1}\right) \mathrm{e}^{-\left(2|t|-\widehat{\mathfrak{c}}_{+}\right)} \widehat{\mathfrak{g}}_{+}}{2 \sqrt{|t|}} \cosh \left(\widehat{\mathfrak{c}}_{-}+\ln \widehat{\mathfrak{g}}_{-}\right)+\mathcal{O}\left(\frac{\underline{c} \mathrm{e}^{-4|t|}}{t}\right),
\end{aligned}
$$

and, for $\varepsilon_{1}=-\varepsilon_{2}$,

$$
\begin{aligned}
& \int_{+\infty}^{x}\left(|u(\xi, t)|^{2}-1\right) \mathrm{d} \xi=\psi^{+}(0)+\frac{\operatorname{sgn}\left(\varepsilon_{1}\right) \mathrm{e}^{-\left(2|t|-\widehat{\mathfrak{c}}_{+}\right)} \widehat{\mathfrak{g}}_{+}}{2 \sqrt{|t|}} \sinh \left(\widehat{\mathfrak{c}}_{-}+\ln \widehat{\mathfrak{g}}_{-}\right)+\mathcal{O}\left(\frac{\underline{c} \mathrm{e}^{-4|t|}}{t}\right) \\
& \int_{-\infty}^{x}\left(|u(\xi, t)|^{2}-1\right) \mathrm{d} \xi=-\psi^{-}(0)+\frac{\operatorname{sgn}\left(\varepsilon_{1}\right) \mathrm{e}^{-\left(2|t|-\widehat{\mathfrak{c}}_{+}\right)} \widehat{\mathfrak{g}}_{+}}{2 \sqrt{|t|}} \sinh \left(\widehat{\mathfrak{c}}_{-}+\ln \widehat{\mathfrak{g}}_{-}\right)+\mathcal{O}\left(\frac{\underline{c} \mathrm{e}^{-4|t|}}{t}\right) .
\end{aligned}
$$

Remark 3.4. In this work, the complete details of the analysis are presented for the case $t \rightarrow+\infty$ and $x \rightarrow-\infty$ such that $z_{0}:=x / t<-2$, and the case $t \rightarrow-\infty$ and $x \rightarrow+\infty$ such that $z_{0}<-2$ is succinctly treated in Section 7: the remaining cases are analogous (see the Appendix). The DZ method has recently been extended to tackle asymptotic problems arising in the theory of random permutations 41], orthogonal polynomials and random matrix theory [34, 42], and perturbation theory for integrable NLEEs [43] (see, also, the recent extension by Kamvissis et al. [44]).

\section{The Auxiliary and Truncated RHPs}

In this section, as $t \rightarrow+\infty$ and $x \rightarrow-\infty$ such that $z_{0}:=x / t<-2$, the RHP formulated in Lemma 2.6 for $m^{c}(x, t ; \zeta)$ on $\sigma_{c}$ (oriented from $-\infty$ to $+\infty$ ) is reformulated as an auxiliary RHP on the augmented contour $\Sigma^{\prime}$ (see Figure 3), which is then dissected to produce an equivalent RHP on the truncated contour $\Sigma^{\sharp}$ (see Figure 4 ). 
Remark 4.1. For notational convenience, except where absolutely necessary and/or where confusion may arise, explicit $x, t$ dependences are suppressed.

As per the DZ method [27], one begins by decomposing the complex plane of the spectral parameter $\zeta$ according to the signature of $\Re\left(\mathrm{i} t \theta^{u}(\zeta)\right)$ (see Figure 1), where, from Eq. (8), $\theta^{u}(\zeta)=\frac{1}{2}\left(\zeta-\frac{1}{\zeta}\right)\left(z_{o}+\zeta+\frac{1}{\zeta}\right)$, with $\left\{\zeta_{i}\right\}_{i=1}^{4}$ defined in Theorem 3.1, Eqs. (16) and (17), $0<\zeta_{2}<\zeta_{1}$, $\left|\zeta_{3}\right|^{2}=1$, and $\pm \leftrightarrow \Re\left(\mathrm{i} t \theta^{u}(\zeta)\right) \gtrless 0$. One now reorients $\sigma_{c}$ (oriented from $-\infty$ to $+\infty$ ) according to, and consistent with, the signature of $\Re\left(\mathrm{i} t \theta^{u}(\zeta)\right.$ ), leading to the reoriented contour $\sigma_{c}^{\prime}$ (see Figure 2). Denoting $m^{c}(\zeta)$ on $\sigma_{c}^{\prime}$ by $\widetilde{m}^{c}(\zeta)$, one shows that $\widetilde{m}^{c}(\zeta): \mathbb{C} \backslash \sigma_{c}^{\prime} \rightarrow \operatorname{SL}(2, \mathbb{C})$ solves the following (normalised at $\infty$ ) RHP: (1) $\widetilde{m}^{c}(\zeta)$ is piecewise holomorphic $\forall \zeta \in \mathbb{C} \backslash \sigma_{c}^{\prime}$; (2)

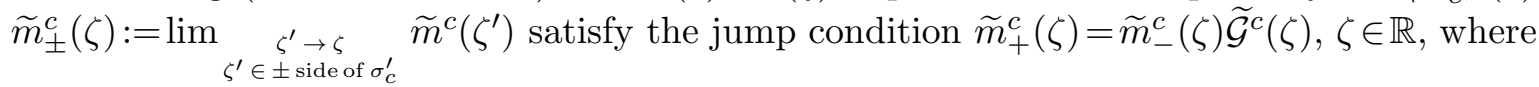

$$
\widetilde{\mathcal{G}}^{c}(\zeta):= \begin{cases}\left(\mathrm{I}-r(\zeta) \mathrm{e}^{2 \mathrm{i} t \theta^{u}(\zeta)} \sigma_{-}\right)\left(\mathrm{I}+\overline{r(\bar{\zeta})} \mathrm{e}^{-2 \mathrm{i} t \theta^{u}(\zeta)} \sigma_{+}\right), & \zeta \in\left(0, \zeta_{2}\right) \cup\left(\zeta_{1},+\infty\right), \\ \left(\mathrm{I}-\overline{r(\bar{\zeta})} \mathrm{e}^{-2 \mathrm{i} t \theta^{u}(\zeta)} \sigma_{+}\right)\left(\mathrm{I}+r(\zeta) \mathrm{e}^{2 \mathrm{i} t \theta^{u}(\zeta)} \sigma_{-}\right), & \zeta \in(-\infty, 0) \cup\left(\zeta_{2}, \zeta_{1}\right)\end{cases}
$$

(3) as $\zeta \rightarrow \infty, \zeta \in \mathbb{C} \backslash \sigma_{c}^{\prime}, \widetilde{m}^{c}(\zeta)=\mathrm{I}+\mathcal{O}\left(\zeta^{-1}\right)$; and $(4) \widetilde{m}^{c}(\zeta)$ satisfies the symmetry reduction $\widetilde{m}^{c}(\zeta)=\sigma_{1} \overline{\widetilde{m}^{c}(\bar{\zeta})} \sigma_{1}$ and the condition $\left(\widetilde{m}^{c}(0) \sigma_{2}\right)^{2}=$ I. One notes from the definition of $\widetilde{\mathcal{G}}^{c}(\zeta)$ that, as $t \rightarrow+\infty$ and $x \rightarrow-\infty$ such that $z_{0}<-2$, for $\zeta \in \mathbb{C}_{\mp} \cap\left\{\zeta ; \Re(\zeta) \in\left(0, \zeta_{2}\right) \cup\left(\zeta_{1},+\infty\right)\right\} \cap$ $\left(\cup_{\star \in\left\{0, \zeta_{2}, \zeta_{1}\right\}}\{\zeta ;|\zeta-\star|>\varepsilon\}\right)$, where $\varepsilon$ is an arbitrarily fixed, sufficiently small positive real number, since $\exp \left(\mp 2 \mathrm{i} t \theta^{u}(\zeta)\right)$ have analytic continuation to $\zeta \mp \mathrm{i} 0,\left|\exp \left(\mp 2 \mathrm{i} t \theta^{u}(\zeta)\right)\right| \rightarrow 0$, but, for $\zeta \in \mathbb{C}_{ \pm} \cap\left\{\zeta ; \Re(\zeta) \in(-\infty, 0) \cup\left(\zeta_{2}, \zeta_{1}\right)\right\} \cap\left(\cup_{\star \in\left\{0, \zeta_{2}, \zeta_{1}\right\}}\{\zeta ;|\zeta-\star|>\varepsilon\}\right)$, since $\exp \left( \pm 2 \mathrm{i} t \theta^{u}(\zeta)\right)$ have analytic continuation to $\zeta \pm \mathrm{i} 0,\left|\exp \left( \pm 2 \mathrm{i} t \theta^{u}(\zeta)\right)\right| \rightarrow \infty$. In order to control the latter exponential growths, the triangular factorisation of $\widetilde{\mathcal{G}}^{c}(\zeta)$ for $\Re(\zeta) \in(-\infty, 0) \cup\left(\zeta_{2}, \zeta_{1}\right)$ must be changed from $\left(\begin{array}{ll}1 & \mathbf{A} \\ 0 & 1\end{array}\right)\left(\begin{array}{ll}1 & 0 \\ \nabla & 1\end{array}\right)$ to $\left(\begin{array}{ll}1 & 0 \\ \nabla & 1\end{array}\right)\left(\begin{array}{ll}1 & \Delta \\ 0 & 1\end{array}\right)$, that is, upper-lower triangular to lower-upper triangular refactorisation: to accomplish this, one introduces the " $\delta(\cdot)$-function" 27].

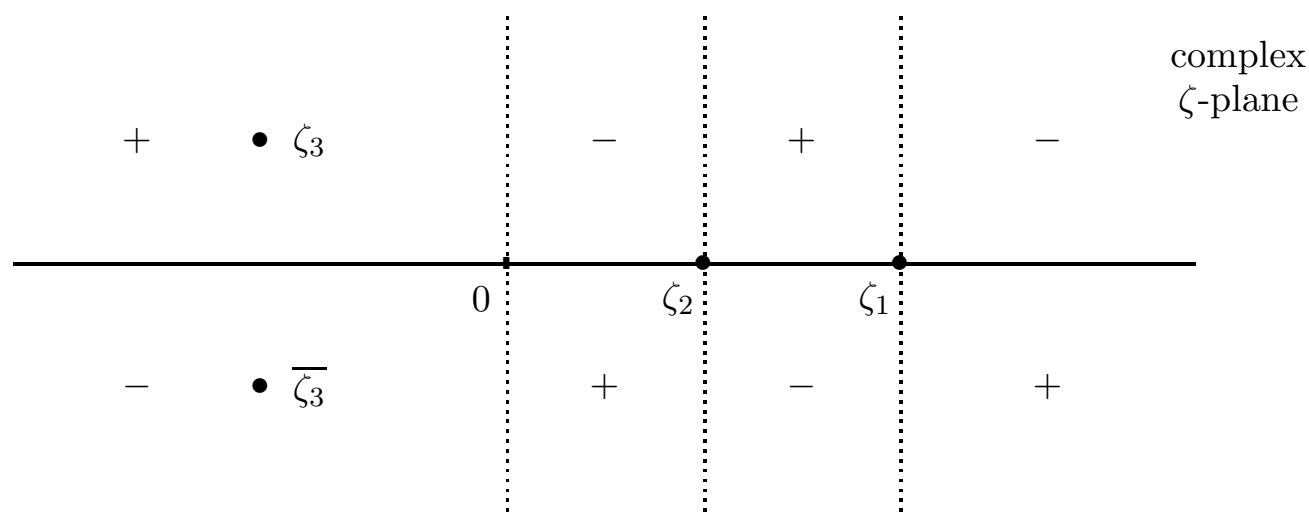

Figure 1: Signature graph of $\Re\left(\mathrm{i} t \theta^{u}(\zeta)\right)$ as $t \rightarrow+\infty$

complex

$\zeta$-plane

Figure 2: Reoriented contour $\sigma_{c}^{\prime}$ 
Proposition 4.1. Let $\delta(\zeta)$ solve the following scalar discontinuous RHP:

$$
\begin{aligned}
\delta_{+}(\zeta) & = \begin{cases}\delta_{-}(\zeta)(1-r(\zeta) \overline{r(\bar{\zeta})}), & \Re(\zeta) \in(-\infty, 0) \cup\left(\zeta_{2}, \zeta_{1}\right), \\
\delta_{-}(\zeta)=\delta(\zeta), & \Re(\zeta) \in\left(0, \zeta_{2}\right) \cup\left(\zeta_{1},+\infty\right),\end{cases} \\
\delta(\zeta) \underset{\zeta \rightarrow \infty}{=} 1+\mathcal{O}\left(\zeta^{-1}\right) . &
\end{aligned}
$$

The unique solution of this RHP can be written as

$$
\delta(\zeta)=\left(\frac{\zeta-\zeta_{1}}{\zeta-\zeta_{2}}\right)^{\mathrm{i} \nu} \exp \left(\int_{-\infty}^{0} \frac{\ln \left(1-|r(\mu)|^{2}\right)}{(\mu-\zeta)} \frac{\mathrm{d} \mu}{2 \pi \mathrm{i}}+\int_{\zeta_{2}}^{\zeta_{1}} \ln \left(\frac{1-|r(\mu)|^{2}}{1-\left|r\left(\zeta_{1}\right)\right|^{2}}\right) \frac{1}{(\mu-\zeta)} \frac{\mathrm{d} \mu}{2 \pi \mathrm{i}}\right),
$$

where $\left\{\zeta_{i}\right\}_{i=1}^{2}$ are defined in Theorem 3.1, Eqs. (16) and (17), and $\nu:=\nu\left(\zeta_{1}\right)=-\frac{1}{2 \pi} \ln (1-$ $\left.\left|r\left(\zeta_{1}\right)\right|^{2}\right) \in \mathbb{R}_{+}$. Furthermore, the function $\delta(\zeta)$ possesses the following properties, $\delta(\zeta) \overline{\delta(\bar{\zeta})}=$ $1, \delta(\zeta) \delta\left(\frac{1}{\zeta}\right)=\delta(0),\left|\delta_{+}(\zeta)\right|^{2} \leqslant 1$ and $\left|\delta_{-}(\zeta)\right|^{2} \leqslant\left(1-\sup _{z \in \mathbb{R}}|r(z)|^{2}\right)^{-1}<\infty \forall \zeta \in \mathbb{R}$, and $\|\left.(\delta(\cdot))^{ \pm 1}\right|_{\mathcal{L}^{\infty}(\mathbb{C})}:=\sup _{\zeta \in \mathbb{C}}\left|(\delta(\zeta))^{ \pm 1}\right|<\infty$.

Proof. Noting that the index, $\kappa$, associated with the RHP for $\delta(\zeta)$ stated in the Proposition is zero, namely, $\kappa:=\frac{1}{2 \pi}\left[\arg \left(1-|r(\zeta)|^{2}\right)\right]_{-\infty}^{+\infty}=0$, it follows from a well-known result [45] (see, also, Theorem A2 in [24]) that it can be solved explicitly (and uniquely) to yield $\delta(\zeta)=$ $\exp \left(\left(\int_{-\infty}^{0}+\int_{\zeta_{2}}^{\zeta_{1}}\right) \frac{\ln \left(1-|r(\mu)|^{2}\right)}{(\mu-\zeta)} \frac{\mathrm{d} \mu}{2 \pi \mathrm{i}}\right)$ : choosing the principal branch of $\ln (\cdot)$ as per item (9) of Notational Conventions, one arrives at the expression for $\delta(\zeta)$ stated in the Proposition. Using the fact that $r(\zeta) \in \mathcal{S}_{\mathbb{C}}^{1}(\mathbb{R})$, one shows from the representation of $\delta(\zeta)$ that $\delta(\zeta)={ }_{\zeta \rightarrow \infty} 1+$ $\mathcal{O}\left(\zeta^{-1}\right)$; moreover, one deduces that $\delta(\zeta)$ satisfies the symmetry reduction $\delta(\zeta) \overline{\delta(\bar{\zeta})}=1$. Using the fact that, for $\zeta \in \mathbb{R}, r\left(\frac{1}{\zeta}\right)=-\overline{r(\bar{\zeta})}=-\overline{r(\zeta)}$, it follows by a change-of-variable argument that $\delta(\zeta) \delta\left(\frac{1}{\zeta}\right)=\delta(0)$, where $\delta(0)=\exp \left(\left(\int_{-\infty}^{0}+\int_{\zeta_{2}}^{\zeta_{1}}\right) \frac{\ln \left(1-|r(\mu)|^{2}\right)}{\mu} \frac{\mathrm{d} \mu}{2 \pi \mathrm{i}}\right)$. Letting $\zeta \rightarrow \zeta \pm \mathrm{i} 0$, one notes from the above symmetry reduction for $\delta(\zeta)$ that $\delta_{ \pm} \overline{\delta_{\mp}(\zeta)}=1$; hence, using the relation $\delta_{+}(\zeta)=\delta_{-}(\zeta)(1-r(\zeta) \overline{r(\bar{\zeta})})$, one deduces that $\left|\delta_{-}(\zeta)\right|^{2} \leqslant\left(1-\sup _{z \in \mathbb{R}}|r(z)|^{2}\right)^{-1}<\infty \forall \zeta \in \mathbb{R}$, from which one also shows that $\left|\delta_{+}(\zeta)\right|^{2} \leqslant 1$. Setting $\zeta:=\xi+\mathrm{i} \eta$, with $\eta \neq 0$, one shows from the representation for $\delta(\zeta)$ that $|\delta(\zeta)|=\exp \left(\left(\int_{-\infty}^{0}+\int_{\zeta_{2}}^{\zeta_{1}}\right) \frac{\eta \ln \left(1-|r(\mu)|^{2}\right)}{(\mu-\xi)^{2}+\eta^{2}} \frac{\mathrm{d} \mu}{2 \pi}\right)$, and, using the fact that $r(\zeta) \in \mathcal{S}_{\mathbb{C}}^{1}(\mathbb{R})$, one shows that $|\delta(\zeta)| \leqslant \exp \left(\frac{\eta \sup _{z \in \mathbb{R}}\left|\ln \left(1-|r(z)|^{2}\right)\right|}{2 \pi} \int_{-\infty}^{+\infty} \frac{\mathrm{d} \mu}{(\mu-\xi)^{2}+\eta^{2}}\right):$ now, recalling that $\int \frac{\mathrm{d} x}{x^{2}+p^{2}}=\frac{1}{p} \arctan (x / p)$, and choosing the principal branch of $\arctan (\cdot)$, one shows that, $\exists \widetilde{M} \in \mathbb{R}_{+}$and bounded such that $\forall \zeta \in \mathbb{C} \backslash \mathbb{R},|\delta(\zeta)| \leqslant \widetilde{M}$ (a similar argument shows that $\left.\left|(\delta(\zeta))^{-1}\right| \leqslant(\widetilde{M})^{-1}\right)$; hence, with the estimates for $\left|\delta_{ \pm}(\zeta)\right|^{2}$, the maximum modulus principle, and the fact that $\{z \in \mathbb{C} ; \delta(z)=0\}=\emptyset$, one shows that $\left\|(\delta(\cdot))^{ \pm 1}\right\|_{\mathcal{L}^{\infty}(\mathbb{C})}<\infty$.

Making use of Proposition 4.1, one changes the triangular factorisation of $\widetilde{\mathcal{G}}^{c}(\zeta)$, with exponential decay of elements like $\exp \left( \pm 2 \mathrm{i} t \theta^{u}(\zeta)\right)$ in their respective domains of analyticity.

Lemma 4.1. Define $\widehat{m}^{c}(\zeta):=\widetilde{m}^{c}(\zeta)(\delta(\zeta))^{-\sigma_{3}}$, where $\delta(\zeta)$ is given in Proposition 4.1. Then $\widehat{m}^{c}(\zeta): \mathbb{C} \backslash \sigma_{c}^{\prime} \rightarrow \mathrm{SL}(2, \mathbb{C})$ solves the following RHP: (1) $\widehat{m}^{c}(\zeta)$ is piecewise holomorphic $\forall \zeta \in$ $\mathbb{C} \backslash \sigma_{c}^{\prime} ;(2) \widehat{m}_{ \pm}^{c}(\zeta):=\lim _{\substack{\zeta^{\prime} \in \pm \text { side of } \sigma_{c}^{\prime} \\ \zeta^{\prime} \rightarrow \zeta}} \widehat{m}^{c}\left(\zeta^{\prime}\right)$ satisfy the jump condition $\widehat{m}_{+}^{c}(\zeta)=\widehat{m}_{-}^{c}(\zeta) \widehat{\mathcal{G}}^{c}(\zeta)$, $\zeta \in \mathbb{R}$, where

$\widehat{\mathcal{G}}^{c}(\zeta):= \begin{cases}\left(\mathrm{I}-\overline{\rho(\bar{\zeta})}(\delta(\zeta))^{-2} \mathrm{e}^{2 \mathrm{i} t \theta^{u}(\zeta)} \sigma_{-}\right)\left(\mathrm{I}+\rho(\zeta)(\delta(\zeta))^{2} \mathrm{e}^{-2 \mathrm{i} t \theta^{u}(\zeta)} \sigma_{+}\right), & \zeta \in\left(0, \zeta_{2}\right) \cup\left(\zeta_{1},+\infty\right), \\ \left(\mathrm{I}-\overline{\rho(\bar{\zeta})}\left(\delta_{-}(\zeta)\right)^{-2} \mathrm{e}^{2 \mathrm{i} t \theta^{u}(\zeta)} \sigma_{-}\right)\left(\mathrm{I}+\rho(\zeta)\left(\delta_{+}(\zeta)\right)^{2} \mathrm{e}^{-2 \mathrm{i} t \theta^{u}(\zeta)} \sigma_{+}\right), & \zeta \in(-\infty, 0) \cup\left(\zeta_{2}, \zeta_{1}\right),\end{cases}$

with

$$
\rho(\zeta):= \begin{cases}\overline{r(\bar{\zeta})}, & \zeta \in\left(0, \zeta_{2}\right) \cup\left(\zeta_{1},+\infty\right) \\ -\overline{r(\bar{\zeta})}(1-r(\zeta) \overline{r(\bar{\zeta})})^{-1}, & \zeta \in(-\infty, 0) \cup\left(\zeta_{2}, \zeta_{1}\right)\end{cases}
$$


(3) as $\zeta \rightarrow \infty, \zeta \in \mathbb{C} \backslash \sigma_{c}^{\prime}, \widehat{m}^{c}(\zeta)=\mathrm{I}+\mathcal{O}\left(\zeta^{-1}\right)$; and (4) $\widehat{m}^{c}(\zeta)$ satisfies the symmetry reduction $\widehat{m}^{c}(\zeta)=\sigma_{1} \overline{\widehat{m}^{c}(\bar{\zeta})} \sigma_{1}$ and the condition $\left(\widehat{m}^{c}(0)(\delta(0))^{\sigma_{3}} \sigma_{2}\right)^{2}=\mathrm{I}$.

Proof. Follows from the RHP for $\widetilde{m}^{c}(\zeta)$ stated at the beginning of Section 4, the definition $\widehat{m}^{c}(\zeta):=\widetilde{m}^{c}(\zeta)(\delta(\zeta))^{-\sigma_{3}}$, with $\delta(\zeta)$ given in Proposition 4.1, and the identity $\sigma_{1} \sigma_{1}=\mathrm{I}$.

The first main objective of this section is to reformulate the RHP for $\widehat{m}^{c}(\zeta)$ as an auxiliary (equivalent) RHP on the augmented (and oriented) contour $\Sigma^{\prime}$ (see Figure 3).

Remark 4.2. The augmented contour $\Sigma^{\prime}$ can be chosen with some degree of flexibility, not necessarily consisting of straight line segments: its crucial characteristic is the position of the rays relative to the lines $\Re\left(\mathrm{i} t \theta^{u}(\zeta)\right)=0$.

Since the jump matrices of the RHP on $\Sigma^{\prime}$ must be written in terms of the jump matrices of the RHP for $\widehat{m}^{c}(\zeta)$ on $\sigma_{c}^{\prime}$, and since, in general, the reflection coefficient, $r(\zeta)$, does not have an analytic continuation to $\mathbb{C} \backslash \mathbb{R}$, one must, as per the DZ method [27], decompose, or split, $r(\zeta)$ into an analytically continuable part and a negligible non-analytic "remainder".

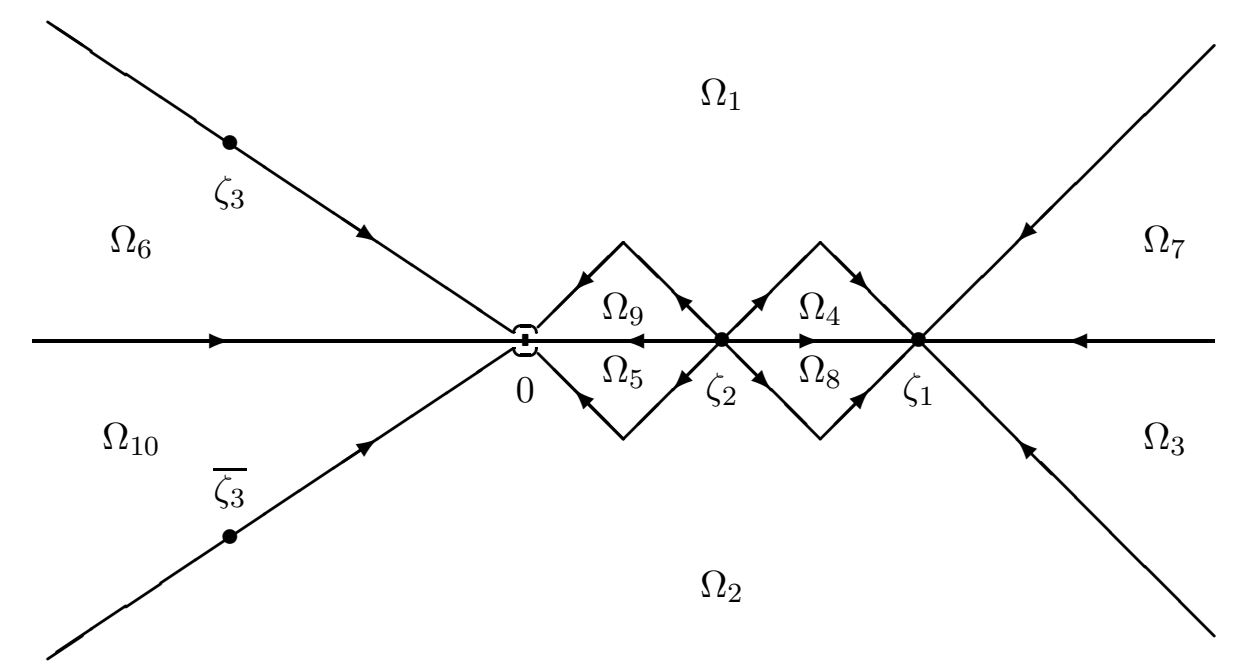

Figure 3: Augmented contour $\Sigma^{\prime}$

Lemma 4.2. Set $\Sigma^{\prime}:=\mathrm{L} \cup \overline{\mathrm{L}} \cup \mathbb{R}$, where $\mathrm{L}=\left\{\zeta ; \zeta=\zeta_{1}+\frac{v}{\sqrt{2}}\left(\zeta_{1}-\zeta_{2}\right) \mathrm{e}^{\frac{3 \pi \mathrm{i}}{4}},-\infty<v \leqslant 1\right\} \cup$ $\left\{\zeta ; \zeta=\zeta_{2}+\frac{v}{\sqrt{2}}\left(\zeta_{1}-\zeta_{2}\right) \mathrm{e}^{\frac{\mathrm{i} \pi}{4}}, v \in[0,1]\right\} \cup\left\{\zeta ; \zeta=\zeta_{2}+\frac{v}{\sqrt{2}} \zeta_{2} \mathrm{e}^{-\frac{3 \pi \mathrm{i}}{4}}, v \in[0,1]\right\} \cup \mathrm{L}_{>} \cup \mathrm{L}_{<}$, with $\mathrm{L}_{>}:=\left\{\zeta ; \zeta=\frac{v}{\sqrt{2}} \zeta_{2} \mathrm{e}^{-\frac{\mathrm{i} \pi}{4}}, v \in(0,1]\right\}$, and $\mathrm{L}_{<}:=\left\{\zeta ; \zeta=v \mathrm{e}^{\mathrm{i} \widetilde{\varphi}_{3}}, \widetilde{\varphi}_{3}:=\arg \left(\zeta_{3}\right) \in\left(\frac{\pi}{2}, \pi\right), v \in \mathbb{R}_{+}\right\}$. Set $\mathrm{L}_{\varepsilon}:=\left\{\zeta ; \zeta=\zeta_{1}+\frac{v}{\sqrt{2}}\left(\zeta_{1}-\zeta_{2}\right) \mathrm{e}^{\frac{3 \pi \mathrm{i}}{4}}, v \in(\varepsilon, 1]\right\} \cup\left\{\zeta ; \zeta=\zeta_{2}+\frac{v}{\sqrt{2}}\left(\zeta_{1}-\zeta_{2}\right) \mathrm{e}^{\frac{\mathrm{i} \pi}{4}}, v \in(\varepsilon, 1]\right\} \cup\{\zeta ; \zeta=$ $\left.\zeta_{2}+\frac{v}{\sqrt{2}} \zeta_{2} \mathrm{e}^{-\frac{3 \pi \mathrm{i}}{4}}, v \in(\varepsilon, 1]\right\} \cup \mathrm{L}_{>}$. Let $l \in \mathbb{Z}_{\geqslant 1}$ be arbitrary, and choose $k=4 q+1$, with $q \in \mathbb{Z}_{\geqslant 1}$ and arbitrarily fixed, so large that $\frac{3 q+2}{2}-\frac{1}{2}>\frac{q}{2}>l$. For each $l \in \mathbb{Z}_{\geqslant 1}$, there exists a decomposition of the function (cf. Lemma 4.1) $\rho(\zeta)$,

$$
\rho(\zeta)=h_{I}(\zeta)+\left(h_{I I}(\zeta)+\mathcal{R}(\zeta)\right), \quad \zeta \in \mathbb{R}
$$

such that $h_{I}(\zeta)$ is analytic on $\mathbb{R}$ (generally, it has no analytic continuation to $\left.\mathbb{C} \backslash \mathbb{R}\right), h_{I I}(\zeta)$ has an analytic continuation to $\mathrm{L}$, and $\mathcal{R}(\zeta)$, with $\mathcal{R}(\zeta) \equiv 0 \forall \zeta<0$, is a piecewise-rational function with the property that $\left.\left(\frac{\mathrm{d}}{\mathrm{d} \zeta}\right)^{j} \rho(\zeta)\right|_{\zeta \in\left\{0, \zeta_{2}, \zeta_{1}\right\}}=\left.\left(\frac{\mathrm{d}}{\mathrm{d} \zeta}\right)^{j} \mathcal{R}(\zeta)\right|_{\zeta \in\left\{0, \zeta_{2}, \zeta_{1}\right\}}, j \in\{0,1, \ldots, k\}$. Then, as $t \rightarrow+\infty$ such that $0<\zeta_{2}<\frac{1}{M}<M<\zeta_{1}$ and $\left|\zeta_{3}\right|^{2}=1$, with $M \in \mathbb{R}_{>1}$ and bounded, the 
following estimates are valid:

$$
\begin{aligned}
\left|\mathrm{e}^{-2 \mathrm{i} t \theta^{u}(\zeta)} h_{I}(\zeta)\right| & \leqslant \frac{\left|\underline{c}\left(\zeta_{1}, \zeta_{2}, \zeta_{3}, \overline{\zeta_{3}}\right)\right|}{\left|\zeta_{1}+\zeta_{2}\right|^{3}\left(|\zeta|^{2}+1\right) t^{l}}, \quad \zeta \in \mathbb{R}, \\
\left|\mathrm{e}^{-2 \mathrm{i} t \theta^{u}(\zeta)}\left(\frac{h_{I}(\zeta)}{\zeta}\right)\right| & \leqslant \frac{\left|\underline{c}\left(\zeta_{1}, \zeta_{2}, \zeta_{3}, \overline{\zeta_{3}}\right)\right|}{\left|\zeta_{1}+\zeta_{2}\right|^{3}\left(|\zeta|^{2}+1\right) t^{l}}, \quad \zeta \in\left(\cos \widetilde{\varphi}_{3}, 0\right) \cup\left(0, \frac{1}{2} \zeta_{2}\right), \\
\left|\mathrm{e}^{-2 \mathrm{i} t \theta^{u}(\zeta)} h_{I I}(\zeta)\right| & \leqslant \frac{\mid \underline{\underline{c}\left(\zeta_{1}, \zeta_{2}, \zeta_{3}, \overline{\zeta_{3}}\right) \mid}}{\left|z_{o}+\zeta_{1}+\zeta_{2}\right|^{l}\left(|\zeta|^{2}+1\right) t}, \quad \zeta \in \mathrm{L} \backslash\left(\mathrm{L}_{>} \cup \mathrm{L}_{<}\right), \\
\left|\mathrm{e}^{-2 \mathrm{i} t \theta^{u}(\zeta)} h_{I I}(\zeta)\right| & \leqslant \frac{\underline{\left|c\left(\zeta_{1}, \zeta_{2}, \zeta_{3}, \overline{\zeta_{3}}\right)\right|}}{\left(|\zeta|^{2}+1\right)} \mathrm{e}^{-\gamma_{I I}^{0} t}, \quad \zeta \in \mathrm{L}_{>} \cup \mathrm{L}_{<}, \\
\left|\mathrm{e}^{-2 \mathrm{i} t \theta^{u}(\zeta)}\left(\frac{h_{I I}(\zeta)}{\zeta}\right)\right| & \leqslant \frac{\mid \underline{\underline{c}\left(\zeta_{1}, \zeta_{2}, \zeta_{3}, \overline{\zeta_{3}}\right) \mid}}{\left(|\zeta|^{2}+1\right)} \mathrm{e}^{-\gamma_{I I}^{1} t}, \quad \zeta \in\left(\mathrm{L}_{>} \cup \mathrm{L}_{<}\right) \backslash\left\{\zeta ; \zeta=v \mathrm{e}^{\mathrm{i} \widetilde{\varphi}_{3}}, v \in \mathbb{R}_{>1}\right\}, \\
\left|\mathrm{e}^{-2 \mathrm{i} t \theta^{u}(\zeta)} \mathcal{R}(\zeta)\right| & \leqslant\left|\underline{c}\left(\zeta_{1}, \zeta_{2}, \zeta_{3}, \overline{\zeta_{3}}\right)\right| \mathrm{e}^{-\varepsilon^{2} \gamma_{\mathcal{R}}^{0} t}, \quad \zeta \in \mathrm{L}_{\varepsilon} \backslash \mathrm{L}_{>}, \\
\left|\mathrm{e}^{-2 \mathrm{i} t \theta^{u}(\zeta)} \mathcal{R}(\zeta)\right| & \leqslant \frac{\underline{\mid c}\left(\zeta_{1}, \zeta_{2}, \zeta_{3}, \overline{\zeta_{3}}\right) \mid}{\left(|\zeta|^{2}+1\right)} \mathrm{e}^{-\gamma_{\mathcal{R}}^{1} t}, \quad \zeta \in \mathrm{L}_{>}, \\
\left|\mathrm{e}^{-2 \mathrm{i} t \theta^{u}(\zeta)}\left(\frac{\mathcal{R}(\zeta)}{\zeta}\right)\right| & \leqslant \frac{\underline{\underline{c}\left(\zeta_{1}, \zeta_{2}, \zeta_{3}, \overline{\zeta_{3}}\right) \mid}}{\left(|\zeta|^{2}+1\right)} \mathrm{e}^{-\gamma_{\mathcal{R}} t}, \quad \zeta \in \mathrm{L}_{>},
\end{aligned}
$$

where $\gamma_{I I}^{0}:=\min \left\{\frac{1}{2}\left(a_{2}-z_{o}\right) \sqrt{4-a_{2}^{2}}, \frac{1}{2}\left|z_{o}\right| \zeta_{1}\left(2-\zeta_{2}^{2}\right),\left(\frac{1}{2}\left|z_{o}\right|-\cos \widetilde{\varphi}_{3}\right) \sin \widetilde{\varphi}_{3}\right\} \quad\left(\in \mathbb{R}_{+}\right), \gamma_{I I}^{1}:=$ $\min \left\{\frac{1}{2}\left|\sin 2 \widetilde{\varphi}_{3}\right|, \frac{1}{2}\left|z_{o}\right| \zeta_{1}\left(2-\zeta_{2}^{2}\right)\right\}\left(\in \mathbb{R}_{+}\right), \gamma_{\mathcal{R}}^{0}:=\min \left\{\frac{1}{2} \zeta_{2}\left|z_{o}+\zeta_{2}\right|\left(1-2 \zeta_{1}^{2}\right)^{2}, \frac{1}{2}\left(\zeta_{1}-\zeta_{2}\right)\left|z_{o}+\zeta_{1}+\zeta_{2}\right|(1-\right.$ $\left.\left.\zeta_{1}^{2}\right)^{2}, \frac{1}{2}\left(\zeta_{1}-\zeta_{2}\right)\left|z_{o}+\zeta_{1}+\zeta_{2}\right|\left(1-\frac{2 \zeta_{1}^{2}}{\zeta_{1}^{4}+1}\right)^{2}\right\}\left(\in \mathbb{R}_{+}\right)$, and $\gamma_{\mathcal{R}}^{1}:=\min \left\{\left|z_{o}\right| \zeta_{1}\left(2-\zeta_{2}^{2}\right),\left(\left|z_{o}\right|-2 \cos \widetilde{\varphi}_{3}\right) \sin \widetilde{\varphi}_{3}\right\}$ $\left(\in \mathbb{R}_{+}\right)$, with $z_{o}:=x / t$, and $\left\{\zeta_{i}\right\}_{i=1}^{3}$ and $a_{2}$ defined in Theorem 3.1, Eqs. (16) and (17). Furthermore, taking conjugates, $\left.\overline{\rho(\bar{\zeta})}=\overline{h_{I}(\zeta)}+\overline{\left(h_{I I}(\bar{\zeta})\right.}+\overline{\mathcal{R}(\bar{\zeta})}\right)$, gives rise to similar estimates for $\mathrm{e}^{2 \mathrm{i} t \theta^{u}(\zeta)} \overline{\star_{I}(\zeta)}, \star_{I}(\zeta) \in\left\{h_{I}(\zeta), h_{I}(\zeta) / \zeta\right\}, \mathrm{e}^{2 \mathrm{i} t \theta^{u}(\zeta)} \overline{\star_{I I}(\bar{\zeta})}, \star_{I I}(\zeta) \in\left\{h_{I I}(\zeta), h_{I I}(\zeta) / \zeta\right\}$, and $\mathrm{e}^{2 \mathrm{i} t \theta^{u}(\zeta)} \overline{\star_{I I I}(\bar{\zeta})}, \star_{I I I}(\zeta) \in\{\mathcal{R}(\zeta), \mathcal{R}(\zeta) / \zeta\}$, on $\overline{\mathrm{L}} \cup \mathbb{R} ;$ moreover, $h_{I}(\zeta)$ (respectively $\left.\overline{h_{I}(\zeta)}\right) \in$ $\cap_{p \in\{1,2, \infty} \mathcal{L}^{p}(\mathbb{R})$ (respectively $\in \cap_{p \in\{1,2, \infty} \mathcal{L}^{p}(\mathbb{R})$ ), and $h_{I I}(\zeta)$ (respectively $\left.\overline{h_{I I}(\bar{\zeta})}\right) \in \cap_{p \in\{1,2, \infty\}}$ $\mathcal{L}^{p}(\mathrm{~L})$ (respectively $\in \cap_{p \in\{1,2, \infty\}} \mathcal{L}^{p}(\overline{\mathrm{L}})$ ).

Proof. Many of the technical details and arguments associated with the full proof of this Lemma are identical; hence, only two representative calculations are presented, and the remaining estimations follow in an analogous manner. Let $\exists M \in \mathbb{R}_{>1}$ and bounded such that, as $t \rightarrow+\infty, 0<\zeta_{2}<\frac{1}{M}<M<\zeta_{1}$ and $\left|\zeta_{3}\right|^{2}=1$. One begins by considering the non-empty domain $\{z ; \Im(z)=0\} \cap\left\{z ; \Re(z) \in\left(\frac{1}{2}\left(\zeta_{1}+\zeta_{2}\right), \zeta_{1}\right)\right\}$. Let $k \in \mathbb{Z}_{\geqslant 1}$ be fixed and sufficiently large with representation [27] $k=4 q+1, q \in \mathbb{Z}_{\geqslant 1}$. Recalling that, from Lemma 4.1, for $\Re(\zeta) \in\left(\zeta_{2}, \zeta_{1}\right)$, $\rho(\zeta)=-\overline{r(\bar{\zeta})}(1-r(\zeta) \overline{r(\bar{\zeta})})^{-1}$, one must estimate, as can be seen from the explicit expression for the jump matrix $\widehat{\mathcal{G}}^{c}(\zeta)$ given in Lemma 4.1, terms of the type $\rho(\zeta)\left(\delta_{+}(\zeta)\right)^{2} \mathrm{e}^{-2 \mathrm{i} t \theta^{u}(\zeta)}$ (respectively $\left.\overline{\rho(\bar{\zeta})}\left(\delta_{-}(\zeta)\right)^{-2} \mathrm{e}^{2 \mathrm{i} t \theta^{u}(\zeta)}\right)$ : in fact, the former will be considered in detail, with analytic continuation to the bounded domain $\Omega_{4}$ (see Figure 3 ) where $\Re\left(\mathrm{i} t \theta^{u}(\zeta)\right)>0$, whilst the latter can be estimated (via a Schwarz symmetry principle argument) analogously with analytic continuation to the bounded domain $\Omega_{8}$ (see Figure 3 ) where $\Re\left(\right.$ it $\left.\theta^{u}(\zeta)\right)<0$. With the above-given choice of $k$, and thus $q$, consider the Taylor expansion with integral remainder term for $\rho(\zeta), \rho(\zeta)=\sum_{n=0}^{k} \frac{1}{n !} \rho^{(n)}\left(\zeta_{1}\right)\left(\zeta-\zeta_{1}\right)^{n}+\frac{1}{k !} \int_{\zeta_{1}}^{\zeta} \rho^{(k+1)}(\xi)(\zeta-\xi)^{k} \mathrm{~d} \xi$, where $\rho^{(n)}\left(\zeta_{1}\right):=$ $\left.-\left(\frac{\mathrm{d}}{\mathrm{d} \zeta}\right)^{n} \overline{r(\bar{\zeta})}(1-r(\zeta) \overline{r(\bar{\zeta})})^{-1}\right)\left.\right|_{\zeta=\zeta_{1}}, n \in\{0,1, \ldots, k\}, k \in \mathbb{Z}_{\geqslant 1}$ : note that, if one considers explicitly, say, the limiting case $\zeta_{1} \rightarrow+\infty\left(\zeta_{2} \rightarrow 0^{+}\right)$, then, as functions of $\zeta_{1}$, the Taylor coefficients, $\rho^{(n)}\left(\zeta_{1}\right), n \in\{0,1, \ldots, k\}, k \in \mathbb{Z}_{\geqslant 1}$, are in the $\mathbb{C}$-valued Schwartz class (since $r(\zeta) \in \mathcal{S}_{\mathbb{C}}(\mathbb{R})$ ). Let $\mathcal{R}(\zeta)$ denote the "polynomial part" of this expansion, and $h(\zeta)$ the "remainder", namely, $\mathcal{R}(\zeta):=\sum_{n=0}^{k} \frac{1}{n !} \rho^{(n)}\left(\zeta_{1}\right)\left(\zeta-\zeta_{1}\right)^{n}$ and $h(\zeta):=\frac{1}{k !} \int_{\zeta_{1}}^{\zeta} \rho^{(k+1)}(\xi)(\zeta-\xi)^{k} \mathrm{~d} \xi$; hence, $\rho(\zeta)=\mathcal{R}(\zeta)+h(\zeta)$. One notes that, with the above-given choice, $\rho(\zeta)-\mathcal{R}(\zeta)=h(\zeta)$ can be interpreted as the error incurred in approximating $\rho(\zeta)$ by a polynomial of degree $k$. Noting that, $\forall j \in\{0,1, \ldots, k\}$, $k \in \mathbb{Z}_{\geqslant 1},\left.\left(\frac{\mathrm{d}}{\mathrm{d} \zeta}\right)^{j} \rho(\zeta)\right|_{\zeta=\zeta_{1}}=\left.\left(\frac{\mathrm{d}}{\mathrm{d} \zeta}\right)^{j} \mathcal{R}(\zeta)\right|_{\zeta=\zeta_{1}}$, it follows that $\left.\left(\frac{\mathrm{d}}{\mathrm{d} \zeta}\right)^{j} h(\zeta)\right|_{\zeta=\zeta_{1}}=0, j \in\{0,1, \ldots, k\}$, $k \in \mathbb{Z}_{\geqslant 1}$; hence, as per the DZ method [27], one uses this latter property to split, further, $h(\zeta)$ as $h(\zeta):=h_{I}(\zeta)+h_{I I}(\zeta)$, which, when combined with $\mathcal{R}(\zeta)$, shows that $\rho(\zeta)=$ 
$h_{I}(\zeta)+\left(h_{I I}(\zeta)+\mathcal{R}(\zeta)\right)$, where $h_{I}(\zeta)$ is defined for $\zeta \in\{z ; \Im(z)=0\} \cap\left\{z ; \Re(z) \in\left(\frac{1}{2}\left(\zeta_{1}+\zeta_{2}\right), \zeta_{1}\right)\right\}$, where $\Re\left(\mathrm{i} t \theta^{u}(\zeta)\right)=0$, and has negligibly "small norm" (see below) as $t \rightarrow+\infty, h_{I I}(\zeta)$ has analytic continuation to $\mathbb{C}_{+} \cap\left\{z ; \Re(z) \in\left(\frac{1}{2}\left(\zeta_{1}+\zeta_{2}\right), \zeta_{1}\right)\right\} \cap \Omega_{4}$, where $\Re\left(\mathrm{i} t \theta^{u}(\zeta)\right)>0$, and $\mathcal{R}(\zeta)$ has trivial analytic continuation to $\mathbb{C}_{+} \cap\left\{z ; \Re(z) \in\left(\frac{1}{2}\left(\zeta_{1}+\zeta_{2}\right), \zeta_{1}\right)\right\} \cap \Omega_{4}$. One notes from the expression for $\theta^{u}(\zeta)$ that $\theta^{u}\left(\zeta_{1}\right)<\theta^{u}\left(\frac{1}{2}\left(\zeta_{1}+\zeta_{2}\right)\right)<0$; hence, considering the auxiliary function (which will be needed below) $\alpha(\zeta):=\zeta^{-3}\left(\zeta-\zeta_{1}\right)^{q}\left(\zeta-\zeta_{2}\right)\left(\zeta-\zeta_{3}\right)\left(\zeta-\overline{\zeta_{3}}\right), q \in \mathbb{Z}_{\geqslant 1}$, set $\left(\frac{h}{\alpha}\right)\left(\theta^{u}\right):=$ $\left\{\frac{h\left(\zeta\left(\theta^{u}\right)\right)}{\alpha\left(\zeta\left(\theta^{u}\right)\right)}, \quad \theta^{u}\left(\zeta_{1}\right)<\theta^{u}<\theta^{u}\left(\frac{1}{2}\left(\zeta_{1}+\zeta_{2}\right)\right)\right.$, $\left\{\begin{array}{ll}\alpha\left(\zeta\left(\theta^{u}\right)\right) & \\ 0, & \theta^{u} \in \mathbb{R} \backslash\left(\theta^{u}\left(\zeta_{1}\right), \theta^{u}\left(\frac{1}{2}\left(\zeta_{1}+\zeta_{2}\right)\right)\right),\end{array}\right.$ and consider the Fourier transform with respect to (w.r.t.) $\theta^{u}(\zeta)$. Before proceeding any further, and for future reference, one notes that $\frac{h(\zeta)}{\alpha(\zeta)}=$ $\frac{\zeta^{3}\left(\zeta-\zeta_{1}\right)^{3 q+2}}{\left(\zeta-\zeta_{2}\right)\left(\zeta-\zeta_{3}\right)\left(\zeta-\overline{\zeta_{3}}\right) k !} \int_{0}^{1} \rho^{(k+1)}\left(\zeta_{1}+\left(\zeta-\zeta_{1}\right) \tau\right)(1-\tau)^{k} \mathrm{~d} \tau$, and, for $0<\zeta_{2}<\frac{1}{M}<M<\zeta_{1}$ and $\left|\zeta_{3}\right|^{3}=1$, since $r(\zeta) \in \mathcal{S}_{\mathbb{C}}(\mathbb{R}),\|r(\cdot)\|_{\mathcal{L}^{\infty}(\mathbb{R})}<1$, and $\sup _{(\tau, k) \in[0,1] \times \mathbb{Z}_{\geqslant 1}}\left|\rho^{(k+1)}\left(\zeta_{1}+\left(\zeta-\zeta_{1}\right) \tau\right)\right|<\infty$, with


$\mathcal{O}\left(\left(\zeta-\zeta_{1}\right)^{-1}\right)$. Define the Fourier transform pair (w.r.t. $\left.\theta^{u}(\zeta)\right):\left(\frac{h}{\alpha}\right)(\zeta):=\int_{-\infty}^{+\infty} \mathrm{e}^{\mathrm{i} s \theta^{u}(\zeta)} \widetilde{\left(\frac{h}{\alpha}\right)}(s) \frac{\mathrm{d} s}{\sqrt{2 \pi}}$, $\frac{1}{2}\left(\zeta_{1}+\zeta_{2}\right)<\Re(\zeta)<\zeta_{1}$, and $\widetilde{\left(\frac{h}{\alpha}\right)}(s):=-\int_{\frac{1}{2}\left(\zeta_{1}+\zeta_{2}\right)}^{\zeta_{1}} \mathrm{e}^{-\mathrm{i} s \theta^{u}(\zeta)}\left(\frac{h}{\alpha}\right)(\zeta) \frac{\mathrm{d} \theta^{u}(\zeta)}{\sqrt{2 \pi}}, s \in \mathbb{R}$. In order to obtain the necessary estimate for $\left(h_{I}(\zeta)+\left(h_{I I}(\zeta)+\mathcal{R}(\zeta)\right)\right)\left(\delta_{+}(\zeta)\right)^{2} \mathrm{e}^{-2 \mathrm{i} t \theta^{u}(\zeta)}$, one needs to show that $\left(\frac{h}{\alpha}\right)\left(\theta^{u}\right):=\frac{h\left(\zeta\left(\theta^{u}\right)\right)}{\alpha\left(\zeta\left(\theta^{u}\right)\right)} \in \mathcal{H}^{j}(\mathbb{R}), 0 \leqslant j \leqslant\left[\frac{3 q+2}{2}\right], q \in \mathbb{Z}_{\geqslant 1}$, where $\mathcal{H}^{j}(\mathbb{R})$ denotes the $L^{2}$ Sobolev space with norm $\|\star(\cdot)\|_{\mathcal{H}(\mathbb{R})}:=\left(\sum_{j=0}^{\left[\frac{3 q+2}{2}\right]}\left\|\left(\frac{\mathrm{d}}{\mathrm{d} \theta^{u}}\right)^{j} \star\left(\theta^{u}\right)\right\|_{\mathcal{L}^{2}(\mathbb{R})}^{2}\right)^{1 / 2}$. In this particular case, one must show that, for $0<\zeta_{2}<\frac{1}{M}<M<\zeta_{1}$ and $\left|\zeta_{3}\right|^{3}=1$, with $\Re(\zeta) \in\left(\frac{1}{2}\left(\zeta_{1}+\zeta_{2}\right), \zeta_{1}\right)$, $\mathrm{I}_{\mathcal{H}}:=\int_{-\infty}^{+\infty}\left|\left(\frac{\mathrm{d}}{\mathrm{d} \theta^{u}}\right)^{j} \frac{h\left(\zeta\left(\theta^{u}\right)\right)}{\alpha\left(\zeta\left(\theta^{u}\right)\right)}\right|^{2} \mathrm{~d} \theta^{u}<\infty, 0 \leqslant j \leqslant\left[\frac{3 q+2}{2}\right], q \in \mathbb{Z}_{\geqslant 1}$ : in fact, since $\frac{h\left(\zeta\left(\theta^{u}\right)\right)}{\alpha\left(\zeta\left(\theta^{u}\right)\right)} \equiv 0 \forall \theta^{u} \in$ $\mathbb{R} \backslash\left(\theta^{u}\left(\zeta_{1}\right), \theta^{u}\left(\frac{1}{2}\left(\zeta_{1}+\zeta_{2}\right)\right)\right)$, the latter integral reduces to $\mathrm{I}_{\mathcal{H}}=\int_{\theta^{u}\left(\zeta_{1}\right)}^{\theta^{u}\left(\frac{1}{2}\left(\zeta_{1}+\zeta_{2}\right)\right)}\left|\left(\frac{\mathrm{d}}{\mathrm{d} \theta^{u}}\right)^{j} \frac{h\left(\zeta\left(\theta^{u}\right)\right)}{\alpha\left(\zeta\left(\theta^{u}\right)\right)}\right|^{2} \mathrm{~d} \theta^{u}$, which, via the chain rule and a change-of-variable argument, is shown to be equal to $\mathrm{I}_{\mathcal{H}}=$ $\int_{\frac{1}{2}\left(\zeta_{1}+\zeta_{2}\right)}^{\zeta_{1}}\left|\frac{\left(\frac{\mathrm{d}}{\mathrm{d} \zeta}\right)^{j} \frac{h(\zeta)}{\alpha(\zeta)}}{\left(\frac{\mathrm{d} \theta^{u}(\zeta)}{\mathrm{d} \zeta}\right)^{j}}\right|^{2}\left(\frac{\mathrm{d} \theta^{u}(\zeta)}{\mathrm{d} \zeta}\right) \mathrm{d} \zeta$. Now, recalling that $\frac{h(\zeta)}{\alpha(\zeta)}=\frac{\zeta^{3}\left(\zeta-\zeta_{1}\right)^{3 q+2}}{\left(\zeta-\zeta_{2}\right)\left(\zeta-\zeta_{3}\right)\left(\zeta-\overline{\zeta_{3}}\right) k !} \int_{0}^{1} \rho^{(k+1)}\left(\zeta_{1}+\right.$ $\left.\left(\zeta-\zeta_{1}\right) \tau\right)(1-\tau)^{k} \mathrm{~d} \tau$ and (from Section 3) $\frac{\mathrm{d} \theta^{u}(\zeta)}{\mathrm{d} \zeta}=\zeta^{-3}\left(\zeta-\zeta_{1}\right)\left(\zeta-\zeta_{2}\right)\left(\zeta-\zeta_{3}\right)\left(\zeta-\overline{\zeta_{3}}\right)$, one shows that, for $0 \leqslant j \leqslant\left[\frac{3 q+2}{2}\right], q \in \mathbb{Z}_{\geqslant 1}, \mathrm{I}_{\mathcal{H}} \leqslant\left|c^{\mathcal{S}}\left(\zeta_{1}\right) \underline{c}\left(\zeta_{2}, \zeta_{3}, \overline{\zeta_{3}}\right)\right|, 0<\zeta_{2}<\frac{1}{M}<M<\zeta_{1}$ and $\left|\zeta_{3}\right|^{2}=1$, that is, $\left\|\left(\frac{h}{\alpha}\right)(\cdot)\right\|_{\mathcal{H}(\mathbb{R})}=\left(\sum_{j=0}^{\left[\frac{3 q+2}{2}\right]}\left\|\left(\frac{\mathrm{d}}{\mathrm{d} \theta^{u}}\right)^{j}\left(\frac{h}{\alpha}\right)\left(\theta^{u}\right)\right\|_{\mathcal{L}^{2}(\mathbb{R})}^{2}\right)^{1 / 2}<\infty$; hence, by Parseval's Theorem, for $M \in \mathbb{R}_{>1}$ and bounded such that $0<\zeta_{2}<\frac{1}{M}<M<\zeta_{1}$ and $\left|\zeta_{3}\right|^{2}=1$, $\int_{-\infty}^{+\infty}\left(1+s^{2}\right)^{j}\left|\widetilde{\left(\frac{h}{\alpha}\right)}(s)\right|^{2} \mathrm{~d} s \leqslant\left|c^{\mathcal{S}}\left(\zeta_{1}\right) \underline{c}\left(\zeta_{2}, \zeta_{3}, \overline{\zeta_{3}}\right)\right|, 0 \leqslant j \leqslant\left[\frac{3 q+2}{2}\right], q \in \mathbb{Z}_{\geqslant 1}$. Recalling from the Fourier transform pair that, for $\frac{1}{2}\left(\zeta_{1}+\zeta_{2}\right)<\Re(\zeta)<\zeta_{1},\left(\frac{h}{\alpha}\right)(\zeta)=\int_{-\infty}^{+\infty} \mathrm{e}^{\mathrm{i} s \theta^{u}(\zeta)} \widetilde{\left(\frac{h}{\alpha}\right)}(s) \frac{\mathrm{d} s}{\sqrt{2 \pi}}$, it follows, by defining $h_{I}(\zeta):=\alpha(\zeta) \int_{t}^{+\infty} \mathrm{e}^{\mathrm{i} s \theta^{u}(\zeta)} \widetilde{\left(\frac{h}{\alpha}\right)}(s) \frac{\mathrm{d} s}{\sqrt{2 \pi}}$ and $h_{I I}(\zeta):=\alpha(\zeta) \int_{-\infty}^{t} \mathrm{e}^{\mathrm{i} s \theta^{u}(\zeta)} \widetilde{\left(\frac{h}{\alpha}\right)}(s) \frac{\mathrm{d} s}{\sqrt{2 \pi}}$, that $h(\zeta)=h_{I}(\zeta)+h_{I I}(\zeta)$. For $\zeta \in\{z ; \Im(z)=0\} \cap\left\{z ; \Re(z) \in\left(\frac{1}{2}\left(\zeta_{1}+\zeta_{2}\right), \zeta_{1}\right)\right\}$, recalling the bounds for $\delta_{+}(\zeta)$ given in Proposition 4.1, and using the Cauchy-Schwarz inequality for integrals, one shows that $\left|\left(\delta_{+}(\zeta)\right)^{2} \mathrm{e}^{-2 \mathrm{i} t \theta^{u}(\zeta)} h_{I}(\zeta)\right|=\left|\left(\delta_{+}(\zeta)\right)^{2} \mathrm{e}^{-2 \mathrm{i} t \theta^{u}(\zeta)} \alpha(\zeta) \int_{t}^{+\infty} \mathrm{e}^{\mathrm{i} s \theta^{u}(\zeta)} \widetilde{\left(\frac{h}{\alpha}\right)}(s) \frac{\mathrm{d} s}{\sqrt{2 \pi}}\right| \leqslant(1-$ $\left.\sup _{z \in \mathbb{R}}|r(z)|^{2}\right) \frac{|\alpha(\zeta)|}{\sqrt{2 \pi}}\left(\int_{t}^{+\infty}\left(1+s^{2}\right)^{-j} \mathrm{~d} s\right)^{1 / 2}\left(\int_{t}^{+\infty}\left(1+s^{2}\right)^{j}\left|\widetilde{\left(\frac{h}{\alpha}\right)}(s)\right|^{2} \mathrm{~d} s\right)^{1 / 2}$ : noting that $\int_{t}^{+\infty}(1+$ $\left.s^{2}\right)^{-j} \mathrm{~d} s \leqslant \frac{t^{-(2 j-1)}}{(2 j-1)}, 2 j-1>0$, and recalling the Parseval estimate $\int_{t}^{+\infty}\left(1+s^{2}\right)^{j}\left|\widetilde{\left(\frac{h}{\alpha}\right)}(s)\right|^{2} \mathrm{~d} s \leqslant$ $\int_{-\infty}^{+\infty}\left(1+s^{2}\right)^{j}\left|\widetilde{\left(\frac{h}{\alpha}\right)}(s)\right|^{2} \mathrm{~d} s \leqslant\left|c^{\mathcal{S}}\left(\zeta_{1}\right) \underline{c}\left(\zeta_{2}, \zeta_{3}, \overline{\zeta_{3}}\right)\right|, 0 \leqslant j \leqslant\left[\frac{3 q+2}{2}\right]$, it follows that, with $\alpha(\zeta)=$


$\frac{1}{2}\left(\zeta_{1}+\zeta_{2}\right)<\Re(\zeta)<\zeta_{1}$, and $0<\zeta_{2}<\frac{1}{M}<M<\zeta_{1}$ and $\left|\zeta_{3}\right|^{2}=1$. Since, for $\zeta \in \mathbb{C}_{+} \cap$ $\left\{z ; \Re(z) \in\left(\frac{1}{2}\left(\zeta_{1}+\zeta_{2}\right), \zeta_{1}\right)\right\} \cap \Omega_{4}, \Re\left(\mathrm{i} t \theta^{u}(\zeta)\right)>0, h_{I I}(\zeta)$ has an analytic continuation to the line (parametrised by $v) \zeta=\zeta(v)=\zeta_{1}+\frac{v}{\sqrt{2}}\left(\zeta_{1}-\zeta_{2}\right) \mathrm{e}^{\frac{3 \pi \mathrm{i}}{4}}, v \in[0,1]$; hence, on this line, and using 
the Cauchy-Schwarz inequality for integrals, it follows that $\mathrm{I}_{h_{I I}}:=\left|\left(\delta_{+}(\zeta)\right)^{2} \mathrm{e}^{-2 \mathrm{i} t \theta^{u}(\zeta)} h_{I I}(\zeta)\right|=$ $\left|\left(\delta_{+}(\zeta)\right)^{2} \mathrm{e}^{-2 \mathrm{i} t \theta^{u}(\zeta)} \alpha(\zeta) \int_{-\infty}^{t} \mathrm{e}^{\mathrm{i} s \theta^{u}(\zeta)} \widetilde{\left(\frac{h}{\alpha}\right)(s)} \frac{\mathrm{d} s}{\sqrt{2 \pi}}\right| \leqslant\left(1-\sup _{z \in \mathbb{R}}|r(z)|^{2}\right) \frac{|\alpha(\zeta)|}{\sqrt{2 \pi}} \mathrm{e}^{-t \Re\left(\mathrm{i} \theta^{u}(\zeta)\right)}\left(\int_{-\infty}^{t}(1+\right.$ $\left.\left.s^{2}\right)^{-j} \mathrm{~d} s\right)^{1 / 2}\left(\int_{-\infty}^{t}\left(1+s^{2}\right)^{j}\left|\widetilde{\left(\frac{h}{\alpha}\right)}(s)\right|^{2} \mathrm{~d} s\right)^{1 / 2}$. Noting that $\int_{-\infty}^{t}\left(1+s^{2}\right)^{-j} \mathrm{~d} s \leqslant \int_{-\infty}^{+\infty}\left(1+s^{2}\right)^{-j} \mathrm{~d} s \leqslant$ $\int_{-\infty}^{+\infty}\left(1+s^{2}\right)^{-1} \mathrm{~d} s=\pi$, and, from the above Parseval estimate $\int_{-\infty}^{t}\left(1+s^{2}\right)^{j}\left|\widetilde{\left(\frac{h}{\alpha}\right)}(s)\right|^{2} \mathrm{~d} s \leqslant$ $\int_{-\infty}^{+\infty}\left(1+s^{2}\right)^{j}\left|\widetilde{\left(\frac{h}{\alpha}\right)}(s)\right|^{2} \mathrm{~d} s \leqslant\left|c^{\mathcal{S}}\left(\zeta_{1}\right) \underline{c}\left(\zeta_{2}, \zeta_{3}, \overline{\zeta_{3}}\right)\right|, 0 \leqslant j \leqslant\left[\frac{3 q+2}{2}\right], q \in \mathbb{Z}_{\geqslant 1}$, it follows from the definition of $\alpha(\zeta)$ that $\mathrm{I}_{h_{I I}} \leqslant \frac{\mid c^{\mathcal{S}}\left(\zeta_{1}\right) \underline{\underline{c}\left(\zeta_{2}, \zeta_{3}, \overline{\zeta_{3}}\right) \mid v^{q}}}{\left|\zeta_{1}+\zeta_{2}\right|^{3} \zeta_{1}-\left.\zeta_{2}\right|^{-q}} \mathrm{e}^{-t \Re\left(\mathrm{i} \theta^{u}(\zeta)\right)}, q \in \mathbb{Z}_{\geqslant 1}, v \in[0,1]$. Recalling that $\theta^{u}(\zeta)=\frac{1}{2}\left(\zeta-\frac{1}{\zeta}\right)\left(z_{o}+\zeta+\frac{1}{\zeta}\right)$, for $\zeta=\zeta(v)=\zeta_{1}+\frac{v}{\sqrt{2}}\left(\zeta_{1}-\zeta_{2}\right) \mathrm{e}^{\frac{3 \pi \mathrm{i}}{4}}, v \in[0,1]$, one shows that $\Im\left(\left(\zeta-\frac{1}{\zeta}\right)\left(z_{O}+\zeta+\frac{1}{\zeta}\right)\right)=\frac{1}{2} v\left(\zeta_{1}-\zeta_{2}\right)\left(\zeta_{1}-\frac{1}{2}\left(\zeta_{1}-\zeta_{2}\right) v\right)\left(1-\frac{1}{\left(\zeta_{1}-\frac{1}{2}\left(\zeta_{1}-\zeta_{2}\right) v\right)^{2}+\left(\frac{1}{2}\left(\zeta_{1}-\zeta_{2}\right) v\right)^{2}}\right)^{2}+\frac{1}{2} v\left(\zeta_{1}-\zeta_{2}\right)\left(\zeta_{1}-\right.$ $\left.\frac{1}{2}\left(\zeta_{1}-\zeta_{2}\right) v\right)\left(1+\frac{1}{\left(\zeta_{1}-\frac{1}{2}\left(\zeta_{1}-\zeta_{2}\right) v\right)^{2}+\left(\frac{1}{2}\left(\zeta_{1}-\zeta_{2}\right) v\right)^{2}}\right)^{2}+\frac{1}{2} v\left(\zeta_{1}-\zeta_{2}\right) z_{o}\left(1+\frac{1}{\left(\zeta_{1}-\frac{1}{2}\left(\zeta_{1}-\zeta_{2}\right) v\right)^{2}+\left(\frac{1}{2}\left(\zeta_{1}-\zeta_{2}\right) v\right)^{2}}\right)$. Setting $\widehat{a}(v):=\left(\zeta_{1}-\frac{1}{2}\left(\zeta_{1}-\zeta_{2}\right) v\right)^{2}+\left(\frac{1}{2}\left(\zeta_{1}-\zeta_{2}\right) v\right)^{2}$, one deduces that, for $v \in[0,1], \widehat{a}(1) \leqslant \widehat{a}(v) \leqslant \widehat{a}(0)$; hence, noting that $\left(\frac{\zeta_{1}^{2}-\zeta_{1}\left(\zeta_{1}-\zeta_{2}\right) v+\frac{1}{2}\left(\zeta_{1}-\zeta_{2}\right)^{2} v^{2}+1}{\zeta_{1}^{2}-\zeta_{1}\left(\zeta_{1}-\zeta_{2}\right) v+\frac{1}{2}\left(\zeta_{1}-\zeta_{2}\right)^{2} v^{2}}\right) \geqslant\left(\frac{\zeta_{1}^{2}-\zeta_{1}\left(\zeta_{1}-\zeta_{2}\right) v+\frac{1}{2}\left(\zeta_{1}-\zeta_{2}\right)^{2} v^{2}-1}{\zeta_{1}^{2}-\zeta_{1}\left(\zeta_{1}-\zeta_{2}\right) v+\frac{1}{2}\left(\zeta_{1}-\zeta_{2}\right)^{2} v^{2}}\right), v \in[0,1]$, one deduces that $\Im\left(\left(\zeta-\frac{1}{\zeta}\right)\left(z_{o}+\zeta+\frac{1}{\zeta}\right)\right) \geqslant v\left(\zeta_{1}-\zeta_{2}\right)\left(\zeta_{1}-\frac{1}{2}\left(\zeta_{1}-\zeta_{2}\right) v\right)\left(1-(\widehat{a}(v))^{-1}\right)^{2}+\frac{1}{2} v\left(\zeta_{1}-\zeta_{2}\right) z_{o}\left(1-(\widehat{a}(v))^{-1}\right)$. Noting that, for $v \in[0,1], 1-(\widehat{a}(1))^{-1} \leqslant 1-(\widehat{a}(v))^{-1} \leqslant 1-(\widehat{a}(0))^{-1}, 1-(\widehat{a}(1))^{-1} \geqslant\left(1-(\widehat{a}(1))^{-1}\right)^{2}$, and $2-v \geqslant v$, it follows that $\Im\left(\left(\zeta-\frac{1}{\zeta}\right)\left(z_{o}+\zeta+\frac{1}{\zeta}\right)\right) \geqslant \frac{1}{2}\left(\zeta_{1}-\zeta_{2}\right)\left(z_{o}+\zeta_{1}+\zeta_{2}\right)\left(1-(\widehat{a}(1))^{-1}\right)^{2} v^{2}$; hence, since $\left(\zeta_{1}-\zeta_{2}\right)>0$ and $\left(z_{o}+\zeta_{1}+\zeta_{2}\right)<0$, it follows that $i \frac{1}{2} \Im\left(\left(\zeta-\frac{1}{\zeta}\right)\left(z_{o}+\zeta+\frac{1}{\zeta}\right)\right) \geqslant$ $\frac{1}{4}\left(\zeta_{1}-\zeta_{2}\right)\left|z_{0}+\zeta_{1}+\zeta_{2}\right|\left(1-(\widehat{a}(1))^{-1}\right)^{2} v^{2}$, whence $-t \Re\left(\mathrm{i} \theta^{u}(\zeta)\right) \leqslant-\frac{1}{4} t\left(\zeta_{1}-\zeta_{2}\right)\left|z_{0}+\zeta_{1}+\zeta_{2}\right|\left(1-(\widehat{a}(1))^{-1}\right)^{2} v^{2}$, $v \in[0,1]$. With this inequality, one deduces that, for $\zeta=\zeta(v)=\zeta_{1}+\frac{v}{\sqrt{2}}\left(\zeta_{1}-\zeta_{2}\right) \mathrm{e}^{\frac{3 \pi \mathrm{i}}{4}}, v \in[0,1]$, $\left|\mathrm{e}^{-2 \mathrm{i} t \theta^{u}(\zeta)} h_{I I}(\zeta)\right| \leqslant \frac{\left|c^{\mathcal{S}}\left(\zeta_{1}\right) \underline{c}\left(\zeta_{2}, \zeta_{3}, \overline{\zeta_{3}}\right)\right|}{\left|\zeta_{1}+\zeta_{2}\right|^{3} t^{q / 2}}, q \in \mathbb{Z}_{\geqslant 1}, 0<\zeta_{2}<\frac{1}{M}<M<\zeta_{1}$ and $\left|\zeta_{3}\right|^{2}=1$. Let $\varepsilon$ be an arbitrarily fixed, sufficiently small positive real number such that $\left\{\zeta ;\left|\zeta-\zeta_{1}\right|<\varepsilon\right\} \cap\left\{\zeta ; \zeta=\zeta_{1}+\right.$ $\left.\frac{v}{\sqrt{2}}\left(\zeta_{1}-\zeta_{2}\right) \mathrm{e}^{\frac{3 \pi \mathrm{i}}{4}}, v \in(\varepsilon, 1]\right\}=\emptyset$, and recall that $\mathcal{R}(\zeta):=\sum_{n=0}^{k} \frac{1}{n !} \rho^{(n)}\left(\zeta_{1}\right)\left(\zeta-\zeta_{1}\right)^{n}, k \in \mathbb{Z}_{\geqslant 1}$. On the line segment $\zeta=\zeta(v)=\zeta_{1}+\frac{v}{\sqrt{2}}\left(\zeta_{1}-\zeta_{2}\right) \mathrm{e}^{\frac{3 \pi \mathrm{i}}{4}}, \varepsilon<v \leqslant 1$, one shows that $\left|\left(\delta_{+}(\zeta)\right)^{2} \mathrm{e}^{-2 \mathrm{i} t \theta^{u}(\zeta)} \mathcal{R}(\zeta)\right| \leqslant$ $\left(1-\sup _{z \in \mathbb{R}}|r(z)|^{2}\right) \mathrm{e}^{-2 t \Re\left(\mathrm{i}^{u}(\zeta)\right)} \sup _{\left(\zeta_{1}, n\right) \in(M,+\infty) \times\{0,1, \ldots, k\}}\left|\rho^{(n)}\left(\zeta_{1}\right)\right| \sum_{n=0}^{k} \frac{\left|\zeta_{1}-\zeta_{2}\right|^{n} v^{n}}{2^{n / 2} n !}:$ now, recalling the above inequality for $-t \Re\left(\mathrm{i} \theta^{u}(\zeta)\right)$, and using the formula $\sum_{n=0}^{k} \star^{n}=\frac{1-\star^{k+1}}{1-\star}$, one shows that, on this line segment, $\left|\mathrm{e}^{-2 \mathrm{i} t \theta^{u}(\zeta)} \mathcal{R}(\zeta)\right| \leqslant\left|\underline{c}\left(\zeta_{1}, \zeta_{2}, \zeta_{3}, \overline{\zeta_{3}}\right)\right| \exp \left(-\frac{1}{2} t \varepsilon^{2}\left(\zeta_{1}-\zeta_{2}\right) \mid z_{o}+\right.$ $\left.\zeta_{1}+\zeta_{2} \mid\left(\frac{\zeta_{1}^{4}-2 \zeta_{1}^{2}+1}{\zeta_{1}^{4}+1}\right)^{2}\right)$

Without loss of generality, and as the second representative calculation, one considers, say, the non-empty domain $\{z ; \Im(z)=0\} \cap\left\{z ; \Re(z) \geqslant \zeta_{1}\right\}$. Once again, let $k \in \mathbb{Z}_{\geqslant 1}$ be fixed and sufficiently large with representation $k=4 q+1, q \in \mathbb{Z}_{\geqslant 1}$. Recalling that, from Lemma 4.1, for $\Re(\zeta) \in\left(\zeta_{1},+\infty\right), \rho(\zeta)=\overline{r(\bar{\zeta})}$, one must estimate terms of the type $\rho(\zeta)(\delta(\zeta))^{2} \mathrm{e}^{-2 \mathrm{i} t \theta^{u}(\zeta)}$ (respectively $\left.\overline{r(\bar{\zeta})}(\delta(\zeta))^{-2} \mathrm{e}^{2 i t \theta^{u}(\zeta)}\right)$ : in fact, the former will be considered in detail, with analytic continuation to the unbounded sector $\Omega_{3}$ (see Figure 3 ) where $\Re\left(\mathrm{i} t \theta^{u}(\zeta)\right)>0$, whilst the latter can be estimated analogously with analytic continuation to the unbounded sector $\Omega_{7}$ (see Figure 3) where $\Re\left(\mathrm{it} t \theta^{u}(\zeta)\right)<0$. For $\Re(\zeta) \geqslant \zeta_{1}$, consider the following (rational) Taylor expansion with integral remainder term for $\rho(\zeta),(\zeta-\mathrm{i})^{k+5} \rho(\zeta)=\sum_{n=0}^{k} \frac{1}{n !} \mu^{(n)}\left(\zeta_{1}\right)\left(\zeta-\zeta_{1}\right)^{n}+\frac{1}{k !} \int_{\zeta_{1}}^{\zeta}((\cdot-$ i $\left.)^{k+5} \rho(\cdot)\right)^{(k+1)}(\xi)(\zeta-\xi)^{k} \mathrm{~d} \xi$, where $\mu^{(n)}\left(\zeta_{1}\right):=\left.\left(\frac{\mathrm{d}}{\mathrm{d} \zeta}\right)^{n}\left((\zeta-\mathrm{i})^{k+5} \rho(\zeta)\right)\right|_{\zeta=\zeta_{1}}, n \in\{0,1, \ldots, k\}$, $k \in \mathbb{Z}_{\geqslant 1}$ : also, if one explicitly considers, say, the limiting case when $\zeta_{1} \rightarrow+\infty\left(\zeta_{2} \rightarrow 0^{+}\right)$, then, as functions of $\zeta_{1}, \mu^{(n)}\left(\zeta_{1}\right), n \in\{0,1, \ldots, k\}, k \in \mathbb{Z}_{\geqslant 1}$, are in the $\mathbb{C}$-valued Schwartz class (since $r(\zeta) \in \mathcal{S}_{\mathbb{C}}(\mathbb{R})$ ). Let $\mathcal{R}(\zeta)$ denote the "rational part" of this expansion, and $h(\zeta)$ the "remainder", namely, $\mathcal{R}(\zeta):=\frac{\sum_{n=0}^{k} \frac{1}{n !} \mu^{(n)}\left(\zeta_{1}\right)\left(\zeta-\zeta_{1}\right)^{n}}{(\zeta-\mathrm{i})^{k+5}}$ and $h(\zeta):=\frac{\int_{\zeta_{1}}^{\zeta}\left((\cdot-\mathrm{i})^{k+5} \rho(\cdot)\right)^{(k+1)}(\xi)(\zeta-\xi)^{k} \mathrm{~d} \xi}{k !(\zeta-\mathrm{i})^{k+5}}$; hence, $\rho(\zeta)=\mathcal{R}(\zeta)+h(\zeta)$, and $\rho(\zeta)-\mathcal{R}(\zeta)=h(\zeta)$ can be interpreted as the error incurred in approximating $\rho(\zeta)$ by a rational function of finite degree. Noting that, $\forall j \in\{0,1, \ldots, k\}, k \in \mathbb{Z}_{\geqslant 1}$, $\left.\left(\frac{\mathrm{d}}{\mathrm{d} \zeta}\right)^{j} \rho(\zeta)\right|_{\zeta=\zeta_{1}}=\left.\left(\frac{\mathrm{d}}{\mathrm{d} \zeta}\right)^{j} \mathcal{R}(\zeta)\right|_{\zeta=\zeta_{1}}$, it follows that $\left.\left(\frac{\mathrm{d}}{\mathrm{d} \zeta}\right)^{j} h(\zeta)\right|_{\zeta=\zeta_{1}}=0, j \in\{0,1, \ldots, k\}, k \in \mathbb{Z}_{\geqslant 1} ;$ 
thence, one splits, further, $h(\zeta)$ as $h(\zeta):=h_{I}(\zeta)+h_{I I}(\zeta)$, whence $\rho(\zeta)=h_{I}(\zeta)+\left(h_{I I}(\zeta)+\mathcal{R}(\zeta)\right)$, where $h_{I}(\zeta)$ is defined for $\zeta \in\{z ; \Im(z)=0\} \cap\left\{z ; \Re(z) \geqslant \zeta_{1}\right\}$, where $\Re\left(\mathrm{i} t \theta^{u}(\zeta)\right)=0$, and has negligibly "small norm" (see below) as $t \rightarrow+\infty$, and $h_{I I}(\zeta)$ has an analytic continuation to $\left\{z ; \Re(z) \geqslant \zeta_{1}\right\} \cap \Omega_{3}$, where $\Re\left(\mathrm{i} t \theta^{u}(\zeta)\right)>0$, and $\mathcal{R}(\zeta)$ is a rational function of finite degree with trivial analytic continuation to $\left\{z ; \Re(z) \geqslant \zeta_{1}\right\} \cap \Omega_{3}$. Considering the auxiliary function (which will be needed below) $\beta(\zeta):=\frac{\left(\zeta-\zeta_{1}\right)^{q}}{(\zeta-\mathrm{i})^{q+2}}, q \in \mathbb{Z}_{\geqslant 1}$, define $\left(\frac{h}{\beta}\right)\left(\theta^{u}\right):=\left\{\begin{array}{ll}\frac{h\left(\zeta\left(\theta^{u}\right)\right)}{\beta\left(\zeta\left(\theta^{u}\right)\right)}, & \theta^{u} \geqslant \theta^{u}\left(\zeta_{1}\right), \\ 0, & \theta^{u}<\theta^{u}\left(\zeta_{1}\right),\end{array}\right.$ and consider the Fourier transform w.r.t. $\theta^{u}(\cdot)$. Via a change of variable argument, one shows that $h(\zeta)=\frac{\left(\zeta-\zeta_{1}\right)^{k+1} g_{k}\left(\zeta, \zeta_{1}\right)}{(\zeta-\mathrm{i})^{k+5}}$, where $g_{k}\left(\zeta, \zeta_{1}\right):=\frac{1}{k !} \int_{0}^{1}\left((\cdot-\mathrm{i})^{k+5} \rho(\cdot)\right)^{(k+1)}\left(\zeta_{1}+\left(\zeta-\zeta_{1}\right) \tau\right)(1-\tau)^{k} \mathrm{~d} \tau$ : since $\rho(\zeta) \in \mathcal{S}_{\mathbb{C}}(\mathbb{R})$ and $\exists M \in \mathbb{R}_{>1}$ and bounded such that $0<\zeta_{2}<\frac{1}{M}<M<\zeta_{1}$ and $\left|\zeta_{3}\right|^{2}=1$, one shows that, $\forall\left(\zeta, \zeta_{1}\right) \in\left[\zeta_{1},+\infty\right) \times(M,+\infty), \exists \hat{\varkappa} \in \mathbb{R}_{+}$and bounded such that $\left|g_{k}^{\left(j_{0}\right)}\left(\zeta, \zeta_{1}\right)\right| \leqslant \hat{\varkappa}$, $\left(j_{o}, k\right) \in \mathbb{Z}_{\geqslant 0} \times \mathbb{Z}_{\geqslant 1}$. With the above choice of $\beta(\zeta)$ (not the only one possible!), one shows that $\frac{h(\zeta)}{\beta(\zeta)}=\frac{\left(\zeta-\zeta_{1}\right)^{3 q+2} g_{k}\left(\zeta, \zeta_{1}\right)}{(\zeta-\mathrm{i})^{3 q+4}}$, with $\frac{h(\zeta)}{\beta(\zeta)}={ }_{\zeta \rightarrow+\infty} \mathcal{O}\left(\zeta^{-2}\right)$; hence, $\left|\int_{\zeta_{1}}^{+\infty} \frac{h(z)}{\beta(z)} \mathrm{d} z\right|<\infty$. Define the


$\int_{\theta^{u}\left(\zeta_{1}\right)}^{+\infty} \mathrm{e}^{-\mathrm{i} s \theta^{u}(\zeta)}\left(\frac{h}{\beta}\right)(\zeta) \frac{\mathrm{d} \theta^{u}(\zeta)}{\sqrt{2 \pi}}$. In order to obtain the necessary estimates for $\left(h_{I}(\zeta)+\left(h_{I I}(\zeta)+\right.\right.$ $\mathcal{R}(\zeta)))(\delta(\zeta))^{2} \mathrm{e}^{-2 \mathrm{i} t \theta^{u}(\zeta)}$, one needs to show that $\left(\frac{h}{\beta}\right)\left(\theta^{u}\right):=\frac{h\left(\zeta\left(\theta^{u}\right)\right)}{\beta\left(\zeta\left(\theta^{u}\right)\right)} \in \mathcal{H}^{j}(\mathbb{R}), 0 \leqslant j \leqslant\left[\frac{3 q+2}{2}\right]$, $q \in \mathbb{Z}_{\geqslant 1}$, where $\mathcal{H}^{j}(\mathbb{R})$ denotes the $L^{2}$-Sobolev space with norm $\|\star(\cdot)\|_{\mathcal{H}(\mathbb{R})}:=\left(\sum_{j=0}^{\left[\frac{3 q+2}{2}\right]} \|\left(\frac{\mathrm{d}}{\mathrm{d} \theta^{u}}\right)^{j} \star\right.$ $\left.\left(\theta^{u}\right) \|_{\mathcal{L}^{2}(\mathbb{R})}^{2}\right)^{1 / 2}$. In this particular case, one must show that, for $0<\zeta_{2}<\frac{1}{M}<M<\zeta_{1}$ and $\left|\zeta_{3}\right|^{2}=1$, with $\zeta \geqslant \zeta_{1}, \widehat{\mathrm{I}}_{\mathcal{H}}:=\int_{-\infty}^{+\infty}\left|\left(\frac{\mathrm{d}}{\mathrm{d} \theta^{u}}\right)^{j} \frac{h\left(\zeta\left(\theta^{u}\right)\right)}{\beta\left(\zeta\left(\theta^{u}\right)\right)}\right|^{2} \mathrm{~d} \theta^{u}<\infty, 0 \leqslant j \leqslant\left[\frac{3 q+2}{2}\right], q \in \mathbb{Z}_{\geqslant 1}$ : in fact, since $\frac{h\left(\zeta\left(\theta^{u}\right)\right)}{\beta\left(\zeta\left(\theta^{u}\right)\right)} \equiv 0 \forall \theta^{u}<\theta^{u}\left(\zeta_{1}\right)$, the latter integral reduces to $\widehat{\mathrm{I}}_{\mathcal{H}}=\int_{\theta^{u}\left(\zeta_{1}\right)}^{+\infty}\left|\left(\frac{\mathrm{d}}{\mathrm{d} \theta^{u}}\right)^{j} \frac{h\left(\zeta\left(\theta^{u}\right)\right)}{\beta\left(\zeta\left(\theta^{u}\right)\right)}\right|^{2} \mathrm{~d} \theta^{u}$, which, via the chain rule and a change-of-variable argument, is shown to be equal to $\widehat{\mathrm{I}}_{\mathcal{H}}=$ $\int_{\zeta_{1}}^{+\infty}\left|\frac{\left(\frac{\mathrm{d}}{\mathrm{d} \zeta}\right)^{j} \frac{h(\zeta)}{\beta(\zeta)}}{\left(\frac{\mathrm{d}^{u}(\zeta)}{\mathrm{d} \zeta}\right)^{j}}\right|^{2}\left(\frac{\mathrm{d} \theta^{u}(\zeta)}{\mathrm{d} \zeta}\right) \mathrm{d} \zeta$. Now, recalling that $\frac{h(\zeta)}{\beta(\zeta)}=\frac{\left(\zeta-\zeta_{1}\right)^{3 q+2}}{(\zeta-\mathrm{i})^{3 q+4} k !} \int_{0}^{1}\left((\cdot-\mathrm{i})^{k+5} \rho(\cdot)\right)^{(k+1)}\left(\zeta_{1}+(\zeta-\right.$ $\left.\left.\zeta_{1}\right) \tau\right)(1-\tau)^{k} \mathrm{~d} \tau$ and (from Section 3) $\frac{\mathrm{d} \theta^{u}(\zeta)}{\mathrm{d} \zeta}=\zeta^{-3}\left(\zeta-\zeta_{1}\right)\left(\zeta-\zeta_{2}\right)\left(\zeta-\zeta_{3}\right)\left(\zeta-\overline{\zeta_{3}}\right)$, one shows that, upon deducing $\left(\frac{h}{\beta}\right)^{(j)}(\zeta)={ }_{\zeta \downarrow \zeta_{1}} \mathcal{O}\left(\left(\zeta-\zeta_{1}\right)^{3 q+2-j}\right), \widehat{\mathrm{I}}_{\mathcal{H}} \leqslant\left|c^{\mathcal{S}}\left(\zeta_{1}\right) \underline{c}\left(\zeta_{2}, \zeta_{3}, \overline{\zeta_{3}}\right)\right|, 0 \leqslant j \leqslant\left[\frac{3 q+2}{2}\right], q \in \mathbb{Z}_{\geqslant 1}$, $0<\zeta_{2}<\frac{1}{M}<M<\zeta_{1}$ and $\left|\zeta_{3}\right|^{2}=1$, that is, $\left\|\left(\frac{h}{\beta}\right)(\cdot)\right\|_{\mathcal{H}(\mathbb{R})}=\left(\sum_{j=0}^{\left[\frac{3 q+2}{2}\right]}\left\|\left(\frac{\mathrm{d}}{\mathrm{d} \theta^{u}}\right)^{j}\left(\frac{h}{\beta}\right)\left(\theta^{u}\right)\right\|_{\mathcal{L}^{2}(\mathbb{R})}^{2}\right)^{1 / 2}<$ $\infty$; hence, by Parseval's Theorem, for $0<\zeta_{2}<\frac{1}{M}<M<\zeta_{1}$ and $\left|\zeta_{3}\right|^{2}=1, \int_{-\infty}^{+\infty}(1+$ $\left.s^{2}\right)^{j}\left|\widetilde{\left(\frac{h}{\beta}\right)}(s)\right|^{2} \mathrm{~d} s \leqslant\left|c^{\mathcal{S}}\left(\zeta_{1}\right) \underline{c}\left(\zeta_{2}, \zeta_{3}, \overline{\zeta_{3}}\right)\right|, 0 \leqslant j \leqslant\left[\frac{3 q+2}{2}\right], q \in \mathbb{Z}_{\geqslant 1}$. Recalling from the Fourier transform pair that, for $\zeta \geqslant \zeta_{1},\left(\frac{h}{\beta}\right)(\zeta)=\int_{-\infty}^{+\infty} \mathrm{e}^{\mathrm{i} s \theta^{u}(\zeta)} \widetilde{\left(\frac{h}{\beta}\right)}(s) \frac{\mathrm{d} s}{\sqrt{2 \pi}}$, it follows, by defining $h_{I}(\zeta):=$ $\beta(\zeta) \int_{t}^{+\infty} \mathrm{e}^{\mathrm{i} s \theta^{u}(\zeta)} \widetilde{\left(\frac{h}{\beta}\right)}(s) \frac{\mathrm{d} s}{\sqrt{2 \pi}}$ and $h_{I I}(\zeta):=\beta(\zeta) \int_{-\infty}^{t} \mathrm{e}^{\mathrm{i} s \theta^{u}(\zeta)} \widetilde{\left(\frac{h}{\beta}\right)(s)} \frac{\mathrm{d} s}{\sqrt{2 \pi}}$, that $h(\zeta)=h_{I}(\zeta)+h_{I I}(\zeta)$. For $\zeta \in\{z ; \Im(z)=0\} \cap\left\{z ; \Re(z) \geqslant \zeta_{1}\right\}$, recalling the estimate for $\delta(\zeta)$ given in Proposition 4.1, using the Cauchy-Schwarz inequality for integrals, as well as the inequality $\left|\frac{\zeta-\zeta_{1}}{\zeta+\zeta_{1}}\right| \leqslant 1$,

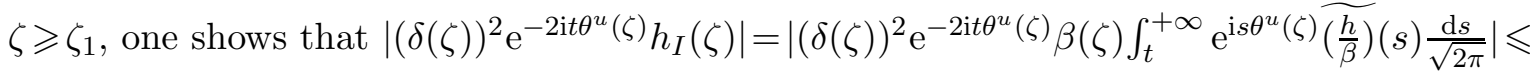
$\left\|(\delta(\cdot))^{2}\right\|_{\mathcal{L}^{\infty}(\mathbb{C})} \frac{|\beta(\zeta)|}{\sqrt{2 \pi}}\left(\int_{t}^{+\infty}\left(1+s^{2}\right)^{-j} \mathrm{~d} s\right)^{1 / 2}\left(\int_{t}^{+\infty}\left(1+s^{2}\right)^{j}\left|\widetilde{\left(\frac{h}{\beta}\right)(s)}\right|^{2} \mathrm{~d} s\right)^{1 / 2}$ : noting that $\int_{t}^{+\infty}(1+$ $\left.s^{2}\right)^{-j} \mathrm{~d} s \leqslant \frac{t^{-(2 j-1)}}{(2 j-1)}, 2 j-1>0$, and recalling the Parseval estimate $\int_{t}^{+\infty}\left(1+s^{2}\right)^{j}\left|\widetilde{\left(\frac{h}{\beta}\right)}(s)\right|^{2} \mathrm{~d} s \leqslant$ $\int_{-\infty}^{+\infty}\left(1+s^{2}\right)^{j}\left|\widetilde{\left(\frac{h}{\beta}\right)}(s)\right|^{2} \mathrm{~d} s \leqslant\left|c^{\mathcal{S}}\left(\zeta_{1}\right) \underline{c}\left(\zeta_{2}, \zeta_{3}, \overline{\zeta_{3}}\right)\right|, 0 \leqslant j \leqslant\left[\frac{3 q+2}{2}\right]$, it follows that, with the choice $\beta(\zeta)=\frac{\left(\zeta-\zeta_{1}\right)^{q}}{(\zeta-\mathrm{i})^{q+2}}, q \in \mathbb{Z}_{\geqslant 1},\left|\mathrm{e}^{-2 \mathrm{i} t \theta^{u}(\zeta)} h_{I}(\zeta)\right| \leqslant \frac{\left|c^{\mathcal{S}}\left(\zeta_{1}\right)\left(\zeta_{2}, \overline{\zeta_{3}} \overline{\zeta_{3}}\right)\right|}{|\zeta-\mathrm{i}|^{2} t^{(j-1 / 2)}}, 1 \leqslant j \leqslant\left[\frac{3 q+2}{2}\right], q \in \mathbb{Z}_{\geqslant 1}, \zeta \geqslant \zeta_{1}$, $0<\zeta_{2}<\frac{1}{M}<M<\zeta_{1}$ and $\left|\zeta_{3}\right|^{2}=1$. Since, for $\zeta \in\left\{z ; \Re(z) \geqslant \zeta_{1}\right\} \cap \Omega_{3}, \Re\left(\mathrm{i} t \theta^{u}(\zeta)\right)>$ $0, h_{I I}(\zeta)$ has an analytic continuation to the ray (parametrised by $\left.v\right) \zeta=\zeta(v)=\zeta_{1}+$ $\frac{v}{\sqrt{2}}\left(\zeta_{1}-\zeta_{2}\right) \mathrm{e}^{-\frac{\mathrm{i} \pi}{4}}, v \in \mathbb{R}_{\geqslant 0}$; hence, on this ray, it follows that $\widehat{\mathrm{I}}_{h_{I I}}:=\left|(\delta(\zeta))^{2} \mathrm{e}^{-2 \mathrm{i} t \theta^{u}(\zeta)} h_{I I}(\zeta)\right|=$ 
$\left|(\delta(\zeta))^{2} \mathrm{e}^{-2 \mathrm{i} t \theta^{u}(\zeta)} \beta(\zeta) \int_{-\infty}^{t} \mathrm{e}^{\mathrm{i} s \theta^{u}(\zeta)} \widetilde{\left(\frac{h}{\beta}\right)}(s) \frac{\mathrm{d} s}{\sqrt{2 \pi}}\right| \leqslant\left\|(\delta(\cdot))^{2}\right\|_{\mathcal{L}^{\infty}(\mathbb{C})}\left|\beta(\zeta)\left\|\mathrm{e}^{-\mathrm{i} t \theta^{u}(\zeta)}\right\| \int_{-\infty}^{t} \widetilde{\left(\frac{h}{\beta}\right)}(s) \frac{\mathrm{d} s}{\sqrt{2 \pi}}\right|$, thus, from the Cauchy-Schwarz inequality for integrals, $\widehat{\mathrm{I}}_{h_{I I}} \leqslant\left\|(\delta(\cdot))^{2}\right\|_{\mathcal{L}^{\infty}(\mathbb{C})} \frac{|\beta(\alpha)|}{\sqrt{2 \pi}} \mathrm{e}^{-t \Re\left(\mathrm{i} \theta^{u}(\zeta)\right)}$ $\cdot\left(\int_{-\infty}^{t}\left(1+s^{2}\right)^{-j} \mathrm{~d} s\right)^{1 / 2}\left(\int_{-\infty}^{t}\left(1+s^{2}\right)^{j}\left|\widetilde{\left(\frac{h}{\beta}\right)}(s)\right|^{2} \mathrm{~d} s\right)^{1 / 2}$. Recalling from the previous calculation that $\int_{-\infty}^{t}\left(1+s^{2}\right)^{-j} \mathrm{~d} s \leqslant \pi$, and deducing, from the above Parseval estimate, that $\int_{-\infty}^{t}(1+$ $\left.s^{2}\right)\left.^{j} \widetilde{\left(\frac{h}{\beta}\right)}(s)\right|^{2} \mathrm{~d} s \leqslant \int_{-\infty}^{+\infty}\left(1+s^{2}\right)^{j}\left|\widetilde{\left(\frac{h}{\beta}\right)}(s)\right|^{2} \mathrm{~d} s \leqslant\left|c^{\mathcal{S}}\left(\zeta_{1}\right) \underline{c}\left(\zeta_{2}, \zeta_{3}, \overline{\zeta_{3}}\right)\right|, 0 \leqslant j \leqslant\left[\frac{3 q+2}{2}\right], q \in \mathbb{Z}_{\geqslant 1}$, it follows from the definition of $\beta(\zeta)$ that $\widehat{\mathrm{I}}_{h_{I I}} \leqslant \frac{\left|c^{\mathcal{S}}\left(\zeta_{1}\right) \underline{c}\left(\zeta_{2}, \zeta_{3}, \overline{\zeta_{3}}\right)\right|\left|\zeta_{1}-\zeta_{2}\right|^{q} v^{q}}{|\zeta-\mathrm{i}|^{2}} \mathrm{e}^{-t \Re\left(\mathrm{i} \theta^{u}(\zeta)\right)}, q \in \mathbb{Z}_{\geqslant 1}, v \geqslant 0$. Recalling that $\theta^{u}(\zeta)=\frac{1}{2}\left(\zeta-\frac{1}{\zeta}\right)\left(z_{o}+\zeta+\frac{1}{\zeta}\right)$, for $\zeta=\zeta(v)=\zeta_{1}+\frac{v}{\sqrt{2}}\left(\zeta_{1}-\zeta_{2}\right) \mathrm{e}^{-\frac{\mathrm{i} \pi}{4}}, v \in \mathbb{R}_{\geqslant 0}$, one shows that $\Re\left(\mathrm{i} \theta^{u}(\zeta)\right)=\frac{1}{4} v\left(\zeta_{1}-\zeta_{2}\right)\left(\zeta_{1}+\frac{1}{2}\left(\zeta_{1}-\zeta_{2}\right) v\right)\left(\frac{\zeta_{1}^{2}+\zeta_{1}\left(\zeta_{1}-\zeta_{2}\right) v+\frac{1}{2}\left(\zeta_{1}-\zeta_{2}\right)^{2} v^{2}-1}{\zeta_{1}^{2}+\zeta_{1}\left(\zeta_{1}-\zeta_{2}\right) v+\frac{1}{2}\left(\zeta_{1}-\zeta_{2}\right)^{2} v^{2}}\right)^{2}+\frac{1}{4} v\left(\zeta_{1}-\zeta_{2}\right)\left(\zeta_{1}+\right.$ $\left.\frac{1}{2}\left(\zeta_{1}-\zeta_{2}\right) v\right)\left(\frac{\zeta_{1}^{2}+\zeta_{1}\left(\zeta_{1}-\zeta_{2}\right) v+\frac{1}{2}\left(\zeta_{1}-\zeta_{2}\right)^{2} v^{2}+1}{\zeta_{1}^{2}+\zeta_{1}\left(\zeta_{1}-\zeta_{2}\right) v+\frac{1}{2}\left(\zeta_{1}-\zeta_{2}\right)^{2} v^{2}}\right)^{2}+\frac{1}{4} v\left(\zeta_{1}-\zeta_{2}\right) z_{o}\left(\frac{\zeta_{1}^{2}+\zeta_{1}\left(\zeta_{1}-\zeta_{2}\right) v+\frac{1}{2}\left(\zeta_{1}-\zeta_{2}\right)^{2} v^{2}+1}{\zeta_{1}^{2}+\zeta_{1}\left(\zeta_{1}-\zeta_{2}\right) v+\frac{1}{2}\left(\zeta_{1}-\zeta_{2}\right)^{2} v^{2}}\right)$. Setting $\widetilde{a}(v):=\left(\zeta_{1}+\frac{1}{2}\left(\zeta_{1}-\zeta_{2}\right) v\right)^{2}+\left(\frac{1}{2}\left(\zeta_{1}-\zeta_{2}\right) v\right)^{2}$, one shows that, for $v \geqslant 0, \widetilde{a}(v) \geqslant \widetilde{a}(0)$; hence, with $\left(\frac{\zeta_{1}^{2}+\zeta_{1}\left(\zeta_{1}-\zeta_{2}\right) v+\frac{1}{2}\left(\zeta_{1}-\zeta_{2}\right)^{2} v^{2}+1}{\zeta_{1}^{2}+\zeta_{1}\left(\zeta_{1}-\zeta_{2}\right) v+\frac{1}{2}\left(\zeta_{1}-\zeta_{2}\right)^{2} v^{2}}\right) \geqslant\left(\frac{\zeta_{1}^{2}+\zeta_{1}\left(\zeta_{1}-\zeta_{2}\right) v+\frac{1}{2}\left(\zeta_{1}-\zeta_{2}\right)^{2} v^{2}-1}{\zeta_{1}^{2}+\zeta_{1}\left(\zeta_{1}-\zeta_{2}\right) v+\frac{1}{2}\left(\zeta_{1}-\zeta_{2}\right)^{2} v^{2}}\right), v \geqslant 0$, one shows that $\Re\left(\mathrm{i} \theta^{u}(\zeta)\right) \geqslant$ $\frac{1}{2} v\left(\zeta_{1}-\zeta_{2}\right)\left(\zeta_{1}+\frac{1}{2}\left(\zeta_{1}-\zeta_{2}\right) v\right)\left(\frac{\zeta_{1}^{2}+\zeta_{1}\left(\zeta_{1}-\zeta_{2}\right) v+\frac{1}{2}\left(\zeta_{1}-\zeta_{2}\right)^{2} v^{2}-1}{\zeta_{1}^{2}+\zeta_{1}\left(\zeta_{1}-\zeta_{2}\right) v+\frac{1}{2}\left(\zeta_{1}-\zeta_{2}\right)^{2} v^{2}}\right)^{2}+\frac{v}{4}\left(\zeta_{1}-\zeta_{2}\right) z_{o}\left(\frac{\zeta_{1}^{2}+\zeta_{1}\left(\zeta_{1}-\zeta_{2}\right) v+\frac{1}{2}\left(\zeta_{1}-\zeta_{2}\right)^{2} v^{2}-1}{\zeta_{1}^{2}+\zeta_{1}\left(\zeta_{1}-\zeta_{2}\right) v+\frac{1}{2}\left(\zeta_{1}-\zeta_{2}\right)^{2} v^{2}}\right)$. Noting that $\left(1-(\widetilde{a}(0))^{-1}\right) \leqslant\left(1-(\widetilde{a}(v))^{-1}\right) \leqslant 1,\left(1-\zeta_{1}^{-2}\right) \geqslant\left(1-\zeta_{1}^{-2}\right)^{2}$, and $2+v \geqslant v, v \geqslant 0$, one establishes that $\Re\left(\mathrm{i} \theta^{u}(\zeta)\right) \geqslant \frac{1}{4} v\left(\zeta_{1}-\zeta_{2}\right)\left(1-\zeta_{1}^{-2}\right)^{2}\left(z_{o}+\left(\zeta_{1}-\zeta_{2}\right) v\right)$. From the identities (valid for $v \geqslant 0$ ) $1 \geqslant(v+1)^{-1}, 1 \geqslant v(v+1)^{-1}$, and $-1 \leqslant \frac{v-1}{v+1} \leqslant 1$, and choosing an arbitrarily fixed, sufficiently small positive real number $\gamma_{o}$ such that $\gamma_{o}<(v+1)^{-1} \leqslant 1, v \geqslant 0$, one deduces that $\Re\left(\mathrm{i} \theta^{u}(\zeta)\right) \geqslant$ $\frac{1}{4} \gamma_{o} v^{2}\left(\zeta_{1}-\zeta_{2}\right)\left(1-\zeta_{1}^{-2}\right)^{2}\left|z_{o}+\zeta_{1}-\zeta_{2}\right|$; hence, $-t \Re\left(\mathrm{i} \theta^{u}(\zeta)\right) \leqslant-\frac{1}{4} t \gamma_{o} v^{2}\left(\zeta_{1}-\zeta_{2}\right)\left(1-\zeta_{1}^{-2}\right)^{2}\left|z_{o}+\zeta_{1}+\zeta_{2}\right|$, $v \in \mathbb{R}_{\geqslant 0}$. Recalling that $\widehat{\mathrm{I}}_{h_{I I}} \leqslant \frac{\left|\mathcal{C}^{\mathcal{S}}\left(\zeta_{1}\right) \underline{c}\left(\zeta_{2}, \zeta_{3}, \overline{\zeta_{3}}\right)\right|\left|\zeta_{1}-\zeta_{2}\right|^{q} v^{q}}{|\zeta-\mathrm{i}|^{2}} \mathrm{e}^{-t \Re\left(\mathrm{i} \theta^{u}(\zeta)\right)}$, it follows from the above inequality for $-t \Re\left(\mathrm{i} \theta^{u}(\zeta)\right)$ (on the ray $\zeta=\zeta(v)=\zeta_{1}+\frac{v}{\sqrt{2}}\left(\zeta_{1}-\zeta_{2}\right) \mathrm{e}^{-\frac{\mathrm{i} \pi}{4}}, v \in \mathbb{R}_{\geqslant 0}$ ) that $\left|\mathrm{e}^{-2 \mathrm{i} t \theta^{u}(\zeta)} h_{I I}(\zeta)\right| \leqslant \frac{\left|c^{\mathcal{S}}\left(\zeta_{1}\right)\left(\zeta_{2}, \overline{\zeta_{3}}, \overline{\zeta_{3}}\right)\right|}{\left.\left|z_{o}+\zeta_{1}-\zeta_{2}\right|\right|^{q / 2}|\zeta-\mathrm{i}|^{2} t^{q / 2}}, q \in \mathbb{Z}_{\geqslant 1}, 0<\zeta_{2}<\frac{1}{M}<M<\zeta_{1}$ and $\left|\zeta_{3}\right|^{2}=1$.

Proceeding in the above-demonstrated manner for the remaining domains and sectors, and setting $\mathcal{R}(\zeta) \equiv 0$ for $\zeta<0$, one obtains the results stated in the Lemma; however, in order to analyse the (complex conjugate pair of) first-order saddle points at $\zeta_{3}$ and $\overline{\zeta_{3}}$, one uses the fact that $r(0)=0$ and the solution of the RHP is bounded outside open neighbourhoods of $\left\{\zeta_{i}\right\}_{i=1}^{4}$, and then proceeds according to the Remark in Section 3.1 of 46 .

From Lemmae 4.1 and 4.2, one derives the following (normalised at $\infty$ ) RHP for $m^{\sharp}(\zeta$ ) on the augmented contour $\Sigma^{\prime}$ :

Lemma 4.3. Let $\widehat{m}^{c}(\zeta)$ be the solution of the RHP formulated in Lemma 4.1. As $t \rightarrow+\infty$ such that $0<\zeta_{2}<\frac{1}{M}<M<\zeta_{1}$ and $\left|\zeta_{3}\right|^{2}=1$, with $M \in \mathbb{R}_{>1}$ and bounded, and for arbitrarily fixed, sufficiently large $l \in \mathbb{Z}_{\geqslant 1}$, let the estimates in Lemma 4.2 be valid. Set

$$
m^{\sharp}(\zeta):= \begin{cases}\widehat{m}^{c}(\zeta), & \zeta \in \Omega_{1} \cup \Omega_{2}, \\ \widehat{m}^{c}(\zeta)\left(\mathrm{I}+\underline{w}_{+}^{a}(\zeta)\right)^{-1}, & \zeta \in \Omega_{3} \cup \Omega_{4} \cup \Omega_{5} \cup \Omega_{6}, \\ \widehat{m}^{c}(\zeta)\left(\mathrm{I}-\underline{w}_{-}^{a}(\zeta)\right)^{-1}, & \zeta \in \Omega_{7} \cup \Omega_{8} \cup \Omega_{9} \cup \Omega_{10},\end{cases}
$$

where $\underline{w}_{ \pm}^{a}(\zeta):=(\delta(\zeta))^{\operatorname{ad}\left(\sigma_{3}\right)} \mathrm{e}^{-\mathrm{i} t \theta^{u}(\zeta) \operatorname{ad}\left(\sigma_{3}\right)} w_{ \pm}^{a}(\zeta)$, with $w_{+}^{a}(\zeta)=\left(h_{I I}(\zeta)+\mathcal{R}(\zeta)\right) \sigma_{+}$and $w_{-}^{a}(\zeta)=$ $-\left(\overline{h_{I I}(\bar{\zeta})}+\overline{\mathcal{R}(\bar{\zeta})}\right) \sigma_{-}$. Then $m^{\sharp}(\zeta): \mathbb{C} \backslash \Sigma^{\prime} \rightarrow \mathrm{SL}(2, \mathbb{C})$ solves the following RHP: (1) $m^{\sharp}(\zeta)$ is piecewise holomorphic $\forall \zeta \in \mathbb{C} \backslash \Sigma^{\prime}$; (2) $m_{ \pm}^{\sharp}(\zeta):=\lim \underset{\substack{\zeta^{\prime} \in \zeta^{\prime} \rightarrow \zeta \\ \text { side of } \Sigma^{\prime}}}{ } m^{\sharp}\left(\zeta^{\prime}\right)$ satisfy the jump condition $m_{+}^{\sharp}(\zeta)=m_{-}^{\sharp}(\zeta) \mathcal{G}^{\sharp}(\zeta), \zeta \in \Sigma^{\prime}$, where $\mathcal{G}^{\sharp}(\zeta):=\left(\mathrm{I}-w_{-}^{\sharp}(\zeta)\right)^{-1}\left(\mathrm{I}+w_{+}^{\sharp}(\zeta)\right)$, with

$$
\left(\mathrm{I}-w_{-}^{\sharp}(\zeta)\right)^{-1}\left(\mathrm{I}+w_{+}^{\sharp}(\zeta)\right)= \begin{cases}\left(\mathrm{I}-\underline{w}_{-}^{o}(\zeta)\right)\left(\mathrm{I}+\underline{w}_{+}^{o}(\zeta)\right)^{-1}, & \zeta \in \mathbb{R}, \\ \left(\mathrm{I}+\underline{w}_{+}^{a}(\zeta)\right), & \zeta \in \mathrm{L}, \\ \left(\mathrm{I}-\underline{w}_{-}^{a}(\zeta)\right)^{-1}, & \zeta \in \overline{\mathrm{L}},\end{cases}
$$


$\underline{w}_{ \pm}^{o}(\zeta):=\left(\delta_{ \pm}(\zeta)\right)^{\operatorname{ad}\left(\sigma_{3}\right)} \mathrm{e}^{-\mathrm{i} t \theta^{u}(\zeta) \operatorname{ad}\left(\sigma_{3}\right)} w_{ \pm}^{o}(\zeta)$, where $w_{+}^{o}(\zeta)=h_{I}(\zeta) \sigma_{+}$and $w_{-}^{o}(\zeta)=-\overline{h_{I}(\zeta)} \sigma_{-}$, and $\underline{w}_{ \pm}^{a}(\zeta)$ are defined above; (3) as $\zeta \rightarrow \infty, \zeta \in \mathbb{C} \backslash \Sigma^{\prime}, m^{\sharp}(\zeta)=\mathrm{I}+\mathcal{O}\left(\zeta^{-1}\right)$; and (4) $m^{\sharp}(\zeta)$ satisfies the symmetry reduction $m^{\sharp}(\zeta)=\sigma_{1} \overline{m^{\sharp}(\bar{\zeta})} \sigma_{1}$ and the condition $\left(m^{\sharp}(0)(\delta(0))^{\sigma_{3}} \sigma_{2}\right)^{2}=$ I. Furthermore, $\underline{w}_{ \pm}^{o}(\zeta) \in \cap_{p \in\{1,2, \infty\}} \mathcal{L}_{\mathrm{M}_{2}(\mathbb{C})}^{p}(\mathbb{R}), \underline{w}_{+}^{a}(\zeta) \in \cap_{p \in\{1,2, \infty\}} \mathcal{L}_{\mathrm{M}_{2}(\mathbb{C})}^{p}(\mathrm{~L})$, and $\underline{w}_{-}^{a}(\zeta) \in$ $\cap_{p \in\{1,2, \infty\}} \mathcal{L}_{\mathrm{M}_{2}(\mathbb{C})}^{p}(\overline{\mathrm{L}})$.

Proof. From Lemma 4.1, one rewrites the jump matrix, $\widehat{\mathcal{G}}^{c}(\zeta)$, in the BC form, $\widehat{\mathcal{G}}^{c}(\zeta)=$ $\left(\mathrm{I}-\widehat{w}_{-}^{c}(\zeta)\right)^{-1}\left(\mathrm{I}+\widehat{w}_{+}^{c}(\zeta)\right)$, where $\widehat{w}_{ \pm}^{c}(\zeta):=\left(\delta_{ \pm}(\zeta)\right)^{\operatorname{ad}\left(\sigma_{3}\right)} \mathrm{e}^{-\mathrm{i} t \theta^{u}(\zeta) \operatorname{ad}\left(\sigma_{3}\right)} w_{ \pm}^{c}(\zeta)$, with $w_{+}^{c}(\zeta)=\rho(\zeta) \sigma_{+}$ and $w_{-}(\zeta)=-\overline{\rho(\bar{\zeta})} \sigma_{-}$, and $\rho(\zeta)$ as defined in Lemma 4.1. Defining $m^{\sharp}(\zeta)$ as in the Lemma, one arrives, as a consequence of the above BC factorisation for the jump matrix, the RHP for $\widehat{m}^{c}(\zeta)$ formulated in Lemma 4.1, the decomposition $\rho(\zeta)=h_{I}(\zeta)+\left(h_{I I}(\zeta)+\mathcal{R}(\zeta)\right)$, and Lemma 4.2, at the RHP for $m^{\sharp}(\zeta)$.

The second main objective of this section is to reformulate the RHP for $m^{\sharp}(\zeta)$ on $\Sigma^{\prime}$ as an equivalent RHP for $m^{\Sigma^{\sharp}}(\zeta)$ (see Lemma 4.6) on the truncated contour (see Figure 4)

$$
\Sigma^{\sharp}=\Sigma^{\prime} \backslash\left(\mathbb{R} \cup\left(\mathrm{L}_{\varepsilon} \cup \mathrm{L}_{<}\right) \cup \overline{\left(\mathrm{L}_{\varepsilon} \cup \mathrm{L}_{<}\right)}\right):=\Sigma_{A^{\prime}} \cup \Sigma_{B^{\prime}},
$$

with $\Sigma_{A^{\prime}} \cap \Sigma_{B^{\prime}}=\emptyset$. In going from the RHP for $m^{\sharp}(\zeta)$ on $\Sigma^{\prime}$ to the RHP for $m^{\Sigma^{\sharp}}(\zeta)$ on $\Sigma^{\sharp}$,
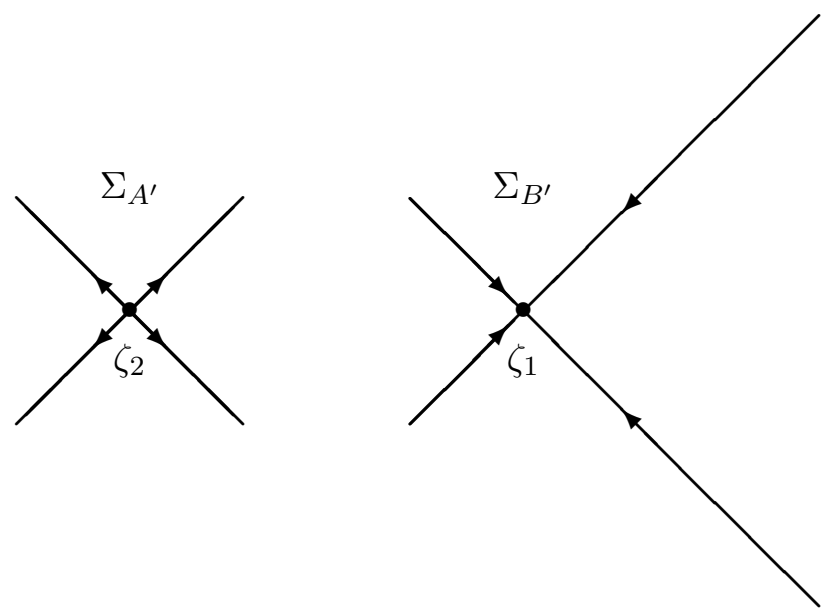

Figure 4: Truncated contour $\Sigma^{\sharp}:=\Sigma_{A^{\prime}} \cup \Sigma_{B^{\prime}}$

the error incurred (as a result of the truncation of the integration contour) will be estimated explicitly, and shown to be, as $t \rightarrow+\infty, \mathcal{O}\left(\frac{\underline{c}\left(\zeta_{1}, \zeta_{2}, \zeta_{3}, \overline{\zeta_{3}}\right) \diamond(\zeta)}{\left|z_{o}+\zeta_{1}+\zeta_{2}\right|^{l} t^{l}}\right), l \in \mathbb{Z}_{\geqslant 1}$ and arbitrarily large, and $\diamond(\zeta) \in \mathcal{L}_{\mathrm{M}_{2}(\mathbb{C})}^{\infty}\left(\mathbb{C} \backslash \Sigma^{\sharp}\right)$. In the course of these estimations, it will be shown that the (asymptotic) contributions of the functions $h_{I}(\zeta): \mathbb{R} \rightarrow \mathbb{C}$ (respectively $\overline{h_{I}(\zeta)}: \mathbb{R} \rightarrow \mathbb{C}$ ) and $h_{I I}(\zeta): \mathrm{L} \rightarrow \mathbb{C}$ (respectively $\overline{h_{I I}(\bar{\zeta})}: \overline{\mathrm{L}} \rightarrow \mathbb{C}$ ) are "negligibly small" (see Lemma 4.4), and the contribution to the leading order asymptotics of the solution of the RHP for $m^{\sharp}(\zeta)$ coming from $\mathbb{R}, \mathrm{L}_{\varepsilon} \cup \overline{\mathrm{L}_{\varepsilon}}$, and $\mathrm{L}_{<} \cup \overline{\mathrm{L}_{<}}$are negligible (see Lemma 4.4). Using Lemma 3.1, the solution of the RHP for $m^{\sharp}(\zeta)$ on $\Sigma^{\prime}$ has the following integral representation,

$$
m^{\sharp}(\zeta)=\mathrm{I}+\int_{\Sigma^{\prime}} \frac{\mu^{\sharp}(z) w^{\sharp}(z)}{(z-\zeta)} \frac{\mathrm{d} z}{2 \pi \mathrm{i}}, \quad \zeta \in \mathbb{C} \backslash \Sigma^{\prime},
$$

where $\mu^{\sharp}(\zeta):=\left(\left(\mathbf{1}-C_{w^{\sharp}}\right)^{-1} \mathrm{I}\right)(\zeta), C_{w^{\sharp}} \star:=C_{+}\left(\star w_{-}^{\sharp}\right)+C_{-}\left(\star w_{+}^{\sharp}\right), \star \in \mathcal{L}_{\mathrm{M}_{2}(\mathbb{C})}^{2}\left(\Sigma^{\prime}\right),\left(C_{ \pm} \star\right)(\zeta):=$ $\lim \underset{\substack{\zeta^{\prime} \rightarrow \zeta \\ \zeta^{\prime} \in \pm \text { side of } \Sigma^{\prime}}}{ } \int_{\Sigma^{\prime}} \frac{\star(z)}{z-\zeta} \frac{\mathrm{d} z}{2 \pi \mathrm{i}}$, with $w_{ \pm}^{\sharp}(\zeta)$ defined in Lemma 4.3, and $w^{\sharp}(\zeta):=\sum_{l \in\{ \pm\}} w_{l}^{\sharp}(\zeta)$. 
One notes from Lemma 4.3 that: (1) for $\zeta \in \mathbb{R}, w^{\sharp}(\zeta)=w_{-}^{\sharp}(\zeta)+w_{+}^{\sharp}(\zeta)=\underline{w}_{-}^{o}(\zeta)+\underline{w}_{+}^{o}(\zeta)=$

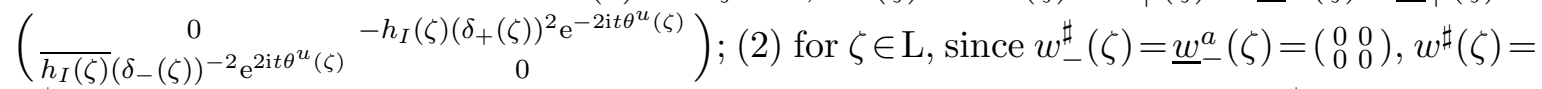
$w_{+}^{\sharp}(\zeta)=\underline{w}_{+}^{a}(\zeta)=\left(h_{I I}(\zeta)+\mathcal{R}(\zeta)\right)(\delta(\zeta))^{2} \mathrm{e}^{-2 \mathrm{i} t \theta^{u}(\zeta)} \sigma_{+}$; and $(3)$ for $\zeta \in \overline{\mathrm{L}}$, since $w_{+}^{\sharp}(\zeta)=\underline{w}_{+}^{a}(\zeta)=$ $\left(\begin{array}{ll}0 & 0 \\ 0 & 0\end{array}\right), w^{\sharp}(\zeta)=w_{-}^{\sharp}(\zeta)=\underline{w}_{-}^{a}(\zeta)=-\left(\overline{h_{I I}(\bar{\zeta})}+\overline{\mathcal{R}(\bar{\zeta})}\right)(\delta(\zeta))^{-2} \mathrm{e}^{2 \mathrm{i} t \theta^{u}(\zeta)} \sigma_{-}$. To carry out the second main objective of this section, and guided by the latter expressions, one decomposes $w^{\sharp}(\zeta)=$ $\sum_{l \in\{ \pm\}} w_{l}^{\sharp}(\zeta)$ as

$$
w^{\sharp}(\zeta)=w^{e}(\zeta)+w^{\prime}(\zeta), \quad w^{e}(\zeta):=w^{\widehat{a}}(\zeta)+w^{b}(\zeta)+w^{c}(\zeta),
$$

where: $(1) w^{\widehat{a}}(\zeta):=\left.w^{\sharp}(\zeta)\right|_{\mathbb{R}}$ has support on $\mathbb{R}$, and consists of the contribution to $w^{\sharp}(\zeta)$ from $h_{I}(\zeta)$ and $\overline{h_{I}(\zeta)} ;(2) w^{b}(\zeta)$ has support on $\mathrm{L} \cup \overline{\mathrm{L}}$, and consists of the contribution to $w^{\sharp}(\zeta)$ from $\left.h_{I I}(\zeta)\right|_{\mathrm{L}}$ and $\overline{h_{I I}(\bar{\zeta}) \Gamma_{\overline{\mathrm{L}}}} ;(3) w^{c}(\zeta)$ has support on $\mathrm{L}_{\varepsilon} \cup \overline{\mathrm{L}_{\varepsilon}}$, and consists of the contribution to $w^{\sharp}(\zeta)$ from $\mathcal{R}(\zeta)\left\lceil_{\mathrm{L}_{\varepsilon}} \text { and } \overline{\mathcal{R}(\bar{\zeta})}\right\rceil_{\overline{\mathrm{L}_{\varepsilon}}}$; and $\left.(4) w^{\prime}(\zeta)\right|_{\Sigma^{\prime} \backslash \Sigma^{\sharp}} \equiv\left(\begin{array}{ll}0 & 0 \\ 0 & 0\end{array}\right)$. It will now be shown that, as $t \rightarrow+\infty, w^{e}(\zeta) \rightarrow\left(\begin{array}{ll}0 & 0 \\ 0 & 0\end{array}\right)$ (see Lemma 4.4), and the contribution to $w^{\sharp}(\zeta)$ from $\mathcal{R}(\zeta)$ and $\overline{\mathcal{R}(\bar{\zeta})}$ (in some regions, polynomials of degree $k \in \mathbb{Z}_{\geqslant 1}$, and in other regions, rational functions of the type $\left.\frac{\text { polynomial of degree } k \in \mathbb{Z}_{\geqslant 1}}{|\zeta \mp i|^{k+5}}\right)$, which is lumped into the factor $w^{\prime}(\zeta)$, and has support on $\Sigma^{\sharp}$, encapsulates the leading-order asymptotics.

Lemma 4.4. For arbitrarily large $l \in \mathbb{Z}_{\geqslant 1}$, and arbitrarily fixed, sufficiently small positive $\varepsilon$, as $t \rightarrow+\infty$ such that $0<\zeta_{2}<\frac{1}{M}<M<\zeta_{1}$ and $\left|\zeta_{3}\right|^{2}=1$, with $M \in \mathbb{R}_{>1}$ and bounded,

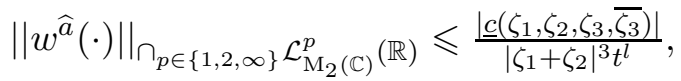

$$
\begin{aligned}
& \| w^{\widehat{a}}(\cdot) /(\cdot)||_{\cap_{p \in\{1,2, \infty\}} \mathcal{L}_{\mathrm{M}_{2}(\mathbb{C})}^{p}\left(\left(\cos \left(\widetilde{\varphi}_{3}\right), 0\right) \cup\left(0, \frac{1}{2} \zeta_{2}\right)\right)} \leqslant \frac{\underline{\left|c\left(\zeta_{1}, \zeta_{2}, \zeta_{3}, \overline{\zeta_{3}}\right)\right|}}{\left|\zeta_{1}+\zeta_{2}\right|^{3} t^{l}}, \\
& \left\|w^{b}(\cdot)\right\|_{\cap_{p \in\{1,2, \infty\}} \mathcal{L}_{\mathrm{M}_{2}(\mathbb{C})}^{p}\left(Q_{0} \cup \overline{Q_{0}}\right)} \leqslant \frac{\mid \underline{\underline{c}\left(\zeta_{1}, \zeta_{2}, \zeta_{3}, \overline{\zeta_{3}}\right) \mid}}{\left|z_{o}+\zeta_{1}+\zeta_{2}\right|^{l} t^{l}}, \\
& \left\|w^{b}(\cdot)\right\|_{\cap_{p \in\{1,2, \infty\}} \mathcal{L}_{\mathrm{M}_{2}(\mathbb{C})}^{p}\left(Q_{1} \cup \overline{Q_{1}}\right)} \leqslant\left|\underline{c}\left(\zeta_{1}, \zeta_{2}, \zeta_{3}, \overline{\zeta_{3}}\right)\right| \exp \left(-2 \gamma_{\mathcal{R}}^{1} t\right), \\
& \left\|w^{b}(\cdot)\right\|_{\cap_{p \in\{1,2, \infty\}} \mathcal{L}_{\mathrm{M}_{2}(\mathbb{C})}^{p}\left(Q_{2} \cup \overline{Q_{2}}\right)} \leqslant \frac{\left|\underline{c}\left(\zeta_{1}, \zeta_{2}, \zeta_{3}, \overline{\zeta_{3}}\right)\right|}{\sqrt{\omega_{1}} \sqrt{t}} \exp \left(-\omega_{0} t\right), \\
& \left\|w^{b}(\cdot) /(\cdot)\right\|_{\cap_{p \in\{1,2, \infty\}}} \mathcal{L}_{\mathrm{M}_{2}(\mathbb{C})}^{p}\left(Q_{1} \cup \overline{Q_{1}}\right) \leqslant\left|\underline{c}\left(\zeta_{1}, \zeta_{2}, \zeta_{3}, \overline{\zeta_{3}}\right)\right| \exp \left(-\gamma_{I I}^{1} t\right), \\
& \|\left. w^{c}(\cdot)\right|_{\cap_{p \in\{1,2, \infty\}} \mathcal{L}_{\mathrm{M}_{2}(\mathbb{C})}^{p}\left(Q_{3} \cup \overline{Q_{3}}\right)} \leqslant\left|\underline{c}\left(\zeta_{1}, \zeta_{2}, \zeta_{3}, \overline{\zeta_{3}}\right)\right| \exp \left(-\varepsilon^{2} \gamma_{\mathcal{R}}^{0} t\right), \\
& \|\left. w^{c}(\cdot)\right|_{\cap_{p \in\{1,2, \infty\}} \mathcal{L}_{\mathrm{M}_{2}(\mathbb{C})}^{p}\left(\mathrm{~L}_{>} \cup \overline{\mathrm{L}>}\right)} \leqslant\left|\underline{c}\left(\zeta_{1}, \zeta_{2}, \zeta_{3}, \overline{\zeta_{3}}\right)\right| \exp \left(-\zeta_{1}\left|z_{o}\right|\left(2-\zeta_{2}^{2}\right) t\right), \\
& || w^{c}(\cdot) /(\cdot)||_{\cap_{p \in\{1,2, \infty\}} \mathcal{L}_{\mathrm{M}_{2}(\mathbb{C})}^{p}\left(\mathrm{~L}_{>} \cup \overline{\mathrm{L}_{>}}\right)} \leqslant\left|\underline{c}\left(\zeta_{1}, \zeta_{2}, \zeta_{3}, \overline{\zeta_{3}}\right)\right| \exp \left(-\zeta_{1}\left|z_{o}\right|\left(2-\zeta_{2}^{2}\right) t\right), \\
& \left\|w^{\prime}(\cdot)\right\|_{\mathcal{L}_{\mathrm{M}_{2}(\mathbb{C})}^{1}\left(\Sigma^{\prime}\right)}=\left\|w^{\prime}(\cdot)\right\|_{\mathcal{L}_{\mathrm{M}_{2}(\mathbb{C})}^{1}\left(\Sigma^{\sharp}\right)} \leqslant \frac{\left|\underline{c}\left(\zeta_{1}, \zeta_{2}, \zeta_{3}, \overline{\zeta_{3}}\right)\right|}{\sqrt{\left(\zeta_{1}-\zeta_{2}\right)\left|z_{o}+\zeta_{1}+\zeta_{2}\right|} \sqrt{t}}, \\
& \left\|w^{\prime}(\cdot)\right\|_{\mathcal{L}_{\mathrm{M}_{2}(\mathbb{C})}^{2}\left(\Sigma^{\prime}\right)}=\left\|w^{\prime}(\cdot)\right\|_{\mathcal{L}_{\mathrm{M}_{2}(\mathbb{C})}^{2}\left(\Sigma^{\sharp}\right)} \leqslant \frac{\left|\underline{c}\left(\zeta_{1}, \zeta_{2}, \zeta_{3}, \overline{\zeta_{3}}\right)\right|}{\left(\left(\zeta_{1}-\zeta_{2}\right)\left|z_{o}+\zeta_{1}+\zeta_{2}\right|\right)^{1 / 4} t^{1 / 4}},
\end{aligned}
$$

where $Q_{0}:=\mathrm{L} \backslash\left(\mathrm{L}_{>} \cup \mathrm{L}_{<}\right), Q_{1}:=\mathrm{L}_{>} \cup\left\{\zeta ; \zeta=v \mathrm{e}^{\mathrm{i} \widetilde{\varphi}_{3}}, \widetilde{\varphi}_{3}:=\arg \left(\zeta_{3}\right) \in\left(\frac{\pi}{2}, \pi\right), v \in(0,1-\varepsilon)\right\}$, $Q_{2}:=\left\{\zeta ; \zeta=v \mathrm{e}^{\mathrm{i} \widetilde{\varphi}_{3}}, \widetilde{\varphi}_{3}:=\arg \left(\zeta_{3}\right) \in\left(\frac{\pi}{2}, \pi\right), v \geqslant 1-\varepsilon\right\}, Q_{3}:=\mathrm{L}_{\varepsilon} \backslash \mathrm{L}_{>}, \omega_{0}:=\frac{1}{2}\left(a_{2}-z_{o}\right)\left(4-a_{2}^{2}\right)^{1 / 2}$ $\left(\in \mathbb{R}_{+}\right)$, and $\omega_{1}:=\frac{1}{2}\left(z_{o}^{2}+32\right)^{1 / 2}\left(4-a_{2}^{2}\right)^{1 / 2}\left(\in \mathbb{R}_{+}\right)$, with $\mathrm{L}, \mathrm{L}_{>}, \mathrm{L}_{<}, \mathrm{L}_{\varepsilon}, \gamma_{\mathcal{R}}^{1}, \gamma_{I I}^{1}$, and $\gamma_{\mathcal{R}}^{0}$ defined in Lemma 4.2.

Proof. Without loss of generality, the bounds for $\left\|w^{b}(\cdot)\right\|_{\mathcal{L}_{\mathrm{M}_{2}(\mathbb{C})}^{p}(*)}$ and $\left\|w^{b}(\cdot) /(\cdot)\right\|_{\mathcal{L}_{\mathrm{M}_{2}(\mathbb{C})}^{p}(*)}$, $p \in\{1,2, \infty\}$, and $\left\|w^{\prime}(\cdot)\right\|_{\mathcal{L}_{\mathrm{M}_{2}(\mathbb{C})}^{p^{\prime}}(*)}, p^{\prime} \in\{1,2\}$, will be derived: the remaining estimates follow 
in an analogous manner. From the proof of Lemma 4.2 and the parametrisations of the respective rays given therein, one shows that, modulo a scalar factor of 2 on the right-hand side (RHS),

$$
\begin{aligned}
\left\|w^{b}(\cdot)\right\|_{\mathcal{L}_{\mathrm{M}_{2}(\mathbb{C})}^{1}\left(Q_{0} \cup \overline{Q_{0}}\right)} & \leqslant \int_{0}^{+\infty} \frac{\left|\underline{c}\left(\zeta_{1}, \zeta_{2}, \zeta_{3}, \overline{\zeta_{3}}\right)\right| v^{q}}{\left|\zeta_{1}+\frac{v}{\sqrt{2}}\left(\zeta_{1}-\zeta_{2}\right) \mathrm{e}^{-\frac{\mathrm{i} \pi}{4}}-\mathrm{i}\right|^{2}} \mathrm{e}^{-\frac{1}{4} t \gamma_{o}\left(\zeta_{1}-\zeta_{2}\right)\left(1-\zeta_{2}^{2}\right)^{2}\left|z_{o}+\zeta_{1}-\zeta_{2}\right| v^{2}} \mathrm{~d} v \\
& +\int_{0}^{1}\left|\underline{c}\left(\zeta_{1}, \zeta_{2}, \zeta_{3}, \overline{\zeta_{3}}\right)\right| v^{q} \mathrm{e}^{-\frac{1}{4} t\left(\zeta_{1}-\zeta_{2}\right)\left(1-\frac{2 \zeta_{1}^{2}}{\zeta_{1}^{4}+1}\right)^{2}\left|z_{o}+\zeta_{1}+\zeta_{2}\right| v^{2}} \mathrm{~d} v \\
& +\int_{0}^{1}\left|\underline{c}\left(\zeta_{1}, \zeta_{2}, \zeta_{3}, \overline{\zeta_{3}}\right)\right| v^{q} \mathrm{e}^{-\frac{1}{4} t\left(\zeta_{1}-\zeta_{2}\right)\left(1-\zeta_{1}^{2}\right)^{2}\left|z_{o}+\zeta_{1}+\zeta_{2}\right| v^{2}} \mathrm{~d} v \\
& +\int_{0}^{1}\left|\underline{c}\left(\zeta_{1}, \zeta_{2}, \zeta_{3}, \overline{\zeta_{3}}\right)\right| v^{q} \mathrm{e}^{-\frac{1}{4} t \zeta_{2}\left(1-2 \zeta_{1}^{2}\right)^{2}\left|z_{o}+\zeta_{2}\right| v^{2}} \mathrm{~d} v,
\end{aligned}
$$

$q \in \mathbb{Z}_{\geqslant 1}$, where $Q_{0}$ is defined in the Lemma. Since, $\forall v \geqslant 0,\left|\zeta_{1}+\frac{v}{\sqrt{2}}\left(\zeta_{1}-\zeta_{2}\right) \mathrm{e}^{-\frac{\mathrm{i} \pi}{4}}-\mathrm{i}\right|^{-2} \leqslant 1$, and the integrands are positive functions of $v$, it follows, by a change-of-variable argument and letting the upper limits of integration tend to $+\infty$, that $\left\|w^{b}(\cdot)\right\|_{\mathcal{L}_{\mathrm{M}_{2}(\mathbb{C})}^{1}\left(Q_{0} \cup \overline{Q_{0}}\right)} \leqslant$ $\left(\frac{\left|\underline{c}\left(\zeta_{1}, \zeta_{2}, \zeta_{3}, \overline{\zeta_{3}}\right)\right| t^{-(q / 2+1 / 2)}}{\left|z_{o}+\zeta_{1}-\zeta_{2}\right|^{q / 2+1 / 2}}+\frac{\mid \underline{\underline{c}\left(\zeta_{1}, \zeta_{2}, \zeta_{3}, \overline{\zeta_{3}}\right) \mid t^{-(q / 2+1 / 2)}}}{\left|z_{o}+\zeta_{1}+\zeta_{2}\right|^{q / 2+1 / 2}}+\frac{\left|\underline{c}\left(\zeta_{1}, \zeta_{2}, \zeta_{3}, \overline{\zeta_{3}}\right)\right| t^{-(q / 2+1 / 2)}}{\left|z_{o}+\zeta_{2}\right|^{q / 2+1 / 2}}\right) \int_{0}^{+\infty} \mathrm{e}^{-\xi} \xi^{\frac{q-1}{2}} \mathrm{~d} \xi$; hence, recalling that 40] $\Gamma(z)=\int_{0}^{+\infty} \mathrm{e}^{-x} x^{z-1} \mathrm{~d} x, \Re(z)>0$, where $\Gamma(\cdot)$ is the gamma function, and $\int_{0}^{+\infty} \mathrm{e}^{-x^{2}} \mathrm{~d} x=\frac{\sqrt{\pi}}{2}$, for $\frac{q}{2}>l, l \in \mathbb{Z}_{\geqslant 1}$ and arbitrarily large, $\left\|w^{b}(\cdot)\right\|_{\mathcal{L}_{\mathrm{M}_{2}(\mathbb{C})}^{1}\left(Q_{0} \cup \overline{Q_{0}}\right)} \leqslant \frac{\left|c\left(\zeta_{1}, \zeta_{2}, \zeta_{3}, \overline{\zeta_{3}}\right)\right|}{\left.\left|z_{o}+\zeta_{1}+\zeta_{2}\right|\right|^{l} t^{l}}$. From the estimates given in Lemma 4.2, it follows that, for $\frac{q}{2}>l,\left\|w^{b}(\cdot)\right\|_{\mathcal{L}_{\mathrm{M}_{2}(\mathbb{C})}^{\infty}\left(Q_{0} \cup \overline{Q_{0}}\right)} \leqslant$

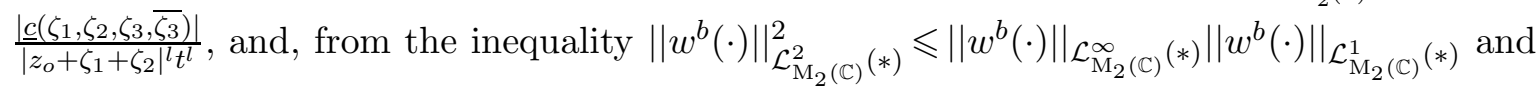
the latter two estimates, one shows that, for $\frac{q}{2}>l, \|\left. w^{b}(\cdot)\right|_{\mathcal{L}_{\mathrm{M}_{2}(\mathbb{C})}^{2}\left(Q_{0} \cup \overline{Q_{0}}\right)} \leqslant \frac{\left|\underline{c}\left(\zeta_{1}, \zeta_{2}, \zeta_{3}, \overline{\zeta_{3}}\right)\right|}{\left|z_{o}+\zeta_{1}+\zeta_{2}\right|^{l} t^{l}}$; hence, recalling that $\left\|w^{b}(\cdot)\right\|_{\cap_{p \in\{1,2, \infty\}} \mathcal{L}_{\mathrm{M}_{2}(\mathbb{C})}^{p}\left(Q_{0} \cup \overline{Q_{0}}\right)}:=\sum_{p \in\{1,2, \infty\}}\left\|w^{b}(\cdot)\right\|_{\mathcal{L}_{\mathrm{M}_{2}(\mathbb{C})}^{p}\left(Q_{0} \cup \overline{Q_{0}}\right)}$, one obtains, for $\frac{q}{2}>l$ and the latter estimates, the result for $\left\|w^{b}(\cdot)\right\|_{\cap_{p \in\{1,2, \infty\}} \mathcal{L}_{\mathrm{M}_{2}(\mathbb{C})}^{p}\left(Q_{0} \cup \overline{Q_{0}}\right)}$ stated in the Lemma. From the proof of Lemma 4.2, one shows that, modulo a scalar factor of 2 on the RHS, $\| w^{b}(\cdot)||_{\mathcal{L}_{\mathrm{M}_{2}(\mathbb{C})}^{1}\left(Q_{1} \cup \overline{Q_{1}}\right)} \leqslant \int_{0}^{1} \frac{\underline{\underline{c}\left(\zeta_{1}, \zeta_{2}, \zeta_{3}, \overline{\zeta_{3}}\right) \mid}}{\xi^{2}+1} \exp \left(-\frac{1}{2} t\left|z_{o}\right| \zeta_{1}\left(2-\zeta_{2}^{2}\right)\right) \mathrm{d} \xi+\int_{0}^{1-\varepsilon} \frac{\underline{\underline{c}\left(\zeta_{1}, \zeta_{2}, \zeta_{3}, \overline{\zeta_{3}}\right) \mid}}{\xi^{2}+1} \exp \left(-t\left(\frac{1}{2}\left|z_{o}\right|\right.\right.$ $\left.\left.-\cos \widetilde{\varphi}_{3}\right) \sin \widetilde{\varphi}_{3}\right) \mathrm{d} \xi$, with $Q_{1}$ as defined in the Lemma: noting that the integrands are positive functions of $\xi$, letting the upper limits of integration tend to $+\infty$, and using the fact that, with the principal branch of $\arctan (\cdot) \operatorname{chosen}, \int_{0}^{+\infty} \frac{\mathrm{d} \xi}{\xi^{2}+1}=\pi / 2$, one shows that $\left\|w^{b}(\cdot)\right\|_{\mathcal{L}_{\mathrm{M}_{2}(\mathbb{C})}^{1}\left(Q_{1} \cup \overline{Q_{1}}\right)} \leqslant\left|\underline{c}\left(\zeta_{1}, \zeta_{2}, \zeta_{3}, \overline{\zeta_{3}}\right)\right| \mathrm{e}^{-2 \gamma_{\mathcal{R}}^{1} t}$, with $\gamma_{\mathcal{R}}^{1}$ defined in Lemma 4.2. The estimate $\left\|w^{b}(\cdot)\right\|_{\mathcal{L}_{\mathrm{M}_{2}(\mathbb{C})}^{\infty}\left(Q_{1} \cup \overline{Q_{1}}\right)} \leqslant\left|\underline{c}\left(\zeta_{1}, \zeta_{2}, \zeta_{3}, \overline{\zeta_{3}}\right)\right| \mathrm{e}^{-2 \gamma_{\mathcal{R}}^{1} t}$ is a consequence of Lemma 4.2: using the latter two estimates and the inequality $\left\|w^{b}(\cdot)\right\|_{\mathcal{L}_{\mathrm{M}_{2}(\mathbb{C})}^{2}(*)}^{2} \leqslant\left\|w^{b}(\cdot)\right\|_{\mathcal{L}_{\mathrm{M}_{2}(\mathbb{C})}^{\infty}(*)}\left\|w^{b}(\cdot)\right\|_{\mathcal{L}_{\mathrm{M}_{2}(\mathbb{C})}^{1}(*)}$, one deduces that $\|\left. w^{b}(\cdot)\right|_{\mathcal{L}_{\mathrm{M}_{2}(\mathbb{C})}\left(Q_{1} \cup \overline{Q_{1}}\right)} \leqslant\left|\underline{c}\left(\zeta_{1}, \zeta_{2}, \zeta_{3}, \overline{\zeta_{3}}\right)\right| \mathrm{e}^{-2 \gamma_{\mathcal{R}}^{1} t}$; hence, one arrives at the estimate for $\left\|w^{b}(\cdot)\right\|_{\cap_{p \in\{1,2, \infty\}} \mathcal{L}_{\mathrm{M}_{2}(\mathbb{C})}^{p}\left(Q_{1} \cup \overline{Q_{1}}\right)}$ stated in the Lemma. From the proof of Lemma 4.2, one shows that, modulo a scalar factor of 2 on the RHS, $\left\|w^{b}(\cdot)\right\|_{\mathcal{L}_{\mathrm{M}_{2}(\mathbb{C})}^{1}\left(Q_{2} \cup \overline{Q_{2}}\right)} \leqslant \int_{1-\varepsilon}^{+\infty} \frac{\mid \underline{\underline{c}\left(\zeta_{1}, \zeta_{2}, \zeta_{3}, \overline{\zeta_{3}}\right) \mid}}{(v+1)^{2}+1} \exp (-t$ $\left.\cdot\left(\omega_{0}+\frac{1}{2} \omega_{1} v^{2}\right)\right) \mathrm{d} v \leqslant \frac{\underline{\mid c}\left(\zeta_{1}, \zeta_{2}, \overline{\zeta_{3}}, \overline{\zeta_{3}}\right) \mid}{\sqrt{\omega_{1} t}} \mathrm{e}^{-\omega_{0} t} \int_{0}^{+\infty} \mathrm{e}^{-\xi^{2}} \mathrm{~d} \xi \leqslant \frac{\mid \underline{\underline{c}\left(\zeta_{1}, \zeta_{2}, \zeta_{3}, \overline{\zeta_{3}}\right) \mid}}{\sqrt{\omega_{1} t}} \mathrm{e}^{-\omega_{0} t}$, with $Q_{2}, \omega_{0}$, and $\omega_{1}$ as defined in the Lemma. The estimate $\|\left. w^{b}(\cdot)\right|_{\mathcal{L}_{\mathrm{M}_{2}(\mathbb{C})}^{\infty}\left(Q_{2} \cup \overline{Q_{2}}\right)} \leqslant \frac{\mid \underline{\underline{c}\left(\zeta_{1}, \zeta_{2}, \zeta_{3}, \overline{\zeta_{3}}\right) \mid}}{\sqrt{\omega_{1} t}} \mathrm{e}^{-\omega_{0} t}$ follows from Lemma 4.2: using the inequality $\left\|w^{b}(\cdot)\right\|_{\mathcal{L}_{\mathrm{M}_{2}(\mathbb{C})}^{2}(*)}^{2} \leqslant\left\|w^{b}(\cdot)\right\|_{\mathcal{L}_{\mathrm{M}_{2}(\mathbb{C})}^{\infty}(*)}\left\|w^{b}(\cdot)\right\|_{\mathcal{L}_{\mathrm{M}_{2}(\mathbb{C})}^{1}(*)}$ and the latter two estimates, one deduces that $\|\left. w^{b}(\cdot)\right|_{\mathcal{L}_{\mathrm{M}_{2}(\mathbb{C})}^{2}\left(Q_{2} \cup \overline{Q_{2}}\right)} \leqslant \frac{\mid \underline{\underline{c}\left(\zeta_{1}, \zeta_{2}, \zeta_{3}, \overline{\zeta_{3}}\right) \mid}}{\sqrt{\omega_{1} t}} \mathrm{e}^{-\omega_{0} t}$; hence, one arrives at the estimate for $\left\|w^{b}(\cdot)\right\|_{\cap_{p \in\{1,2, \infty\}} \mathcal{L}_{\mathrm{M}_{2}(\mathbb{C})}^{p}\left(Q_{2} \cup \overline{Q_{2}}\right)}$ stated in the Lemma. From the proof of 
Lemma 4.2, one shows that, modulo a scalar factor of 2 on the RHS, $\left\|w^{b}(\cdot) /(\cdot)\right\|_{\mathcal{L}_{\mathrm{M}_{2}(\mathbb{C})}^{1}\left(Q_{1} \cup \overline{Q_{1}}\right)} \leqslant$ $\int_{0}^{1} \frac{\left.\underline{\underline{c}\left(\zeta_{1}, \zeta_{2}, \zeta_{3}, \overline{\zeta_{3}}\right.}\right) \mid v^{-3 q}}{\left|\zeta_{2} \frac{v}{\sqrt{2}} \mathrm{e}^{-\frac{i \pi}{4}}\right|} \exp \left(-\frac{1}{2} t\left|z_{o}\right| \zeta_{1}\left(2-\zeta_{2}^{2}\right)\right) \exp \left(-\frac{t}{v^{2}} \zeta_{1}^{2}\left(1-\frac{1}{2} \zeta_{2}^{2}\right)^{2}\right) \mathrm{d}\left(\frac{\zeta_{2} v}{\sqrt{2}}\right)+\int_{0}^{1-\varepsilon} \frac{\left|\underline{c}\left(\zeta_{1}, \zeta_{2}, \zeta_{3}, \overline{\zeta_{3}}\right)\right|}{\left(\left|\mathrm{e}^{i \varphi_{3}} v\right|^{2}+1\right) v} \exp (-$ $\left.\frac{t\left|z_{o}\right|}{2 v^{2}} \sin \widetilde{\varphi}_{3}\right) \exp \left(-\frac{t}{2}\left|\sin 2 \widetilde{\varphi}_{3}\right|\right) \leqslant \frac{\left|\underline{c}\left(\zeta_{1}, \zeta_{2}, \zeta_{3}, \overline{\zeta_{3}}\right)\right|}{\zeta_{1}^{3 q}\left(1-\frac{1}{2} \zeta_{2}^{2}\right)^{3 q} t^{3 q}} \exp \left(-\frac{1}{2} t\left|z_{o}\right| \zeta_{1}\left(2-\zeta_{2}^{2}\right)\right) \int_{0}^{+\infty} \mathrm{e}^{-\xi} \xi^{\frac{3 q}{2}-1} \mathrm{~d} \xi+\mid \underline{c}\left(\zeta_{1}, \zeta_{2}\right.$, $\left.\zeta_{3}, \overline{\zeta_{3}}\right)\left|\mathrm{e}^{-\frac{1}{2} t\left|\sin 2 \widetilde{\varphi}_{3}\right|} \int_{0}^{+\infty} \mathrm{e}^{-\xi} \xi^{-1} \mathrm{~d} \xi \leqslant\right| \underline{c}\left(\zeta_{1}, \zeta_{2}, \zeta_{3}, \overline{\zeta_{3}}\right) \mid \mathrm{e}^{-\gamma_{I I}^{1} t}, q \in \mathbb{Z}_{\geqslant 1}$, with $\gamma_{I I}^{1}$ defined in Lemma 4.2. Also, $\| w^{b}(\cdot) /\left.(\cdot)\right|_{\mathcal{L}_{\mathrm{M}_{2}(\mathbb{C})}^{\infty}\left(Q_{1} \cup \overline{Q_{1}}\right)} \leqslant\left|\underline{c}\left(\zeta_{1}, \zeta_{2}, \zeta_{3}, \overline{\zeta_{3}}\right)\right| \mathrm{e}^{-\gamma_{I I}^{1} t}$, and, from the latter two estimates and the inequality $\left\|w^{b}(\cdot) /(\cdot)\right\|_{\mathcal{L}_{\mathrm{M}_{2}(\mathbb{C})}^{2}(*)}^{2} \leqslant\left\|w^{b}(\cdot) /(\cdot)\right\|_{\mathcal{L}_{\mathrm{M}_{2}(\mathbb{C})}^{\infty}(*)}\left\|w^{b}(\cdot) /(\cdot)\right\|_{\mathcal{L}_{\mathrm{M}_{2}(\mathbb{C})}^{1}(*)}$, one deduces that $\left\|w^{b}(\cdot) /(\cdot)\right\|_{\mathcal{L}_{\mathrm{M}_{2}(\mathbb{C})}^{2}\left(Q_{1} \cup \overline{Q_{1}}\right)} \leqslant\left|\underline{c}\left(\zeta_{1}, \zeta_{2}, \zeta_{3}, \overline{\zeta_{3}}\right)\right| \mathrm{e}^{-\gamma_{I I}^{1} t}$; hence, one arrives at the estimate for $\left\|w^{b}(\cdot) /(\cdot)\right\|_{\cap_{p \in\{1,2, \infty\}} \mathcal{L}_{\mathrm{M}_{2}(\mathbb{C})}^{p}\left(Q_{1} \cup \overline{Q_{1}}\right)}$ stated in the Lemma. From Eq. (102), the fact that $\left.w^{\prime}(\zeta)\right|_{\Sigma^{\prime} \backslash \Sigma^{\sharp}}=\left(\begin{array}{ll}0 & 0 \\ 0 & 0\end{array}\right)$, and the proof of Lemma 4.2, one shows that $\left\|w^{\prime}(\cdot)\right\|_{\mathcal{L}_{\mathrm{M}_{2}(\mathbb{C})}^{1}\left(\Sigma^{\prime}\right)}=$ $\left\|w^{\prime}(\cdot)\right\|_{\mathcal{L}_{\mathrm{M}_{2}(\mathbb{C})}^{1}\left(\Sigma^{\prime} \backslash \Sigma^{\sharp} \cup \Sigma^{\sharp}\right)}=\left\|w^{\prime}(\cdot)\right\|_{\mathcal{L}_{\mathrm{M}_{2}(\mathbb{C})}^{1}\left(\Sigma^{\prime} \backslash \Sigma^{\sharp}\right)}+\left\|w^{\prime}(\cdot)\right\|_{\mathcal{L}_{\mathrm{M}_{2}(\mathbb{C})}^{1}\left(\Sigma^{\sharp}\right)}=\left\|w^{\prime}(\cdot)\right\|_{\mathcal{L}_{\mathrm{M}_{2}(\mathbb{C})}^{1}\left(\Sigma^{\sharp}\right)} \leqslant \int_{0}^{+\infty} 2^{-\frac{1}{2}}$ $\cdot\left|\underline{c}\left(\zeta_{1}, \zeta_{2}, \zeta_{3}, \overline{\zeta_{3}}\right)\right|\left(\zeta_{1}-\zeta_{2}\right) \exp \left(-\frac{1}{2} t\left(\zeta_{1}-\zeta_{2}\right)\left(1-\frac{2 \zeta_{1}^{2}}{\zeta_{1}^{4}+1}\right)^{2}\left|z_{0}+\zeta_{1}+\zeta_{2}\right| v^{2}\right) \mathrm{d} v+\int_{0}^{+\infty} 2^{-\frac{1}{2}}\left|\underline{c}\left(\zeta_{1}, \zeta_{2}, \zeta_{3}, \overline{\zeta_{3}}\right)\right|\left(\zeta_{1}-\right.$ $\left.\zeta_{2}\right) \exp \left(-\frac{t}{2} \gamma_{o}\left(\zeta_{1}-\zeta_{2}\right)\left(1-\zeta_{2}^{2}\right)^{2}\left|z_{o}+\zeta_{1}+\zeta_{2}\right| v^{2}\right) \mathrm{d} v \leqslant\left(\left|\underline{c}\left(\zeta_{1}, \zeta_{2}, \zeta_{3}, \overline{\zeta_{3}}\right)\right|\left(\zeta_{1}-\zeta_{2}\right) /\left(\sqrt{t\left(\zeta_{1}-\zeta_{2}\right)\left|z_{o}+\zeta_{1}+\zeta_{2}\right|}\right.\right.$ $\left.\left.\cdot\left(1-\frac{2 \zeta_{1}^{2}}{\zeta_{1}^{4}+1}\right)\right)+\left|\underline{c}\left(\zeta_{1}, \zeta_{2}, \zeta_{3}, \overline{\zeta_{3}}\right)\right|\left(\zeta_{1}-\zeta_{2}\right) /\left(\sqrt{t \gamma_{o}\left(\zeta_{1}-\zeta_{2}\right)\left|z_{o}+\zeta_{1}+\zeta_{2}\right|}\left(1-\zeta_{2}^{2}\right)\right)\right) \int_{0}^{+\infty} \mathrm{e}^{-\xi^{2}} \mathrm{~d} \xi \leqslant \mid \underline{c}\left(\zeta_{1}, \zeta_{2}, \zeta_{3}\right.$, $\left.\overline{\zeta_{3}}\right) \mid\left(t\left(\zeta_{1}-\zeta_{2}\right)\left|z_{0}+\zeta_{1}+\zeta_{2}\right|\right)^{-1 / 2}$; hence, $\left\|w^{\prime}(\cdot)\right\|_{\mathcal{L}_{\mathrm{M}_{2}(\mathbb{C})}^{2}\left(\Sigma^{\prime}\right)}=\left\|w^{\prime}(\cdot)\right\|_{\mathcal{L}_{\mathrm{M}_{2}(\mathbb{C})}^{2}\left(\Sigma^{\sharp}\right)} \leqslant\left|\underline{c}\left(\zeta_{1}, \zeta_{2}, \zeta_{3}, \overline{\zeta_{3}}\right)\right|\left(t\left(\zeta_{1}\right.\right.$ $\left.\left.-\zeta_{2}\right)\left|z_{0}+\zeta_{1}+\zeta_{2}\right|\right)^{-1 / 4}$.

Definition 4.1. Let $\mathcal{N}(*)$ denote the space of bounded linear operators acting from $\mathcal{L}_{\mathrm{M}_{2}(\mathbb{C})}^{2}(*)$ into $\mathcal{L}_{\mathrm{M}_{2}(\mathbb{C})}^{2}(*)$.

The following Lemma will be proven a posteriori (see Section 5, Lemma 5.4):

Lemma 4.5. As $t \rightarrow+\infty$ such that $0<\zeta_{2}<\frac{1}{M}<M<\zeta_{1}$ and $\left|\zeta_{3}\right|^{2}=1$, with $M \in \mathbb{R}_{>1}$ and bounded, $\left(\mathbf{1}-C_{w^{\prime}}\right)^{-1} \in \mathcal{N}\left(\Sigma^{\prime}\right)\left(\left\|\left(\mathbf{1}-C_{w^{\prime}}\right)^{-1}\right\|_{\mathcal{N}\left(\Sigma^{\prime}\right)}<\infty\right)$.

Remark 4.3. Actually, the operator $\left(1-C_{w^{\prime}}\right)^{-1}$ acts in $\mathrm{I}+\mathcal{L}_{\mathrm{M}_{2}(\mathbb{C})}^{2}\left(\Sigma^{\prime}\right)$; however, due to a result of Zhou [31], using the Fredholm alternative, if $\left(\mathbf{1}-C_{w^{\prime}}\right)^{-1}$ is invertible on $\mathcal{L}_{\mathrm{M}_{2}(\mathbb{C})}^{2}\left(\Sigma^{\prime}\right)$, then it is invertible on every space set theoretically contained in the span of constant functions and $\mathcal{L}_{\mathrm{M}_{2}(\mathbb{C})}^{2}\left(\Sigma^{\prime}\right)$ (which is the case here); hence, one can consider $\left(\mathbf{1}-C_{w^{\prime}}\right)^{-1} \uparrow_{\mathcal{L}_{\mathrm{M}_{2}(\mathbb{C})}^{2}}\left(\Sigma^{\prime}\right)$. The result stated in Lemma 4.5 should not, after all, come as a surprise, since the jump matrix of the RHP formulated in Lemma 2.6, within the framework of the BC formulation introduced in Section 3, admits a (bounded) algebraic factorisation of the form $\mathcal{G}(\zeta)=$ $\left(\mathrm{I}-w_{-}^{\mathcal{G}}(\zeta)\right)^{-1}\left(\mathrm{I}+w_{+}^{\mathcal{G}}(\zeta)\right), \zeta \in \mathbb{R}$ (oriented from $-\infty$ to $\left.+\infty\right)$, and due to another result of Zhou [32] (see, in particular, Proposition 2.16 and the arguments thereafter), one has that, for $r(\zeta) \in \mathcal{S}_{\mathbb{C}}^{1}(\mathbb{R}),\left\|\left(\mathbf{1}-C_{w^{\mathcal{G}}}\right)^{-1}\right\|_{\mathcal{N}\left(\sigma_{c}\right)} \leqslant \frac{\left(\lambda_{\max }+1\right)+\sqrt{\left(\lambda_{\max }+1\right)^{2}-4 \lambda_{\min }}}{2 \lambda_{\min }}\left\|\left(\mathrm{I}-w_{-}^{\mathcal{G}}(\cdot)\right)^{-1}\right\|_{\mathcal{L}_{\mathrm{M}_{2}(\mathbb{C})}^{\infty}\left(\sigma_{c}\right)}$, with $\lambda_{\max }:=\sup _{\zeta \in \mathbb{R}}\left\{\right.$ maximal eigenvalue of $\left.\left(\mathcal{G}(\zeta) \mathcal{G}^{\dagger}(\zeta)\right)^{1 / 2}\right\}$, where ${ }^{\dagger}$ denotes Hermitian conjugation, and $\lambda_{\min }:=\inf _{\zeta \in \mathbb{R}}\left\{\right.$ minimal eigenvalue of $\left.\frac{1}{2}\left(\mathcal{G}(\zeta)+\mathcal{G}^{\dagger}(\zeta)\right)\right\}$ (see, also, Lemma 2.31 in [33]).

Proposition 4.2. As $t \rightarrow+\infty$ such that $0<\zeta_{2}<\frac{1}{M}<M<\zeta_{1}$ and $\left|\zeta_{3}\right|^{2}=1$, with $M \in \mathbb{R}_{>1}$ and bounded, $\left(\mathbf{1}-C_{w^{\sharp}}\right)^{-1} \in \mathcal{N}\left(\Sigma^{\prime}\right) \Leftrightarrow\left(\mathbf{1}-C_{w^{\prime}}\right)^{-1} \in \mathcal{N}\left(\Sigma^{\prime}\right)$.

Proof. For $\left(1-C_{w^{\sharp}}\right)^{-1}: \mathcal{L}_{\mathrm{M}_{2}(\mathbb{C})}^{2}\left(\Sigma^{\prime}\right) \rightarrow \mathcal{L}_{\mathrm{M}_{2}(\mathbb{C})}^{2}\left(\Sigma^{\prime}\right)$ and $\left(1-C_{w^{\prime}}\right)^{-1}: \mathcal{L}_{\mathrm{M}_{2}(\mathbb{C})}^{2}\left(\Sigma^{\prime}\right) \rightarrow \mathcal{L}_{\mathrm{M}_{2}(\mathbb{C})}^{2}\left(\Sigma^{\prime}\right)$, from the second resolvent identity, $\left(\mathbf{1}-C_{w^{\sharp}}\right)^{-1}=\left(\mathbf{1}-C_{w^{\prime}}\right)^{-1}+\left(\mathbf{1}-C_{w^{\sharp}}\right)^{-1}\left(C_{w^{\sharp}}-C_{w^{\prime}}\right)\left(\mathbf{1}-C_{w^{\prime}}\right)^{-1}$; hence, $\left\|\left(\mathbf{1}-C_{w^{\sharp}}\right)^{-1}\right\|_{\mathcal{N}\left(\Sigma^{\prime}\right)} \leqslant\left\|\left(\mathbf{1}-C_{w^{\prime}}\right)^{-1}\right\|_{\mathcal{N}\left(\Sigma^{\prime}\right)}+\left\|\left(\mathbf{1}-C_{w^{\sharp}}\right)^{-1}\right\|_{\mathcal{N}\left(\Sigma^{\prime}\right)}\left\|\left(C_{w^{\sharp}}-C_{w^{\prime}}\right)\right\|_{\mathcal{N}\left(\Sigma^{\prime}\right)} \|(\mathbf{1}-$ 
$\left.C_{w^{\prime}}\right)^{-1} \|_{\mathcal{N}\left(\Sigma^{\prime}\right)}$. Recalling from Section 3 that, for $\star \in \mathcal{L}_{\mathrm{M}_{2}(\mathbb{C})}^{2}(*), C_{w} \star:=C_{+}\left(\star w_{-}\right)+C_{-}\left(\star w_{+}\right)$, using the linearity of the Cauchy operators, $C_{ \pm}$, and recalling Eq. (102), one deduces that $\left(C_{w^{\sharp}}-C_{w^{\prime}}\right) \star=C_{+}\left(\star w_{-}^{e}\right)+C_{-}\left(\star w_{+}^{e}\right)=C_{w^{e}} \star$; hence, $\left\|\left(\mathbf{1}-C_{w^{\sharp}}\right)^{-1}\right\|_{\mathcal{N}\left(\Sigma^{\prime}\right)} \leqslant\left\|\left(\mathbf{1}-C_{w^{\prime}}\right)^{-1}\right\|_{\mathcal{N}\left(\Sigma^{\prime}\right)}+$ $\left\|\left(\mathbf{1}-C_{w^{\sharp}}\right)^{-1}\right\|_{\mathcal{N}\left(\Sigma^{\prime}\right)}|| w^{e}(\cdot)\left\|_{\mathcal{L}_{\mathrm{M}_{2}(\mathbb{C})}^{\infty}\left(\Sigma^{\prime}\right)}\right\|\left(\mathbf{1}-C_{w^{\prime}}\right)^{-1} \|_{\mathcal{N}\left(\Sigma^{\prime}\right)}$. Recalling from Eq. (102) that $w^{e}(\zeta)=$ $w^{\widehat{a}}(\zeta)+w^{b}(\zeta)+w^{c}(\zeta)$, using the bounds in Lemma 4.4, one shows that $\left\|w^{e}(\cdot)\right\|_{\mathcal{L}_{\mathrm{M}_{2}(\mathbb{C})}^{\infty}\left(\Sigma^{\prime}\right)} \leqslant$ $\frac{\left|\underline{\underline{c}}\left(\zeta_{1}, \zeta_{2}, \zeta_{3}, \overline{\zeta_{3}}\right)\right|}{\left|z_{o}+\zeta_{1}+\zeta_{2}\right|{ }^{l} t^{l}}+\mathcal{O}\left(\left|\underline{c}\left(\zeta_{1}, \zeta_{2}, \zeta_{3}, \overline{\zeta_{3}}\right)\right| \exp \left(-t \min \left\{2 \gamma_{\mathcal{R}}^{1}, \omega_{0}, \varepsilon^{2} \gamma_{\mathcal{R}}^{0},\left|z_{o}\right| \zeta_{1}\left(2-\zeta_{2}^{2}\right)\right\}\right)\right)$, with $l \in \mathbb{Z}_{\geqslant 1}$ and arbitrarily large; thus, from Lemma 4.5 and the estimate for $\left\|w^{e}(\cdot)\right\|_{\mathcal{L}_{\mathrm{M}_{2}(\mathbb{C})}^{\infty}\left(\Sigma^{\prime}\right)}$, one shows that $\left\|\left(\mathbf{1}-C_{w^{\sharp}}\right)^{-1}\right\|_{\mathcal{N}\left(\Sigma^{\prime}\right)}<\infty$.

Proposition $4.3(27)$. For $\zeta \in \mathbb{C} \backslash \Sigma^{\prime}$,

$$
\int_{\Sigma^{\prime}} \frac{\left(\left(\mathbf{1}-C_{w^{\sharp}}\right)^{-1} \mathrm{I}\right)(z) w^{\sharp}(z)}{(z-\zeta)} \frac{\mathrm{d} z}{2 \pi \mathrm{i}}=\int_{\Sigma^{\prime}} \frac{\left(\left(\mathbf{1}-C_{w^{\prime}}\right)^{-1} \mathrm{I}\right)(z) w^{\prime}(z)}{(z-\zeta)} \frac{\mathrm{d} z}{2 \pi \mathrm{i}}+\mathcal{A}+\mathcal{B}+\mathcal{C}+\mathcal{D},
$$

where

$$
\begin{gathered}
\mathcal{A}:=\int_{\Sigma^{\prime}} \frac{w^{e}(z)}{(z-\zeta)} \frac{\mathrm{d} z}{2 \pi \mathrm{i}}, \quad \mathcal{B}:=\int_{\Sigma^{\prime}} \frac{\left(\left(\mathbf{1}-C_{w^{\prime}}\right)^{-1}\left(C_{w^{e}} \mathrm{I}\right)\right)(z) w^{\sharp}(z)}{(z-\zeta)} \frac{\mathrm{d} z}{2 \pi \mathrm{i}}, \\
\mathcal{C}:=\int_{\Sigma^{\prime}} \frac{\left(\left(\mathbf{1}-C_{w^{\prime}}\right)^{-1}\left(C_{w^{\prime}} \mathrm{I}\right)\right)(z) w^{e}(z)}{(z-\zeta)} \frac{\mathrm{d} z}{2 \pi \mathrm{i}}, \\
\mathcal{D}:=\int_{\Sigma^{\prime}} \frac{\left(\left(\mathbf{1}-C_{w^{\prime}}\right)^{-1} C_{w^{e}}\left(\mathbf{1}-C_{w^{\sharp}}\right)^{-1}\left(C_{w^{\sharp}} \mathrm{I}\right)\right)(z) w^{\sharp}(z)}{(z-\zeta)} \frac{\mathrm{d} z}{2 \pi \mathrm{i}} .
\end{gathered}
$$

Remark 4.4. Hereafter, all exponentially small error terms of the type $\mathcal{O}(\exp (-\diamond t)), \diamond \in$ $\mathbb{R}_{+}$, will be neglected, and only leading order error terms will be retained.

Proposition 4.4. If $\left(1-C_{w^{\prime}}\right)^{-1} \in \mathcal{N}\left(\Sigma^{\prime}\right)$, then, for $\zeta \in \mathbb{C} \backslash \Sigma^{\prime}$ and arbitrarily large $l \in \mathbb{Z}_{\geqslant 1}$, as $t \rightarrow+\infty$ such that $0<\zeta_{2}<\frac{1}{M}<M<\zeta_{1}$ and $\left|\zeta_{3}\right|^{2}=1$, with $M \in \mathbb{R}_{>1}$ and bounded,

$$
\int_{\Sigma^{\prime}} \frac{\left(\left(\mathbf{1}-C_{w^{\sharp}}\right)^{-1} \mathrm{I}\right)(z) w^{\sharp}(z)}{(z-\zeta)} \frac{\mathrm{d} z}{2 \pi \mathrm{i}}=\int_{\Sigma^{\prime}} \frac{\left(\left(\mathbf{1}-C_{w^{\prime}}\right)^{-1} \mathrm{I}\right)(z) w^{\prime}(z)}{(z-\zeta)} \frac{\mathrm{d} z}{2 \pi \mathrm{i}}+\mathcal{O}\left(\frac{c}{\left.\mid \zeta_{0}+\zeta_{1}, \zeta_{2}, \zeta_{3}, \overline{\zeta_{3}}\right)\left.f_{2}\right|^{\prime} t^{l}}\right),
$$

with $f^{\prime}(\zeta) \in \mathcal{L}_{\mathrm{M}_{2}(\mathbb{C})}^{\infty}\left(\mathbb{C} \backslash \Sigma^{\prime}\right)$.

Proof. Modulo exponentially small terms (cf. Remark 4.4), one must show that $\mathcal{A}, \mathcal{B}, \mathcal{C}$ and $\mathcal{D}$ have, respectively, for arbitrarily large $l \in \mathbb{Z}_{\geqslant 1}$, the estimate $\mathcal{O}\left(\frac{\underline{c}\left(\zeta_{1}, \zeta_{2}, \zeta_{3}, \overline{\zeta_{3}}\right) \diamond(\zeta)}{\left|z_{o}+\zeta_{1}+\zeta_{2}\right|^{l} t^{l}}\right)$, where

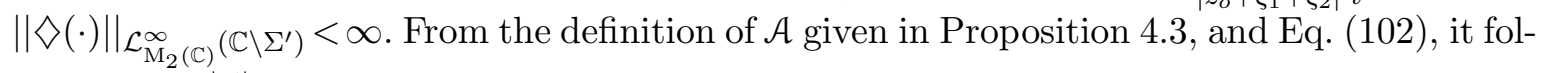

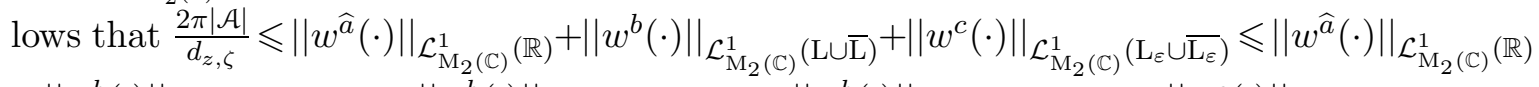
$+\left\|w^{b}(\cdot) \mid\right\|_{\mathcal{L}_{\mathrm{M}_{2}(\mathbb{C})}^{1}\left(Q_{0} \cup \overline{Q_{0}}\right)}+\left\|w^{b}(\cdot)\right\|_{\mathcal{L}_{\mathrm{M}_{2}(\mathbb{C})}^{1}\left(Q_{1} \cup \overline{Q_{1}}\right)}+\left\|w^{b}(\cdot)\right\|_{\mathcal{L}_{\mathrm{M}_{2}(\mathbb{C})}^{1}\left(Q_{2} \cup \overline{Q_{2}}\right)}+\left\|w^{c}(\cdot)\right\|_{\mathcal{L}_{\mathrm{M}_{2}(\mathbb{C})}^{1}\left(Q_{3} \cup \overline{Q_{3}}\right)}+$ $\left\|w^{c}(\cdot)\right\|_{\mathcal{L}_{\mathrm{M}_{2}(\mathbb{C})}^{1}\left(\mathrm{~L}_{>} \cup \overline{\left.\mathrm{L}_{>}\right)}\right.}$, where $d_{z, \zeta}:=\sup _{(z, \zeta) \in \Sigma^{\prime} \times \mathbb{C} \backslash \Sigma^{\prime}}\left|(z-\zeta)^{-1}\right|(<\infty)$; hence, from the estimates given in Lemma 4.4, modulo exponentially small terms, one deduces that $|\mathcal{A}| \leqslant \frac{\left|\underline{c}\left(\zeta_{1}, \zeta_{2}, \zeta_{3}, \overline{\zeta_{3}}\right)\right| d_{z, \zeta}}{\left|z_{o}+\zeta \zeta_{1}+\zeta_{2}\right|{ }^{l} t^{l}}$. From the definition of $\mathcal{B}$ given in Proposition 4.3, Eq. (102), and Lemma 4.5, $2 \pi|\mathcal{B}|\left(d_{z, \zeta}\right)^{-1} \leqslant$ $\left\|\left(\mathbf{1}-C_{w^{\prime}}\right)^{-1}||_{\mathcal{N}\left(\Sigma^{\prime}\right)}|| C_{w^{e}} \mathrm{I}\right\|_{\mathcal{L}_{\mathrm{M}_{2}(\mathbb{C})}^{2}\left(\Sigma^{\prime}\right)}|| w^{\sharp}(\cdot) \|_{\mathcal{L}_{\mathrm{M}_{2}(\mathbb{C})}^{2}\left(\Sigma^{\prime}\right)} \leqslant\left|\underline{c}\left(\zeta_{1}, \zeta_{2}, \zeta_{3}, \overline{\zeta_{3}}\right)\right|\left(\left\|w^{\widehat{a}}(\cdot)\right\|_{\mathcal{L}_{\mathrm{M}_{2}(\mathbb{C})}^{2}(\mathbb{R})}+\| w^{b}(\cdot)\right.$ \|\|$\left._{\mathcal{L}_{\mathrm{M}_{2}(\mathbb{C})}^{2}(\mathrm{~L} \cup \overline{\mathrm{L}})}+\left\|w^{c}(\cdot)\right\|_{\mathcal{L}_{\mathrm{M}_{2}(\mathbb{C})}^{2}\left(\mathrm{~L}_{\varepsilon} \cup \overline{\mathrm{L}_{\varepsilon}}\right)}\right)\left(\left\|w^{\prime}(\cdot)\right\|_{\mathcal{L}_{\mathrm{M}_{2}(\mathbb{C})}^{2}\left(\Sigma^{\prime}\right)}+\left\|w^{\widehat{a}(\cdot)}\right\|_{\mathcal{L}_{\mathrm{M}_{2}(\mathbb{C})}^{2}(\mathbb{R})}+\left\|w^{b}(\cdot)\right\|_{\mathcal{L}_{\mathrm{M}_{2}(\mathbb{C})}^{2}(\mathrm{~L} \cup \overline{\mathrm{L}})}+\right.$ $\left.\left\|w^{c}(\cdot)\right\|_{\mathcal{L}_{\mathrm{M}_{2}(\mathbb{C})}^{2}\left(\mathrm{~L}_{\varepsilon} \cup \overline{\mathrm{L}_{\varepsilon}}\right)}\right)$; using the estimates given in Lemma 4.4, and recalling that $\left.w^{\prime}(\zeta)\right|_{\Sigma^{\prime} \backslash \Sigma^{\sharp}}=$ $\left(\begin{array}{ll}0 & 0 \\ 0 & 0\end{array}\right)$, one shows that $2 \pi|\mathcal{B}|\left(d_{z, \zeta}\right)^{-1} \leqslant \frac{\mid \underline{\underline{c}\left(\zeta_{1}, \zeta_{2}, \zeta_{3}, \overline{\zeta_{3}}\right) \mid}}{\left|z_{o}+\zeta_{1}+\zeta_{2}\right|^{l} t^{l}}\left(\frac{\left|\underline{\underline{c}}\left(\zeta_{1}, \zeta_{2}, \zeta_{3}, \overline{\zeta_{3}}\right)\right|}{\left(\left(\zeta_{1}-\zeta_{2}\right)\left|z_{o}+\zeta_{1}+\zeta_{2}\right|\right)^{1 / 4} t^{1 / 4}}+\frac{\left|\underline{\underline{c}}\left(\zeta_{1}, \zeta_{2}, \zeta_{3}, \overline{\zeta_{3}}\right)\right|}{\left|z_{o}+\zeta_{1}+\zeta_{2}\right|^{l} t^{l}}\right)$, 
whence $|\mathcal{B}| \leqslant \frac{\left|\underline{c}\left(\zeta_{1}, \zeta_{2}, \overline{\zeta_{3}}, \overline{\zeta_{3}}\right)\right| d_{z, \zeta}}{\left|z_{o}+\zeta_{1}+\zeta_{2}\right|^{l} t^{l}}$. From the definition of $\mathcal{C}$ given in Proposition 4.3, Eq. (102), Lemma 4.5, and recalling that $\left.w^{\prime}(\zeta)\right|_{\Sigma^{\prime} \backslash \Sigma^{\sharp}}=\left(\begin{array}{ll}0 & 0 \\ 0 & 0\end{array}\right), \frac{2 \pi|\mathcal{C}|}{d_{z, \zeta}} \leqslant\left\|\left(\mathbf{1}-C_{w^{\prime}}\right)^{-1}\right\|_{\mathcal{N}\left(\Sigma^{\prime}\right)}\left\|C_{w^{\prime}} \mathrm{I}\right\|_{\mathcal{L}_{\mathrm{M}_{2}(\mathbb{C})}^{2}\left(\Sigma^{\prime}\right)}$ $\cdot\left\|w^{e}(\cdot)\right\|_{\mathcal{L}_{\mathrm{M}_{2}(\mathbb{C})}^{2}\left(\Sigma^{\prime}\right)} \leqslant\left|\underline{c}\left(\zeta_{1}, \zeta_{2}, \zeta_{3}, \overline{\zeta_{3}}\right)\right|\left\|w^{\prime}(\cdot)\right\|_{\mathcal{L}_{\mathrm{M}_{2}(\mathbb{C})}^{2}\left(\Sigma^{\sharp}\right)}\left(\left\|w^{\widehat{a}(\cdot)}\right\|_{\mathcal{L}_{\mathrm{M}_{2}(\mathbb{C})}^{2}(\mathbb{R})}+\left\|w^{b}(\cdot)\right\|_{\mathcal{L}_{\mathrm{M}_{2}(\mathbb{C})}^{2}(\mathrm{~L} \cup \overline{\mathrm{L}})}+\right.$ $\left.\left\|w^{c}(\cdot)\right\|_{\mathcal{L}_{\mathrm{M}_{2}(\mathbb{C})}^{2}\left(\mathrm{~L}_{\varepsilon} \cup \overline{\mathrm{L}_{\varepsilon}}\right)}\right)$; thus, using the estimates given in Lemma 4.4, one shows that $\frac{2 \pi|\mathcal{C}|}{d_{z, \zeta}} \leqslant$ $\frac{\left|\underline{c}\left(\zeta_{1}, \zeta_{2}, \overline{\zeta_{3}}, \overline{\zeta_{3}}\right)\right|}{\left|z_{o}+\zeta_{1}+\zeta_{2}\right|^{l} t^{l}} \frac{\left|\underline{c}\left(\zeta_{1}, \zeta_{2}, \zeta_{3}, \overline{\zeta_{3}}\right)\right|}{\left(\left(\zeta_{1}-\zeta_{2}\right)\left|z_{o}+\zeta_{1}+\zeta_{2}\right|\right)^{1 / 4} t^{1 / 4}}$, whence, $|\mathcal{C}| \leqslant \frac{\left|\underline{c}\left(\zeta_{1}, \zeta_{2}, \zeta_{3}, \overline{\zeta_{3}}\right)\right| d_{z, \zeta}}{\left|z_{o}+\zeta_{1}+\zeta_{2}\right|^{l} t^{l}}$. From the definition of $\mathcal{D}$ given in Proposition 4.3, Eq. (102), Lemma 4.5, and noting that $w^{\prime}(\zeta) \Gamma_{\Sigma^{\prime} \backslash \Sigma^{\sharp}}=\left(\begin{array}{ll}0 & 0 \\ 0 & 0\end{array}\right)$, it follows that

$$
\begin{aligned}
& \frac{2 \pi|\mathcal{D}|}{d_{z, \zeta}} \leqslant\left.\left\|\left.\left(\mathbf{1}-C_{w^{\prime}}\right)^{-1}\right|_{\mathcal{N}\left(\Sigma^{\prime}\right)}\right\| C_{w^{e}}\left\|_{\mathcal{N}\left(\Sigma^{\prime}\right)}||\left(\mathbf{1}-C_{w^{\sharp}}\right)^{-1}\right\|_{\mathcal{N}\left(\Sigma^{\prime}\right)}|| C_{w^{\sharp}} \mathrm{I}\right|_{\mathcal{L}_{\mathrm{M}_{2}(\mathbb{C})}^{2}\left(\Sigma^{\prime}\right)} \\
& \times\left\|\left.w^{\sharp}(\cdot)\right|_{\mathcal{L}_{\mathrm{M}_{2}(\mathbb{C})}^{2}\left(\Sigma^{\prime}\right)} \leqslant\left|\underline{c}\left(\zeta_{1}, \zeta_{2}, \zeta_{3}, \overline{\zeta_{3}}\right)\right||| w^{e}(\cdot)\right\|_{\mathcal{L}_{\mathrm{M}_{2}(\mathbb{C})}^{\infty}\left(\Sigma^{\prime}\right)} \|\left. w^{\sharp}(\cdot)\right|_{\mathcal{L}_{\mathrm{M}_{2}(\mathbb{C})}^{2}\left(\Sigma^{\prime}\right)} ^{2} \\
& \leqslant\left|\underline{c}\left(\zeta_{1}, \zeta_{2}, \zeta_{3}, \overline{\zeta_{3}}\right)\right|\left(\left.\left\|\left.w^{\widehat{a}}(\cdot)\right|_{\mathcal{L}_{\mathrm{M}_{2}(\mathbb{C})}^{\infty}(\mathbb{R})}+\right\| w^{b}(\cdot)\right|_{\mathcal{L}_{\mathrm{M}_{2}(\mathbb{C})}^{\infty}(\mathrm{L} \cup \overline{\mathrm{L}})}+\|\left. w^{c}(\cdot)\right|_{\mathcal{L}_{\mathrm{M}_{2}(\mathbb{C})}^{\infty}\left(\mathrm{L}_{\varepsilon} \cup \overline{\mathrm{L}_{\varepsilon}}\right)}\right) \\
& \left.\times \sum_{\substack{n_{1}+n_{2}+n_{3}+n_{4}=2 \\
0 \leqslant n_{i} \leqslant 2}} \frac{2 !}{n_{1} ! n_{2} ! n_{3} ! n_{4} !}\left\|w^{\prime}(\cdot)\right\|_{\mathcal{L}_{\mathrm{M}_{2}(\mathbb{C})}^{2}\left(\Sigma^{\sharp}\right)}^{n_{1}}\left\|w^{\widehat{a}}(\cdot)\right\|_{\mathcal{L}_{\mathrm{M}_{2}(\mathbb{C})}^{2}}^{n_{2}} \| \mathbb{R}^{2}\right) w^{b}(\cdot) \|_{\mathcal{L}_{\mathrm{M}_{2}(\mathbb{C})}^{2}(\mathrm{~L} \cup \overline{\mathrm{L}})}^{n_{3}} \\
& \times\left\|w^{c}(\cdot)\right\|_{\mathcal{L}_{\mathrm{M}_{2}(\mathbb{C})}^{2}\left(\mathrm{~L}_{\varepsilon} \cup \overline{\mathrm{L}_{\varepsilon}}\right)}^{n_{4}},
\end{aligned}
$$

whence, using Chebyshev's inequality', one shows that

$$
\begin{aligned}
\frac{\pi|\mathcal{D}|\left(2 d_{z, \zeta}\right)^{-1}}{\left|\underline{c}\left(\zeta_{1}, \zeta_{2}, \zeta_{3}, \overline{\zeta_{3}}\right)\right|} & \leqslant\left(\left.\left\|\left.w^{\widehat{a}}(\cdot)\right|_{\mathcal{L}_{\mathrm{M}_{2}(\mathbb{C})}^{\infty}(\mathbb{R})}+\right\| w^{b}(\cdot)\right|_{\mathcal{L}_{\mathrm{M}_{2}(\mathbb{C})}^{\infty}(\mathrm{L} \cup \overline{\mathrm{L}})}+\left\|w^{c}(\cdot)\right\|_{\mathcal{L}_{\mathrm{M}_{2}(\mathbb{C})}^{\infty}\left(\mathrm{L}_{\varepsilon} \cup \overline{\mathrm{L}_{\varepsilon}}\right)}\right) \\
& \times\left(\left\|\left.w^{\prime}(\cdot)\right|_{\mathcal{L}_{\mathrm{M}_{2}(\mathbb{C})}^{2}\left(\Sigma^{\sharp}\right)} ^{2}+\right\| w^{\widehat{a}}(\cdot)\left\|_{\mathcal{L}_{\mathrm{M}_{2}(\mathbb{C})}^{2}(\mathbb{R})}^{2}+\right\| w^{b}(\cdot) \|_{\mathcal{L}_{\mathrm{M}_{2}(\mathbb{C})}^{2}(\mathrm{~L} \cup \overline{\mathrm{L}})}^{2}\right. \\
& \left.+\|\left. w^{c}(\cdot)\right|_{\mathcal{L}_{\mathrm{M}_{2}(\mathbb{C})}^{2}\left(\mathrm{~L}_{\varepsilon} \cup \overline{\mathrm{L}_{\varepsilon}}\right)} ^{2}\right)
\end{aligned}
$$

hence, from the estimates given in Lemma 4.4, $\frac{\pi|\mathcal{D}|}{2 d_{z, \zeta}} \leqslant \frac{\left|\underline{\underline{c}}\left(\zeta_{1}, \zeta_{2}, \zeta_{3}, \overline{\zeta_{3}}\right)\right|}{\left.\left|z_{o}+\zeta_{1}+\zeta_{2}\right|\right|^{l} t^{l}} \frac{\left|\underline{\underline{c}}\left(\zeta_{1}, \zeta_{2}, \zeta_{3}, \overline{\zeta_{3}}\right)\right|}{\sqrt{\left(\zeta_{1}-\zeta_{2}\right)\left|z_{o}+\zeta_{1}+\zeta_{2}\right|} \sqrt{t}}$, whence $|\mathcal{D}| \leqslant \frac{\left|\underline{c}\left(\zeta_{1}, \zeta_{2}, \zeta_{3}, \overline{\zeta_{3}}\right)\right| d_{z, \zeta}}{\left|z_{o}+\zeta_{1}+\zeta_{2}\right|^{l} t^{l}}$. From the above-derived bounds for $\mathcal{A}, \mathcal{B}, \mathcal{C}$, and $\mathcal{D}$, one arrives at the result stated in the Proposition.

Definition 4.2. Recall the definition of the BC operator: $C_{\star} \diamond:=C_{+}\left(\nabla_{\star_{-}}\right)+C_{-}\left(\nabla_{\star_{+}}\right), \diamond \in$ $\mathcal{L}_{\mathrm{M}_{2}(\mathbb{C})}^{2}(*)$, where $C_{ \pm}: \mathcal{L}_{\mathrm{M}_{2}(\mathbb{C})}^{2}(*) \rightarrow \mathcal{L}_{\mathrm{M}_{2}(\mathbb{C})}^{2}(*)$ are the (bounded) Cauchy operators introduced at the beginning of Section 3. Let: (1) $C_{w^{\sharp}}:=C_{w^{\sharp}}^{\Sigma^{\prime}}: \mathcal{L}_{\mathrm{M}_{2}(\mathbb{C})}^{2}\left(\Sigma^{\prime}\right) \rightarrow \mathcal{L}_{\mathrm{M}_{2}(\mathbb{C})}^{2}\left(\Sigma^{\prime}\right)$ denote the BC operator with $\star \leftrightarrow w^{\sharp} ;$ (2) $C_{w^{\prime}}:=C_{w^{\prime}}^{s}: \mathcal{L}_{\mathrm{M}_{2}(\mathbb{C})}^{2}(s) \rightarrow \mathcal{L}_{\mathrm{M}_{2}(\mathbb{C})}^{2}(s), s \in\left\{\Sigma^{\prime}, \Sigma^{\sharp}\right\}$, denotes the BC operator with $\star \leftrightarrow w^{\prime} ;$ (3) $\mathbf{1}_{s}, s \in\left\{\Sigma^{\prime}, \Sigma^{\sharp}\right\}$, denotes the identity operator on $\mathcal{L}_{\mathrm{M}_{2}(\mathbb{C})}^{2}(s)$; and (4) $w_{\Sigma^{\prime}}^{\sharp}(\zeta):=\left.w^{\sharp}(\zeta)\right|_{\Sigma^{\prime}}$ and $w^{\Sigma^{\sharp}}(\zeta):=\left.w^{\prime}(\zeta)\right|_{\Sigma^{\sharp}}$.

Note also that, since $\left.w^{\prime}(\zeta)\right|_{\Sigma^{\prime} \backslash \Sigma^{\sharp}}=\left(\begin{array}{ll}0 & 0 \\ 0 & 0\end{array}\right), C_{w^{\prime}}^{\Sigma^{\prime} \star}=C_{+}^{\Sigma^{\prime} \backslash \Sigma^{\sharp} \cup \Sigma^{\sharp}}\left(\star w_{-}^{\prime}\right)+C_{-}^{\Sigma^{\prime} \backslash \Sigma^{\sharp} \cup \Sigma^{\sharp}}\left(\star w_{+}^{\prime}\right)=$ $C_{+}^{\Sigma^{\sharp}}\left(\star w_{-}^{\prime}\right)+C_{-}^{\Sigma^{\sharp}}\left(\star w_{+}^{\prime}\right)=C_{w^{\prime}}^{\Sigma^{\sharp} \star}$.

Corollary 4.1. If $\left(\mathbf{1}_{\Sigma^{\prime}}-C_{w^{\prime}}^{\Sigma^{\prime}}\right)^{-1} \in \mathcal{N}\left(\Sigma^{\prime}\right)$, then, for $\zeta \in \mathbb{C} \backslash \Sigma^{\sharp}$ and arbitrarily large $l \in \mathbb{Z}_{\geqslant 1}$, as $t \rightarrow+\infty$ such that $0<\zeta_{2}<\frac{1}{M}<M<\zeta_{1}$ and $\left|\zeta_{3}\right|^{2}=1$, with $M \in \mathbb{R}_{>1}$ and bounded,

$$
\begin{aligned}
\int_{\Sigma^{\prime}} \frac{\left(\left(\mathbf{1}_{\Sigma^{\prime}}-C_{w^{\sharp}}^{\Sigma^{\prime}}\right)^{-1} \mathrm{I}\right)(z) w_{\Sigma^{\prime}}^{\sharp}(z)}{(z-\zeta)} \frac{\mathrm{d} z}{2 \pi \mathrm{i}} & =\int_{\Sigma^{\sharp}} \frac{\left(\left(\mathbf{1}_{\Sigma^{\sharp}}-C_{w^{\Sigma^{\sharp}}}^{\Sigma^{\sharp}}\right)^{-1} \mathrm{I}\right)(z) w^{\Sigma^{\sharp}}(z)}{(z-\zeta)} \frac{\mathrm{d} z}{2 \pi \mathrm{i}} \\
& +\mathcal{O}\left(\frac{\underline{c}\left(\zeta_{1}, \zeta_{2}, \zeta_{3}, \overline{\zeta_{3}}\right) f^{\sharp}(\zeta)}{\left|z_{o}+\zeta_{1}+\zeta_{2}\right|^{l} t^{l}}\right),
\end{aligned}
$$

${ }^{3}$ If $a_{1} \geqslant a_{2} \geqslant \cdots \geqslant a_{n}$ and $b_{1} \geqslant b_{2} \geqslant \cdots \geqslant b_{n},\left(a_{1}+a_{2}+\cdots+a_{n}\right)\left(b_{1}+b_{2}+\cdots+b_{n}\right) \leqslant n \sum_{i=1}^{n} a_{i} b_{i}$. 
with $f^{\sharp}(\zeta) \in \mathcal{L}_{\mathrm{M}_{2}(\mathbb{C})}^{\infty}\left(\mathbb{C} \backslash \Sigma^{\sharp}\right)$.

Proof. Set $\mu^{\Sigma^{\prime}}(\zeta):=\left(\left(\mathbf{1}_{\Sigma^{\prime}}-C_{w^{\sharp}}^{\Sigma^{\prime}}\right)^{-1} \mathrm{I}\right)(\zeta)$ and $\mu^{\Sigma^{\sharp}}(\zeta):=\left(\left(\mathbf{1}_{\Sigma^{\sharp}}-C_{w^{\Sigma^{\sharp}}}^{\Sigma^{\sharp}}\right)^{-1} \mathrm{I}\right)(\zeta)$. Then, from Proposition 4.4, Definition 4.2, and the fact that $\left.w^{\prime}(\zeta)\right|_{\Sigma^{\prime} \backslash \Sigma^{\sharp}}=\left(\begin{array}{ll}0 & 0 \\ 0 & 0\end{array}\right)$,

$$
\begin{aligned}
& \int_{\Sigma^{\prime}} \frac{\mu^{\Sigma^{\prime}}(z) w_{\Sigma^{\prime}}^{\sharp}(z)}{(z-\zeta)} \frac{\mathrm{d} z}{2 \pi \mathrm{i}}=\int_{\Sigma^{\prime} \backslash \Sigma^{\sharp} \cup \Sigma^{\sharp}} \frac{\left(\left(\mathbf{1}_{\Sigma^{\prime}}-C_{w^{\prime}}^{\Sigma^{\prime}}-\right)^{-1} \mathrm{I}\right)(z) w^{\prime}(z)}{(z-\zeta)} \frac{\mathrm{d} z}{2 \pi \mathrm{i}}+\mathcal{E} \\
& =\int_{\Sigma^{\prime} \backslash \Sigma^{\sharp}} \frac{\left(\left.\left(\left(\mathbf{1}_{\Sigma^{\prime}}-C_{w^{\prime}}^{\Sigma^{\prime}}\right)^{-1} \mathrm{I}\right)(z)\right|_{\Sigma^{\prime} \backslash \Sigma^{\sharp}}\right)\left(\left.w^{\prime}(z)\right|_{\Sigma^{\prime} \backslash \Sigma^{\sharp}}\right)}{(z-\zeta)} \frac{\mathrm{d} z}{2 \pi \mathrm{i}}+\int_{\Sigma^{\sharp}} \frac{\left(\left(\left(\mathbf{1}_{\Sigma^{\prime}}-C_{w^{\prime}}^{\Sigma^{\prime}}\right)^{-1} \mathrm{I}\right)(z) \Gamma_{\Sigma^{\sharp}}\right)\left(w^{\prime}(z) \Gamma_{\Sigma^{\sharp}}\right)}{(z-\zeta)} \frac{\mathrm{d} z}{2 \pi \mathrm{i}}+\mathcal{E}
\end{aligned}
$$

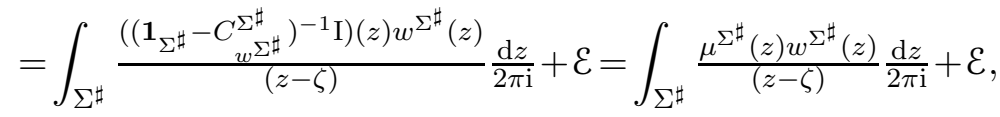

where $\mathcal{E}:=\mathcal{O}\left(\frac{\underline{c}\left(\zeta_{1}, \zeta_{2}, \overline{\zeta_{3}}, \overline{\zeta_{3}}\right) f^{\sharp}(\zeta)}{\left.\left|z_{o}+\zeta_{1}+\zeta_{2}\right|\right|^{l} t^{l}}\right)$, with $\|\left. f^{\sharp}(\cdot)\right|_{\mathcal{L}_{\mathrm{M}_{2}(\mathbb{C})}^{\infty}\left(\mathbb{C} \backslash \Sigma^{\sharp}\right)}<\infty$.

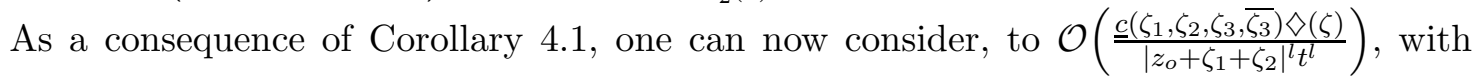
arbitrarily large $l \in \mathbb{Z}_{\geqslant 1}$, and $\|\diamond(\cdot)\|_{\mathcal{L}_{\mathrm{M}_{2}(\mathbb{C})}^{\infty}\left(\mathbb{C} \backslash \Sigma^{\sharp}\right)}<\infty$, the (normalised at $\infty$ ) RHP for $m^{\Sigma^{\sharp}}(\zeta):=\left.m^{\sharp}(\zeta)\right|_{\Sigma^{\sharp}}$ on $\Sigma^{\sharp}$, thus realising the second main objective of this section, and culminating in the following

Lemma 4.6. As $t \rightarrow+\infty$ such that $0<\zeta_{2}<\frac{1}{M}<M<\zeta_{1}$ and $\left|\zeta_{3}\right|^{2}=1$, with $M \in \mathbb{R}_{>1}$ and bounded, $m^{\Sigma^{\sharp}}(\zeta):=m^{\sharp}(\zeta) \Gamma_{\Sigma^{\sharp}}$ solves the following RHP: (1) $m^{\Sigma^{\sharp}}(\zeta)$ is piecewise holomorphic $\forall \zeta \in \mathbb{C} \backslash \Sigma^{\sharp}$; (2) $m_{ \pm}^{\Sigma_{ \pm}^{\sharp}}(\zeta):=\lim \underset{\substack{\zeta^{\prime} \rightarrow \zeta \\ \zeta^{\prime} \in \pm \text { side of } \Sigma^{\sharp}}}{ } m^{\Sigma^{\sharp}}\left(\zeta^{\prime}\right)$ satisfy the jump condition $m_{+}^{\Sigma^{\sharp}}(\zeta)=$ $m_{-}^{\Sigma^{\sharp}}(\zeta)\left(\mathrm{I}-w_{-}^{\Sigma^{\sharp}}(\zeta)\right)^{-1}\left(\mathrm{I}+w_{+}^{\Sigma^{\sharp}}(\zeta)\right), \zeta \in \Sigma^{\sharp}$, where

$$
\begin{gathered}
w_{+}^{\Sigma^{\sharp}}(\zeta)=(\delta(\zeta))^{\operatorname{ad}\left(\sigma_{3}\right)} \exp \left(-\mathrm{i} t \theta^{u}(\zeta) \operatorname{ad}\left(\sigma_{3}\right)\right) \mathcal{R}(\zeta) \sigma_{+}, \quad w_{-}^{\Sigma^{\sharp}}(\zeta)=\left(\begin{array}{ll}
0 & 0 \\
0 & 0
\end{array}\right), \quad \zeta \in \mathrm{L} \backslash \widetilde{\mathrm{L}}_{\varepsilon} \subset \Sigma^{\sharp}, \\
w_{+}^{\Sigma^{\sharp}}(\zeta)=\left(\begin{array}{ll}
0 & 0 \\
0 & 0
\end{array}\right), \quad w_{-}^{\Sigma^{\sharp}}(\zeta)=-(\delta(\zeta))^{\operatorname{ad}\left(\sigma_{3}\right)} \exp \left(-\mathrm{i} t \theta^{u}(\zeta) \operatorname{ad}\left(\sigma_{3}\right)\right) \overline{\mathcal{R}(\zeta)} \sigma_{-}, \quad \zeta \in \overline{\mathrm{L}} \backslash \overline{\widetilde{\mathrm{L}}_{\varepsilon}} \subset \Sigma^{\sharp},
\end{gathered}
$$

with $\widetilde{\mathrm{L}}_{\varepsilon}:=\mathrm{L}_{\varepsilon} \cup \mathrm{L}_{>} \cup \mathrm{L}_{<} ;$(3) as $\zeta \rightarrow \infty, \zeta \in \mathbb{C} \backslash \Sigma^{\sharp}, m^{\Sigma^{\sharp}}(\zeta)=\mathrm{I}+\mathcal{O}\left(\zeta^{-1}\right)$; and (4) $m^{\Sigma^{\sharp}}(\zeta)$ satisfies the symmetry reduction $m^{\Sigma^{\sharp}}(\zeta)=\sigma_{1} \overline{m^{\Sigma^{\sharp}}(\bar{\zeta})} \sigma_{1}$ and the condition $\left(m^{\Sigma^{\sharp}}(0)(\delta(0))^{\sigma_{3}} \sigma_{2}\right)^{2}=\mathrm{I}$. Furthermore, $w_{ \pm}^{\Sigma^{\sharp}}(\zeta) \in \cap_{p \in\{1,2, \infty\}} \mathcal{L}_{\mathrm{M}_{2}(\mathbb{C})}^{p}\left(\Sigma^{\sharp}\right)$.

Proof. Follows from Lemma 4.3, Lemma 4.5, Corollary 4.1, and the definition of $m^{\Sigma^{\sharp}}(\zeta)$ given in the Lemma.

Remark 4.5. In Lemma 4.6, $\overline{\mathcal{R}(\zeta)}$ denotes the piecewise-rational function $\mathcal{R}(\zeta)$ with the complex conjugated coefficients.

Using Lemma 3.1, the solution of the RHP for $m^{\Sigma^{\sharp}}(\zeta)$ on $\Sigma^{\sharp}$ stated in Lemma 4.6 has the integral representation

$$
m^{\Sigma^{\sharp}}(\zeta)=\mathrm{I}+\int_{\Sigma^{\sharp}} \frac{\mu^{\Sigma^{\sharp}}(z) w^{\Sigma^{\sharp}}(z)}{(z-\zeta)} \frac{\mathrm{d} z}{2 \pi \mathrm{i}}, \quad \zeta \in \mathbb{C} \backslash \Sigma^{\sharp},
$$

where $\mu^{\Sigma^{\sharp}}(\zeta):=\left(\left(\mathbf{1}_{\Sigma^{\sharp}}-C_{w^{\Sigma^{\sharp}}}^{\Sigma^{\sharp}}\right)^{-1} \mathrm{I}\right)(\zeta)$, and $w^{\Sigma^{\sharp}}(\zeta):=\sum_{l \in\{ \pm\}} w_{l}^{\Sigma^{\sharp}}(\zeta)$.

\section{Towards the Model RHP}

In this section, the RHP for $m^{\Sigma^{\sharp}}(\zeta)$ on $\Sigma^{\sharp}$ stated in Lemma 4.6 is reduced to RHPs on the two disjoint crosses $\Sigma_{A^{\prime}}$ and $\Sigma_{B^{\prime}}$, and it is shown that, as $t \rightarrow+\infty$, the leading term of asymptotics of the (singular) integral representation for $m^{\Sigma^{\sharp}}(\zeta)$ can be written as the 
linear superposition of two (singular) integrals corresponding to the solution of two auxiliary RHPs, each of which is defined on one of the disjoint crosses. Furthermore, the basic bound on $\left(\mathbf{1}_{\Sigma^{\sharp}}-C_{w^{\Sigma^{\sharp}}}^{\Sigma^{\sharp}}\right)^{-1}$, namely, $\left(\mathbf{1}_{\Sigma^{\sharp}}-C_{w^{\Sigma^{\sharp}}}^{\Sigma^{\sharp}}\right)^{-1} \in \mathcal{N}\left(\Sigma^{\sharp}\right)$, is proved, whence, as a consequence of Corollary 4.1, Definition 4.2, Proposition 4.4, and Proposition 4.2, the basic bound on $\left(\mathbf{1}-C_{w^{\prime}}\right)^{-1}$, that is, $\left(\mathbf{1}-C_{w^{\prime}}\right)^{-1} \in \mathcal{N}\left(\Sigma^{\prime}\right)$, follows.

To formulate a number of exact results, some notational preamble is necessary. Recalling that, for $\diamond(\zeta) \in \mathcal{L}_{\mathrm{M}_{2}(\mathbb{C})}^{2}\left(\Sigma^{\sharp}\right)$, the BC operator is defined as $C_{w^{\Sigma^{\sharp}}}^{\Sigma^{\sharp}} \diamond:=C_{+}\left(\diamond w_{-}^{\Sigma^{\sharp}}\right)+$ $C_{-}\left(\diamond w_{+}^{\Sigma^{\sharp}}\right)$, where $\left(C_{ \pm} \diamond\right)(\zeta):=\lim _{\substack{\zeta^{\prime} \rightarrow \zeta \\ \zeta^{\prime} \in \pm \text { side of } \Sigma^{\sharp}}} \int_{\Sigma^{\sharp}} \frac{\diamond(z)}{\left(z-\zeta^{\prime}\right)} \frac{\mathrm{d} z}{2 \pi \mathrm{i}}$, one shows that, for $w^{\Sigma^{\sharp}}(\zeta):=$ $\sum_{l \in\left\{A^{\prime}, B^{\prime}\right\}} w^{\Sigma_{l}}(\zeta)$, where $w^{\Sigma_{l}}(\zeta):=\left.w^{\Sigma^{\sharp}}(\zeta)\right|_{\Sigma_{l}}$ and $w^{\Sigma_{j}}(\zeta)=\left(\begin{array}{ll}0 & 0 \\ 0 & 0\end{array}\right), \zeta \in \Sigma_{l}, l \neq j \in\left\{A^{\prime}, B^{\prime}\right\}$, $C_{w^{\Sigma^{\sharp}}}^{\Sigma^{\sharp}} \diamond=C_{w^{\Sigma_{A^{\prime}}}}^{\Sigma^{\sharp}} \diamond+C_{w^{\Sigma_{B^{\prime}}}}^{\Sigma^{\sharp}} \diamond$, where $C_{w^{\Sigma_{l}}}^{\Sigma^{\sharp}} \diamond:=\left(C_{+}\left(\diamond w_{-}^{\Sigma^{\sharp}}\right)+C_{-}\left(\diamond w_{+}^{\Sigma_{+}^{\sharp}}\right)\right)\left\lceil\Sigma_{l}, l \in\left\{A^{\prime}, B^{\prime}\right\}\right.$; hence, as an operator on $\mathcal{L}_{\mathrm{M}_{2}(\mathbb{C})}^{2}\left(\Sigma^{\sharp}\right), C_{w^{\Sigma^{\sharp}}}^{\Sigma^{\sharp}}:=\sum_{l \in\left\{A^{\prime}, B^{\prime}\right\}} C_{w^{\Sigma_{l}}}^{\Sigma_{l}}$. Writing $\Sigma^{\sharp}=\Sigma_{A^{\prime}} \cup \Sigma_{B^{\prime}}$, with $\Sigma_{A^{\prime}} \cap \Sigma_{B^{\prime}}=\emptyset$, extend the (oriented) contours $\Sigma_{A^{\prime}}$ and $\Sigma_{B^{\prime}}$ (with orientations unchanged), respectively, to the oriented contours

$$
\begin{aligned}
\widehat{\Sigma}_{A^{\prime}} & :=\left\{\zeta(v) ; \zeta(v)=\zeta_{2}+\frac{v}{\sqrt{2}}\left(\zeta_{1}-\zeta_{2}\right) \exp \left( \pm \frac{\mathrm{i} \pi}{4}\right), v \in[0,+\infty)\right\} \\
\cup\left\{\zeta(v) ; \zeta(v)=\zeta_{2}+\frac{v}{\sqrt{2}} \zeta_{2} \exp \left( \pm \frac{3 \pi \mathrm{i}}{4}\right), v \in[0,+\infty)\right\} & \\
\widehat{\Sigma}_{B^{\prime}}: & =\left\{\zeta(v) ; \zeta(v)=\zeta_{1}+\frac{v}{\sqrt{2}}\left(\zeta_{1}-\zeta_{2}\right) \exp \left( \pm \frac{3 \pi \mathrm{i}}{4}\right), v \in \mathbb{R}\right\}
\end{aligned}
$$

and denote by $\Sigma_{A}$ and $\Sigma_{B}$, respectively, the contours $\left\{\varpi(v) ; \varpi(v)=v\left(\zeta_{1}-\zeta_{2}\right) \exp \left( \pm \frac{\mathrm{i} \pi}{4}\right), v \in \mathbb{R}\right\}$ oriented "outward", as in $\Sigma_{A^{\prime}}$ and $\widehat{\Sigma}_{A^{\prime}}$, and "inward", as in $\Sigma_{B^{\prime}}$ and $\widehat{\Sigma}_{B^{\prime}}$. For $l \in\left\{A^{\prime}, B^{\prime}\right\}$, define $\widehat{w}^{\widehat{\Sigma}_{l}}(\zeta)=\sum_{k \in\{ \pm\}} \widehat{w}_{k}^{\widehat{\Sigma}_{l}}(\zeta)$ on $\widehat{\Sigma}_{l}$ via

$$
\widehat{w}^{\widehat{\Sigma}_{l}}(\zeta):= \begin{cases}w^{\Sigma_{l}}(\zeta)=\sum_{k \in\{ \pm\}} w_{k}^{\Sigma_{l}}(\zeta), & \zeta \in \Sigma_{l} \subset \widehat{\Sigma}_{l} \\
\left(\begin{array}{ll}
0 & 0 \\
0 & 0
\end{array}\right), & \zeta \in \widehat{\Sigma}_{l} \backslash \Sigma_{l} .\end{cases}
$$

The corresponding $\mathrm{BC}$ operators on $\Sigma_{l}, l \in\{A, B\}$, are denoted by $C_{w^{\Sigma_{l}}}^{\Sigma_{l}}$, and, on $\widehat{\Sigma}_{l^{\prime}}$, by $C_{\widehat{w}^{\Sigma_{l^{\prime}}}}^{\widehat{\Sigma}_{l^{\prime}}}$. Introduce the following scaling-shifting operators:

$$
\begin{aligned}
\mathcal{N}_{A}: \mathcal{L}^{2}\left(\widehat{\Sigma}_{A^{\prime}}\right) \rightarrow \mathcal{L}^{2}\left(\Sigma_{A}\right), & f(\zeta) \mapsto\left(\mathcal{N}_{A} f\right)(\widetilde{w})=f\left(\zeta_{2}+\varepsilon_{A}(\widetilde{w})\right), \\
\mathcal{N}_{B}: \mathcal{L}^{2}\left(\widehat{\Sigma}_{B^{\prime}}\right) \rightarrow \mathcal{L}^{2}\left(\Sigma_{B}\right), & g(\zeta) \mapsto\left(\mathcal{N}_{B} g\right)(\widetilde{w})=g\left(\zeta_{1}+\varepsilon_{B}(\widetilde{w})\right),
\end{aligned}
$$

where

$$
\varepsilon_{A}(\widetilde{w}):=\widetilde{w} / \frac{\left|\zeta_{2}-\zeta_{3}\right|}{\zeta_{2}}\left(\frac{2 t\left(\zeta_{1}-\zeta_{2}\right)}{\zeta_{2}}\right)^{1 / 2}, \quad \varepsilon_{B}(\widetilde{w}):=\widetilde{w} / \frac{\left|\zeta_{1}-\zeta_{3}\right|}{\zeta_{1}}\left(\frac{2 t\left(\zeta_{1}-\zeta_{2}\right)}{\zeta_{1}}\right)^{1 / 2}
$$

Noting from the expressions for $w_{ \pm}^{\Sigma^{\sharp}}(\zeta)$ given in Lemma 4.6 that, modulo factors like $\mathcal{R}(\zeta)$ and $\overline{\mathcal{R}(\zeta)}$, the elements of the jump matrix for $m^{\Sigma^{\sharp}}(\zeta)$ are proportional to $(\delta(\zeta))^{ \pm 2} \exp \left(\mp 2 \mathrm{i} t \theta^{u}(\zeta)\right)$, one considers the "action" of $\mathcal{N}_{k}, k \in\{A, B\}$, on such terms.

Proposition 5.1. Let $\mathcal{N}_{k}, k \in\{A, B\}$, be the operators defined in Eq. (105). Then, for $k \in\{A, B\}$, with $\varepsilon_{k}(\widetilde{w})$ defined in Eq. (106),

$$
\left(\mathcal{N}_{k}\left(\delta^{ \pm 2} \mathrm{e}^{\mp 2 \mathrm{i} t \theta^{u}}\right)\right)(\widetilde{w})=\left(\delta_{k}^{0}\right)^{ \pm 2}\left(\delta_{k}^{1}(\widetilde{w})\right)^{ \pm 2}
$$


where

$$
\begin{aligned}
& \delta_{A}^{0}:=\left|\zeta_{2}-\zeta_{3}\right|^{\mathrm{i} \nu}\left(2 t\left(\zeta_{1}-\zeta_{2}\right)^{3} \zeta_{2}^{-3}\right)^{\frac{\mathrm{i} \nu}{2}} \mathrm{e}^{\chi\left(\zeta_{2}\right)} \exp \left(\frac{\mathrm{i} t}{2}\left(\zeta_{1}-\zeta_{2}\right)\left(z_{O}+\zeta_{1}+\zeta_{2}\right)\right), \\
& \delta_{B}^{0}:=\left|\zeta_{1}-\zeta_{3}\right|^{-\mathrm{i} \nu}\left(2 t\left(\zeta_{1}-\zeta_{2}\right)^{3} \zeta_{1}^{-3}\right)^{-\frac{\mathrm{i} \nu}{2}} \mathrm{e}^{\chi\left(\zeta_{1}\right)} \exp \left(-\frac{\mathrm{i} t}{2}\left(\zeta_{1}-\zeta_{2}\right)\left(z_{0}+\zeta_{1}+\zeta_{2}\right)\right), \\
& \delta_{A}^{1}(\widetilde{w}):=(-\widetilde{w})^{-\mathrm{i} \nu}\left(\frac{-\left(\zeta_{1}-\zeta_{2}\right)+\varepsilon_{A}(\widetilde{w})}{-\left(\zeta_{1}-\zeta_{2}\right)}\right)^{\mathrm{i} \nu} \mathrm{e}^{\chi\left(\zeta_{2}+\varepsilon_{A}(\widetilde{w})\right)-\chi\left(\zeta_{2}\right)} \mathrm{e}^{\frac{\mathrm{i} \widetilde{w}^{2}}{4}} \exp \left(\frac{\mathrm{i} \theta_{3}^{u}\left(\zeta_{2}\right) \zeta_{2}^{9 / 2} \widetilde{w}^{3}}{3 !\left(2\left(\zeta_{1}-\zeta_{2}\right)\right)^{3 / 2}\left|\zeta_{2}-\zeta_{3}\right|^{3} \sqrt{t}}\right), \\
& \delta_{B}^{1}(\widetilde{w}):=(\widetilde{w})^{\mathrm{i} \nu}\left(\frac{\left(\zeta_{1}-\zeta_{2}\right)}{\left(\zeta_{1}-\zeta_{2}\right)+\varepsilon_{B}(\widetilde{w})}\right)^{\mathrm{i} \nu} \mathrm{e}^{\chi\left(\zeta_{1}+\varepsilon_{B}(\widetilde{w})\right)-\chi\left(\zeta_{1}\right)} \mathrm{e}^{-\frac{\mathrm{i} \widetilde{w}^{2}}{4}} \exp \left(-\frac{\mathrm{i} \theta_{3}^{u}\left(\zeta_{1}\right) \zeta_{1}^{9 / 2} \widetilde{w}^{3}}{3 !\left(2\left(\zeta_{1}-\zeta_{2}\right)\right)^{3 / 2}\left|\zeta_{1}-\zeta_{3}\right|^{3} \sqrt{t}}\right),
\end{aligned}
$$

with $\nu$ defined in Proposition 4.1,

$$
\chi(\zeta):=\int_{-\infty}^{0} \frac{\ln \left(1-|r(\mu)|^{2}\right)}{(\mu-\zeta)} \frac{\mathrm{d} \mu}{2 \pi \mathrm{i}}+\int_{\zeta_{2}}^{\zeta_{1}} \ln \left(\frac{1-|r(\mu)|^{2}}{1-\left|r\left(\zeta_{1}\right)\right|^{2}}\right) \frac{1}{(\mu-\zeta)} \frac{\mathrm{d} \mu}{2 \pi \mathrm{i}},
$$

$\theta_{3}^{u}\left(\zeta_{n}\right):=2 \zeta_{n}^{-3}\left(2\left(\zeta_{1}-\zeta_{2}\right)\left(\zeta_{n}-\cos \widetilde{\varphi}_{3}\right)+(-1)^{n+1}\left|\zeta_{n}-\zeta_{3}\right|^{2}\left(1+3(-1)^{n} \zeta_{n}^{-1}\left(\zeta_{1}-\zeta_{2}\right)\right)\right), n \in\{1,2\}$, $\widetilde{\varphi}_{3}:=\arg \left(\zeta_{3}\right) \in\left(\frac{\pi}{2}, \pi\right)$, and $( \pm \widetilde{w})^{ \pm \mathrm{i} \nu}:=\exp ( \pm \mathrm{i} \nu \ln ( \pm \widetilde{w}))$ with branch cuts along $\mp \mathbb{R}_{+}$.

Proof. Consequence of the expression for $\delta(\zeta)$ given in Proposition 4.1, the formula (Eq. (8)) $\theta^{u}(\zeta)=\frac{1}{2}\left(\zeta-\frac{1}{\zeta}\right)\left(z_{o}+\zeta+\frac{1}{\zeta}\right)$, the definition of the operators $\mathcal{N}_{k}, k \in\{A, B\}$, given in Eq. (105), and Eq. (106).

For $k \in\{A, B\}$, define

$$
\Delta_{k}^{0}:=\left(\delta_{k}^{0}\right)^{\sigma_{3}}
$$

and let $\widetilde{\Delta}_{k}^{0}$ denote (the operator of) right multiplication by $\Delta_{k}^{0}$ :

$$
\widetilde{\Delta}_{k}^{0} \phi:=\phi \Delta_{k}^{0}
$$

Remark 5.1. One notes from Proposition 5.1 that, for $k \in\{A, B\}$, since $\chi\left(\zeta_{k}\right)$ are pure imaginary, $\left|\delta_{k}^{0}\right|=1$; furthermore, from Eq. (108) and the aforementioned, one also shows that $\widetilde{\Delta}_{k}^{0}$ are unitary operators, namely, $\left(\widetilde{\Delta}_{k}^{0}\right)^{\dagger}=\left(\widetilde{\Delta}_{k}^{0}\right)^{-1}$.

Proposition 5.2. For $k \in\{A, B\}$,

$$
C_{\widehat{w}^{\Sigma_{k^{\prime}}}}^{\widehat{\Sigma}_{k^{\prime}}}=\left(\mathcal{N}_{k}\right)^{-1}\left(\widetilde{\Delta}_{k}^{0}\right)^{-1} C_{w^{\Sigma} \Sigma_{k}}^{\Sigma_{k}}\left(\widetilde{\Delta}_{k}^{0}\right) \mathcal{N}_{k}, \quad w^{\Sigma_{k}}=w^{\Sigma_{k}}(\cdot):=\left(\left(\Delta_{k}^{0}\right)^{-1}\left(\mathcal{N}_{k} \widehat{w}^{\widehat{\Sigma}_{k^{\prime}}}\right)\left(\Delta_{k}^{0}\right)\right)(\cdot),
$$

where

$$
\begin{array}{r}
C_{\left.w^{\Sigma_{k}}\right\rceil_{\mathcal{L}_{\mathrm{M}_{2}(\mathbb{C})}^{2}}^{\Sigma_{k}}\left(\widetilde{\mathrm{L}}_{k}\right)}=C_{-}\left(\cdot\left(\left(\delta_{k}^{1}(\widetilde{w})\right)^{2} \mathcal{R}\left(\zeta_{s(k)}+\varepsilon_{k}(\widetilde{w})\right) \sigma_{+}\right)\right), \\
C_{\left.\left.w^{\Sigma_{k}}\right\rceil_{\mathcal{L}_{\mathrm{M}_{2}(\mathbb{C})}^{2}}^{\Sigma_{\mathrm{L}_{k}}}\right)}=-C_{+}\left(\cdot\left(\left(\delta_{k}^{1}(\widetilde{w})\right)^{-2} \overline{\mathcal{R}\left(\zeta_{s(k)}+\varepsilon_{k}(\widetilde{w})\right)} \sigma_{-}\right)\right),
\end{array}
$$

$s(A)=2, s(B)=1$, and the rays $\widetilde{\mathrm{L}}_{k}$ are defined as

$$
\begin{aligned}
\widetilde{\mathrm{L}}_{A} & :=\left\{\widetilde{w} ; \widetilde{w}=v\left(t\left(\zeta_{1}-\zeta_{2}\right)^{3} \zeta_{2}^{-3}\right)^{1 / 2}\left|\zeta_{2}-\zeta_{3}\right| \exp \left(\frac{\mathrm{i} \pi}{4}\right), v \in[0,+\infty)\right\} \\
\cup\{\widetilde{w} ; \widetilde{w} & \left.=v\left(t\left(\zeta_{1}-\zeta_{2}\right) \zeta_{2}^{-1}\right)^{1 / 2}\left|\zeta_{2}-\zeta_{3}\right| \exp \left(-\frac{3 \pi \mathrm{i}}{4}\right), v \in[0,+\infty)\right\}, \\
\widetilde{\mathrm{L}}_{B} & :=\left\{\widetilde{w} ; \widetilde{w}=v\left(t\left(\zeta_{1}-\zeta_{2}\right)^{3} \zeta_{1}^{-3}\right)^{1 / 2}\left|\zeta_{1}-\zeta_{3}\right| \exp \left(\frac{3 \pi \mathrm{i}}{4}\right), v \in \mathbb{R}\right\},
\end{aligned}
$$

so that $\Sigma_{k}=\widetilde{\mathrm{L}}_{k} \cup \overline{\widetilde{\mathrm{L}}_{k}}$. 
Proof. The case $k=B$ is considered: the case $k=A$ is analogous. Recalling the definition of the Cauchy operators, $C_{ \pm}$, the BC operator, applying the operator $\mathcal{N}_{B}$ defined in Eq. (105), using Eq. (107) (in particular, the $\widetilde{w}$-independence of $\delta_{B}^{0}$ ), and the action (Eq. (108)) and unitarity (Remark 5.1) of $\widetilde{\Delta}_{B}^{0}$, one obtains, via a change-of-variable argument, the expression for $C_{\widehat{w}^{\Sigma_{B^{\prime}}}}^{\widehat{\Sigma}_{B^{\prime}}}$ stated in the Proposition, where $C_{w^{\Sigma} B}^{\Sigma_{B}}=C_{\left(\Delta_{B}^{0}\right)^{-1}\left(\mathcal{N}_{B} \widehat{w}^{\left.\widehat{\Sigma}_{B^{\prime}}\right)\left(\Delta_{B}^{0}\right)}\right.}=$ $C_{+}\left(\cdot\left(\Delta_{B}^{0}\right)^{-1}\left(\mathcal{N}_{B} \widehat{w}_{-}^{\widehat{\Sigma}_{B^{\prime}}}\right)\left(\Delta_{B}^{0}\right)\right)+C_{-}\left(\cdot\left(\Delta_{B}^{0}\right)^{-1}\left(\mathcal{N}_{B} \widehat{w}_{+} \widehat{\Sigma}_{B^{\prime}}\right)\left(\Delta_{B}^{0}\right)\right)$. From the definition of $\widehat{w}^{\widehat{\Sigma}_{B^{\prime}}}(\zeta)$ given in Eq. (104) and recalling that $w^{\Sigma_{B}}(\zeta):=\left.w^{\Sigma^{\sharp}}(\zeta)\right|_{\Sigma_{B}}$, one shows, from the expression for $w^{\Sigma^{\sharp}}(\zeta)=\sum_{l \in\{ \pm\}} w_{l}^{\Sigma^{\sharp}}(\zeta)$ given in Lemma 4.6, that: (1) $\left(\left(\Delta_{B}^{0}\right)^{-1}\left(\mathcal{N}_{B}{\widehat{w_{-}}}_{B^{\prime}}\right)\left(\Delta_{B}^{0}\right)\right)(\widetilde{w}) \Gamma_{\widetilde{L}_{B}}=$ $\left.\left(\left(\Delta_{B}^{0}\right)^{-1}\left(\mathcal{N}_{B} \widehat{w}_{+}^{\widehat{\Sigma}_{B^{\prime}}}\right)\left(\Delta_{B}^{0}\right)\right)(\widetilde{w})\right|_{\widetilde{\mathrm{L}}_{B}}=\left(\begin{array}{cc}0 & 0 \\ 0 & 0\end{array}\right) ;(2)$ for $\widetilde{w} \in\left\{z ; z=v\left(t\left(\zeta_{1}-\zeta_{2}\right)^{3} \zeta_{1}^{-3}\right)^{1 / 2}\left|\zeta_{1}-\zeta_{3}\right| \mathrm{e}^{\frac{3 \pi \mathrm{i}}{4}},-\infty\right.$ $<v<\varepsilon\} \subset \widetilde{\mathrm{L}}_{B},\left(\left(\Delta_{B}^{0}\right)^{-1}\left(\mathcal{N}_{B} \widehat{w}_{+}^{\widehat{\Sigma}_{B^{\prime}}}\right)\left(\Delta_{B}^{0}\right)\right)(\widetilde{w})=\left(\delta_{B}^{1}(\widetilde{w})\right)^{2} \mathcal{R}\left(\zeta_{1}+\varepsilon_{B}(\widetilde{w})\right) \sigma_{+}$, and, for $\widetilde{w} \in \widetilde{\mathrm{L}}_{B} \backslash$ $\left\{z ; z=v\left(t\left(\zeta_{1}-\zeta_{2}\right)^{3} \zeta_{1}^{-3}\right)^{1 / 2}\left|\zeta_{1}-\zeta_{3}\right| \mathrm{e}^{\frac{3 \pi \mathrm{i}}{4}},-\infty<v<\varepsilon\right\},\left(\left(\Delta_{B}^{0}\right)^{-1}\left(\mathcal{N}_{B} \widehat{w}_{+}^{\widehat{\Sigma}_{B^{\prime}}}\right)\left(\Delta_{B}^{0}\right)\right)(\widetilde{w})=\left(\begin{array}{ll}0 & 0 \\ 0 & 0\end{array}\right)$; and (3) for $\widetilde{w} \in\left\{z ; z=v\left(t\left(\zeta_{1}-\zeta_{2}\right)^{3} \zeta_{1}^{-3}\right)^{1 / 2}\left|\zeta_{1}-\zeta_{3}\right| \mathrm{e}^{-\frac{3 \pi \mathrm{i}}{4}},-\infty<v<\varepsilon\right\} \subset \overline{\widetilde{\mathrm{L}}_{B}},\left(\left(\Delta_{B}^{0}\right)^{-1}\left(\mathcal{N}_{B} \widehat{w}_{-}^{\widehat{\Sigma}_{B^{\prime}}}\right)\left(\Delta_{B}^{0}\right)\right)$ $(\widetilde{w})=-\left(\delta_{B}^{1}(\widetilde{w})\right)^{-2} \overline{\mathcal{R}\left(\zeta_{1}+\varepsilon_{B}(\widetilde{w})\right)} \sigma_{-}$, and, for $\widetilde{w} \in \overline{\widetilde{\mathrm{L}}_{B}} \backslash\left\{z ; z=v\left(t\left(\zeta_{1}-\zeta_{2}\right)^{3} \zeta_{1}^{-3}\right)^{1 / 2} \mid \zeta_{1}-\right.$ $\left.\zeta_{3} \mid \mathrm{e}^{-\frac{3 \pi \mathrm{i}}{4}},-\infty<v<\varepsilon\right\},\left(\left(\Delta_{B}^{0}\right)^{-1}\left(\mathcal{N}_{B} \widehat{w}_{-}^{\widehat{\Sigma}_{B^{\prime}}}\right)\left(\Delta_{B}^{0}\right)\right)(\widetilde{w})=\left(\begin{array}{ll}0 & 0 \\ 0 & 0\end{array}\right)$. With the expressions for $\left(\left(\Delta_{B}^{0}\right)^{-1}\right.$ $\left.\cdot\left(\mathcal{N}_{B} \widehat{w}_{ \pm}^{\widehat{\Sigma}_{B^{\prime}}}\right)\left(\Delta_{B}^{0}\right)\right)(\widetilde{w}), \widetilde{w} \in \Sigma_{B}\left(=\widetilde{\mathrm{L}}_{B} \cup \overline{\widetilde{\mathrm{L}}_{B}}\right)$, and the formula for $C_{w^{\Sigma_{B}}}^{\Sigma_{B}}$ given earlier in the proof, one arrives at the expression for $\left.C_{w^{\Sigma_{B}}}^{\Sigma_{B}}\right\rceil_{\mathcal{L}_{\mathrm{M}_{2}(\mathbb{C})}^{2}(*)}, * \in\left\{\widetilde{\mathrm{L}}_{B}, \overline{\widetilde{\mathrm{L}}_{B}}\right\}$, stated in the Proposition.

From the formulae stated in Proposition 5.1 and the definition of the rays $\widetilde{\mathrm{L}}_{k}, k \in\{A, B\}$, given in Proposition 5.2, as $t \rightarrow+\infty$ : (1) for $\widetilde{w} \in \widetilde{\mathrm{L}}_{k}, k \in\{A, B\},\left(\delta_{k}^{1}(\widetilde{w})\right)^{2} \mathcal{R}\left(\zeta_{s(k)}+\varepsilon_{k}(\widetilde{w})\right) \rightarrow$ $(\operatorname{sgn}(k) \widetilde{w})^{2 \operatorname{isgn}(k) \nu} \exp \left(-\frac{\mathrm{i}}{2} \operatorname{sgn}(k) \widetilde{w}^{2}\right) \mathcal{R}\left(\zeta_{s(k)}^{ \pm}\right)$, with $s(A)=2, s(B)=1$, and $-\operatorname{sgn}(A)=\operatorname{sgn}(B)=$ 1 ; and $(2)$ for $\widetilde{w} \in \overline{\widetilde{\mathrm{L}}_{k}}, k \in\{A, B\},\left(\delta_{k}^{1}(\widetilde{w})\right)^{-2} \overline{\mathcal{R}\left(\zeta_{s(k)}+\varepsilon_{k}(\widetilde{w})\right)} \rightarrow(\operatorname{sgn}(k) \widetilde{w})^{-2 \operatorname{sisgn}(k) \nu} \exp \left(\frac{\mathrm{i}}{2} \operatorname{sgn}(k)\right.$ - $\widetilde{w}^{2} \overline{\mathcal{R}\left(\zeta_{s(k)}^{ \pm}\right)}$(see Lemma 5.1 for the definition of $\left.\mathcal{R}\left(\zeta_{s(k)}^{ \pm}\right)\right)$.

Lemma 5.1. Let $\gamma \in\left(0, \frac{1}{2}\right)$ be an arbitrarily fixed, sufficiently small real number, $s(A)=2$, $s(B)=1, \widehat{s}(A)=1, \widehat{s}(B)=2,-\operatorname{sgn}(A)=\operatorname{sgn}(B)=1, \widetilde{\mathrm{L}}_{k}, k \in\{A, B\}$, be the rays defined in Proposition 5.2, and $\varepsilon_{k}(\widetilde{w})$ be defined by $E q$. (106). Then, for $k \in\{A, B\}$, as $t \rightarrow+\infty$ such that $0<\zeta_{2}<\frac{1}{M}<M<\zeta_{1}$ and $\left|\zeta_{3}\right|^{2}=1$, with $M \in \mathbb{R}_{>1}$ and bounded,

$$
\begin{aligned}
\|\left(\delta_{k}^{1}(\widetilde{w})\right)^{2} \mathcal{R}\left(\zeta_{s(k)}+\varepsilon_{k}(\widetilde{w})\right) & -\left.(\operatorname{sgn}(k) \widetilde{w})^{2 \operatorname{isgn}(k) \nu} \exp \left(-\frac{\mathrm{i}}{2} \operatorname{sgn}(k) \widetilde{w}^{2}\right) \mathcal{R}\left(\zeta_{s(k)}^{ \pm}\right)\right|_{\mathcal{L}^{\infty}\left(\widetilde{\mathrm{L}}_{k}\right)} \\
& \leqslant \frac{\left|c^{\mathcal{S}}\left(\zeta_{s(k)}\right)\right|\left|\underline{c}\left(\zeta_{\tilde{s}(k)}, \zeta_{3}, \overline{\zeta_{3}}\right)\right|}{\left|\zeta_{s(k)}-\zeta_{3}\right| \sqrt{\left(\zeta_{1}-\zeta_{2}\right)}} \frac{\ln (t)}{\sqrt{t}} \exp \left(-\frac{1}{2} \gamma \widetilde{E}(k) v_{k}^{2} t\right), \quad \widetilde{w} \in \widetilde{\mathrm{L}}_{k}, \\
\|\left(\delta_{k}^{1}(\widetilde{w})\right)^{-2} \overline{\mathcal{R}\left(\zeta_{s(k)}+\varepsilon_{k}(\widetilde{w})\right)} & -(\operatorname{sgn}(k) \widetilde{w})^{-2 i \operatorname{sinn}(k) \nu} \exp \left(\frac{i}{2} \operatorname{sgn}(k) \widetilde{w}^{2}\right) \overline{\mathcal{R}\left(\zeta_{s(k)}^{ \pm}\right)}||_{\mathcal{L}^{\infty}\left(\overline{\widetilde{\mathrm{L}}_{k}}\right)} \\
\leqslant & \frac{\left|c^{\mathcal{S}}\left(\zeta_{s(k)}\right)\right|\left|\underline{c}\left(\zeta_{\widetilde{s}(k)}, \zeta_{3}, \overline{\zeta_{3}}\right)\right|}{\left|\zeta_{s(k)}-\zeta_{3}\right| \sqrt{\left(\zeta_{1}-\zeta_{2}\right)}} \frac{\ln (t)}{\sqrt{t}} \exp \left(-\frac{1}{2} \gamma \widetilde{E}(k) v_{k}^{2} t\right), \quad \widetilde{w} \in \overline{\widetilde{\mathrm{L}}_{k}},
\end{aligned}
$$

where $\mathcal{R}\left(\zeta_{1}^{+}\right):=\lim _{\Re(\zeta) \downarrow \zeta_{1}} \mathcal{R}(\zeta)=\overline{r\left(\zeta_{1}\right)}, \mathcal{R}\left(\zeta_{1}^{-}\right):=\lim _{\Re(\zeta) \uparrow \zeta_{1}} \mathcal{R}(\zeta)=-\overline{r\left(\zeta_{1}\right)}\left(1-\left|r\left(\zeta_{1}\right)\right|^{2}\right)^{-1}$, $\mathcal{R}\left(\zeta_{2}^{+}\right):=\lim _{\Re(\zeta) \downarrow \zeta_{2}} \mathcal{R}(\zeta)=r\left(\zeta_{1}\right)\left(1-\left|r\left(\zeta_{1}\right)\right|^{2}\right)^{-1}, \mathcal{R}\left(\zeta_{2}^{-}\right):=\lim _{\Re(\zeta) \uparrow \zeta_{2}} \mathcal{R}(\zeta)=-r\left(\zeta_{1}\right), \widetilde{E}(A):=$ $\zeta_{2}^{-1}\left(\zeta_{1}-\zeta_{2}\right)\left|\zeta_{2}-\zeta_{3}\right|^{2} \min \left\{\zeta_{2}^{-2}\left(\zeta_{1}-\zeta_{2}\right)^{2}, 1\right\}, \widetilde{E}(B):=\zeta_{1}^{-3}\left(\zeta_{1}-\zeta_{2}\right)^{3}\left|\zeta_{1}-\zeta_{3}\right|^{2}, 0<v_{A}<\widetilde{\varepsilon}$, and $-\infty<v_{B}<\widetilde{\varepsilon}$, with $\widetilde{\varepsilon}$ some judiciously fixed small positive real number.

Proof. Without loss of generality, the $\mathcal{L}^{\infty}(*)$ bound for the case $k=B$ and $\widetilde{w} \in \widetilde{\mathrm{L}}_{B}$ is considered: the remaining cases follow in an analogous manner. One begins by writing, for $\gamma \in\left(0, \frac{1}{2}\right)$ and $\widetilde{w} \in \widetilde{\mathrm{L}}_{B}$,

$$
\left(\delta_{B}^{1}(\widetilde{w})\right)^{2} \mathcal{R}\left(\zeta_{1}+\varepsilon_{B}(\widetilde{w})\right)-(\widetilde{w})^{2 \mathrm{i} \nu} \mathrm{e}^{-\frac{\mathrm{i} \widetilde{w}^{2}}{2}} \mathcal{R}\left(\zeta_{1}^{ \pm}\right)=\mathrm{e}^{-\frac{\mathrm{i} \gamma \widetilde{w}^{2}}{2}}\left(\mathrm { e } ^ { - \frac { \mathrm { i } \gamma \widetilde { w } ^ { 2 } } { 2 } } \left[\mathcal{R}\left(\zeta_{1}+\varepsilon_{B}(\widetilde{w})\right)\left(\frac{\left(\zeta_{1}-\zeta_{2}\right)}{\left(\zeta_{1}-\zeta_{2}\right)+\varepsilon_{B}(\widetilde{w})}\right)^{2 \mathrm{i} \nu}\right.\right.
$$


$\left.\left.\cdot(\widetilde{w})^{2 \mathrm{i} \nu} \mathrm{e}^{-\frac{\mathrm{i}(1-2 \gamma) \tilde{w}^{2}}{2}\left(1+\frac{\theta_{3}^{u}\left(\zeta_{1}\right) \zeta_{1}^{9 / 2} \widetilde{w}}{3 \sqrt{2}(1-2 \gamma) \sqrt{t}\left(\zeta_{1}-\zeta_{2}\right)^{3 / 2}\left|\zeta_{1}-\zeta_{3}\right|^{3}}\right)} \mathrm{e}^{2\left(\chi\left(\zeta_{1}+\varepsilon_{B}(\widetilde{w})\right)-\chi\left(\zeta_{1}\right)\right)}-\mathcal{R}\left(\zeta_{1}^{ \pm}\right)(\widetilde{w})^{2 \mathrm{i} \nu} \mathrm{e}^{-\frac{\mathrm{i}(1-2 \gamma) \widetilde{w}^{2}}{2}}\right]\right)$.

One notes that $\mathrm{e}^{-\frac{\mathrm{i} \gamma}{2} \widetilde{w}^{2}}=\exp \left(-\frac{1}{2} \gamma \zeta_{1}^{-3}\left(\zeta_{1}-\zeta_{2}\right)^{3}\left|\zeta_{1}-\zeta_{3}\right|^{2} v_{B}^{2} t\right),-\infty<v_{B}<\widetilde{\varepsilon}$, which gives rise to the exponential factor stated in the Lemma, and $\mathrm{e}^{-\mathrm{i}(1-2 \gamma) \frac{\widetilde{w}^{2}}{2}}=\exp \left(-\left(\frac{1}{2}-\gamma\right) \zeta_{1}^{-3}\left(\zeta_{1}-\zeta_{2}\right)^{3} \mid \zeta_{1}-\right.$ $\left.\left.\zeta_{3}\right|^{2} v_{B}^{2} t\right)$. From the definition of $\mathcal{R}(\zeta)$ given in the formulation and proof of Lemma 4.2 , and the fact that $r(\zeta) \in \mathcal{S}_{\mathbb{C}}(\mathbb{R}) \cap\left\{h(z) ; \|\left. h(\cdot)\right|_{\mathcal{L}^{\infty}(\mathbb{R})}:=\sup _{z \in \mathbb{R}}|h(z)|<1\right\}$, one shows that, for $v_{B} \in(-\infty, \widetilde{\varepsilon}),\left|\mathcal{R}\left(\zeta_{1}+\varepsilon_{B}(\widetilde{w})\right)\right|=\left|\mathcal{R}\left(\zeta_{1}+\frac{1}{\sqrt{2}} v_{B}\left(\zeta_{1}-\zeta_{2}\right) \mathrm{e}^{\frac{3 \pi \mathrm{i}}{4}}\right)\right| \leqslant\left|\underline{c}\left(\zeta_{1}, \zeta_{2}, \zeta_{3}, \overline{\zeta_{3}}\right)\right|$. One shows that $\sup _{-\infty<v_{B}<\widetilde{\varepsilon}}\left|\left(\frac{\left(\zeta_{1}-\zeta_{2}\right)}{\left(\zeta_{1}-\zeta_{2}\right)+\varepsilon_{B}(\widetilde{w})}\right)^{2 \mathrm{i} \nu}\right|=\sup _{-\infty<v_{B}<\widetilde{\varepsilon}}\left|\mathrm{e}^{2 \nu \arg \left(1+\frac{1}{\sqrt{2}} v_{B} \mathrm{e}^{\frac{3 \pi \mathrm{i}}{4}}\right)}\right| \leqslant \mathrm{e}^{2 \pi \nu_{m}} \leqslant\left|\underline{c}\left(\zeta_{1}, \zeta_{2}, \zeta_{3}, \overline{\zeta_{3}}\right)\right|$, with $0<\nu:=\nu\left(\zeta_{1}\right) \leqslant \nu_{m}:=-\frac{1}{2 \pi} \ln \left(1-\sup _{z \in \mathbb{R}}|r(z)|^{2}\right)$, since $\arg \left(1+\frac{1}{\sqrt{2}} v_{B} \mathrm{e}^{\frac{3 \pi \mathrm{i}}{4}}\right) \in(-\pi, \pi)$, $-\infty<v_{B}<\widetilde{\varepsilon}$ : also, $\left|(\widetilde{w})^{2 \mathrm{i} \nu}\right| \leqslant \mathrm{e}^{2 \pi \nu_{m}} \leqslant\left|\underline{c}\left(\zeta_{1}, \zeta_{2}, \zeta_{3}, \overline{\zeta_{3}}\right)\right|$. For the exponential term $E:=$ $\exp \left(-\mathrm{i}\left(\frac{1}{2}-\gamma\right) \widetilde{w}^{2}\left(1+\frac{\theta_{3}^{u}\left(\zeta_{1}\right) \zeta_{1}^{9 / 2} \widetilde{w}}{3 \sqrt{2}(1-2 \gamma) \sqrt{t}\left(\zeta_{1}-\zeta_{2}\right)^{3 / 2}\left|\zeta_{1}-\zeta_{3}\right|^{3}}\right)\right)$, in light of the estimation for $\mathrm{e}^{-\mathrm{i}\left(\frac{1}{2}-\gamma\right) \widetilde{w}^{2}}$ given earlier in the proof, one must study the sign of $\widetilde{\mathcal{R}}:=\Re\left(1+\frac{\theta_{3}^{u}\left(\zeta_{1}\right) \zeta_{1}^{9 / 2} \widetilde{w}}{3 \sqrt{2}(1-2 \gamma) \sqrt{t}\left(\zeta_{1}-\zeta_{2}\right)^{3 / 2}\left|\zeta_{1}-\zeta_{3}\right|^{3}}\right)$. One shows that, for $-\infty<v_{B}<\widetilde{\varepsilon}, \widetilde{\mathcal{R}}=1-\frac{\theta_{3}^{u}\left(\zeta_{1}\right) \zeta_{1}^{3} v_{B}}{6(1-2 \gamma)\left|\zeta_{1}-\zeta_{3}\right|^{2}}$. From Eqs. (16) and (17), and the formula for $\theta_{3}^{u}\left(\zeta_{1}\right)$ given in Proposition 5.1, one shows that, for $z_{0}<-2, \frac{\theta_{3}^{u}\left(\zeta_{1}\right) \zeta_{1}^{3}}{\left|\zeta_{1}-\zeta_{3}\right|^{2}}>$ 0 , whence $\frac{\theta_{3}^{u}\left(\zeta_{1}\right) \zeta_{1}^{3}}{6(1-2 \gamma)\left|\zeta_{1}-\zeta_{3}\right|^{2}}>0$; hence, for $-\infty<v_{B}<\widetilde{\varepsilon}$, and choosing $\widetilde{\varepsilon}$ so (small) that $1-\frac{\theta_{3}^{u}\left(\zeta_{1}\right) \zeta_{1}^{3} \widetilde{\varepsilon}}{6(1-2 \gamma)\left|\zeta_{1}-\zeta_{3}\right|^{2}}>0$, one deduces that $\widetilde{\mathcal{R}}>0$, from which it follows that $|E| \leqslant \exp \left(-\left(\frac{1}{2}-\right.\right.$ $\left.\gamma) \zeta_{1}^{-3}\left(\zeta_{1}-\zeta_{2}\right)^{3}\left|\zeta_{1}-\zeta_{3}\right|^{2} \widetilde{\mathcal{R}} v_{B}^{2} t\right) \leqslant\left|\underline{c}\left(\zeta_{1}, \zeta_{2}, \zeta_{3}, \overline{\zeta_{3}}\right)\right|$. The boundedness of $\mathrm{e}^{2\left(\chi\left(\zeta_{1}+\varepsilon_{B}(\widetilde{w})\right)-\chi\left(\zeta_{1}\right)\right)}$ is a consequence of the inequality $\left\|(\delta(\cdot))^{ \pm 1}\right\|_{\mathcal{L}^{\infty}(\mathbb{C})}<\infty$ (Proposition 4.1) and the formula for $\chi(\zeta)$ given in Proposition 5.1. Via a Taylor expansion argument, one shows that $\mid \mathrm{e}^{-\frac{\mathrm{i} \gamma}{2} \widetilde{w}^{2}}\left(\mathcal{R}\left(\zeta_{1}+\right.\right.$ $\left.\left.\varepsilon_{B}(\widetilde{w})\right)-\mathcal{R}\left(\zeta_{1}\right)\right) \mid \leqslant \frac{\left|\underline{c}\left(\zeta_{1}, \zeta_{2}, \zeta_{3}, \overline{\zeta_{3}}\right)\right||| \partial_{\bullet} \mathcal{R}(\bullet) \|_{\mathcal{L}} \infty(\mathbb{R})}{\left|\zeta_{1}-\zeta_{3}\right| \sqrt{t\left(\zeta_{1}-\zeta_{2}\right)^{3}}}$. For $\mathrm{e}^{-\frac{\mathrm{i} \gamma}{2} \widetilde{w}^{2}}\left(\left(\frac{\left(\zeta_{1}-\zeta_{2}\right)}{\left(\zeta_{1}-\zeta_{2}\right)+\varepsilon_{B}(\widetilde{w})}\right)^{2 \mathrm{i} \nu}-1\right)$, one shows that $\left|\mathrm{e}^{-\frac{\mathrm{i} \gamma}{2} \widetilde{w}^{2}}\left(\left(\frac{\left(\zeta_{1}-\zeta_{2}\right)}{\left(\zeta_{1}-\zeta_{2}\right)+\varepsilon_{B}(\widetilde{w})}\right)^{2 \mathrm{i} \nu}-1\right)\right| \leqslant 2\left|\mathrm{e}^{-\frac{\mathrm{i} \gamma}{2} \widetilde{w}^{2}}\right|\left|\nu \int_{1}^{\varepsilon_{B}(\widetilde{w}) /\left(\zeta_{1}-\zeta_{2}\right)} \xi^{-2 \mathrm{i} \nu-1} \mathrm{~d} \xi\right| \leqslant \sqrt{2} \nu_{m} \exp \left(-\frac{1}{2} \gamma \zeta_{1}^{-3}\left(\zeta_{1}-\right.\right.$ $\left.\left.\zeta_{2}\right)^{3}\left|\zeta_{1}-\zeta_{3}\right|^{2} v_{B}^{2} t\right) v_{B} \sup _{s \in[0,1]}\left\{\left|z^{-2 \mathrm{i} \nu-1}\right| ; z=1+\frac{1}{\sqrt{2}} s v_{B} \mathrm{e}^{\frac{3 \pi \mathrm{i}}{4}},-\infty<v_{B}<\widetilde{\varepsilon}\right\} \leqslant \frac{\left|c\left(\zeta_{1}, \zeta_{2}, \zeta_{3}, \overline{\zeta_{3}}\right)\right|}{\left|\zeta_{1}-\zeta_{3}\right| \sqrt{t\left(\zeta_{1}-\zeta_{2}\right)^{3}}}$, since $\left|z^{-2 \mathrm{i} \nu-1}\right| \leqslant \mathrm{e}^{2 \pi \nu_{m}}\left(\left(1-\frac{1}{2} s v_{B}\right)^{2}+\left(\frac{1}{2} s v_{B}\right)^{2}\right)^{-1 / 2}<\infty,\left(s, v_{B}\right) \in[0,1] \times(-\infty, \widetilde{\varepsilon})$. Using the inequality $\left|\mathrm{e}^{\diamond}-1\right| \leqslant \sup _{s \in[0,1]}\left|\mathrm{e}^{s \diamond}\right||\diamond|$,

$$
\begin{aligned}
& \left|\mathrm{e}^{-\frac{\mathrm{i} \gamma}{2} \widetilde{w}^{2}}\left(\mathrm{e}^{2\left(\chi\left(\zeta_{1}+\varepsilon_{B}(\widetilde{w})\right)-\chi\left(\zeta_{1}\right)\right)}-1\right)\right| \leqslant 2 \exp \left(-\frac{1}{2} \gamma \zeta_{1}^{-3}\left(\zeta_{1}-\zeta_{2}\right)^{3}\left|\zeta_{1}-\zeta_{3}\right|^{2} v_{B}^{2} t\right) \\
& \times \sup _{s \in[0,1]}\left|\mathrm{e}^{2 s\left(\chi\left(\zeta_{1}+\varepsilon_{B}(\widetilde{w})\right)-\chi\left(\zeta_{1}\right)\right)}\right|\left|\chi\left(\zeta_{1}+\varepsilon_{B}(\widetilde{w})\right)-\chi\left(\zeta_{1}\right)\right| \\
& \leqslant\left|\underline{c}\left(\zeta_{1}, \zeta_{2}, \zeta_{3}, \overline{\zeta_{3}}\right)\right| \exp \left(-\frac{1}{2} \gamma \zeta_{1}^{-3}\left(\zeta_{1}-\zeta_{2}\right)^{3}\left|\zeta_{1}-\zeta_{3}\right|^{2} v_{B}^{2} t\right) \\
& \times\left|\chi\left(\zeta_{1}+\varepsilon_{B}(\widetilde{w})\right)-\chi\left(\zeta_{1}\right)\right| \text {. }
\end{aligned}
$$

Recalling the definition of $\chi(\zeta)$ given in Proposition 5.1, one writes, via an integration by parts argument, $\chi\left(\zeta_{1}+\varepsilon_{B}(\widetilde{w})\right)-\chi\left(\zeta_{1}\right)=\frac{i}{2 \pi}\left(\int_{-\infty}^{-M_{o}}+\int_{-M_{o}}^{-\delta_{o}}+\int_{-\delta_{o}}^{0}+\int_{\zeta_{2}}^{\zeta_{1}}\right)\left(\ln \left(\mu-\zeta_{1}-\varepsilon_{B}(\widetilde{w})\right)-\ln (\mu-\right.$ $\left.\left.\zeta_{1}\right)\right) \mathrm{d} \ln \left(\frac{1-|r(\mu)|^{2}}{1-\left|r\left(\zeta_{1}\right)\right|^{2}}\right):=\widetilde{\mathcal{J}}_{1}+\widetilde{\mathcal{J}}_{2}+\widetilde{\mathcal{J}}_{3}+\widetilde{\mathcal{J}}_{4}$, with $M_{o}$ (respectively $\delta_{o}$ ) an arbitrarily fixed, finite (respectively sufficiently small) positive real number. Using the fact that $r(\zeta) \in \mathcal{S}_{\mathbb{C}}(\mathbb{R})$, one shows that $\left|\widetilde{\mathcal{J}}_{j}\right| \leqslant \mathcal{O}\left(\left|\varepsilon_{B}(\widetilde{w})\right|\right), j \in\{1,2\}$; hence, $\exp \left(-\frac{1}{2} \gamma \zeta_{1}^{-3}\left(\zeta_{1}-\zeta_{2}\right)^{3}\left|\zeta_{1}-\zeta_{3}\right|^{2} v_{B}^{2} t\right)\left(\left|\widetilde{\mathcal{J}}_{1}\right|+\left|\widetilde{\mathcal{J}}_{2}\right|\right) \leqslant$ $\frac{\left|\underline{c}\left(\zeta_{1}, \zeta_{2}, \zeta_{3}, \overline{\zeta_{3}}\right)\right|}{\left|\zeta_{1}-\zeta_{3}\right| \sqrt{t\left(\zeta_{1}-\zeta_{2}\right)}}$. Recalling that $r(0)=0$ and $\|\left. r(\cdot)\right|_{\mathcal{L}^{\infty}(\mathbb{R})}<1$, one shows that $\exp \left(-\frac{1}{2} \gamma \zeta_{1}^{-3}\left(\zeta_{1}-\right.\right.$ $\left.\left.\zeta_{2}\right)^{3}\left|\zeta_{1}-\zeta_{3}\right|^{2} v_{B}^{2} t\right)\left|\widetilde{\mathcal{J}}_{3}\right| \leqslant \frac{\left|\underline{c}\left(\zeta_{1}, \zeta_{2}, \zeta_{3}, \overline{\zeta_{3}}\right)\right|}{\left|\zeta_{1}-\zeta_{3}\right| \sqrt{t\left(\zeta_{1}-\zeta_{2}\right)}}$. Using the Lipschitz property of the reflection coefficient, namely, $\left|r\left(z_{1}\right)-r\left(z_{2}\right)\right| \leqslant \mathrm{A}_{r}\left|z_{1}-z_{2}\right|$, with Lipschitz constant $\mathrm{A}_{r}>0$, and the fact that $r(\zeta) \in \mathcal{S}_{\mathbb{C}}^{1}(\mathbb{R})$, one shows that $\exp \left(-\frac{1}{2} \gamma \zeta_{1}^{-3}\left(\zeta_{1}-\zeta_{2}\right)^{3}\left|\zeta_{1}-\zeta_{3}\right|^{2} v_{B}^{2} t\right)\left|\widetilde{\mathcal{J}}_{4}\right| \leqslant \frac{\left|\underline{c}\left(\zeta_{1}, \zeta_{2}, \zeta_{3}, \overline{\zeta_{3}}\right)\right|}{\left|\zeta_{1}-\zeta_{3}\right| \sqrt{t\left(\zeta_{1}-\zeta_{2}\right)}}+$ 
$\frac{\left|\mathcal{C}^{\mathcal{S}}\left(\zeta_{1}\right)\right| \mid \underline{c}\left(\zeta_{2}, \zeta_{3}, \overline{\zeta_{3}}\right)}{\left|\zeta_{1}-\zeta_{3}\right| \sqrt{\left(\zeta_{1}-\zeta_{2}\right)}} \frac{\ln t}{\sqrt{t}} ;$ hence

$$
\begin{aligned}
\exp \left(-\frac{1}{2} \gamma \zeta_{1}^{-3}\left(\zeta_{1}-\zeta_{2}\right)^{3}\left|\zeta_{1}-\zeta_{3}\right|^{2} v_{B}^{2} t\right)\left|\chi\left(\zeta_{1}+\varepsilon_{B}(\widetilde{w})\right)-\chi\left(\zeta_{1}\right)\right| & \leqslant \frac{\left|\underline{c}\left(\zeta_{1}, \zeta_{2}, \zeta_{3}, \overline{\zeta_{3}}\right)\right|}{\left|\zeta_{1}-\zeta_{3}\right| \sqrt{t\left(\zeta_{1}-\zeta_{2}\right)}} \\
& +\frac{\left|c \mathcal{S}\left(\zeta_{1}\right)\right|\left|\underline{c}\left(\zeta_{2}, \zeta_{3}, \overline{\zeta_{3}}\right)\right|}{\left|\zeta_{1}-\zeta_{3}\right| \sqrt{\left(\zeta_{1}-\zeta_{2}\right)}} \frac{\ln t}{\sqrt{t}}
\end{aligned}
$$

Using the inequality $\left|\mathrm{e}^{\diamond}-1\right| \leqslant \sup _{s \in[0,1]}\left|\frac{\mathrm{d}}{\mathrm{d} s} \mathrm{e}^{s \diamond}\right|$

$$
\begin{aligned}
& \left|\mathrm{e}^{-\frac{\mathrm{i} \gamma}{2} \widetilde{w}^{2}}(\widetilde{w})^{2 \mathrm{i} \nu} \mathrm{e}^{-\mathrm{i}\left(\frac{1}{2}-\gamma\right) \widetilde{w}^{2}}\left(\mathrm{e}^{-\mathrm{i}}-1\right)\right| \leqslant \exp \left(-\frac{1}{2}(1-\gamma) \zeta_{1}^{-3}\left(\zeta_{1}-\zeta_{2}\right)^{3}\left|\zeta_{1}-\zeta_{3}\right|^{2} v_{B}^{2} t\right) \\
& \times \mathrm{e}^{2 \pi \nu_{m}}\left|\sup _{s \in[0,1]}\right| \mathrm{e}^{-\mathrm{i} s} \mid \leqslant \frac{\left|\underline{c}\left(\zeta_{1}, \zeta_{2}, \zeta_{3}, \overline{\zeta_{3}}\right)\right|}{\left|\zeta_{1}-\zeta_{3}\right|^{3} \sqrt{t\left(\zeta_{1}-\zeta_{2}\right)^{3}}},
\end{aligned}
$$

where $\bullet:=\frac{\theta_{3}^{u}\left(\zeta_{1}\right) \zeta_{1}^{9 / 2} \widetilde{w}^{3}}{6 \sqrt{2} \sqrt{t}\left(\zeta_{1}-\zeta_{2}\right)^{3 / 2}\left|\zeta_{1}-\zeta_{3}\right|^{3}}$. Gathering the above-derived bounds, one deduces that, for $v_{B} \in(-\infty, \widetilde{\varepsilon})$,

$$
\begin{aligned}
\|\left(\delta_{B}^{1}(\widetilde{w})\right)^{2} \mathcal{R}\left(\zeta_{1}+\varepsilon_{B}(\widetilde{w})\right) & -(\widetilde{w})^{2 \mathrm{i} \nu} \exp \left(-\frac{\mathrm{i}}{2} \widetilde{w}^{2}\right) \mathcal{R}\left(\zeta_{1}^{ \pm}\right) \|_{\mathcal{L}^{\infty}\left(\widetilde{\mathrm{L}}_{B}\right)} \leqslant \frac{\left|c^{\mathcal{S}}\left(\zeta_{1}\right)\right|\left|\underline{c}\left(\zeta_{2}, \zeta_{3}, \overline{\zeta_{3}}\right)\right|}{\left|\zeta_{1}-\zeta_{3}\right| \sqrt{\left(\zeta_{1}-\zeta_{2}\right)}} \\
& \times \exp \left(-\frac{1}{2} \gamma \zeta_{1}^{-3}\left(\zeta_{1}-\zeta_{2}\right)^{3}\left|\zeta_{1}-\zeta_{3}\right|^{2} v_{B}^{2} t\right) \frac{\ln t}{\sqrt{t}}
\end{aligned}
$$

Proposition 5.3. For general operators $C_{w^{\Sigma_{k^{\prime}}}}^{\Sigma^{\sharp}}, k \in\{1,2, \ldots, N\}$, if $\left(\mathbf{1}_{\Sigma^{\sharp}}-C_{w^{\Sigma} k^{\prime}}^{\Sigma^{\sharp}}\right)^{-1}$ exists, then

$$
\begin{aligned}
\left(\mathbf{1}_{\Sigma^{\sharp}}\right. & \left.+\sum_{i=1}^{N} C_{w^{\Sigma_{i^{\prime}}}}^{\Sigma^{\sharp}}\left(\mathbf{1}_{\Sigma^{\sharp}}-C_{w^{\Sigma_{i^{\prime}}}}^{\Sigma^{\sharp}}\right)^{-1}\right)\left(\mathbf{1}_{\Sigma^{\sharp}}-\sum_{j=1}^{N} C_{w^{\Sigma_{j^{\prime}}}}^{\Sigma^{\sharp}}\right)=\mathbf{1}_{\Sigma^{\sharp}} \\
& -\sum_{i=1}^{N} \sum_{j=1}^{N}\left(1-\delta_{i j}\right)\left(\mathbf{1}_{\Sigma^{\sharp}}-C_{w^{\Sigma_{i^{\prime}}}}^{\Sigma^{\sharp}}\right)^{-1} C_{w^{\Sigma_{i^{\prime}}}}^{\Sigma^{\sharp}} C_{w^{\Sigma_{j^{\prime}}}}^{\Sigma^{\sharp}}, \\
\left(\mathbf{1}_{\Sigma^{\sharp}}\right. & \left.-\sum_{j=1}^{N} C_{w^{\Sigma_{j^{\prime}}}}^{\Sigma^{\sharp}}\right)\left(\mathbf{1}_{\Sigma^{\sharp}}+\sum_{i=1}^{N} C_{w^{\Sigma_{i^{\prime}}}}^{\Sigma^{\sharp}}\left(\mathbf{1}_{\Sigma^{\sharp}}-C_{w^{\Sigma_{i^{\prime}}}}^{\Sigma^{\sharp}}\right)^{-1}\right)=\mathbf{1}_{\Sigma^{\sharp}} \\
& -\sum_{i=1}^{N} \sum_{j=1}^{N}\left(1-\delta_{i j}\right) C_{w^{\Sigma_{i^{\prime}}}}^{\Sigma^{\sharp}} C_{w^{\Sigma_{j^{\prime}}}}^{\Sigma^{\sharp}}\left(\mathbf{1}_{\Sigma^{\sharp}}-C_{w^{\Sigma_{j^{\prime}}}}^{\Sigma^{\sharp}}\right)^{-1},
\end{aligned}
$$

where $\delta_{i j}$ is the Kronecker delta.

Proof. Follows from the assumption of the existence of the general operators $\left(\mathbf{1}_{\Sigma^{\sharp}}-\right.$ $\left.C_{w^{\Sigma} k^{\prime}}^{\Sigma^{\sharp}}\right)^{-1}, k \in\{1,2, \ldots, N\}$, the second resolvent identity, and an induction argument.

Lemma 5.2. For $\alpha \neq \beta \in\left\{A^{\prime}, B^{\prime}\right\}$, as $t \rightarrow+\infty$ such that $0<\zeta_{2}<\frac{1}{M}<M<\zeta_{1}$ and $\left|\zeta_{3}\right|^{2}=1$, with $M \in \mathbb{R}_{>1}$ and bounded,

$$
\begin{gathered}
\left\|C_{w^{\Sigma_{\alpha}}}^{\Sigma^{\sharp}} C_{w^{\Sigma_{\beta}}}^{\Sigma^{\sharp}}\right\|_{\mathcal{N}\left(\Sigma^{\sharp}\right)} \leqslant \frac{\left|\underline{c}\left(\zeta_{1}, \zeta_{2}, \zeta_{3}, \overline{\zeta_{3}}\right)\right|}{\left(\zeta_{1}-\zeta_{2}\right)^{3 / 2} \sqrt{\left|z_{o}+\zeta_{1}+\zeta_{2}\right| t}}, \\
\|\left. C_{w^{\Sigma_{\alpha}}}^{\Sigma^{\sharp}} C_{w^{\Sigma_{\beta}}}^{\Sigma^{\sharp}}\right|_{\mathcal{L}_{\mathrm{M}_{2}(\mathbb{C})}^{\infty}\left(\Sigma^{\sharp}\right) \rightarrow \mathcal{L}_{\mathrm{M}_{2}(\mathbb{C})}^{2}\left(\Sigma^{\sharp}\right)} \leqslant \frac{\left|\underline{c}\left(\zeta_{1}, \zeta_{2}, \zeta_{3}, \overline{\zeta_{3}}\right)\right|}{\left(\zeta_{1}-\zeta_{2}\right)^{7 / 4}\left(\left|z_{o}+\zeta_{1}+\zeta_{2}\right| t\right)^{3 / 4}} .
\end{gathered}
$$

Proof. Without loss of generality, the bounds for $C_{w^{\Sigma} A^{\prime}}^{\Sigma^{\sharp}} C_{w^{\Sigma} B^{\prime}}^{\Sigma^{\sharp}}$ are proved. For $\phi(z) \in$ $\mathcal{L}_{\mathrm{M}_{2}(\mathbb{C})}^{2}\left(\Sigma^{\sharp}\right)$, from the definition of the $\mathrm{BC}$ and Cauchy operators (and their associativity 
properties), it follows that

$$
\begin{aligned}
\mathcal{X}(z) & :=\left(C_{w^{\Sigma} A^{\prime}}^{\Sigma^{\sharp}} C_{w^{\Sigma} B^{\prime}}^{\Sigma^{\sharp}} \phi\right)(z) \\
& =\left(C_{-}^{\Sigma^{\sharp}}\left(C_{+}^{\Sigma^{\sharp}}\left(\phi w_{-}^{\Sigma_{B^{\prime}}}\right) w_{+}^{\Sigma_{A^{\prime}}}\right)\right)(z)+\left(C_{+}^{\Sigma^{\sharp}}\left(C_{-}^{\Sigma^{\sharp}}\left(\phi w_{+}^{\Sigma_{B^{\prime}}}\right) w_{-}^{\Sigma_{A^{\prime}}}\right)\right)(z) \\
& +\lim _{\substack{\zeta^{\prime \prime} \rightarrow z \\
\zeta^{\prime \prime} \in+\text { side of } \Sigma_{A^{\prime}}}} \int_{\Sigma_{A^{\prime}}} \lim _{\substack{\zeta^{\prime} \rightarrow \zeta \\
\zeta^{\prime} \in+\text { side of } \Sigma_{B^{\prime}}}} \int_{\Sigma_{B^{\prime}}} \frac{\phi(\xi) w_{-}^{\Sigma_{B^{\prime}}}(\xi) w_{-}^{\Sigma_{A^{\prime}}}(\zeta)}{\left(\xi-\zeta^{\prime}\right)\left(\zeta-\zeta^{\prime \prime}\right)} \frac{\mathrm{d} \xi}{2 \pi \mathrm{i}} \frac{\mathrm{d} \zeta}{2 \pi \mathrm{i}} \\
& +\lim _{\zeta^{\prime \prime} \in-\text { side of } \Sigma_{A^{\prime}}} \int_{\Sigma_{A^{\prime}}} \lim _{\substack{\zeta^{\prime} \rightarrow \zeta \\
\zeta^{\prime} \in \text { side of } \Sigma_{B^{\prime}}}} \int_{\Sigma_{B^{\prime}}} \frac{\phi(\xi) w_{+}^{\Sigma_{B^{\prime}}}(\xi) w_{+}^{\Sigma_{A^{\prime}}}(\zeta)}{\left(\xi-\zeta^{\prime}\right)\left(\zeta-\zeta^{\prime \prime}\right)} \frac{\mathrm{d} \xi}{2 \pi \mathrm{i}} \frac{\mathrm{d} \zeta}{2 \pi \mathrm{i}} ;
\end{aligned}
$$

but $w_{-}^{\Sigma_{B^{\prime}}}(\xi) w_{-}^{\Sigma_{A^{\prime}}}(\zeta)=w_{+}^{\Sigma_{B^{\prime}}}(\xi) w_{+}^{\Sigma_{A^{\prime}}}(\zeta)=\left(\begin{array}{ll}0 & 0 \\ 0 & 0\end{array}\right)$, thus

$$
\mathcal{X}(z)=\left(C_{-}^{\Sigma^{\sharp}}\left(C_{+}^{\Sigma^{\sharp}}\left(\phi w_{-}^{\Sigma_{B^{\prime}}}\right) w_{+}^{\Sigma_{A^{\prime}}}\right)\right)(z)+\left(C_{+}^{\Sigma^{\sharp}}\left(C_{-}^{\Sigma^{\sharp}}\left(\phi w_{+}^{\Sigma_{B^{\prime}}}\right) w_{-}^{\Sigma_{A^{\prime}}}\right)\right)(z) .
$$

Now,

$$
\begin{aligned}
& \|\mathcal{X}(\bullet)\|_{\mathcal{L}_{\mathrm{M}_{2}(\mathbb{C})}^{2}\left(\Sigma^{\sharp}\right)} \leqslant\left\|\int_{\Sigma_{A^{\prime}}}\left(\int_{\Sigma_{B^{\prime}}} \frac{\phi(\xi) w_{-}^{\Sigma_{B^{\prime}}(\xi)}}{\left(\xi-\zeta_{+}\right)} \frac{\mathrm{d} \xi}{2 \pi \mathrm{i}}\right) \frac{w_{+}^{\Sigma_{A^{\prime}}(\zeta)}}{\left(\zeta^{-\bullet}-\right)} \frac{\mathrm{d} \zeta}{2 \pi \mathrm{i}}\right\|_{\mathcal{L}_{\mathrm{M}_{2}(\mathbb{C})}^{2}\left(\Sigma^{\sharp}\right)} \\
& +\left\|\int_{\Sigma_{A^{\prime}}}\left(\int_{\Sigma_{B^{\prime}}} \frac{\phi(\xi) w_{+}^{\Sigma_{B^{\prime}}}(\xi)}{\left(\xi-\zeta_{-}\right)} \frac{\mathrm{d} \xi}{2 \pi \mathrm{i}}\right) \frac{w_{-}^{\Sigma_{A^{\prime}}}(\zeta)}{(\zeta-\bullet+)} \frac{\mathrm{d} \zeta}{2 \pi \mathrm{i}}\right\|_{\mathcal{L}_{\mathrm{M}_{2}(\mathbb{C})}^{2}\left(\Sigma^{\sharp}\right)}
\end{aligned}
$$

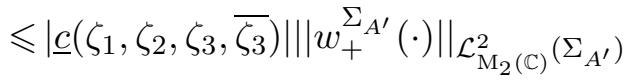

$$
\begin{aligned}
& \times \sup _{\zeta \in \Sigma_{A^{\prime}}}\left|\int_{\Sigma_{B^{\prime}}} \frac{\phi(\xi) w_{-}^{\Sigma_{B^{\prime}}}(\xi)}{(\xi-\zeta)} \frac{\mathrm{d} \xi}{2 \pi \mathrm{i}}\right|+\left|\underline{c}\left(\zeta_{1}, \zeta_{2}, \zeta_{3}, \overline{\zeta_{3}}\right)\right|
\end{aligned}
$$

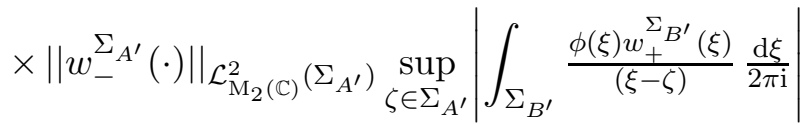

$$
\begin{aligned}
& \leqslant\left(\left(\zeta_{1}-\zeta_{2}\right)^{-1}\left|\underline{c}\left(\zeta_{1}, \zeta_{2}, \zeta_{3}, \overline{\zeta_{3}}\right)\right||| w_{+}^{\Sigma_{A^{\prime}}(\cdot) \|_{\mathcal{L}_{\mathrm{M}_{2}(\mathbb{C})}^{2}}\left(\Sigma_{A^{\prime}}\right)}\right.
\end{aligned}
$$

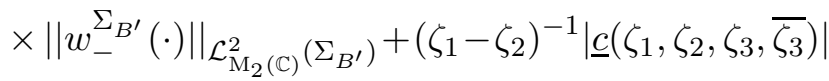

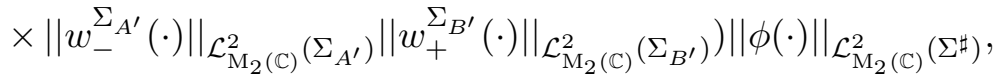

since $\sup _{(\zeta, \xi) \in \Sigma_{A^{\prime}} \times \Sigma_{B^{\prime}}}\left|(\xi-\zeta)^{-1}\right| \leqslant \frac{\left|\underline{c}\left(\zeta_{1}, \zeta_{2}, \zeta_{3}, \overline{\zeta_{3}}\right)\right|}{\left(\zeta_{1}-\zeta_{2}\right)} ;$ thus, from the bound for $\left\|w^{\prime}(\cdot)\right\|_{\mathcal{L}_{\mathrm{M}_{2}(\mathbb{C})}^{2}\left(\Sigma^{\sharp}\right)}$ given in Lemma 4.4, one arrives at $\|\left.\mathcal{X}(\cdot)\right|_{\mathcal{L}_{\mathrm{M}_{2}(\mathbb{C})}^{2}\left(\Sigma^{\sharp}\right)} \leqslant\left.\frac{\left|\underline{c}\left(\zeta_{1}, \zeta_{2}, \zeta_{3}, \overline{\zeta_{3}}\right)\right|}{\left(\zeta_{1}-\zeta_{2}\right)^{3 / 2} \sqrt{t\left|z_{o}+\zeta_{1}+\zeta_{2}\right|}}|| \phi(\cdot)\right|_{\mathcal{L}_{\mathrm{M}_{2}(\mathbb{C})}^{2}\left(\Sigma^{\sharp}\right)}$, whence, one deduces the bound for $\left\|C_{w^{\Sigma} A^{\prime}}^{\Sigma^{\sharp}} C_{w^{\Sigma^{\prime}}}^{\Sigma^{\sharp}}\right\|_{\mathcal{N}\left(\Sigma^{\sharp}\right)}$ stated in the Lemma. Proceeding analogously as above, but considering, instead, the $\mathcal{L}_{\mathrm{M}_{2}(\mathbb{C})}^{\infty}\left(\Sigma^{\sharp}\right)$ bound on $\phi(z)$, one shows that

$$
\begin{aligned}
& \|\mathcal{X}(\cdot)\|_{\mathcal{L}_{\mathrm{M}_{2}(\mathbb{C})}^{\infty}\left(\Sigma^{\sharp}\right) \rightarrow \mathcal{L}_{\mathrm{M}_{2}(\mathbb{C})}^{2}\left(\Sigma^{\sharp}\right)} \leqslant\left(\left(\zeta_{1}-\zeta_{2}\right)^{-1}\left|\underline{c}\left(\zeta_{1}, \zeta_{2}, \zeta_{3}, \overline{\zeta_{3}}\right)\right|\left\|w_{+}^{\Sigma_{A^{\prime}}}(\cdot)\right\|_{\mathcal{L}_{\mathrm{M}_{2}(\mathbb{C})}^{2}\left(\Sigma_{A^{\prime}}\right)}\right.
\end{aligned}
$$

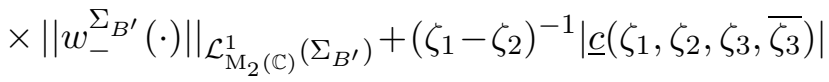

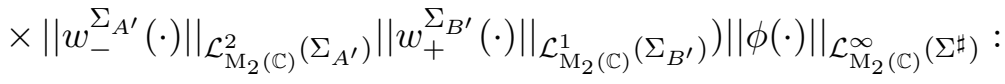

now, using, for $p \in\{1,2\}$, the bounds for $\left\|w^{\prime}(\cdot)\right\|_{\mathcal{L}_{\mathrm{M}_{2}(\mathbb{C})}^{p}\left(\Sigma^{\sharp}\right)}$ given in Lemma 4.4, one arrives at $\|\mathcal{X}(\cdot)\|_{\mathcal{L}_{\mathrm{M}_{2}(\mathbb{C})}^{\infty}\left(\Sigma^{\sharp}\right) \rightarrow \mathcal{L}_{\mathrm{M}_{2}(\mathbb{C})}^{2}\left(\Sigma^{\sharp}\right)} \leqslant\left(\zeta_{1}-\zeta_{2}\right)^{-7 / 4}\left|\underline{c}\left(\zeta_{1}, \zeta_{2}, \zeta_{3}, \overline{\zeta_{3}}\right)\right|\left(t\left|z_{o}+\zeta_{1}+\zeta_{2}\right|\right)^{-3 / 4}\|\phi(\cdot)\|_{\mathcal{L}_{\mathrm{M}_{2}(\mathbb{C})}^{\infty}\left(\Sigma^{\sharp}\right)} ;$ hence, one deduces the bound for $\left\|C_{w^{\Sigma} A^{\prime}}^{\Sigma^{\sharp}} C_{w^{\Sigma_{B^{\prime}}}}^{\Sigma^{\sharp}}\right\|_{\mathcal{L}_{\mathrm{M}_{2}(\mathbb{C})}^{\infty}\left(\Sigma^{\sharp}\right) \rightarrow \mathcal{L}_{\mathrm{M}_{2}(\mathbb{C})}^{2}\left(\Sigma^{\sharp}\right)}$. 
Lemma 5.3. If, for $k \in\{A, B\},\left(\mathbf{1}_{\Sigma_{k^{\prime}}}-C_{w^{\Sigma_{k^{\prime}}}}^{\Sigma_{k^{\prime}}}\right)^{-1} \in \mathcal{N}\left(\Sigma_{k^{\prime}}\right)$, then, as $t \rightarrow+\infty$ such that $0<\zeta_{2}<\frac{1}{M}<M<\zeta_{1}$ and $\left|\zeta_{3}\right|^{2}=1$, with $M \in \mathbb{R}_{>1}$ and bounded, for $\zeta \in \mathbb{C} \backslash \Sigma^{\sharp}$,

$$
\begin{aligned}
\int_{\Sigma^{\sharp}} \frac{\left(\left(\mathbf{1}_{\Sigma^{\sharp}}-C_{w^{\Sigma^{\sharp}}}^{\Sigma^{\sharp}}\right)^{-1} \mathrm{I}\right)(z) w^{\Sigma^{\sharp}}(z)}{(z-\zeta)} \frac{\mathrm{d} z}{2 \pi \mathrm{i}} & =\sum_{k \in\{A, B\}} \int_{\Sigma_{k^{\prime}}} \frac{\left(\left(\mathbf{1}_{\Sigma_{k^{\prime}}}-C_{w^{\Sigma_{k^{\prime}}}}^{\Sigma_{k^{\prime}}}\right)^{-1} \mathrm{I}\right)(z) w^{\Sigma_{k^{\prime}}}(z)}{(z-\zeta)} \frac{\mathrm{d} z}{2 \pi \mathrm{i}} \\
& +\mathcal{O}\left(\frac{\underline{c}\left(\zeta_{1}, \zeta_{2}, \zeta_{3}, \overline{\zeta_{3}}\right) f^{\Sigma^{\sharp}}(\zeta)}{\left(\zeta_{1}-\zeta_{2}\right)^{2}\left|z_{o}+\zeta_{1}+\zeta_{2}\right| t}\right),
\end{aligned}
$$

with $f^{\Sigma^{\sharp}}(\zeta) \in \mathcal{L}_{\mathrm{M}_{2}(\mathbb{C})}^{\infty}\left(\mathbb{C} \backslash \Sigma^{\sharp}\right)$.

Proof. From Proposition 5.3 (with associations $N=2,1 \leftrightarrow A$, and $2 \leftrightarrow B$ ) and the second resolvent identity, one obtains $\left(\mathbf{1}_{\Sigma^{\sharp}}-\sum_{k \in\{A, B\}} C_{w^{\Sigma_{k^{\prime}}}}^{\Sigma_{\prime^{\prime}}}\right)^{-1}=D_{\Sigma^{\sharp}}+D_{\Sigma^{\sharp}}\left(\mathbf{1}_{\Sigma^{\sharp}}-\mathbf{E}_{\Sigma^{\sharp}}\right)^{-1} \mathbf{E}_{\Sigma^{\sharp}}$, where $D_{\Sigma^{\sharp}}:=\mathbf{1}_{\Sigma^{\sharp}}+\sum_{k \in\{A, B\}} C_{w^{\Sigma} k^{\prime}}^{\Sigma_{k^{\prime}}}\left(\mathbf{1}_{\Sigma_{k^{\prime}}}-C_{w^{\Sigma} k^{\prime}}^{\Sigma_{k^{\prime}}}\right)^{-1}$, and $\mathbf{E}_{\Sigma^{\sharp}}:=\sum_{\alpha, \beta \in\{A, B\}}\left(1-\delta_{\alpha \beta}\right) C_{w^{\Sigma} \alpha^{\prime}}^{\Sigma_{\alpha^{\prime}}} C_{w^{\Sigma_{\beta^{\prime}}}}^{\Sigma_{\beta^{\prime}}}\left(\mathbf{1}_{\Sigma_{\beta^{\prime}}}-\right.$ $\left.C_{w^{\Sigma_{\beta^{\prime}}}}^{\Sigma_{\beta^{\prime}}}\right)^{-1}$, with $\delta_{\alpha \beta}$ the Kronecker delta; hence, for $\zeta \in \mathbb{C} \backslash \Sigma^{\sharp}, I^{\sharp}:=\int_{\Sigma^{\sharp}} \frac{\left(\left(\mathbf{1}_{\Sigma^{\sharp}}-C_{w^{\Sigma^{\sharp}}}^{\Sigma^{\sharp}}\right)^{-1} \mathrm{I}\right)(z) w^{\Sigma^{\sharp}}(z)}{(z-\zeta)}$ $\cdot \frac{\mathrm{d} z}{2 \pi \mathrm{i}}=\int_{\Sigma^{\sharp}} \frac{\left(D_{\Sigma^{\sharp}} \mathrm{I}\right)(z) w^{\Sigma^{\sharp}}(z)}{(z-\zeta)} \frac{\mathrm{d} z}{2 \pi \mathrm{i}}+\int_{\Sigma^{\sharp}} \frac{\left(\left(D_{\Sigma^{\sharp}}\left(\mathbf{1}_{\Sigma^{\sharp}}-\mathbf{E}_{\Sigma^{\sharp}}\right)^{-1} \mathbf{E}_{\Sigma^{\sharp}}\right) \mathrm{I}\right)(z) w^{\Sigma^{\sharp}}(z)}{(z-\zeta)} \frac{\mathrm{d} z}{2 \pi \mathrm{i}}$. Recall that $\Sigma^{\sharp}=\Sigma_{A^{\prime}} \cup \Sigma_{B^{\prime}}$.

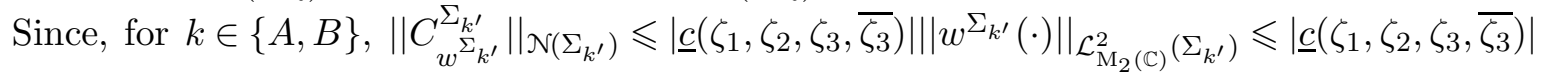
$\cdot\left(\left(\zeta_{1}-\zeta_{2}\right)\left|z_{o}+\zeta_{1}+\zeta_{2}\right| t\right)^{-1 / 4} \leqslant\left|\underline{c}\left(\zeta_{1}, \zeta_{2}, \zeta_{3}, \overline{\zeta_{3}}\right)\right|$ (Lemma 4.4), and, by assumption, $\left(\mathbf{1}_{\Sigma_{k^{\prime}}}-C_{w^{\Sigma} k^{\prime}}^{\Sigma_{k^{\prime}}}\right)^{-1}$ $\in \mathcal{N}\left(\Sigma_{k^{\prime}}\right)$, it follows, from the bounds given in Lemma 5.2 and the second resolvent identity, that $\|\left. D_{\Sigma^{\sharp}}\right|_{\mathcal{N}\left(\Sigma^{\sharp}\right)} \leqslant\left|\underline{c}\left(\zeta_{1}, \zeta_{2}, \zeta_{3}, \overline{\zeta_{3}}\right)\right|$ and $\|\left.\left(\mathbf{1}_{\Sigma^{\sharp}}-\mathbf{E}_{\Sigma^{\sharp}}\right)^{-1}\right|_{\mathcal{N}\left(\Sigma^{\sharp}\right)} \leqslant\left|\underline{c}\left(\zeta_{1}, \zeta_{2}, \zeta_{3}, \overline{\zeta_{3}}\right)\right|$. From the second resolvent identity, the definition of $\mathbf{E}_{\Sigma^{\sharp}}$, the fact that $\left\|\left(C_{w^{\Sigma_{k^{\prime}}}}^{\Sigma_{k^{\prime}}} \mathrm{I}\right)(\cdot)\right\|_{\mathcal{L}_{\mathrm{M}_{2}(\mathbb{C})}^{2}\left(\Sigma^{\sharp}\right)} \leqslant$

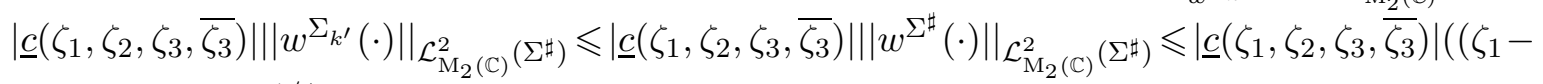
$\left.\left.\zeta_{2}\right)\left|z_{0}+\zeta_{1}+\zeta_{2}\right| t\right)^{-1 / 4}$ (Lemma 4.4), $k \in\{A, B\}$, and the Cauchy-Schwarz inequality for integrals, one shows that, modulo the term $d_{z}(\zeta):=\sup _{(z, \zeta) \in \Sigma^{\sharp} \times \mathbb{C} \backslash \Sigma^{\sharp}}\left|(z-\zeta)^{-1}\right|,|| \frac{\left(\mathbf{E}_{\Sigma^{\sharp}} \mathrm{I}\right)(\cdot) w^{\Sigma^{\sharp}}(\cdot)}{(\cdot-\zeta)} \|_{\mathcal{L}_{\mathrm{M}_{2}(\mathbb{C})}^{2}\left(\Sigma^{\sharp}\right)}$ $\leqslant \sum_{\alpha, \beta \in\{A, B\}}\left(1-\delta_{\alpha \beta}\right)\left\|\left(C_{w^{\alpha_{\alpha^{\prime}}}}^{\Sigma_{\alpha^{\prime}}} C_{w^{\Sigma_{\beta^{\prime}}}}^{\Sigma_{\beta^{\prime}}} \mathrm{I}\right)(\cdot)\right\|_{\mathcal{L}_{\mathrm{M}_{2}(\mathbb{C})}^{2}\left(\Sigma^{\sharp}\right)}\left\|w^{\Sigma^{\sharp}}(\cdot)\right\|_{\mathcal{L}_{\mathrm{M}_{2}(\mathbb{C})}^{2}\left(\Sigma^{\sharp}\right)}+\sum_{\alpha, \beta \in\{A, B\}}\left(1-\delta_{\alpha \beta}\right)$ $\cdot\left\|C_{w^{\Sigma_{\alpha^{\prime}}}}^{\Sigma_{\alpha^{\prime}}} C_{w^{\Sigma_{\beta^{\prime}}}}^{\Sigma_{\beta^{\prime}}}\right\|_{\mathcal{N}\left(\Sigma^{\sharp}\right)}\left\|\left(\mathbf{1}_{\Sigma_{\beta^{\prime}}}-C_{w^{\Sigma_{\beta^{\prime}}}}^{\Sigma_{\beta^{\prime}}}\right)^{-1}\right\|_{\mathcal{N}\left(\Sigma^{\sharp}\right)}\left\|w^{\Sigma^{\sharp}}(\cdot)\right\|_{\mathcal{L}_{\mathrm{M}_{2}(\mathbb{C})}^{2}\left(\Sigma^{\sharp}\right)}^{2}$ : using the bounds given in Lemmae 4.4 and 5.2 , one arrives at $\left\|\frac{\left(\mathbf{E}_{\Sigma^{\sharp}} \mathrm{I}\right)(\cdot) w^{\Sigma^{\sharp}}(\cdot)}{(\cdot-\zeta)}\right\|_{\mathcal{L}_{\mathrm{M}_{2}(\mathbb{C})}^{2}\left(\Sigma^{\sharp}\right)} \leqslant \frac{\left|\underline{c}\left(\zeta_{1}, \zeta_{2}, \zeta_{3}, \overline{\zeta_{3}}\right)\right| d_{z}(\zeta)}{\left(\zeta_{1}-\zeta_{2}\right)^{2} \mid z_{o}+\overline{\zeta_{1}+\zeta_{2} \mid t}}$; hence, $\left(\mathbf{1}_{\Sigma^{\sharp}}-\sum_{k \in\{A, B\}} C_{w^{\Sigma_{k^{\prime}}}}^{\Sigma_{k^{\prime}}}\right)^{-1} \in \mathcal{N}\left(\Sigma^{\sharp}\right)$, and $\mathrm{I}^{\sharp}=\int_{\Sigma^{\sharp}} \frac{\left(D_{\Sigma^{\sharp}} \mathrm{I}\right)(z) w^{\Sigma^{\sharp}}(z)}{(z-\zeta)} \frac{\mathrm{d} z}{2 \pi \mathrm{i}}+\mathcal{O}\left(\frac{\underline{c}\left(\zeta_{1}, \zeta_{2}, \zeta_{3}, \overline{\zeta_{3}}\right) d_{z}(\zeta)}{\left(\zeta_{1}-\zeta_{2}\right)^{2}\left|z_{0}+\zeta_{1}+\zeta_{2}\right| t}\right)$. From the definition of $D_{\Sigma^{\sharp}}$ and an application of the second resolvent identity, one shows that, for $\zeta \in \mathbb{C} \backslash \Sigma^{\sharp}$,

$$
\begin{aligned}
\mathrm{I}^{\sharp} & =\sum_{k \in\{A, B\}} \int_{\Sigma_{k^{\prime}}} \frac{\left(\left(\mathbf{1}_{\Sigma_{k^{\prime}}}-C_{w^{\Sigma_{k^{\prime}}}}^{\Sigma_{k^{\prime}}}\right)^{-1} \mathrm{I}\right)(z) w^{\Sigma_{k^{\prime}}}(z)}{(z-\zeta)} \frac{\mathrm{d} z}{2 \pi \mathrm{i}}+\sum_{\alpha, \beta \in\{A, B\}}\left(1-\delta_{\alpha \beta}\right) \\
& \times \int_{\Sigma^{\sharp}} \frac{\left(C_{w^{\Sigma_{\alpha^{\prime}}}}^{\Sigma_{\alpha^{\prime}}}\left(\mathbf{1}_{\Sigma_{\alpha^{\prime}}}-C_{w^{\Sigma_{\alpha^{\prime}}}}^{\Sigma_{\alpha^{\prime}}}\right)^{-1} \mathrm{I}\right)(z) w^{\Sigma_{\beta^{\prime}}(z)}}{(z-\zeta)} \frac{\mathrm{d} z}{2 \pi \mathrm{i}}+\mathcal{O}\left(\frac{\underline{c}\left(\zeta_{1}, \zeta_{2}, \zeta_{3}, \bar{\zeta} \zeta_{3}\right) d_{z}(\zeta)}{\left(\zeta_{1}-\zeta_{2}\right)^{2}\left|z_{o}+\zeta_{1}+\zeta_{2}\right| t}\right) .
\end{aligned}
$$

The latter two integrals are now estimated: applying, again, the second resolvent identity, 
one shows that, for $\alpha \neq \beta \in\{A, B\}$ and $\zeta \in \mathbb{C} \backslash \Sigma^{\sharp}$,

$$
\begin{aligned}
& \left|\int_{\Sigma \sharp} \frac{\left(C_{w^{\prime}}^{\Sigma_{\alpha^{\prime}}}\left(\mathbf{1}_{\Sigma_{\alpha^{\prime}}}-C_{w_{\alpha^{\prime}} \Sigma_{\alpha^{\prime}}{ }^{\Sigma^{\prime}}}\right)^{-1} \mathrm{I}\right)(z) w^{\Sigma_{\beta^{\prime}}(z)}}{(z-\zeta)} \frac{\mathrm{d} z}{2 \pi \mathrm{i}}\right| \leqslant\left|\int_{\Sigma^{\sharp}} \frac{\left(C_{w^{\prime}}^{\Sigma_{\alpha^{\prime}}} \mathrm{I}\right)(z) w^{\Sigma_{\beta^{\prime}}}(z)}{(z-\zeta)} \frac{\mathrm{d} z}{2 \pi \mathrm{i}}\right|
\end{aligned}
$$

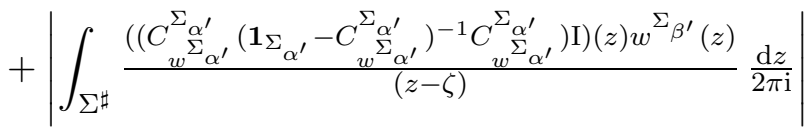

$$
\begin{aligned}
& \leqslant\left|\int_{\Sigma_{\beta^{\prime}}}\left(\int_{\Sigma_{\alpha^{\prime}}} \frac{w^{\Sigma_{\alpha^{\prime}}(\mu)}}{(\mu-z)} \frac{\mathrm{d} \mu}{2 \pi \mathrm{i}}\right) \frac{w^{\Sigma_{\beta^{\prime}}(z)}}{(z-\zeta)} \frac{\mathrm{d} z}{2 \pi \mathrm{i}}\right|
\end{aligned}
$$

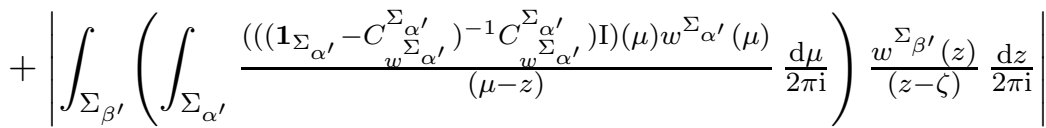

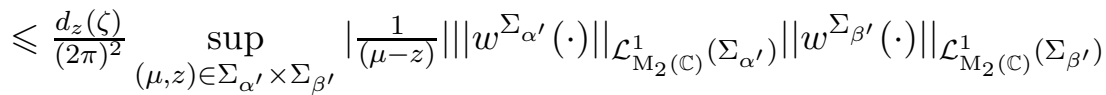

$$
\begin{aligned}
& +\frac{d_{z}(\zeta)}{(2 \pi)^{2}} \sup _{(\mu, z) \in \Sigma_{\alpha^{\prime}} \times \Sigma_{\beta^{\prime}}}\left|\frac{1}{(\mu-z)}\right|||\left(\left(\left(\mathbf{1}_{\Sigma_{\alpha^{\prime}}}-C_{w^{\Sigma_{\alpha^{\prime}}}}^{\Sigma_{\alpha^{\prime}}}\right)^{-1} C_{w^{\Sigma_{\alpha^{\prime}}}}^{\Sigma_{\alpha^{\prime}}}\right) \mathrm{I}\right)(\cdot) \|_{\mathcal{L}_{\mathrm{M}_{2}(\mathbb{C})}^{2}\left(\Sigma_{\alpha^{\prime}}\right)}
\end{aligned}
$$

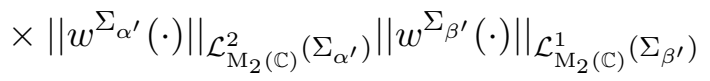

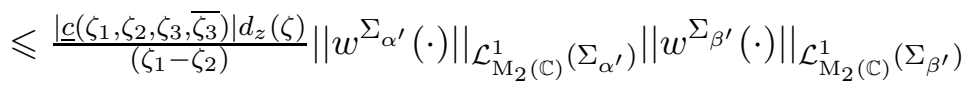

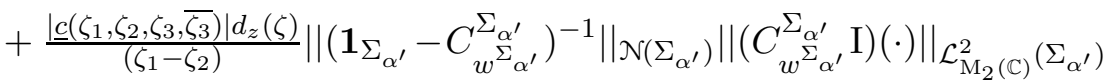

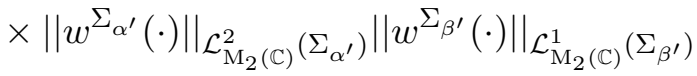

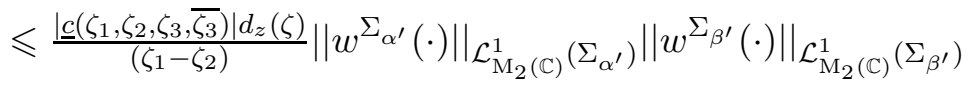

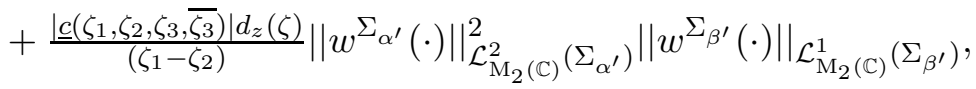

since $\sup _{(\mu, z) \in \Sigma_{\alpha^{\prime}} \times \Sigma_{\beta^{\prime}}}\left|(\mu-z)^{-1}\right| \leqslant\left|\underline{c}\left(\zeta_{1}, \zeta_{2}, \zeta_{3}, \overline{\zeta_{3}}\right)\right|\left(\zeta_{1}-\zeta_{2}\right)^{-1},\left\|\left(\mathbf{1}_{\Sigma_{\alpha^{\prime}}}-C_{w^{\Sigma_{\alpha^{\prime}}}}^{\Sigma_{\alpha^{\prime}}}\right)^{-1}\right\|_{\mathcal{N}\left(\Sigma_{\alpha^{\prime}}\right)} \leqslant$

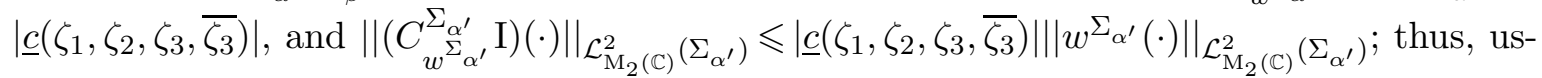

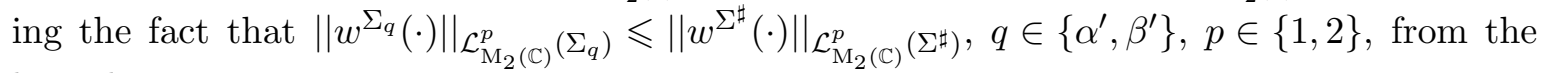
bounds given in Lemma 4.4, one arrives at

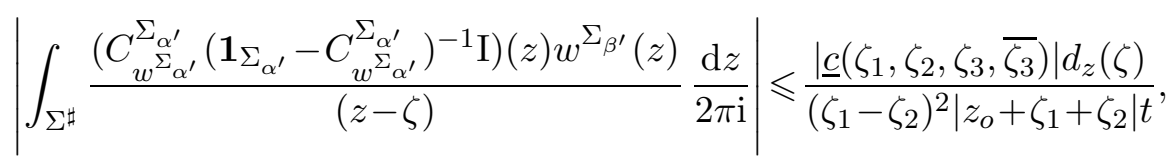

whence, recalling the expression for $I^{\sharp}$ given earlier in the proof, one obtains, for $\zeta \in \mathbb{C} \backslash \Sigma^{\sharp}$, the result stated in the Lemma.

Proposition 5.4. As $t \rightarrow+\infty$ such that $0<\zeta_{2}<\frac{1}{M}<M<\zeta_{1}$ and $\left|\zeta_{3}\right|^{2}=1$, with $M \in \mathbb{R}_{>1}$ and bounded, the solution of the RHP for $m^{\sharp}(\zeta): \mathbb{C} \backslash \Sigma^{\prime} \rightarrow \mathrm{SL}(2, \mathbb{C})$ formulated in Lemma 4.3 has the integral representation

$$
\begin{aligned}
m^{\sharp}(\zeta) & =\mathrm{I}+\sum_{k \in\{A, B\}} \int_{\Sigma_{k^{\prime}}} \frac{\left(\left(\mathbf{1}_{\Sigma_{k^{\prime}}}-C_{w^{\Sigma} k^{\prime}}^{\Sigma_{k^{\prime}}}\right)^{-1} \mathrm{I}\right)(z) w^{\Sigma_{k^{\prime}}}(z)}{(z-\zeta)} \frac{\mathrm{d} z}{2 \pi \mathrm{i}} \\
& +\mathcal{O}\left(\frac{\underline{c}\left(\zeta_{1}, \zeta_{2}, \zeta_{3}, \overline{\zeta_{3}}\right) \diamond^{\sharp}(\zeta)}{\left(\zeta_{1}-\zeta_{2}\right)^{2}\left|z_{o}+\zeta_{1}+\zeta_{2}\right| t}\right), \quad \zeta \in \mathbb{C} \backslash \Sigma_{A^{\prime}} \cup \Sigma_{B^{\prime}},
\end{aligned}
$$

where $w^{\Sigma_{k^{\prime}}}(\zeta)=\sum_{l \in\{ \pm\}} w_{l}^{\Sigma_{k^{\prime}}}(\zeta)$, with $w_{ \pm}^{\Sigma_{k^{\prime}}}(\zeta):=w_{ \pm}^{\Sigma_{ \pm}^{\sharp}}(\zeta)\left\lceil\Sigma_{k^{\prime}}\right.$ and $w_{ \pm}^{\Sigma^{\sharp}}(\zeta)$ given in Lemma 4.6, and $\diamond^{\sharp}(\zeta) \in \mathcal{L}_{\mathrm{M}_{2}(\mathbb{C})}^{\infty}\left(\mathbb{C} \backslash \Sigma_{A^{\prime}} \cup \Sigma_{B^{\prime}}\right)$. 
Proof. From Lemma 5.3 and the (singular) integral representation for the solution of the RHP for $m^{\Sigma^{\sharp}}(\zeta): \mathbb{C} \backslash \Sigma^{\sharp} \rightarrow \mathrm{SL}(2, \mathbb{C})$ formulated in Lemma 4.6 (cf. Eq. (103)), one shows that, as $t \rightarrow+\infty$ such that $0<\zeta_{2}<\frac{1}{M}<M<\zeta_{1}$ and $\left|\zeta_{3}\right|^{2}=1$, with $M \in \mathbb{R}_{>1}$ and bounded, $m^{\Sigma^{\sharp}}(\zeta)=\mathrm{I}+\int_{\Sigma^{\sharp}} \frac{\left(\left(\mathbf{1}_{\Sigma^{\sharp}}-C_{w^{\Sigma^{\sharp}}}^{\Sigma^{\sharp}}\right)^{-1} \mathrm{I}\right)(z) w^{\Sigma^{\sharp}}(z)}{(z-\zeta)} \frac{\mathrm{d} z}{2 \pi \mathrm{i}}=\sum_{k \in\{A, B\}} \int_{\Sigma_{k^{\prime}}} \frac{\left(\left(\mathbf{1}_{\Sigma_{k^{\prime}}}-C_{w^{\Sigma_{k^{\prime}}}}^{\Sigma_{k^{\prime}}}\right)^{-1} \mathrm{I}\right)(z) w^{\Sigma_{k^{\prime}}(z)}}{(z-\zeta)} \frac{\mathrm{d} z}{2 \pi \mathrm{i}}+$ $\mathcal{O}\left(\frac{\underline{c}\left(\zeta_{1}, \zeta_{2}, \zeta_{3}, \overline{\zeta_{3}}\right) \diamond^{\sharp}(\zeta)}{\left(\zeta_{1}-\zeta_{2}\right)^{2}\left|z_{o}+\zeta_{1}+\zeta_{2}\right| t}\right), \zeta \in \mathbb{C} \backslash \Sigma^{\sharp}:$ now, from the RHP for $m^{\sharp}(\zeta): \mathbb{C} \backslash \Sigma^{\prime} \rightarrow \operatorname{SL}(2, \mathbb{C})$ formulated in Lemma 4.3 and the corresponding integral representation $m^{\sharp}(\zeta)=\mathrm{I}+\int_{\Sigma^{\prime}} \frac{\left(\left(\mathbf{1}-C_{w^{\sharp}}\right)^{-1} \mathrm{I}\right)(z) w^{\sharp}(z)}{(z-\zeta)} \frac{\mathrm{d} z}{2 \pi \mathrm{i}}$, $\zeta \in \mathbb{C} \backslash \Sigma^{\prime}$, Proposition 4.4, Corollary 4.1, the definition of $w^{\Sigma_{k^{\prime}}}(\zeta), k \in\{A, B\}$, given in the


$l \in \mathbb{Z}_{\geqslant 1}$ and arbitrarily large, one arrives at the result stated in the Proposition.

Heretofore, it has been shown that (Lemma 5.3, Proposition 5.2, Corollary 4.1, Definition 4.2, Proposition 4.4, and Proposition 4.2) $\left\{\left(\mathbf{1}_{\Sigma_{k}}-C_{w^{\Sigma_{k}}}^{\Sigma_{k}}\right)^{-1} \in \mathcal{N}\left(\Sigma_{k}\right)\right\}_{k \in\{A, B\}} \Rightarrow\left\{\left(\mathbf{1}_{\widehat{\Sigma}_{k^{\prime}}}-\right.\right.$ $\left.\left.C_{\widehat{w}_{k_{k^{\prime}}}^{\widehat{\Sigma}_{k^{\prime}}}}^{\widehat{\widehat{c}}}\right)^{-1} \in \mathcal{N}\left(\widehat{\Sigma}_{k^{\prime}}\right)\right\}_{k \in\{A, B\}} \Rightarrow\left\{\left(\mathbf{1}_{\Sigma_{k^{\prime}}}-C_{w^{\Sigma_{k^{\prime}}}}^{\Sigma_{k^{\prime}}}\right)^{-1} \in \mathcal{N}\left(\Sigma_{k^{\prime}}\right)\right\}_{k \in\{A, B\}} \Rightarrow\left(\mathbf{1}_{\Sigma^{\sharp}}-C_{w^{\Sigma^{\sharp}}}^{\Sigma^{\sharp}}\right)^{-1} \in \mathcal{N}\left(\Sigma^{\sharp}\right) \Rightarrow$ $\left(\mathbf{1}-C_{w^{\prime}}\right)^{-1} \in \mathcal{N}\left(\Sigma^{\prime}\right) \Rightarrow\left(\mathbf{1}-C_{w^{\sharp}}\right)^{-1} \in \mathcal{N}\left(\Sigma^{\prime}\right)$; thus, in order to prove the basic bound $\|(\mathbf{1}-$ $\left.C_{w^{\prime}}\right)^{-1} \|_{\mathcal{N}\left(\Sigma^{\prime}\right)}<\infty$, one must show that $\left\|\left(\mathbf{1}_{\Sigma_{k}}-C_{w^{\Sigma_{k}}}^{\Sigma_{k}}\right)^{-1}\right\|_{\mathcal{N}\left(\Sigma_{k}\right)}<\infty, k \in\{A, B\}$ : this is the programme of the following Lemma.

Remark 5.2. Essentially, Lemma 5.4 (see below) was proved in 27] (see Proposition 3.109, pp. 340-346); however, it is succinctly reworked here because a very important model RHP which arises in it, and which is solved asymptotically in Section 6 and is essential for the proof of Lemma 6.1 (see below), is necessary in order to obtain the results stated in Theorem 3.1.

Lemma 5.4. As $t \rightarrow+\infty$ such that $0<\zeta_{2}<\frac{1}{M}<M<\zeta_{1}$ and $\left|\zeta_{3}\right|^{2}=1$, with $M \in \mathbb{R}_{>1}$ and bounded, $\left(\mathbf{1}_{\Sigma_{k}}-C_{w^{\Sigma_{k}}}^{\Sigma_{k}}\right)^{-1} \in \mathcal{N}\left(\Sigma_{k}\right), k \in\{A, B\}$.

Proof. Without loss of generality, the case $k=B$ is considered: the case $k=A$ follows in an analogous manner. From the bounds given in Lemma 5.1, it is to be understood (for the purposes of this proof) that $\Sigma_{B}$-related quantities encapsulate the $\frac{\left|c^{\mathcal{S}}\left(\zeta_{1}\right)\right|\left|\underline{c}\left(\zeta_{2}, \zeta_{3}, \overline{\zeta_{3}}\right)\right|}{\left|\zeta_{1}-\zeta_{3}\right| \sqrt{\left(\zeta_{1}-\zeta_{2}\right)}} \frac{\ln t}{\sqrt{t}}$ error terms (since $\left.\exp \left(-\frac{1}{2} \gamma \zeta_{1}^{-3}\left(\zeta_{1}-\zeta_{2}\right)^{3}\left|\zeta_{1}-\zeta_{3}\right|^{2} v_{B}^{2} t\right) \leqslant 1, \gamma \in\left(0, \frac{1}{2}\right), v_{B} \in(-\infty, \widetilde{\varepsilon})\right)$; therefore, by $\Sigma_{B^{0-}}$ related quantities, one denotes the "master", or leading, terms of the $\Sigma_{B}$-related quantities, such that, symbolically, $\|\left(\Sigma_{B}-\right.$ related $)-\left(\Sigma_{B^{0}}-\right.$ related $)\|\|_{\cap_{p \in\{1,2, \infty\}} \mathcal{L}^{p}\left(\Sigma_{B}\right)} \leqslant \frac{\left|c^{\mathcal{S}}\left(\zeta_{1}\right)\right|\left|\underline{c}\left(\zeta_{2}, \zeta_{3}, \overline{\zeta_{3}}\right)\right|}{\left|\zeta_{1}-\zeta_{3}\right| \sqrt{\left(\zeta_{1}-\zeta_{2}\right)}} \frac{\ln t}{\sqrt{t}}$, and associated with the master, or $\Sigma_{B^{0}}$-related, terms, is the master, and normalised at $\infty\left(m^{\Sigma_{B^{0}}}(\infty)=\mathrm{I}\right) \operatorname{RHP} m_{+}^{\Sigma_{B^{0}}}(\widetilde{w})=m_{-}^{\Sigma_{B^{0}}}(\widetilde{w})\left(\mathrm{I}-w_{-}^{\Sigma_{B^{0}}}(\widetilde{w})\right)^{-1}\left(\mathrm{I}+w_{+}^{\Sigma_{B^{0}}}(\widetilde{w})\right), \widetilde{w} \in \Sigma_{B}$ (see Figure 5(a)), where


Figure 5: (a) $\Sigma_{B}$; (b) $\Sigma_{B, r}$; and (c) $\Sigma_{e}:=\Sigma_{B, r} \cup \mathbb{R}$. 


$$
\begin{aligned}
& w_{+}^{\Sigma_{B^{0}}}(\widetilde{w})=\left\{\begin{array}{ll}
(\widetilde{w})^{2 \mathrm{i} \nu} \mathrm{e}^{-\frac{\mathrm{i}}{2} \widetilde{w}^{2} \overline{r\left(\zeta_{1}\right)} \sigma_{+},} & \widetilde{w} \in \Sigma_{B}^{1}, \\
-(\widetilde{w})^{2 \mathrm{i} \nu} \mathrm{e}^{-\frac{\mathrm{i}}{2} \widetilde{w}^{2}} \frac{\frac{r\left(\zeta_{1}\right)}{\left(1-\mid r\left(\left.\zeta_{1}\right|^{2}\right)\right.}}{\left(\sigma_{+},\right.}, & \widetilde{w} \in \Sigma_{B}^{3},
\end{array} \quad w_{-}^{\Sigma_{B^{0}}}(\widetilde{w})=\left(\begin{array}{ll}
0 & 0 \\
0 & 0
\end{array}\right), \quad \widetilde{w} \in \Sigma_{B}^{1} \cup \Sigma_{B}^{3},\right. \\
& w_{-}^{\Sigma_{B^{0}}}(\widetilde{w})=\left\{\begin{array}{ll}
-(\widetilde{w})^{-2 \mathrm{i} \nu} \mathrm{e}^{\frac{\mathrm{i}}{2} \widetilde{w}^{2}} r\left(\zeta_{1}\right) \sigma_{-}, & \widetilde{w} \in \Sigma_{B}^{2}, \\
(\widetilde{w})^{-2 \mathrm{i} \nu} \mathrm{e}^{\frac{\mathrm{i}}{2} \widetilde{w}^{2}} \frac{r\left(\zeta_{1}\right)}{\left(1-\left|r\left(\zeta_{1}\right)\right|^{2}\right)} \sigma_{-}, & \widetilde{w} \in \Sigma_{B}^{4},
\end{array} \quad w_{+}^{\Sigma_{B^{0}}}(\widetilde{w})=\left(\begin{array}{ll}
0 & 0 \\
0 & 0
\end{array}\right), \quad \widetilde{w} \in \Sigma_{B}^{2} \cup \Sigma_{B}^{4} .\right.
\end{aligned}
$$

Define the functions (Proposition 5.2) $w_{ \pm}^{\Sigma_{B}}(\widetilde{w}):=\left(\left(\Delta_{B}^{0}\right)^{-1}\left(\mathcal{N}_{B} \widehat{w}_{ \pm}^{\widehat{\Sigma}_{B^{\prime}}}\right)\left(\Delta_{B}^{0}\right)\right)(\widetilde{w})$ and $w_{ \pm}^{\Sigma_{B^{0}}}(\widetilde{w}):=$ $\left(\left(\Delta_{B}^{0}\right)^{-1}\left(\mathcal{N}_{B} \widehat{w}_{ \pm}^{\widehat{\Sigma}_{B}^{0}}\right)\left(\Delta_{B}^{0}\right)\right)(\widetilde{w})$, with $\Delta_{B}^{0}$ defined by Eq. (107) and Proposition 5.1, and $\mathcal{N}_{B}$ defined by Eq. (105). Recall that $C_{w^{\Sigma_{B}}}^{\Sigma_{\Sigma_{B}}}:=C_{+}\left(\cdot w_{-}^{\Sigma_{B}}\right)+C_{-}\left(\cdot w_{+}^{\Sigma_{B}}\right)$, and set $C_{w^{\Sigma_{B}}}^{\Sigma_{B}}:=C_{+}\left(\cdot w_{-}^{\Sigma_{B^{0}}}\right)+$ $C_{-}\left(\cdot w_{+}^{\Sigma_{B^{0}}}\right)$. Since $w^{\Sigma_{q}}(\widetilde{w})=\sum_{l \in\{ \pm\}} w_{l}^{\Sigma_{q}}(\widetilde{w}), q \in\left\{B, B^{0}\right\}$, it follows from the above expressions for $w_{ \pm}^{\Sigma_{B^{0}}}(\widetilde{w})$, Lemma 5.1, and the linearity of $\mathcal{N}_{B}$ that, as $t \rightarrow+\infty$ such that $0<\zeta_{2}<\frac{1}{M}<$ $M<\zeta_{1}$ and $\left|\zeta_{3}\right|^{2}=1$, with $M \in \mathbb{R}_{>1}$ and bounded, $\left\|\left(w^{\Sigma_{B}}-w^{\Sigma_{B^{0}}}\right)(\cdot)\right\|_{\cap_{p \in\{1,2, \infty\}} \mathcal{L}_{\mathrm{M}_{2}(\mathbb{C})}^{p}\left(\Sigma_{B}\right)}=$ $\|\left.\left(\left(\Delta_{B}^{0}\right)^{-1}\left(\mathcal{N}_{B} \widehat{w}^{\widehat{\Sigma}_{B^{\prime}}}\right)\left(\Delta_{B}^{0}\right)-\left(\Delta_{B}^{0}\right)^{-1}\left(\mathcal{N}_{B} \widehat{w}^{\widehat{\Sigma}_{B^{0}}}\right)\left(\Delta_{B}^{0}\right)\right)(\cdot)\right|_{\cap_{p \in\{1,2, \infty\}} \mathcal{L}_{\mathrm{M}_{2}(\mathbb{C})}^{p}\left(\Sigma_{B}\right)} \leqslant \frac{\left|\mathcal{c}^{\mathcal{S}}\left(\zeta_{1}\right)\right|\left|\underline{c}\left(\zeta_{2}, \zeta_{3}, \overline{\zeta_{3}}\right)\right|}{\left|\zeta_{1}-\zeta_{3}\right| \sqrt{\left(\zeta_{1}-\zeta_{2}\right)}}$ $\cdot \frac{\ln t}{\sqrt{t}}$; hence, from the linearity of the Cauchy operators, $\left\|C_{w^{\Sigma_{B}}}^{\Sigma_{B}}-C_{w^{\Sigma_{B}}}^{\Sigma_{B}}\right\|_{\mathcal{N}\left(\Sigma_{B}\right)}=\| C_{+}\left(\cdot\left(w_{-}^{\Sigma_{B}}\right.\right.$ $\left.\left.w_{-}^{\Sigma_{B^{0}}}\right)\right)+\left.C_{-}\left(\cdot\left(w_{+}^{\Sigma_{B}}-w_{+}^{\Sigma_{B^{0}}}\right)\right)\right|_{\mathcal{N}\left(\Sigma_{B}\right)} \leqslant \frac{\left|c^{\mathcal{S}}\left(\zeta_{1}\right)\right|\left|\underline{\underline{c}\left(\zeta_{2}, \zeta_{3}, \overline{\zeta_{3}}\right)}\right|}{\left|\zeta_{1}-\zeta_{3}\right| \sqrt{\left(\zeta_{1}-\zeta_{2}\right)}} \frac{\ln t}{\sqrt{t}}$, and, consequently, via the second resolvent identity, one deduces that $\left(\mathbf{1}_{\Sigma_{B}}-C_{w^{\Sigma_{B}}}^{\Sigma_{B}}\right)^{-1} \in \mathcal{N}\left(\Sigma_{B}\right) \Rightarrow\left(\mathbf{1}_{\Sigma_{B}}-C_{w^{\Sigma_{B}}}^{\Sigma_{B}}\right)^{-1} \in \mathcal{N}\left(\Sigma_{B}\right)$. Reorient $\Sigma_{B}$ as in Figure $5(\mathrm{~b})$, and denote the reoriented contour as $\Sigma_{B, r}$. Define $w^{\Sigma_{B, r}}(\widetilde{w}):=$ $\sum_{l \in\{ \pm\}} w_{l}^{\Sigma_{B, r}}(\widetilde{w})$, where $w_{ \pm}^{\Sigma_{B, r}}(\widetilde{w})=\left\{\begin{array}{ll}w_{ \pm}^{\Sigma_{B^{0}}}(\widetilde{w}), & \widetilde{w} \in \Sigma_{B, r}^{1} \cup \Sigma_{B, r}^{2}, \\ -w_{\mp}^{\Sigma_{B^{0}}}(\widetilde{w}), & \widetilde{w} \in \Sigma_{B, r}^{3} \cup \Sigma_{B, r}^{4},\end{array}\right.$ and consider the operator $C_{w_{B, r}^{\Sigma_{B}}}^{\Sigma_{\Sigma_{1}}}:=C_{+}\left(\cdot w_{-}^{\Sigma_{B, r}}\right)+C_{-}\left(\cdot w_{+}^{\Sigma_{B, r}}\right)\left(=C_{w^{\Sigma} B^{0}}^{\Sigma_{B}}\right)$, where the Cauchy operators are now taken with respect to the oriented contour $\Sigma_{B, r}$; hence, from the second resolvent identity, $\left(\mathbf{1}_{\Sigma_{B, r}}-C_{w^{\Sigma_{B, r}}}^{\Sigma_{B, r}}\right)^{-1} \in \mathcal{N}\left(\Sigma_{B, r}\right) \Rightarrow\left(\mathbf{1}_{\Sigma_{B}}-C_{w^{\Sigma_{B}}}^{\Sigma_{B}}\right)^{-1} \in \mathcal{N}\left(\Sigma_{B}\right)$. Extend $\Sigma_{B, r} \rightarrow \Sigma_{e}:=\Sigma_{B, r} \cup \mathbb{R}$, with the orientation in Figure $5(\mathrm{c})$, and set $w_{ \pm}^{\Sigma_{e}}(\widetilde{w}):=\left\{\begin{array}{ll}w_{ \pm}^{\Sigma_{B, r}}(\widetilde{w}), & \widetilde{w} \in \Sigma_{B, r} \subset \Sigma_{e}, \\ \left(\begin{array}{cc}0 & 0 \\ 0 & 0\end{array}\right), & \widetilde{w} \in \Sigma_{e} \backslash \Sigma_{B, r} .\end{array}\right.$ Define $C_{w^{\Sigma_{e}}}^{\Sigma_{e}}:=C_{+}\left(\cdot w_{-}^{\Sigma_{e}}\right)+C_{-}\left(\cdot w_{+}^{\Sigma_{e}}\right)$, with the Cauchy operators now taken with respect to the oriented contour $\Sigma_{e}$; hence, via the second resolvent identity, it follows that $\left(\mathbf{1}_{\Sigma_{e}}-C_{w^{\Sigma_{e}}}^{\Sigma_{e}}\right)^{-1} \in$ $\mathcal{N}\left(\Sigma_{e}\right) \Rightarrow\left(\mathbf{1}_{\Sigma_{B, r}}-C_{w^{\Sigma_{B, r}}}^{\Sigma_{B, r}}\right)^{-1} \in \mathcal{N}\left(\Sigma_{B, r}\right)$. Define the following piecewise-analytic $2 \times 2$ matrixvalued function:

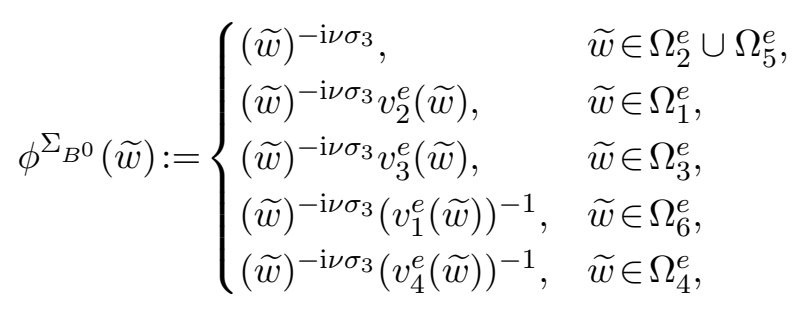

where

$$
\begin{aligned}
& v_{1}^{e}(\widetilde{w})=(\widetilde{w})^{\mathrm{i} \nu \operatorname{ad}\left(\sigma_{3}\right)} \exp \left(-\frac{\mathrm{i}}{4} \widetilde{w}^{2} \operatorname{ad}\left(\sigma_{3}\right)\right)\left(\mathrm{I}+\overline{r\left(\zeta_{1}\right)} \sigma_{+}\right), \\
& v_{2}^{e}(\widetilde{w})=(\widetilde{w})^{\mathrm{i} \nu \operatorname{ad}\left(\sigma_{3}\right)} \exp \left(-\frac{\mathrm{i}}{4} \widetilde{w}^{2} \operatorname{ad}\left(\sigma_{3}\right)\right)\left(\mathrm{I}-r\left(\zeta_{1}\right) \sigma_{-}\right), \\
& v_{3}^{e}(\widetilde{w})=(\widetilde{w})^{\mathrm{i} \nu \operatorname{ad}\left(\sigma_{3}\right)} \exp \left(-\frac{\mathrm{i}}{4} \widetilde{w}^{2} \operatorname{ad}\left(\sigma_{3}\right)\right)\left(\mathrm{I}+\frac{\overline{r\left(\zeta_{1}\right)}}{\left(1-\left|r\left(\zeta_{1}\right)\right|^{2}\right)} \sigma_{+}\right), \\
& v_{4}^{e}(\widetilde{w})=(\widetilde{w})^{\mathrm{i} \nu \operatorname{ad}\left(\sigma_{3}\right)} \exp \left(-\frac{\mathrm{i}}{4} \widetilde{w}^{2} \operatorname{ad}\left(\sigma_{3}\right)\right)\left(\mathrm{I}-\frac{r\left(\zeta_{1}\right)}{\left(1-\left|r\left(\zeta_{1}\right)\right|^{2}\right)} \sigma_{-}\right),
\end{aligned}
$$


with

$$
v_{1}^{e}(\widetilde{w})-\mathrm{I} \underset{\substack{\widetilde{w} \rightarrow \infty \\ \widetilde{w} \in \Omega_{6}^{e}}}{=} o(1), \quad v_{2}^{e}(\widetilde{w})-\mathrm{I} \underset{\substack{\widetilde{w} \rightarrow \infty \\ \widetilde{w} \in \Omega_{1}^{e}}}{=} o(1), \quad v_{3}^{e}(\widetilde{w})-\mathrm{I}_{\substack{\widetilde{w} \rightarrow \infty \\ \widetilde{w} \in \Omega_{3}^{e}}}^{=} o(1), \quad v_{4}^{e}(\widetilde{w})-\mathrm{I}_{\substack{\widetilde{w} \rightarrow \infty \\ w \in \Omega_{4}^{e}}}^{=} o(1) .
$$

For $\widetilde{w} \in \Sigma_{e}$, set $\mathcal{V}^{e, \phi}(\widetilde{w}):=\phi_{-}^{\Sigma_{B^{0}}}(\widetilde{w})\left(\mathrm{I}-w_{-}^{\Sigma_{e}}(\widetilde{w})\right)^{-1}\left(\mathrm{I}+w_{+}^{\Sigma_{e}}(\widetilde{w})\right)\left(\phi_{+}^{\Sigma_{B^{0}}}(\widetilde{w})\right)^{-1}$, where $\phi_{ \pm}^{\Sigma_{B^{0}}}(\widetilde{w}):=$

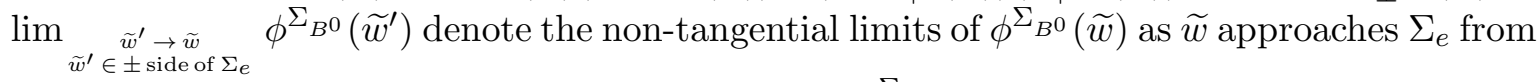
the " \pm " sides, respectively. From the definition of $w_{ \pm}^{\Sigma_{e}}(\widetilde{w})$ given above, for $\widetilde{w} \in \Sigma_{B, r}\left(\subset \Sigma_{e}\right)$, $\mathcal{V}^{e, \phi}(\widetilde{w})=\mathrm{I}$, and, for $\widetilde{w} \in \Sigma_{e} \backslash \Sigma_{B, r}(=\mathbb{R}), \mathcal{V}^{e, \phi}(\widetilde{w})=\phi_{-}^{\Sigma_{B^{0}}}(\widetilde{w})\left(\phi_{+}^{\Sigma_{B^{0}}}(\widetilde{w})\right)^{-1}$; hence, from the definition of $\phi^{\Sigma_{B^{0}}}(\widetilde{w})$ and the formulae for $v_{k}^{e}(\widetilde{w}), k \in\{1,2,3,4\}$, given above, taking the principal branch of the logarithm for $\widetilde{w}<0$, one shows that $\mathcal{V}^{e, \phi}(\widetilde{w})= \begin{cases}\mathrm{e}^{-\frac{\mathrm{i}}{4} \widetilde{w}^{2} \operatorname{ad}\left(\sigma_{3}\right)} V\left(\zeta_{1}\right), & \widetilde{w} \in \mathbb{R}_{+}, \\ \mathrm{e}^{-\frac{i}{4} \widetilde{w}^{2} \operatorname{ad}\left(\sigma_{3}\right)} V\left(\zeta_{1}\right), & -\widetilde{w} \in \mathbb{R}_{+},\end{cases}$


$\left.\|r(\cdot)\|_{\mathcal{L}^{\infty}(\mathbb{R})}<1\right)$. Hence, the jump matrix, $\mathcal{V}^{e, \phi}(\widetilde{w})$, is characterised as follows: $\mathcal{V}^{e, \phi}(\widetilde{w})=$ $\left\{\begin{array}{ll}\mathrm{I}, & \widetilde{w} \in \Sigma_{B, r}, \\ \mathrm{e}^{-\frac{\mathrm{i}}{4} \widetilde{w}^{2} \operatorname{ad}\left(\sigma_{3}\right)}\left(\begin{array}{cc}1-\left|r\left(\zeta_{1}\right)\right|^{2}-\overline{r\left(\zeta_{1}\right)} \\ r\left(\zeta_{1}\right) & 1\end{array}\right), & \widetilde{w} \in \Sigma_{e} \backslash \Sigma_{B, r} .\end{array}\right.$ On $\mathbb{R}$, one has that $\mathcal{V}^{e, \phi}(\widetilde{w})=\left(\mathrm{I}-\overline{r\left(\zeta_{1}\right)} \mathrm{e}^{-\frac{\mathrm{i}}{2} \widetilde{w}^{2}}\right.$ $\left.\cdot \sigma_{+}\right)\left(\mathrm{I}+r\left(\zeta_{1}\right) \mathrm{e}^{\frac{\mathrm{i}}{2} \widetilde{w}^{2}} \sigma_{-}\right):=\left(\mathrm{I}-w_{-}^{e, \phi}(\widetilde{w})\right)^{-1}\left(\mathrm{I}+w_{+}^{e, \phi}(\widetilde{w})\right)$. Let $C_{w_{e}^{e, \phi}}^{\Sigma_{e}}:=C_{+}\left(\cdot w_{-}^{e, \phi}\right)+C_{-}\left(\cdot w_{+}^{e, \phi}\right)$ be the associated operator on $\Sigma_{e}$, with $w^{e, \phi}(\widetilde{w}):=\sum_{l \in\{ \pm\}} w_{l}^{e, \phi}(\widetilde{w})$, and the orientation is that of $\Sigma_{e}$ : the boundedness of $\left\|\left(1_{\Sigma_{e}}-C_{w^{\Sigma_{e}}}^{\Sigma_{e}}\right)^{-1}\right\|_{\mathcal{N}\left(\Sigma_{e}\right)}$ follows from the boundedness of $\|\left(\mathbf{1}_{\Sigma_{e}} \mathbb{R}-\right.$ $\left.C_{w_{e}^{e, \phi} \mathbb{R}}^{\Sigma_{e} \mathbb{R}}\right)^{-1} \|_{\mathcal{N}\left(\Sigma_{e} \mathbb{R}\right)}$, that is, $\left(\mathbf{1}_{\Sigma_{e} \mid \mathbb{R}}-C_{w^{e, \phi} \mathbb{R}}^{\Sigma_{e} \mathbb{R}}\right)^{-1} \in \mathcal{N}\left(\Sigma_{e} \mid \mathbb{R}\right) \Rightarrow\left(1_{\Sigma_{e}}-C_{w^{\Sigma_{e}}}^{\Sigma_{e}}\right)^{-1} \in \mathcal{N}\left(\Sigma_{e}\right)$, where $C_{w^{e, \phi} \mathbb{R}}^{\Sigma_{e} \uparrow \mathbb{R}}: \mathcal{L}_{\mathrm{M}_{2}(\mathbb{C})}^{2}(\mathbb{R}) \rightarrow \mathcal{L}_{\mathrm{M}_{2}(\mathbb{C})}^{2}(\mathbb{R})$ is the operator associated with the restriction of $w^{e, \phi}(\widetilde{w})$ to $\mathbb{R}$. Now, ||$C_{w^{e, \phi} \mathbb{R}}^{\Sigma_{e} \mathbb{R}}||_{\mathcal{N}(\mathbb{R})} \leqslant \max _{1 \leqslant i, j \leqslant 2} \sup _{\widetilde{w} \in \mathbb{R}}\left|w_{+}^{e, \phi}(\widetilde{w})+w_{-}^{e, \phi}(\widetilde{w})\right| \leqslant \sup _{\widetilde{w} \in \mathbb{R}}\left|\mathrm{e}^{-\frac{i}{2} \widetilde{w}^{2}} \overline{r\left(\zeta_{1}\right)}\right|$ $\left(=\sup _{\widetilde{w} \in \mathbb{R}}\left|\mathrm{e}^{\frac{\mathrm{i}}{2} \widetilde{w}^{2}} r\left(\zeta_{1}\right)\right|\right) \leqslant\|r(\cdot)\|_{\mathcal{L}^{\infty}(\mathbb{R})}<1$; hence, by the second resolvent identity, $\|\left(\mathbf{1}_{\Sigma_{e} \mid \mathbb{R}}-\right.$ $\left.C_{w^{e, \phi} \mid \mathbb{R}}^{\Sigma_{e} \mathbb{R}}\right)^{-1} \|_{\mathcal{N}\left(\Sigma_{e} \mid \mathbb{R}\right)} \leqslant\left|\underline{c}\left(\zeta_{1}, \zeta_{2}, \zeta_{3}, \overline{\zeta_{3}}\right)\right|\left(1-\|r(\cdot)\|_{\mathcal{L}^{\infty}(\mathbb{R})}\right)^{-1}<\infty$, whence, $\left(\mathbf{1}_{\Sigma_{e}}-C_{w^{\Sigma_{e}}}^{\Sigma_{\Sigma_{e}}}\right)^{-1} \in \mathcal{N}\left(\Sigma_{e}\right)$ : this completes the proof.

\section{Asymptotic Solution of the Model RHP}

In this section the $\mathcal{O}(1)$ asymptotics of $\mu^{\Sigma^{\sharp}}(\zeta):=\left(\left(\mathbf{1}_{\Sigma^{\sharp}}-C_{w^{\Sigma^{\sharp}}}^{\Sigma^{\sharp}}\right)^{-1} \mathrm{I}\right)(\zeta)$ (Eq. (103)), having an explicit representation in terms of parabolic cylinder functions, is obtained, and the RHP for $m^{c}(\zeta)$ formulated in Lemma 2.6 is solved asymptotically, whence, the leading-order asymptotics of $u(x, t)$ and related integrals are derived.

Lemma 6.1. Let $\varepsilon$ be an arbitrarily fixed, sufficiently small positive real number, and, for $\lambda \in\left\{\zeta_{2}, \zeta_{1}\right\}$, set $\mathbb{U}(\lambda ; \varepsilon):=\{z ;|z-\lambda|<\varepsilon\}$. Then, as $t \rightarrow+\infty$ such that $0<\zeta_{2}<\frac{1}{M}<M<\zeta_{1}$ and $\left|\zeta_{3}\right|^{2}=1$, with $M \in \mathbb{R}_{>1}$ and bounded, for $\zeta \in \mathbb{C} \backslash \cup_{\lambda \in\left\{\zeta_{2}, \zeta_{1}\right\}} \mathbb{U}(\lambda ; \varepsilon), m^{c}(\zeta)$ has the following 
asymptotics,

$$
\begin{aligned}
& m_{11}^{c}(\zeta)=\delta(\zeta)\left(1+\mathcal{O}\left(\left(\frac{c^{\mathcal{S}}\left(\zeta_{1}\right) \underline{c}\left(\zeta_{2}, \zeta_{3}, \overline{\zeta_{3}}\right)}{\sqrt{\zeta_{2}\left(z_{o}^{2}+32\right)}\left(\zeta-\zeta_{1}\right)}+\frac{c^{\mathcal{S}}\left(\zeta_{2}\right) \underline{c}\left(\zeta_{1}, \zeta_{3}, \overline{\zeta_{3}}\right)}{\sqrt{\zeta_{1}\left(z_{o}^{2}+32\right)}\left(\zeta-\zeta_{2}\right)}\right) \frac{\ln t}{\left(\zeta_{1}-\zeta_{2}\right) t}\right)\right) \\
& m_{12}^{c}(\zeta)=\frac{\mathrm{e}^{\frac{\mathrm{i} \Omega^{+}(0)}{2}}}{\delta(\zeta)}\left(\frac{\sqrt{\nu\left(\zeta_{1}\right)} \zeta_{1}^{2 \mathrm{i} \nu\left(\zeta_{1}\right)}}{\sqrt{t\left(\zeta_{1}-\zeta_{2}\right)}\left(z_{o}^{2}+32\right)^{1 / 4}}\left(\frac{\zeta_{1} \mathrm{e}^{-\mathrm{i}\left(\Theta^{+}\left(z_{o}, t\right)+\frac{\pi}{4}\right)}}{\left(\zeta-\zeta_{1}\right)}+\frac{\zeta_{2} \mathrm{e}^{\mathrm{i}\left(\Theta^{+}\left(z_{o}, t\right)+\frac{\pi}{4}\right)}}{\left(\zeta-\zeta_{2}\right)}\right)\right. \\
& \left.+\mathcal{O}\left(\left(\frac{c^{\mathcal{S}}\left(\zeta_{1}\right) \underline{c}\left(\zeta_{2}, \zeta_{3}, \overline{\zeta_{3}}\right)}{\sqrt{\zeta_{2}\left(z_{o}^{2}+32\right)}\left(\zeta-\zeta_{1}\right)}+\frac{c^{\mathcal{S}}\left(\zeta_{2}\right) \underline{c}\left(\zeta_{1}, \zeta_{3}, \overline{\zeta_{3}}\right)}{\sqrt{\zeta_{1}\left(z_{o}^{2}+32\right)}\left(\zeta-\zeta_{2}\right)}\right) \frac{\ln t}{\left(\zeta_{1}-\zeta_{2}\right) t}\right)\right), \\
& m_{21}^{c}(\zeta)=\frac{\delta(\zeta)}{\mathrm{e}^{\frac{\mathrm{i} \Omega^{+}(0)}{2}}}\left(\frac{\sqrt{\nu\left(\zeta_{1}\right)} \zeta_{1}^{-2 \mathrm{i} \nu\left(\zeta_{1}\right)}}{\sqrt{t\left(\zeta_{1}-\zeta_{2}\right)}\left(z_{o}^{2}+32\right)^{1 / 4}}\left(\frac{\zeta_{1} \mathrm{e}^{\mathrm{i}\left(\Theta^{+}\left(z_{o}, t\right)+\frac{\pi}{4}\right)}}{\left(\zeta-\zeta_{1}\right)}+\frac{\zeta_{2} \mathrm{e}^{-\mathrm{i}\left(\Theta^{+}\left(z_{o}, t\right)+\frac{\pi}{4}\right)}}{\left(\zeta-\zeta_{2}\right)}\right)\right. \\
& \left.+\mathcal{O}\left(\left(\frac{c^{\mathcal{S}}\left(\zeta_{1}\right) \underline{c}\left(\zeta_{2}, \zeta_{3}, \overline{\zeta_{3}}\right)}{\sqrt{\zeta_{2}\left(z_{o}^{2}+32\right)}\left(\zeta-\zeta_{1}\right)}+\frac{c^{\mathcal{S}}\left(\zeta_{2}\right) \underline{c}\left(\zeta_{1}, \zeta_{3}, \overline{\zeta_{3}}\right)}{\sqrt{\zeta_{1}\left(z_{o}^{2}+32\right)}\left(\zeta-\zeta_{2}\right)}\right) \frac{\ln t}{\left(\zeta_{1}-\zeta_{2}\right) t}\right)\right),
\end{aligned}
$$

$$
m_{22}^{c}(\zeta)=\frac{1}{\delta(\zeta)}\left(1+\mathcal{O}\left(\left(\frac{c^{\mathcal{S}}\left(\zeta_{1}\right) \underline{c}\left(\zeta_{2}, \zeta_{3}, \overline{\zeta_{3}}\right)}{\sqrt{\zeta_{2}\left(z_{o}^{2}+32\right)}\left(\zeta-\zeta_{1}\right)}+\frac{c^{\mathcal{S}}\left(\zeta_{2}\right) \underline{c}\left(\zeta_{1}, \zeta_{3}, \overline{\zeta_{3}}\right)}{\sqrt{\zeta_{1}\left(z_{o}^{2}+32\right)}\left(\zeta-\zeta_{2}\right)}\right) \frac{\ln t}{\left(\zeta_{1}-\zeta_{2}\right) t}\right)\right)
$$

where $\delta(\zeta)$ is given in Proposition 4.1, $\nu(z), \Theta^{+}\left(z_{o}, t\right), \Omega^{+}(z)$, and $\left\{\zeta_{i}\right\}_{i=1}^{3}$ are defined in Theorem 3.1, Eqs. (12)-(14), (16), and (17), $\sup _{\zeta \in \mathbb{C} \backslash \cup_{\lambda \in\left\{\zeta_{2}, \zeta_{1}\right\}} \mathbb{U}(\lambda ; \varepsilon)}\left|\left(\zeta-\zeta_{k}\right)^{-1}\right| \leqslant M^{c}$, with $M^{c} \in \mathbb{R}_{+}$(and bounded), $k \in\{1,2\}, m^{c}(\zeta)=\sigma_{1} \overline{m^{c}(\bar{\zeta})} \sigma_{1}$, and $\left(m^{c}(0) \sigma_{2}\right)^{2}=\mathrm{I}$.

Proof. From Lemma 5.3, the fact that $w_{ \pm}^{\Sigma_{k^{\prime}}}(\zeta):=w_{ \pm}^{\Sigma^{\sharp}}(\zeta)\left\lceil\Sigma_{k^{\prime}}, k \in\{A, B\}, C_{w^{\Sigma^{\sharp}}}^{\Sigma^{\sharp}}:=\right.$ $\sum_{k \in\{A, B\}} C_{w^{\Sigma} k^{\prime}}^{\Sigma_{k^{\prime}}}$, and Lemma 4.6, one shows that, as $t \rightarrow+\infty$ such that $0<\zeta_{2}<\frac{1}{M}<M<\zeta_{1}$ 
and $\left|\zeta_{3}\right|^{2}=1$, with $M \in \mathbb{R}_{>1}$ and bounded, for $\zeta \in \mathbb{C} \backslash \Sigma_{A^{\prime}} \cup \Sigma_{B^{\prime}}$,

$$
\begin{aligned}
& m_{11}^{\Sigma^{\sharp}}(\zeta)=1-\left(\int_{\zeta_{1}+\varepsilon \mathrm{e}^{-\frac{3 \pi \mathrm{i}}{4}}}^{\zeta_{1}+0^{-} \mathrm{e}^{-\frac{3 \pi \mathrm{i}}{4}}}+\int_{\zeta_{1}+\infty \mathrm{e}^{\frac{\mathrm{i} \pi}{4}}}^{\zeta_{1}+0^{+} \mathrm{e}^{\frac{\mathrm{i} \pi}{4}}}\right) \frac{\mu_{12}^{\Sigma_{B^{\prime}}}(\xi) \overline{\mathcal{R}(\xi)}(\delta(\xi))^{-2} \mathrm{e}^{2 \mathrm{i} t \theta^{u}(\xi)}}{(\xi-\zeta)} \frac{\mathrm{d} \xi}{2 \pi \mathrm{i}} \\
& -\left(\int_{\zeta_{2}+0^{+} \mathrm{e}^{-\frac{\mathrm{i} \pi}{4}}}^{\zeta_{2}+\varepsilon \mathrm{e}^{-\frac{\mathrm{i} \pi}{4}}}+\int_{\zeta_{2}+0^{-} \mathrm{e}^{\frac{3 \pi \mathrm{i}}{4}}}^{\zeta_{2}+\varepsilon \mathrm{e}^{\frac{3 \pi \mathrm{i}}{4}}}\right) \frac{\mu_{12}^{\Sigma_{A^{\prime}}}(\xi) \overline{\mathcal{R}(\xi)}(\delta(\xi))^{-2} \mathrm{e}^{2 \mathrm{i} t \theta^{u}(\xi)}}{(\xi-\zeta)} \frac{\mathrm{d} \xi}{2 \pi \mathrm{i}}+\mathcal{E}_{11}^{\Sigma^{\sharp}}(\zeta), \\
& m_{12}^{\Sigma^{\sharp}}(\zeta)=\left(\int_{\zeta_{1}+\infty \mathrm{e}^{-\frac{\mathrm{i} \pi}{4}}}^{\zeta_{1}+0^{+} \mathrm{e}^{-\frac{\mathrm{i} \pi}{4}}}+\int_{\zeta_{1}+\varepsilon \mathrm{e}^{\frac{3 \pi \mathrm{i}}{4}}}^{\zeta_{1}+0^{-} \mathrm{e}^{\frac{3 \pi \mathrm{i}}{4}}}\right) \frac{\mu_{11}^{\Sigma_{B^{\prime}}}(\xi) \mathcal{R}(\xi)(\delta(\xi))^{2} \mathrm{e}^{-2 \mathrm{i} t \theta^{u}(\xi)}}{(\xi-\zeta)} \frac{\mathrm{d} \xi}{2 \pi \mathrm{i}}
\end{aligned}
$$

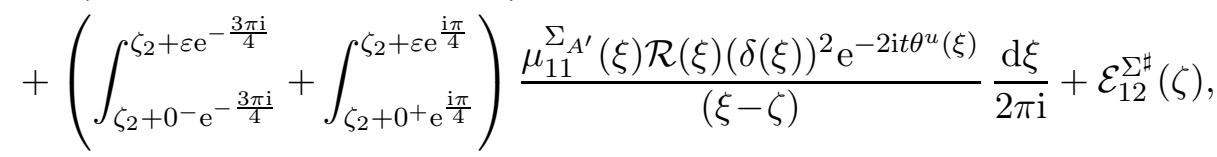

$$
\begin{aligned}
& m_{21}^{\Sigma^{\sharp}}(\zeta)=-\left(\int_{\zeta_{1}+\varepsilon \mathrm{e}^{-\frac{3 \pi \mathrm{i}}{4}}}^{\zeta_{1}+0^{-} \mathrm{e}^{-\frac{3 \pi \mathrm{i}}{4}}}+\int_{\zeta_{1}+\infty \mathrm{e}^{\frac{\mathrm{i} \pi}{4}}}^{\zeta_{1}+0^{+} \mathrm{e}^{\frac{\mathrm{i} \pi}{4}}}\right) \frac{\mu_{22}^{\Sigma_{B^{\prime}}}(\xi) \overline{\mathcal{R}(\xi)}(\delta(\xi))^{-2} \mathrm{e}^{2 \mathrm{i} t \theta^{u}(\xi)}}{(\xi-\zeta)} \frac{\mathrm{d} \xi}{2 \pi \mathrm{i}} \\
& -\left(\int_{\zeta_{2}+0^{+} \mathrm{e}^{-\frac{\mathrm{i} \pi}{4}}}^{\zeta_{2}+\varepsilon \mathrm{e}^{-\frac{\mathrm{i} \pi}{4}}}+\int_{\zeta_{2}+0^{-} \mathrm{e}^{\frac{3 \pi \mathrm{i}}{4}}}^{\zeta_{2}+\varepsilon \mathrm{e}^{\frac{3 \pi \mathrm{i}}{4}}}\right) \frac{\mu_{22}^{\Sigma_{A^{\prime}}}(\xi) \overline{\mathcal{R}(\xi)}(\delta(\xi))^{-2} \mathrm{e}^{2 \mathrm{i} t \theta^{u}(\xi)}}{(\xi-\zeta)} \frac{\mathrm{d} \xi}{2 \pi \mathrm{i}}+\mathcal{E}_{21}^{\Sigma^{\sharp}}(\zeta), \\
& m_{22}^{\Sigma^{\sharp}}(\zeta)=1+\left(\int_{\zeta_{1}+\infty \mathrm{e}^{-\frac{\mathrm{i} \pi}{4}}}^{\zeta_{1}+0^{+} \mathrm{e}^{-\frac{\mathrm{i} \pi}{4}}}+\int_{\zeta_{1}+\varepsilon \mathrm{e}^{\frac{3 \pi \mathrm{i}}{4}}}^{\zeta_{1}+0^{-} \mathrm{e}^{\frac{3 \pi \mathrm{i}}{4}}}\right) \frac{\mu_{21}^{\Sigma_{B^{\prime}}}(\xi) \mathcal{R}(\xi)(\delta(\xi))^{2} \mathrm{e}^{-2 \mathrm{i} t \theta^{u}(\xi)}}{(\xi-\zeta)} \frac{\mathrm{d} \xi}{2 \pi \mathrm{i}} \\
& +\left(\int_{\zeta_{2}+0^{-} \mathrm{e}^{-\frac{3 \pi \mathrm{i}}{4}}}^{\zeta_{2}+\varepsilon \mathrm{e}^{-\frac{3 \pi \mathrm{i}}{4}}}+\int_{\zeta_{2}+0^{+} \mathrm{e}^{\mathrm{i} \pi}}^{\zeta_{2}+\varepsilon \mathrm{e}^{\frac{\mathrm{i} \pi}{4}}}\right) \frac{\sum_{21}^{\Sigma_{A^{\prime}}}(\xi) \mathcal{R}(\xi)(\delta(\xi))^{2} \mathrm{e}^{-2 \mathrm{i} t \theta^{u}(\xi)}}{2 \pi \mathrm{i}}+\mathcal{E}_{22}^{\Sigma^{\sharp}}(\zeta),
\end{aligned}
$$

where $\mu^{\Sigma_{l}}(\zeta):=\left(\left(\mathbf{1}_{\Sigma_{l}}-C_{w^{\Sigma_{l}}}^{\Sigma_{l}}\right)^{-1} \mathrm{I}\right)(\zeta), l \in\{A, B\}, \varepsilon$ is an arbitrarily fixed, sufficiently small positive real number, and $\mathcal{E}_{i j}^{\Sigma^{\sharp}}(\zeta):=\mathcal{O}\left(\frac{\underline{c}\left(\zeta_{1}, \zeta_{2}, \zeta_{3}, \overline{\zeta_{3}}\right) f_{i j}^{\Sigma_{i j}^{\sharp}}(\zeta)}{\left(\zeta_{1}-\zeta_{2}\right)^{2}\left|z_{o}+\zeta_{1}+\zeta_{2}\right| t}\right)$, with $\left\|f_{i j}^{\Sigma^{\sharp}}(\cdot)\right\|_{\mathcal{L}^{\infty}\left(\mathbb{C} \backslash \Sigma_{A^{\prime}} \cup \Sigma_{B^{\prime}}\right)}<\infty$. In order to proceed, explicit expressions for the $\mathcal{O}(1)$ asymptotics of $\mu^{\Sigma_{k^{\prime}}}(\zeta), k \in\{A, B\}$, are needed: without loss of generality, $\mu^{\Sigma_{B^{\prime}}}\left(\zeta\right.$ ), associated with $\zeta_{1}$, is considered in detail (an analogous argument applies for $\mu^{\Sigma_{A^{\prime}}}(\zeta)$, associated with $\left.\zeta_{2}\right)$. From the proof of Lemma 5.4, introduce the $2 \times 2$ matrix-valued function $\mathcal{D}^{\Sigma_{B^{0}}}(\widetilde{w}):=m^{\Sigma_{B^{0}}}(\widetilde{w})\left(\phi^{\Sigma_{B^{0}}}(\widetilde{w})\right)^{-1} \exp \left(-\frac{\mathrm{i}}{4} \widetilde{w}^{2} \sigma_{3}\right), \widetilde{w} \in$ $\mathbb{C} \backslash \Sigma_{e}$, and note that, for $\widetilde{w} \in \Sigma_{B}\left(=\Sigma_{e} \backslash \mathbb{R}\right), \mathcal{D}_{+}^{\Sigma_{B^{0}}}(\widetilde{w})=\mathcal{D}_{-}^{\Sigma_{B^{0}}}(\widetilde{w})$, and, for $\widetilde{w} \in \mathbb{R}\left(=\Sigma_{e} \backslash \Sigma_{B}\right)$, oriented from $-\infty$ to $+\infty, \mathcal{D}_{+}^{\Sigma_{B} 0}(\widetilde{w})=\mathcal{D}_{-}^{\Sigma_{B^{0}}}(\widetilde{w}) V\left(\zeta_{1}\right)$, where $V\left(\zeta_{1}\right):=\left(\begin{array}{c}1-\left|r\left(\zeta_{1}\right)\right|^{2}-\overline{r\left(\zeta_{1}\right)} \\ r\left(\zeta_{1}\right)\end{array}\right)$, with (cf. the asymptotics for $v_{k}^{e}(\widetilde{w}), k \in\{1,2,3,4\}$, given in the proof of Lemma 5.4) asymptotics $\mathcal{D}^{\Sigma_{B^{0}}}(\widetilde{w})=\underset{\substack{\widetilde{w} \rightarrow \infty \\ \widetilde{w} \in \mathbb{C} \backslash \mathbb{R}}}{ }\left(\mathrm{I}-\frac{1}{\widetilde{w}} m_{1}^{\Sigma_{B^{0}}}+\mathcal{O}\left(\frac{1}{\widetilde{w}^{2}}\right)\right)(\widetilde{w})^{\mathrm{i} \nu \sigma_{3}} \exp \left(-\frac{\mathrm{i}}{4} \widetilde{w}^{2} \sigma_{3}\right)$, and $\mathcal{D}^{\Sigma_{B^{0}}}(\widetilde{w})=\sigma_{1} \overline{\mathcal{D}^{\Sigma_{B^{0}}}(\overline{\widetilde{w}})} \sigma_{1} ;$

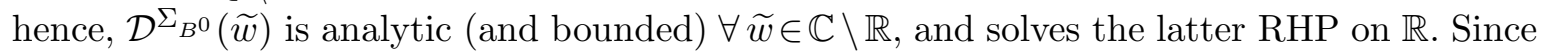
$\operatorname{det}\left(\mathcal{D}^{\Sigma_{B^{0}}}(\widetilde{w})\right)$ is an analytic and bounded $\mathbb{C}$-valued function, it is, by Liouville's Theorem, a constant: in this case, since $\operatorname{det}\left(V\left(\zeta_{1}\right)\right)=1, \operatorname{det}\left(\mathcal{D}^{\Sigma_{B} 0}(\widetilde{w})\right)=1$. By (partial) differentiation, it follows that $\partial_{\widetilde{w}} \mathcal{D}_{+}^{\Sigma_{B^{0}}}(\widetilde{w})=\partial_{\widetilde{w}} \mathcal{D}_{-}^{\Sigma_{B^{0}}}(\widetilde{w}) V\left(\zeta_{1}\right), \widetilde{w} \in \mathbb{R}$; hence, $\partial_{\widetilde{w}} \mathcal{D}^{\Sigma_{B^{0}}}(\widetilde{w})\left(\mathcal{D}^{\Sigma_{B^{0}}}(\widetilde{w})\right)^{-1}$ has no jumps across $\mathbb{R}$, and is an entire function of $\widetilde{w}$. Recalling the definition of $\mathcal{D}^{\Sigma_{B}}(\widetilde{w})$ given above, and its asymptotics, one shows that $\left(\partial_{\widetilde{w}} \mathcal{D}^{\Sigma_{B^{0}}}(\widetilde{w})+\frac{i}{2} \widetilde{w} \sigma_{3} \mathcal{D}^{\Sigma_{B^{0}}}(\widetilde{w})\right)\left(\mathcal{D}^{\Sigma_{B^{0}}}(\widetilde{w})\right)^{-1}=\underset{\substack{\widetilde{w} \rightarrow \infty \\ \widetilde{c} \backslash \mathbb{R}}}{\underset{w}{w}}$ $-\frac{i}{2}\left[\sigma_{3}, m_{1}^{\Sigma_{B^{0}}}\right]+\mathcal{O}\left(\widetilde{w}^{-1}\right)$ : applying, now, a generalisation of Liouville's Theorem to the lefthand side of the latter asymptotics, one arrives at the following (linear) matrix ODE for 
$\mathcal{D}^{\Sigma_{B^{0}}}(\widetilde{w})$

$$
\partial_{\widetilde{w}} \mathcal{D}^{\Sigma_{B^{0}}}(\widetilde{w})+\frac{i}{2} \widetilde{w} \sigma_{3} \mathcal{D}^{\Sigma_{B^{0}}}(\widetilde{w})=\beta^{\Sigma_{B^{0}}} \mathcal{D}^{\Sigma_{B^{0}}}(\widetilde{w})
$$

where $\beta^{\Sigma_{B^{0}}}:=-\frac{\mathrm{i}}{2}\left[\sigma_{3}, m_{1}^{\Sigma_{B^{0}}}\right]=\beta_{21}^{\Sigma_{B^{0}}} \sigma_{-}+\beta_{12}^{\Sigma_{B^{0}}} \sigma_{+}$, whence, since $\left(m_{1}^{\Sigma_{B^{0}}}\right)_{12}=\overline{\left(m_{1}^{\Sigma_{B^{0}}}\right)_{21}}$, $\beta_{12}^{\Sigma_{B^{0}}}=\overline{\beta_{21}^{\Sigma_{B^{0}}}}$. The method of solution for such matrix ODEs is well known (see, for example, 6, 27, 47]): following [27] (see Section 4, pp. 350-353), and recalling that $\mathcal{D}_{+}^{\Sigma_{B}}(\widetilde{w})=$ $\mathcal{D}_{-}^{\Sigma_{B^{0}}}(\widetilde{w})\left(\begin{array}{c}1-\left|r\left(\zeta_{1}\right)\right|^{2} \\ r\left(\zeta_{1}\right)\end{array}-\overline{r\left(\zeta_{1}\right)}\right), \widetilde{w} \in \mathbb{R}$, one shows that the solution of the above matrix ODE for $\mathcal{D}^{\Sigma_{B^{0}}}(\widetilde{w})$ is $\left( \pm \leftrightarrow \widetilde{w} \in \mathbb{C}_{ \pm}\right)$

$$
\begin{gathered}
\left(\mathcal{D}_{+}^{\Sigma_{B^{0}}}(\widetilde{w})\right)_{11}=\mathrm{e}^{-\frac{3 \pi \nu}{4}} \mathbf{D}_{\mathrm{i} \nu}\left(\mathrm{e}^{-\frac{3 \pi \mathrm{i}}{4}} \widetilde{w}\right), \quad\left(\mathcal{D}_{+}^{\Sigma_{B^{0}}}(\widetilde{w})\right)_{22}=\mathrm{e}^{\frac{\pi \nu}{4}} \mathbf{D}_{-\mathrm{i} \nu}\left(\mathrm{e}^{-\frac{\mathrm{i} \pi}{4}} \widetilde{w}\right), \\
\left(\mathcal{D}_{+}^{\Sigma_{B^{0}}}(\widetilde{w})\right)_{12}=\left(\beta_{21}^{\Sigma_{B^{0}}}\right)^{-1} \mathrm{e}^{\frac{\pi \nu}{4}}\left(\partial_{\widetilde{w}} \mathbf{D}_{-\mathrm{i} \nu}\left(\mathrm{e}^{-\frac{\mathrm{i} \pi}{4}} \widetilde{w}\right)-\frac{\mathrm{i}}{2} \widetilde{w} \mathbf{D}_{-\mathrm{i} \nu}\left(\mathrm{e}^{-\frac{\mathrm{i} \pi}{4}} \widetilde{w}\right)\right) \\
\left(\mathcal{D}_{+}^{\Sigma_{B^{0}}}(\widetilde{w})\right)_{21}=\left(\beta_{12}^{\Sigma_{B^{0}}}\right)^{-1} \mathrm{e}^{-\frac{3 \pi \nu}{4}}\left(\partial_{\widetilde{w}} \mathbf{D}_{\mathrm{i} \nu}\left(\mathrm{e}^{-\frac{3 \pi \mathrm{i}}{4}} \widetilde{w}\right)+\frac{\mathrm{i}}{2} \widetilde{w} \mathbf{D}_{\mathrm{i} \nu}\left(\mathrm{e}^{-\frac{3 \pi \mathrm{i}}{4}} \widetilde{w}\right)\right) \\
\left(\mathcal{D}_{-}^{\Sigma_{B^{0}}}(\widetilde{w})\right)_{11}=\mathrm{e}^{\frac{\pi \nu}{4}} \mathbf{D}_{\mathrm{i} \nu}\left(\mathrm{e}^{\frac{\mathrm{i} \pi}{4}} \widetilde{w}\right), \quad\left(\mathcal{D}_{-}^{\Sigma_{B^{0}}}(\widetilde{w})\right)_{22}=\mathrm{e}^{-\frac{3 \pi \nu}{4}} \mathbf{D}_{-\mathrm{i} \nu}\left(\mathrm{e}^{\frac{3 \pi \mathrm{i}}{4}} \widetilde{w}\right) \\
\left(\mathcal{D}_{-}^{\Sigma_{B^{0}}}(\widetilde{w})\right)_{12}=\left(\beta_{21}^{\Sigma_{B^{0}}}\right)^{-1} \mathrm{e}^{-\frac{3 \pi \nu}{4}}\left(\partial_{\widetilde{w}} \mathbf{D}_{-\mathrm{i} \nu}\left(\mathrm{e}^{\frac{3 \pi \mathrm{i}}{4}} \widetilde{w}\right)-\frac{\mathrm{i}}{2} \widetilde{w} \mathbf{D}_{-\mathrm{i} \nu}\left(\mathrm{e}^{\frac{3 \pi \mathrm{i}}{4}} \widetilde{w}\right)\right) \\
\left(\mathcal{D}_{-}^{\Sigma_{B^{0}}}(\widetilde{w})\right)_{21}=\left(\beta_{12}^{\Sigma_{B^{0}}}\right)^{-1} \mathrm{e}^{\frac{\pi \nu}{4}}\left(\partial_{\widetilde{w}} \mathbf{D}_{\mathrm{i} \nu}\left(\mathrm{e}^{\mathrm{i} \pi} \widetilde{w}\right)+\frac{\mathrm{i}}{2} \widetilde{w} \mathbf{D}_{\mathrm{i} \nu}\left(\mathrm{e}^{\frac{\mathrm{i} \pi}{4}} \widetilde{w}\right)\right)
\end{gathered}
$$

where $\mathbf{D}_{*}(\cdot)$ is the parabolic cylinder function [40], and $\beta_{12}^{\Sigma_{B^{0}}}=\overline{\beta_{21}^{\Sigma_{B^{0}}}}=\frac{\sqrt{2 \pi} \mathrm{e}^{-\frac{\pi \nu}{2}} \mathrm{e}^{\frac{\mathrm{i} \pi}{4}}}{r\left(\zeta_{1}\right) \overline{\Gamma(\mathrm{i} \nu)}}$; using the identity [40] $|\Gamma(\mathrm{i} \nu)|^{2}=\frac{\pi}{\nu \sinh (\pi \nu)}$, and recalling that $\nu:=\nu\left(\zeta_{1}\right)=-\frac{1}{2 \pi} \ln \left(1-\left|r\left(\zeta_{1}\right)\right|^{2}\right)(>0)$, one deduces that $\left|\beta_{12}^{\Sigma_{B^{0}}}\right|^{2}=\left|\beta_{21}^{\Sigma_{B^{0}}}\right|^{2}=\nu$. From the latter expressions, one obtains explicit formulae

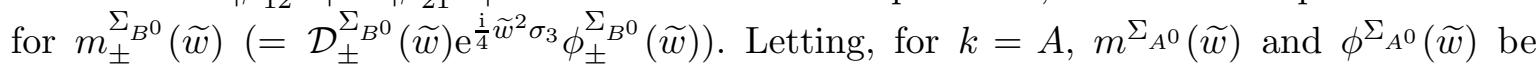
the analogues of $m^{\Sigma_{B^{0}}}(\widetilde{w})$ and $\phi^{\Sigma_{B^{0}}}(\widetilde{w})$, and carrying through with an analysis analogous to that presented in the proof of Lemma 5.4 and above, one shows that, for $\mathcal{D}^{\Sigma_{A 0}}(\widetilde{w}):=$

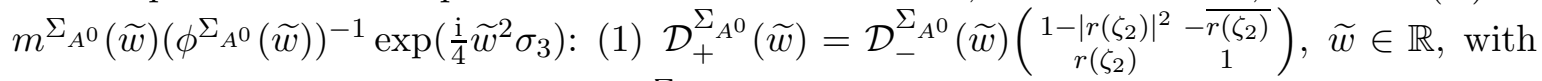

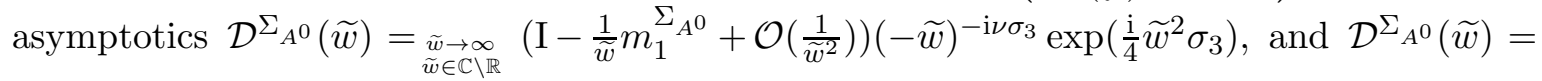
$\sigma_{1} \overline{\mathcal{D}^{\Sigma_{A^{0}}(\overline{\widetilde{w}})}} \sigma_{1} ;$ and $(2)$

$$
\partial_{\widetilde{w}} \mathcal{D}^{\Sigma_{A^{0}}}(\widetilde{w})-\frac{\mathrm{i}}{2} \widetilde{w} \sigma_{3} \mathcal{D}^{\Sigma_{A^{0}}}(\widetilde{w})=\beta^{\Sigma_{A^{0}}} \mathcal{D}^{\Sigma_{A^{0}}}(\widetilde{w})
$$

$\underline{\text { where }} \beta^{\Sigma_{A^{0}}}:=\frac{\mathrm{i}}{2}\left[\sigma_{3}, m_{1}^{\Sigma_{A^{0}}}\right]=\beta_{21}^{\Sigma_{A^{0}}} \sigma_{-}+\beta_{12}^{\Sigma_{A^{0}}} \sigma_{+}$, whence, since $\left(m_{1}^{\Sigma_{A^{0}}}\right)_{12}=\overline{\left(m_{1}^{\Sigma_{A^{0}}}\right)_{21}}, \beta_{12}^{\Sigma_{A^{0}}}=$ $\overline{\beta_{21}^{\Sigma_{A} 0}}$, with solution given by $\left( \pm \leftrightarrow \widetilde{w} \in \mathbb{C}_{ \pm}\right)$

$$
\begin{aligned}
& \left(\mathcal{D}_{+}^{\Sigma_{A 0} 0}(\widetilde{w})\right)_{11}=\mathrm{e}^{-\frac{3 \pi \nu}{4}} \mathbf{D}_{-\mathrm{i} \nu}\left(\mathrm{e}^{-\frac{\mathrm{i} \pi}{4}} \widetilde{w}\right), \quad\left(\mathcal{D}_{+}^{\Sigma_{A} 0}(\widetilde{w})\right)_{22}=\mathrm{e}^{\frac{\pi \nu}{4}} \mathbf{D}_{\mathrm{i} \nu}\left(\mathrm{e}^{-\frac{3 \pi \mathrm{i}}{4}} \widetilde{w}\right), \\
& \left(\mathcal{D}_{+}^{\Sigma_{A^{0}}}(\widetilde{w})\right)_{12}=\left(\beta_{21}^{\Sigma_{A^{0}}}\right)^{-1} \mathrm{e}^{\frac{\pi \nu}{4}}\left(\partial_{\widetilde{w}} \mathbf{D}_{\mathrm{i} \nu}\left(\mathrm{e}^{-\frac{3 \pi \mathrm{i}}{4}} \widetilde{w}\right)+\frac{\mathrm{i}}{2} \widetilde{w} \mathbf{D}_{\mathrm{i} \nu}\left(\mathrm{e}^{-\frac{3 \pi \mathrm{i}}{4}} \widetilde{w}\right)\right), \\
& \left(\mathcal{D}_{+}^{\Sigma_{A^{0}}}(\widetilde{w})\right)_{21}=\left(\beta_{12}^{\Sigma_{A^{0}}}\right)^{-1} \mathrm{e}^{-\frac{3 \pi \nu}{4}}\left(\partial_{\widetilde{w}} \mathbf{D}_{-\mathrm{i} \nu}\left(\mathrm{e}^{-\frac{\mathrm{i} \pi}{4}} \widetilde{w}\right)-\frac{\mathrm{i}}{2} \widetilde{w} \mathbf{D}_{-\mathrm{i} \nu}\left(\mathrm{e}^{-\frac{\mathrm{i} \pi}{4}} \widetilde{w}\right)\right), \\
& \left(\mathcal{D}_{-}^{\Sigma_{A^{0}}}(\widetilde{w})\right)_{11}=\mathrm{e}^{\frac{\pi \nu}{4}} \mathbf{D}_{-\mathrm{i} \nu}\left(\mathrm{e}^{\frac{3 \pi \mathrm{i}}{4}} \widetilde{w}\right), \quad\left(\mathcal{D}_{-}^{\Sigma_{A^{0}}}(\widetilde{w})\right)_{22}=\mathrm{e}^{-\frac{3 \pi \nu}{4}} \mathbf{D}_{\mathrm{i} \nu}\left(\mathrm{e}^{\frac{\mathrm{i} \pi}{4}} \widetilde{w}\right), \\
& \left(\mathcal{D}_{-}^{\Sigma_{A^{0}}}(\widetilde{w})\right)_{12}=\left(\beta_{21}^{\Sigma_{A 0}}\right)^{-1} \mathrm{e}^{-\frac{3 \pi \nu}{4}}\left(\partial_{\widetilde{w}} \mathbf{D}_{\mathrm{i} \nu}\left(\mathrm{e}^{\frac{\mathrm{i} \pi}{4}} \widetilde{w}\right)+\frac{\mathrm{i}}{2} \widetilde{w} \mathbf{D}_{\mathrm{i} \nu}\left(\mathrm{e}^{\frac{\mathrm{i} \pi}{4}} \widetilde{w}\right)\right), \\
& \left(\mathcal{D}_{-}^{\Sigma_{A^{0}}}(\widetilde{w})\right)_{21}=\left(\beta_{12}^{\Sigma_{A^{0}}}\right)^{-1} \mathrm{e}^{\frac{\pi \nu}{4}}\left(\partial_{\widetilde{w}} \mathbf{D}_{-\mathrm{i} \nu}\left(\mathrm{e}^{\frac{3 \pi \mathrm{i}}{4}} \widetilde{w}\right)-\frac{\mathrm{i}}{2} \widetilde{w} \mathbf{D}_{-\mathrm{i} \nu}\left(\mathrm{e}^{\frac{3 \pi \mathrm{i}}{4}} \widetilde{w}\right)\right),
\end{aligned}
$$

where $\beta_{12}^{\Sigma_{A}}=\overline{\beta_{21}^{\Sigma_{A^{0}}}}=\frac{\sqrt{2 \pi} \mathrm{e}^{-\frac{\pi \nu}{2}} \mathrm{e}^{-\frac{\mathrm{i} \pi}{4}}}{\overline{r\left(\zeta_{1}\right)} \Gamma(\mathrm{i} \nu)}$, and $\left|\beta_{12}^{\Sigma_{A^{0}}}\right|^{2}=\left|\beta_{21}^{\Sigma_{A^{0}}}\right|^{2}=\nu$. From the latter results, one obtains explicit formulae for $m_{ \pm}^{\Sigma_{A^{0}}}(\widetilde{w})\left(=\mathcal{D}_{ \pm}^{\Sigma_{A^{0}}}(\widetilde{w}) \mathrm{e}^{-\frac{i}{4} \widetilde{w}^{2} \sigma_{3}} \phi_{ \pm}^{\Sigma_{A^{0}}}(\widetilde{w})\right)$. Thus, from the above 
analysis and the proof of Lemma 5.4, one collects the following leading-order asymptotic results (as $t \rightarrow+\infty$ such that $0<\zeta_{2}<\frac{1}{M}<M<\zeta_{1}$ and $\left|\zeta_{3}\right|^{2}=1$, with $M \in \mathbb{R}_{>1}$ and bounded)

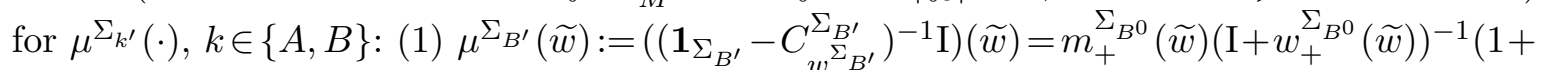
$o(1))=\mathcal{D}_{+}^{\Sigma_{B^{0}}}(\widetilde{w}) \mathrm{e}^{\frac{\mathrm{i}}{4} \widetilde{w}^{2} \sigma_{3}} \phi_{+}^{\Sigma_{B^{0}}}(\widetilde{w})\left(\mathrm{I}+w_{+}^{\Sigma_{B^{0}}}(\widetilde{w})\right)^{-1}(\mathrm{I}+o(1))=m_{-}^{\Sigma_{B^{0}}}(\widetilde{w})\left(\mathrm{I}-w_{-}^{\Sigma_{B^{0}}}(\widetilde{w})\right)^{-1}(1+o(1))=$ $\mathcal{D}_{-}^{\Sigma_{B^{0}}}(\widetilde{w}) \mathrm{e}^{\frac{\mathrm{i}}{4} \widetilde{w}^{2} \sigma_{3}} \phi_{-}^{\Sigma_{B^{0}}}(\widetilde{w})\left(\mathrm{I}-w_{-}^{\Sigma_{B^{0}}}(\widetilde{w})\right)^{-1}(\mathrm{I}+o(1)), \widetilde{w} \in \Sigma_{B} ;$ and $(2) \mu^{\Sigma_{A^{\prime}}}(\widetilde{w}):=\left(\left(\mathbf{1}_{\Sigma_{A^{\prime}}}-C_{w^{\Sigma_{A^{\prime}}}}^{\Sigma_{A^{\prime}}}\right)^{-1}\right.$ $\cdot \mathrm{I})(\widetilde{w})=m_{+}^{\Sigma_{A^{0}}}(\widetilde{w})\left(\mathrm{I}+w_{+}^{\Sigma_{A^{0}}}(\widetilde{w})\right)^{-1}(1+o(1))=\mathcal{D}_{+}^{\Sigma_{A^{0}}}(\widetilde{w}) \mathrm{e}^{-\frac{\mathrm{i}}{4} \widetilde{w}^{2} \sigma_{3}} \phi_{+}^{\Sigma_{A^{0}}}(\widetilde{w})\left(\mathrm{I}+w_{+}^{\Sigma_{A^{0}}}(\widetilde{w})\right)^{-1}(\mathrm{I}+o(1))=$ $m_{-}^{\Sigma_{A^{0}}}(\widetilde{w})\left(\mathrm{I}-w_{-}^{\Sigma_{A^{0}}}(\widetilde{w})\right)^{-1}(1+o(1))=\mathcal{D}_{-}^{\Sigma_{A^{0}}}(\widetilde{w}) \mathrm{e}^{-\frac{i}{4} \widetilde{w}^{2} \sigma_{3}} \phi_{-}^{\Sigma_{A^{0}}}(\widetilde{w})\left(\mathrm{I}-w_{-}^{\Sigma_{A^{0}}}(\widetilde{w})\right)^{-1}(\mathrm{I}+o(1)), \widetilde{w} \in \Sigma_{A}$. In fact, using the method of successive approximations and proceeding as in Section 2 of 48 (see, also, Section 3 of [49], and the proof of Lemma 4.1 in [50]), one can expand $\mu^{\Sigma_{k^{\prime}}}(\widetilde{w})$, $k \in\{A, B\}$, into Neumann-type series, and improve the $o(1)$ estimate to $\mathcal{O}\left(\mu_{1,1}^{\Sigma_{k^{\prime}}}(\widetilde{w}) \frac{\ln t}{\sqrt{t}}\right)$,

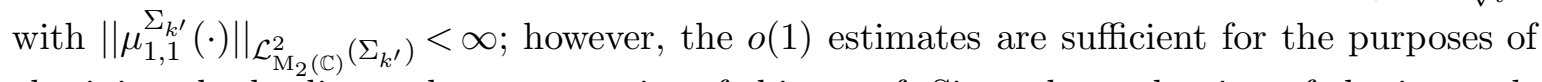
obtaining the leading order asymptotics of this proof. Since the evaluation of the integrals appearing in the formulae for $m_{i j}^{\Sigma^{\sharp}}(\zeta), i, j \in\{1,2\}$, are analogous, consider, say, and without loss of generality, the following integral (appearing in the expression for $m_{12}^{\Sigma^{\sharp}}(\zeta)$ ):

$$
\mathrm{I}^{\Sigma^{\sharp}}:=\int_{\zeta_{1}+\infty \mathrm{e}^{-\frac{\mathrm{i} \pi}{4}}}^{\zeta_{1}+0^{+} \mathrm{e}^{-\frac{\mathrm{i} \pi}{4}}} \frac{\mu_{11}^{\Sigma_{B^{\prime}}}(\xi) \mathcal{R}(\xi)(\delta(\xi))^{2} \exp \left(-2 \mathrm{i} t \theta^{u}(\xi)\right)}{(\xi-\zeta)} \frac{\mathrm{d} \xi}{2 \pi \mathrm{i}} .
$$

Making the change of variable (Eqs. (105) and (106)) $\xi=\zeta_{1}+\varepsilon_{B}(\widetilde{w})$, where $\varepsilon_{B}(\widetilde{w}):=\frac{\widetilde{w}}{\mathcal{X}_{B} \sqrt{t}}$, with $\mathcal{X}_{B}=\left(\frac{2\left(\zeta_{1}-\zeta_{2}\right)}{\zeta_{1}}\right)^{1 / 2} \frac{\left|\zeta_{1}-\zeta_{3}\right|}{\zeta_{1}}$, setting $\mu_{11}^{\Sigma_{B^{\prime}}}\left(\zeta_{1}+\varepsilon_{B}(\widetilde{w})\right):=\mu_{11}^{\Sigma_{B^{\prime}}}(\widetilde{w})$, and recalling the action of $\mathcal{N}_{B}$ given in Proposition 5.1, namely, $\left(\mathcal{N}_{B}\left(\delta^{2} \mathrm{e}^{-2 \mathrm{i} t \theta^{u}}\right)\right)(\widetilde{w})=\left(\delta_{B}^{0}\right)^{2}\left(\delta_{B}^{1}(\widetilde{w})\right)^{2}$, with $\delta_{B}^{0}$ and $\delta_{B}^{1}(\widetilde{w})$ defined in Proposition 5.1,

$$
\begin{aligned}
& \mathrm{I}^{\Sigma^{\sharp}}=\int_{\infty \mathrm{e}^{-\frac{\mathrm{i} \pi}{4}}}^{0^{+}} \frac{\mu_{11}^{\Sigma_{B^{\prime}}}\left(\zeta_{1}+\varepsilon_{B}(\widetilde{w})\right) \mathcal{R}\left(\zeta_{1}+\varepsilon_{B}(\widetilde{w})\right)\left(\delta\left(\zeta_{1}+\varepsilon_{B}(\widetilde{w})\right)\right)^{2} \mathrm{e}^{-2 \mathrm{i} t \theta^{u}\left(\zeta_{1}+\varepsilon_{B}(\widetilde{w})\right)}}{\left(\zeta_{1}+\varepsilon_{B}(\widetilde{w})-\zeta\right)} \frac{\mathrm{d} \varepsilon_{B}(\widetilde{w})}{2 \pi \mathrm{i}}
\end{aligned}
$$



$$
\begin{aligned}
& =\frac{1}{\left(\zeta-\zeta_{1}\right)} \int_{0^{+}}^{\infty \mathrm{e}^{-\frac{\mathrm{i} \pi}{4}}} \frac{\mu_{11}^{\Sigma_{B^{\prime}}}(\widetilde{w}) \mathcal{R}\left(\zeta_{1}+\varepsilon_{B}(\widetilde{w})\left(\mathcal{N}_{B}\left(\delta^{2} \mathrm{e}^{-2 \mathrm{i} t \theta^{u}}\right)\right)(\widetilde{w})\right.}{\left(1-\varepsilon_{B}(\widetilde{w})\left(\zeta-\zeta_{1}\right)^{-1}\right)} \frac{\mathrm{d} \varepsilon_{B}(\widetilde{w})}{2 \pi \mathrm{i}} \\
& =\frac{1}{\left(\zeta-\zeta_{1}\right)} \int_{0^{+}}^{\infty \mathrm{e}^{-\frac{\mathrm{i} \pi}{4}}} \frac{\mu_{11}^{\Sigma_{B^{\prime}}}(\widetilde{w}) \mathcal{R}\left(\zeta_{1}+\varepsilon_{B}(\widetilde{w})\right)\left(\delta_{B}^{0}\right)^{2}\left(\delta_{B}^{1}(\widetilde{w})\right)^{2}}{\left(1-\varepsilon_{B}(\widetilde{w})\left(\zeta-\zeta_{1}\right)^{-1}\right)} \frac{\mathrm{d} \varepsilon_{B}(\widetilde{w})}{2 \pi \mathrm{i}} \\
& =\frac{\left(\delta_{B}^{0}\right)^{2}}{\left(\zeta-\zeta_{1}\right)} \int_{0^{+}}^{\infty \mathrm{e}^{-\frac{\mathrm{i} \pi}{4}}} \frac{\mu_{11}^{\Sigma_{B^{\prime}}}(\widetilde{w})\left(\delta_{B}^{1}(\widetilde{w})\right)^{2} \mathcal{R}\left(\zeta_{1}+\varepsilon_{B}(\widetilde{w})\right)}{\left(1-\varepsilon_{B}(\widetilde{w})\left(\zeta-\zeta_{1}\right)^{-1}\right)} \frac{\mathrm{d} \varepsilon_{B}(\widetilde{w})}{2 \pi \mathrm{i}} .
\end{aligned}
$$

Recalling from Lemma 5.1 that

$$
\left(\delta_{B}^{1}(\widetilde{w})\right)^{2} \mathcal{R}\left(\zeta_{1}+\varepsilon_{B}(\widetilde{w})\right)=(\widetilde{w})^{2 \mathrm{i} \nu} \exp \left(-\frac{\mathrm{i} \widetilde{w}^{2}}{2}\right) \mathcal{R}\left(\zeta_{1}^{+}\right)+\mathcal{O}\left(\frac{c^{\mathcal{S}}\left(\zeta_{1}\right) \underline{c}\left(\zeta_{2}, \zeta_{3}, \overline{\zeta_{3}}\right)}{\left|\zeta_{1}-\zeta_{3}\right| \sqrt{\left(\zeta_{1}-\zeta_{2}\right)}} \frac{\ln (t)}{\sqrt{t}} \exp \left(-\frac{\mathrm{i} \gamma \widetilde{w}^{2}}{2}\right)\right)
$$

with $\mathcal{R}\left(\zeta_{1}^{+}\right):=\lim _{\Re(\zeta) \downarrow \zeta_{1}} \mathcal{R}(\zeta)=\overline{r\left(\zeta_{1}\right)}$, and $\gamma \in\left(0, \frac{1}{2}\right)$, and noting the exponential decay of the factors $\mathrm{e}^{-\frac{\mathrm{i}}{2} \widetilde{w}^{2}}$ and $\mathrm{e}^{-\frac{\mathrm{i} \gamma}{2} \widetilde{w}^{2}}$ along the (unbounded) ray $0^{+}$to $\infty \mathrm{e}^{-\frac{\mathrm{i} \pi}{4}}$, one expands the factor $1 /\left(1-\varepsilon_{B}(\widetilde{w})\left(\zeta-\zeta_{1}\right)^{-1}\right)$ in a geometric progression and, recalling that $\varepsilon_{B}(\widetilde{w})=\frac{\widetilde{w}}{\mathcal{X}_{B} \sqrt{t}}$, using the following rules from asymptotic analysis, $\mathcal{O}\left(\star_{1}\right) o\left(\star_{2}\right)=o\left(\star_{1} \star_{2}\right)$ and $\mathcal{O}(\star)+o(\star)=\mathcal{O}(\star)$, 
along with the fact that, for $\epsilon$ some arbitrarily fixed, sufficiently small positive real number, $t^{\epsilon} \ln t>1$, one arrives at the following (limiting) result:

$$
\begin{aligned}
& \mathrm{I}^{\Sigma^{\sharp}}=\frac{\overline{r\left(\zeta_{1}\right)}\left(\delta_{B}^{0}\right)^{2}}{2 \pi \mathrm{i}\left(\zeta-\zeta_{1}\right) \mathcal{X}_{B} \sqrt{t}} \int_{0}^{\infty \mathrm{e}^{-\frac{\mathrm{i} \pi}{4}}} \mu_{11}^{\Sigma_{B^{\prime}}}(\widetilde{w})(\widetilde{w})^{2 \mathrm{i} \nu} \exp \left(-\frac{\mathrm{i} \widetilde{w}^{2}}{2}\right) \mathrm{d} \widetilde{w} \\
& +\mathcal{O}\left(\frac{c^{\mathcal{S}}\left(\zeta_{1}\right) \underline{c}\left(\zeta_{2}, \zeta_{3}, \overline{\zeta_{3}}\right)\left(\delta_{B}^{0}\right)^{2}}{\left(\zeta-\zeta_{1}\right)\left|\zeta_{1}-\zeta_{3}\right| \sqrt{\left(\zeta_{1}-\zeta_{2}\right)} \mathcal{X}_{B}} \frac{\ln (t)}{t} \int_{0}^{\infty \mathrm{e}^{-\frac{\mathrm{i} \pi}{4}}} \mu_{11}^{\Sigma_{B^{\prime}}}(\widetilde{w}) \exp \left(-\frac{\mathrm{i} \gamma \widetilde{w}^{2}}{2}\right) \mathrm{d} \widetilde{w}\right) .
\end{aligned}
$$

Recalling the formula for $\mu^{\Sigma_{B^{\prime}}}(\widetilde{w})$ given heretofore in the proof, using the definition of $\phi^{\Sigma_{B^{0}}}(\widetilde{w})$ given in the proof of Lemma 5.4, and noting that $w_{+}^{\Sigma_{B^{0}}}(\widetilde{w})$ is nilpotent, with degree of nilpotency 2 , one deduces that $\mu_{11}^{\Sigma_{B^{\prime}}}(\widetilde{w})=\left(\mathcal{D}_{-}^{\Sigma_{B^{0}}}(\widetilde{w})\right)_{11} \mathrm{e}^{\frac{\mathrm{i}}{4} \widetilde{w}^{2}}(\widetilde{w})^{-\mathrm{i} \nu}=\mathrm{e}^{\frac{\pi \nu}{4}} \mathbf{D}_{\mathrm{i} \nu}\left(\mathrm{e}^{\frac{\mathrm{i} \pi}{4}} \widetilde{w}\right) \mathrm{e}^{\frac{\mathrm{i}}{4} \widetilde{w}^{2}}(\widetilde{w})^{-\mathrm{i} \nu}$ : making one more change of variable, namely, $z=\mathrm{e}^{\frac{\mathrm{i} \pi}{4}} \widetilde{w}$, one arrives at

$$
\mathrm{I}^{\Sigma^{\sharp}}=\frac{\overline{r\left(\zeta_{1}\right)}\left(\delta_{B}^{0}\right)^{2} \mathrm{e}^{\frac{\pi \nu}{2}} \mathrm{e}^{-\frac{\mathrm{i} \pi}{4}}}{2 \pi \mathrm{i}\left(\zeta-\zeta_{1}\right) \mathcal{X}_{B} \sqrt{t}} \int_{0}^{+\infty} \mathbf{D}_{\mathrm{i} \nu}(z) z^{\mathrm{i} \nu} \mathrm{e}^{-\frac{z^{2}}{4}} \mathrm{~d} z+\mathcal{O}\left(\frac{c^{\mathcal{S}}\left(\zeta_{1}\right) \underline{c}\left(\zeta_{2}, \zeta_{3}, \overline{\zeta_{3}}\right)\left(\delta_{B}^{0}\right)^{2}}{\left(\zeta-\zeta_{1}\right)\left|\zeta_{1}-\zeta_{3}\right| \sqrt{\left(\zeta_{1}-\zeta_{2}\right)} \mathcal{X}_{B}} \frac{\ln (t)}{t} \mathrm{I}_{\gamma}^{\Sigma^{\sharp}}\right),
$$

where

$$
\mathrm{I}_{\gamma}^{\Sigma^{\sharp}}:=\int_{0}^{+\infty} \mathbf{D}_{\mathrm{i} \nu}(z) z^{-\mathrm{i} \nu} \exp \left(\frac{1}{2}\left(\frac{1}{2}-\gamma\right) z^{2}\right) \mathrm{d} z .
$$

Recall from the proof of Lemma 5.1 that $0<\nu:=\nu\left(\zeta_{1}\right) \leqslant \nu_{m}:=-\frac{1}{2 \pi} \ln \left(1-\sup _{z \in \mathbb{R}}|r(z)|^{2}\right)$ : let $\nu_{m}^{\sharp}$ be an arbitrarily fixed, sufficiently large positive real number with $\nu_{m}^{\sharp} \gg \nu_{m}$ (in particular, choose $\nu_{m}^{\sharp} \gg \sqrt{2}$ ). Set

$$
\mathrm{I}_{\gamma}^{\Sigma^{\sharp}}=\left(\int_{0}^{\nu_{m}}+\int_{\nu_{m}}^{\nu_{m}^{\sharp}}+\int_{\nu_{m}^{\sharp}}^{+\infty}\right) \mathbf{D}_{\mathrm{i} \nu}(z) z^{-\mathrm{i} \nu} \exp \left(\frac{1}{2}\left(\frac{1}{2}-\gamma\right) z^{2}\right) \mathrm{d} z:=\mathrm{I}_{\gamma}^{1}+\mathrm{I}_{\gamma}^{2}+\mathrm{I}_{\gamma}^{3} .
$$

Via a change-of-variable argument and the fact that, for $(z, \gamma) \in\left[0, \nu_{m}\right) \times\left(0, \frac{1}{2}\right), \mathrm{e}^{-\frac{\gamma}{2} z^{2}} \leqslant 1$, one deduces that $\left|\mathrm{I}_{\gamma}^{1}\right| \leqslant \nu_{m} \sup _{x \in[0,1)}\left|\mathbf{D}_{\mathrm{i} \nu}\left(\nu_{m} x\right)\right| \int_{0}^{1} \mathrm{e}^{\frac{\nu_{m}^{2} x^{2}}{4}} \mathrm{~d} x<\infty$. For $z \in\left[\nu_{m}, \nu_{m}^{\sharp}\right)$, one uses the following representation for the parabolic cylinder function 40$], \mathbf{D}_{\mathrm{i} \nu}(z)=2^{\frac{\mathrm{i} \nu}{2}} \mathrm{e}^{-\frac{z^{2}}{4}}\left(\frac{\sqrt{\pi}}{\Gamma\left(\frac{1-\mathrm{i} \nu}{2}\right)} \mathbf{F}\left(-\frac{\mathrm{i} \nu}{2}\right.\right.$, $\left.\left.\frac{1}{2} ; \frac{z^{2}}{2}\right)-\frac{\sqrt{2 \pi} z}{\Gamma\left(-\frac{\mathrm{i} \nu}{2}\right)} \mathbf{F}\left(\frac{1-\mathrm{i} \nu}{2}, \frac{3}{2} ; \frac{z^{2}}{2}\right)\right)$, where $\mathbf{F}(a, b ; x):=\sum_{n=0}^{\infty} \frac{(a)_{n} x^{n}}{(b)_{n} n !}$ is the confluent hypergeometric function, and $(\star)_{n}:=\star(\star+1) \cdots(\star+n-1)=\frac{\Gamma(\star+n)}{\Gamma(\star)}$ is the Pochhammer symbol; hence, $\mathrm{I}_{\gamma}^{2}=$ $\frac{2^{\frac{\mathrm{i} \nu}{2}} \sqrt{\pi}}{\Gamma\left(\frac{1-\mathrm{i} \nu}{2}\right)} \sum_{n=0}^{\infty} \frac{\left(-\frac{\mathrm{i} \nu}{2}\right)_{n} 2^{-n}}{\left(\frac{1}{2}\right)_{n} n !} \int_{\nu_{m}}^{\nu_{m}^{\sharp}} z^{-\mathrm{i} \nu} z^{2 n} \mathrm{e}^{-\frac{\gamma}{2} z^{2}} \mathrm{~d} z-\frac{2^{\frac{\mathrm{i} \nu}{2}} \sqrt{2 \pi}}{\Gamma\left(-\frac{\mathrm{i} \nu}{2}\right)} \sum_{n=0}^{\infty} \frac{\left(\frac{1-\mathrm{i} \nu}{2}\right)_{n} 2^{-n}}{\left(\frac{3}{2}\right)_{n} n !} \int_{\nu_{m}}^{\nu_{m}^{\sharp}} z^{-\mathrm{i} \nu} z^{2 n+1} \mathrm{e}^{-\frac{\gamma}{2} z^{2}} \mathrm{~d} z$. Recall that $\mathrm{e}^{\star}=\sum_{n=0}^{\infty} \frac{\star^{n}}{n !}$, where the convergence of the series is absolute and uniform for $\star$ in compact subsets of $\mathbb{R}$ : using the series expansion for $\mathrm{e}^{\star}$ and integrating term-by-term, noting that, $\forall(m, n) \in \mathbb{Z}_{\geqslant 0} \times \mathbb{Z}_{\geqslant 0},\left((2(n+m)+s)^{2}+\nu^{2}\right)^{-1 / 2} \leqslant\left(1+\nu^{2}\right)^{-1 / 2}, s \in\{1,2\}$, and using the identities [40] $\left|\Gamma\left(\frac{1-\mathrm{i} \nu}{2}\right)\right|=\sqrt{\frac{\pi}{\cosh (\pi \nu / 2)}}$ and $\left|\Gamma\left(-\frac{\mathrm{i} \nu}{2}\right)\right|=\sqrt{\frac{2 \pi}{\nu \sinh (\pi \nu / 2)}}$, and the functional relation $\Gamma(1+x)=x \Gamma(x)$, via the well-known result $\Gamma(1 / 2)=\sqrt{\pi}$ and a majorisation argument, one shows that

$$
\left|\mathrm{I}_{\gamma}^{2}\right| \leqslant \sqrt{\frac{\nu \sinh (\pi \nu)}{2\left(1+\nu^{2}\right)}} \sum_{s \in\left\{\nu_{m}, \nu_{m}^{\sharp}\right\}} \sum_{n=0}^{\infty}\left(\frac{1}{\sqrt{2}}\left|\Gamma\left(n-\frac{\mathrm{i} \nu}{2}\right)\right|+s\left|\Gamma\left(n+\frac{1}{2}-\frac{\mathrm{i} \nu}{2}\right)\right|\right) \frac{s^{2 n+1} \mathrm{e}^{\gamma s^{2} / 2}}{2^{n} n ! \Gamma\left(n+\frac{1}{2}\right)} .
$$

From the above gamma function identities and repeated application of the functional relation $\Gamma(1+x)=x \Gamma(x)$, one shows that, for $n \in \mathbb{Z}_{\geqslant 1}, \frac{\left|\Gamma\left(n-\frac{\mathrm{i} \nu}{2}\right)\right|}{\Gamma\left(n+\frac{1}{2}\right)} \leqslant \frac{\left((n-1)^{2}+\left(\frac{\nu}{2}\right)^{2}\right)^{\frac{n}{2}} 2^{n+\frac{1}{2}}}{(2 n-1) ! ! \sqrt{\nu \sinh (\pi \nu / 2)}}$ and $\frac{\left|\Gamma\left(n+\frac{1}{2}-\frac{\mathrm{i} \nu}{2}\right)\right|}{\Gamma\left(n+\frac{1}{2}\right)} \leqslant$ 
$\frac{\left(\left(n-\frac{1}{2}\right)^{2}+\left(\frac{\nu}{2}\right)^{2}\right)^{\frac{n}{2}} 2^{n}}{(2 n-1) ! ! \sqrt{\cosh (\pi \nu / 2)}}$, where $(2 n-1) ! !=(2 n-1) \cdots 3 \cdot 1$ : using the latter inequalities, one arrives at

$$
\begin{aligned}
\left|\mathrm{I}_{\gamma}^{2}\right| & \leqslant \sqrt{\frac{\cosh (\pi \nu / 2)}{1+\nu^{2}}} \sum_{s \in\left\{\nu_{m}, \nu_{m}^{\sharp}\right\}}\left(1+\sum_{n=1}^{\infty} \frac{\left((n-1)^{2}+\left(\frac{\nu}{2}\right)^{2}\right)^{n / 2} s^{2 n}}{n !(2 n-1) ! !}\right) s \mathrm{e}^{\gamma s^{2} / 2} \\
& +\sqrt{\frac{\nu \sinh (\pi \nu / 2)}{1+\nu^{2}}} \sum_{s \in\left\{\nu_{m}, \nu_{m}^{\sharp}\right\}}\left(1+\sum_{n=1}^{\infty} \frac{\left(\left(n-\frac{1}{2}\right)^{2}+\left(\frac{\nu}{2}\right)^{2}\right)^{n / 2} s^{2 n}}{n !(2 n-1) ! !}\right) s^{2} \mathrm{e}^{\gamma s^{2} / 2} .
\end{aligned}
$$

A straightforward application of the Ratio Test shows that the latter two (infinite) series converge absolutely (hence converge); thus, $\left|\mathrm{I}_{\gamma}^{2}\right|<\infty$. For $z \in\left[\nu_{m}^{\sharp},+\infty\right)$, with $|\arg z|<\frac{3 \pi}{4}$, one uses the following asymptotic expansion for $\mathbf{D}_{\mathrm{i} \nu}(z)$ [40]:

$$
\mathbf{D}_{\mathrm{i} \nu}(z) \underset{\substack{z \rightarrow \infty \\|\arg z|<\frac{3 \pi}{4}}}{=} z^{\mathrm{i} \nu} \mathrm{e}^{-\frac{z^{2}}{4}}\left(\sum_{n=0}^{N} \frac{(-1)^{n} \prod_{k=0}^{2 n-1}(\mathrm{i} \nu-k)}{(2 n) ! ! z^{2 n}}+\mathcal{O}\left(\frac{\prod_{k=0}^{2 N+1}(\mathrm{i} \nu-k)}{(2 N+2) ! z^{2 N+2}}\right)\right),
$$

where $N \in \mathbb{N},(2 n) ! !=(2 n) \cdots 4 \cdot 2$, and $\prod_{k=0}^{-1}(\mathrm{i} \nu-k):=1$. With this asymptotic representation for $\mathbf{D}_{\mathrm{i} \nu}(z)$, one presents $\mathrm{I}_{\gamma}^{3}$ as $\mathrm{I}_{\gamma}^{3}=\widetilde{\mathrm{I}}_{\gamma}^{3}+\widehat{\mathrm{I}}_{\gamma}^{3}$, where $\widetilde{\mathrm{I}}_{\gamma}^{3}:=\int_{\nu_{m}^{\sharp}}^{+\infty} \mathrm{e}^{-\frac{\gamma}{2} z^{2}} \mathrm{~d} z$, and $\widehat{\mathrm{I}}_{\gamma}^{3}:=$ $\int_{\nu_{m}^{\sharp}}^{+\infty} \mathrm{e}^{-\frac{\gamma}{2} z^{2}}\left(\sum_{n=1}^{N} \frac{(-1)^{n} \prod_{k=0}^{2 n-1}(\mathrm{i} \nu-k)}{(2 n) ! ! z^{2 n}}+\mathcal{O}\left(\frac{\prod_{k=0}^{2 N+1}(\mathrm{i} \nu-k)}{(2 N+2) ! ! z^{2 N+2}}\right)\right) \mathrm{d} z$. Write $\int_{\nu_{m}^{\sharp}}^{+\infty} \mathrm{e}^{-\frac{\gamma}{2} z^{2}} \mathrm{~d} z=\int_{0}^{+\infty} \mathrm{e}^{-\frac{\gamma}{2} z^{2}}$ $\cdot \mathrm{d} z-\int_{0}^{\nu_{m}^{\sharp}} \mathrm{e}^{-\frac{\gamma}{2} z^{2}} \mathrm{~d} z$. Recalling that $\Gamma(1 / 2)=\sqrt{\pi}$, via a change-of-variable argument, one shows that $\int_{0}^{+\infty} \mathrm{e}^{-\frac{\gamma}{2} z^{2}} \mathrm{~d} z=(\pi / 2 \gamma)^{1 / 2}$. Recalling that $\mathrm{e}^{\star}=\sum_{n=0}^{\infty} \frac{\star^{n}}{n !}$, one integrates (the second integral) term-by-term and, using the fact that, for $n \in \mathbb{Z}_{\geqslant 0},(2 n+1)^{-1} \leqslant 1$ and $2^{-n} \leqslant 1$, via a change-of-variable and majorisation argument, one shows that $\left|\int_{0}^{\nu_{m}^{\sharp}} \mathrm{e}^{-\frac{\gamma}{2} z^{2}} \mathrm{~d} z\right| \leqslant$ $\nu_{m}^{\sharp} \mathrm{e}^{\gamma\left(\nu_{m}^{\sharp}\right)^{2}}$; hence, $\left|\widetilde{\mathrm{I}}_{\gamma}^{3}\right| \leqslant(\pi / 2 \gamma)^{1 / 2}+\nu_{m}^{\sharp} \mathrm{e}^{\gamma\left(\nu_{m}^{\sharp}\right)^{2}}<\infty$ (one can also use the fact that the second integral has an explicit representation in terms of the incomplete gamma function 40] to obtain a similar estimate). Via a change-of-variable argument, one arrives at $\widehat{\mathrm{I}}_{\gamma}^{3} \sim$ $\frac{1}{\sqrt{2 \gamma}} \sum_{n \geqslant 1} \frac{(-1)^{n}\left(\prod_{k=0}^{2 n-1}(\mathrm{i} \nu-k)\right) \gamma^{n}}{(2 n) ! 2^{n}} \int_{\frac{1}{2} \gamma\left(\nu_{m}^{\sharp}\right)^{2}}^{+\infty} x^{-(n+1 / 2)} \mathrm{e}^{-x} \mathrm{~d} x$ : using the following definite integral 40] $\int_{u}^{+\infty} x^{-\nu^{\prime}} \mathrm{e}^{-x} \mathrm{~d} x=u^{-\nu^{\prime} / 2} \mathrm{e}^{-u / 2} \mathbf{W}_{-\frac{\nu^{\prime}}{2}, \frac{\left(1-\nu^{\prime}\right)}{2}}(u), u>0$, where $\mathbf{W}_{z_{1}, z_{2}}(z)$ is the Whittaker function, $\mathbf{W}_{z_{1}, z_{2}}(z):=\frac{\Gamma\left(-2 z_{2}\right)}{\Gamma\left(\frac{1}{2}-z_{1}-z_{2}\right)} \mathrm{e}^{-z / 2} z^{1 / 2+z_{2}} \mathbf{F}\left(z_{2}-z_{1}+\frac{1}{2}, 2 z_{2}+1 ; z\right)+\frac{\Gamma\left(2 z_{2}\right)}{\Gamma\left(\frac{1}{2}-z_{1}+z_{2}\right)} \mathrm{e}^{-z / 2} z^{1 / 2-z_{2}}$ $\cdot \mathbf{F}\left(-z_{2}-z_{1}+\frac{1}{2},-2 z_{2}+1 ; z\right)$, and the gamma function identity [40] $\Gamma\left(\frac{1}{2}-n\right)=\frac{(-1)^{n} 2^{n} \sqrt{\pi}}{(2 n-1) ! !}, n \in \mathbb{Z}_{\geqslant 1}$, one shows, upon noting the identity $(2 n) ! !(2 n-1) ! !=(2 n) !$, that

$$
\begin{aligned}
\left|\widehat{\mathrm{I}}_{\gamma}^{3}\right| & \leqslant \frac{\sqrt{\pi} \nu_{m}^{\sharp} \mathrm{e}^{-\frac{\gamma\left(\nu_{m}^{\sharp}\right)^{2}}{2}}}{2}\left(\sum_{n=1}^{N} \frac{2^{n} \prod_{k=0}^{2 n-1}\left(\nu^{2}+k^{2}\right)^{\frac{1}{2}}}{(2 n) !\left(\nu_{m}^{\sharp}\right)^{2 n}}\right) \sum_{m=0}^{\infty} \frac{\left(\gamma\left(\nu_{m}^{\sharp}\right)^{2}\right)^{m}}{2^{m}\left|\Gamma\left(m-n+\frac{3}{2}\right)\right|}+\left(\frac{\pi}{2 \gamma}\right)^{1 / 2} \sum_{n=1}^{N} \frac{\gamma^{n} \prod_{k=0}^{2 n-1}\left(\nu^{2}+k^{2}\right)^{\frac{1}{2}}}{(2 n) !} \\
& +\mathcal{O}\left(\frac{2^{N} \prod_{k=0}^{2 N+1}\left(\nu^{2}+k^{2}\right)^{\frac{1}{2}}}{(2 N+2) !\left(\nu_{m}^{\sharp}\right)^{2 N}} \sum_{m=0}^{\infty} \frac{\left(\gamma\left(\nu_{m}^{\sharp}\right)^{2}\right)^{m}}{2^{m}\left|\Gamma\left(m-N+\frac{1}{2}\right)\right|}+\frac{\gamma^{N} \prod_{k=0}^{2 N+1}\left(\nu^{2}+k^{2}\right)^{\frac{1}{2}}}{(2 N+2) !}\right) .
\end{aligned}
$$

Recalling that $\nu_{m}^{\sharp} \gg \sqrt{2}$ and $\gamma \in\left(0, \frac{1}{2}\right)$, a straightforward application of the Ratio Test shows that the respective series above are absolutely convergent (hence convergent); thus, via the inequality $\left|\star_{1} \star_{2}\right| \leqslant\left|\star_{1}\right|\left|\star_{2}\right|$, one deduces that $\left|\widehat{\mathrm{I}}_{\gamma}^{3}\right|<\infty$. Collecting the above-derived estimates, one arrives at $\mathrm{I}_{\gamma}^{\Sigma^{\sharp}}=\sum_{k=1}^{3} \mathrm{I}_{\gamma}^{k} \leqslant \underline{c}\left(\zeta_{1}, \zeta_{2}, \zeta_{3}, \overline{\zeta_{3}}\right)(=\mathcal{O}(1))$; hence,

$$
\mathrm{I}^{\Sigma^{\sharp}}=\frac{\overline{r\left(\zeta_{1}\right)}\left(\delta_{B}^{0}\right)^{2} \mathrm{e}^{\frac{\pi \nu}{2}} \mathrm{e}^{-\frac{\mathrm{i} \pi}{4}}}{2 \pi \mathrm{i}\left(\zeta-\zeta_{1}\right) \mathcal{X}_{B} \sqrt{t}} \int_{0}^{+\infty} \mathbf{D}_{\mathrm{i} \nu}(z) z^{\mathrm{i} \nu} \mathrm{e}^{-\frac{z^{2}}{4}} \mathrm{~d} z+\mathcal{O}\left(\frac{c^{\mathcal{S}}\left(\zeta_{1}\right) \underline{c}\left(\zeta_{2}, \zeta_{3}, \overline{\zeta_{3}}\right)\left(\delta_{B}^{0}\right)^{2}}{\left(\zeta-\zeta_{1}\right)\left|\zeta_{1}-\zeta_{3}\right| \sqrt{\left(\zeta_{1}-\zeta_{2}\right)} \mathcal{X}_{B}} \frac{\ln t}{t}\right) .
$$


Proceeding as above for the remaining integrals for $m_{i j}^{\Sigma^{\sharp}}(\zeta), i, j \in\{1,2\}$, given at the beginning of the proof, letting $\varepsilon \rightarrow+\infty$ in the limits of integration, and neglecting exponentially small terms (cf. Remark 4.4), one arrives at

$$
\begin{aligned}
& m_{11}^{\Sigma^{\sharp}}(\zeta)=1-\frac{r\left(\zeta_{1}\right)\left(\delta_{B}^{0}\right)^{-2} \mathrm{e}^{\frac{\pi \nu}{2}} \mathrm{e}^{\frac{\mathrm{i} \pi}{4}}}{2 \pi \mathrm{i}\left(\zeta-\zeta_{1}\right) \beta_{21}^{\Sigma} B^{0} \mathcal{X}_{B} \sqrt{t}} \int_{0}^{+\infty}\left(\mathrm{e}^{-\frac{\mathrm{i} \pi}{4}} \partial_{z} \mathbf{D}_{-\mathrm{i} \nu}(z)-\frac{\mathrm{i}}{2} \mathrm{e}^{\frac{\mathrm{i} \pi}{4}} z \mathbf{D}_{-\mathrm{i} \nu}(z)\right) z^{-\mathrm{i} \nu} \mathrm{e}^{-\frac{z^{2}}{4}} \mathrm{~d} z \\
& +\frac{r\left(\zeta_{1}\right)\left(1-\left|r\left(\zeta_{1}\right)\right|^{2}\right)^{-1}\left(\delta_{B}^{0}\right)^{-2} \mathrm{e}^{-\frac{3 \pi \mathrm{i}}{4}}}{2 \pi \mathrm{i}\left(\zeta-\zeta_{1}\right) \beta_{21}^{2} B^{0} \mathrm{e}^{\frac{3 \pi \nu}{2}} \mathcal{X}_{B} \sqrt{t}} \int_{0}^{+\infty}\left(\mathrm{e}^{\frac{3 \pi \mathrm{i}}{4}} \partial_{z} \mathbf{D}_{-\mathrm{i} \nu}(z)-\frac{\mathrm{i}}{2} \mathrm{e}^{-\frac{3 \pi \mathrm{i}}{4}} z \mathbf{D}_{-\mathrm{i} \nu}(z)\right) z^{-\mathrm{i} \nu} \mathrm{e}^{-\frac{z^{2}}{4}} \mathrm{~d} z \\
& -\frac{\overline{r\left(\zeta_{1}\right)}\left(\delta_{A}^{0}\right)^{-2} \mathrm{e}^{-\frac{\pi \nu}{2}}(-1)^{\mathrm{i} \nu} \mathrm{e}^{\frac{3 \pi \mathrm{i}}{4}}}{2 \pi \mathrm{i}\left(\zeta-\zeta_{2}\right) \beta_{21}^{\Sigma_{A}} \mathcal{X}_{A} \sqrt{t}} \int_{0}^{+\infty}\left(\mathrm{e}^{-\frac{3 \pi \mathrm{i}}{4}} \partial_{z} \mathbf{D}_{\mathrm{i} \nu}(z)+\frac{\mathrm{i}}{2} \mathrm{e}^{\frac{3 \pi \mathrm{i}}{4}} z \mathbf{D}_{\mathrm{i} \nu}(z)\right) z^{\mathrm{i} \nu} \mathrm{e}^{-\frac{z^{2}}{4}} \mathrm{~d} z \\
& +\frac{\overline{r\left(\zeta_{1}\right)}\left(1-\left|r\left(\zeta_{1}\right)\right|^{2}\right)^{-1}\left(\delta_{A}^{0}\right)^{-2}(-1)^{\mathrm{i} \nu} \mathrm{e}^{-\frac{\mathrm{i} \pi}{4}}}{2 \pi \mathrm{i}\left(\zeta-\zeta_{2}\right) \beta_{21}^{\Sigma^{0}} \mathrm{e}^{\frac{\pi \nu}{2}} \mathcal{X}_{A} \sqrt{t}} \int_{0}^{+\infty}\left(\mathrm{e}^{\frac{\mathrm{i} \pi}{4}} \partial_{z} \mathbf{D}_{\mathrm{i} \nu}(z)+\frac{\mathrm{i}}{2} \mathrm{e}^{-\frac{\mathrm{i} \pi}{4}} z \mathbf{D}_{\mathrm{i} \nu}(z)\right) z^{\mathrm{i} \nu} \mathrm{e}^{-\frac{z^{2}}{4}} \mathrm{~d} z \\
& +\mathcal{O}\left(\left(\frac{c^{\mathcal{S}}\left(\zeta_{1}\right) \underline{c}\left(\zeta_{2}, \zeta_{3}, \overline{\zeta_{3}}\right)\left(\delta_{B}^{0}\right)^{-2}}{\left(\zeta-\zeta_{1}\right)\left|\zeta_{1}-\zeta_{3}\right| \sqrt{\left(\zeta_{1}-\zeta_{2}\right)} \mathcal{X}_{B}}+\frac{c^{\mathcal{S}}\left(\zeta_{2}\right) \underline{c}\left(\zeta_{1}, \zeta_{3}, \overline{\zeta_{3}}\right)\left(\delta_{A}^{0}\right)^{-2}}{\left(\zeta-\zeta_{2}\right)\left|\zeta_{2}-\zeta_{3}\right| \sqrt{\left(\zeta_{1}-\zeta_{2}\right)} \mathcal{X}_{A}}\right) \frac{\ln t}{t}\right), \\
& m_{12}^{\sum^{\sharp}}(\zeta)=\left(\frac{\overline{r\left(\zeta_{1}\right)}\left(\delta_{B}^{0}\right)^{2} \mathrm{e}^{\frac{\pi \nu}{2}} \mathrm{e}^{-\frac{\mathrm{i} \pi}{4}}}{2 \pi \mathrm{i}\left(\zeta-\zeta_{1}\right) \mathcal{X}_{B} \sqrt{t}}-\frac{\overline{r\left(\zeta_{1}\right)}\left(1-\left|r\left(\zeta_{1}\right)\right|^{2}\right)^{-1}\left(\delta_{B}^{0}\right)^{2} \mathrm{e}^{\frac{3 \pi \mathrm{i}}{4}}}{2 \pi \mathrm{i}\left(\zeta-\zeta_{1}\right) \mathrm{e}^{\frac{3 \pi \nu}{2}} \mathcal{X}_{B} \sqrt{t}}\right) \int_{0}^{+\infty} \mathbf{D}_{\mathrm{i} \nu}(z) z^{\mathrm{i} \nu} \mathrm{e}^{-\frac{z^{2}}{4}} \mathrm{~d} z \\
& +\left(\frac{r\left(\zeta_{1}\right)\left(\delta_{A}^{0}\right)^{2} \mathrm{e}^{-\frac{\pi \nu}{2}} \mathrm{e}^{-\frac{3 \pi \mathrm{i}}{4}}}{2 \pi \mathrm{i}\left(\zeta-\zeta_{2}\right)(-1)^{\mathrm{i} \nu} \mathcal{X}_{A} \sqrt{t}}-\frac{r\left(\zeta_{1}\right)\left(1-\left|r\left(\zeta_{1}\right)\right|^{2}\right)^{-1}\left(\delta_{A}^{0}\right)^{2} \mathrm{e}^{\frac{\mathrm{i} \pi}{4}}}{2 \pi \mathrm{i}\left(\zeta-\zeta_{2}\right) \mathrm{e}^{\frac{\pi \nu}{2}}(-1)^{\mathrm{i} \nu} \mathcal{X}_{A} \sqrt{t}}\right) \int_{0}^{+\infty} \mathbf{D}_{-\mathrm{i} \nu}(z) z^{-\mathrm{i} \nu} \mathrm{e}^{-\frac{z^{2}}{4}} \mathrm{~d} z \\
& +\mathcal{O}\left(\left(\frac{c^{\mathcal{S}}\left(\zeta_{1}\right) \underline{c}\left(\zeta_{2}, \zeta_{3}, \overline{\zeta_{3}}\right)\left(\delta_{B}^{0}\right)^{2}}{\left(\zeta-\zeta_{1}\right)\left|\zeta_{1}-\zeta_{3}\right| \sqrt{\left(\zeta_{1}-\zeta_{2}\right)} \mathcal{X}_{B}}+\frac{c^{\mathcal{S}}\left(\zeta_{2}\right) \underline{c}\left(\zeta_{1}, \zeta_{3}, \overline{\zeta_{3}}\right)\left(\delta_{A}^{0}\right)^{2}}{\left(\zeta-\zeta_{2}\right)\left|\zeta_{2}-\zeta_{3}\right| \sqrt{\left(\zeta_{1}-\zeta_{2}\right)} \mathcal{X}_{A}}\right) \frac{\ln t}{t}\right), \\
& m_{21}^{\Sigma^{\sharp}}(\zeta)=-\left(\frac{r\left(\zeta_{1}\right)\left(\delta_{B}^{0}\right)^{-2} \mathrm{e}^{\frac{\pi \nu}{2}} \mathrm{e}^{\frac{\mathrm{i} \pi}{4}}}{2 \pi \mathrm{i}\left(\zeta-\zeta_{1}\right) \mathcal{X}_{B} \sqrt{t}}-\frac{r\left(\zeta_{1}\right)\left(1-\left|r\left(\zeta_{1}\right)\right|^{2}\right)^{-1}\left(\delta_{B}^{0}\right)^{-2} \mathrm{e}^{-\frac{3 \pi \mathrm{i}}{4}}}{2 \pi \mathrm{i}\left(\zeta-\zeta_{1}\right) \mathrm{e}^{\frac{3 \pi \nu}{2}} \mathcal{X}_{B} \sqrt{t}}\right) \int_{0}^{+\infty} \mathbf{D}_{-\mathrm{i} \nu}(z) z^{-\mathrm{i} \nu} \mathrm{e}^{-\frac{z^{2}}{4}} \mathrm{~d} z \\
& -\left(\frac{\overline{r\left(\zeta_{1}\right)}\left(\delta_{A}^{0}\right)^{-2} \mathrm{e}^{-\frac{\pi \nu}{2}} \mathrm{e}^{\frac{3 \pi \mathrm{i}}{4}}}{2 \pi \mathrm{i}\left(\zeta-\zeta_{2}\right)(-1)^{-\mathrm{i} \nu} \mathcal{X}_{A} \sqrt{t}}-\frac{\overline{r\left(\zeta_{1}\right)}\left(1-\left|r\left(\zeta_{1}\right)\right|^{2}\right)^{-1}\left(\delta_{A}^{0}\right)^{-2} \mathrm{e}^{-\frac{\mathrm{i} \pi}{4}}}{2 \pi \mathrm{i}\left(\zeta-\zeta_{2}\right) \mathrm{e}^{\frac{\pi \nu}{2}}(-1)^{-\mathrm{i} \nu} \mathcal{X}_{A} \sqrt{t}}\right) \int_{0}^{+\infty} \mathbf{D}_{\mathrm{i} \nu}(z) z^{\mathrm{i} \nu} \mathrm{e}^{-\frac{z^{2}}{4}} \mathrm{~d} z \\
& +\mathcal{O}\left(\left(\frac{c^{\mathcal{S}}\left(\zeta_{1}\right) \underline{c}\left(\zeta_{2}, \zeta_{3}, \overline{\zeta_{3}}\right)\left(\delta_{B}^{0}\right)^{-2}}{\left(\zeta-\zeta_{1}\right)\left|\zeta_{1}-\zeta_{3}\right| \sqrt{\left(\zeta_{1}-\zeta_{2}\right)} \mathcal{X}_{B}}+\frac{c^{\mathcal{S}}\left(\zeta_{2}\right) \underline{c}\left(\zeta_{1}, \zeta_{3}, \overline{\zeta_{3}}\right)\left(\delta_{A}^{0}\right)^{-2}}{\left(\zeta-\zeta_{2}\right)\left|\zeta_{2}-\zeta_{3}\right| \sqrt{\left(\zeta_{1}-\zeta_{2}\right)} \mathcal{X}_{A}}\right) \frac{\ln t}{t}\right), \\
& m_{22}^{\sum^{\sharp}}(\zeta)=1+\frac{\overline{r\left(\zeta_{1}\right)}\left(\delta_{B}^{0}\right)^{2} \mathrm{e}^{\frac{\pi \nu}{2}} \mathrm{e}^{-\frac{\mathrm{i} \pi}{4}}}{2 \pi \mathrm{i}\left(\zeta-\zeta_{1}\right) \beta_{12}^{\Sigma_{B}} \mathcal{X}_{B} \sqrt{t}} \int_{0}^{+\infty}\left(\mathrm{e}^{\frac{\mathrm{i} \pi}{4}} \partial_{z} \mathbf{D}_{\mathrm{i} \nu}(z)+\frac{\mathrm{i}}{2} \mathrm{e}^{-\frac{\mathrm{i} \pi}{4}} z \mathbf{D}_{\mathrm{i} \nu}(z)\right) z^{\mathrm{i} \nu} \mathrm{e}^{-\frac{z^{2}}{4}} \mathrm{~d} z \\
& -\frac{\overline{r\left(\zeta_{1}\right)}\left(1-\left|r\left(\zeta_{1}\right)\right|^{2}\right)^{-1}\left(\delta_{B}^{0}\right)^{2} \mathrm{e}^{\frac{3 \pi \mathrm{i}}{4}}}{2 \pi \mathrm{i}\left(\zeta-\zeta_{1}\right) \beta_{12}^{\Sigma} B^{0} \mathrm{e}^{\frac{3 \pi \nu}{2}} \mathcal{X}_{B} \sqrt{t}} \int_{0}^{+\infty}\left(\mathrm{e}^{-\frac{3 \pi \mathrm{i}}{4}} \partial_{z} \mathbf{D}_{\mathrm{i} \nu}(z)+\frac{\mathrm{i}}{2} \mathrm{e}^{\frac{3 \pi \mathrm{i}}{4}} z \mathbf{D}_{\mathrm{i} \nu}(z)\right) z^{\mathrm{i} \nu} \mathrm{e}^{-\frac{z^{2}}{4}} \mathrm{~d} z \\
& +\frac{r\left(\zeta_{1}\right)\left(\delta_{A}^{0}\right)^{2} \mathrm{e}^{-\frac{\pi \nu}{2}} \mathrm{e}^{-\frac{3 \pi \mathrm{i}}{4}}}{2 \pi \mathrm{i}\left(\zeta-\zeta_{2}\right) \beta_{12}^{\Sigma} A^{0}(-1)^{\mathrm{i} \nu} \mathcal{X}_{A} \sqrt{t}} \int_{0}^{+\infty}\left(\mathrm{e}^{\frac{3 \pi \mathrm{i}}{4}} \partial_{z} \mathbf{D}_{-\mathrm{i} \nu}(z)-\frac{\mathrm{i}}{2} \mathrm{e}^{-\frac{3 \pi \mathrm{i}}{4}} z \mathbf{D}_{-\mathrm{i} \nu}(z)\right) z^{-\mathrm{i} \nu} \mathrm{e}^{-\frac{z^{2}}{4}} \mathrm{~d} z \\
& -\frac{r\left(\zeta_{1}\right)\left(1-\left|r\left(\zeta_{1}\right)\right|^{2}\right)^{-1}\left(\delta_{A}^{0}\right)^{2} \mathrm{e}^{\frac{\mathrm{i} \pi}{4}}}{2 \pi \mathrm{i}\left(\zeta-\zeta_{2}\right) \beta_{12}^{{ }^{2} A^{0}} \mathrm{e}^{\frac{\pi \nu}{2}}(-1)^{\mathrm{i} \nu} \mathcal{X}_{A} \sqrt{t}} \int_{0}^{+\infty}\left(\mathrm{e}^{-\frac{\mathrm{i} \pi}{4}} \partial_{z} \mathbf{D}_{-\mathrm{i} \nu}(z)-\frac{\mathrm{i}}{2} \mathrm{e}^{\frac{\mathrm{i} \pi}{4}} z \mathbf{D}_{-\mathrm{i} \nu}(z)\right) z^{-\mathrm{i} \nu} \mathrm{e}^{-\frac{z^{2}}{4}} \mathrm{~d} z \\
& +\mathcal{O}\left(\left(\frac{c^{\mathcal{S}}\left(\zeta_{1}\right) \underline{c}\left(\zeta_{2}, \zeta_{3}, \overline{\zeta_{3}}\right)\left(\delta_{B}^{0}\right)^{2}}{\left(\zeta-\zeta_{1}\right)\left|\zeta_{1}-\zeta_{3}\right| \sqrt{\left(\zeta_{1}-\zeta_{2}\right)} \mathcal{X}_{B}}+\frac{c^{\mathcal{S}}\left(\zeta_{2}\right) \underline{c}\left(\zeta_{1}, \zeta_{3}, \overline{\zeta_{3}}\right)\left(\delta_{A}^{0}\right)^{2}}{\left(\zeta-\zeta_{2}\right)\left|\zeta_{2}-\zeta_{3}\right| \sqrt{\left(\zeta_{1}-\zeta_{2}\right)} \mathcal{X}_{A}}\right) \frac{\ln t}{t}\right),
\end{aligned}
$$

where $\delta_{A}^{0}$ and $\delta_{B}^{0}$ are defined in Proposition 5.1,

$$
\begin{aligned}
& \chi\left(\zeta_{1}\right):=\frac{\mathrm{i}}{2 \pi} \int_{-\infty}^{0} \ln \left|\mu-\zeta_{1}\right| \mathrm{d} \ln \left(1-|r(\mu)|^{2}\right)+\frac{\mathrm{i}}{2 \pi} \int_{\zeta_{2}}^{\zeta_{1}} \ln \left|\mu-\zeta_{1}\right| \mathrm{d} \ln \left(1-|r(\mu)|^{2}\right), \\
& \chi\left(\zeta_{2}\right):=-\chi\left(\zeta_{1}\right)+\frac{\mathrm{i}}{2 \pi} \int_{-\infty}^{0} \ln |\mu| \mathrm{d} \ln \left(1-|r(\mu)|^{2}\right)+\frac{\mathrm{i}}{2 \pi} \int_{\zeta_{2}}^{\zeta_{1}} \ln |\mu| \mathrm{d} \ln \left(1-|r(\mu)|^{2}\right), \\
& \mathcal{X}_{B}=\frac{\left|\zeta_{1}-\zeta_{3}\right|}{\zeta_{1}} \sqrt{\frac{2\left(\zeta_{1}-\zeta_{2}\right)}{\zeta_{1}}}, \quad \mathcal{X}_{A}=\frac{\left|\zeta_{2}-\zeta_{3}\right|}{\zeta_{2}} \sqrt{\frac{2\left(\zeta_{1}-\zeta_{2}\right)}{\zeta_{2}}}, \\
& \beta_{12}^{\Sigma_{B^{0}}}=\overline{\beta_{21}^{\Sigma_{B^{0}}}}=\frac{\sqrt{2 \pi} \mathrm{e}^{-\frac{\pi \nu}{2}} \mathrm{e}^{\frac{\mathrm{i} \pi}{4}}}{r\left(\zeta_{1}\right) \overline{\Gamma(\mathrm{i} \nu)}}, \quad \beta_{12}^{\Sigma_{A^{0}}}=\overline{\beta_{21}^{\Sigma_{A^{0}}}}=\frac{\sqrt{2 \pi} \mathrm{e}^{-\frac{\pi \nu}{2}} \mathrm{e}^{-\frac{\mathrm{i} \pi}{4}}}{\overline{r\left(\zeta_{1}\right)} \Gamma(\mathrm{i} \nu)} .
\end{aligned}
$$


From the above expressions for $\mathcal{X}_{B}$ and $\mathcal{X}_{A}$, one notes that the terms $\left|\zeta_{1}-\zeta_{3}\right| \zeta_{1}^{-1}$ and $\mid \zeta_{2}-$ $\zeta_{3} \mid \zeta_{2}^{-1}$ must be calculated: using the expressions for $\left\{\zeta_{i}\right\}_{i=1}^{3}$ defined in Theorem 3.1, Eqs. (16) and (17), along with the fact that $\zeta_{1} \zeta_{2}=\zeta_{3} \overline{\zeta_{3}}=1$, one shows that $\left|\zeta_{k}-\zeta_{3}\right| \zeta_{k}^{-1}=\left(2 \zeta_{k}\right)^{-1 / 2}\left(z_{o}^{2}+\right.$ $32)^{1 / 4}, k \in\{1,2\}$. Finally, assembling the above formulae, using the identities [40] $\partial_{z} \mathbf{D}_{z_{1}}(z)=$ $\frac{1}{2}\left(z_{1} \mathbf{D}_{z_{1}-1}(z)-\mathbf{D}_{z_{1}+1}(z)\right), z \mathbf{D}_{z_{1}}(z)=\mathbf{D}_{z_{1}+1}(z)+z_{1} \mathbf{D}_{z_{1}-1}(z)$, and $|\Gamma(\mathrm{i} \nu)|^{2}=\frac{\pi}{\nu \sinh (\pi \nu)}$, and the integral 40]

$$
\int_{0}^{+\infty} \mathbf{D}_{-z_{1}}(z) z^{z_{2}-1} \exp \left(-\frac{z^{2}}{4}\right) \mathrm{d} z=\frac{\sqrt{\pi} \exp \left(-\frac{1}{2}\left(z_{1}+z_{2}\right) \ln 2\right) \Gamma\left(z_{2}\right)}{\Gamma\left(\frac{1}{2}\left(z_{1}+z_{2}\right)+\frac{1}{2}\right)}, \quad \Re\left(z_{2}\right)>0,
$$

one obtains, upon using (repeatedly) the relation $\left|r\left(\zeta_{1}\right)\right||\Gamma(\mathrm{i} \nu)| \nu \mathrm{e}^{\frac{\pi \nu}{2}}=\sqrt{2 \pi \nu}$, and the fact that, for $\zeta \in\left(\mathbb{C} \backslash \cup_{\lambda \in\left\{\zeta_{2}, \zeta_{1}\right\}} \mathbb{U}(\lambda ; \varepsilon)\right) \cap\left(\Omega_{1} \cup \Omega_{2}\right), m^{c}(\zeta)=m^{\Sigma^{\sharp}}(\zeta)(\delta(\zeta))^{\sigma_{3}}\left(\mathrm{I}+\mathcal{O}\left(\frac{\underline{c}\left(\zeta_{1}, \zeta_{2}, \zeta_{3}, \overline{\zeta_{3}}\right) \triangleleft(\zeta)}{\left|z_{o}+\zeta_{1}+\zeta_{2}\right|^{l} t^{l}}\right)\right)$, with arbitrarily large $l \in \mathbb{Z}_{\geqslant 1}, \|\left.\diamond(\cdot)\right|_{\mathcal{L}_{\mathrm{M}_{2}(\mathbb{C})}^{\infty}\left(\mathbb{C} \backslash \cup_{\lambda \in\left\{\zeta_{2}, \zeta_{1}\right\}} \mathbb{U}(\lambda ; \varepsilon)\right)}<\infty$, and $\delta(\zeta)$ given in Proposition 4.1, the result stated in the Lemma; furthermore, one shows that the symmetry reduction $m^{c}(\zeta)=$ $\sigma_{1} \overline{m^{c}(\bar{\zeta})} \sigma_{1}$ is satisfied, and verifies that, to $\mathcal{O}\left(t^{-1} \ln t\right),\left(m^{c}(0) \sigma_{2}\right)^{2}=\mathrm{I}$.

Proposition 6.1. As $t \rightarrow+\infty$ such that $0<\zeta_{2}<\frac{1}{M}<M<\zeta_{1}$ and $\left|\zeta_{3}\right|^{2}=1$, with $M \in \mathbb{R}_{>1}$ and bounded,

$$
\begin{aligned}
& \left(\Delta_{o}\right)_{11}=-\frac{2 \mathrm{i} \sqrt{\nu\left(\zeta_{1}\right)} \cos \left(\Theta^{+}\left(z_{o}, t\right)+\frac{\pi}{4}\right)}{\sqrt{t\left(\zeta_{1}-\zeta_{2}\right)}\left(z_{o}^{2}+32\right)^{1 / 4}}+\mathcal{O}\left(\left(\frac{c^{\mathcal{S}}\left(\zeta_{1}\right) \underline{c}\left(\zeta_{2}, \zeta_{3}, \overline{\zeta_{3}}\right)}{\sqrt{\zeta_{1}\left(z_{o}^{2}+32\right)}}+\frac{c^{\mathcal{S}}\left(\zeta_{2}\right) \underline{c}\left(\zeta_{1}, \zeta_{3}, \overline{\zeta_{3}}\right)}{\sqrt{\zeta_{2}\left(z_{o}^{2}+32\right)}}\right) \frac{\ln t}{\left(\zeta_{1}-\zeta_{2}\right) t}\right), \\
& \left(\Delta_{o}\right)_{12}=-\mathrm{i} \exp \left(-\mathrm{i}\left(\int_{-\infty}^{0} \frac{\ln \left(1-|r(\mu)|^{2}\right)}{\mu} \frac{\mathrm{d} \mu}{2 \pi}+\int_{\zeta_{2}}^{\zeta_{1}} \frac{\ln \left(1-|r(\mu)|^{2}\right)}{\mu} \frac{\mathrm{d} \mu}{2 \pi}\right)\right) \\
& \times\left(1+\mathcal{O}\left(\left(\frac{c^{\mathcal{S}}\left(\zeta_{1}\right) \underline{c}\left(\zeta_{2}, \zeta_{3}, \overline{\zeta_{3}}\right)}{\sqrt{\zeta_{1}\left(z_{o}^{2}+32\right)}}+\frac{c^{\mathcal{S}}\left(\zeta_{2}\right) \underline{c}\left(\zeta_{1}, \zeta_{3}, \overline{\zeta_{3}}\right)}{\sqrt{\zeta_{2}\left(z_{o}^{2}+32\right)}}\right) \frac{\ln t}{\left(\zeta_{1}-\zeta_{2}\right) t}\right)\right), \\
& \left(\Delta_{o}\right)_{21}=\mathrm{i} \exp \left(\mathrm{i}\left(\int_{-\infty}^{0} \frac{\ln \left(1-|r(\mu)|^{2}\right)}{\mu} \frac{\mathrm{d} \mu}{2 \pi}+\int_{\zeta_{2}}^{\zeta_{1}} \frac{\ln \left(1-|r(\mu)|^{2}\right)}{\mu} \frac{\mathrm{d} \mu}{2 \pi}\right)\right) \\
& \times\left(1+\mathcal{O}\left(\left(\frac{c^{\mathcal{S}}\left(\zeta_{1}\right) \underline{c}\left(\zeta_{2}, \zeta_{3}, \overline{\zeta_{3}}\right)}{\sqrt{\zeta_{1}\left(z_{o}^{2}+32\right)}}+\frac{c^{\mathcal{S}}\left(\zeta_{2}\right) \underline{c}\left(\zeta_{1}, \zeta_{3}, \overline{\zeta_{3}}\right)}{\sqrt{\zeta_{2}\left(z_{o}^{2}+32\right)}}\right) \frac{\ln t}{\left(\zeta_{1}-\zeta_{2}\right) t}\right)\right), \\
& \left(\Delta_{o}\right)_{22}=\frac{2 \mathrm{i} \sqrt{\nu\left(\zeta_{1}\right)} \cos \left(\Theta^{+}\left(z_{o}, t\right)+\frac{\pi}{4}\right)}{\sqrt{t\left(\zeta_{1}-\zeta_{2}\right)}\left(z_{o}^{2}+32\right)^{1 / 4}}+\mathcal{O}\left(\left(\frac{c^{\mathcal{S}}\left(\zeta_{1}\right) \underline{c}\left(\zeta_{2}, \zeta_{3}, \overline{\zeta_{3}}\right)}{\sqrt{\zeta_{1}\left(z_{o}^{2}+32\right)}}+\frac{c^{\mathcal{S}}\left(\zeta_{2}\right) \underline{\underline{c}}\left(\zeta_{1}, \zeta_{3}, \overline{\zeta_{3}}\right)}{\sqrt{\zeta_{2}\left(z_{o}^{2}+32\right)}}\right) \frac{\ln t}{\left(\zeta_{1}-\zeta_{2}\right) t}\right),
\end{aligned}
$$

where $\nu(z)$ and $\Theta^{+}\left(z_{o}, t\right)$ are given in Theorem 3.1, Eqs. (12)-(14).

Proof. One recalls from Lemma 2.6 that $\left(x, t\right.$ dependences suppressed) $\Delta_{o} m^{c}(0)=\sigma_{2}$ : one deduces from this that $\left(\Delta_{o}\right)_{11}=\mathrm{i} m_{21}^{c}(0),\left(\Delta_{o}\right)_{12}=-\mathrm{i} m_{11}^{c}(0),\left(\Delta_{o}\right)_{21}=\mathrm{i} m_{22}^{c}(0)$, and $\left(\Delta_{o}\right)_{22}=-\mathrm{i} m_{12}^{c}(0)$. Using the formulae for $m_{i j}^{c}(\zeta), i, j \in\{1,2\}$, given in Lemma 6.1 , and the fact that, via an integration by parts argument, $\delta(0) \zeta_{1}^{-2 \mathrm{i} \nu\left(\zeta_{1}\right)} \exp \left(-\frac{\mathrm{i} \Omega^{+}(0)}{2}\right)=1$, one obtains the result stated in the Proposition; furthermore, one also verifies that, to $\mathcal{O}\left(t^{-1} \ln t\right), \Delta_{o}$ has the order 2 matrix involutive structure stated in Lemma 2.6, and $\operatorname{det}\left(\Delta_{o}\right)=-1$.

Lemma 6.2. As $t \rightarrow+\infty$ such that $0<\zeta_{2}<\frac{1}{M}<M<\zeta_{1}$ and $\left|\zeta_{3}\right|^{2}=1$, with $M \in$ $\mathbb{R}_{>1}$ and bounded, $u(x, t)$, the solution of the Cauchy problem for the $\mathrm{D}_{f} \mathrm{NLSE}$, has the asymptotics stated in Theorem 3.1, Eqs. (9), (10), (12), (13), (14), (16), and (17), and $\int_{ \pm \infty}^{x}\left(|u(\xi, t)|^{2}-1\right) \mathrm{d} \xi$ have the asymptotics stated in Theorem 3.1, Eqs. (25) and (26), with $\theta^{+}(z)$ (respectively $\theta^{-}(z)$ ) defined in Theorem 3.1, Eq. (10) (respectively Eq. (11)).

Proof. From the proof of Lemma 2.4, one recalls that ( $x, t$ dependences suppressed) $m(\zeta)=\underset{\zeta \in \mathbb{C} \backslash \mathbb{R}}{\ln } \mathrm{I}+\frac{1}{\zeta}\left(\begin{array}{cc}\mathrm{i} \int_{+\infty}^{x}\left(|u(\xi, t)|^{2}-1\right) \mathrm{d} \xi & -\mathrm{i} u(x, t) \\ \mathrm{i} u(x, t) & -\mathrm{i} \int_{+\infty}^{x}\left(|u(\xi, t)|^{2}-1\right) \mathrm{d} \xi\end{array}\right)+\mathcal{O}\left(\zeta^{-2}\right) ;$ in particular, and without loss of generality, the $\zeta \rightarrow \infty$ asymptotics are taken in the domain $\Omega_{1} \cup \Omega_{2} \subset \mathbb{C} \backslash \mathbb{R}$ 
(Figure 3). One recalls the ordered factorisation for $m(\zeta)$ given in Lemma 2.6, Eq. (7), namely, $m(\zeta)=\left(\mathrm{I}+\Delta_{o} \zeta^{-1}\right) m^{c}(\zeta)$, with $m^{c}(\zeta)$ (respectively $\Delta_{o}$ ) given in Lemma 6.1 (respectively Proposition 6.1). From the $\zeta \rightarrow \infty, \zeta \in \Omega_{1} \cup \Omega_{2}$, asymptotics of $m(\zeta)$ and its ordered factorisation (both given above), one arrives at (and the complex conjugates of) $-\mathrm{i} u(x, t)=$ $\lim _{\substack{\zeta \rightarrow \infty \\ \zeta \in \Omega_{1} \cup \Omega_{2}}}(\zeta(m(\zeta)-\mathrm{I}))_{12}=\left(\Delta_{o}\right)_{12}+\lim _{\substack{\zeta \rightarrow \infty \\ \zeta \in \Omega_{1} \cup \Omega_{2}}}\left(\zeta\left(m^{c}(\zeta)-\mathrm{I}\right)\right)_{12}$ and $\mathrm{i} \int_{+\infty}^{x}\left(|u(\xi, t)|^{2}-1\right) \mathrm{d} \xi=$ $\lim _{\substack{\zeta \rightarrow \infty \\ \zeta \in \Omega_{1} \cup \Omega_{2}}}(\zeta(m(\zeta)-\mathrm{I}))_{11}=\left(\Delta_{o}\right)_{11}+\lim _{\substack{\zeta \in \Omega_{1} \cup \Omega_{2} \\ \zeta \rightarrow \Omega^{\prime}}}\left(\zeta\left(m^{c}(\zeta)-\mathrm{I}\right)\right)_{11}$ : using the expressions for $m_{i j}^{c}(\zeta)$ and $\left(\Delta_{o}\right)_{i j}, i, j \in\{1,2\}$, given in Lemma 6.1 and Proposition 6.1, respectively, the asymp-

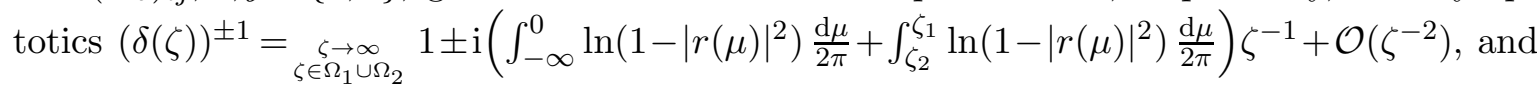
the trace identity (cf. Corollary 2.4) $\int_{-\infty}^{+\infty}\left(|u(\xi, t)|^{2}-1\right) \mathrm{d} \xi=-\int_{-\infty}^{+\infty} \ln \left(1-|r(\mu)|^{2}\right) \frac{\mathrm{d} \mu}{2 \pi}$, one obtains the result stated in the Lemma.

\section{$7 \quad$ Asymptotics as $t \rightarrow-\infty$}

In this section, as $t \rightarrow-\infty$ and $x \rightarrow+\infty$ such that $z_{o}:=x / t<-2$, the RHP for $m^{c}(\zeta)$ on $\sigma_{c}$ formulated in Lemma 2.6 is discussed succinctly, and the asymptotics of $u(x, t)$ and related integrals are obtained. As the calculations subsumed in this section are analogous to those presented in Sections 4-6, only final results, with in one instance a sketch of a proof, are given.

As $t \rightarrow-\infty$ and $x \rightarrow+\infty$ such that $z_{o}<-2$, one begins by decomposing the complex plane of the spectral parameter $\zeta$ according to the signature of $\Re\left(\mathrm{i} t \theta^{u}(\zeta)\right.$ ) (see Figure 6 ), where, from Eq. (8), $\theta^{u}(\zeta)=\frac{1}{2}\left(\zeta-\frac{1}{\zeta}\right)\left(z_{o}+\zeta+\frac{1}{\zeta}\right)$, with $\left\{\zeta_{i}\right\}_{i=1}^{4}$ defined in Theorem 3.1, Eqs. (16) and (17), $0<\zeta_{2}<\zeta_{1},\left|\zeta_{3}\right|^{2}=1$, and $\pm \leftrightarrow \Re\left(\mathrm{i} t \theta^{u}(\zeta)\right) \gtrless 0$. One now reorients $\sigma_{c}$, oriented from

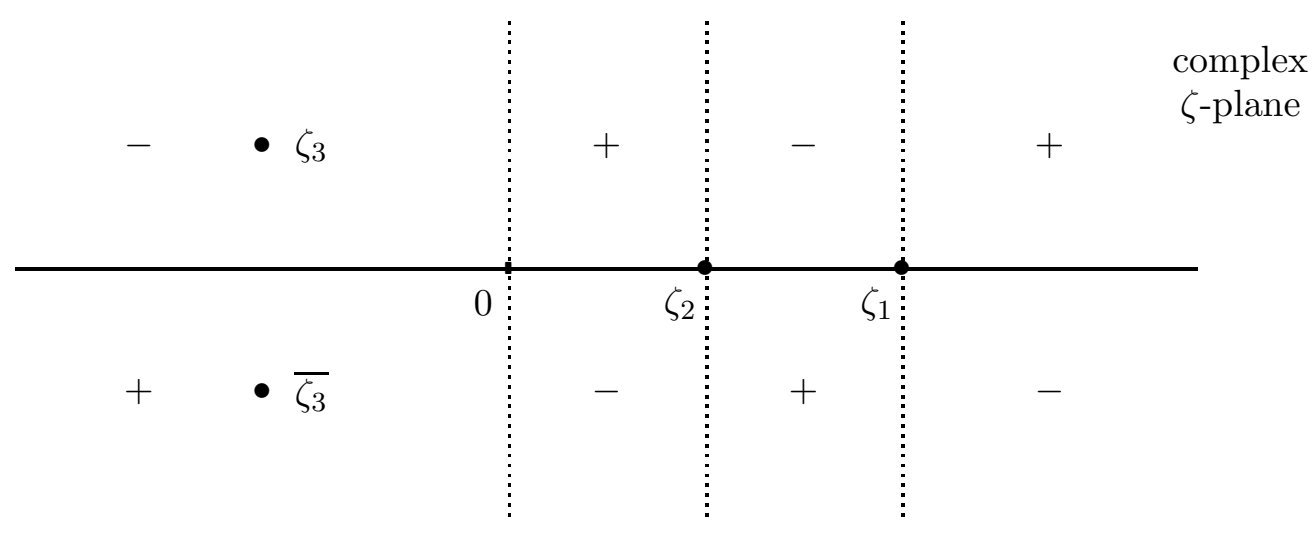

Figure 6: Signature graph of $\Re\left(\mathrm{it} \theta^{u}(\zeta)\right)$ as $t \rightarrow-\infty$

$-\infty$ to $+\infty$, according to, and consistent with, the signature graph of $\Re\left(\mathrm{it} \theta^{u}(\zeta)\right.$ ), leading to the reoriented contour $\sigma_{c}^{\prime \prime}$ (see Figure 7). Denoting $m^{c}(\zeta)$ on $\sigma_{c}^{\prime \prime}$ by $\mathcal{M}^{c}(\zeta)$, one shows that

complex

$\zeta$-plane

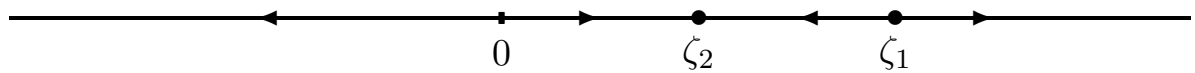

Figure 7: Reoriented contour $\sigma_{c}^{\prime \prime}$

(recalling Lemma 2.6) $\mathcal{M}^{c}(\zeta): \mathbb{C} \backslash \sigma_{c}^{\prime \prime} \rightarrow \mathrm{SL}(2, \mathbb{C}$ ) solves the following (normalised at $\infty$ ) RHP: 
(1) $\mathcal{M}^{c}(\zeta)$ is piecewise holomorphic $\forall \zeta \in \mathbb{C} \backslash \sigma_{c}^{\prime \prime} ;(2) \mathcal{M}_{ \pm}^{c}(\zeta):=\lim _{\substack{\zeta^{\prime} \in \pm \text { sideof } \sigma_{c}^{\prime \prime} \\ \zeta^{\prime} \rightarrow \zeta}} \mathcal{M}^{c}\left(\zeta^{\prime}\right)$ satisfy the jump condition $\mathcal{M}_{+}^{c}(\zeta)=\mathcal{M}_{-}^{c}(\zeta) \mathcal{G}^{c}(\zeta), \zeta \in \sigma_{c}^{\prime \prime}$, where

$$
\mathcal{G}^{c}(\zeta):= \begin{cases}\left(\mathrm{I}-\overline{r(\bar{\zeta})} \mathrm{e}^{-2 \mathrm{i} t \theta^{u}(\zeta)} \sigma_{+}\right)\left(\mathrm{I}+r(\zeta) \mathrm{e}^{2 \mathrm{i} t \theta^{u}(\zeta)} \sigma_{-}\right), & \zeta \in\left(0, \zeta_{2}\right) \cup\left(\zeta_{1},+\infty\right), \\ \left(\mathrm{I}-r(\zeta) \mathrm{e}^{2 \mathrm{i} t \theta^{u}(\zeta)} \sigma_{-}\right)\left(\mathrm{I}+\overline{r(\bar{\zeta})} \mathrm{e}^{-2 \mathrm{i} t \theta^{u}(\zeta)} \sigma_{+}\right), & \zeta \in(-\infty, 0) \cup\left(\zeta_{2}, \zeta_{1}\right)\end{cases}
$$

(3) as $\zeta \rightarrow \infty, \zeta \in \mathbb{C} \backslash \sigma_{c}^{\prime \prime}, \mathcal{M}^{c}(\zeta)=\mathrm{I}+\mathcal{O}\left(\zeta^{-1}\right)$; and (4) $\mathcal{M}^{c}(\zeta)$ satisfies the symmetry reduction $\mathcal{M}^{c}(\zeta)=\sigma_{1} \overline{\mathcal{M}^{c}(\bar{\zeta})} \sigma_{1}$ and the condition $\left(\mathcal{M}^{c}(0) \sigma_{2}\right)^{2}=\mathrm{I}$. The analogue, therefore, of Proposition 4.1 and Lemma 4.1 is the following

Lemma 7.1. Let $\widetilde{\delta}(\zeta)$ solve the following scalar discontinuous RHP:

$$
\begin{aligned}
\widetilde{\delta}_{+}(\zeta) & = \begin{cases}\widetilde{\delta}_{-}(\zeta)(1-r(\zeta \overline{r(\bar{\zeta})}), & \Re(\zeta) \in\left(0, \zeta_{2}\right) \cup\left(\zeta_{1},+\infty\right), \\
\widetilde{\delta}_{-}(\zeta)=\widetilde{\delta}(\zeta), & \Re(\zeta) \in(-\infty, 0) \cup\left(\zeta_{2}, \zeta_{1}\right),\end{cases} \\
\widetilde{\delta}(\zeta) \underset{\zeta \rightarrow \infty}{=} 1+\mathcal{O}\left(\zeta^{-1}\right), &
\end{aligned}
$$

with index $\kappa:=\frac{1}{2 \pi}[\arg (1-r(\zeta) \overline{r(\bar{\zeta})})]_{-\infty}^{+\infty}=0$. The unique solution of this $\mathrm{RHP}$ can be written as

$$
\widetilde{\delta}(\zeta)=\left(\frac{\zeta-\zeta_{2}}{\zeta-\zeta_{1}}\right)^{\mathrm{i} \nu} \exp \left(\frac{\mathrm{i}}{2 \pi}\left(\int_{0}^{\zeta_{2}}+\int_{\zeta_{1}}^{+\infty}\right) \ln (\mu-\zeta) \mathrm{d} \ln \left(1-|r(\mu)|^{2}\right)\right)
$$

where $\left\{\zeta_{i}\right\}_{i=1}^{2}$ are defined in Theorem 3.1, Eqs. (16) and (17), $\nu:=\nu\left(\zeta_{1}\right)=-\frac{1}{2 \pi} \ln (1-$ $\left.\left|r\left(\zeta_{1}\right)\right|^{2}\right) \in \mathbb{R}_{+}, \widetilde{\delta}(\zeta) \overline{\widetilde{\delta}(\bar{\zeta})}=1, \widetilde{\delta}(\zeta) \widetilde{\delta}\left(\frac{1}{\zeta}\right)=\widetilde{\delta}(0)=\exp \left(\left(\int_{0}^{\zeta_{2}}+\int_{\zeta_{1}}^{+\infty}\right) \frac{\ln \left(1-|r(\mu)|^{2}\right)}{\mu} \frac{\mathrm{d} \mu}{2 \pi}\right),\left|\widetilde{\delta}_{+}(\zeta)\right|^{2} \leqslant 1$ and $\left|\widetilde{\delta}_{-}(\zeta)\right|^{2} \leqslant\left(1-\sup _{z \in \mathbb{R}}|r(z)|^{2}\right)^{-1}<\infty \forall \zeta \in \mathbb{R}$, and ||$(\widetilde{\delta}(\cdot))^{ \pm 1}||_{\mathcal{L}^{\infty}(\mathbb{C})}:=\sup _{\zeta \in \mathbb{C}}\left|(\widetilde{\delta}(\zeta))^{ \pm 1}\right|<$ $\infty$. Set $\widetilde{\mathcal{M}}^{c}(\zeta):=\mathcal{M}^{c}(\zeta)(\widetilde{\delta}(\zeta))^{-\sigma_{3}}$. Then $\widetilde{\mathcal{M}}^{c}(\zeta): \mathbb{C} \backslash \sigma_{c}^{\prime \prime} \rightarrow \operatorname{SL}(2, \mathbb{C})$ solves the following RHP: (1) $\tilde{\mathcal{M}}^{c}(\zeta)$ is piecewise holomorphic $\forall \zeta \in \mathbb{C} \backslash \sigma_{c}^{\prime \prime}$; (2) $\tilde{\mathcal{M}}_{ \pm}^{c}(\zeta):=\lim _{\substack{\zeta^{\prime} \rightarrow \zeta \\ \zeta^{\prime} \in \pm \text { side of } \sigma_{c}^{\prime \prime}}} \tilde{\mathcal{M}}^{c}\left(\zeta^{\prime}\right)$ satisfy the jump condition $\tilde{\mathcal{M}}_{+}^{c}(\zeta)=\widetilde{\mathcal{M}}_{-}^{c}(\zeta) \widetilde{\mathcal{G}}^{c}(\zeta), \zeta \in \sigma_{c}^{\prime \prime}$, where

$\widetilde{\mathcal{G}}^{c}(\zeta):= \begin{cases}\left(\mathrm{I}-\overline{\widetilde{\rho}(\bar{\zeta})}\left(\widetilde{\delta}_{-}(\zeta)\right)^{-2} \mathrm{e}^{2 \mathrm{i} t \theta^{u}(\zeta)} \sigma_{-}\right)\left(\mathrm{I}+\widetilde{\rho}(\zeta)\left(\widetilde{\delta}_{+}(\zeta)\right)^{2} \mathrm{e}^{-2 \mathrm{i} t \theta^{u}(\zeta)} \sigma_{+}\right), & \zeta \in\left(0, \zeta_{2}\right) \cup\left(\zeta_{1},+\infty\right), \\ \left(\mathrm{I}-\overline{\widetilde{\rho}(\bar{\zeta})}(\widetilde{\delta}(\zeta))^{-2} \mathrm{e}^{2 \mathrm{i} t \theta^{u}(\zeta)} \sigma_{-}\right)\left(\mathrm{I}+\widetilde{\rho}(\zeta)(\widetilde{\delta}(\zeta))^{2} \mathrm{e}^{-2 \mathrm{i} t \theta^{u}(\zeta)} \sigma_{+}\right), & \zeta \in(-\infty, 0) \cup\left(\zeta_{2}, \zeta_{1}\right),\end{cases}$

with

$$
\widetilde{\rho}(\zeta):= \begin{cases}-\overline{r(\bar{\zeta})}(1-r(\zeta) \overline{r(\bar{\zeta})})^{-1}, & \zeta \in\left(0, \zeta_{2}\right) \cup\left(\zeta_{1},+\infty\right) \\ r(\bar{\zeta}), & \zeta \in(-\infty, 0) \cup\left(\zeta_{2}, \zeta_{1}\right)\end{cases}
$$

(3) as $\zeta \rightarrow \infty, \zeta \in \mathbb{C} \backslash \sigma_{c}^{\prime \prime}, \tilde{\mathcal{M}}^{c}(\zeta)=\mathrm{I}+\mathcal{O}\left(\zeta^{-1}\right)$; and (4) $\tilde{\mathcal{M}}^{c}(\zeta)$ satisfies the symmetry reduction $\widetilde{\mathcal{M}}^{c}(\zeta)=\sigma_{1} \overline{\widetilde{\mathcal{M}}^{c}(\bar{\zeta})} \sigma_{1}$ and the condition $\left(\widetilde{\mathcal{M}}^{c}(0)(\widetilde{\delta}(0))^{\sigma_{3}} \sigma_{2}\right)^{2}=\mathrm{I}$.

Now, proceeding according to an analysis analogous to that presented in Section 4, one arrives at the following "model" RHP on $\widetilde{\Sigma}$ (see Figure 8) for $\widetilde{\mathcal{M}}^{\widetilde{\Sigma}}(\zeta)$ (analogue of Lemma 4.6):

Lemma 7.2. Set $\widetilde{\Sigma}:=\widehat{\mathcal{L}} \cup \overline{\mathcal{L}}$, where $\widehat{\mathcal{L}}=\left\{\zeta ; \zeta=\zeta_{1}+\frac{v}{\sqrt{2}}\left(\zeta_{1}-\zeta_{2}\right) \mathrm{e}^{\frac{3 \pi \mathrm{i}}{4}},-\infty<v<\varepsilon\right\} \cup\{\zeta ; \zeta=$ $\left.\zeta_{2}+\frac{v}{\sqrt{2}}\left(\zeta_{1}-\zeta_{2}\right) \mathrm{e}^{\frac{\mathrm{i} \pi}{4}}, 0 \leqslant v<\varepsilon\right\} \cup\left\{\zeta ; \zeta=\zeta_{2}+\frac{v}{\sqrt{2}} \zeta_{2} \mathrm{e}^{-\frac{3 \pi \mathrm{i}}{4}}, 0 \leqslant v<\varepsilon\right\}$, and $\varepsilon$ is an arbitrarily fixed, sufficiently small positive real number. As $t \rightarrow-\infty$ such that $0<\zeta_{2}<\frac{1}{M}<M<\zeta_{1}$ and 

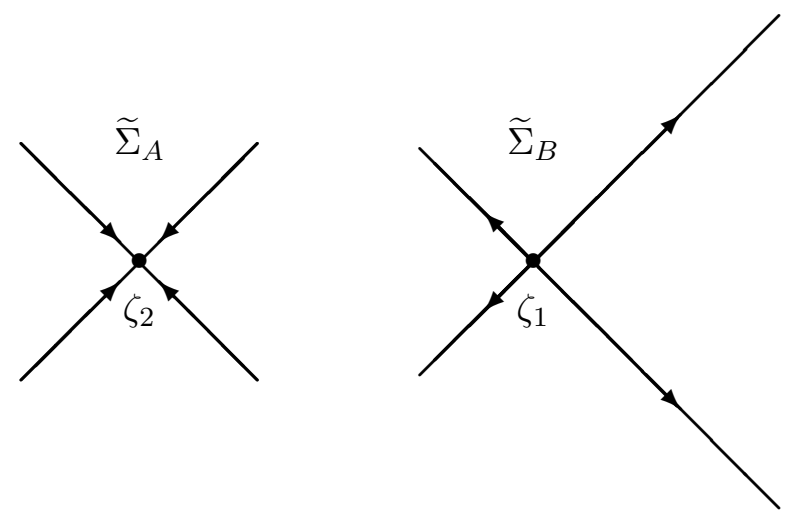

Figure 8: Truncated contour $\widetilde{\Sigma}:=\widetilde{\Sigma}_{A} \cup \widetilde{\Sigma}_{B}$

$\left|\zeta_{3}\right|^{2}=1$, with $M \in \mathbb{R}_{>1}$ and bounded, $\widetilde{\mathcal{M}}^{\widetilde{\Sigma}}(\zeta): \mathbb{C} \backslash \widetilde{\Sigma} \rightarrow \mathrm{SL}(2, \mathbb{C})$ solves the following RHP: (1)

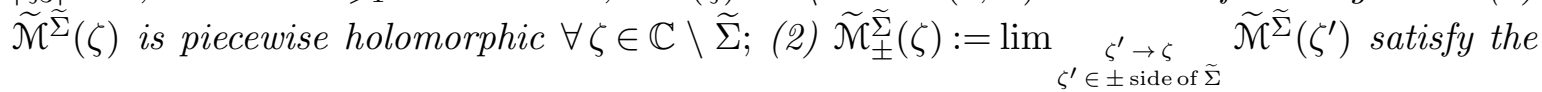
jump condition $\widetilde{\mathcal{M}}_{+}^{\widetilde{\Sigma}}(\zeta)=\widetilde{\mathcal{M}}_{-}^{\widetilde{\Sigma}}(\zeta)\left(\mathrm{I}-\widetilde{w}_{-}^{\widetilde{\Sigma}}(\zeta)\right)^{-1}\left(\mathrm{I}+\widetilde{w}_{+}^{\widetilde{\Sigma}}(\zeta)\right), \zeta \in \widetilde{\Sigma}$, where

$$
\begin{aligned}
& \widetilde{w}_{+}^{\widetilde{\Sigma}}(\zeta)=\left(\begin{array}{ll}
0 & 0 \\
0 & 0
\end{array}\right), \quad \widetilde{w}_{-}^{\widetilde{\Sigma}}(\zeta)=-(\widetilde{\delta}(\zeta))^{\operatorname{ad}\left(\sigma_{3}\right)} \exp \left(-\mathrm{i} t \theta^{u}(\zeta) \operatorname{ad}\left(\sigma_{3}\right)\right) \overline{\widetilde{\mathcal{R}}(\zeta)} \sigma_{-}, \quad \zeta \in \widehat{\mathcal{L}} \subset \widetilde{\Sigma} \\
& \widetilde{w}_{+}^{\widetilde{\Sigma}}(\zeta)=(\widetilde{\delta}(\zeta))^{\operatorname{ad}\left(\sigma_{3}\right)} \exp \left(-\mathrm{i} t \theta^{u}(\zeta) \operatorname{ad}\left(\sigma_{3}\right)\right) \widetilde{\mathcal{R}}(\zeta) \sigma_{+}, \quad \widetilde{w}_{-}^{\widetilde{\Sigma}}(\zeta)=\left(\begin{array}{ll}
0 & 0 \\
0 & 0
\end{array}\right), \quad \zeta \in \overline{\widehat{\mathcal{L}}} \subset \widetilde{\Sigma}
\end{aligned}
$$

with $\widetilde{\mathcal{R}}(\zeta)$ the analogue of $\mathcal{R}(\zeta)$ defined in Lemma $4.2 ;$ (3) as $\zeta \rightarrow \infty, \zeta \in \mathbb{C} \backslash \widetilde{\Sigma}, \widetilde{\mathcal{M}}^{\widetilde{\Sigma}}(\zeta)=$ $\mathrm{I}+\mathcal{O}\left(\zeta^{-1}\right)$; and (4) $\tilde{\mathcal{M}}^{\widetilde{\Sigma}}(\zeta)$ satisfies the symmetry reduction $\widetilde{\mathcal{M}}^{\widetilde{\Sigma}}(\zeta)=\sigma_{1} \overline{\widetilde{\mathcal{M}}^{\widetilde{\Sigma}}(\bar{\zeta})} \sigma_{1}$ and the condition $\left(\widetilde{\mathcal{M}}^{\widetilde{\Sigma}}(0)(\widetilde{\delta}(0))^{\sigma_{3}} \sigma_{2}\right)^{2}=\mathrm{I}$. Furthermore, $\widetilde{w}_{ \pm}^{\widetilde{\Sigma}}(\zeta) \in \cap_{p \in\{1,2, \infty\}} \mathcal{L}_{\mathrm{M}_{2}(\mathbb{C})}^{p}(\widetilde{\Sigma})$.

Remark 7.1. As per the analysis of Section 4, one shows that the relation between $\tilde{\mathcal{M}}^{\widetilde{\Sigma}}(\zeta)$ and $\tilde{\mathcal{M}}^{c}(\zeta)$ is $\tilde{\mathcal{M}}^{c}(\zeta)=\tilde{\mathcal{M}}^{\widetilde{\Sigma}}(\zeta)\left(\mathrm{I}+\mathcal{O}\left(\frac{\underline{c}\left(\zeta_{1}, \zeta_{2}, \zeta_{3}, \overline{\zeta_{3}}\right) \widetilde{\gamma}(\zeta)}{\left|z_{o}+\zeta_{1}+\zeta_{2}\right|^{l} t^{l}}\right)\right)$, with arbitrarily large $l \in \mathbb{Z}_{\geqslant 1}$, and $\widetilde{\diamond}(\zeta) \in \mathcal{L}_{\mathrm{M}_{2}(\mathbb{C})}^{\infty}(\mathbb{C} \backslash \widetilde{\Sigma})$.

Using Lemma 3.1, the solution of the RHP for $\widetilde{\mathcal{M}}^{\Sigma}(\zeta)$ on $\widetilde{\Sigma}$ stated in Lemma 7.2 has the integral representation

$$
\tilde{\mathcal{M}}^{\widetilde{\Sigma}}(\zeta)=\mathrm{I}+\int_{\widetilde{\Sigma}} \frac{\widetilde{\mu}^{\widetilde{\Sigma}}(z) \widetilde{w}^{\widetilde{\Sigma}}(z)}{(z-\zeta)} \frac{\mathrm{d} z}{2 \pi \mathrm{i}}, \quad \zeta \in \mathbb{C} \backslash \widetilde{\Sigma},
$$

where $\widetilde{\mu}^{\widetilde{\Sigma}}(\zeta):=\left(\left(\mathbf{1}_{\widetilde{\Sigma}}-C_{\widetilde{w}^{\widetilde{\Sigma}}}^{\widetilde{\Sigma}}\right)^{-1} \mathrm{I}\right)(\zeta)$, and $\widetilde{w}^{\widetilde{\Sigma}}(\zeta):=\sum_{l \in\{ \pm\}} \widetilde{w}_{l}^{\widetilde{\Sigma}}(\zeta)$.

Now, proceeding as per the analysis of Section 5 , one solves, asymptotically, the model RHP for $\widetilde{\mathcal{M}}^{\widetilde{\Sigma}}(\zeta)$ on $\widetilde{\Sigma}$ formulated in Lemma 7.2, and arrives at the following (analogue of Lemma 6.1)

Lemma 7.3. Let $\varepsilon$ be an arbitrarily fixed, sufficiently small positive real number, and, for $\lambda \in\left\{\zeta_{2}, \zeta_{1}\right\}$, set $\mathbb{U}(\lambda ; \varepsilon):=\{z ;|z-\lambda|<\varepsilon\}$. Then, as $t \rightarrow-\infty$ such that $0<\zeta_{2}<\frac{1}{M}<M<\zeta_{1}$ and $\left|\zeta_{3}\right|^{2}=1$, with $M \in \mathbb{R}_{>1}$ and bounded, for $\zeta \in \mathbb{C} \backslash \cup_{\lambda \in\left\{\zeta_{2}, \zeta_{1}\right\}} \mathbb{U}(\lambda ; \varepsilon), m^{c}(\zeta)$ has the following 
asymptotics,

$$
\begin{aligned}
& m_{11}^{c}(\zeta)=\widetilde{\delta}(\zeta)\left(1+\mathcal{O}\left(\left(\frac{c^{\mathcal{S}}\left(\zeta_{1}\right) \underline{c}\left(\zeta_{2}, \zeta_{3}, \overline{\zeta_{3}}\right)}{\sqrt{\zeta_{2}\left(z_{o}^{2}+32\right)}\left(\zeta-\zeta_{1}\right)}+\frac{c^{\mathcal{S}}\left(\zeta_{2}\right) \underline{c}\left(\zeta_{1}, \zeta_{3}, \overline{\zeta_{3}}\right)}{\sqrt{\zeta_{1}\left(z_{o}^{2}+32\right)}\left(\zeta-\zeta_{2}\right)}\right) \frac{\ln |t|}{\left(\zeta_{1}-\zeta_{2}\right) t}\right)\right) \\
& m_{12}^{c}(\zeta)=\frac{\mathrm{e}^{\frac{\mathrm{i} \Omega^{-}(0)}{2}}}{\widetilde{\delta}(\zeta)}\left(\frac{\sqrt{\nu\left(\zeta_{1}\right)} \zeta_{1}^{-2 \mathrm{i} \nu\left(\zeta_{1}\right)}}{\sqrt{|t|\left(\zeta_{1}-\zeta_{2}\right)}\left(z_{o}^{2}+32\right)^{1 / 4}}\left(\frac{\zeta_{1} \mathrm{e}^{\mathrm{i}\left(\Theta^{-}\left(z_{o}, t\right)-\frac{3 \pi}{4}\right)}}{\left(\zeta-\zeta_{1}\right)}+\frac{\zeta_{2} \mathrm{e}^{-\mathrm{i}\left(\Theta^{-}\left(z_{o}, t\right)-\frac{3 \pi}{4}\right)}}{\left(\zeta-\zeta_{2}\right)}\right)\right.
\end{aligned}
$$

$$
\begin{aligned}
& \left.+\mathcal{O}\left(\left(\frac{c^{\mathcal{S}}\left(\zeta_{1}\right) \underline{c}\left(\zeta_{2}, \zeta_{3}, \overline{\zeta_{3}}\right)}{\sqrt{\zeta_{2}\left(z_{o}^{2}+32\right)}\left(\zeta-\zeta_{1}\right)}+\frac{c^{\mathcal{S}}\left(\zeta_{2}\right) \underline{c}\left(\zeta_{1}, \zeta_{3}, \overline{\zeta_{3}}\right)}{\sqrt{\zeta_{1}\left(z_{o}^{2}+32\right)}\left(\zeta-\zeta_{2}\right)}\right) \frac{\ln |t|}{\left(\zeta_{1}-\zeta_{2}\right) t}\right)\right), \\
m_{21}^{c}(\zeta) & =\frac{\widetilde{\delta}(\zeta)}{\mathrm{e}^{\frac{\mathrm{i} \Omega}{2}(0)}}\left(\frac{\sqrt{\nu\left(\zeta_{1}\right)} \zeta_{1}^{2 \mathrm{i} \nu\left(\zeta_{1}\right)}}{\sqrt{|t|\left(\zeta_{1}-\zeta_{2}\right)}\left(z_{o}^{2}+32\right)^{1 / 4}}\left(\frac{\zeta_{1} \mathrm{e}^{-\mathrm{i}\left(\Theta^{-}\left(z_{o}, t\right)-\frac{3 \pi}{4}\right)}}{\left(\zeta-\zeta_{1}\right)}+\frac{\zeta_{2} \mathrm{e}^{\mathrm{i}\left(\Theta^{-}\left(z_{o}, t\right)-\frac{3 \pi}{4}\right)}}{\left(\zeta-\zeta_{2}\right)}\right)\right. \\
& \left.+\mathcal{O}\left(\left(\frac{c^{\mathcal{S}}\left(\zeta_{1}\right) \underline{c}\left(\zeta_{2}, \zeta_{3}, \bar{\zeta}\right)}{\sqrt{\zeta_{2}\left(z_{o}^{2}+32\right)}\left(\zeta-\zeta_{1}\right)}+\frac{c^{\mathcal{S}}\left(\zeta_{2}\right) \underline{c}\left(\zeta_{1}, \zeta_{3}, \overline{\zeta_{3}}\right)}{\sqrt{\zeta_{1}\left(z_{o}^{2}+32\right)}\left(\zeta-\zeta_{2}\right)}\right) \frac{\ln |t|}{\left(\zeta_{1}-\zeta_{2}\right) t}\right)\right), \\
m_{22}^{c}(\zeta) & =\frac{1}{\widetilde{\delta}(\zeta)}\left(1+\mathcal{O}\left(\left(\frac{c^{\mathcal{S}}\left(\zeta_{1}\right) \underline{c}\left(\zeta_{2}, \zeta_{3}, \overline{\zeta_{3}}\right)}{\sqrt{\zeta_{2}\left(z_{o}^{2}+32\right)}\left(\zeta-\zeta_{1}\right)}+\frac{c^{\mathcal{S}}\left(\zeta_{2}\right) \underline{c}\left(\zeta_{1}, \zeta_{3}, \overline{\zeta_{3}}\right)}{\sqrt{\zeta_{1}\left(z_{o}^{2}+32\right)}\left(\zeta-\zeta_{2}\right)}\right) \frac{\ln |t|}{\left(\zeta_{1}-\zeta_{2}\right) t}\right)\right),
\end{aligned}
$$

where $\widetilde{\delta}(\zeta)$ is given in Lemma 7.1, $\nu(z), \Theta^{-}\left(z_{o}, t\right), \Omega^{-}(z)$, and $\left\{\zeta_{i}\right\}_{i=1}^{3}$ are defined in Theorem 3.1, Eqs. (12), (13), (15), (16), and (17), $\sup _{\zeta \in \mathbb{C} \backslash \cup_{\lambda \in\left\{\zeta_{2}, \zeta_{1}\right\}} \mathbb{U}(\lambda ; \varepsilon)}\left|\left(\zeta-\zeta_{k}\right)^{-1}\right| \leqslant M^{\widetilde{c}}$, with $M^{\widetilde{c}} \in \mathbb{R}_{+}$(and bounded), $k \in\{1,2\}, m^{c}(\zeta)=\sigma_{1} \overline{m^{c}(\bar{\zeta})} \sigma_{1}$, and $\left(m^{c}(0) \sigma_{2}\right)^{2}=\mathrm{I}$.

Sketch of Proof. Proceeding as in the proof of Lemma 6.1, as $t \rightarrow-\infty$ such that $0<\zeta_{2}<$ $\frac{1}{M}<M<\zeta_{1}$ and $\left|\zeta_{3}\right|^{2}=1$, with $M \in \mathbb{R}_{>1}$ and bounded, for $\zeta \in \mathbb{C} \backslash \cup_{\lambda \in\left\{\zeta_{2}, \zeta_{1}\right\}} \mathbb{U}(\lambda ; \varepsilon)$, one 
arrives at

$$
\begin{aligned}
& \widetilde{\mathcal{M}}_{11}^{\widetilde{\Sigma}}(\zeta)=1+\frac{r\left(\zeta_{1}\right)\left(\widetilde{\delta}_{B}^{0}\right)^{-2} \mathrm{e}^{-\frac{3 \pi \nu}{2}} \mathrm{e}^{\frac{3 \pi \mathrm{i}}{4}}}{2 \pi \mathrm{i}\left(\zeta-\zeta_{1}\right) \widetilde{\beta}_{21}^{\widetilde{\Sigma}_{B}} \widetilde{\mathcal{X}}_{B} \sqrt{|t|}} \int_{0}^{+\infty}\left(\mathrm{e}^{-\frac{3 \pi \mathrm{i}}{4}} \partial_{z} \mathbf{D}_{\mathrm{i} \nu}(z)+\frac{\mathrm{i}}{2} \mathrm{e}^{\frac{3 \pi \mathrm{i}}{4}} z \mathbf{D}_{\mathrm{i} \nu}(z)\right) z^{\mathrm{i} \nu} \mathrm{e}^{-\frac{z^{2}}{4}} \mathrm{~d} z \\
& -\frac{r\left(\zeta_{1}\right)\left(1-\left|r\left(\zeta_{1}\right)\right|^{2}\right)^{-1}\left(\widetilde{\delta}_{B}^{0}\right)^{-2} \mathrm{e}^{-\frac{\mathrm{i} \pi}{4}}}{2 \pi \mathrm{i}\left(\zeta-\zeta_{1}\right) \widetilde{\beta}_{21}^{\Sigma_{B}{ }^{0}} \mathrm{e}^{-\frac{\pi \nu}{2}} \widetilde{\mathcal{X}}_{B} \sqrt{|t|}} \int_{0}^{+\infty}\left(\mathrm{e}^{\frac{\mathrm{i} \pi}{4}} \partial_{z} \mathbf{D}_{\mathrm{i} \nu}(z)+\frac{\mathrm{i}}{2} \mathrm{e}^{-\frac{\mathrm{i} \pi}{4}} z \mathbf{D}_{\mathrm{i} \nu}(z)\right) z^{\mathrm{i} \nu} \mathrm{e}^{-\frac{z^{2}}{4}} \mathrm{~d} z \\
& +\frac{\overline{r\left(\zeta_{1}\right)}\left(\widetilde{\delta}_{A}^{0}\right)^{-2} \mathrm{e}^{-\frac{\pi \nu}{2}}(-1)^{-\mathrm{i} \nu} \mathrm{e}^{\frac{\mathrm{i} \pi}{4}}}{2 \pi \mathrm{i}\left(\zeta-\zeta_{2}\right) \widetilde{\beta}_{21}^{\Sigma} A^{0} \widetilde{\mathcal{X}}_{A} \sqrt{|t|}} \int_{0}^{+\infty}\left(\mathrm{e}^{-\frac{\mathrm{i} \pi}{4}} \partial_{z} \mathbf{D}_{-\mathrm{i} \nu}(z)-\frac{\mathrm{i}}{2} \mathrm{e}^{\frac{\mathrm{i} \pi}{4}} z \mathbf{D}_{-\mathrm{i} \nu}(z)\right) z^{-\mathrm{i} \nu} \mathrm{e}^{-\frac{z^{2}}{4}} \mathrm{~d} z \\
& -\frac{\overline{r\left(\zeta_{1}\right)}\left(1-\left|r\left(\zeta_{1}\right)\right|^{2}\right)^{-1}\left(\widetilde{\delta}_{A}^{0}\right)^{-2} \mathrm{e}^{-\frac{3 \pi \mathrm{i}}{4}}}{2 \pi \mathrm{i}\left(\zeta-\zeta_{2}\right) \widetilde{\beta}_{21}^{\Sigma} \mathrm{e}^{0} \mathrm{e}^{\frac{\pi \nu}{2}}(-1)^{\mathrm{i} \nu} \widetilde{\mathcal{X}}_{A} \sqrt{|t|}} \int_{0}^{+\infty}\left(\mathrm{e}^{\frac{3 \pi \mathrm{i}}{4}} \partial_{z} \mathbf{D}_{-\mathrm{i} \nu}(z)-\frac{\mathrm{i}}{2} \mathrm{e}^{-\frac{3 \pi \mathrm{i}}{4}} z \mathbf{D}_{-\mathrm{i} \nu}(z)\right) z^{-\mathrm{i} \nu} \mathrm{e}^{-\frac{z^{2}}{4}} \mathrm{~d} z \\
& +\mathcal{O}\left(\left(\frac{c^{\mathcal{S}}\left(\zeta_{1}\right) \underline{c}\left(\zeta_{2}, \zeta_{3}, \overline{\zeta_{3}}\right)\left(\tilde{\delta}_{B}^{0}\right)^{-2}}{\left(\zeta-\zeta_{1}\right)\left|\zeta_{1}-\zeta_{3}\right| \sqrt{\left(\zeta_{1}-\zeta_{2}\right)} \widetilde{\mathcal{X}}_{B}}+\frac{c^{\mathcal{S}}\left(\zeta_{2}\right) \underline{c}\left(\zeta_{1}, \zeta_{3}, \overline{\zeta_{3}}\right)\left(\widetilde{\delta}_{A}^{0}\right)^{-2}}{\left(\zeta-\zeta_{2}\right)\left|\zeta_{2}-\zeta_{3}\right| \sqrt{\left(\zeta_{1}-\zeta_{2}\right)} \widetilde{\mathcal{X}}_{A}}\right) \frac{\ln |t|}{t}\right) \\
& \tilde{\mathcal{M}}_{12}^{\widetilde{\Sigma}}(\zeta)=\left(\frac{\overline{r\left(\zeta_{1}\right)}\left(1-\left|r\left(\zeta_{1}\right)\right|^{2}\right)^{-1}\left(\widetilde{\delta}_{B}^{0}\right)^{2} \mathrm{e}^{\mathrm{i} \frac{\pi}{4}}}{2 \pi \mathrm{i}\left(\zeta-\zeta_{1}\right) \mathrm{e}^{-\frac{\pi \nu}{2}} \tilde{\mathcal{X}}_{B} \sqrt{|t|}}-\frac{\overline{r\left(\zeta_{1}\right)}\left(\widetilde{\delta}_{B}^{0}\right)^{2} \mathrm{e}^{-\frac{3 \pi \nu}{2}} \mathrm{e}^{-\frac{3 \pi \mathrm{i}}{4}}}{2 \pi \mathrm{i}\left(\zeta-\zeta_{1}\right) \tilde{\mathcal{X}}_{B} \sqrt{|t|}}\right) \int_{0}^{+\infty} \mathbf{D}_{-\mathrm{i} \nu}(z) z^{-\mathrm{i} \nu} \mathrm{e}^{-\frac{z^{2}}{4}} \mathrm{~d} z \\
& +\left(\frac{r\left(\zeta_{1}\right)\left(1-\left|r\left(\zeta_{1}\right)\right|^{2}\right)^{-1}\left(\widetilde{\delta}_{A}^{0}\right)^{2} \mathrm{e}^{\frac{3 \pi \mathrm{i}}{4}}}{2 \pi \mathrm{i}\left(\zeta-\zeta_{2}\right) \mathrm{e}^{\frac{\pi \nu}{2}}(-1)^{-\mathrm{i} \nu} \widetilde{\mathcal{X}}_{A} \sqrt{|t|}}-\frac{r\left(\zeta_{1}\right)\left(\widetilde{\delta}_{A}^{0}\right)^{2} \mathrm{e}^{-\frac{\pi \nu}{2}} \mathrm{e}^{-\frac{\mathrm{i} \pi}{4}}}{2 \pi \mathrm{i}\left(\zeta-\zeta_{2}\right)(-1)^{-\mathrm{i} \nu} \widetilde{\mathcal{X}}_{A} \sqrt{|t|}}\right) \int_{0}^{+\infty} \mathbf{D}_{\mathrm{i} \nu}(z) z^{\mathrm{i} \nu} \mathrm{e}^{-\frac{z^{2}}{4}} \mathrm{~d} z \\
& +\mathcal{O}\left(\left(\frac{c^{\mathcal{S}}\left(\zeta_{1}\right) \underline{c}\left(\zeta_{2}, \zeta_{3}, \overline{\zeta_{3}}\right)\left(\widetilde{\delta}_{B}^{0}\right)^{2}}{\left(\zeta-\zeta_{1}\right)\left|\zeta_{1}-\zeta_{3}\right| \sqrt{\left(\zeta_{1}-\zeta_{2}\right)} \widetilde{\mathcal{X}}_{B}}+\frac{c^{\mathcal{S}}\left(\zeta_{2}\right) \underline{c}\left(\zeta_{1}, \zeta_{3}, \overline{\zeta_{3}}\right)\left(\tilde{\delta}_{A}^{0}\right)^{2}}{\left(\zeta-\zeta_{2}\right)\left|\zeta_{2}-\zeta_{3}\right| \sqrt{\left(\zeta_{1}-\zeta_{2}\right)} \widetilde{\mathcal{X}}_{A}}\right) \frac{\ln |t|}{t}\right) \\
& \tilde{\mathcal{M}}_{21}^{\widetilde{\Sigma}}(\zeta)=-\left(\frac{r\left(\zeta_{1}\right)\left(1-\left|r\left(\zeta_{1}\right)\right|^{2}\right)^{-1}\left(\widetilde{\delta}_{B}^{0}\right)^{-2} \mathrm{e}^{-\frac{\mathrm{i} \pi}{4}}}{2 \pi \mathrm{i}\left(\zeta-\zeta_{1}\right) \mathrm{e}^{-\frac{\pi \nu}{2}} \widetilde{\mathcal{X}}_{B} \sqrt{|t|}}-\frac{r\left(\zeta_{1}\right)\left(\widetilde{\delta}_{B}^{0}\right)^{-2} \mathrm{e}^{-\frac{3 \pi \nu}{2}} \mathrm{e}^{\frac{3 \pi \mathrm{i}}{4}}}{2 \pi \mathrm{i}\left(\zeta-\zeta_{1}\right) \widetilde{\mathcal{X}}_{B} \sqrt{|t|}}\right) \int_{0}^{+\infty} \mathbf{D}_{\mathrm{i} \nu}(z) z^{\mathrm{i} \nu} \mathrm{e}^{-\frac{z^{2}}{4}} \mathrm{~d} z \\
& -\left(\frac{\overline{r\left(\zeta_{1}\right)}\left(1-\left|r\left(\zeta_{1}\right)\right|^{2}\right)^{-1}\left(\widetilde{\delta}_{A}^{0}\right)^{-2} \mathrm{e}^{-\frac{3 \pi \mathrm{i}}{4}}}{2 \pi \mathrm{i}\left(\zeta-\zeta_{2}\right) \mathrm{e}^{\frac{\pi \nu}{2}}(-1)^{\mathrm{i} \nu} \widetilde{\mathcal{X}}_{A} \sqrt{|t|}}-\frac{\overline{r\left(\zeta_{1}\right)}\left(\widetilde{\delta}_{A}^{0}\right)^{-2} \mathrm{e}^{-\frac{\pi \nu}{2}} \mathrm{e}^{\frac{\mathrm{i} \pi}{4}}}{2 \pi \mathrm{i}\left(\zeta-\zeta_{2}\right)(-1)^{\mathrm{i} \nu} \widetilde{\mathcal{X}}_{A} \sqrt{|t|}}\right) \int_{0}^{+\infty} \mathbf{D}_{-\mathrm{i} \nu}(z) z^{-\mathrm{i} \nu} \mathrm{e}^{-\frac{z^{2}}{4}} \mathrm{~d} z \\
& +\mathcal{O}\left(\left(\frac{c^{\mathcal{S}}\left(\zeta_{1}\right) \underline{c}\left(\zeta_{2}, \zeta_{3}, \overline{\zeta_{3}}\right)\left(\tilde{\delta}_{B}^{0}\right)^{-2}}{\left(\zeta-\zeta_{1}\right)\left|\zeta_{1}-\zeta_{3}\right| \sqrt{\left(\zeta_{1}-\zeta_{2}\right)} \tilde{\mathcal{X}}_{B}}+\frac{c^{\mathcal{S}}\left(\zeta_{2}\right) \underline{c}\left(\zeta_{1}, \zeta_{3}, \overline{\zeta_{3}}\right)\left(\widetilde{\delta}_{A}^{0}\right)^{-2}}{\left(\zeta-\zeta_{2}\right)\left|\zeta_{2}-\zeta_{3}\right| \sqrt{\left(\zeta_{1}-\zeta_{2}\right)} \tilde{\mathcal{X}}_{A}}\right) \frac{\ln |t|}{t}\right) \\
& \widetilde{\mathcal{M}}_{22}^{\widetilde{\Sigma}}(\zeta)=1-\frac{\overline{r\left(\zeta_{1}\right)}\left(\widetilde{\delta}_{B}^{0}\right)^{2} \mathrm{e}^{-\frac{3 \pi \nu}{2}} \mathrm{e}^{-\frac{3 \pi \mathrm{i}}{4}}}{2 \pi \mathrm{i}\left(\zeta-\zeta_{1}\right) \widetilde{\beta}_{12}^{\Sigma_{B}} \tilde{\mathcal{X}}_{B} \sqrt{|t|}} \int_{0}^{+\infty}\left(\mathrm{e}^{\frac{3 \pi \mathrm{i}}{4}} \partial_{z} \mathbf{D}_{-\mathrm{i} \nu}(z)-\frac{\mathrm{i}}{2} \mathrm{e}^{-\frac{3 \pi \mathrm{i}}{4}} z \mathbf{D}_{-\mathrm{i} \nu}(z)\right) z^{-\mathrm{i} \nu} \mathrm{e}^{-\frac{z^{2}}{4}} \mathrm{~d} z \\
& +\frac{\overline{r\left(\zeta_{1}\right)}\left(1-\left|r\left(\zeta_{1}\right)\right|^{2}\right)^{-1}\left(\widetilde{\delta}_{B}^{0}\right)^{2} \mathrm{e}^{\frac{\mathrm{i} \pi}{4}}}{2 \pi \mathrm{i}\left(\zeta-\zeta_{1}\right) \widetilde{\beta}_{12}^{\Sigma_{B}} \mathrm{e}^{-\frac{\pi \nu}{2}} \widetilde{\mathcal{X}}_{B} \sqrt{|t|}} \int_{0}^{+\infty}\left(\mathrm{e}^{-\frac{\mathrm{i} \pi}{4}} \partial_{z} \mathbf{D}_{-\mathrm{i} \nu}(z)-\frac{\mathrm{i}}{2} \mathrm{e}^{\frac{\mathrm{i} \pi}{4}} z \mathbf{D}_{-\mathrm{i} \nu}(z)\right) z^{-\mathrm{i} \nu} \mathrm{e}^{-\frac{z^{2}}{4}} \mathrm{~d} z \\
& -\frac{r\left(\zeta_{1}\right)\left(\widetilde{\delta}_{A}^{0}\right)^{2} \mathrm{e}^{-\frac{\pi \nu}{2}}(-1)^{\mathrm{i} \nu} \mathrm{e}^{-\frac{\mathrm{i} \pi}{4}}}{2 \pi \mathrm{i}\left(\zeta-\zeta_{2}\right) \widetilde{\tilde{\Sigma}}_{12}^{\tilde{\Sigma}_{A}^{0}} \tilde{\mathcal{X}}_{A} \sqrt{|t|}} \int_{0}^{+\infty}\left(\mathrm{e}^{\frac{\mathrm{i} \pi}{4}} \partial_{z} \mathbf{D}_{\mathrm{i} \nu}(z)+\frac{\mathrm{i}}{2} \mathrm{e}^{-\frac{\mathrm{i} \pi}{4}} z \mathbf{D}_{\mathrm{i} \nu}(z)\right) z^{\mathrm{i} \nu} \mathrm{e}^{-\frac{z^{2}}{4}} \mathrm{~d} z \\
& +\frac{r\left(\zeta_{1}\right)\left(1-\left|r\left(\zeta_{1}\right)\right|^{2}\right)^{-1}\left(\widetilde{\delta}_{A}^{0}\right)^{2}(-1)^{\mathrm{i} \nu} \mathrm{e}^{\frac{3 \pi \mathrm{i}}{4}}}{2 \pi \mathrm{i}\left(\zeta-\zeta_{2}\right) \widetilde{\beta}_{12} \tilde{\Sigma}^{0} \mathrm{e}^{\frac{\pi \nu}{2}} \widetilde{\mathcal{X}}_{A} \sqrt{|t|}} \int_{0}^{+\infty}\left(\mathrm{e}^{-\frac{3 \pi \mathrm{i}}{4}} \partial_{z} \mathbf{D}_{\mathrm{i} \nu}(z)+\frac{\mathrm{i}}{2} \mathrm{e}^{\frac{3 \pi \mathrm{i}}{4}} z \mathbf{D}_{\mathrm{i} \nu}(z)\right) z^{\mathrm{i} \nu} \mathrm{e}^{-\frac{z^{2}}{4}} \mathrm{~d} z \\
& +\mathcal{O}\left(\left(\frac{c^{\mathcal{S}}\left(\zeta_{1}\right) \underline{c}\left(\zeta_{2}, \zeta_{3}, \overline{\zeta_{3}}\right)\left(\widetilde{\delta}_{B}^{0}\right)^{2}}{\left(\zeta-\zeta_{1}\right)\left|\zeta_{1}-\zeta_{3}\right| \sqrt{\left(\zeta_{1}-\zeta_{2}\right)} \widetilde{\mathcal{X}}_{B}}+\frac{c^{\mathcal{S}}\left(\zeta_{2}\right) \underline{c}\left(\zeta_{1}, \zeta_{3}, \overline{\zeta_{3}}\right)\left(\widetilde{\delta}_{A}^{0}\right)^{2}}{\left(\zeta-\zeta_{2}\right)\left|\zeta_{2}-\zeta_{3}\right| \sqrt{\left(\zeta_{1}-\zeta_{2}\right)} \widetilde{\mathcal{X}}_{A}}\right) \frac{\ln |t|}{t}\right)
\end{aligned}
$$

where

$$
\begin{aligned}
& \widetilde{\delta}_{B}^{0}:=\left|\zeta_{1}-\zeta_{3}\right|^{\mathrm{i} \nu}\left(2|t|\left(\zeta_{1}-\zeta_{2}\right)^{3} \zeta_{1}^{-3}\right)^{\frac{\mathrm{i} \nu}{2}} \mathrm{e}^{\widetilde{\chi}\left(\zeta_{1}\right)} \exp \left(-\frac{\mathrm{i} t}{2}\left(\zeta_{1}-\zeta_{2}\right)\left(z_{O}+\zeta_{1}+\zeta_{2}\right)\right), \\
& \widetilde{\delta}_{A}^{0}:=\left|\zeta_{2}-\zeta_{3}\right|^{-\mathrm{i} \nu}\left(2|t|\left(\zeta_{1}-\zeta_{2}\right)^{3} \zeta_{2}^{-3}\right)^{-\frac{\mathrm{i} \nu}{2}} \mathrm{e}^{\widetilde{\chi}\left(\zeta_{2}\right)} \exp \left(\frac{\mathrm{i} t}{2}\left(\zeta_{1}-\zeta_{2}\right)\left(z_{o}+\zeta_{1}+\zeta_{2}\right)\right), \\
& \widetilde{\chi}\left(\zeta_{1}\right):=\frac{\mathrm{i}}{2 \pi} \int_{0}^{\zeta_{2}} \ln \left|\mu-\zeta_{1}\right| \mathrm{d} \ln \left(1-|r(\mu)|^{2}\right)+\frac{\mathrm{i}}{2 \pi} \int_{\zeta_{1}}^{+\infty} \ln \left|\mu-\zeta_{1}\right| \mathrm{d} \ln \left(1-|r(\mu)|^{2}\right), \\
& \widetilde{\chi}\left(\zeta_{2}\right):=-\widetilde{\chi}\left(\zeta_{1}\right)+\frac{\mathrm{i}}{2 \pi} \int_{0}^{\zeta_{2}} \ln |\mu| \mathrm{d} \ln \left(1-|r(\mu)|^{2}\right)+\frac{\mathrm{i}}{2 \pi} \int_{\zeta_{1}}^{+\infty} \ln |\mu| \mathrm{d} \ln \left(1-|r(\mu)|^{2}\right), \\
& \widetilde{\mathcal{X}}_{B}=\mathcal{X}_{B}, \quad \widetilde{\mathcal{X}}_{A}=\mathcal{X}_{A}, \quad \widetilde{\beta}_{12}^{\widetilde{\Sigma}_{B}}=\overline{\widetilde{\beta}_{21}^{\widetilde{\Sigma}_{B^{0}}}}:=\frac{\sqrt{2 \pi} \mathrm{e}^{-\frac{\pi \nu}{2}} \mathrm{e}^{\frac{3 \pi \mathrm{i}}{4}}}{r\left(\zeta_{1}\right) \Gamma(\mathrm{i} \nu)}, \quad \widetilde{\beta}_{12}^{\widetilde{\Sigma}_{A 0}}=\overline{\widetilde{\beta}_{21}^{\widetilde{\Sigma}_{A^{0}}}}:=\frac{\sqrt{2 \pi} \mathrm{e}^{-\frac{\pi \nu}{2}} \mathrm{e}^{-\frac{3 \pi \mathrm{i}}{4}}}{\overline{r\left(\zeta_{1}\right)} \overline{\Gamma(\mathrm{i} \nu)}},
\end{aligned}
$$


and $\mathbf{D}_{*}(\cdot)$ is the parabolic cylinder function 40 . Using the relations $\left|\zeta_{k}-\zeta_{3}\right| \zeta_{k}^{-1}=\left(2 \zeta_{k}\right)^{-1 / 2}\left(z_{o}^{2}+\right.$ $32)^{1 / 4}, k \in\{1,2\}$, the identities [40] $\partial_{z} \mathbf{D}_{z_{1}}(z)=\frac{1}{2}\left(z_{1} \mathbf{D}_{z_{1}-1}(z)-\mathbf{D}_{z_{1}+1}(z)\right), z \mathbf{D}_{z_{1}}(z)=\mathbf{D}_{z_{1}+1}(z)$ $+z_{1} \mathbf{D}_{z_{1}-1}(z)$, and $|\Gamma(\mathrm{i} \nu)|^{2}=\frac{\pi}{\nu \sinh (\pi \nu)}$, the integral [40] $\int_{0}^{+\infty} \mathbf{D}_{-z_{1}}(z) z^{z_{2}-1} \mathrm{e}^{-\frac{z^{2}}{4}} \mathrm{~d} z=\sqrt{\pi} \Gamma\left(z_{2}\right)$ $\cdot 2^{-\frac{1}{2}\left(z_{1}+z_{2}\right)}\left(\Gamma\left(\frac{1}{2}\left(z_{1}+z_{2}\right)+\frac{1}{2}\right)\right)^{-1}, \Re\left(z_{2}\right)>0$, the relation $\left|r\left(\zeta_{1}\right)\right||\Gamma(\mathrm{i} \nu)| \nu \mathrm{e}^{\frac{\pi \nu}{2}}=\sqrt{2 \pi \nu}$, and the fact that (Lemma 7.1 and Remark 7.1), for $\zeta \in\left(\mathbb{C} \backslash \cup_{\lambda \in\left\{\zeta_{2}, \zeta_{1}\right\}} \mathbb{U}(\lambda ; \varepsilon)\right) \cap\left(\Omega_{1} \cup \Omega_{2}\right)$, $m^{c}(\zeta)=\widetilde{\mathcal{M}}^{\widetilde{\Sigma}}(\zeta)(\widetilde{\delta}(\zeta))^{\sigma_{3}}\left(\mathrm{I}+\mathcal{O}\left(\frac{\underline{\underline{c}\left(\zeta_{1}, \zeta_{2}, \zeta_{3}, \overline{\zeta_{3}}\right) \widehat{\nabla}(\zeta)}}{\left.\left|z_{0}+\zeta_{1}+\zeta_{2}\right|\right|^{l} t^{l}}\right)\right)$, with arbitrarily large $l \in \mathbb{Z}_{\geqslant 1}, \widehat{\diamond}(\zeta) \in \mathcal{L}_{\mathrm{M}_{2}(\mathbb{C})}^{\infty}(\mathbb{C} \backslash$ $\cup_{\lambda \in\left\{\zeta_{2}, \zeta_{1}\right\}} \mathbb{U}(\lambda ; \varepsilon)$ ), and $\widetilde{\delta}(\zeta)$ given in Lemma 7.1 , one obtains the result stated in the Lemma; furthermore, one shows that the symmetry reduction $m^{c}(\zeta)=\sigma_{1} \overline{m^{c}(\bar{\zeta})} \sigma_{1}$ is satisfied, and verifies that, to $\mathcal{O}\left(t^{-1} \ln |t|\right),\left(m^{c}(0) \sigma_{2}\right)^{2}=\mathrm{I}$.

From the relation $\Delta_{o} m^{c}(0)=\sigma_{2}$ and Lemma 7.3, one obtains the following (analogue of Proposition 6.1)

Proposition 7.1. As $t \rightarrow-\infty$ such that $0<\zeta_{2}<\frac{1}{M}<M<\zeta_{1}$ and $\left|\zeta_{3}\right|^{2}=1$, with $M \in \mathbb{R}_{>1}$ and bounded,

$$
\begin{aligned}
\left(\Delta_{o}\right)_{11}= & -\frac{2 \mathrm{i} \sqrt{\nu\left(\zeta_{1}\right)} \cos \left(\Theta^{-}\left(z_{o}, t\right)-\frac{3 \pi}{4}\right)}{\sqrt{|t|\left(\zeta_{1}-\zeta_{2}\right)}\left(z_{o}^{2}+32\right)^{1 / 4}}+\mathcal{O}\left(\left(\frac{c^{\mathcal{S}}\left(\zeta_{1}\right) \underline{c}\left(\zeta_{2}, \zeta_{3}, \overline{\zeta_{3}}\right)}{\sqrt{\zeta_{1}\left(z_{o}^{2}+32\right)}}+\frac{c^{\mathcal{S}}\left(\zeta_{2}\right) \underline{c}\left(\zeta_{1}, \zeta_{3}, \overline{\zeta_{3}}\right)}{\sqrt{\zeta_{2}\left(z_{o}^{2}+32\right)}}\right) \frac{\ln |t|}{\left(\zeta_{1}-\zeta_{2}\right) t}\right) \\
\left(\Delta_{o}\right)_{12}= & -\mathrm{i} \exp \left(-\mathrm{i}\left(\int_{0}^{\zeta_{2}} \frac{\ln \left(1-|r(\mu)|^{2}\right)}{\mu} \frac{\mathrm{d} \mu}{2 \pi}+\int_{\zeta_{1}}^{+\infty} \frac{\ln \left(1-|r(\mu)|^{2}\right)}{\mu} \frac{\mathrm{d} \mu}{2 \pi}\right)\right) \\
& \times\left(1+\mathcal{O}\left(\left(\frac{c^{\mathcal{S}}\left(\zeta_{1}\right) \underline{c}\left(\zeta_{2}, \zeta_{3}, \overline{\zeta_{3}}\right)}{\sqrt{\zeta_{1}\left(z_{o}^{2}+32\right)}}+\frac{c^{\mathcal{S}}\left(\zeta_{2}\right) \underline{c}\left(\zeta_{1}, \zeta_{3}, \overline{\zeta_{3}}\right)}{\sqrt{\zeta_{2}\left(z_{o}^{2}+32\right)}}\right) \frac{\ln |t|}{\left(\zeta_{1}-\zeta_{2}\right) t}\right)\right) \\
\left(\Delta_{o}\right)_{21}= & \mathrm{i} \exp \left(\mathrm{i}\left(\int_{0}^{\zeta_{2}} \frac{\ln \left(1-|r(\mu)|^{2}\right)}{\mu} \frac{\mathrm{d} \mu}{2 \pi}+\int_{\zeta_{1}}^{+\infty} \frac{\ln \left(1-|r(\mu)|^{2}\right)}{\mu} \frac{\mathrm{d} \mu}{2 \pi}\right)\right) \\
& \times\left(1+\mathcal{O}\left(\left(\frac{c^{\mathcal{S}}\left(\zeta_{1}\right) \underline{c}\left(\zeta_{2}, \zeta_{3}, \overline{\zeta_{3}}\right)}{\sqrt{\zeta_{1}\left(z_{o}^{2}+32\right)}}+\frac{c^{\mathcal{S}}\left(\zeta_{2}\right) \underline{c}\left(\zeta_{1}, \zeta_{3}, \overline{\zeta_{3}}\right)}{\sqrt{\zeta_{2}\left(z_{o}^{2}+32\right)}}\right) \frac{\ln |t|}{\left(\zeta_{1}-\zeta_{2}\right) t}\right)\right) \\
\left(\Delta_{o}\right)_{22}= & \frac{2 \mathrm{i} \sqrt{\nu\left(\zeta_{1}\right)} \cos \left(\Theta^{-}\left(z_{o}, t\right)-\frac{3 \pi}{4}\right)}{\sqrt{|t|\left(\zeta_{1}-\zeta_{2}\right)}\left(z_{o}^{2}+32\right)^{1 / 4}}+\mathcal{O}\left(\left(\frac{c^{\mathcal{S}}\left(\zeta_{1}\right) \underline{c}\left(\zeta_{2}, \zeta_{3}, \overline{\zeta_{3}}\right)}{\sqrt{\zeta_{1}\left(z_{o}^{2}+32\right)}}+\frac{c^{\mathcal{S}}\left(\zeta_{2}\right) \underline{c}\left(\zeta_{1}, \zeta_{3}, \overline{\zeta_{3}}\right)}{\sqrt{\zeta_{2}\left(z_{o}^{2}+32\right)}}\right) \frac{\ln |t|}{\left(\zeta_{1}-\zeta_{2}\right) t}\right)
\end{aligned}
$$

where $\nu(z)$ and $\Theta^{-}\left(z_{o}, t\right)$ are given in Theorem 3.1, Eqs. (12), (13), and (15).

Finally, the analogue of Lemma 6.2 is

Lemma 7.4. As $t \rightarrow-\infty$ such that $0<\zeta_{2}<\frac{1}{M}<M<\zeta_{1}$ and $\left|\zeta_{3}\right|^{2}=1$, with $M \in$ $\mathbb{R}_{>1}$ and bounded, $u(x, t)$, the solution of the Cauchy problem for the $\mathrm{D}_{f} \mathrm{NLSE}$, has the asymptotics stated in Theorem 3.1, Eqs. (9), (11), (12), (13), (15), (16), and (17), and $\int_{ \pm \infty}^{x}\left(|u(\xi, t)|^{2}-1\right) \mathrm{d} \xi$ have the asymptotics stated in Theorem 3.2, Eqs. (27) and (28), with $\theta^{+}(z)$ (respectively $\theta^{-}(z)$ ) given in Theorem 3.1, Eq. (10) (respectively Eq. (11)).

\section{Acknowledgements}

The author is very grateful to K. T.-R. McLaughlin and X. Zhou for the invitation to the AMS Special Session on Integrable Systems and Riemann-Hilbert Problems (Birmingham, Alabama, U. S. A., Nov. 10-12, 2000), where partial results of this work were presented, A. V. Kitaev for fruitful discussions, X. Zhou for a copy of [43], and M. Kovalyov and R. Saxton for encouragement. The author is especially grateful to K. T.-R. McLaughlin, S. Shipman, A. Tovbis, S. Venakides and X. Zhou for many helpful comments and suggestions. The author is also very grateful to the referee for a meticulous reading of the manuscript and for many helpful suggestions. The author dedicates this work to C. Roth. 


\section{Appendix}

In order to obtain the results stated in Theorems 3.2 and 3.3, the following Lemmae and Propositions, which are the analogues of Lemmae 6.1 and 7.3 and Propositions 6.1 and 7.1, are necessary.

Lemma A.1. Let $\varepsilon$ be an arbitrarily fixed, sufficiently small positive real number, and, for $\lambda \in \widetilde{\mathfrak{J}}:=\left\{\left(s_{1}\right)^{ \pm 1},\left(s_{2}\right)^{ \pm 1}\right\}$, where $s_{1}:=\zeta_{1}^{\sharp}=\exp \left(\mathrm{i} \widehat{\varphi}_{1}\right)$ and $s_{2}:=\zeta_{3}^{\sharp}=\exp \left(\mathrm{i} \widehat{\varphi}_{3}\right)$, with $\zeta_{n}^{\sharp}$ and $\widehat{\varphi}_{n}$, $n \in\{1,3\}$, defined in Theorem 3.2, Eqs. (36) and (37), set $\mathbb{U}(\lambda ; \varepsilon):=\{z ;|z-\lambda|<\varepsilon\}$. Then, for $r\left(s_{1}\right)=\exp \left(-\frac{\mathrm{i} \varepsilon_{1} \pi}{2}\right)\left|r\left(s_{1}\right)\right|, \varepsilon_{1} \in\{ \pm 1\}, r\left(\overline{s_{2}}\right)=\exp \left(\frac{\mathrm{i} \varepsilon_{2} \pi}{2}\right)\left|r\left(\overline{s_{2}}\right)\right|, \varepsilon_{2} \in\{ \pm 1\}, 0<r\left(s_{2}\right) \overline{r\left(\overline{s_{2}}\right)}<1$, and $\zeta \in \mathbb{C} \backslash \cup_{\lambda \in \widetilde{\mathfrak{J}}} \mathbb{U}^{2}(\lambda ; \varepsilon)$, as $t \rightarrow+\infty$ and $x \rightarrow-\infty$ such that $z_{0}:=x / t \in(-2,0), m^{c}(\zeta)$ has the following asymptotics,

$$
\begin{aligned}
& m_{11}^{c}(\zeta)=\delta(\zeta)\left(1+\mathcal{O}\left(\left(\frac{\underline{c}\left(z_{o}\right)}{\left(\zeta-s_{1}\right)}+\frac{\underline{c}\left(z_{o}\right)}{\left(\zeta-\overline{s_{2}}\right)}\right) \frac{\mathrm{e}^{-4 \alpha t}}{\beta t}\right)\right), \\
& m_{12}^{c}(\zeta)=\frac{1}{\delta(\zeta)}\left(\frac{n_{1} \mathrm{e}^{-\left(2 a_{0} t+\sin \left(\widehat{\varphi}_{1}\right) \int_{-\infty}^{0} \frac{\ln \left(1-|r(\mu)|^{2}\right)}{\left(\mu-\cos \widehat{\varphi}_{1}\right)^{2}+\sin ^{2} \widehat{\varphi}_{1}} \frac{\mathrm{d} \mu}{\pi}\right)} \mathrm{e}^{-\mathrm{i}\left(\widehat{\varphi}_{1}+\int_{-\infty}^{0} \frac{\left(\mu-\cos \widehat{\varphi}_{1}\right) \ln \left(1-|r(\mu)|^{2}\right)}{\left(\mu-\cos \widehat{\varphi}_{1}\right)^{2}+\sin ^{2} \widehat{\varphi}_{1}} \frac{\mathrm{d} \mu}{\pi}\right)}}{2\left(\left|r\left(s_{1}\right)\right|\right)^{-1}\left(b_{0} t\right)^{1 / 2}\left(\zeta-\overline{s_{1}}\right)}\right. \\
& +\frac{n_{2} \mathrm{e}^{-\left(2 \widehat{a}_{0} t-\sin \left(\widehat{\varphi}_{3}\right) \int_{-\infty}^{0} \frac{\ln \left(1-|r(\mu)|^{2}\right)}{\left(\mu-\cos \widehat{\varphi}_{3}\right)^{2}+\sin ^{2} \widehat{\varphi}_{3}} \frac{\mathrm{d} \mu}{\pi}\right)} \mathrm{e}^{\mathrm{i}\left(\widehat{\varphi}_{3}-\int_{-\infty}^{0} \frac{\left(\mu-\cos \widehat{\varphi}_{3}\right) \ln \left(1-|r(\mu)|^{2}\right)}{\left(\mu-\cos \widehat{\varphi}_{3}\right)^{2}+\sin ^{2} \widehat{\varphi}_{3}} \frac{\mathrm{d} \mu}{\pi}\right)}}{2\left(\left|r\left(\overline{s_{2}}\right)\right|\right)^{-1}\left(1-r\left(s_{2}\right) \overline{r\left(\overline{s_{2}}\right)}\right)\left(\widehat{b}_{0} t\right)^{1 / 2}\left(\zeta-s_{2}\right)} \\
& \left.+\mathcal{O}\left(\left(\frac{\underline{c}\left(z_{o}\right)}{\left(\zeta-\overline{s_{1}}\right)}+\frac{\underline{c}\left(z_{o}\right)}{\left(\zeta-s_{2}\right)}\right) \frac{\mathrm{e}^{-4 \alpha t}}{\beta t}\right)\right) \text {, } \\
& m_{21}^{c}(\zeta)=\delta(\zeta)\left(\frac{n_{1} \mathrm{e}^{-\left(2 a_{0} t+\sin \left(\widehat{\varphi}_{1}\right) \int_{-\infty}^{0} \frac{\ln \left(1-|r(\mu)|^{2}\right)}{\left(\mu-\cos \widehat{\varphi}_{1}\right)^{2}+\sin ^{2} \widehat{\varphi}_{1}} \frac{\mathrm{d} \mu}{\pi}\right)} \mathrm{e}^{\mathrm{i}\left(\widehat{\varphi}_{1}+\int_{-\infty}^{0} \frac{\left(\mu-\cos \widehat{\varphi}_{1}\right) \ln \left(1-|r(\mu)|^{2}\right)}{\left(\mu-\cos \widehat{\varphi}_{1}\right)^{2}+\sin ^{2} \widehat{\varphi}_{1}} \frac{\mathrm{d} \mu}{\pi}\right)}}{2\left(\left|r\left(s_{1}\right)\right|\right)^{-1}\left(b_{0} t\right)^{1 / 2}\left(\zeta-s_{1}\right)}\right. \\
& +\frac{n_{2} \mathrm{e}^{-\left(2 \widehat{a}_{0} t-\sin \left(\widehat{\varphi}_{3}\right) \int_{-\infty}^{0} \frac{\ln \left(1-|r(\mu)|^{2}\right)}{\left(\mu-\cos \widehat{\varphi}_{3}\right)^{2}+\sin ^{2} \widehat{\varphi}_{3}} \frac{\mathrm{d} \mu}{\pi}\right)} \mathrm{e}^{\mathrm{i}\left(-\widehat{\varphi}_{3}+\int_{-\infty}^{0} \frac{\left(\mu-\cos \widehat{\varphi}_{3}\right) \ln \left(1-|r(\mu)|^{2}\right)}{\left(\mu-\cos \widehat{\varphi}_{3}\right)^{2}+\sin ^{2} \widehat{\varphi}_{3}} \frac{\mathrm{d} \mu}{\pi}\right)}}{2\left(\left|r\left(\overline{s_{2}}\right)\right|\right)^{-1}\left(1-r\left(s_{2}\right) \overline{r\left(\overline{s_{2}}\right)}\right)\left(\widehat{b}_{0} t\right)^{1 / 2}\left(\zeta-\overline{s_{2}}\right)} \\
& \left.+\mathcal{O}\left(\left(\frac{\underline{c}\left(z_{o}\right)}{\left(\zeta-s_{1}\right)}+\frac{\underline{c}\left(z_{o}\right)}{\left(\zeta-\overline{s_{2}}\right)}\right) \frac{\mathrm{e}^{-4 \alpha t}}{\beta t}\right)\right), \\
& m_{22}^{c}(\zeta)=\frac{1}{\delta(\zeta)}\left(1+\mathcal{O}\left(\left(\frac{\underline{c}\left(z_{o}\right)}{\left(\zeta-\overline{s_{1}}\right)}+\frac{\underline{c}\left(z_{o}\right)}{\left(\zeta-s_{2}\right)}\right) \frac{\mathrm{e}^{-4 \alpha t}}{\beta t}\right)\right) \text {, }
\end{aligned}
$$

where $n_{1}:=\operatorname{sgn}\left(\varepsilon_{1}\right), n_{2}:=\operatorname{sgn}\left(\varepsilon_{2}\right)$,

$$
\begin{array}{ccc}
\delta(\zeta):=\exp \left(\int_{-\infty}^{0} \frac{\ln \left(1-|r(\mu)|^{2}\right)}{(\mu-\zeta)} \frac{\mathrm{d} \mu}{2 \pi \mathrm{i}}\right), & a_{0}:=\frac{1}{2}\left(z_{o}-a_{1}\right)\left(4-a_{1}^{2}\right)^{1 / 2}(>0), \\
\widehat{a}_{0}:=-\frac{1}{2}\left(z_{o}-a_{2}\right)\left(4-a_{2}^{2}\right)^{1 / 2}(>0), & b_{0}:=\frac{1}{2}\left(z_{o}^{2}+32\right)^{1 / 2}\left(4-a_{1}^{2}\right)^{1 / 2}(>0), \\
\widehat{b}_{0}:=\frac{1}{2}\left(z_{o}^{2}+32\right)^{1 / 2}\left(4-a_{2}^{2}\right)^{1 / 2}(>0), & \alpha:=\min \left\{a_{0}, \widehat{a}_{0}\right\}, \quad \beta:=\min \left\{b_{0}, \widehat{b}_{0}\right\},
\end{array}
$$

with $a_{1}$ and $a_{2}$ given in Theorem 3.1, Eq. (17), $\sup _{\zeta \in \mathbb{C} \backslash \cup_{\lambda \in \in \mathbb{\mathfrak { J }}} \mathbb{U}(\lambda ; \varepsilon)}\left|\left(\zeta-\left(\zeta_{n}^{\sharp}\right)^{l}\right)^{-1}\right| \leqslant \widetilde{\mathfrak{M}}$, with $\widetilde{\mathfrak{M}} \in \mathbb{R}_{+}$(and bounded), $n \in\{1,3\}, l \in\{ \pm 1\}, m^{c}(\zeta)=\sigma_{1} \overline{m^{c}(\bar{\zeta})} \sigma_{1}$, and $\left(m^{c}(0) \sigma_{2}\right)^{2}=\mathrm{I}$.

Proposition A.1. Let $s_{1}:=\zeta_{1}^{\sharp}=\exp \left(\mathrm{i} \widehat{\varphi}_{1}\right)$ and $s_{2}:=\zeta_{3}^{\sharp}=\exp \left(\mathrm{i} \widehat{\varphi}_{3}\right)$, where $\zeta_{n}^{\sharp}$ and $\widehat{\varphi}_{n}, n \in\{1,3\}$, are defined in Theorem 3.2, Eqs. (36) and (37). For $r\left(s_{1}\right)=\exp \left(-\frac{\mathrm{i} \varepsilon_{1} \pi}{2}\right)\left|r\left(s_{1}\right)\right|, \varepsilon_{1} \in\{ \pm 1\}$, $r\left(\overline{s_{2}}\right)=\exp \left(\frac{\mathrm{i} \varepsilon_{2} \pi}{2}\right)\left|r\left(\overline{s_{2}}\right)\right|, \varepsilon_{2} \in\{ \pm 1\}, 0<r\left(s_{2}\right) \overline{r\left(\overline{s_{2}}\right)}<1$, and $\varepsilon_{1}=\varepsilon_{2}$, as $t \rightarrow+\infty$ and $x \rightarrow-\infty$ such that $z_{0}:=x / t \in(-2,0)$,

$$
\left(\Delta_{o}\right)_{11}=-\frac{n_{1} \mathrm{ie}^{-\left(\widetilde{a}_{+} t+\widetilde{c}_{+}\right)} \beth_{+}}{\widetilde{b} \sqrt{t}} \cosh \left(\widetilde{a}_{-} t+\widetilde{c}_{-}+\frac{1}{8} \ln \left(\frac{4-a_{1}^{2}}{4-a_{2}^{2}}\right)-\ln \beth_{-}\right)+\mathcal{O}\left(\frac{\underline{c}\left(z_{o}\right) \mathrm{e}^{-4 \alpha t}}{\beta t}\right),
$$




$$
\begin{gathered}
\left(\Delta_{o}\right)_{12}=-\mathrm{ie}^{-\mathrm{i} \psi^{+}(1)}\left(1+\mathcal{O}\left(\frac{\underline{c}\left(z_{o}\right) \mathrm{e}^{-4 \alpha t}}{\beta t}\right)\right), \quad\left(\Delta_{o}\right)_{21}=\mathrm{ie}^{\mathrm{i} \psi^{+}(1)}\left(1+\mathcal{O}\left(\frac{\underline{c}\left(z_{o}\right) \mathrm{e}^{-4 \alpha t}}{\beta t}\right)\right), \\
\left(\Delta_{o}\right)_{22}=\frac{n_{1} \mathrm{ie}^{-\left(\widetilde{a}_{+} t+\widetilde{c}_{+}\right)} \beth_{+}}{\widetilde{b} \sqrt{t}} \cosh \left(\widetilde{a}_{-} t+\widetilde{c}_{-}+\frac{1}{8} \ln \left(\frac{4-a_{1}^{2}}{4-a_{2}^{2}}\right)-\ln \beth_{-}\right)+\mathcal{O}\left(\frac{\underline{c}\left(z_{o}\right) \mathrm{e}^{-4 \alpha t}}{\beta t}\right),
\end{gathered}
$$

and, for $\varepsilon_{1}=-\varepsilon_{2}$,

$$
\begin{aligned}
& \left(\Delta_{o}\right)_{11}=\frac{n_{1} \mathrm{ie}^{-\left(\widetilde{a}_{+} t+\widetilde{c}_{+}\right)} \beth_{+}}{\widetilde{b} \sqrt{t}} \sinh \left(\widetilde{a}_{-} t+\widetilde{c}_{-}+\frac{1}{8} \ln \left(\frac{4-a_{1}^{2}}{4-a_{2}^{2}}\right)-\ln \beth_{-}\right)+\mathcal{O}\left(\frac{\underline{c}\left(z_{o}\right) \mathrm{e}^{-4 \alpha t}}{\beta t}\right), \\
& \left(\Delta_{o}\right)_{12}=-\mathrm{ie}^{-\mathrm{i} \psi^{+}(1)}\left(1+\mathcal{O}\left(\frac{\underline{c}\left(z_{o}\right) \mathrm{e}^{-4 \alpha t}}{\beta t}\right)\right), \quad\left(\Delta_{o}\right)_{21}=\mathrm{ie}^{\mathrm{i} \psi^{+}(1)}\left(1+\mathcal{O}\left(\frac{\underline{c}\left(z_{o}\right) \mathrm{e}^{-4 \alpha t}}{\beta t}\right)\right), \\
& \left(\Delta_{o}\right)_{22}=-\frac{n_{1} \mathrm{ie}^{-\left(\widetilde{a}_{+} t+\widetilde{c}_{+}\right)} \beth_{+}}{\widetilde{b} \sqrt{t}} \sinh \left(\widetilde{a}_{-} t+\widetilde{c}_{-}+\frac{1}{8} \ln \left(\frac{4-a_{1}^{2}}{4-a_{2}^{2}}\right)-\ln \beth_{-}\right)+\mathcal{O}\left(\frac{\underline{c}\left(z_{o}\right) \mathrm{e}^{-4 \alpha t}}{\beta t}\right),
\end{aligned}
$$

where $n_{1}:=\operatorname{sgn}\left(\varepsilon_{1}\right), \psi^{+}(\cdot), \widetilde{a}_{ \pm}, \widetilde{c}_{ \pm}, \widetilde{b}, \beth_{ \pm}, \alpha$, and $\beta$ are defined in Theorem 3.2, Eqs. (35), (38), (39), (40), (41), (42), and (43), and $a_{1}$ and $a_{2}$ are given in Theorem 3.1, Eq. (17).

Lemma A.2. Let $\varepsilon$ be an arbitrarily fixed, sufficiently small positive real number, and, for $\lambda \in \widetilde{\mathfrak{J}}:=\left\{\left(s_{1}\right)^{ \pm 1},\left(s_{2}\right)^{ \pm 1}\right\}$, where $s_{1}:=\zeta_{1}^{\sharp}=\exp \left(\mathrm{i} \widehat{\varphi}_{1}\right)$ and $s_{2}:=\zeta_{3}^{\sharp}=\exp \left(\mathrm{i} \widehat{\varphi}_{3}\right)$, with $\zeta_{n}^{\sharp}$ and $\widehat{\varphi}_{n}$, $n \in\{1,3\}$, defined in Theorem 3.2, Eqs. (36) and (37), set $\mathbb{U}(\lambda ; \varepsilon):=\{z ;|z-\lambda|<\varepsilon\}$. Then, for $r\left(\overline{s_{1}}\right)=\exp \left(\frac{\mathrm{i} \varepsilon_{1} \pi}{2}\right)\left|r\left(\overline{s_{1}}\right)\right|, \varepsilon_{1} \in\{ \pm 1\}, r\left(s_{2}\right)=\exp \left(-\frac{\mathrm{i} \varepsilon_{2} \pi}{2}\right)\left|r\left(s_{2}\right)\right|, \varepsilon_{2} \in\{ \pm 1\}, 0<r\left(s_{1}\right) \overline{r\left(\overline{s_{1}}\right)}<1$, and $\zeta \in \mathbb{C} \backslash \cup_{\lambda \in \widetilde{\mathfrak{J}}} \mathbb{U}(\lambda ; \varepsilon)$, as $t \rightarrow-\infty$ and $x \rightarrow+\infty$ such that $z_{o}:=x / t \in(-2,0), m^{c}(\zeta)$ has the following asymptotics,

$$
\begin{aligned}
& m_{11}^{c}(\zeta)=\widetilde{\delta}(\zeta)\left(1+\mathcal{O}\left(\left(\frac{\underline{c}\left(z_{o}\right)}{\left(\zeta-\overline{s_{1}}\right)}+\frac{\underline{c}\left(z_{o}\right)}{\left(\zeta-s_{2}\right)}\right) \frac{\mathrm{e}^{-4 \alpha|t|}}{\beta t}\right)\right) \\
& m_{12}^{c}(\zeta)=-\frac{1}{\widetilde{\delta}(\zeta)}\left(\frac{n_{1} \mathrm{e}^{-\left(2 a_{0}|t|-\sin \left(\widehat{\varphi}_{1}\right) \int_{0}^{+\infty} \frac{\ln \left(1-|r(\mu)|^{2}\right)}{\left(\mu-\cos \widehat{\varphi}_{1}\right)^{2}+\sin ^{2} \widehat{\varphi}_{1}} \frac{\mathrm{d} \mu}{\pi}\right)} \mathrm{e}^{\mathrm{i}\left(\widehat{\varphi}_{1}-\int_{0}^{+\infty} \frac{\left(\mu-\cos \widehat{\varphi}_{1}\right) \ln \left(1-|r(\mu)|^{2}\right)}{\left(\mu-\cos \widehat{\varphi}_{1}\right)^{2}+\sin ^{2} \widehat{\varphi}_{1}} \frac{\mathrm{d} \mu}{\pi}\right)}}{2\left(\left|r\left(\overline{s_{1}}\right)\right|\right)^{-1}\left(1-r\left(s_{1}\right) \overline{r\left(\overline{s_{1}}\right)}\right)\left(b_{0}|t|\right)^{1 / 2}\left(\zeta-s_{1}\right)}\right. \\
& +\frac{n_{2} \mathrm{e}^{-\left(2 \widehat{a}_{0}|t|+\sin \left(\widehat{\varphi}_{3}\right) \int_{0}^{+\infty} \frac{\ln \left(1-|r(\mu)|^{2}\right)}{\left(\mu-\cos \widehat{\varphi}_{3}\right)^{2}+\sin ^{2} \widehat{\varphi}_{3}} \frac{\mathrm{d} \mu}{\pi}\right)} \mathrm{e}^{-\mathrm{i}\left(\widehat{\varphi}_{3}+\int_{0}^{+\infty} \frac{\left(\mu-\cos \widehat{\varphi}_{3}\right) \ln \left(1-|r(\mu)|^{2}\right)}{\left(\mu-\cos \widehat{\varphi}_{3}\right)^{2}+\sin ^{2} \widehat{\varphi}_{3}} \frac{\mathrm{d} \mu}{\pi}\right)}}{2\left(\left|r\left(s_{2}\right)\right|\right)^{-1}\left(\widehat{b}_{0}|t|\right)^{1 / 2}\left(\zeta-\overline{s_{2}}\right)} \\
& \left.+\mathcal{O}\left(\left(\frac{\underline{c}\left(z_{o}\right)}{\left(\zeta-s_{1}\right)}+\frac{\underline{c}\left(z_{o}\right)}{\left(\zeta-\overline{s_{2}}\right)}\right) \frac{\mathrm{e}^{-4 \alpha|t|}}{\beta t}\right)\right) \text {, } \\
& m_{21}^{c}(\zeta)=-\widetilde{\delta}(\zeta)\left(\frac{n_{1} \mathrm{e}^{-\left(2 a_{0}|t|-\sin \left(\widehat{\varphi}_{1}\right) \int_{0}^{+\infty} \frac{\ln \left(1-|r(\mu)|^{2}\right)}{\left(\mu-\cos \widehat{\varphi}_{1}\right)^{2}+\sin ^{2} \widehat{\varphi}_{1}} \frac{\mathrm{d} \mu}{\pi}\right)} \mathrm{e}^{-\mathrm{i}\left(\widehat{\varphi}_{1}-\int_{0}^{+\infty} \frac{\left(\mu-\cos \widehat{\varphi}_{1}\right) \ln \left(1-|r(\mu)|^{2}\right)}{\left(\mu-\cos \widehat{\varphi}_{1}\right)^{2}+\sin ^{2} \widehat{\varphi}_{1}} \frac{\mathrm{d} \mu}{\pi}\right)}}{2\left(\left|r\left(\overline{s_{1}}\right)\right|\right)^{-1}\left(1-r\left(s_{1}\right) \overline{r\left(\overline{s_{1}}\right)}\right)\left(b_{0}|t|\right)^{1 / 2}\left(\zeta-\overline{s_{1}}\right)}\right. \\
& +\frac{n_{2} \mathrm{e}^{-\left(2 \widehat{a}_{0}|t|+\sin \left(\widehat{\varphi}_{3}\right) \int_{0}^{+\infty} \frac{\ln \left(1-|r(\mu)|^{2}\right)}{\left(\mu-\cos \widehat{\varphi}_{3}\right)^{2}+\sin ^{2} \widehat{\varphi}_{3}} \frac{\mathrm{d} \mu}{\pi}\right)} \mathrm{e}^{\mathrm{i}\left(\widehat{\varphi}_{3}+\int_{0}^{+\infty} \frac{\left(\mu-\cos \widehat{\varphi}_{3}\right) \ln \left(1-|r(\mu)|^{2}\right)}{\left(\mu-\cos \widehat{\varphi}_{3}\right)^{2}+\sin ^{2} \widehat{\varphi}_{3}} \frac{\mathrm{d} \mu}{\pi}\right)}}{2\left(\left|r\left(s_{2}\right)\right|\right)^{-1}\left(\widehat{b}_{0}|t|\right)^{1 / 2}\left(\zeta-s_{2}\right)} \\
& \left.+\mathcal{O}\left(\left(\frac{\underline{c}\left(z_{o}\right)}{\left(\zeta-\overline{s_{1}}\right)}+\frac{\underline{c}\left(z_{o}\right)}{\left(\zeta-s_{2}\right)}\right) \frac{\mathrm{e}^{-4 \alpha|t|}}{\beta t}\right)\right) \text {, } \\
& m_{22}^{c}(\zeta)=\frac{1}{\widetilde{\delta}(\zeta)}\left(1+\mathcal{O}\left(\left(\frac{\underline{c}\left(z_{o}\right)}{\left(\zeta-s_{1}\right)}+\frac{\underline{c}\left(z_{o}\right)}{\left(\zeta-\overline{s_{2}}\right)}\right) \frac{\mathrm{e}^{-4 \alpha|t|}}{\beta t}\right)\right) \text {, }
\end{aligned}
$$

where $n_{1}:=\operatorname{sgn}\left(\varepsilon_{1}\right), n_{2}:=\operatorname{sgn}\left(\varepsilon_{2}\right)$,

$$
\widetilde{\delta}(\zeta):=\exp \left(\int_{0}^{+\infty} \frac{\ln \left(1-|r(\mu)|^{2}\right)}{(\mu-\zeta)} \frac{\mathrm{d} \mu}{2 \pi \mathrm{i}}\right),
$$


$a_{0}, \widehat{a}_{0}, b_{0}, \widehat{b}_{0}, \alpha$, and $\beta$ are defined in Lemma A.1, $\sup _{\zeta \in \mathbb{C} \backslash \cup_{\lambda \in \tilde{\mathfrak{J}}} \mathbb{U}(\lambda ; \varepsilon)}\left|\left(\zeta-\left(\zeta_{n}^{\sharp}\right)^{l}\right)^{-1}\right| \leqslant \widehat{\mathfrak{M}}$, with $\widehat{\mathfrak{M}} \in \mathbb{R}_{+}$(and bounded), $n \in\{1,3\}, l \in\{ \pm 1\}, m^{c}(\zeta)=\sigma_{1} \overline{m^{c}(\bar{\zeta})} \sigma_{1}$, and $\left(m^{c}(0) \sigma_{2}\right)^{2}=\mathrm{I}$.

Proposition A.2. Let $s_{1}:=\zeta_{1}^{\sharp}=\exp \left(\mathrm{i} \widehat{\varphi}_{1}\right)$ and $s_{2}:=\zeta_{3}^{\sharp}=\exp \left(\mathrm{i} \widehat{\varphi}_{3}\right)$, where $\zeta_{n}^{\sharp}$ and $\widehat{\varphi}_{n}, n \in\{1,3\}$, are defined in Theorem 3.2, Eqs. (36) and (37). For $r\left(\overline{s_{1}}\right)=\exp \left(\frac{\mathrm{i} \varepsilon_{1} \pi}{2}\right)\left|r\left(\overline{s_{1}}\right)\right|, \varepsilon_{1} \in\{ \pm 1\}$, $r\left(s_{2}\right)=\exp \left(-\frac{\mathrm{i} \varepsilon_{2} \pi}{2}\right)\left|r\left(s_{2}\right)\right|, \varepsilon_{2} \in\{ \pm 1\}, 0<r\left(s_{1}\right) \overline{r\left(\overline{s_{1}}\right)}<1$, and $\varepsilon_{1}=\varepsilon_{2}$, as $t \rightarrow-\infty$ and $x \rightarrow+\infty$ such that $z_{0}:=x / t \in(-2,0)$,

$$
\begin{aligned}
& \left.\left(\Delta_{o}\right)_{11}=\frac{\left.n_{1} \mathrm{ie}^{-\left(\widetilde{a}_{+}|t|-\widehat{c}_{+}\right)}\right\rceil_{+}}{\widetilde{b} \sqrt{|t|}} \cosh \left(\widetilde{a}_{-}|t|-\widehat{c}_{-}+\frac{1}{8} \ln \left(\frac{4-a_{1}^{2}}{4-a_{2}^{2}}\right)+\ln \right\urcorner_{-}\right)+\mathcal{O}\left(\frac{c}{\left(z_{o}\right) \mathrm{e}^{-4 \alpha|t|}} \beta_{t}\right), \\
& \left(\Delta_{o}\right)_{12}=-\mathrm{ie}^{-\mathrm{i} \psi^{-}(1)}\left(1+\mathcal{O}\left(\frac{\underline{c}\left(z_{o}\right) \mathrm{e}^{-4 \alpha|t|}}{\beta t}\right)\right), \quad\left(\Delta_{o}\right)_{21}=\mathrm{ie}^{\mathrm{i} \psi^{-}(1)}\left(1+\mathcal{O}\left(\frac{\underline{c}\left(z_{o}\right) \mathrm{e}^{-4 \alpha|t|}}{\beta t}\right)\right), \\
& \left.\left(\Delta_{o}\right)_{22}=-\frac{\left.n_{1} \mathrm{ie}^{-\left(\widetilde{a}_{+}|t|-\widehat{c}_{+}\right)}\right\rceil_{+}}{\widetilde{b} \sqrt{|t|}} \cosh \left(\widetilde{a}_{-}|t|-\widehat{c}_{-}+\frac{1}{8} \ln \left(\frac{4-a_{1}^{2}}{4-a_{2}^{2}}\right)+\ln \right\urcorner_{-}\right)+\mathcal{O}\left(\frac{\underline{c}\left(z_{o}\right) \mathrm{e}^{-4 \alpha|t|}}{\beta t}\right),
\end{aligned}
$$

and, for $\varepsilon_{1}=-\varepsilon_{2}$,

$$
\begin{aligned}
& \left.\left(\Delta_{o}\right)_{11}=-\frac{\left.n_{1} \mathrm{ie}^{-\left(\widetilde{a}_{+}|t|-\widehat{c}_{+}\right)}\right\rceil_{+}}{\widetilde{b} \sqrt{|t|}} \sinh \left(\widetilde{a}_{-}|t|-\widehat{c}_{-}+\frac{1}{8} \ln \left(\frac{4-a_{1}^{2}}{4-a_{2}^{2}}\right)+\ln \right\urcorner_{-}\right)+\mathcal{O}\left(\frac{\underline{c}\left(z_{o}\right) \mathrm{e}^{-4 \alpha|t|}}{\beta t}\right), \\
& \left(\Delta_{o}\right)_{12}=-\mathrm{ie}^{-\mathrm{i} \psi^{-}(1)}\left(1+\mathcal{O}\left(\frac{\underline{c}\left(z_{o}\right) \mathrm{e}^{-4 \alpha|t|}}{\beta t}\right)\right), \quad\left(\Delta_{o}\right)_{21}=\mathrm{ie}^{\mathrm{i} \psi^{-}(1)}\left(1+\mathcal{O}\left(\frac{\underline{c}\left(z_{o}\right) \mathrm{e}^{-4 \alpha|t|}}{\beta t}\right)\right), \\
& \left.\left(\Delta_{o}\right)_{22}=\frac{\left.n_{1} \mathrm{ie}^{-\left(\widetilde{a}_{+}|t|-\widehat{c}_{+}\right)}\right\urcorner_{+}}{\widetilde{b} \sqrt{|t|}} \sinh \left(\widetilde{a}_{-}|t|-\widehat{c}_{-}+\frac{1}{8} \ln \left(\frac{4-a_{1}^{2}}{4-a_{2}^{2}}\right)+\ln \right\urcorner_{-}\right)+\mathcal{O}\left(\frac{c}{\left(z_{o}\right) \mathrm{e}^{-4 \alpha|t|}} \beta_{t}\right),
\end{aligned}
$$

where $n_{1}:=\operatorname{sgn}\left(\varepsilon_{1}\right), \widetilde{a}_{ \pm}, \widetilde{b}, \alpha, \beta, \psi^{-}(\cdot), \widehat{c}_{ \pm}$, and $\urcorner_{ \pm}$are defined in Theorem 3.2, Eqs. (38), (40), (42), (43), (46), (47), and (48), and $a_{1}$ and $a_{2}$ are given in Theorem 3.1, Eq. (17).

Lemma A.3. Let $\varepsilon$ be an arbitrarily fixed, sufficiently small positive real number, and, for $\lambda \in \widehat{\mathfrak{J}}:=\left\{\left(s_{1}\right)^{ \pm 1},\left(s_{2}\right)^{ \pm 1}\right\}$, where $s_{1}:=\exp \left(\frac{\mathrm{i} \pi}{4}\right)$ and $s_{2}:=\exp \left(\frac{3 \pi \mathrm{i}}{4}\right)$, set $\mathbb{U}(\lambda ; \varepsilon):=\{z ;|z-\lambda|<\varepsilon\}$. Then, for $r\left(s_{1}\right)=\exp \left(-\frac{\mathrm{i} \varepsilon_{1} \pi}{2}\right)\left|r\left(s_{1}\right)\right|, \varepsilon_{1} \in\{ \pm 1\}, r\left(\overline{s_{2}}\right)=\exp \left(\frac{\mathrm{i} \varepsilon_{2} \pi}{2}\right)\left|r\left(\overline{s_{2}}\right)\right|, \varepsilon_{2} \in\{ \pm 1\}, 0<$ $r\left(s_{2}\right) \overline{r\left(\overline{s_{2}}\right)}<1$, and $\zeta \in \mathbb{C} \backslash \cup_{\lambda \in \widehat{\mathfrak{J}}} \mathbb{U}(\lambda ; \varepsilon)$, as $t \rightarrow+\infty$ and $x \rightarrow-\infty$ such that $z_{0}:=x / t \rightarrow 0^{-}$, 
$m^{c}(\zeta)$ has the following asymptotics,

$$
\begin{aligned}
& m_{11}^{c}(\zeta)=\delta(\zeta)\left(1+\mathcal{O}\left(\left(\frac{\underline{c}}{\left(\zeta-s_{1}\right)}+\frac{\underline{c}}{\left(\zeta-\overline{s_{2}}\right)}\right) \frac{\mathrm{e}^{-4 t}}{t}\right)\right) \\
& m_{12}^{c}(\zeta)=\frac{1}{\delta(\zeta)}\left(\frac{\operatorname{sgn}\left(\varepsilon_{1}\right) \mathrm{e}^{-\left(2 t+\sqrt{2} \int_{-\infty}^{0} \frac{\ln \left(1-|r(\mu)|^{2}\right)}{(\sqrt{2} \mu-1)^{2}+1} \frac{\mathrm{d} \mu}{\pi}\right)} \mathrm{e}^{-\mathrm{i}\left(\frac{\pi}{4}+\sqrt{2} \int_{-\infty}^{0} \frac{(\sqrt{2} \mu-1) \ln \left(1-|r(\mu)|^{2}\right)}{(\sqrt{2} \mu-1)^{2}+1} \frac{\mathrm{d} \mu}{\pi}\right)}}{4\left(\left|r\left(s_{1}\right)\right|\right)^{-1} \sqrt{t}\left(\zeta-\overline{s_{1}}\right)}\right. \\
&+ \frac{\operatorname{sgn}\left(\varepsilon_{2}\right) \mathrm{e}^{-\left(2 t-\sqrt{2} \int_{-\infty}^{0} \frac{\ln \left(1-|r(\mu)|^{2}\right)}{(\sqrt{2} \mu+1)^{2}+1} \frac{\mathrm{d} \mu}{\pi}\right)} \mathrm{e}^{\mathrm{i}\left(\frac{3 \pi}{4}-\sqrt{2} \int_{-\infty}^{0} \frac{(\sqrt{2} \mu+1) \ln \left(1-|r(\mu)|^{2}\right)}{(\sqrt{2} \mu+1)^{2}+1} \frac{\mathrm{d} \mu}{\pi}\right)}}{4\left(\left|r\left(\overline{s_{2}}\right)\right|\right)^{-1}\left(1-r\left(s_{2}\right) \overline{r(\bar{s})}\right) \sqrt{t}\left(\zeta-s_{2}\right)} \\
&+\left.\mathcal{O}\left(\left(\frac{\underline{c}}{\left(\zeta-\overline{s_{1}}\right)}+\frac{\underline{c}}{\left(\zeta-s_{2}\right)}\right) \frac{\mathrm{e}^{-4 t}}{t}\right)\right), \\
& m_{21}^{c}(\zeta)= \delta(\zeta)\left(\frac{\operatorname{sgn}\left(\varepsilon_{1}\right) \mathrm{e}^{-\left(2 t+\sqrt{2} \int_{-\infty}^{0} \frac{\ln \left(1-|r(\mu)|^{2}\right)}{(\sqrt{2} \mu-1)^{2}+1} \frac{\mathrm{d} \mu}{\pi}\right)} \mathrm{e}^{\mathrm{i}\left(\frac{\pi}{4}+\sqrt{2} \int_{-\infty}^{0} \frac{(\sqrt{2} \mu-1) \ln \left(1-|r(\mu)|^{2}\right)}{(\sqrt{2} \mu-1)^{2}+1} \frac{\mathrm{d} \mu}{\pi}\right)}}{4\left(\left|r\left(s_{1}\right)\right|\right)^{-1} \sqrt{t}\left(\zeta-s_{1}\right)}\right. \\
&+ \frac{\operatorname{sgn}\left(\varepsilon_{2}\right) \mathrm{e}^{-\left(2 t-\sqrt{2} \int_{-\infty}^{0} \frac{\ln \left(1-|r(\mu)|^{2}\right.}{(\sqrt{2} \mu+1)^{2}+1} \frac{\mathrm{d} \mu}{\pi}\right)} \mathrm{e}^{\mathrm{i}\left(-\frac{3 \pi}{4}+\sqrt{2} \int_{-\infty}^{0} \frac{(\sqrt{2} \mu+1) \ln \left(1-|r(\mu)|^{2}\right)}{(\sqrt{2} \mu+1)^{2}+1} \frac{\mathrm{d} \mu}{\pi}\right)}}{4\left(\left|r\left(\overline{s_{2}}\right)\right|\right)^{-1}\left(1-r\left(s_{2}\right) \overline{r\left(\overline{s_{2}}\right)}\right) \sqrt{t}\left(\zeta-\overline{s_{2}}\right)} \\
&\left.+\mathcal{O}\left(\left(\frac{\underline{c}}{\left(\zeta-s_{1}\right)}+\frac{\underline{c}}{\left(\zeta-\overline{s_{2}}\right)}\right) \frac{\mathrm{e}^{-4 t}}{t}\right)\right) \\
& m_{22}^{c}(\zeta)=\frac{1}{\delta(\zeta)}\left(1+\mathcal{O}\left(\left(\frac{\underline{c}}{\left(\zeta-\overline{s_{1}}\right)}+\frac{\underline{c}}{\left(\zeta-s_{2}\right)}\right) \frac{\mathrm{e}^{-4 t}}{t}\right)\right),
\end{aligned}
$$

where $\delta(\zeta)$ is defined in Lemma A.1, $\sup _{\zeta \in \mathbb{C} \backslash \cup_{\lambda \in \widehat{\mathfrak{J}}} \mathbb{U}(\lambda ; \varepsilon)}\left|(\zeta-\underline{\widehat{\zeta}})^{-1}\right| \leqslant \mathfrak{M}^{\natural}$, with $\mathfrak{M}^{\natural} \in \mathbb{R}_{+}$(and bounded), $\underline{\widehat{\zeta}} \in \widehat{\mathfrak{J}}, m^{c}(\zeta)=\sigma_{1} \overline{m^{c}(\bar{\zeta})} \sigma_{1}$, and $\left(m^{c}(0) \sigma_{2}\right)^{2}=\mathrm{I}$.

Proposition A.3. Let $s_{1}:=\exp \left(\frac{\mathrm{i} \pi}{4}\right)$ and $s_{2}:=\exp \left(\frac{3 \pi \mathrm{i}}{4}\right)$. For $r\left(s_{1}\right)=\exp \left(-\frac{\mathrm{i} \varepsilon_{1} \pi}{2}\right)\left|r\left(s_{1}\right)\right|, \varepsilon_{1} \in$ $\{ \pm 1\}, r\left(\overline{s_{2}}\right)=\exp \left(\frac{\mathrm{i} \varepsilon_{2} \pi}{2}\right)\left|r\left(\overline{s_{2}}\right)\right|, \varepsilon_{2} \in\{ \pm 1\}, 0<r\left(s_{2}\right) \overline{r\left(\overline{s_{2}}\right)}<1$, and $\varepsilon_{1}=\varepsilon_{2}$, as $t \rightarrow+\infty$ and $x \rightarrow-\infty$ such that $z_{0}:=x / t \rightarrow 0^{-}$,

$$
\begin{gathered}
\left(\Delta_{o}\right)_{11}=-\frac{\operatorname{sgn}\left(\varepsilon_{1}\right) \mathrm{ie}^{-\left(2 t+\widetilde{\mathfrak{c}}_{+}\right)} \mathfrak{b}_{+}}{2 \sqrt{t}} \cosh \left(\widetilde{\mathfrak{c}}_{-}-\ln \mathfrak{b}_{-}\right)+\mathcal{O}\left(\frac{\underline{c} \mathrm{e}^{-4 t}}{t}\right), \\
\left(\Delta_{o}\right)_{12}=-\mathrm{ie}^{-\mathrm{i} \psi^{+}(1)}\left(1+\mathcal{O}\left(\frac{\underline{c} \mathrm{e}^{-4 t}}{t}\right)\right), \quad\left(\Delta_{o}\right)_{21}=\mathrm{ie}^{\mathrm{i} \psi^{+}(1)}\left(1+\mathcal{O}\left(\frac{\underline{c} \mathrm{e}^{-4 t}}{t}\right)\right), \\
\left(\Delta_{o}\right)_{22}=\frac{\operatorname{sgn}\left(\varepsilon_{1}\right) \mathrm{ie}^{-\left(2 t+\widetilde{\mathfrak{c}}_{+}\right)} \mathfrak{b}_{+}}{2 \sqrt{t}} \cosh \left(\widetilde{\mathfrak{c}}_{-}-\ln \mathfrak{b}_{-}\right)+\mathcal{O}\left(\frac{\underline{c} \mathrm{e}^{-4 t}}{t}\right),
\end{gathered}
$$

and, for $\varepsilon_{1}=-\varepsilon_{2}$,

$$
\begin{gathered}
\left(\Delta_{o}\right)_{11}=\frac{\operatorname{sgn}\left(\varepsilon_{1}\right) \mathrm{ie}^{-\left(2 t+\widetilde{\mathfrak{c}}_{+}\right)} \mathfrak{b}_{+}}{2 \sqrt{t}} \sinh \left(\widetilde{\mathfrak{c}}_{-}-\ln \mathfrak{b}_{-}\right)+\mathcal{O}\left(\frac{\underline{c} \mathrm{e}^{-4 t}}{t}\right), \\
\left(\Delta_{o}\right)_{12}=-\mathrm{ie}^{-\mathrm{i} \psi^{+}(1)}\left(1+\mathcal{O}\left(\frac{\underline{c} \mathrm{e}^{-4 t}}{t}\right)\right), \quad\left(\Delta_{o}\right)_{21}=\mathrm{ie}^{\mathrm{i} \psi^{+}(1)}\left(1+\mathcal{O}\left(\frac{\underline{c} \mathrm{e}^{-4 t}}{t}\right)\right), \\
\left(\Delta_{o}\right)_{22}=-\frac{\operatorname{sgn}\left(\varepsilon_{1}\right) \mathrm{ie}^{-\left(2 t+\widetilde{\mathfrak{c}}_{+}\right)} \mathfrak{b}_{+}}{2 \sqrt{t}} \sinh \left(\widetilde{\mathfrak{c}}_{-}-\ln \mathfrak{b}_{-}\right)+\mathcal{O}\left(\frac{\underline{c} \mathrm{e}^{-4 t}}{t}\right),
\end{gathered}
$$

where $\psi^{+}(\cdot)$ is defined in Theorem 3.2, Eq. (35), and $\widetilde{\mathfrak{c}}_{ \pm}$and $\mathfrak{b}_{ \pm}$are defined in Theorem 3.3, Eqs. (73) and (74). 
Lemma A.4. Let $\varepsilon$ be an arbitrarily fixed, sufficiently small positive real number, and, for $\lambda \in \widehat{\mathfrak{J}}:=\left\{\left(s_{1}\right)^{ \pm 1},\left(s_{2}\right)^{ \pm 1}\right\}$, where $s_{1}:=\exp \left(\frac{\mathrm{i} \pi}{4}\right)$ and $s_{2}:=\exp \left(\frac{3 \pi \mathrm{i}}{4}\right)$, set $\mathbb{U}(\lambda ; \varepsilon):=\{z ;|z-\lambda|<\varepsilon\}$. Then, for $r\left(\overline{s_{1}}\right)=\exp \left(\frac{\mathrm{i} \varepsilon_{1} \pi}{2}\right)\left|r\left(\overline{s_{1}}\right)\right|, \varepsilon_{1} \in\{ \pm 1\}, r\left(s_{2}\right)=\exp \left(-\frac{\mathrm{i} \varepsilon_{2} \pi}{2}\right)\left|r\left(s_{2}\right)\right|, \varepsilon_{2} \in\{ \pm 1\}, 0<$ $r\left(s_{1}\right) \overline{r\left(\overline{s_{1}}\right)}<1$, and $\zeta \in \mathbb{C} \backslash \cup_{\lambda \in \widehat{\mathfrak{J}}} \mathbb{U}(\lambda ; \varepsilon)$, as $t \rightarrow-\infty$ and $x \rightarrow+\infty$ such that $z_{0}:=x / t \rightarrow 0^{-}$, $m^{c}(\zeta)$ has the following asymptotics,

$$
\begin{aligned}
& m_{11}^{c}(\zeta)=\widetilde{\delta}(\zeta)\left(1+\mathcal{O}\left(\left(\frac{\underline{c}}{\left(\zeta-\overline{s_{1}}\right)}+\frac{\underline{c}}{\left(\zeta-s_{2}\right)}\right) \frac{\mathrm{e}^{-4|t|}}{t}\right)\right) \\
& m_{12}^{c}(\zeta)=-\frac{1}{\widetilde{\delta}(\zeta)}\left(\frac{\operatorname{sgn}\left(\varepsilon_{1}\right) \mathrm{e}^{-\left(2|t|-\sqrt{2} \int_{0}^{+\infty} \frac{\ln \left(1-|r(\mu)|^{2}\right)}{(\sqrt{2} \mu-1)^{2}+1} \frac{\mathrm{d} \mu}{\pi}\right)} \mathrm{e}^{\mathrm{i}\left(\frac{\pi}{4}-\sqrt{2} \int_{0}^{+\infty} \frac{(\sqrt{2} \mu-1) \ln \left(1-|r(\mu)|^{2}\right)}{(\sqrt{2} \mu-1)^{2}+1} \frac{\mathrm{d} \mu}{\pi}\right)}}{4\left(\left|r\left(\overline{s_{1}}\right)\right|\right)^{-1}\left(1-r\left(s_{1}\right) \overline{r\left(\overline{s_{1}}\right)}\right) \sqrt{|t|}\left(\zeta-s_{1}\right)}\right. \\
& +\frac{\operatorname{sgn}\left(\varepsilon_{2}\right) \mathrm{e}^{-\left(2|t|+\sqrt{2} \int_{0}^{+\infty} \frac{\ln \left(1-|r(\mu)|^{2}\right)}{(\sqrt{2} \mu+1)^{2}+1} \frac{\mathrm{d} \mu}{\pi}\right)} \mathrm{e}^{-\mathrm{i}\left(\frac{3 \pi}{4}+\sqrt{2} \int_{0}^{+\infty} \frac{(\sqrt{2} \mu+1) \ln \left(1-|r(\mu)|^{2}\right)}{(\sqrt{2} \mu+1)^{2}+1} \frac{\mathrm{d} \mu}{\pi}\right)}}{4\left(\left|r\left(s_{2}\right)\right|\right)^{-1} \sqrt{|t|}\left(\zeta-\overline{s_{2}}\right)} \\
& \left.+\mathcal{O}\left(\left(\frac{\underline{c}}{\left(\zeta-s_{1}\right)}+\frac{\underline{c}}{\left(\zeta-\overline{s_{2}}\right)}\right) \frac{\mathrm{e}^{-4|t|}}{t}\right)\right) \\
& m_{21}^{c}(\zeta)=-\widetilde{\delta}(\zeta)\left(\frac{\operatorname{sgn}\left(\varepsilon_{1}\right) \mathrm{e}^{-\left(2|t|-\sqrt{2} \int_{0}^{+\infty} \frac{\ln \left(1-|r(\mu)|^{2}\right)}{(\sqrt{2} \mu-1)^{2}+1} \frac{\mathrm{d} \mu}{\pi}\right)} \mathrm{e}^{-\mathrm{i}\left(\frac{\pi}{4}-\sqrt{2} \int_{0}^{+\infty} \frac{(\sqrt{2} \mu-1) \ln \left(1-|r(\mu)|^{2}\right)}{(\sqrt{2} \mu-1)^{2}+1} \frac{\mathrm{d} \mu}{\pi}\right)}}{4\left(\left|r\left(\overline{s_{1}}\right)\right|\right)^{-1}\left(1-r\left(s_{1}\right) \overline{r\left(\overline{s_{1}}\right)}\right) \sqrt{|t|}\left(\zeta-\overline{s_{1}}\right)}\right. \\
& +\frac{\operatorname{sgn}\left(\varepsilon_{2}\right) \mathrm{e}^{-\left(2|t|+\sqrt{2} \int_{0}^{+\infty} \frac{\ln \left(1-|r(\mu)|^{2}\right.}{(\sqrt{2} \mu+1)^{2}+1} \frac{\mathrm{d} \mu}{\pi}\right)} \mathrm{e}^{\mathrm{i}\left(\frac{3 \pi}{4}+\sqrt{2} \int_{0}^{+\infty} \frac{(\sqrt{2} \mu+1) \ln \left(1-|r(\mu)|^{2}\right)}{(\sqrt{2} \mu+1)^{2}+1} \frac{\mathrm{d} \mu}{\pi}\right)}}{4\left(\left|r\left(s_{2}\right)\right|\right)^{-1} \sqrt{|t|}\left(\zeta-s_{2}\right)} \\
& \left.+\mathcal{O}\left(\left(\frac{\underline{c}}{\left(\zeta-\overline{s_{1}}\right)}+\frac{\underline{c}}{\left(\zeta-s_{2}\right)}\right) \frac{\mathrm{e}^{-4|t|}}{t}\right)\right) \\
& m_{22}^{c}(\zeta)=\frac{1}{\widetilde{\delta}(\zeta)}\left(1+\mathcal{O}\left(\left(\frac{\underline{c}}{\left(\zeta-s_{1}\right)}+\frac{\underline{c}}{\left(\zeta-\overline{s_{2}}\right)}\right) \frac{\mathrm{e}^{-4|t|}}{t}\right)\right) \text {, }
\end{aligned}
$$

where $\widetilde{\delta}(\zeta)$ is defined in Lemma A.2, $\sup _{\zeta \in \mathbb{C} \backslash \cup_{\lambda \in \widehat{\mathfrak{J}}} \mathbb{U}(\lambda ; \varepsilon)}\left|(\zeta-\underline{\zeta})^{-1}\right| \leqslant \mathfrak{M}^{\sharp}$, with $\mathfrak{M}^{\sharp} \in \mathbb{R}_{+}$(and bounded), $\widehat{\widehat{\zeta}} \in \widehat{\mathfrak{J}}, m^{c}(\zeta)=\sigma_{1} \overline{m^{c}(\bar{\zeta})} \sigma_{1}$, and $\left(m^{c}(0) \sigma_{2}\right)^{2}=\mathrm{I}$.

Proposition A.4. Let $s_{1}:=\exp \left(\frac{\mathrm{i} \pi}{4}\right)$ and $s_{2}:=\exp \left(\frac{3 \pi \mathrm{i}}{4}\right)$. For $r\left(\overline{s_{1}}\right)=\exp \left(\frac{\mathrm{i} \varepsilon_{1} \pi}{2}\right)\left|r\left(\overline{s_{1}}\right)\right|, \varepsilon_{1} \in$ $\{ \pm 1\}, r\left(s_{2}\right)=\exp \left(-\frac{\mathrm{i} \varepsilon_{2} \pi}{2}\right)\left|r\left(s_{2}\right)\right|, \varepsilon_{2} \in\{ \pm 1\}, 0<r\left(s_{1}\right) \overline{r\left(\overline{s_{1}}\right)}<1$, and $\varepsilon_{1}=\varepsilon_{2}$, as $t \rightarrow-\infty$ and $x \rightarrow+\infty$ such that $z_{0}:=x / t \rightarrow 0^{-}$,

$$
\begin{gathered}
\left(\Delta_{o}\right)_{11}=\frac{\operatorname{sgn}\left(\varepsilon_{1}\right) \mathrm{ie}^{-\left(2|t|-\widehat{\mathfrak{c}}_{+}\right)} \mathfrak{d}_{+}}{2 \sqrt{|t|}} \cosh \left(\widehat{\mathfrak{c}}_{-}-\ln \mathfrak{d}_{-}\right)+\mathcal{O}\left(\frac{\underline{c} \mathrm{e}^{-4|t|}}{t}\right) \\
\left(\Delta_{o}\right)_{12}=-\mathrm{ie}^{-\mathrm{i} \psi^{-}(1)}\left(1+\mathcal{O}\left(\frac{\underline{c} \mathrm{e}^{-4|t|}}{t}\right)\right), \quad\left(\Delta_{o}\right)_{21}=\mathrm{ie}^{\mathrm{i} \psi^{-}(1)}\left(1+\mathcal{O}\left(\frac{\underline{c} \mathrm{e}^{-4|t|}}{t}\right)\right) \\
\left(\Delta_{o}\right)_{22}=-\frac{\operatorname{sgn}\left(\varepsilon_{1}\right) \mathrm{ie}^{-\left(2|t|-\widehat{\mathfrak{c}}_{+}\right)} \mathfrak{d}_{+}}{2 \sqrt{|t|}} \cosh \left(\widehat{\mathfrak{c}}_{-}-\ln \mathfrak{d}_{-}\right)+\mathcal{O}\left(\frac{\underline{c} \mathrm{e}^{-4|t|}}{t}\right)
\end{gathered}
$$


and, for $\varepsilon_{1}=-\varepsilon_{2}$,

$$
\begin{gathered}
\left(\Delta_{o}\right)_{11}=\frac{\operatorname{sgn}\left(\varepsilon_{1}\right) \mathrm{ie}^{-\left(2|t|-\widehat{\mathfrak{c}}_{+}\right)} \mathfrak{d}_{+}}{2 \sqrt{|t|}} \sinh \left(\widehat{\mathfrak{c}}_{-}-\ln \mathfrak{d}_{-}\right)+\mathcal{O}\left(\frac{\underline{c} \mathrm{e}^{-4|t|}}{t}\right), \\
\left(\Delta_{o}\right)_{12}=-\mathrm{ie}^{-\mathrm{i} \psi^{-}(1)}\left(1+\mathcal{O}\left(\frac{\underline{c} \mathrm{e}^{-4|t|}}{t}\right)\right), \quad\left(\Delta_{o}\right)_{21}=\mathrm{ie}^{\mathrm{i} \psi^{-(1)}}\left(1+\mathcal{O}\left(\frac{\underline{c} \mathrm{e}^{-4|t|}}{t}\right)\right), \\
\left(\Delta_{o}\right)_{22}=-\frac{\operatorname{sgn}\left(\varepsilon_{1}\right) \mathrm{ie}^{-\left(2|t|-\widehat{\mathfrak{c}}_{+}\right)} \mathfrak{d}_{+}}{2 \sqrt{|t|}} \sinh \left(\widehat{\mathfrak{c}}_{-}-\ln \mathfrak{d}_{-}\right)+\mathcal{O}\left(\frac{\underline{c} \mathrm{e}^{-4|t|}}{t}\right),
\end{gathered}
$$

where $\psi^{-}(\cdot)$ is defined in Theorem 3.2, Eq. (46), and $\widehat{\mathfrak{c}}_{ \pm}$and $\mathfrak{d}_{ \pm}$are defined in Theorem 3.3, Eqs. (77) and (78). 


\section{References}

[1] G. P. Agrawal, 2nd edn., Nonlinear Fiber Optics, Academic Press, San Diego, 1995.

[2] A. M. Weiner, "Dark optical solitons", pp. 378-408, in J. R. Taylor, ed., Optical Solitons - Theory and Experiment, Cambridge Studies in Modern Optics, Vol. 10, Cambridge University Press, Cambridge, 1992.

[3] Y. Kodama, "The Whitham Equations for Optical Communications: Mathematical Theory of NRZ", SIAM J. Appl. Math., Vol. 59, No. 6, pp. 2162-2192, 1999.

[4] D. Sh. Lundina and V. A. Marchenko, "Compactness of the Set of Multisoliton Solutions of the Nonlinear Schrödinger Equation", Russian Acad. Sci. Sb. Math., Vol. 75, No. 2, pp. 429-443, 1993.

[5] P. D. Miller, "Zero-crosstalk junctions made from dark solitons", Phys. Rev. E, Vol. 53, No. 4, pp. 4137-4142, 1996. P. D. Miller and N. N. Akhmediev, "Transfer matrices for multiport devices made from solitons", Phys. Rev. E, Vol. 53, No. 4, pp. 4098-4106, 1996.

[6] S. P. Novikov, S. V. Manakov, L. P. Pitaevskii, and V. E. Zakharov, Theory of Solitons: The Inverse Scattering Method, Plenum, New York, 1984.

[7] M. J. Ablowitz and P. A. Clarkson, Solitons, Nonlinear Evolution Equations and Inverse Scattering, LMS 149, Cambridge University Press, Cambridge, 1991.

[8] R. Beals, P. Deift, and X. Zhou, "The Inverse Scattering Transform on the Line", pp. 732, in A. S. Fokas and V. E. Zakharov, eds., Important Developments in Soliton Theory, Springer Series in Nonlinear Dynamics, Springer-Verlag, New York, 1993.

[9] L. D. Faddeev and L. A. Takhtajan, Hamiltonian Methods in the Theory of Solitons, Springer-Verlag, Berlin, 1987.

[10] V. E. Zakharov and A. B. Shabat, "Interaction between solitons in a stable medium", Sov. Phys. JETP, Vol. 37, No. 5, pp. 823-828, 1973.

[11] T. Kawata and H. Inoue, "Inverse Scattering Method for the Nonlinear Evolution Equations under Nonvanishing Conditions", J. Phys. Soc. Japan, Vol. 44, No. 5, pp. 1722 $1729,1978$.

[12] N. Asano and Y. Kato, "Non-self-adjoint Zakharov-Shabat operator with a potential of the finite asymptotic values. II. Inverse problem", J. Math. Phys., Vol. 25, No. 3, pp. 570-588, 1984. N. Asano and Y. Kato, "Non-self-adjoint Zakharov-Shabat operator with a potential of the finite asymptotic values. I. Direct spectral and scattering problems", J. Math. Phys., Vol. 22, No. 12, pp. 2780-2793, 1981.

[13] A. Boutet de Monvel and V. Marchenko, "The Cauchy problem for nonlinear Schrödinger equation with bounded initial data", Mat. Fiz. Anal. Geom., Vol. 4, No. $1 / 2$, pp. 3-45, 2000.

[14] M. Boiti and F. Pempinelli, "The Spectral Transform for the NLS Equation with LeftRight Asymmetric Boundary Conditions", Il Nuovo Cimento, Vol. 69B, No. 2, pp. 213$227,1982$.

[15] T. Kawata, J. Sakai, and N. Kobayashi, "Inverse Method for the Mixed Nonlinear Schrödinger Equation and Soliton Solutions", J. Phys. Soc. Japan, Vol. 48, No. 4, pp. 1371-1379, 1980. T. Kawata and H. Inoue, "Exact Solutions of the Derivative Nonlinear Schrödinger Equation under the Nonvanishing Conditions", J. Phys. Soc. Japan, Vol. 44, No. 6, pp. 1968-1976, 1978.

[16] A. Cohen and T. Kappeler, "Scattering and Inverse Scattering for Steplike Potentials in the Schrödinger Equation", Indiana Univ. Math. J., Vol. 34, No. 1, pp. 127-180, 1985. 
[17] V. A. Marchenko, "The Cauchy Problem for the KdV Equation with Non-Decreasing Initial Data", pp. 273-318, in What is Integrability?, V. E. Zakharov, ed., Springer Series in Nonlinear Dynamics, Springer-Verlag, Berlin, 1991.

[18] A. Boutet de Monvel, E. Ya. Khruslov, and V. P. Kotlyarov, "The Cauchy problem for the sinh-Gordon equation and regular solitons", Inverse Problems, Vol. 14, No. 6, pp. 1403-1427, 1998. A. B. Borisov and V. V. Kiseliev, "Inverse problem for an elliptic sine-Gordon equation with an asymptotic behaviour of the cnoidal-wave type", Inverse Problems, Vol. 5, No. 6, pp. 959-982, 1989.

[19] A. R. Its and A. F. Ustinov, "The time asymptotics of the solution of the Cauchy problem for the nonlinear Schrödinger equation with finite density boundary conditions", Dokl. Akad. Nauk SSSR, Vol. 291, No. 1, pp. 91-95, 1986 (in Russian).

[20] A. R. Its and A. F. Ustinov, "Formulation of the Scattering Theory for the Nonlinear Schrödinger Equation with Boundary Conditions of the Finite Density Type in a Soliton-Free Sector", J. Sov. Math., Vol. 54, No. 3, pp. 900-905, 1991.

[21] V. E. Zakharov and A. B. Shabat, "Integration of the non-linear equations of mathematical physics by the method of the inverse scattering transform. II", Funct. Anal. Appl., Vol. 13, No. 3, pp. 166-173, 1980.

[22] P. A. Deift, S. Kamvissis, T. Kriecherbauer, and X. Zhou, "The Toda Rarefaction Problem", Comm. Pure Appl. Math., Vol. 49, No. 1, pp. 35-83, 1996.

[23] K. Clancey and I. Gohberg, Factorization of Matrix Functions and Singular Integral Operators, Operator Theory: Advances and Applications, Vol. 3, Birkhäuser, Basel, 1981.

[24] R. Beals and R. R. Coifman, "Scattering and Inverse Scattering for First Order Systems", Comm. Pure Appl. Math., Vol. 37, No. 1, pp. 39-90, 1984.

[25] P. Deift, Orthogonal Polynomials and Random Matrices: A Riemann-Hilbert Approach, Courant Lecture Notes in Mathematics, Vol. 3, CIMS, New York, 1999.

[26] A. S. Fokas, "On the integrability of linear and nonlinear partial differential equations", J. Math. Phys., Vol. 41, No. 6, pp. 4188-4237, 2000.

[27] P. Deift and X. Zhou, "A Steepest descent method for oscillatory Riemann-Hilbert problems. Asymptotics for the MKdV equation", Ann. of Math., Vol. 137, No. 2, pp. 295368, 1993.

[28] X. Zhou, "Direct and Inverse Scattering Transforms with Arbitrary Spectral Singularities", Comm. Pure Appl. Math., Vol. 42, No. 7, pp. 895-938, 1989.

[29] X. Zhou, "Inverse Scattering Transform for Systems with Rational Spectral Dependence", J. Differential Equations, Vol. 115, No. 2, pp. 277-303, 1995.

[30] N.-N. Huang and Z.-Y. Chen, "Zakharov-Shabat Equations for Dark Solitons to the NLS Equation", Commun. Theor. Phys., Vol. 20, No. 2, pp. 187-194, 1993.

[31] X. Zhou, "The Riemann-Hilbert Problem and Inverse Scattering", SIAM J. Math. Anal., Vol. 20, No. 4, pp. 966-986, 1989.

[32] X. Zhou, "Strong Regularizing Effect of Integrable Systems", Comm. PDE, Vol. 22, Nos. 3 \& 4, pp. 503-526, 1997.

[33] X. Zhou, " $L^{2}$-Sobolev Space Bijectivity of the Scattering and Inverse Scattering Transforms", Comm. Pure Appl. Math., Vol. 51, No. 7, pp. 697-731, 1998. 
[34] P. Deift, T. Kriecherbauer, K. T.-R. McLaughlin, S. Venakides, and X. Zhou, "Strong Asymptotics of Orthogonal Polynomials with Respect to Exponential Weights", Comm. Pure Appl. Math., Vol. 52, No. 12, pp. 1491-1552, 1999.

[35] H. Flaschka and A. C. Newell, "Monodromy- and Spectrum-Preserving Deformations I", Comm. Math. Phys., Vol. 76, No. 1, pp. 65-116, 1980.

[36] A. S. Fokas and M. J. Ablowitz, "On the Initial Value Problem of the Second Painlevé Transcendent", Comm. Math. Phys., Vol. 91, No. 3, pp. 381-403, 1983.

[37] A. R. Its and V. Yu. Novokshenov, The Isomonodromy Deformation Method in the Theory of Painlevé Equations, LNM 1191, Springer-Verlag, Berlin, 1986.

[38] A. R. Its and A. A. Kapaev, "The Method of Isomonodromy Deformations and Connection Formulas for the Second Painlevé Transcendent", Math. USSR Izvestiya, Vol. 31, No. 1, pp. 193-207, 1988.

[39] P. Deift and X. Zhou, "Asymptotics for the Painlevé II Equation", Comm. Pure Appl. Math., Vol. 48, No. 3, pp. 277-337, 1995.

[40] I. S. Gradshteyn and I. M. Ryzhik, Tables of Integrals, Series, and Products, 5th edn., A. Jeffrey, ed., Academic Press, San Diego, 1994.

[41] J. Baik, P. Deift, and K. Johansson, "On the Distribution of the Length of the Longest Increasing Subsequence of Random Permutations", J. Amer. Math. Soc., Vol. 12, No. 4, pp. 1119-1178, 1999.

[42] P. Deift, T. Kriecherbauer, K. T.-R. McLaughlin, S. Venakides, and X. Zhou, "Uniform Asymptotics for Polynomials Orthogonal with Respect to Varying Exponential Weights and Applications to Universality Questions in Random Matrix Theory", Comm. Pure Appl. Math., Vol. 52, No. 11, pp. 1335-1425, 1999.

[43] P. Deift and X. Zhou, "Perturbation theory for infinite dimensional integrable systems on the line. A case study", Preprint, 2000.

[44] S. Kamvissis, K. T.-R. McLaughlin, and P. D. Miller, "Semiclassical Soliton Ensembles for the Focusing Nonlinear Schrödinger Equation", arXiv:nlin.SI/0012034.

[45] F. D. Gakhov, Boundary Value Problems, Dover, New York, 1990.

[46] P.-J. Cheng, S. Venakides, and X. Zhou, "Long-Time Asymptotics for the Pure Radiation Solution of the Sine-Gordon Equation", Comm. PDE, Vol. 24, Nos. 7 \& 8, pp. 1195-1262, 1999.

[47] A. R. Its, "Asymptotics of Solutions of the Nonlinear Schrödinger Equation and Isomonodromic Deformations of Systems of Linear Differential Equations", Soviet Math. Dokl., Vol. 24, No. 3, pp. 452-456, 1982.

[48] P. Deift and X. Zhou, "Long-time Asymptotics for Integrable Systems. Higher Order Theory", Comm. Math. Phys., Vol. 165, No. 1, pp. 175-191, 1994.

[49] A. H. Vartanian, "Higher Order Asymptotics of the Modified Non-Linear Schrödinger Equation", Comm. PDE, Vol. 25, Nos. 5 \& 6, pp. 1043-1098, 2000.

[50] A. V. Kitaev and A. H. Vartanian, "Asymptotics of Solutions to the Modified Nonlinear Schrödinger Equation: Solitons on a Nonvanishing Continuous Background", SIAM J. Math. Anal., Vol. 30, No. 4, pp. 787-832, 1999. 